Final Technical Report

\title{
PREFERRED WATERFLOOD MANAGEMENT PRACTICES FOR THE SPRABERRY TREND AREA
}

DOE Contract No.: DE-FC26-01BC15274

Harold Vance Department of Petroleum Engineering

Texas A\& M University

3116 TAMU

College Station, TX 77843-3116

(979) 845-2241

Contract Date:

Anticipated Completion Date:

Program Manager:

Principal Investigator:

Contracting Officer's Representative:

Report Period:
September 1, 2001

Augustus 31, 2004

C. M. Sizemore

Pioneer Natural Resources

David S. Schechter

Harold Vance Department of Petroleum Engineering

Dan Ferguson

National Petroleum Technology Office

Sept. 1, 2001- Aug. 31, 2004

US/DOE Patent Clearance is not required prior to the publication of this document. 


\section{DISCLAIMER}

This report was prepared as an account of work sponsored by an agency of the United States Government. Neither the United States Government nor any agency thereof, nor any their employees, makes any warranty, express or implied, or assumes any legal liability or responsibility for the accuracy, completeness, or usefulness of any information, apparatus, product, or process disclosed, or represents that its use would not infringe privately owned rights. Reference herein to any specific commercial product, process, or service by trade name, trademark, manufacturer, or otherwise does not necessarily constitute or imply its endorsement, recommendation, or favoring by the United States Government or any agency thereof. The views and opinions of authors expressed herein do not necessarily state or reflect those of the United States Government or any agency thereof. 


\begin{abstract}
The naturally fractured Spraberry Trend Area is one of the largest reservoirs in the domestic U.S. and is the largest reservoir in area extent in the world. Production from Spraberry sands is found over a 2,500 sq. mile area and Spraberry reservoirs can be found in an eight county area in west Texas. Over 150 operators produce 65,000 barrels of oil per day (bopd) from the Spraberry Trend Area from more than 9,000 production wells. Recovery is poor, on the order of $7-10 \%$ due to the profoundly complicated nature of the reservoir, yet billions of barrels of hydrocarbons remain. We estimate over $15 \%$ of remaining reserves in domestic Class III reservoirs are in Spraberry Trend Area reservoirs. This tremendous domestic asset is a prime example of an endangered hydrocarbon resource in need of immediate technological advancements before thousands of wells are permanently abandoned. This report describes the final work of the project, "Preferred Waterflood Management Practices for the Spraberry Trend Area." The objective of this project is to significantly increase field-wide production in the Spraberry Trend in a short time frame through the application of preferred practices for managing and optimizing water injection. Our goal is to dispel negative attitudes and lack of confidence in water injection and to document the methodology and results for public dissemination to motivate waterflood expansion in the Spraberry Trend. This objective has been accomplished through research in three areas: 1) detail historical review and extensive reservoir characterization, 2) production data management, and 3) field demonstration. This provides results of the final year of the three-year project for each of the three areas.

In the first area, we reviewed several waterflood pilots which can be used to justify the reason of poor performance of Lower Spraberry and to propose a method to determine the location of the injection and production wells in order to increase the oil recovery from Spraberry area. We found that the reasons of poor waterflood performance in lower Spraberry are due to low water intake capacity indicating probability of lesser extend of fracture system, low volume of water injection (bbl/per acre) and High oil shrinkage factor. The result from four waterflood pilots indicates that the on-trend wells respond favorably compared to the off-trend wells. Thus, placing production and injection wells along the same fracture orientation may increase oil recovery in the Spraberry area.

We proposed the location of new injection wells in the GSU pilot that are parallel along major fracture trend to have fast responses and viable waterfloods. We recommended that the injection wells GSU-408A and GSU-10 should be relocated because they are located on old injector path.

We reviewed wellbore status and forecasted the incremental oil recovery based on waterflooding performance in other waterflood pilot area in order to demonstrate the benefit of waterflooding in Germania unit area. Based on the high WOR map, production wells GSU-1, GSU-315A and GSU-5 indicates that these wells may have casing leaks. We also demonstrated that waterflooding in Germania Unit would be economical and competing with other secondary projects.
\end{abstract}

The need for characterization of the Germania unit has emerged as a first step in the review, understanding and enhancement of the production practices applicable within the unit and the trend area in general. Petrophysical characterization of the Germania 
Spraberry units requires a unique approach for a number of reasons - limited core data, lack of modern log data and absence of directed studies within the unit. In the absence of the afore mentioned resources, an approach that will rely heavily on previous petrophysical work carried out in the neighboring ET O’Daniel unit (6.2 miles away), and normalization of the old log data prior to conventional interpretation techniques will be used. A log-based rock model has been able to guide successfully the prediction of pay and non-pay intervals within the ET O'Daniel unit, and will be useful if found applicable within the Germania unit. A novel multiple regression technique utilizing non-parametric transformations to achieve better correlations in predicting a dependent variable (permeability) from multiple independent variables (rock type, shale volume and porosity) will also be investigated in this study. A log data base includes digitized formats of gamma ray, cased hole neutron, limited resistivity and neutron/density/sonic porosity logs over a considerable wide area.

Consequently, this project provides a significant reservoir characterization and evaluates the performance of previous and current waterflooding activities. The latter will provide facts, information and knowledge to obtain the maximum economic recovery from this reservoir. Thus, attempts are made to describe the reservoir, understand the performance of the reservoir under the current waterflooding project, and controlled surveillance will be carried out to improve field performance. The results indicate that the production performance in Germania Spraberry Unit is clearly dominated by the presence of natural fractures and the wettability of the rock. The average Voidage Replacement observed from 1969 to 1975 indicates that the water injection rate was too high in proportion to the fluid production rate. This may explain the high water cut and rapid breakthrough observed in some wells and is perhaps one of the most responsible factors for the poor performance of unit. The log-log plot of WOR and its derivative provide more insight and information for well performance evaluation and surveillance system. Using this surveillance technique, coning and channeling can be discerned and normal displacement, and breakthrough behavior can be differentiated. Results obtained with this type pf plots, indicate that wells GSU and GSU-5 may be experiencing casing leak. Based on declinecurve analysis for active wells, a bubble map showing the areas with the most opportunities (most remaining reserves) was displayed. The map showed that the areas with the most remaining reserves are located towards the north-east part of the unit. Heterogeneity Indexing is a useful surveillance tool for ranking and identifying specific wells with poor or superior performance in Germania Unit. It can also be used as a quick screening tool to identify opportunities in the area. The results of the application of this screening technique suggest that wells GSU-2, GSU-127, GSU-114, GSU-128, GSU-116, GSU-123, GSU-412, GSU-328, and GSU-5 are good candidates for the application of water control techniques. Wells drilled in the first campaign ( from 1957 to 1964) have shown the highest value of average initial rate ( 48 BOPD) and the performance shown by wells drilled during the third campaign (from 1990 to 1996) demonstrate the importance and impact of infill drilling in this unit. Areas having the best oil-producing wells ("sweet spots") and their adjacent water injection wells formed trends parallel to the main set of natural fractures ( $\mathrm{N} 56^{\circ} \mathrm{E}$ ) and are also correlative with axes of maximum net pay. 
In the second area, we developed a production database management to control a vast data from the pilot and to perform precise reservoir engineering techniques such as decline curve analysis, gas and oil material balances, bubble map plot and PVT analysis. We believe by proper data acquisition and precise reservoir engineering techniques, any lack of confidence in waterflooding can be overcome. The manual for this software is listed in the Appendix-A.

Lastly, we reported a final field demonstration status on GSU waterflood pilot. 


\section{TABLE OF CONTENTS}

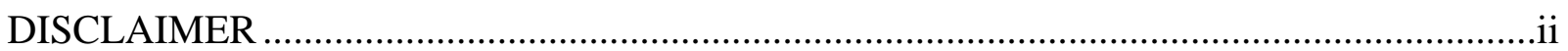

ABSTRACT ................................................................................................................

TABLE OF CONTENTS...................................................................................................

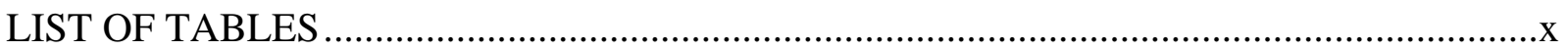

LIST OF FIGURES …………………………………..............................................

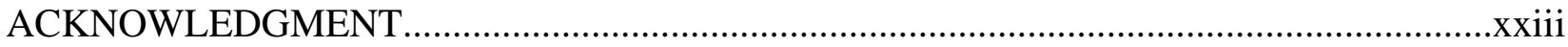

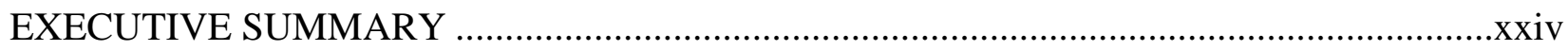

1.1. Review of Upper and Lower Pilots in the Spraberry Area ....................................................1

1.2. Germania Unit Rate Forecasting Based on Other Waterflood Pilots in the Spraberry

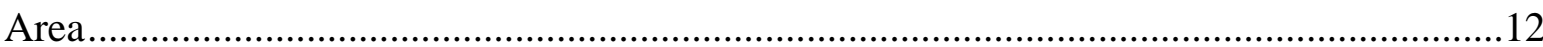

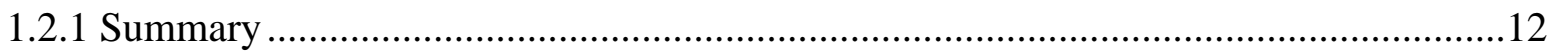

1.2.2 Waterflood History of Germania Unit ..........................................................................12

1.2.3 Waterflooding performances in the previous Germania unit and other units...............15

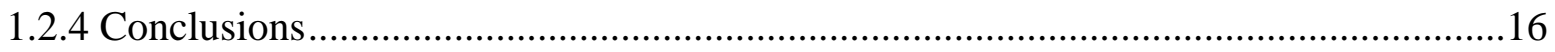

1.3 Characterization of the Germania Sparberry Unit from Analog Studies and Cased-

Hole Neutron Log Data..................................................................................................41

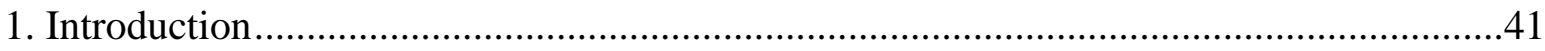

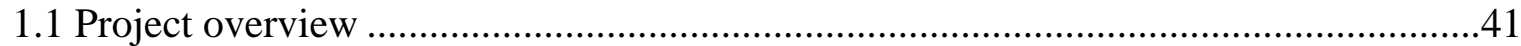

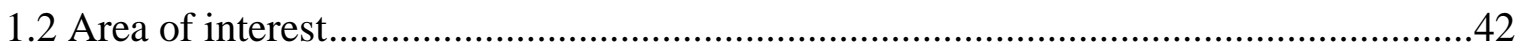

1.3 Rock-log model....................................................................................................43

1.4 Lithofacies based model ..........................................................................................4

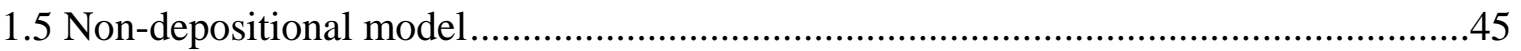

1.6 Permeability estimation techniques ............................................................................4

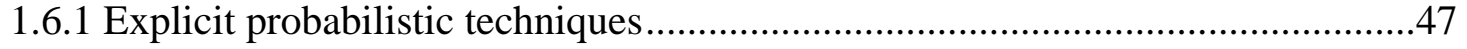

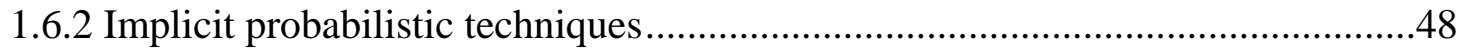

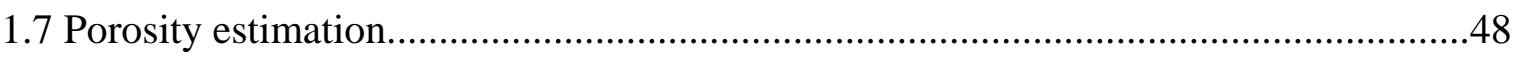

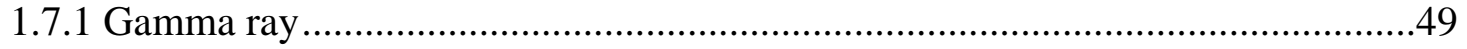

1.7.2 Clay content ...............................................................................................49

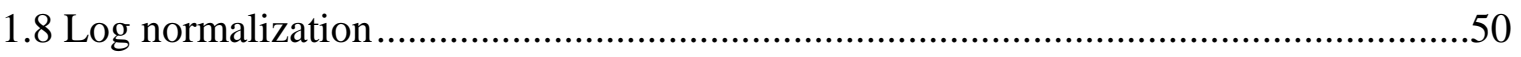

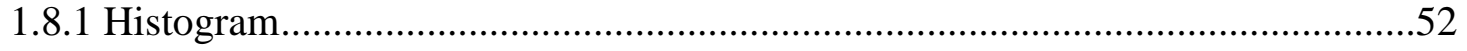




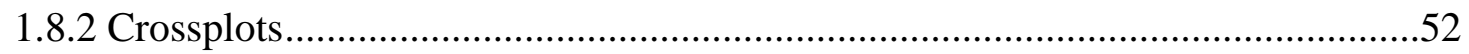

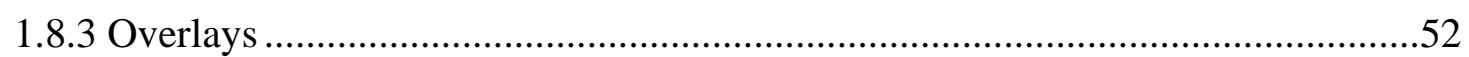

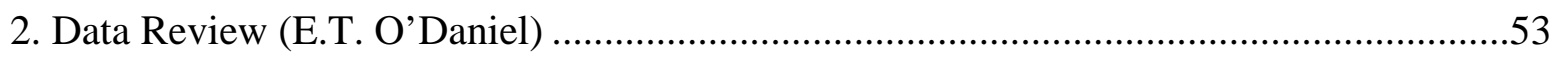

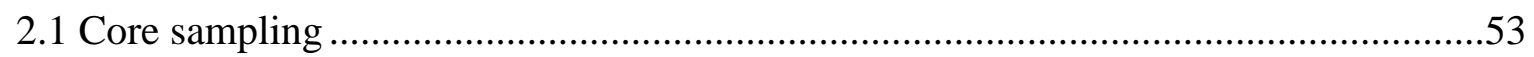

2.2 Depth matched core-log playback ...............................................................................54

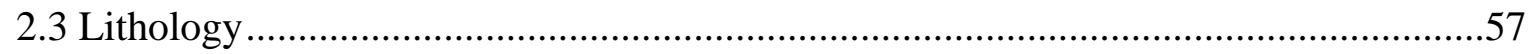

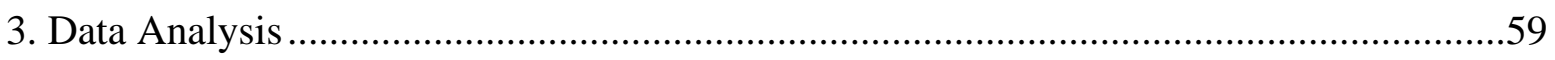

3.1 Log conversions and normalization .......................................................................59

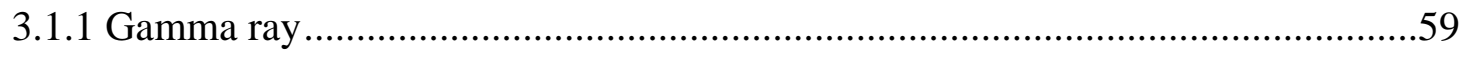

3.1.1.1 Gamma ray maps ..............................................................................59

3.1.1.2 Gamma ray normalization........................................................................62

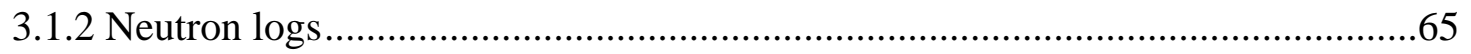

3.1.2.1 Standardization of neutron log units ........................................................65

3.1.2.2 Conversion from neutron units to linear porosity units .............................66

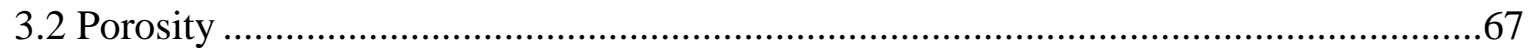

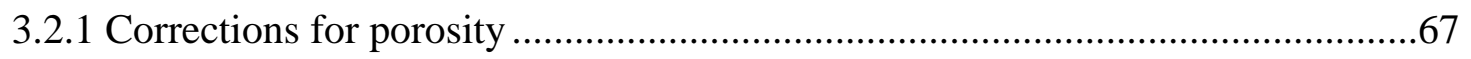

3.3 ET O’Daniel log-core model ...............................................................................69

3.3.1 Log porosity - core porosity x-plots ................................................................69

3.4 Variables influencing permeability .........................................................................72

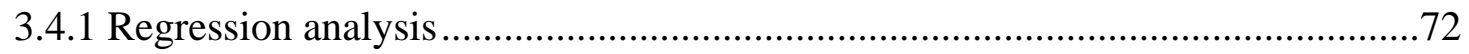

3.4.2 Shale effects on porosity and permeability …………..........................................73

3.4.3 Data conditioning ('ACE') ...................................................................................

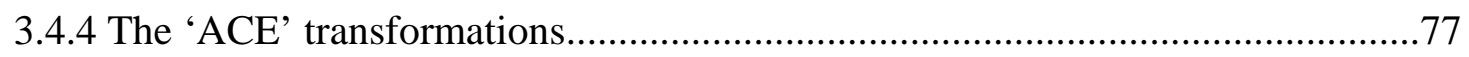

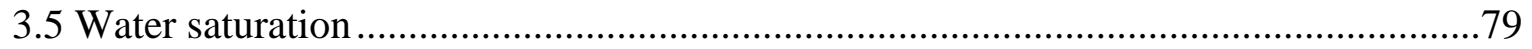

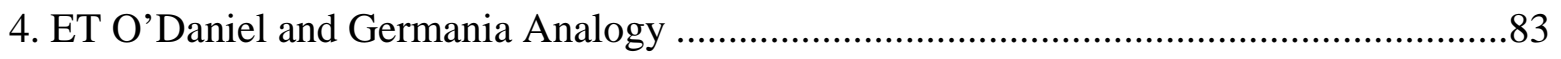

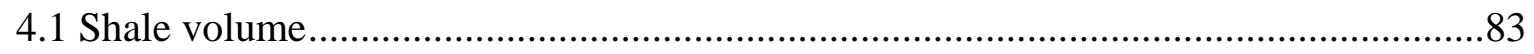

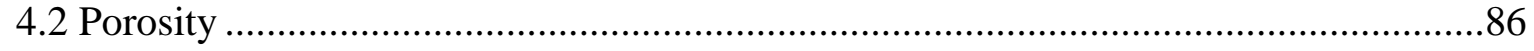

4.3 Kolmogorov - Smirnov test for porosity and shale volume ...........................................88

4.4 Litho- stratigraphic section .....................................................................................90

5. Model Application in Germania Unit ..............................................................................93

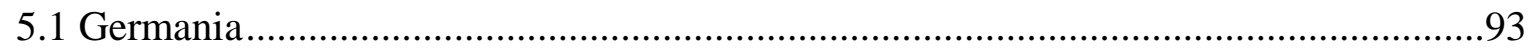




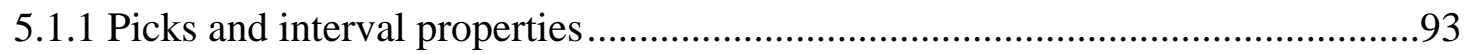

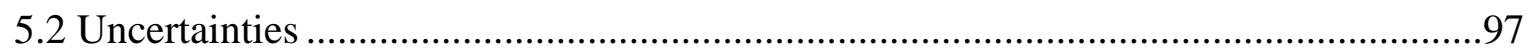

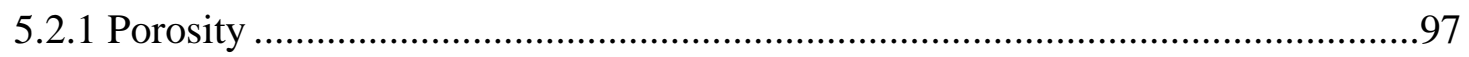

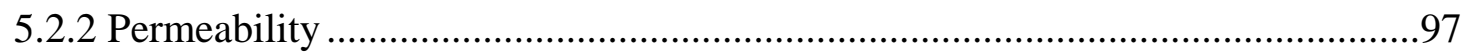

5.2.3 Water saturation ............................................................................................

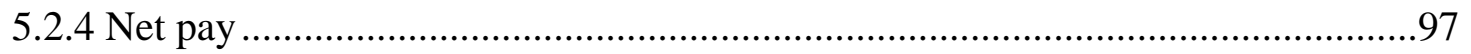

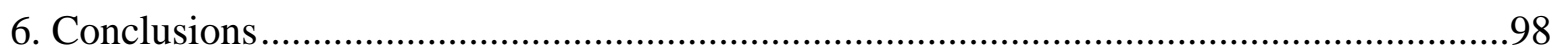

APPENDIX A BACKGROUND GEOLOGY AND STRATIGRAPHY ..........................105

APPENDIX B MAPS FOR ET O’DANIEL AND GERMANIA …………………….......118

1.4. Evaluation of Waterflooding Performance in Germania Spraberry Unit ..............................129

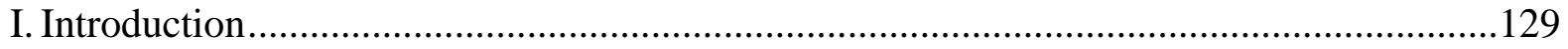

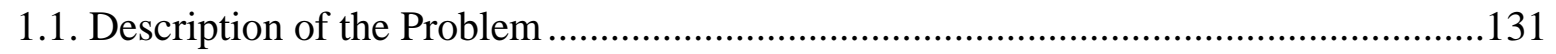

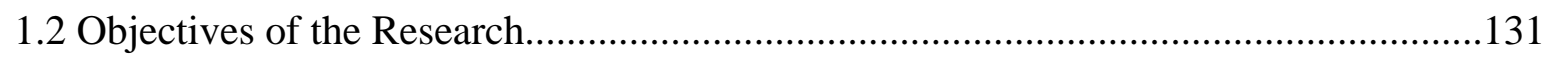

1.3 Research Methodology …………………………..............................................132

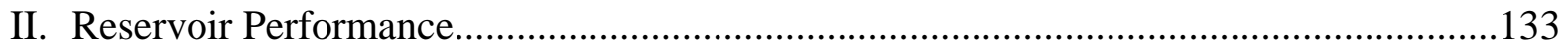

2.1 Primary Performance in Germania Spraberry Unit ......................................................133

2.2 Secondary Performance in Germania Spraberry Unit (Waterflooding). .......................134

III. Reservoir Monitoring and Surveillance System ..............................................................137

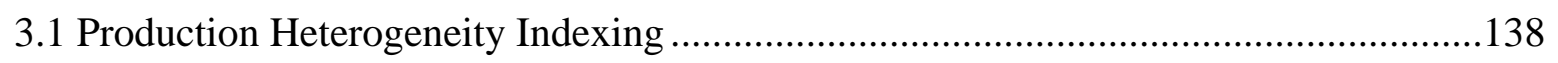

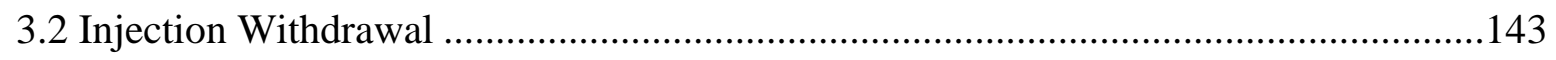

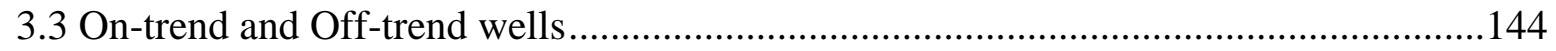

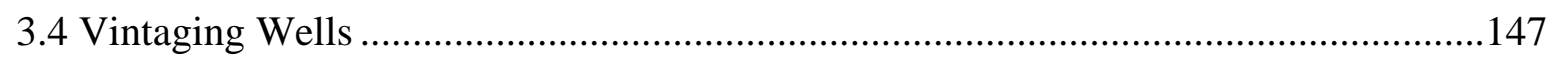

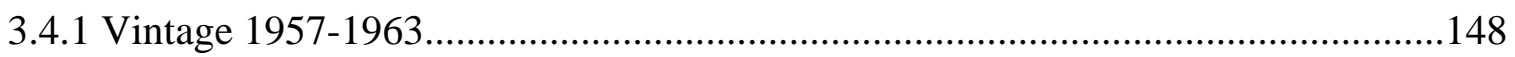

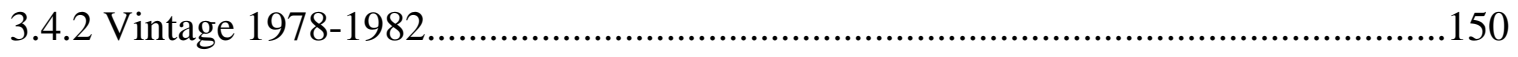

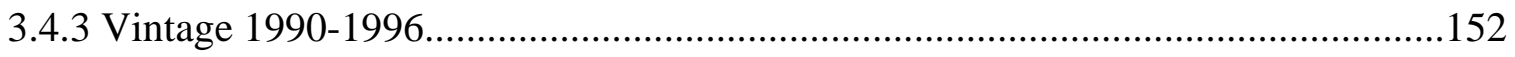

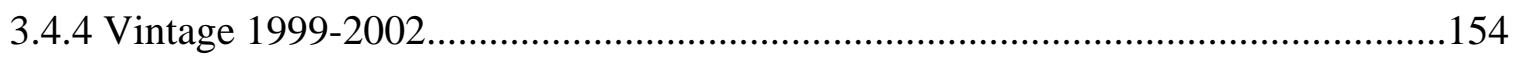

3.4.5 Comparative Analysis for Vintages ......................................................................156

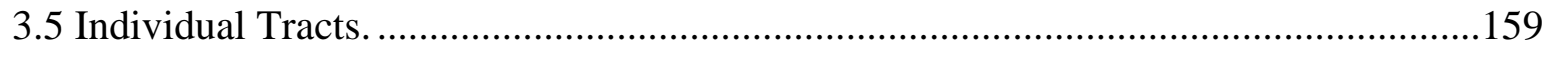

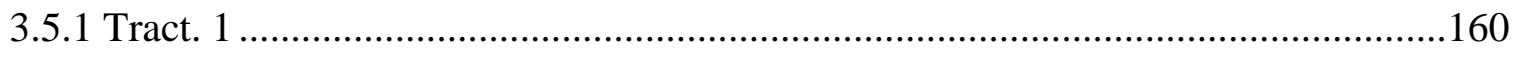

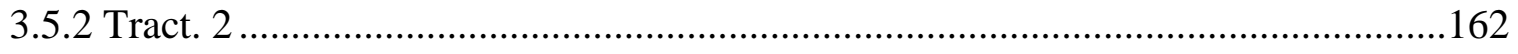

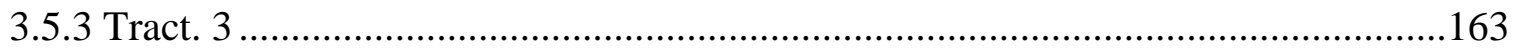




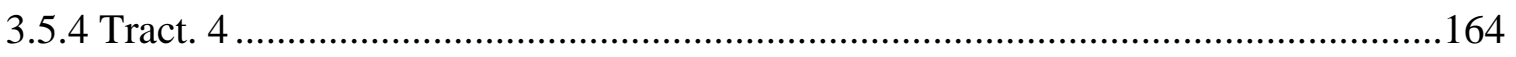

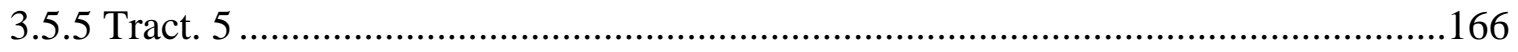

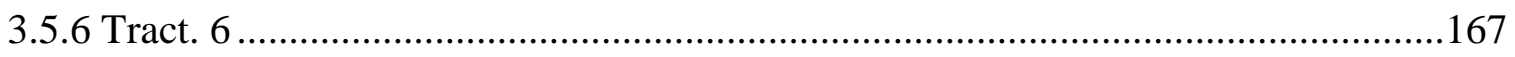

3.5.7 Comparative Analysis for Tracts. .......................................................................168

3.6 Well Performance Monitoring System. ......................................................................171

3.6.1 Water Control Diagnostic Plots ..........................................................................171

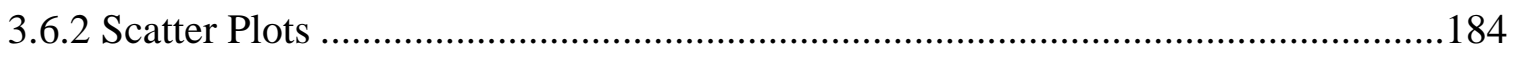

3.7 Flood Front Maps and Bypassed Oil. ………………................................................186

IV. Production Forecast and Reserve Estimation ..................................................................189

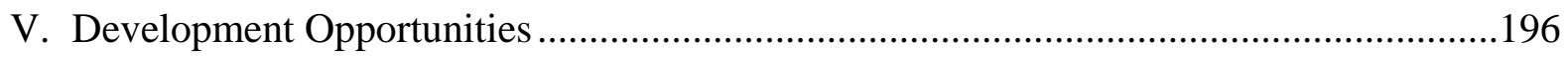

VI. Conclusion and Recommendations ...............................................................................197

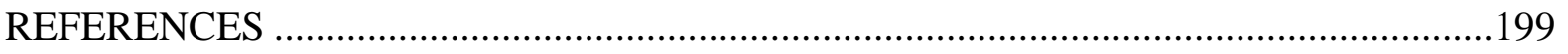

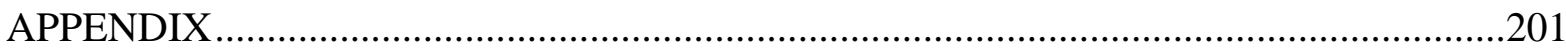

2. Development of Production and Database Management .....................................................210

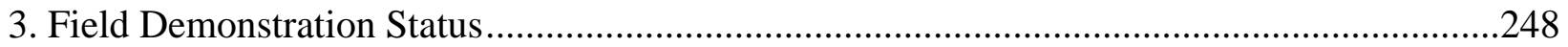

3.1 Voidage Replacement Ratio (VRR)...............................................................................248

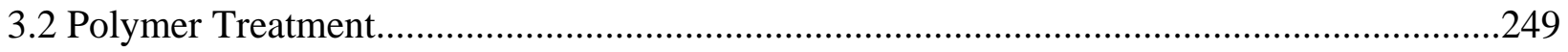

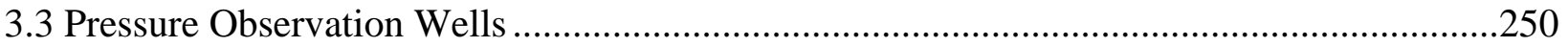

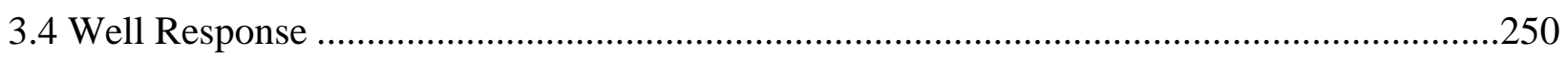

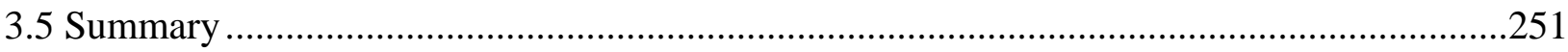

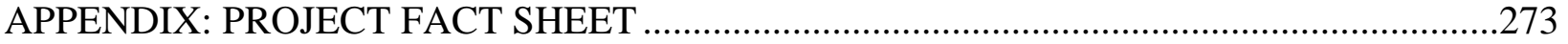




\section{LIST OF TABLES}

Table 1.2.1 Coupling between injector and producers..................................................18

Table 1.2.2 Summary of waterflood projects .............................................................18

Table 1.3.1 Criteria for pay identification in the ET O’Daniel unit...........................43

Table 1.3.2 Summary of rock properties and saturations for ET O’Daniel wells...............54

Table 1.3.3 Regression from crossplots of core - log porosity for cored

ET O’Daniel wells......................................................71

Table 1.3.4 Archie parameters used in determining saturation in the upper

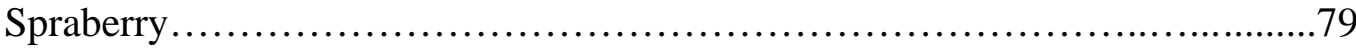

Table 1.3.5 Interval averaged water saturations for well with resistivity curves................80

Table 1.3.6 Kolmogorov-Smirnov test for porosity and shale volume.......................89

Table 1.4.1 Category of Active Wells Based on the Current Production Performance .........142

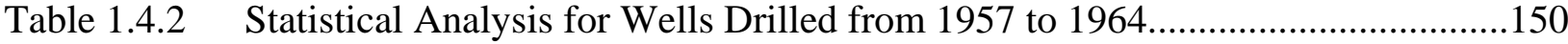

Table 1.4.3 Statistical Analysis for Wells Drilled from 1978 to 1982 ................................152

Table 1.4.4 Statistical Analysis for Wells Drilled from 1990 to 1996 .................................154

Table 1.4.5 Statistical Analysis for Wells Drilled from 1999 to 2002_...............................156

Table 1.4.6 Oil Recovery for Different Tracts of Germania Spraberry Unit........................171

Table 1.4.7 Summary from Water Control Diagnostic Plots for Wells in Germania

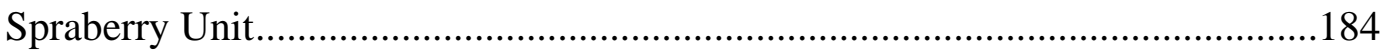

Table 1.4.A-1 Remaining Reserve and Estimated Ultimate Recovery for Active Wells ...........208 


\section{LIST OF FIGURES}

Fig. 1.1.1 Location of On-trend and Off-trend wells in the Lower Spraberry of Midkiff Unit “McDonald” Waterflood Pilot.

Fig. 1.1.2 The off-trend production response of Lower Spraberry (Midkiff Unit “McDonald” Waterflood Pilot) from Wells \#2805, 3003, 3210, 3215A, 4004 and 3103.

Fig. 1.1.3 The on-trend production response of Lower Spraberry (Midkiff Unit “McDonald” Waterflood Pilot) from Wells \#2801, 2804, 4701, 4702, 4712, 4714, 4715 and 4716A

Fig. 1.1.4 Location of On-trend and Off-trend wells in Upper Spraberry of the Midkiff Unit “McDonald” Waterflood Pilot.

Fig. 1.1.5 Off-trend production response of Upper Spraberry (Midkiff Unit “McDonald Waterflood Pilot”) from Wells \#1206, 1208, 1401, 1406, 1807 and 1905

Fig. 1.1.6 On-trend production response of Upper Spraberry (Midkiff Unit “McDonald” Waterflood Pilot) from Wells \#1205, 1207, 1209, 1211, 1212, 2008, 2009, 2010, 2105, 2108, 2109, PU\#3415A, PU\#4407A, PU\#4408A and PU\#4409A.

Fig. 1.1.7 Incremental oil recovery of Upper Spraberry (Midkiff Unit "McDonald" Waterflood Pilot) from on-trend wells after waterflood baseline 8

Fig. 1.1.8 Location of On-trend and Off-trend wells in the Lower and Upper of Midkiff Unit “Heckman” Waterflood Pilot

Fig. 1.1.9 On-trend production response of Upper and Lower Spraberry (Midkiff Unit “Heckman” Waterflood Pilot) from wells \#2413A, 2513A, 3405, 3506, 3507, 3508, 3509, 3510, 3511, 3611, 3613, 3703, 3705, 3808, 3810, 3905, 3906 and 4910

Fig. 1.1.10 Off-trend production response of Upper and Lower Spraberry (Midkiff Unit “Heckman” Waterflood Pilot) from wells \#3612, 3614, 3616, 3704, 3706, 4908, 4909, 6706 and 6807.

Fig. 1.1.11 Comparison of incremental oil recovery between off-trend and on-trend wells of Upper and Lower Spraberry (Midkiff Unit "Heckman" 
Waterflood Pilot) 10

Fig. 1.1.12 Comparison between on-trend and off-trend production rate of Upper Spraberry in the ET O'Daniel Pilot

Fig. 1.1.13 On-trend incremental oil recovery of Upper Spraberry in the ET O’Daniel Pilot .11

Fig. 1.2.1 The new and old waterflood patterns applied in Naturally Spraberry Trend Area .19

Fig. 1.2.2 The Germania Unit Layout showing couple between injectors and producers taken from old waterflood history. 20

Fig. 1.2.3 Cumulative water injection map ....................................................................21

Fig. 1.2.4 Cumulative oil production map ……………...............................................22

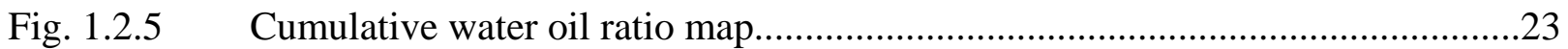

Fig. 1.2.6 Cumulative water oil ratio in bubble map............................................................24

Fig. 1.2.7 Cumulative water cut map …………………...................................................25

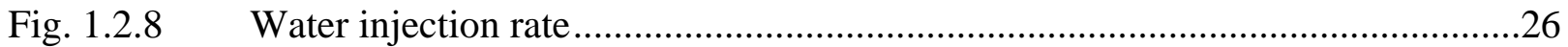

Fig. 1.2.9 Production summary of Germania Unit......................................................26

Fig. 1.2.10 Oil production history and decline curve analysis ...............................................27

Fig. 1.2.11 Comparison of cumulative production between infill wells and waterflood

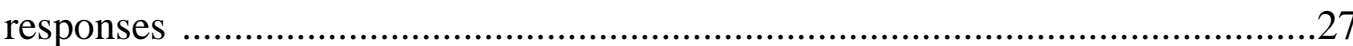

Fig. 1.2.12 Decline curve analysis taken from all data points and continued with the forecasting incremental oil from current ET'O'Daniel waterflood performance.

Fig. 1.2.13 Decline curve analysis taken from all data points and continued with the forecasting incremental oil from old ET'O'Daniel waterflood performance.

Fig. 1.2.14 Nominal decline rate above and below base case decline curve for current ET'O’Daniel waterflood performance.

Fig. 1.2.15 Nominal decline rate above and below base case decline curve for old ET'O'Daniel waterflood performance.

Fig. 1.2.16 Decline curve analysis taken from last data points .30

Fig. 1.2.17 Decline curve analysis taken from last data points and continued with the 
forecasting incremental oil from old and current ET'O’Daniel waterflood

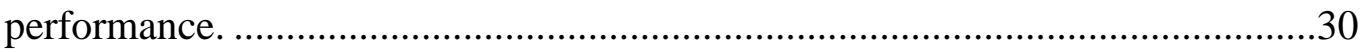

Fig. 1.2.18 Production summary of GSU-21 …………....................................................

Fig. 1.2.19 Decline curve analysis of GSU-21................................................................

Fig. 1.2.20 Cumulative oil recovery due to waterflood in the Germania unit ........................32

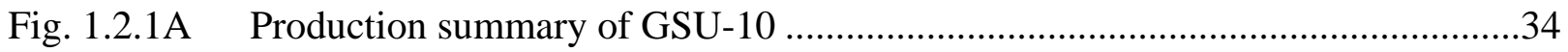

Fig. 1.2.2A Decline curve analysis of GSU-10....................................................................

Fig. 1.2.3A Production summary of GSU-12 ……...............................................................35

Fig. 1.2.4A Decline curve analysis of GSU-12...................................................................35

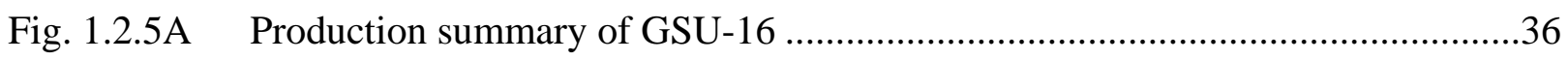

Fig. 1.2.6A Decline curve analysis of GSU-16....................................................................

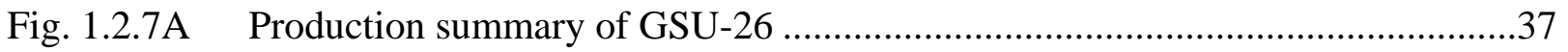

Fig. 1.2.8A Decline curve analysis of GSU-26....................................................................3

Fig. 1.2.1B Production summary of old ET O’Daniel unit.......................................................39

Fig. 1.2.2B Incremental oil recovery of old ET O'Daniel unit................................................39

Fig. 1.2.3B Cumulative oil production of old ET O'Daniel unit.............................................40

Fig. 1.3.1 Unit locations within the Spraberry trend area...............................42

Fig. 1.3.2 Crossplot of shale volume and porosity for well ET 47, 1U sand..................44

Fig. 1.3.3 Porosity - permeability crossplot for the primary rock types identified...............45

Fig. 1.3.4 Identification of pay based on shale volume and effective porosity

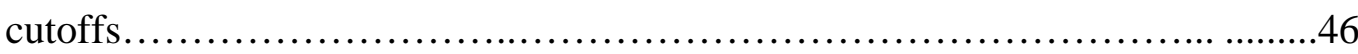

Fig. 1.3.5 Non-linearity of the different models for estimating clay content

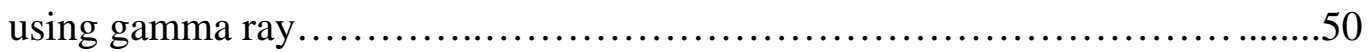

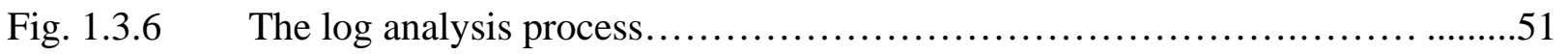

Fig. 1.3.7 Map of cored well locations in the ET O’Daniel pilot area..........................53

Fig. 1.3.8 Typical core - log playback in the $1 \mathrm{U}$ interval...................................55

Fig. 1.3.9 Crossplot of shale volume and porosity for well ET 37, $1 \mathrm{U}$ sand..................56

Fig. 1.3.10 Typical core - log playback in the $5 \mathrm{U}$ interval.................................56

Fig. 1.3.11 Crossplot of shale volume and porosity for well ET 37, 5U sand...................57

Fig. 1.3.12a Crossplot for lithology identification in $1 \mathrm{U}$ sand for well ET 37...................58 
Fig. 1.3.12b Crossplot for lithology identification in 5U sand for well ET 37.................58

Fig. 1.3.13 Minimum gamma ray values for ET O’Daniel unit in the $1 \mathrm{U}$

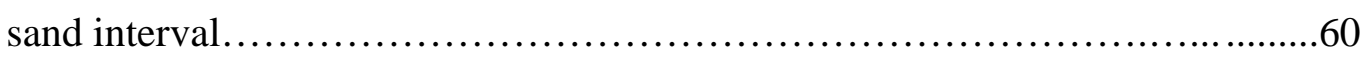

Fig. 1.3.14 Maximum gamma ray values for ET O’Daniel unit in the 1U

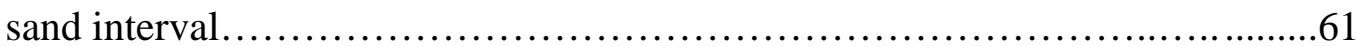

Fig. 1.3.15 Variations in response from the gamma ray curves in ET O’Daniel...............62

Fig. 1.3.16 Histogram and CDF for wells 36, before and after normalizing

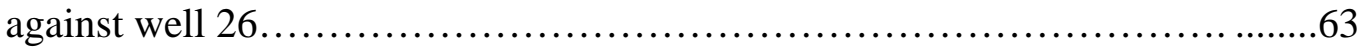

Fig. 1.3.17 Corrected gamma ray distribution for ET O’Daniel wells.......................64

Fig. 1.3.18 Normalized gamma ray values in $1 \mathrm{U}$ and $5 \mathrm{U}$ regions of upper

Spraberry, ET O’Daniel unit................................................65

Fig. 1.3.19 Effects on quality of porosity data from density and neutron

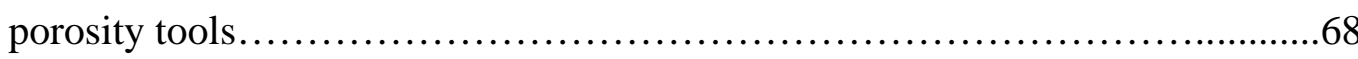

Fig. 1.3.20 Crossplot of core porosity and log porosity for ET 39, 1U sand................69

Fig. 1.3.21 Crossplot of core porosity and log porosity for ET 39, 5U sand.................70

Fig. 1.3.22 Playback of log and core porosity for ET 39, $1 \mathrm{U}$ sand........................71

Fig. 1.3.23 Playback of log and core porosity for ET 39, 5U sand........................71

Fig. 1.3.24a ET 39 crossplot for shale volume and permeability..........................73

Fig. 1.3.24b ET 47 crossplot for shale volume and permeability...........................73

Fig. 1.3.25a ET 39 crossplot for log porosity and permeability............................74

Fig. 1.3.25b ET 47 crossplot for log porosity and permeability...........................75

Fig. 1.3.26a ET 39 crossplot for shale corrected porosity and permeability...................76

Fig. 1.3.26b ET 47 crossplot for shale corrected porosity and permeability...................76

Fig. 1.3.27 Playback of results from conventional regression and ACE regression.............78

Fig. 1.3.28 Saturation profile matched for ET 38, 1U using Rw 0.035 ohm-m

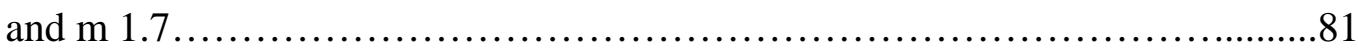

Fig. 1.3.29 Saturation profile matched for ET 40, 1U using Rw 0.035 ohm-m

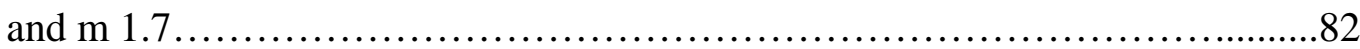

Fig. 1.3.30 Statistics of Vsh values for ET O’Daniel, $1 \mathrm{U}$ sand...........................83

Fig. 1.3.31 Statistics of Vsh values for ET O’Daniel, 5U sand............................84

Fig. 1.3.32 Statistics of Vsh values for Germania, $1 \mathrm{U}$ sand.............................84 
Fig. 1.3.33 Statistics of Vsh values for Germania, 5U sand.................................85

Fig. 1.3.34 IQR and mean values of shale volume and porosity for ET O’Daniel

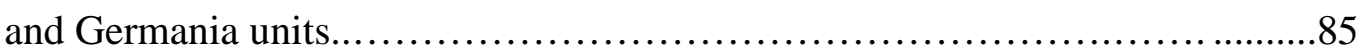

Fig. 1.3.35 Statistics of porosity values for ET O'Daniel, 1U sand..............................86

Fig. 1.3.36 Statistics of porosity values for ET O'Daniel, 5U sand..............................87

Fig. 1.3.37 Statistics of porosity values for Germania, $1 \mathrm{U}$ sand..............................87

Fig. 1.3.38 Statistics of porosity values for Germania, $5 \mathrm{U}$ sand.............................88

Fig. 1.3.39 Kolmogorov - Smirnov test on porosity function, 1U sand...........................89

Fig. 1.3.40 Gross thickness map of the $1 \mathrm{U}$ sand........................................91

Fig. 1.3.41 Lithostratigraphic section A-A' with datum at top of $1 \mathrm{U}$ interval...................92

Fig. 1.3.42 Payzone prediction based on rock model for GSU 146A, 1U sand...................93

Fig. 1.3.43 Estimate of rock types in GSU146A, 1U sand from shale volume -

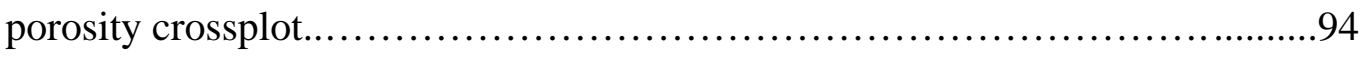

Fig. 1.3.44 Payzone prediction based on rock model for GSU214A, 1U sand..................94

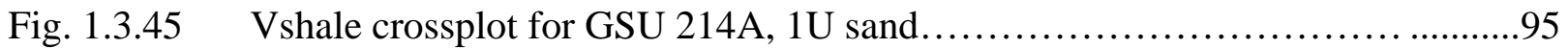

Fig. 1.3.46 Payzone prediction for GSU214A, 5U sand................................95

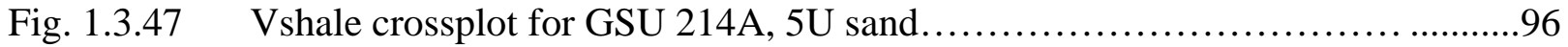

Fig. 1.3.A1 Structure contour data, top of Spraberry sandstone Midland Basin, west

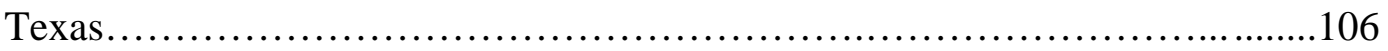

Fig. 1.3.A2 Regional geologic setting of Permian Basin, west Texas...........................107

Fig. 1.3.A3 West -east stratigraphic cross section BB' ...................................108

Fig. 1.3.A4 Lithofacies map, four County area with Spraberry isopach contours................110

Fig. 1.3.A5 Facies model of clastic and carbonate dominated shelf margin systems.............111

Fig. 1.3.A6 Distribution of environments, geometry of deposits, and channel

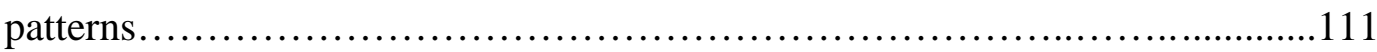

Fig. 1.3.A7 Sections of onlapping clastics and downdip widening of channels.................112

Fig. 1.3.A8 Isopach map of upper Spraberry unit in Midland County..........................114

Fig. 1.3.A9 Clastic ratio map of upper Spraberry unit in Midland County.......................115

Fig. 1.3.A10 Operational units within the upper Spraberry formation............................116

Fig. 1.3.A11 Location of class A \& B Spraberry reservoirs of west Texas.......................117

Fig. 1.3.B1 Minimum gamma ray map of ET O’Daniel, 5U sand............................118 
Fig. 1.3.B2 Maximum gamma ray map of ET O’Daniel, 5U sand............................119

Fig. 1.3.B3 Paleo-structure map of ET O’Daniel, 1U sand...............................120

Fig. 1.3.B4 Paleo-structure map of ET O’Daniel, 5U sand..............................121

Fig. 1.3.B5 Paleo-structure map of Germania, 1U sand..................................122

Fig. 1.3.B6 Paleo-structure map of Germania, 5U sand...............................123

Fig. 1.3.B7 Porosity distribution map of ET O’Daniel, 1U sand............................124

Fig. 1.3.B8 Porosity distribution map of ET O’Daniel, 5U sand..............................125

Fig. 1.3.B9 Porosity distribution map of Germania, 1U sand.............................126

Fig. 1.3.B10 Porosity distribution map of Germania, 5U sand..............................127

Fig. 1.3.B11 Effect of sample sizes on Kolmogorov - Smirnov distribution tests..................128

Fig.1.4.1 Location of Germania Spraberry Unit ......................................................130

Fig.1.4.2 Location of New Water Injectors Wells in Germania Spraberry Unit................131

Fig. 1.4.3 Oil Rate and Water Cut during Primary Depletion of Germania Spraberry Unit

Fig. 1.4.4 Oil Rate per well, Liquid Rate per well and Active wells during Primary Depletion for Germania Spraberry Unit .134

Fig. 1.4.5 Base Map of Germania Spraberry Unit showing the wells injecting water

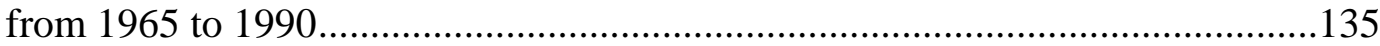

Fig. 1.4.6 Cumulative Water Injection for the five wells injecting from 1965 to 1990.......135

Fig. 1.4.7 Oil Rate and Water Cut during Primary and Secondary Depletion of Germania Spraberry Unit.

Fig. 1.4.8 Base Map of Germania Spraberry Unit showing wells injecting water under the new Injection Pattern

Fig. 1.4.9 Oil Rate per well, Liquid Rate per well and Active wells during Primary and Secondary Depletion for Germania Spraberry Unit.

Fig. 1.4.10 Cross-Plot showing the Production Heterogeneity Index for Oil and Water in 64 active wells of Germania Spraberry Unit

Fig. 1.4.11 Base Map showing the 64 Active Wells in Germania Spraberry Unit and its position with regards to the injectors (old injection pattern).

Fig. 1.4.12 Fracture Orientation from Core Analysis .141

Fig. 1.4.13 Voidage Replacement Ratio for the First Period of Injection. .144 
Fig. 1.4.14 Voidage Replacement Ratio for the Second Period of Injection ........................144

Fig. 1.4.15 Base Map Showing the On-trend and Off-trend wells.....................................145

Fig. 1.4.16 Oil Rate per well for On-trend and Off-trend wells........................................146

Fig. 1.4.17 Average water-Oil ratio for On-trend and Off-trend wells ...............................147

Fig. 1.4.18 Different Drilling Campaigns for Development of Germania............................148

Fig. 1.4.19 Base Map showing the Location of Wells Drilled from 1957 to 1963...............149

Fig. 1.4.20 Histogram of Initial Oil Rate for Wells Drilled from 1957 to 1963...................149

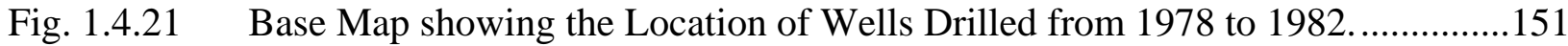

Fig. 1.4.22 Histogram of Initial Oil Rate for Wells Drilled from 1978 to 1982...................151

Fig. 1.4.23 Base Map showing the Location of Wells Drilled from 1990 to 1996...............153

Fig. 1.4.24 Histogram of Initial Oil Rate for Wells Drilled from 1990 to 1996...................153

Fig. 1.4.25 Base Map showing the Location of Wells Drilled from 1999 to 2002...............155

Fig. 1.4.26 Histogram of Initial Oil Rate for Wells Drilled from 1999 to 2002 ...................155

Fig. 1.4.27 Histogram of Initial Oil Rate for Wells Drilled from 1999 to 2002 ..................157

Fig. 1.4.28 Historical Oil Rate per well for Different Campaigns of wells during the Development of Germania Spraberry Unit.

Fig. 1.4.29 Historical Water-Oil Ratio for Different Campaigns of wells during the Development of Germania Spraberry Unit. .158

Fig. 1.4.30 Cumulative Oil Production for Different Campaigns of wells during the Development of Germania Spraberry Unit. .159

Fig. 1.4.31 Location of Individual Tracts in Germania Spraberry Unit..............................160

Fig. 1.4.32 Oil Rate and Water Cut for Tract 1. (Germania Spraberry Unit.) .....................161

Fig. 1.4.33 Cumulative Oil Production and Active wells for Tract 1 (Germania Spraberry Unit) .161

Fig. 1.4.34 Oil Rate and Water Cut for Tract 2. (Germania Spraberry Unit.) ......................162

Fig. 1.4.35 Cumulative Oil Production and Active wells for Tract 2 (Germania Spraberry Unit) .163

Fig. 1.4.36 Oil Rate and Water Cut for Tract 2. (Germania Spraberry Unit.) 164

Fig. 1.4.37 Cumulative Oil Production and Active wells for Tract 2 (Germania Spraberry Unit) .164

Fig. 1.4.38 Oil Rate and Water Cut for Tract 3. (Germania Spraberry Unit) .165 
Fig. 1.4.39 Cumulative Oil Production and Active wells for Tract 3 (Germania

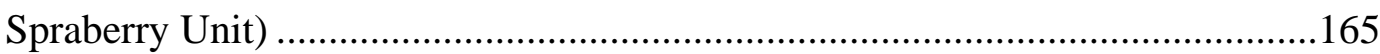

Fig. 1.4.40 Oil Rate and Water Cut for Tract 4. (Germania Spraberry Unit.) .........................166

Fig. 1.4.41 Cumulative Oil Production and Active wells for Tract 4 (Germania Spraberry Unit) ................................................................................................167

Fig. 1.4.42 Oil Rate and Water Cut for Tract 6. (Germania Spraberry Unit) .........................168

Fig. 1.4.43 Cumulative Oil Production and Active wells for Tract 6 (Germania

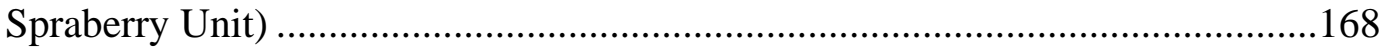

Fig. 1.4.44 Historical Oil Rate for Different Tracts of Germania Spraberry Unit .................169

Fig. 1.4.45 Historical Oil Rate per well for Different Tracts of Germania Spraberry Unit .170

Fig. 1.4.46 Historical Water-Oil Ratio for Different Tracts of Germania Spraberry Unit

Fig. 1.4.47 WOR and WOR Derivative for well GSU-1: Experiencing near Wellbore Water Channeling.

Fig. 1.4.48 WOR and WOR Derivative for well GSU-114: Experiencing Near Wellbore Water Channeling. .172

Fig. 1.4.49 WOR and WOR Derivative for well GSU-115: Experiencing Near Wellbore Water Channeling.

Fig. 1.4.50 WOR and WOR Derivative for well GSU-117: Experiencing Near Wellbore Water Channeling.

Fig. 1.4.51 WOR and WOR Derivative for well GSU-118: Experiencing Near Wellbore Water Channeling.

Fig. 1.4.52 WOR and WOR Derivative for well GSU-119: Experiencing Near Wellbore Water Channeling

Fig. 1.4.53 WOR and WOR Derivative for well GSU-120: Experiencing Near Wellbore Water Channeling

Fig. 1.4.54 WOR and WOR Derivative for well GSU-2: Experiencing Near Wellbore Water Channeling. .175

Fig. 1.4.55 WOR and WOR Derivative for well GSU-309: Experiencing Bottom Water Coning with Late Time Channeling. 
Fig. 1.4.56 WOR and WOR Derivative for well GSU-3: Experiencing Bottom Water Coning with Late Time Channeling.

Fig. 1.4.57 WOR and WOR Derivative for well GSU-11: Experiencing Rapid Channeling. .....

Fig. 1.4.58 WOR and WOR Derivative for well GSU-16: Experiencing Rapid Channeling.

Fig. 1.4.59 WOR and WOR Derivative for well GSU-16: Experiencing Rapid Channeling.

Fig. 1.4.60 WOR and WOR Derivative for well GSU-21: Experiencing Rapid Channeling.

Fig. 1.4.61 WOR and WOR Derivative for well GSU-22: Experiencing Rapid Channeling

Fig. 1.4.62 WOR and WOR Derivative for well GSU-321: Experiencing Rapid Channeling.

Fig. 1.4.63 WOR and WOR Derivative for well GSU-5: Experiencing Rapid Channeling.

Fig. 1.4.64 WOR and WOR Derivative for well GSU-20: Experiencing Normal Displacement with High WOR.

Fig. 1.4.65 WOR and WOR Derivative for well GSU-13: Experiencing Normal Displacement with High WOR. .181

Fig. 1.4.66 WOR and WOR Derivative for well GSU-23: Experiencing Normal Displacement with High WOR. .181

Fig. 1.4.67 WOR and WOR Derivative for well GSU-25: Experiencing Normal Displacement with High WOR.

Fig. 1.4.68 WOR and WOR Derivative for well GSU-26: Experiencing Normal Displacement with High WOR. .182

Fig. 1.4.69 WOR and WOR Derivative for well GSU-28: Experiencing Normal Displacement with High WOR.

Fig. 1.4.70 WOR and WOR Derivative for well GSU-205: Experiencing Normal Displacement with High WOR.

Fig. 1.4.71 WOR and WOR Derivative for well GSU-206: Experiencing Normal 
Displacement with High WOR. 184

Fig. 1.4.72 Scatter plot showing the performance of Cumulative Oil vs Cumulative Water for active wells in Germania Spraberry Unit 185

Fig. 1.4.73 Bubble Maps for different periods of exploitation. Figure Shows the Evolution of the Cumulative Oil Production for every well Completed in Germania Spraberry Unit and the Response Observed around the injectors .187

Fig. 1.4.74 Bubble Maps of Cumulative Oil Production. Figure Shows the Cumulative Oil Production for every well Completed in Germania Spraberry Unit and the most drained areas of the Unit

Fig. 1.4.75 Bubble Maps of Cumulative Water Production. Figure Shows the Cumulative Water Production for every well Completed in Germania Spraberry Unit and the most drained areas of the Unit 188

Fig. 1.4.76 Bubble Maps of Cumulative Gas Production. Figure Shows the Cumulative Gas Production for every well Completed in Germania Spraberry Unit and the most drained areas of the Unit 189

Fig. 1.4.77 Remaining and Produced Reserves for wells GSU-113,GSU-114,GSU115,GSU-116,GSU-117,GSU-118,GSU-119,GSU-120,GSU-121,GSU122, and GSU-122. 191

Fig. 1.4.78 Remaining and Produced Reserves for wells GSU-124,GSU-125,GSU126,GSU-127,GSU-128,GSU-13,GSU-131,GSU-132,GSU-133,GSU-134, and GSU-13.

Fig. 1.4.79 Remaining and Produced Reserves for wells GSU-2,GSU-20,GSU205,GSU-206,GSU-207,GSU-208,GSU-212,GSU-25,GSU-26,GSU-28, and GSU-308.

Fig. 1.4.80 Remaining and Produced Reserves for wells GSU-309,GSU-31,GSU310,GSU-311,GSU-312,GSU-313,GSU-314,GSU-316,GSU-317,GSU318, and GSU-321. 192

Fig. 1.4.81 Remaining and Produced Reserves for wells GSU-322, GSU-323, GSU324, GSU-325, GSU-326, GSU-327, GSU-328, GSU-405, GSU-408, and GSU-409. .193

Fig. 1.4.82 Remaining and Produced Reserves for wells GSU-411,GSU-412,GSU- 
5,GSU-502,GSU-503,GSU-602, and GSU-602.

Fig. 1.4.83 Bubble Maps of Remaining Reserves. Figure shows the remaining reserves for every well active in Germania Spraberry Unit.

Fig. 1.4.84 Bubble Maps of Estimated Ultimate Recovery (EUR). Figure shows the remaining reserves for every well active in Germania Spraberry Unit. 195

Fig. 1.4.85 WOR behavior and Cumulative Oil Production due to Infill drilling and Waterflooding in Germania Spraberry Unit ............................................................196

Fig. 1.4.A. 1 Decline Curve Analysis for Well GSU-114.........................................................202

Fig. 1.4.A. 2 Decline Curve Analysis for Well GSU-115...........................................................202

Fig. 1.4.A. 3 Decline Curve Analysis for Well GSU-117 ……………………….....................203

Fig. 1.4.A. 4 Decline Curve Analysis for Well GSU-120...........................................................203

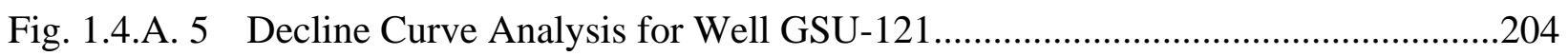

Fig. 1.4.A. 6 Decline Curve Analysis for Well GSU-123........................................................204

Fig. 1.4.A. 7 Decline Curve Analysis for Well GSU-127 …………………………................205

Fig. 1.4.A. 8 Decline Curve Analysis for Well GSU-405............................................................205

Fig. 1.4.A. 9 Decline Curve Analysis for Well GSU-406............................................................206

Fig. 1.4.A. 10 Decline Curve Analysis for Well GSU-408..........................................................206

Fig. 1.4.A. 11 Decline Curve Analysis for Well GSU-502..........................................................20

Fig. 2.1 Front Page of Field Data Management Software.................................................211

Fig. $2.2 \quad$ Well Data Form.............................................................................................211

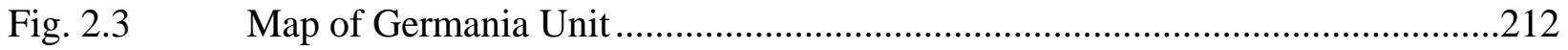

Fig.2.4 Zoom-in view of particular cluster …….......................................................212

Fig. 2.5 Tabulated Production History Data of Well 207A …………………………......213

Fig. 2.6 Graph of Oil Production of Well 207A.................................................................213

Fig. $3.7 \quad$ Hyperbolic Decline Curve of Well 207A …….................................................214

Fig. 3.1 Location of new wells drilled in 2003 (red dot symbol).......................................254

Fig. 3.2 GSU daily water injection from six injectors ……………………….................255

Fig. $3.3 \quad$ Total GSU water injection ...............................................................................25

Fig. $3.4 \quad$ Volume of water injection until on-trend production

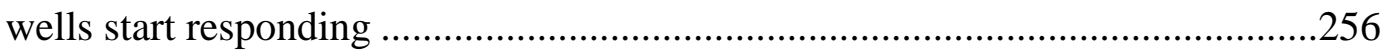

Fig. $3.5 \quad$ Voidage Replacement Ratio (1965-1989) …………………………......................256 
Fig. $3.6 \quad$ Voidage Replacement Ratio (VRR) in GSU ................................................257

Fig. 3.7 Voidage Replacement Ratio (VRR) in ET O’Daniel.......................................257

Fig. 3.8 FL and PIP performances of GSU 406A polymer squeezed well .....................258

Fig. $3.9 \quad$ FL and PIP performances of GSU 408A polymer squeezed well .....................258

Fig. 3.10 FL and PIP performances of GSU 409A polymer squeezed well ......................259

Fig. $3.11 \quad$ FL and PIP performances of GSU 5 observation well....................................259

Fig. 3.12 FL and PIP performances of GSU 20 observation well..................................260

Fig. 3.13 FL and PIP performances of GSU 20 observation well...................................260

Fig. $3.14 \quad$ Production performance in Tract 2 .............................................................261

Fig. 3.15 Production performance in Tract 3 ..........................................................261

Fig. 3.16 Production performance in Tract 4 .............................................................262

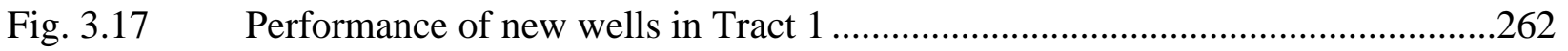

Fig. 3.18 Performance of new wells in Tracts 2 and 4 .................................................263

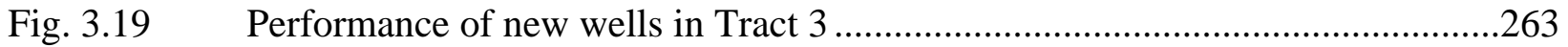

Fig. $3.20 \quad$ Wells experience high water production rate.................................................264

Fig. $3.21 \quad$ Wells have a good oil response....................................................................264

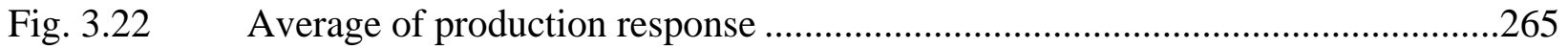

Fig. 3.23 Total of production response ..................................................................265

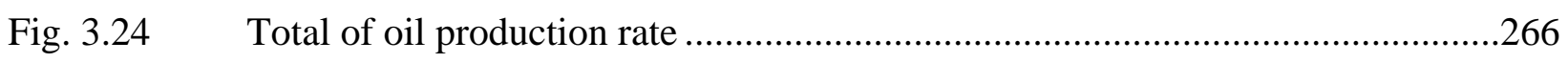

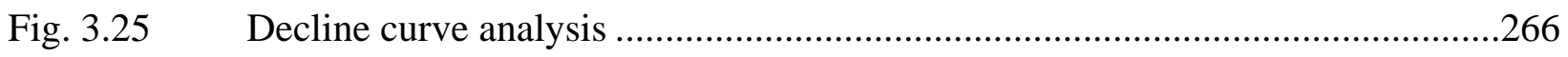

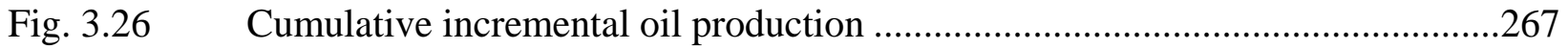

Appendix 3.A Wells produce high water rate during water injection in GSU waterflood

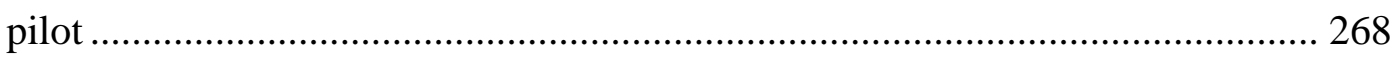

Appendix 3.B Wells that have oil response during water injection in GSU waterflood pilot 


\section{ACKNOWLEDGMENTS}

Financial support for this work is graciously provided by the United States Department of Energy (NETL/National Petroleum Technology Office).

This support is gratefully acknowledged, as well as that of GeoGraphix and Oil Field Manager (OFM), which donated software used in this study. I greatly appreciate diligent efforts from the following individuals who contributed to this project: Erwinsyah Putra helped with the technical part of this research along with writing field demonstration status as described in Chapter 1.1, 1.2 and 3. Babajide A. Olumide conducted Germania unit characterization described in Chapter 1.3. Erwin Hernandez conducted evalution of waterflooding in Germania unit as described in Chapter 1.4. Zuher Syihab developed a Field Data Management Software as summarized in Chapters 2 and Dicman Alfred wrote the manual for the software as found in the Appendix. I also appreciate the thorough review and grammatical corrections of this manuscript by Matthew E. Gross. 


\section{EXECUTIVE SUMMARY}

This report describes the final work of the project, "Preferred Waterflood Management Practices for the Spraberry Trend Area." The objective of this project is to significantly increase field-wide production in the Spraberry Trend in a short time frame through the application of preferred practices for managing and optimizing water injection. Our goal is to dispel negative attitudes and lack of confidence in water injection and to document the methodology and results for public dissemination to motivate waterflood expansion in the Spraberry Trend.

This report provides results of the final technical report in three areas: 1) detail historical review and extensive reservoir characterization, 2) production data management, and 3) field demonstration. In this report we present the results of the final year of the three-year project for each of the three areas. The following headings and subsequent findings outline the work that appears in this report.

Review of Upper and Lower Pilots in the Spraberry Area. The objectives of this study are to review the performance of Lower Spraberry. A number of papers have been published concerning the performance in the Lower Spraberry. The intake capacity and the oil recovery per acre in Lower Spraberry are lesser than those in Upper Spraberry. The reasons of poor waterflood performance are because the Lower Spraberry has higher oil shrinkage factor and less fracture intensity. In addition, Lower Spraberry in Preston Unit had severe corrosion problem. The waterflooding in Lower Sparbbery in other pilot area, E.T O’Daniel waterflood Pilot also showed that the Lower Spraberry was not commercial. In contrarily, the positive response of water injection in Lower Spraberry was observed in the JoMill (Spraberry) Field. In this study, we reviewed waterflooding performance in the four recent waterflood pilots. Three pilots were performed in the Midkiff Unit and the other one was conducted in the E.T O'Daniel Pilot. In the Midkiff Unit, it is divided into "Lower-McDonald" pilot, "Upper-McDonald" pilot and "Upper and Lower-Heckman" pilot. We divided the wells as on-trend and off-trend wells to investigate the effect of placing the injection and production wells to the oil production rate.

The off-trend and on-trend production wells in the "Lower-McDonald" pilot show no response to waterflood. The positive response was only due to adding more wells. We are, thus, unable to quantify additional oil recovery from wells that completed in the Lower zone. In the "Upper-McDonald" pilot, no waterflood response was observed in the off-trend wells whereas a significant waterflood response was noted in the on-trend wells. Even though one well was added during the water injection but the production response was obviously from waterflood response alone. Both on-trend and off-trend wells in the "Heckman" pilot show favorable response to waterflood After initiation of water injection, immediate increase in oil production was noted in the on-trend wells whereas the response in off-trend wells was delayed as expected. Higher production response is obtained in the on-trend wells than in the off-trend wells. The last pilot is the E.T O'Daniel Pilot as part of DOE project. It shows that the on-trend wells respond favorably to waterflood while the off-trend wells have no significant response. 
Germania Unit Rate Forecasting Based on Other Waterflood Pilots in the Spraberry Area. Based on recent technological development and intensive research, both basic and applied, we have gained a new understanding of proper procedures for waterflooding Spraberry reservoirs. The Germania unit as well as other waterflood units in the Spraberry Area had been waterflooded with the conventional waterflood techniques applied in natural fractured reservoirs elsewhere, where all injection wells were aligned parallel along major fracture trend in order to force the oil to flow in a direction perpendicular to the fracture trend towards a line of production wells. Based on our experience in this field and responses from successful waterflood projects, the injection wells should be aligned to production wells along the fracture orientation to have fast responses and viable waterfloods. The objectives of this study are to propose the location of new injection wells in the Germania unit, to review wellbore status in Germania unit and finally, to forecast the incremental oil recovery based on waterflooding performance in other waterflood pilot area in order to demonstrate the benefit of waterflooding in Germania unit area. To propose the location of new injection wells, we divided the on-trend and off-trend wells based on the location of injection wells to the production wells and fracture orientation. Any changes in water, oil and gas production during the waterflood period are classified as waterflood responses. We indicated a communication (coupling) between injection and production wells which are located along fracture orientation in NE-SW direction. Based on this criterion, the new injection wells were located in the pilot area. To achieve the second objective, we collected and organized injection and production data followed by developing a database using software donated by Schlumberger (OFM). Using this software, we were able to see the old waterflood performance and identify casing failure due to the corrosive nature of San Andreas water resulting in premature abandonment of Spraberry well bores by plotting the cumulative water oil ratio (CWOR). This OFM software can also be interfaced with real time data acquisition so that daily analysis of response can be monitored and analyzed from remote location. The last objective of this particular study is to forecast the incremental oil recovery. Waterflooding in the naturally fractured Spraberry Trend Area reservoirs has always been scorned resulting from ambiguity of performance data after water injection or results that did not conform to previously conceived notions. However, upon closer inspection, Spraberry water injection projects have all responded in a similar manner. In order to analyze the old waterflood performances in Germania unit and other units, we gathered and compiled all production and injection data. Then reconstructed and developed injection/production data using Oil Field Manager (OFM) database. A decline analysis was conducted to quantify the waterflood response. All production wells in this unit have been analyzed and the waterflood response has been quantified. A total of 80,000 bbls oil has been recovered due to waterflooding in this area over 25 years with total injection of 2.5 MMbbls. The waterflood response in this unit resulted in a lack of confidence in water injection as a result many wells face abandonment. The small amount of oil that has been recovered due to waterflooding and negligible amount of water that has been injected for 25 years warrant that this unit would be potential for recovering oil with proper management practices during water injection. 
Germania Unit Characterization using an Analog Field and Old Cased Hole Neutron. Extensive reservoir characterization work has been carried out in the ET O’Daniel based on recent core and log data acquisition, production and injection data, welltesting results, tracer analysis and simulation studies. The GSU on the other hand lacks core and modern log data, and has not been characterized for predicting rock type and pay zones. In this study, we focus on the petropysical evaluation of the upper Spraberry unit derived from a database of 85 log suites, primarily consisting of Gamma ray and Old Cased Hole Neutron logs. Core based relationships developed in the ET O’Daniel unit are borrowed upon to aid the characterization of this field, and will generally suffice due to the similar depositional environment and proximity of the units from one another. We summarize our findings as follows:

1. The Isopach maps indicate channel deposits and interdistributary flat features that are consistent with the depositional evaluation proposed by Handford et al. The facies indicate detrital clasts of both depositional episodes as evident in the lithology charts in chapter II which indicate that the $1 \mathrm{U}$ and $5 \mathrm{U}$ intervals are made up of terrigenous sandstones as well as dolomitic carbonate facies.

2. Particularly evident in the ET O'Daniel Isopach maps are the north - south thinning of the sandstone intervals, this is indicative of an energy source north of the Spraberry.

3. The Germania unit isopach (net thickness) is conservative in its estimation of net pay thickness, this is can be attributed to the limitations of the log based rock model in predicting payzones based on normalized cased hole neutron logs. Neutron logs alone do not lend themselves to corrections (environmental etc). Porosity correction techniques require a multi-dimensional array of porosity data i.e. density and neutron or sonic. Ideally, the density and neutron porosity logging devices should be run together so as to allow for necessary compensatory corrections in log data.

4. Optimal non-parametric regression techniques did not clearly improve the estimations of permeability based on shale volume and porosity. The method in itself is efficient, but alternative or more appropriate relationships specific to the Spraberry units need to be investigated. Additional studies on pore throat distribution based on core analysis may offer opportunities to improve the predictions.

5. Techniques for determining water saturation in the spraberry or shaly sands do not recommend using the Archie's equation. The Archie model is simplistic in its approach and is only considered a means of 'estimating' water saturation for this study.

6. Acquisition of core data within the Germania unit is not an option. To enable further evaluation of the GSU, core derived parameters must be available to guide simulation studies and inform fracture characterization to yield an integrated reservoir model

Evaluation of Waterflooding Performance in Germania Spraberry Unit. The Germania unit as well as other units in the Spraberry Area has been waterflooded using the conventional waterflood techniques applied in natural fractured reservoirs, where all injection wells are aligned parallel along major fracture trend to force the oil to flow perpendicular to the fracture trend towards a line of producer wells.

In the past, several studies have been conducted to propose different waterflooding techniques and development plans for Germania Unit; however none of the previous 
studies, have addressed the reservoir characterization and monitoring of the waterflooding project and propose alternatives of development taking into account the current and future conditions of the reservoir. Within the project objective, the specific goals for this period are to (1) integrate the production and injection data to characterize the reservoir, (2) evaluate development opportunities with emphasis toward preventing trapped oil, and maximizing recovery, (3) identify bypassed oil and flood front to locate infill wells, (4) identify problems in some wells by using the concept of water-oil ratio and its derivative to differentiate whether the wells are experiencing coning problems, layer breakthrough or near wellbore channeling, (5) estimate the remaining reserves associated to the drainage radius of every well by performing decline curve analysis of individual wells, and (6) analyze the historical relationship between reservoir withdrawals and the water injection rate in different areas of the unit to optimize the performance of the waterflood.

Consequently, this project will be addressed to provide a significant reservoir characterization and evaluate the performance of the waterflooding to provide facts, information and knowledge to obtain the maximum economic recovery from this reservoir. Thus, attempts are made to describe the reservoir, understand the performance of the reservoir under the current waterflooding project, and controlled surveillance will be carried out to improve field performance. The following methodology was used to achieve the objectives of the project:

1. The data needed was collected, reviewed, and validated and data base constructed using the software Oil Field Manager (OFM). Since the data was obtained from different related sources, it was reviewed, re-organized, and finally reduced to a format manageable in OFM. The data collected comprises: production and injection, coordinates, dates and events, wellbore, limits of leasing, logs, PVT analyses, etc. The calculations and processes were done using the main modules of the program (Decline Curve Analysis, System Functions, Calculated Variables, Plots, Reports, Bubble Maps, Grid Maps and Scatter Plots) and the interrelation among them, was also considered.

2. The study was approached by considering the overall performance of the Germania Unit as well as the performance and experiences obtained in others areas of Spraberry Unit. Under a full field scope surveillance system, the different modules of OFM were used and statistical analyses for different wells were also considered.

3. Based on this study, recommendations for future field operation and developing plan were provided.

Development of Reservoir Management Database Software. Properly managing a reservoir that is so large and communicates, via the fractures, over great distances, poses a complicated technological and data management constraint. This problem acts as a deterrent for waterflood operations in Spraberry reservoirs. Reservoir engineering, by definition, requires precise injection, production and pressure data. Acquisition and control of this data has always been a constraint to providing the optimum method for water injection. The result is large volumes of oil that could have been recovered via water injection remain untapped. We believe by proper data acquisition and precise reservoir engineering techniques, any lack of confidence in waterflooding can be overcome. We developed the production management software in several stages. The 
first software was developed for specific Spraberry field only and under excel visual basic platform. We found a Graphic User Interface (GUI) is limited in this platform thus we changed our platform to visual basic-object oriented programming language. In the current version, the software can be used for general purposes, so it can be used to manage and analyze production and injection data in other field. The software has the capability to perform similar tasks as the OFM database system such as decline curve analysis, material balance, and buble map plot. In addition it has more unique features in grouping the wells, performing decline curve analysis for well by well and a group of wells, displaying the graph in $\mathrm{x}-\mathrm{y}$ plot, 2D and 3D map and much more.

There are many other features or applications of this software such as bubble map, isobaric map, PVT application, Material Balances of this software that can be found in the manual/tutorial presented in Appendix A.

Germania Spraberry Field Demostration Status. In the previous section, we forecasted the incremental oil recovery due to waterflood in other pilot area, which then was applied to the Germania Spraberry Unit (GSU). We proposed the new location of injectors based on the existing injectors' location and response of previous injectors to producers. We also identified the wells that have casing leaks using OFM based on the plot of water-oil ratio. In this section, we present the status of on-going field activities in the GSU pilot. We presented a report on maintaining the injection rate based on Voidage Replacement Ratio (VRR), polymer treatment and pressure observation results, and gathering the production data for each production wells, predicting the waterflood response and incremental oil recovery due to current waterflood. The VRR is estimated periodically. From the VRR calculation we found that the present of new nine wells altered the energy balance in this pilot. The amount of fluid produced was much higher than the total amount of water being injected. During this period, we suggested to increase the water injection rate at each six injectors. At current date, more water is produced from on-trend wells. It is required to increase injection rate to sustain the energy balance. In order to reduce water production, three wells (406A, 408A and 409A) that had high water production located close to the injectors were treated with polymer. After the polymer squeezing, it is anticipated that the water being injected will find different paths around these wells and increase the overall sweep efficiency of the waterflood project. The fluid level (FL) and pump intake pressure (PIP) were measured every three days in these treatment wells. After the polymer treatment, we expect that FL will increase and PIP will decrease. We found that GSU 406A and 408A respond as expectedly indicating the successful of polymer treatment. However, we found inconsistency of FL and PIP trends in GSU 409A indicating ineffective polymer treatment.

In order to understand the response outside the pilot area, we monitor the pressure at three observation wells (GSU 5, 20 and 317). Each observation well had been tested for casing leaks. The FL was shot every 2 weeks. During injection period, we found that FL and PIP responses of GSU 5 and GSU 317 follow the expected trend indicating that these wells have good responder to water injection. For GSU 20, we found that the waterflood responses are delayed.

The response of water injection was observed through each of production well. We gathered the production data and recorded them in two production data base management 
software, Oil Field Manager $\left(\mathrm{OFM}^{\mathrm{TM}}\right)$ and Field Management Database Software (FMDS). The FMDS software is the software we developed for better managing, predicting and tracking the production for this area. We are able to make the software more general and robust in handling more sporadic data set. Using this software, we summarize our findings as follows: 1) Most of the sudden increases in oil production are due to the new production wells drilled in 2003. 2) Two new wells (213A and 413A) located in off-trend direction and near injection wells, are not affected by waterflooding. Both wells produced at high initial oil production and later on declined rapidly. Water production rates decrease significantly with time indicating no response of water injection to these wells. 3) No significant response observes in the tract. 4) We observe the response in individual production well. We observed that the on-trend wells that are located close to the injectors experience high water production rate as expected. We found that most of the wells in Track 3 have responded well to water injection. It looks like the water pushes the oil more to the N-E direction than to the S-W direction. 5) Finally, using the FMDS software, we combined the sporadic production data from 22 wells that have good oil responses. We found that the incremental oil production on current date is about 30,000 bbls.

\section{Project Fact Sheet}

Progress work efforts at Project Fact Sheet are listed in the Appendix. 


\section{I.1 Review of Upper and Lower Pilots in the Spraberry Area}

Up to now, most of the oil production in the Spraberry area has been recovered from the Upper Spraberry. Several pilots have attempted to establish production from the Lower Spraberry. The objectives of this study are to review several waterflood pilots which can be used to justify the poor performance of Lower Spraberry and to propose a method to determine the location of the injection and production wells in order to increase the oil recovery from Spraberry area. A number of papers have been published concerning the performance in the Lower Spraberry concerning waterflooding as presented below.

In 1963 Elkins and Skov ${ }^{1}$ compared the water injection into Lower and Upper Spraberry in the Driver Unit. They found that the water injection into Lower Spraberry was less than Upper Spraberry because Lower Spraberry had one third-less injection wells, lower intake capacity and less volume of water injection.

No significant effect of injection had been noted in any lower Spraberry producing wells after injecting about 1,000 bbl per acre. They suggested that at least 2,000 bbl per acre of water injection were required to fill up the Lower Spraberry. The water injection volume required to fill up the Upper Spraberry is less, only 900 bbl per acre, because Upper Spraberry has lower saturation pressure and lower formation factor compared to what the Lower Spraberry has.

Even though the properties of the matrix and the presence of vertical fractures in the Lower sand are similar to those of the Upper sand, they doubted the oil recovery per acre would be the same due to the greater oil shrinkage and less fracture intensity.

Again in 1968, Elkins et $a l^{2}$ analyzed the effectiveness of water injection into the Lower Spraberry in the Area 1 of the Driver Unit. The water was injected separately into the Upper and Lower Spraberry. The first water injection began on March 31, 1961. Through June 1968 about 1,640 bbl/acre of water had been injected into the Lower Spraberry. Some of the water injection had been lost through leaks to the Upper Spraberry. Production performances before and after water injection indicate no significant water breakthrough had occurred in any producing wells from Lower Spraberry. The low injection capacities of about 200 to $1,000 \mathrm{~b} / \mathrm{d}$ with 300 to 400 psi surface pressure in Lower Spraberry compared to 10,000 to $15,000 \mathrm{~b} / \mathrm{d}$ on vacuum or at moderate surface pressure in Upper Spraberry suggested that continuity and intensity of natural fractures is less in the Lower Spraberry than in the Upper.

In the same year, Guidroz ${ }^{3}$ reviewed the performance of the water injection in Lower Spraberry of the Preston Unit. In this Unit the water was injected at the Upper and Lower Spraberry simultaneously with approximately constant injection rate of 1000 bpd per injection well. The water injection was started in February 1965. It was divided equally between the Upper and Lower zones from start of injection to June 1968. Almost all injection wells were dually completed to permit independent injection into the Upper and Lower Spraberry zones. Injection into the lower zone was through the tubing and injection into the upper zone was down the tubing-casing annulus. Even though no significant problems had been encountered in obtaining desired injection rates, the corrosion problem existed particularly in the tubing-casing annulus. The corrosion 
problem affected at least six injection wells such that the tubing and tubing-casing annulus were in communication with one another. Cumulative water injected to August 1, 1968 was $614 \mathrm{bbl} /$ acre into the upper zone and $477 \mathrm{bbl} /$ acre into the lower zone. Again, there was no positive indication that the lower zone was responding to injection. Only the upper zone had responded to injection and had produced $80 \%$ of the production capacity. Due to the poor response of the Lower Spraberry and the corrosion problem in the injection well, he proposed discontinuing injection into the Lower Spraberry and ending production from Lower Spraberry by recompleting all injection wells as single Upper Spraberry wells and isolating the Lower zone in all production wells.

In the other pilot area, E.T O’Daniel Pilot Area, three wells, i.e. wells 17, 18 and 21 there was an attempt to establish Lower Spraberry production in that area. The results showed that the Lower Spraberry was non-commercial. Well 17 had been P\&A and well 21 was recompleted as an Upper Spraberry producer. Well 18 was plugged back to the Upper Spraberry and was converted into an injector in 1961.

The positive response of water injection in Lower Spraberry was observed in the JoMill (Spraberry) Field. ${ }^{5}$ The JoMill Field was discovered in February 1954 and unitized by Texaco in February 1, 1969. The Unit was originally developed on 80-acre proration units, with 40-acre development beginning in 1978. The waterflood pilot was initiated in September 1969 and consisted of 13 injection wells forming peripheral injection pattern. Following the improved production performance under the pilot, the peripheral water injection was expanded to the entire unit with conversion of another 31 injection wells in 1973. Starting in 1986, a total of 55 production wells were converted to injectors to establish a line drive injection pattern. In 1996 the unit had 173 producers and 92 injectors. The production had steadily increased from an average of $2000 \mathrm{bpd}$, before pilot waterflooding was implemented, to about 6000 bpd in 1996. In 1996 the first horizontal injection well (well 1425H) was drilled between two rows of producers. A significant response of oil production was observed. The oil production rate from offset wells increased from an average of 100 bpd in May ' 97 to an average of 425 bpd in Nov '98.

This paper reviews the waterflooding performance in the four recent waterflood pilots. Three pilots were attempted in the Midkiff Unit and the other one was conducted in the E.T O'Daniel Pilot. Three recent water injection pilots were attempted in the Midkiff Unit to help establish Upper and Lower Spraberry production. They are "LowerMcDonald" pilot, "Upper-McDonald" pilot and "Upper and Lower-Heckman” pilot. We have divided the wells as on-trend and off-trend wells to investigate the relationship between placement of the injection and production wells to the oil production rate.

The "Lower-McDonald" was started in 18 July 1993 and continues until now. An average of $500 \mathrm{bwpd} /$ well was injected through four injectors. About 600,000 bbls of water was injected on 80-acre pilot in a five spot pattern. The location of the wells is shown in Fig. 1.1.1. The wells are grouped into on-trend and off-trend wells following the fracture orientation obtained from core analysis. The "star" symbol indicates an off-trend well and the "circle" symbol indicates an on-trend well. Six wells were taken as off-trend wells and eight wells were taken as on-trend wells. The off-trend and on-trend production 
responses are shown in Figs. 1.1.2 and 1.1.3. To differentiate the cause of production response, either from waterflooding or adding more wells, the number of wells is plotted in the same graph. The off-trend and on-trend production wells show no response to waterflooding. The positive response was only due to adding more wells. We are, thus, unable to quantify additional oil recovery from wells that were completed in the Lower zone.

Another McDonald pilot was started in 4 July 1993 and completed only in the Upper zone. The location of the pilot and wells is shown Fig. 1.1.4. Six wells were taken as offtrend wells and 15 wells were taken as on-trend wells. A total of 1,405,695 bbls of water with an average 500 bwpd were injected during the water injection period. The off-trend and on-trend responses are shown in Figs. 1.1.5 and 1.1.6. No waterflood response was observed in the off-trend wells whereas a significant waterflood response was noted in the on-trend wells. Even though one well was added during the water injection, the production response was obviously from waterflood response alone. A decline curve was made to delineate the incremental oil recovery. The incremental oil recovery from the Upper Spraberry in the "Upper-McDonald" pilot after the waterflood baseline is shown in Fig. 1.1.7.

The third waterflood pilot reviewed in this study is the "Heckman" pilot. The injection wells were completed in the Upper and Lower zones. The 80-acre pilot location is located in Tr36 of the Midkiff Unit as shown in Fig. 1.1.8. Water injection was started in 1996. The number of wells grouped as on-trend and off-trend wells are 18 wells and nine wells, respectively. Both on-trend and off-trend wells show response to waterflooding as shown in Figs. 1.1.9 and 1.1.10. After the initiation of water injection, an immediate increase in oil production was noted in the on-trend wells whereas the response in offtrend wells was delayed as expected. Higher production response is obtained in the ontrend wells than in the off-trend wells. The incremental gain of oil recovery after the waterflood baseline is about $5-8$ bbls for each of the on-trend wells that responded and about 0-5 bbls for each of the off-trend wells as shown Fig. 1.1.11.

The last pilot is the E.T O'Daniel Pilot as part of the DOE project. Additional information of this project can be found in our annual reports. ${ }^{6}$ Water injection started in October 1999. We have divided oil production into 23 off-trend and 7 on-trend wells and the results are shown in Fig. 1.1.12. Incremental oil production has steadily increased in the on-trend wells to a current level near 150 bopd (Fig. 1.1.13). This represents an incremental gain of $20-25$ barrels for each of the seven wells that responded.

\section{Conclusion}

1. Based on the comparison performance with Upper Spraberry, the probable reason of poor performance of the lower Spraberry can be summarized as follows:

o Low water intake capacity indicates probability of the lesser extent of the fracture system.

o Low volume of water injection (bbl/per acre).

o High oil shrinkage factor. 
2. The result from four waterflood pilots indicates that the on-trend wells respond favorably compared to the off-trend wells. Thus, placing production and injection wells along the same fracture orientation may increase oil recovery in the Spraberry area.

\section{References}

1. Elkins, L.F and Skov, A. M.: "Cyclic Water Flooding the Spraberry Utilizes "End Effect” to Increase Oil Production Rate,” JPT (Aug., 1963), 877.

2. Elkins, L.F., Skov, A. M., and Gould, R.C.: "Progress Report on Spraberry Waterflood Reservoir Performance, Well Stimulation and Water Treating and Handling” Trans., AIME (1968) 243.

3. Guidroz, G.M.: Proposal to Discontinue Injection into and Production from Lower Spraberry, Preston Spraberry Unit. Mobil Oil Corporation, Interim report (Oct., 1968)

4. Carr, L.A. and Thiebaud, R.L.: “E.T. O’Daniel Performance Review, Spraberry Trend Area Field, Midland County, Texas,” Interoffice Correspondence (Jan 15, 1979).

5. Texaco Exploration and Production Inc.: "Modern Reservoir Management: Improved Recovery in The JoMill (Spraberry) Field, West Texas” U.S. DOE Proposal No. DEPS26-99BC15144 (May 1999).

6. Schechter, D.S.: “Advanced Reservoir Characterization and Evaluation of $\mathrm{CO}_{2}$ Gravity Drainage in the Naturally Fractured Spraberry Trend Area,” Six Annual Technical Progress Report, U.S. DOE Contract No. DE-FC22-95BC14942 (Oct 2001). 


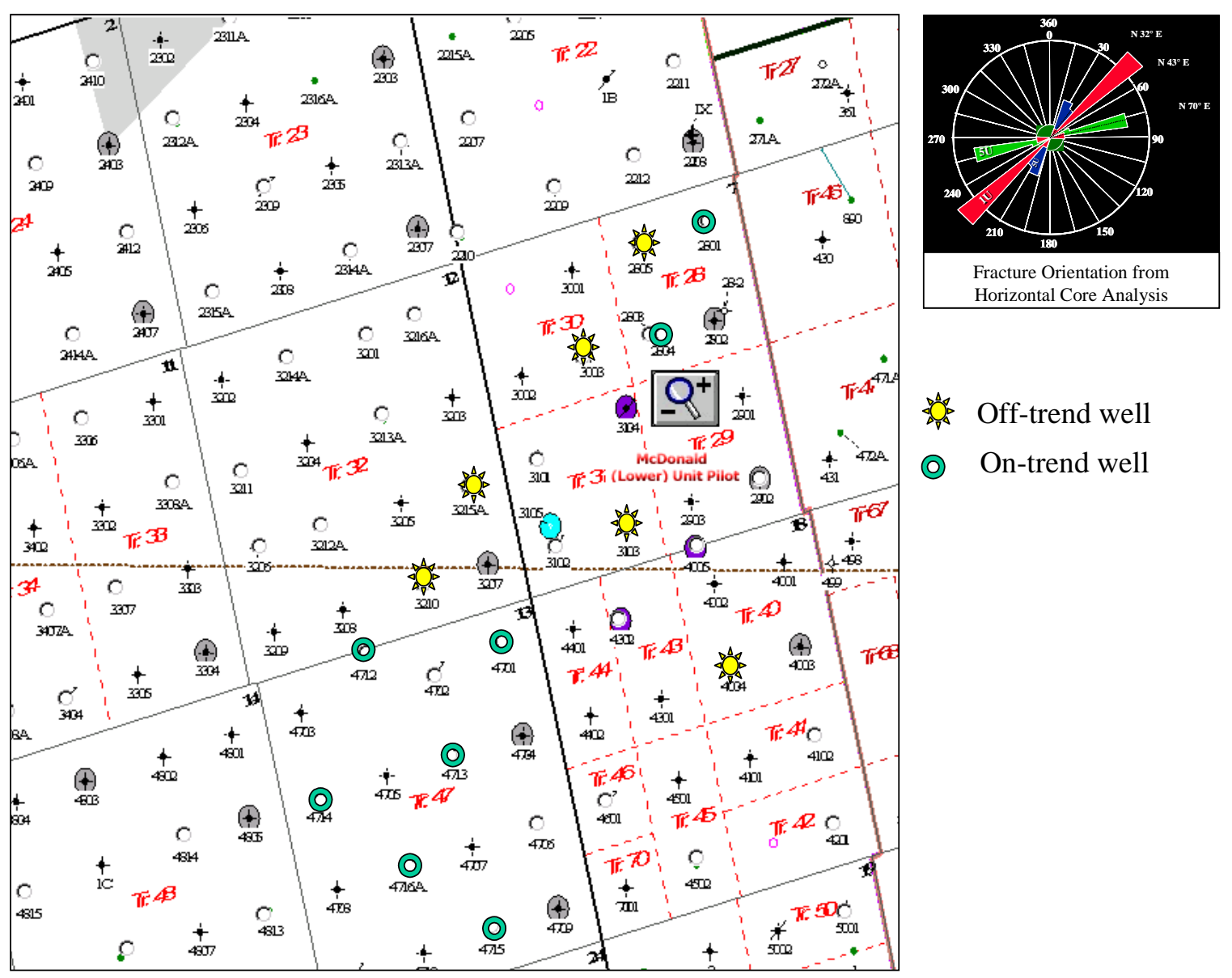

Fig. 1.1.1 - Location of On-trend and Off-trend wells in the Lower Spraberry of Midkiff Unit “McDonald” Waterflood Pilot. 


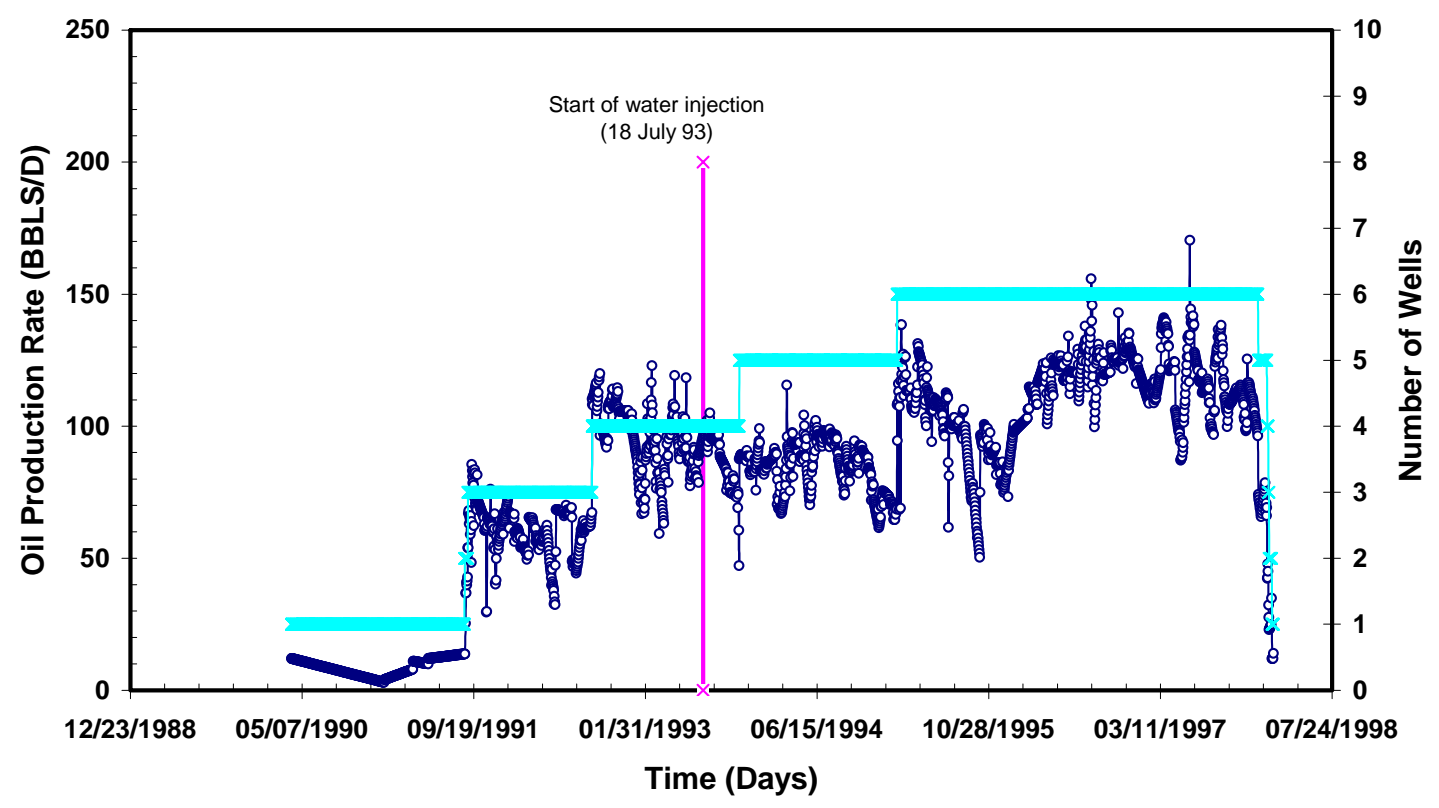

Fig. 1.1.2 - The off-trend production response of Lower Spraberry (Midkiff Unit “McDonald” Waterflood Pilot) from Wells \#2805, 3003, 3210, 3215A, 4004 and 3103.

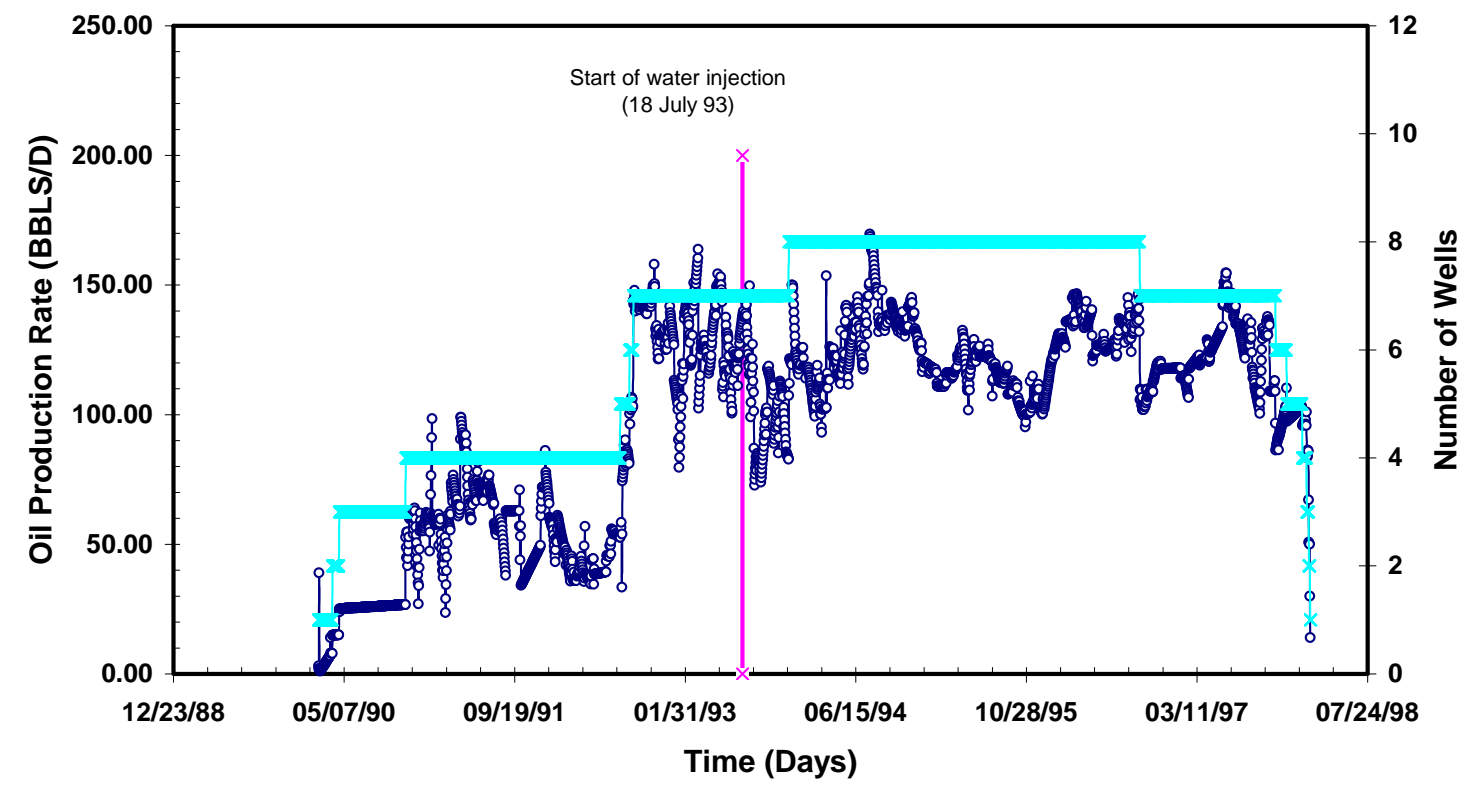

Fig. 1.1.3 - The on-trend production response of Lower Spraberry (Midkiff Unit “McDonald” Waterflood Pilot) from Wells \#2801, 2804, 4701, 4702, 4712, 4714, 4715 and 4716A. 


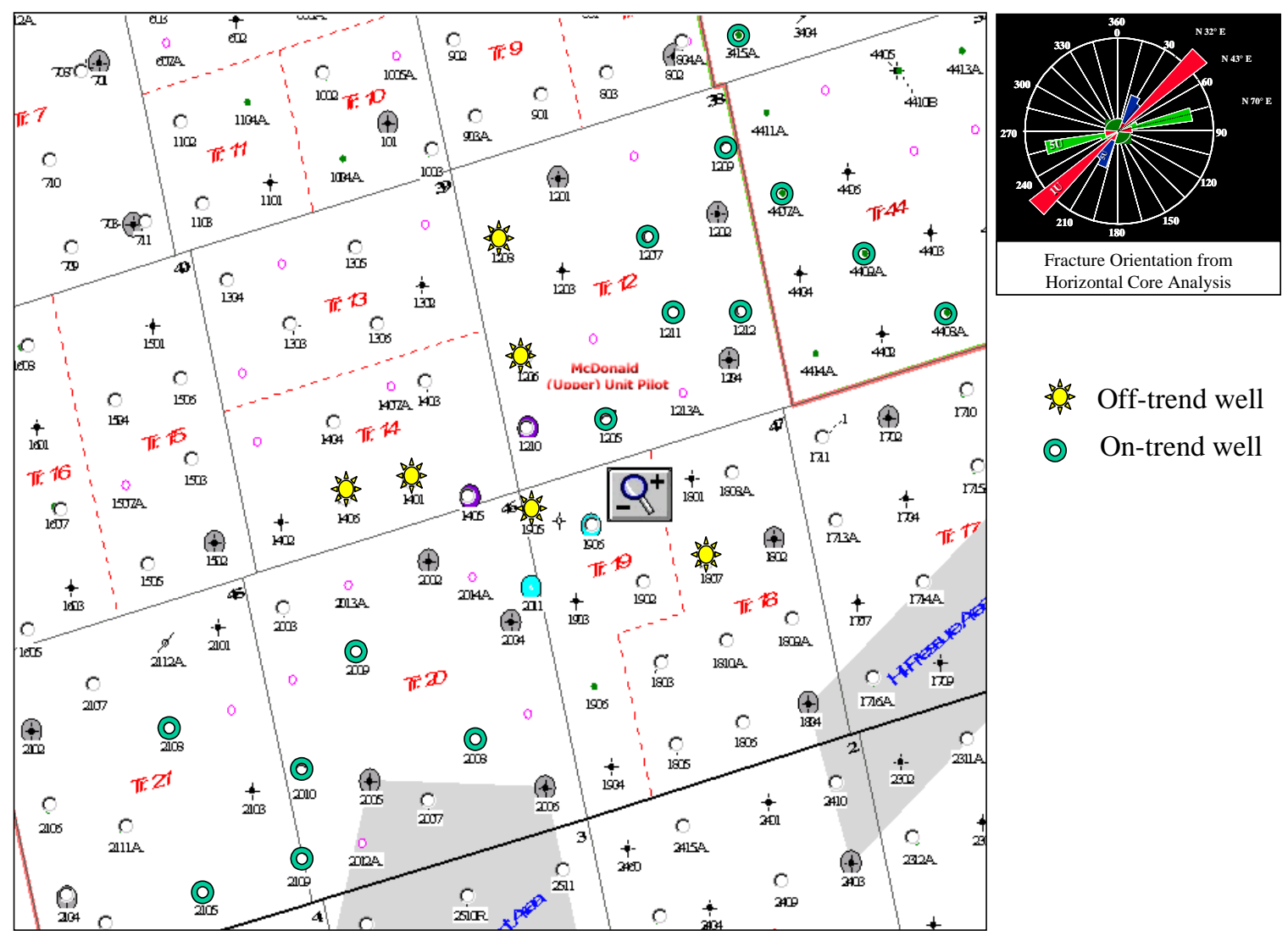

Fig. 1.1.4 - Location of On-trend and Off-trend wells in Upper Spraberry of the Midkiff Unit “McDonald” Waterflood Pilot.

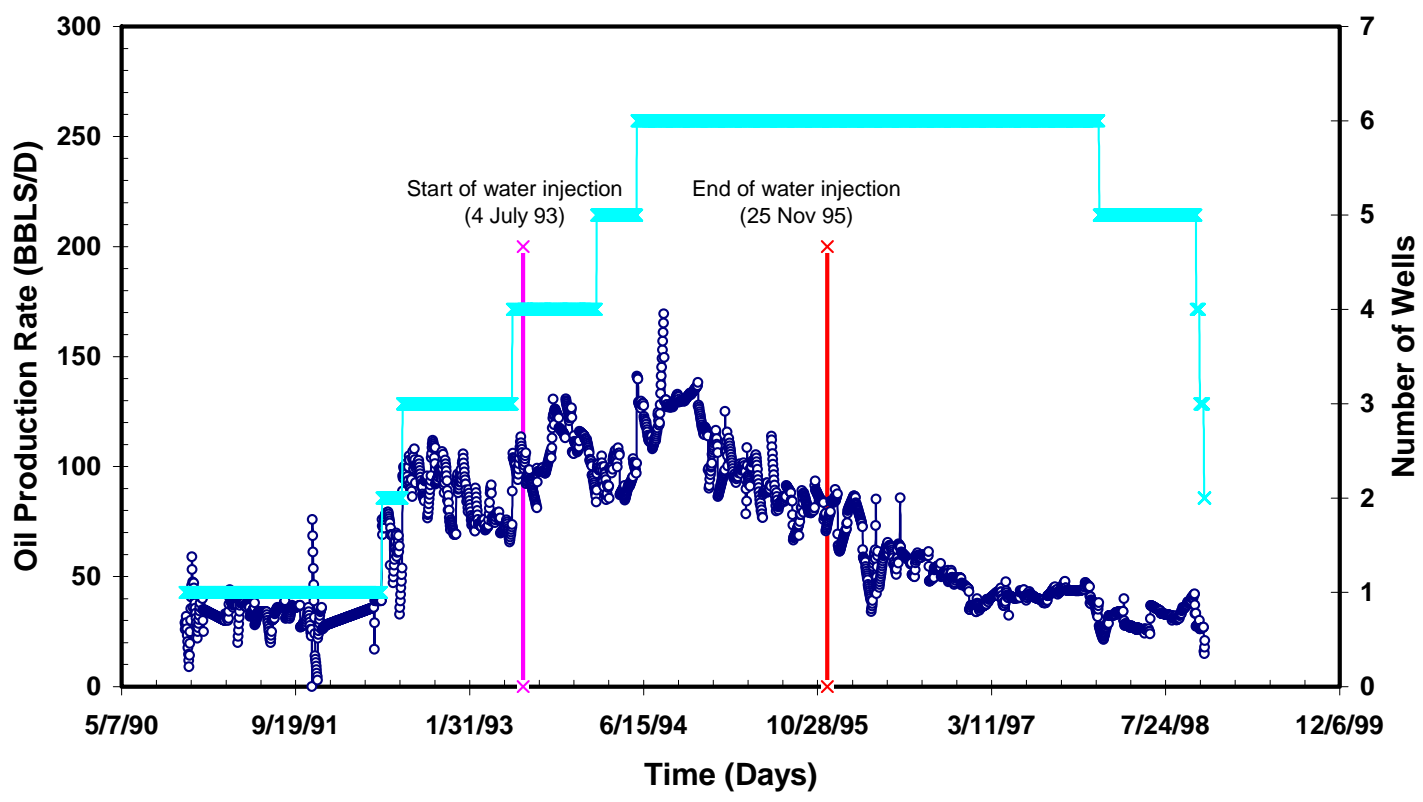

Fig. 1.1.5 - Off-trend production response of Upper Spraberry (Midkiff Unit "McDonald Waterflood Pilot”) from Wells \#1206, 1208, 1401, 1406, 1807 and 1905. 


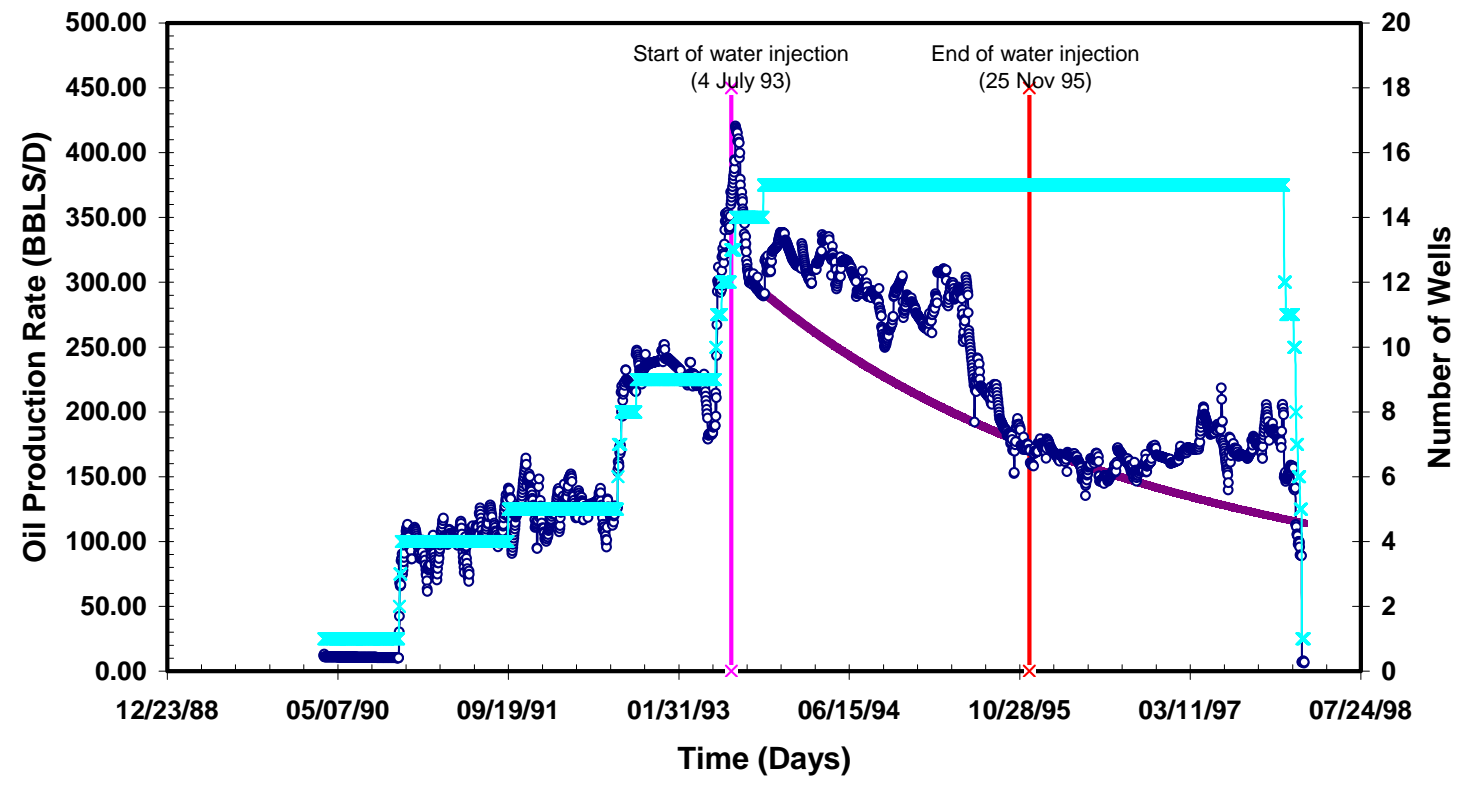

Fig. 1.1.6 - On-trend production response of Upper Spraberry (Midkiff Unit “McDonald” Waterflood Pilot) from Wells \#1205, 1207, 1209, 1211, 1212, 2008, 2009, 2010, 2105, 2108, 2109, PU\#3415A, PU\#4407A, PU\#4408A and PU\#4409A.

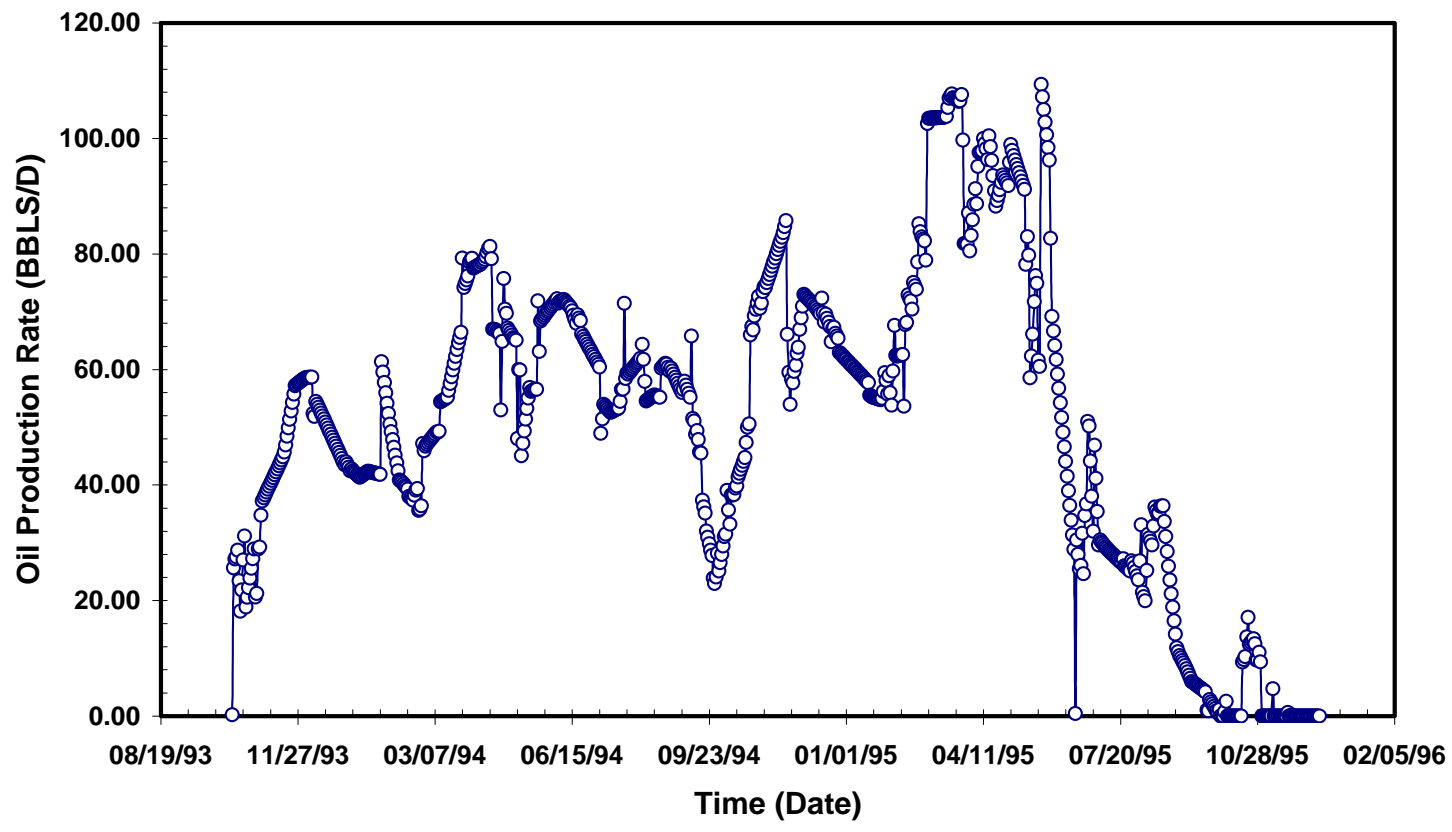

Fig. 1.1.7 - Incremental oil recovery of Upper Spraberry (Midkiff Unit “McDonald” Waterflood Pilot) from on-trend wells after waterflood baseline. 


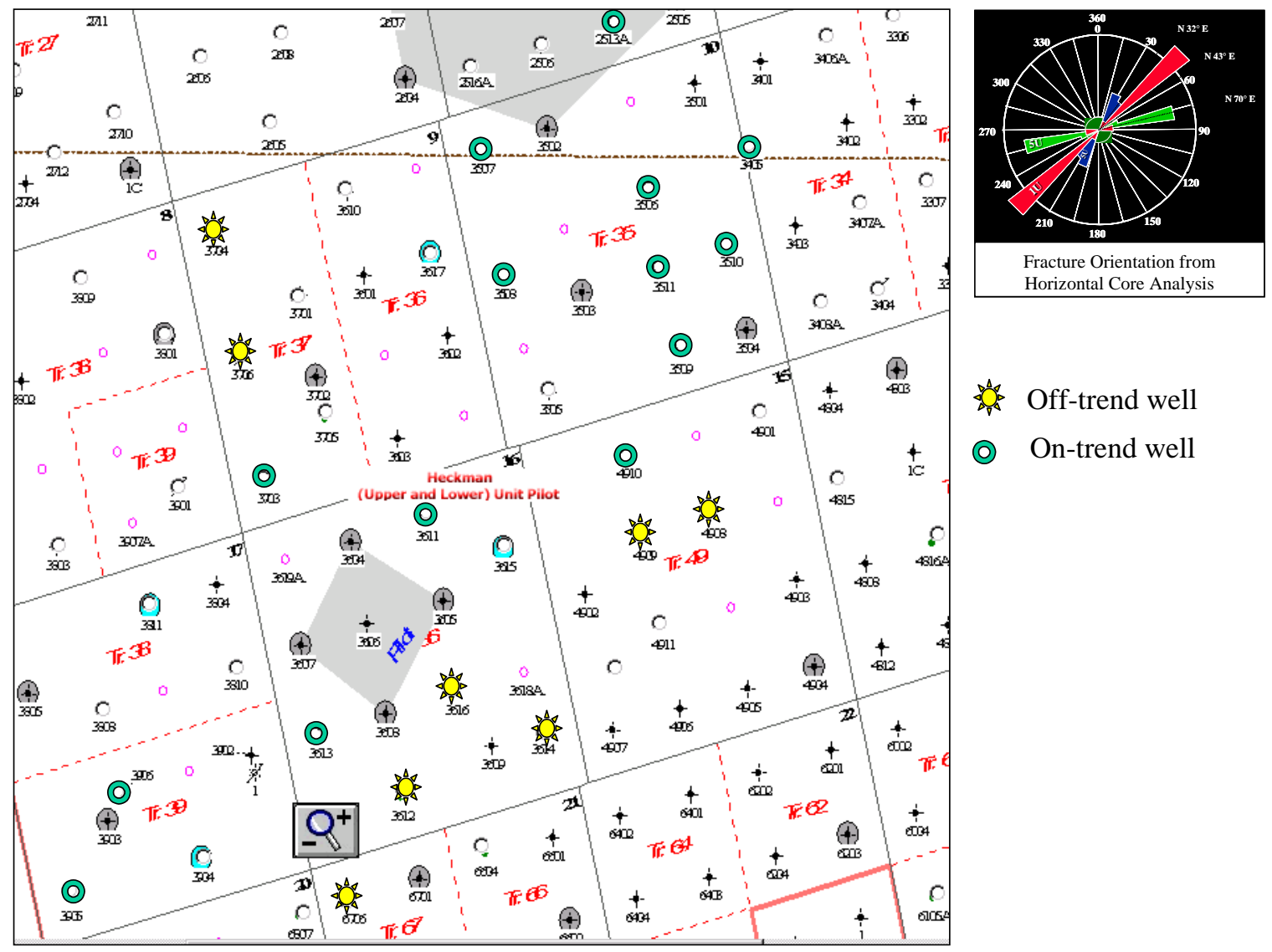

Fig. 1.1.8 - Location of On-trend and Off-trend wells in the Lower and Upper of Midkiff Unit “Heckman” Waterflood Pilot.

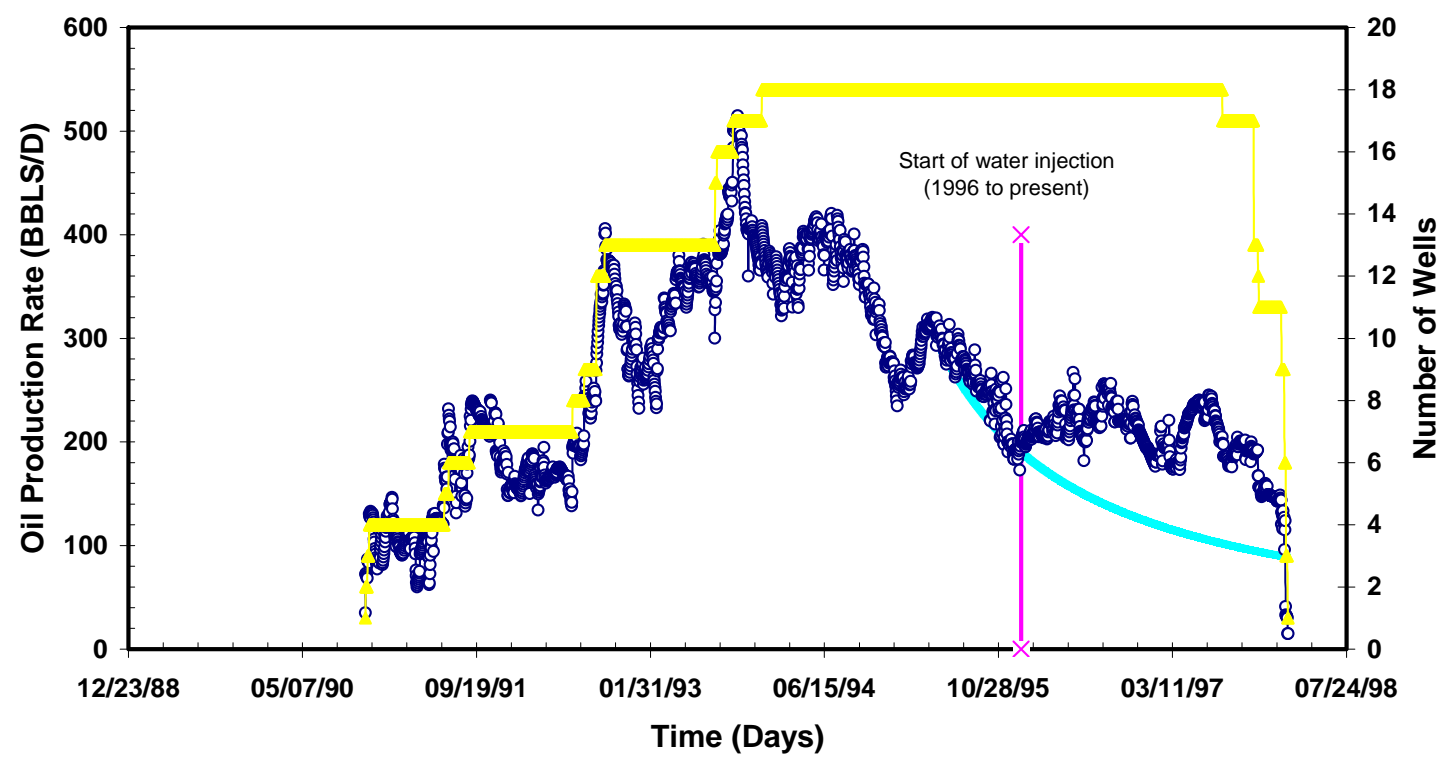

Fig. 1.1.9 - On-trend production response of Upper and Lower Spraberry (Midkiff Unit “Heckman” Waterflood Pilot) from wells \#2413A, 2513A, 3405, 3506, 3507, 3508, 3509, 3510, 3511, 3611, 3613, 3703, 3705, 3808, 3810, 3905, 3906 and 4910. 


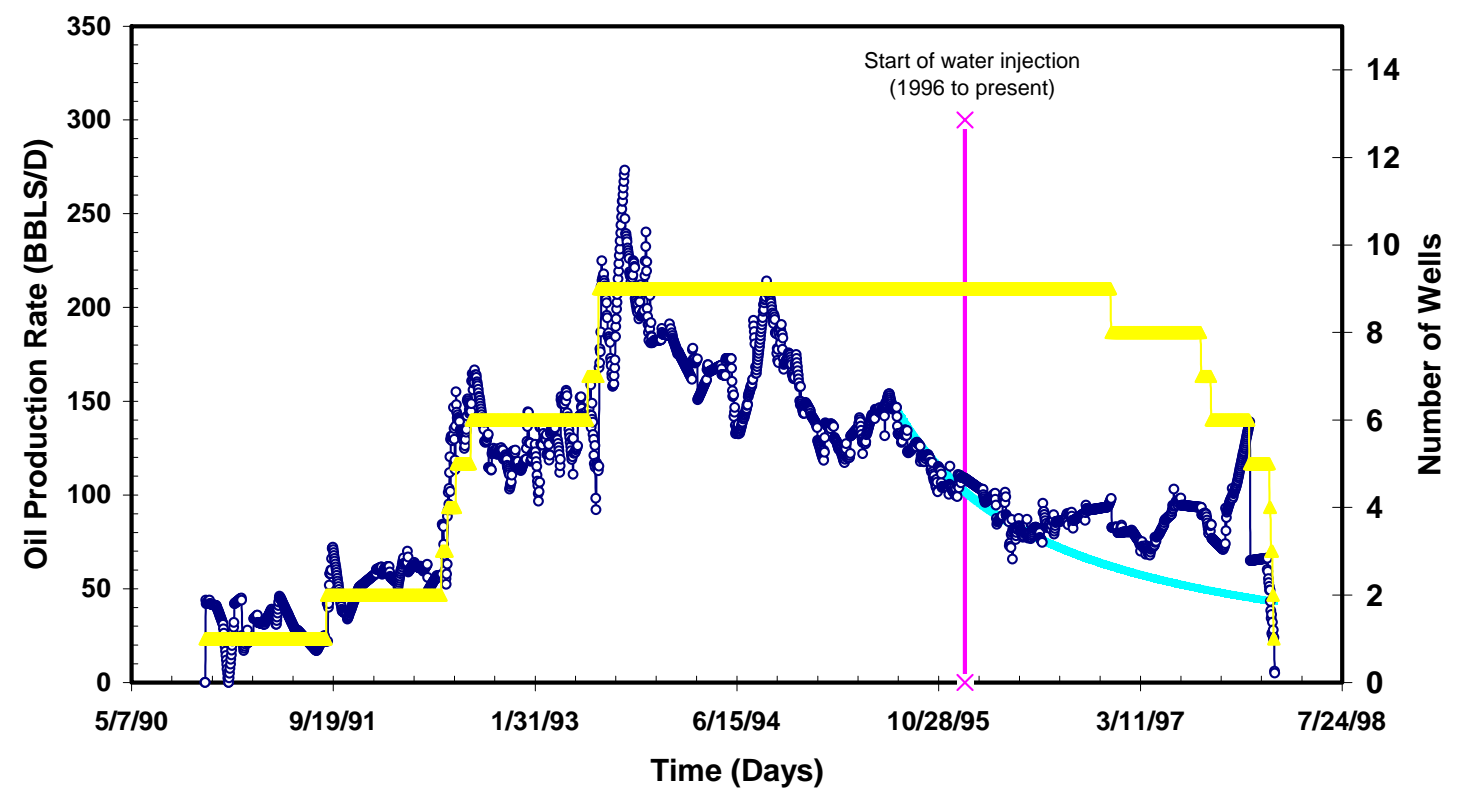

Fig. 1.1.10 - Off-trend production response of Upper and Lower Spraberry (Midkiff Unit “Heckman” Waterflood Pilot) from wells \#3612, 3614, 3616, 3704, 3706, 4908, 4909, 6706 and 6807.

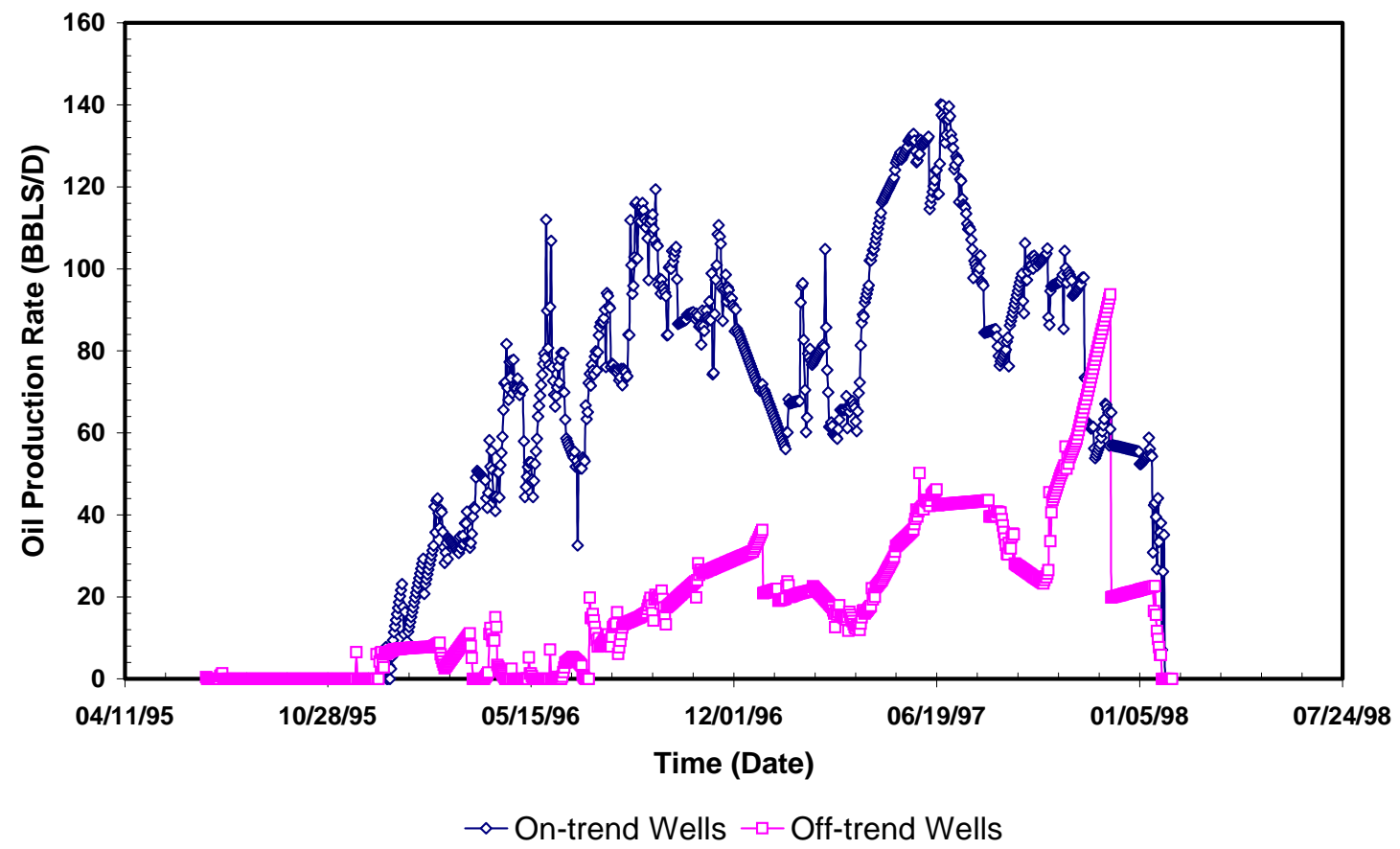

Fig. 1.1.11 - Comparison of incremental oil recovery between off-trend and on-trend wells of Upper and Lower Spraberry (Midkiff Unit “Heckman” Waterflood Pilot). 


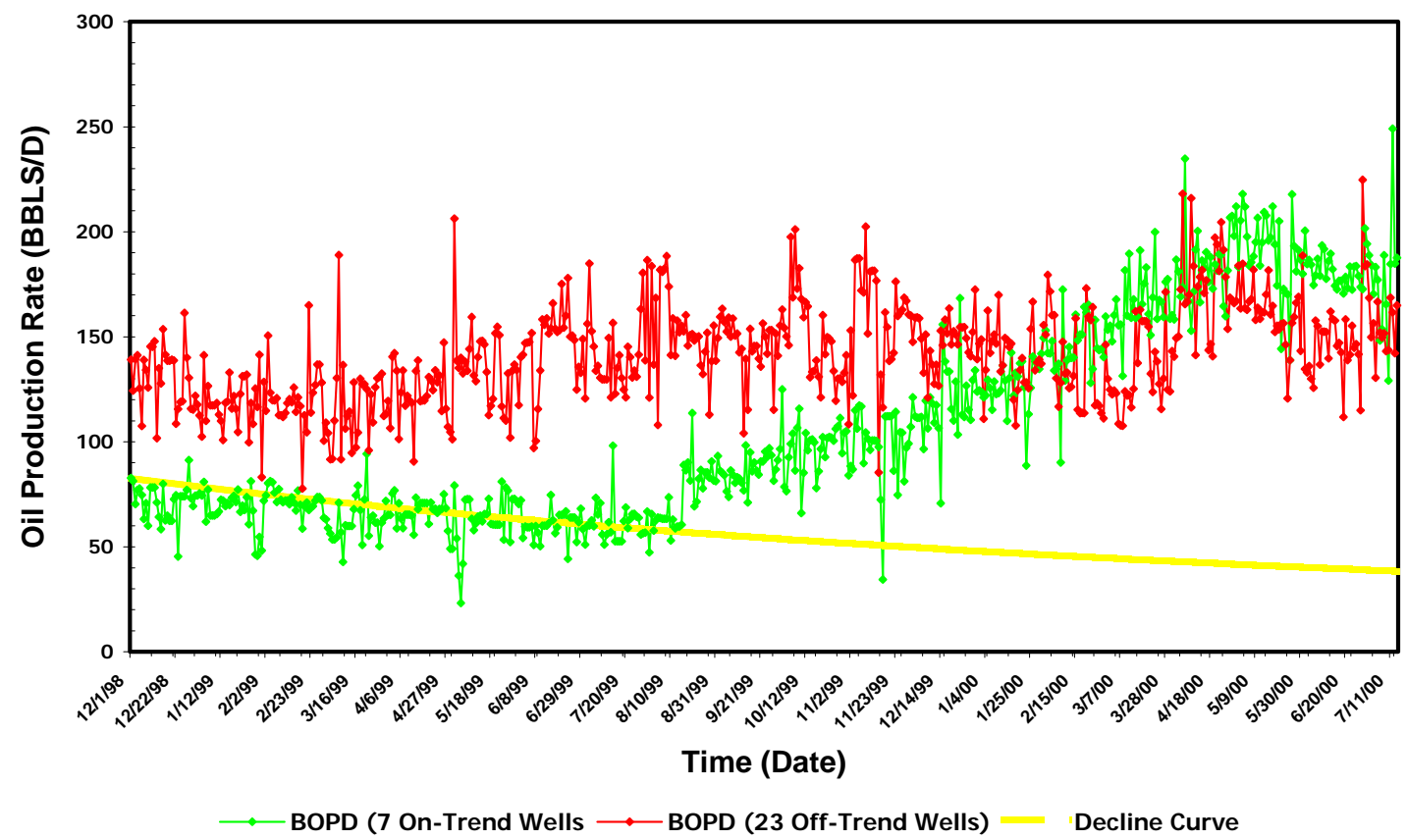

Fig. 1.1.12 - Comparison between on-trend and off-trend production rate of Upper Spraberry in the ET O'Daniel Pilot.

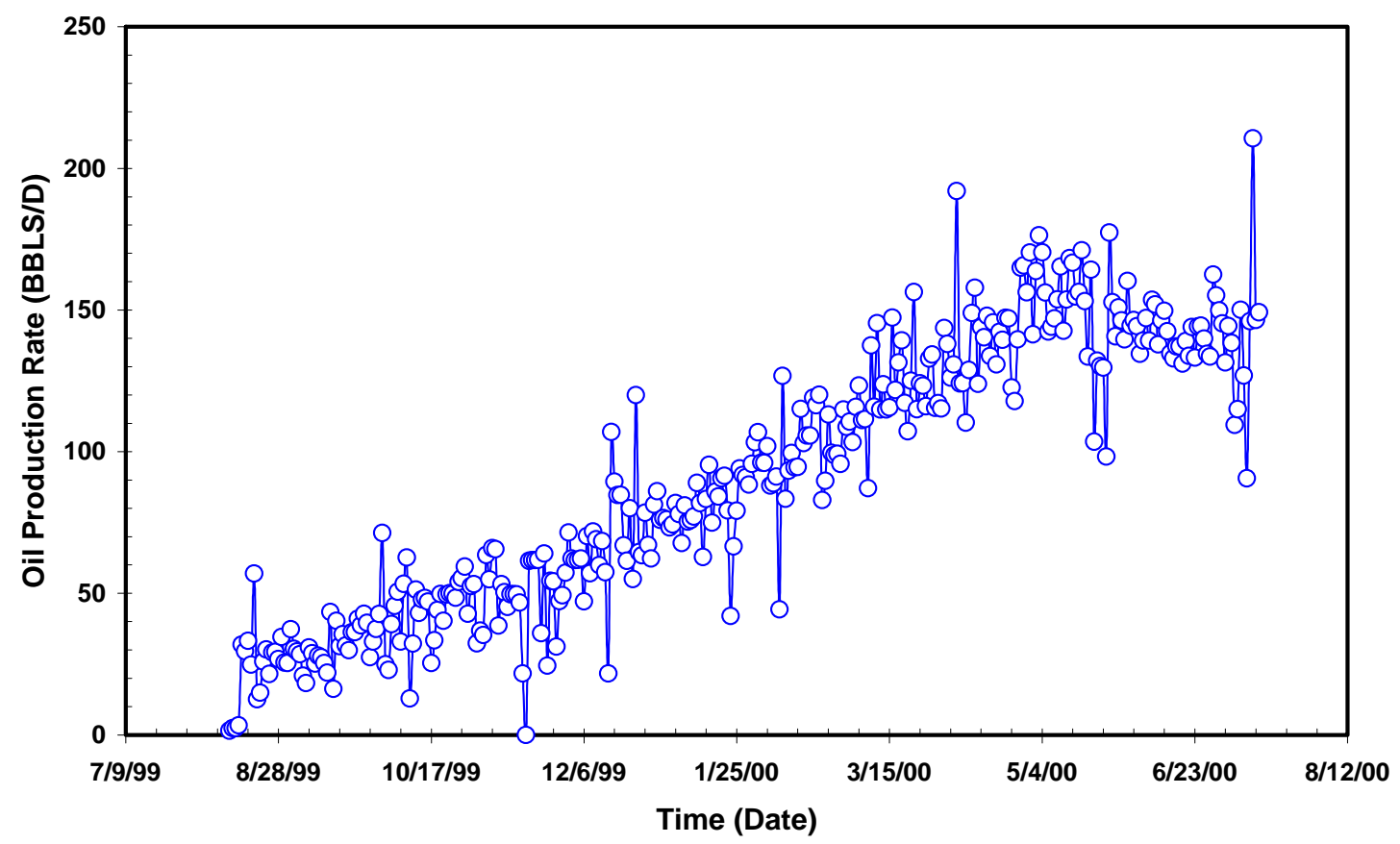

Fig. 1.1.13 - On-trend incremental oil recovery of Upper Spraberry in the ET O’Daniel Pilot. 


\section{I.2 Germania Unit Rate Forecasting Based on Other Waterflood Pilots in the Spraberry Area}

\subsubsection{Summary}

Due to the favorable results from DOE/NPTO Class III Field Demonstration Project in E. T O’Daniel waterflood pilot (Schechter et al, 2002), we have extended our waterflood techniques in the Germania unit. Based on recent technological development and intensive research, both basic and applied, we have gained a new understanding of proper procedures for waterflooding Spraberry reservoirs.

The Germania unit is selected for a waterflood pilot candidate based on several quantitative and qualitative criteria such as history of waterflood (cumulative injection and production per acre), condition and number of existing injection and production wells, source of water injection, surface facilities etc. The Germania unit as well as other waterflood units in the Spraberry Area had been waterflooded with the conventional waterflood techniques applied in natural fractured reservoirs elsewhere, where all injection wells were aligned parallel along major fracture trend in order to force the oil to flow in a direction perpendicular to the fracture trend towards a line of production wells. Based on our experience in this field and responses from successful waterflood projects, the injection wells should be aligned to production wells along the fracture orientation to have fast responses and viable waterfloods. The pattern configuration for both waterflood techniques is presented in Fig. 1.2.1.

Many wells have been pre-maturely abandoned in the Germania Unit as well as in other units in the Spraberry Trend. Abandonment is the result of either low productivity or casing failures due to corrosive nature of San Andreas water. We catalog all the production and injection wells since casing failures imply that unrecovered reserved are near these wells and should be aligned immediately and directly along the fracture trend to mobilize and sweep this oil to production wells that are still active (Schechter et al., 2000).

The objectives of this report are, thus, to propose the location of new injection wells, to review wellbore status in Germania unit and to forecast the incremental oil recovery based on waterflooding performance in other waterflood pilot area in order to demonstrate the benefit of waterflooding in Germania unit area.

\subsubsection{Waterflood History of Germania Unit}

Figure 1.2.2 is the Germania unit layout with 31 wells that have available injection and production data. The waterfloding was initiated at the end of 1965 and continued until Jan 1992. We divided the on-trend and off-trend wells based on the location of injection 
wells to the production wells and fracture orientation. For example, water injection GSU6 is in the on-trend location to GSU-12 and off-trend location to GSU-3 and GSU- 13.

Any changes in water, oil and gas production during the waterflood period are classified as waterflood responses. The responses of waterflood to off-trend and on-trend wells are tabulated in Table 1.2.1. Sometimes it is difficult to differentiate which injection wells affected the production wells. For example, injection wells GSU-11 and GSU-22 that are located in off-trend direction to the production wells GSU-12 and GSU-21, respectively, seems to be affecting those production wells. By analyzing the response in details, we found that those production wells were affected by on-trend injection wells GSU-6 and GSU-27, respectively.

The line drawn between injection and production wells indicates a communication between those wells (coupling) during waterflood period. Couplings between injection and production wells obtained from this study are oriented to the known fracture orientation in NE-SW direction (Schechter et al, 2002). Not all injection wells affected the offset production wells. Injection wells GSU-8, GSU-11 and GSU-15 did not have significant effect on surrounding oil production wells, either in the on-trend or off-trend production wells. The reasons behind this are still unclear. It may be inadequate volume of water that has been injected or lack of fracture density around this area.

Based on some criteria discussed previously, Germania unit has been chosen as a waterflooding pilot. The Pioneer Natural Resources proposed the location of new injection wells as shown in Fig. 1.2.2. The seven proposed injection wells have a blue circle symbol. From the results of coupling between injection and production wells show that the location of proposed injection wells GSU-408A and GSU-10 should be relocated because they are located on old injector path.

To have a better analysis of production data from Germania Unit and avoid the ambiguity analysis we collected and organized injection and production data followed by developing a database using software donated by Schlumberger (OFM). Using this software, we were able to see the old waterflood performance and identify casing failure due to the corrosive nature of San Andreas water resulting in premature abandonment of Spraberry well bores. This OFM software can also be interfaced with real time data acquisition so that daily analysis of response can be monitored and analyzed from remote location.

Figure 1.2.3 shows the history of cumulative water injection and the results of water injection on oil production (Fig. 1.2.4). It shows that wells located in the NE-SW location responded to water injection and water injection improved oil recovery in this area. The water injection not only affected the oil production but also increased the water production. Production wells GSU-10 and GSU-16 had the highest cumulative water production. When we plotted the cumulative water oil ratio (CWOR), it shows that the production wells GSU-1, GSU-315A and GSU-5 had the highest CWOR (Fig. 1.2.5). The high CWOR indicates that the wells might have casing leaks. When we consulted the casing leaks problem to the Pioneer, they also found the same problem in the field. Well 
GSU-1 has been plugged and abandoned. Well GSU-315A has been temporarily abandoned. The leaks in well GSU-5 have been fixed. Near these wells, the oil bucket is still unrecovered and should be mobilized to production wells that are still active.

Figure 1.2.6 shows the CWOR in the bubble plot map. The difference between this map and the previous contour map is that the bubble map shows actual magnitude of the number to identify trends and anomalies in a project while the contour map uses interpolation techniques i.e. kriging, spline and nearest neighbor to create the isolines, or areas, for a map image.

To identify the location of new wells (infill wells) and oil spot, we can employ the water cut map as our base analysis (Fig. 1.2.7). It becomes apparent from the map that the Germania unit has un-swept areas. Wells GSU-13, GSU-25 and GSU-17 would be good producers when the new water injection pattern concept is applied since the water cuts around those wells are still low. Low total oil production and insignificant total water injection indicate that the Germania unit is an excellent target for waterflooding. Figure 1.2.8 shows the water injection history of four injection wells and the cumulative water injection. The total water that had been injected was about 2.5 MMbbls for about 20 years. The production summary and the number of wells in the unit area are shown in Fig. 1.2.9. The average oil production per well was ranging from 4 to 29 bopd. The infill wells show instantaneous increase in oil production but later the production faded away. In Sep-92, the average production rate was 8.5 bopd and after adding a total of 23 wells in March-93, the production jumped to 28.7 bopd (Fig. 1.2.10). Similar production response was exhibited at successive infill wells.

The decline curve analysis was conducted to define the baseline of waterflooding so that the excess production due to infill wells and waterflooding can be quantified. Due to the nature of oil production rate from the naturally fractured reservoir, a hyperbolic-type decline curve was used to fit the production trend and forecast the future production rate. The following well-known decline curve equation is presented below:

$$
q_{i}=q_{o}\left(1+n d_{i} T\right)^{\frac{1}{n}}
$$

where $q_{o}$ is the initial rate (bopd), $n$ is the fractional power exponential decline (dimensionless), $d_{i}$ is the nominal decline rate (1/day) and $T$ is cumulative time (day).

Three cases were conducted in decline analysis:

1. Fitting decline curve to data points before the infill wells started to compare the performance of infill wells versus waterflooding.

2. Fitting decline curve to all data points after peak rate to analyze the future waterflood scenarios.

3. Starting decline curve analysis from the last data point.

The first case: Since the waterflooding in this unit was terminated around May-90 and the infill wells were started on Sep-92, thus, all incremental oil was a result of infill wells 
only. The result of decline curve analysis is depicted in Fig. 1.2.10. The decline curve was fitted until Mar-97 data points in order to take into account the production response due to infill wells. The incremental oil recovery obtained as a result of infill well was compared to the results of waterflooding in the ET O'Daniel Pilot. We divided two successful waterflood performances, old ET O’Daniel performance (Guidroz, 1967) and current ET O’Daniel waterflood pilot performance (1995-now) (Schechter et al, 2002). These two production performances were added on Sep-02 by assuming that the Germania unit would behave with a similarly response during water injection. The abrupt change shown in decline curve on Sep-02 was conducted to take into account the losing production rate due to conversion of 5 producers to injectors.

The cumulative oil comparisons between infill well and waterflooding scenarios are demonstrated in Fig. 1.2.11. The incremental oil due to 15 infill wells is similar at about 1100 days with incremental oil due to waterflood response from current ET O’Daniel performance. Meanwhile, the waterflood response from old ET O’Daniel performance needs only 400 days to reach the same production response due to infill wells. Even before conducting the economic calculation, we can justify that the waterflooding would be more economical than the incremental rate obtained from infill wells.

The second case: Again, we fitted the decline curve to all data points to analyze the future waterflood scenarios as shown in Figs. 1.2.12 and 1.2.13. We also used the old and current ET O'Daniel waterflood performance for our prediction. We did a sensitivity study to forecast the resulting incremental oil rate by changing nominal decline rate above and below base case decline as the case may be used for running project economics (Figs. 1.2.14 and 1.2.15).

As our last case, we did the decline analysis from the last data point as shown in Fig. 1.2.16. The old and current ET O'Daniel waterflood performances were also included for forecasting our incremental oil. We used current ET O’Daniel data as our best and pessimistic guesses and old ET O’Daniel data as our optimistic guess. This case also includes -14 bopd oil rate reduction due to negative impact of converting three known existing producers to injectors (Fig.1.2.17). If this unit follows the incremental oil recovery from ET O’Daniel waterflood, either old or current waterflood performances, we justify that the waterflooding would be economical and competing with other secondary projects.

\subsubsection{Waterflooding performances in the previous Germania unit and other units}

Waterflooding in the naturally fractured Spraberry Trend Area reservoirs has always been scorned resulting from ambiguity of performance data after water injection or results that did not conform to previously conceived notions. However, upon closer inspection, Spraberry water injection projects have all responded in a similar manner. Indeed, incremental oil was always recovered and production rates increased (Schechter et al., 2000). 
In order to analyze the old waterflood performances in Germania unit and other units, we gathered and compiled all production and injection data. Then reconstructed and developed injection/production data using Oil Field Manager (OFM) database. The example of production data from GSU-21 that was used as an input parameter for OFM database is presented in Fig. 1.2.18. A decline analysis was conducted to quantify the waterflood response (Fig. 1.2.19). All production wells in this unit have been analyzed and the waterflood response has been quantified (Appendix-A). A total of 80,000 bbls oil has been recovered due to waterflooding in this area over 25 years with total injection of 2.5 MMbbls (Fig. 1.2.20). The waterflood response in this unit resulted in a lack of confidence in water injection as a result many wells face abandonment. The small amount of oil that has been recovered due to waterflooding and negligible amount of water that has been injected for 25 years warrant that this unit would be potential for recovering oil with proper management practices during water injection.

We have reviewed waterflood performances in other units in order to demonstrate the benefit of waterflooding in this area and to reach a stage of development of management practices that could eventually help for conducting a successful water injection in Germania Units. The previous summary of waterflood projects in other units is summarized in Table 1.2.2 and can be found in PUMP semi annual report (Putra and Schechter, 2002). ET O’Daniel waterflood performance has been reviewed in PUMP proposal (Schechter and Putra, 2000) and Guidroz's paper (1967). We provide a production summary and decline analysis for this area as found in Appendix-B. An updated review of current ET O’Daniel pilot is presented in the next section.

\subsubsection{Conclusions}

1. Proposed injection wells GSU-408A and GSU-10 should be relocated because they are located on old injector path

2. Based on the high WOR map, production wells GSU-1, GSU-315A and GSU-5 indicates that these wells may have casing leaks.

3. Waterflooding in Germania Unit would be economical and competing with other secondary projects.

4. Spraberry water injection projects in other waterflood units recovered and increased oil production rates.

5. The amount of water injection rate per day in Germania unit was very low compared to the water injection rate in other waterflood units. Based on the injection rate in other pilots and by aligning the water injection parallel to production wells along the fracture orientation, this unit could be successfully flooded with total injection rates between 2000 and 2500 bwpd. 


\section{References}

McDonald, P., Lorenz, J. C., Sizemore, C., Schechter, D.S., and Sheffield, T.: "Fracture Characterization Based on Oriented Horizontal Core From the Spraberry Trend Reservoir: A Case Study” paper SPE 38664 presented at the 1997 Annual Technical Conference and Exhibition, San Antonio, Tx, 5-8 October.

Guidroz, G.M.: “E.T. O’Daniel Project - A Successful Spraberry Flood,” paper SPE 1791 presented at the 1967 Sixth Permian Basin Oil Recovery Conference, Midland, 8-9 May.

Schechter, D.S., Putra, E. and Knight, W: "Preferred Waterflood Management Practices for the Spraberry Trend Area," Proposal submitted to DOE contract No.: DE-FC2295BC14942 (August 2000).

Schechter, D.S.: “Advanced Reservoir Characterization and Evaluation of $\mathrm{CO}_{2}$ Gravity Drainage in the Naturally Fractured Spraberry Trend Area," Final Technical Report, DOE contract No.: DE-FC22-95BC14942 (June 2002).

Putra, E. and Schechter, D.S.: "Preferred Waterflood Management Practices for the Spraberry Trend Area,” Semi Annual Technical Report, DOE Contract No.: DE-FC2601BC15274 (Mar 2002) 
Table 1.2.1 - Coupling between injector and producers

\begin{tabular}{|c|c|c|c|c|c|c|}
\hline Injector & $\begin{array}{l}\text { Waterflood } \\
\text { period }\end{array}$ & $\begin{array}{l}\text { On-trend } \\
\text { well }\end{array}$ & Responding & $\begin{array}{l}\text { Off-trend } \\
\text { well }\end{array}$ & Responding & Remarks \\
\hline G.S.U. \#6 & $01 / 72-05 / 89$ & GSU\#12 & $\begin{array}{l}\text { Oil, gas and } \\
\text { water }\end{array}$ & $\begin{array}{l}\text { GSU\#3 } \\
\text { GSU\#13 }\end{array}$ & $\begin{array}{l}\text { None } \\
\text { None }\end{array}$ & \\
\hline G.S.U. \#8 & $08 / 71-02 / 77$ & GSU\#2 & None & $\begin{array}{l}\text { GSU \#1 } \\
\text { GSU \#7 }\end{array}$ & $\begin{array}{l}\text { Water } \\
\text { None }\end{array}$ & \\
\hline G.S.U. \#11 & $\begin{array}{l}12 / 65-01 / 68 \\
02 / 70-02 / 75\end{array}$ & $\begin{array}{l}\text { GSU\#31 } \\
\text { GSU\#13 } \\
\text { GSU\#3 } \\
\text { GSU\#20 } \\
\text { GSU\#19 } \\
\end{array}$ & $\begin{array}{l}\text { None } \\
\text { None } \\
\text { None } \\
\text { None } \\
\text { None } \\
\end{array}$ & $\begin{array}{l}\text { GSU\#10 } \\
\text { GSU\#17 } \\
\text { GSU\#12 } \\
\text { GSU\#7 } \\
\end{array}$ & $\begin{array}{l}\text { None } \\
\text { None } \\
\text { Oil, gas and } \\
\text { water } \\
\text { None } \\
\end{array}$ & $\begin{array}{l}\text { \#19 was } \\
\text { converted to } \\
\text { WIW } \\
\text { \#12 was affected } \\
\text { by WIW\#6 }\end{array}$ \\
\hline G.S.U. \#15 & 09/71 - 01/92 & GSU\#14 & $\begin{array}{l}\text { None } \\
\text { None }\end{array}$ & $\begin{array}{l}\text { GSU\#13 } \\
\text { GSU\#16 }\end{array}$ & $\begin{array}{l}\text { None } \\
\text { None }\end{array}$ & \\
\hline G.S.U. \#19 & $06 / 70-01 / 77$ & GSU\#10 & $\begin{array}{l}\text { Oil, gas and } \\
\text { water }\end{array}$ & $\begin{array}{l}\text { GSU\#20 } \\
\text { GSU\#9 }\end{array}$ & $\begin{array}{l}\text { None } \\
\text { Oil, gas and } \\
\text { water ??? }\end{array}$ & \\
\hline G.S.U. \#22 & $06 / 70-01 / 76$ & $\begin{array}{l}\text { GSU\#16 } \\
\text { GSU\#26 }\end{array}$ & $\begin{array}{l}\text { Oil, gas and } \\
\text { water } \\
\text { Oil, gas and } \\
\text { water }\end{array}$ & $\begin{array}{l}\text { GSU\#21 } \\
\text { GSU\#23 } \\
\text { GSU\#17 } \\
\text { GSU\#25 }\end{array}$ & $\begin{array}{l}\text { Water } \\
\text { None } \\
\text { None } \\
\text { None }\end{array}$ & $\begin{array}{l}\text { \#21 was affected } \\
\text { by WIW\#27 }\end{array}$ \\
\hline G.S.U. \#27 & $06 / 70-01 / 77$ & GSU\#21 & water & $\begin{array}{l}\text { GSU\#26 } \\
\text { GSU\#20 }\end{array}$ & $\begin{array}{l}\text { None } \\
\text { None }\end{array}$ & \\
\hline & & & & & & \\
\hline
\end{tabular}

Table 1.2.2 - Summary of waterflood projects

\begin{tabular}{|c|c|c|c|}
\hline Pilot area & $\begin{array}{c}\text { Duration of } \\
\text { waterflooding }\end{array}$ & $\begin{array}{c}\text { Cumulative water } \\
\text { injection/No. of } \\
\text { injection wells }\end{array}$ & $\begin{array}{c}\text { Cumulative oil } \\
\text { production/No. of } \\
\text { production wells }\end{array}$ \\
\hline Germania unit & $\sim 19$ & $\begin{array}{c}\sim 2,500,000(4) \\
(\sim 1000 \text { bwipd })\end{array}$ & $\sim 80,000$ (5) \\
\hline Current E.T O'Daniel & $\sim 3$ & $\begin{array}{c}\sim 1,600,000(6) \\
(\sim 2000 \text { bwipd) }\end{array}$ & $\sim 110,000$ (4) \\
\hline Old E.T O’Daniel & $\sim 26$ & $\begin{array}{c}\sim 17,600,600 \\
(\sim 2500 \text { bwipd })\end{array}$ & $\sim 2,200,000$ \\
\hline Midkiff “Mc Donald" unit & $\sim 2$ & $\begin{array}{c}\sim 1,400,000 *(4) \\
(\sim 2000 \text { bwipd) }\end{array}$ & $\sim 40,000$ (15) \\
\hline Midkiff “Heckman” unit & Since 1996 & - & $\sim 60,000$ (18) \\
\hline
\end{tabular}

*) injected only in Upper Spraberry layer 


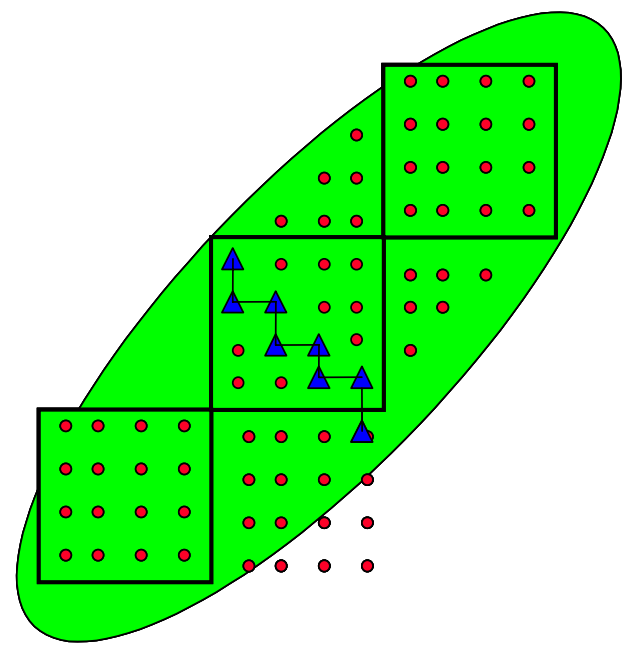

a. New waterflood technique

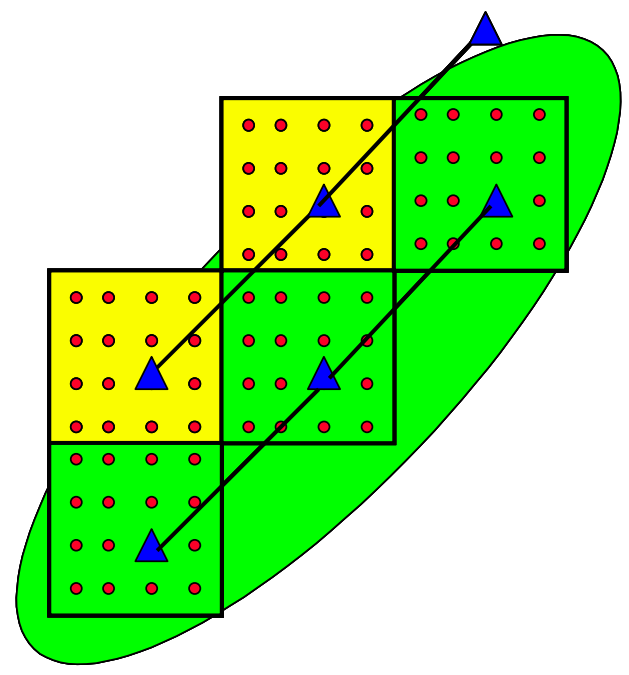

b. Old waterflood technique

Fig. 1.2.1 - The new and old waterflood patterns applied in Naturally Spraberry Trend Area. 


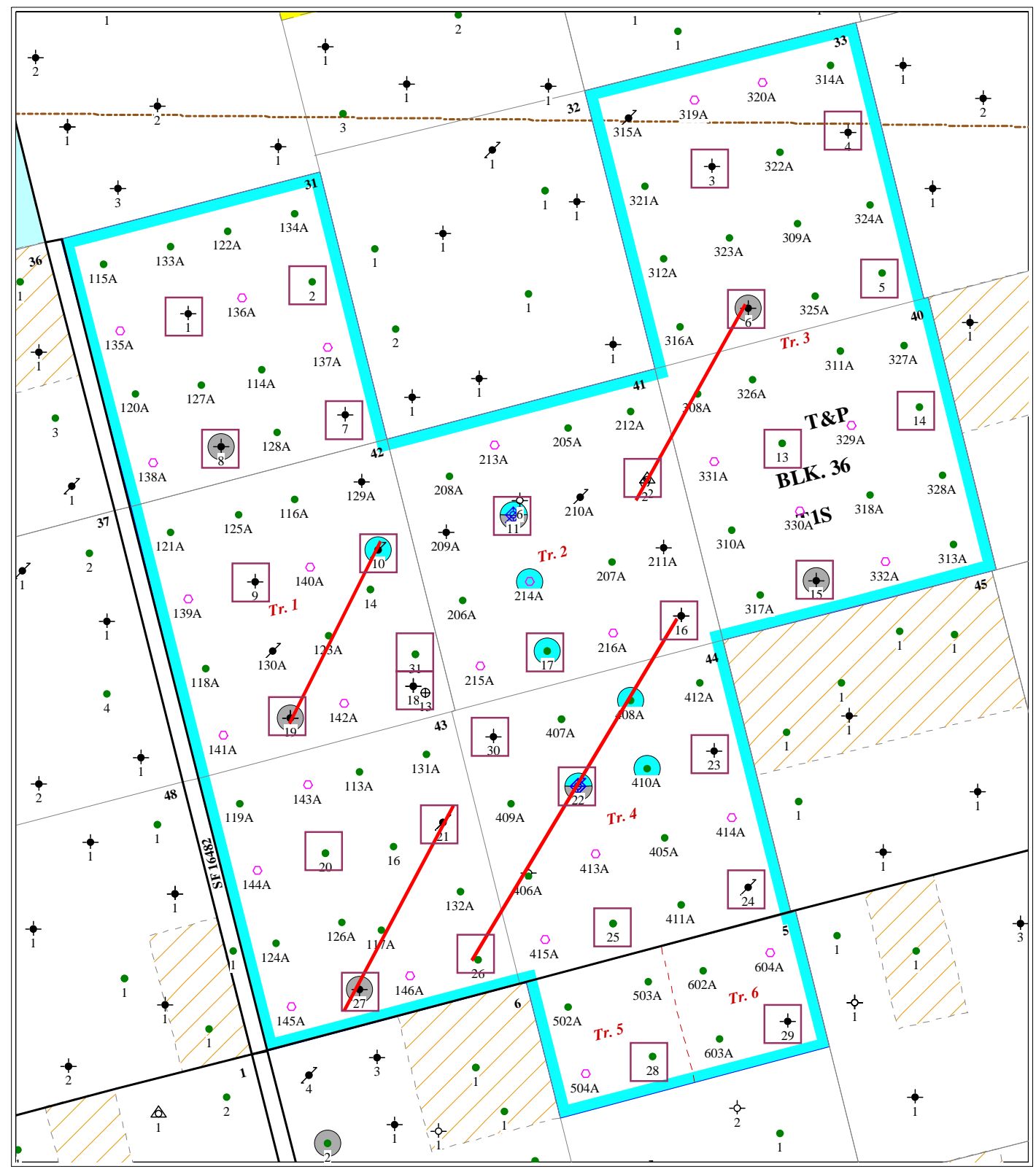

Fig. 1.2.2 - The Germania Unit Layout showing couple between injectors and producers taken from old waterflood history. 


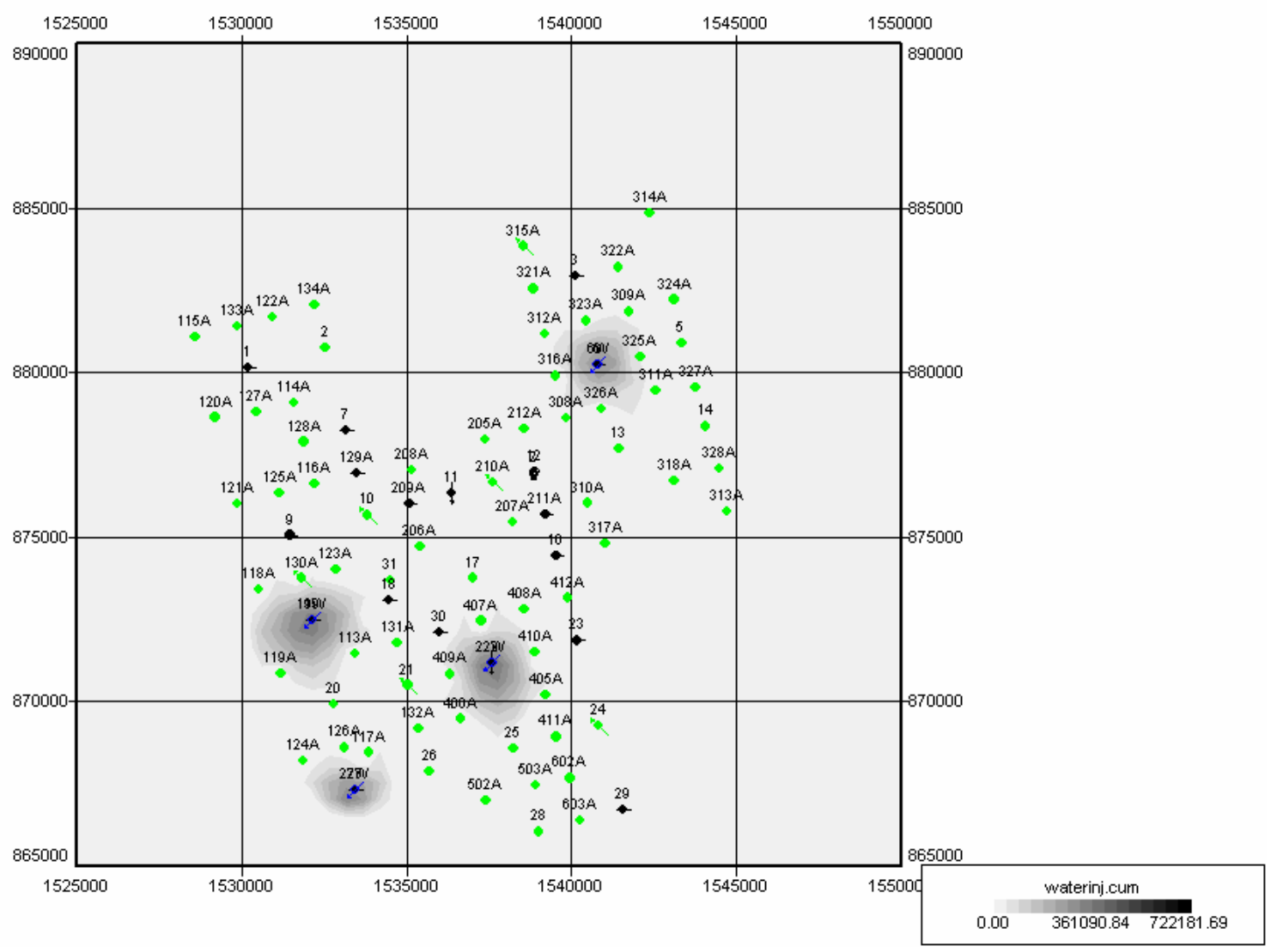

Fig. 1.2.3 - Cumulative water injection map. 


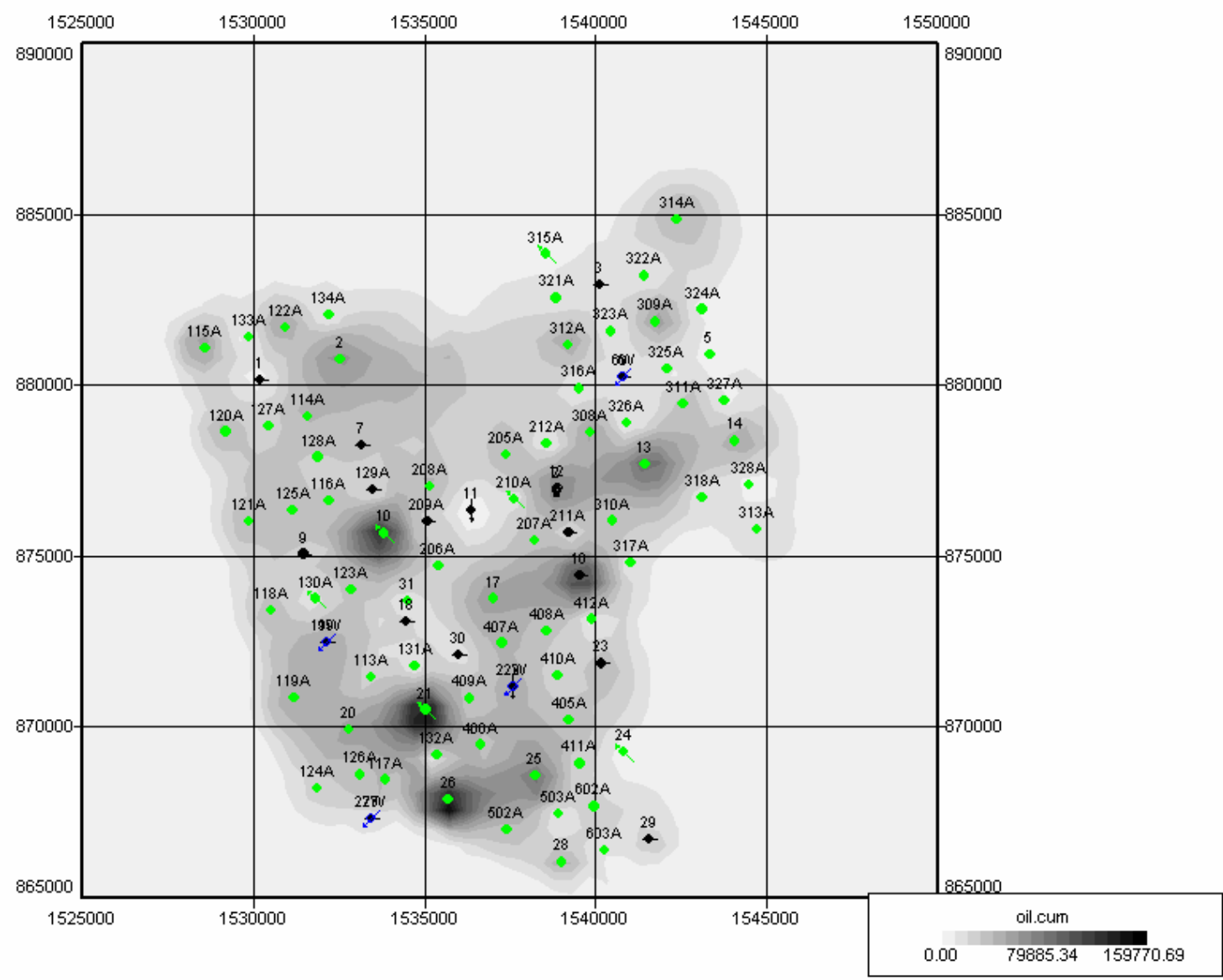

Fig. 1.2.4 - Cumulative oil production map. 


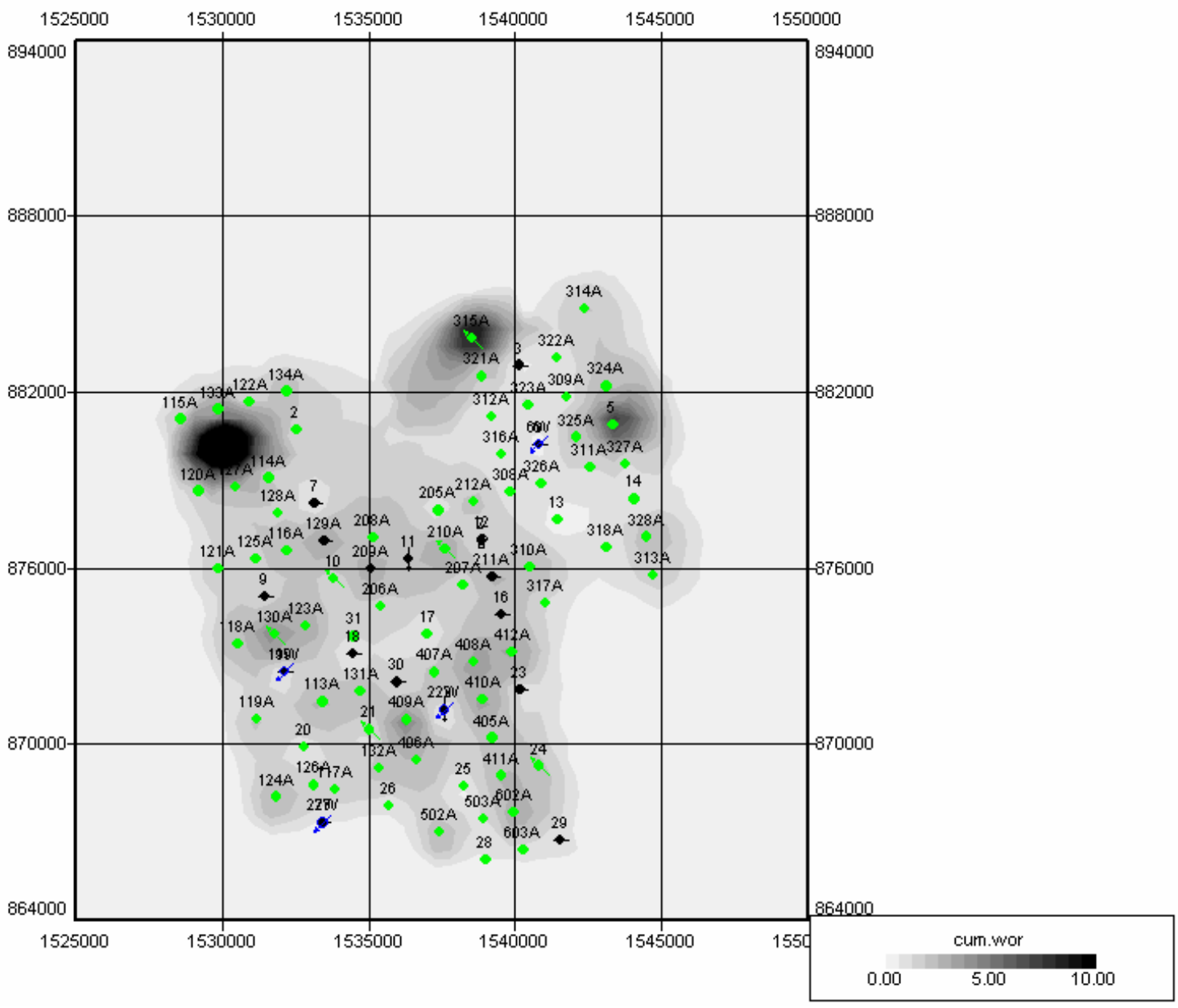

Fig. 1.2.5 - Cumulative water oil ratio map. 


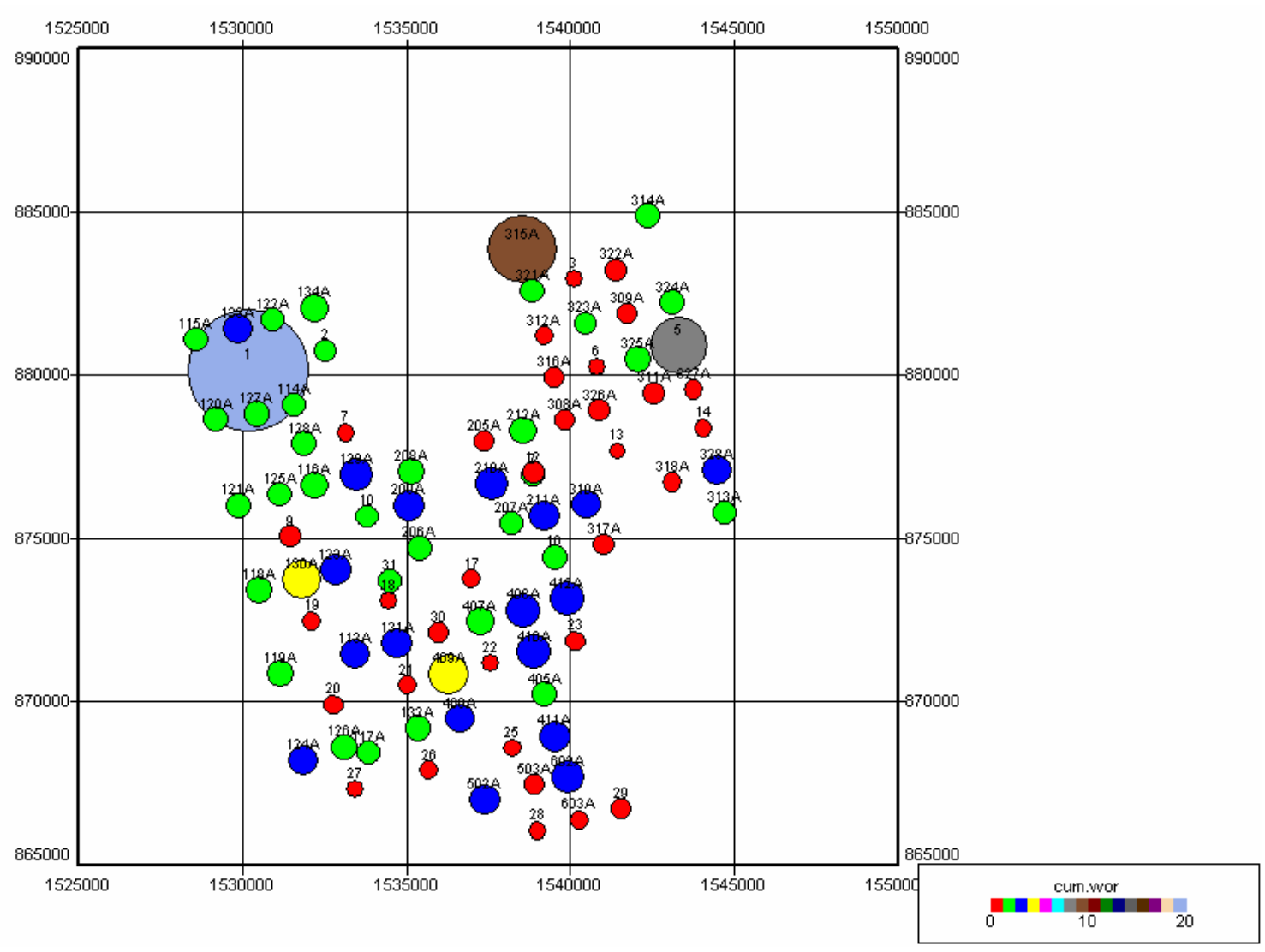

Fig. 1.2.6 - Cumulative water oil ratio in bubble map. 


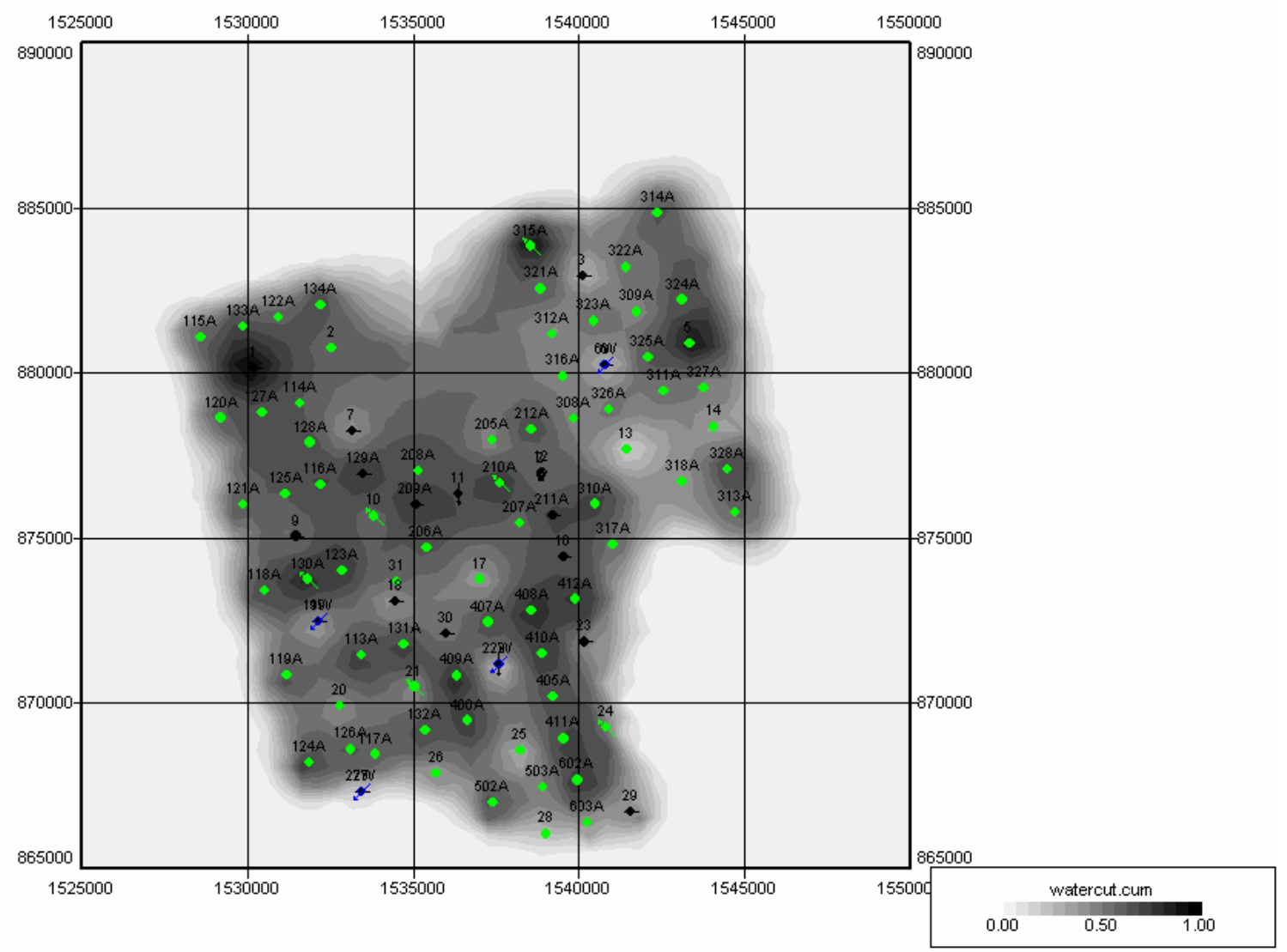

Fig. 1.2.7 - Cumulative water cut map. 


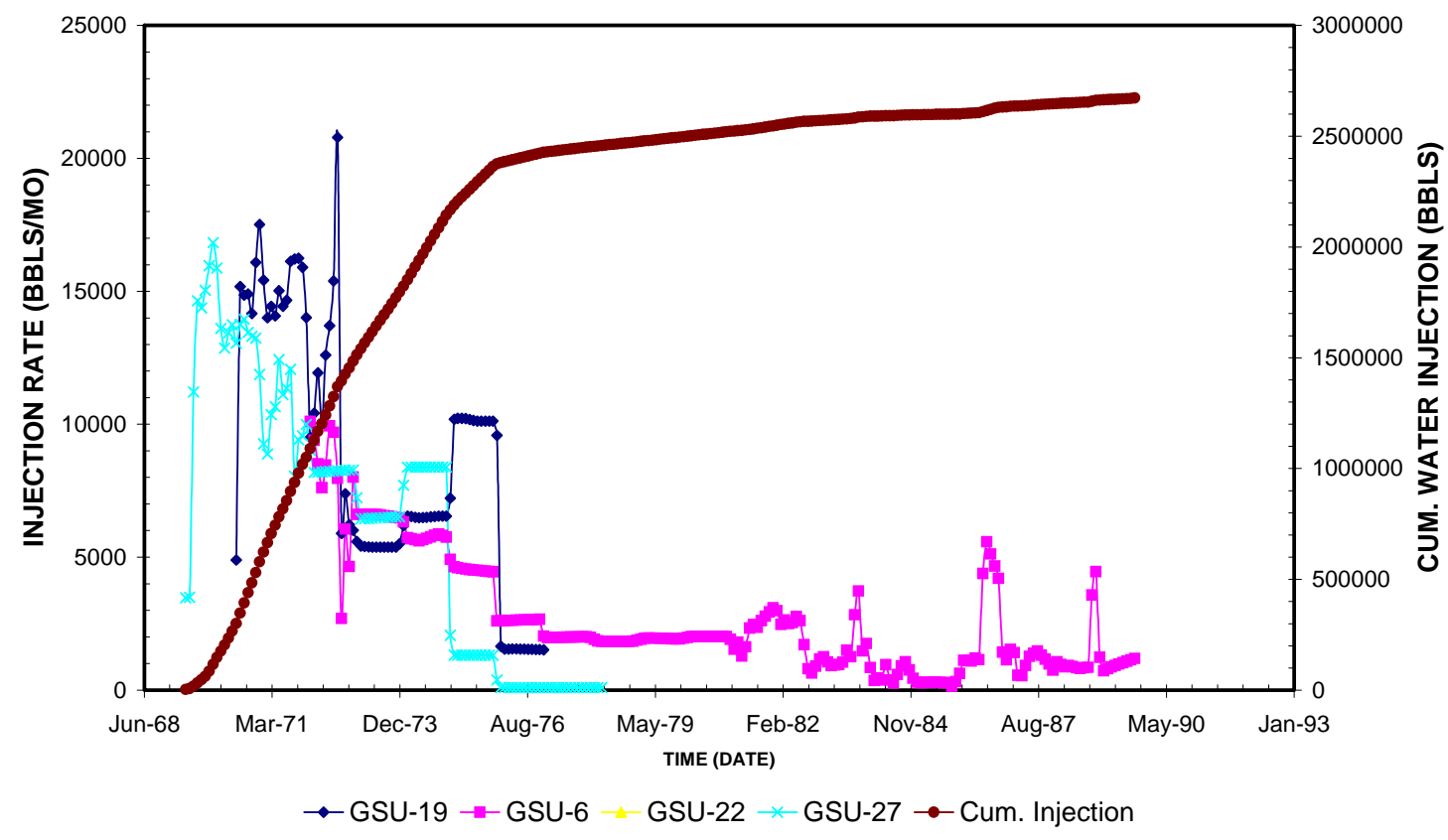

Fig. 1.2.8 - Water injection rate.

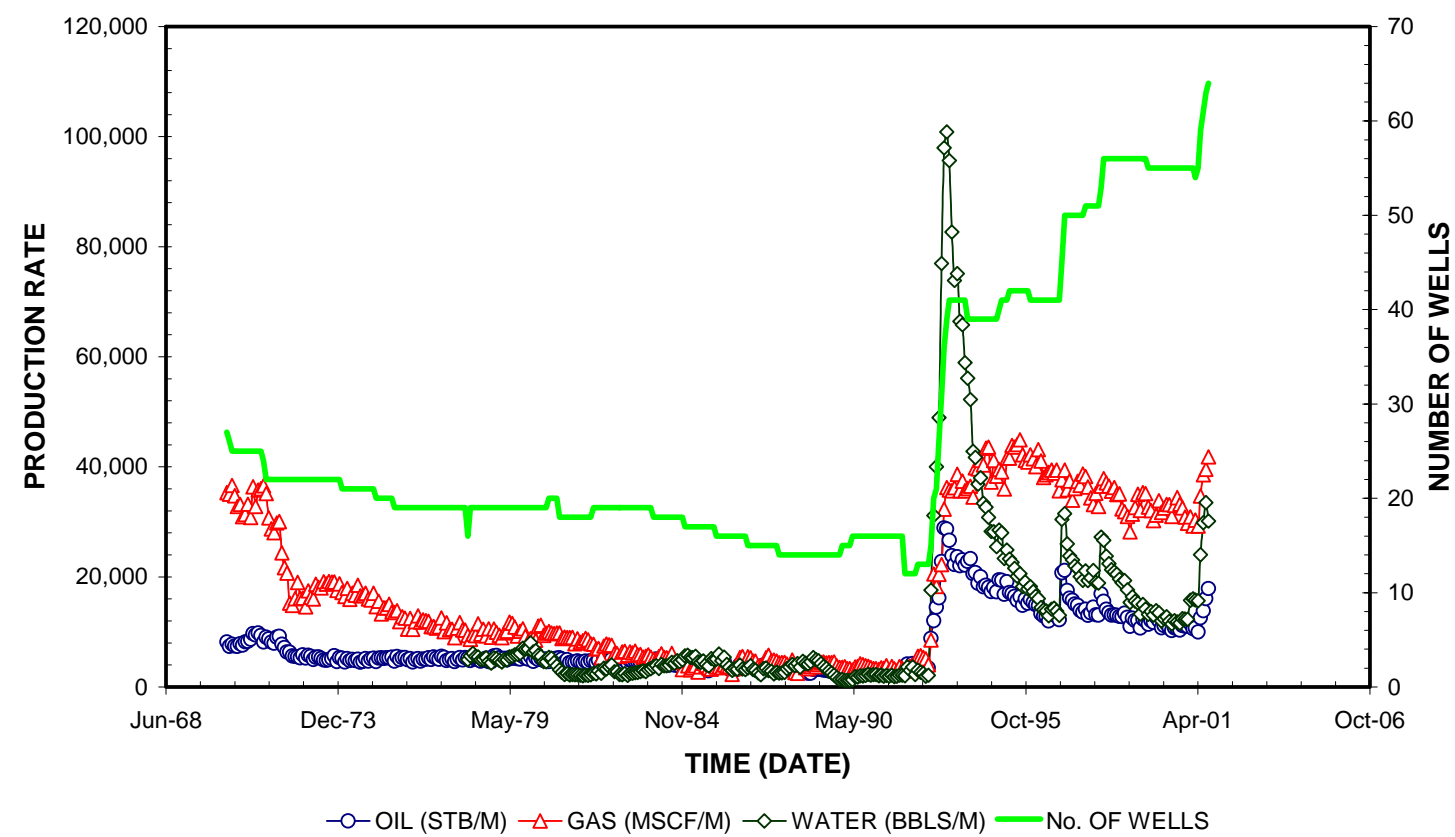

Fig. 1.2.9 - Production summary of Germania Unit. 


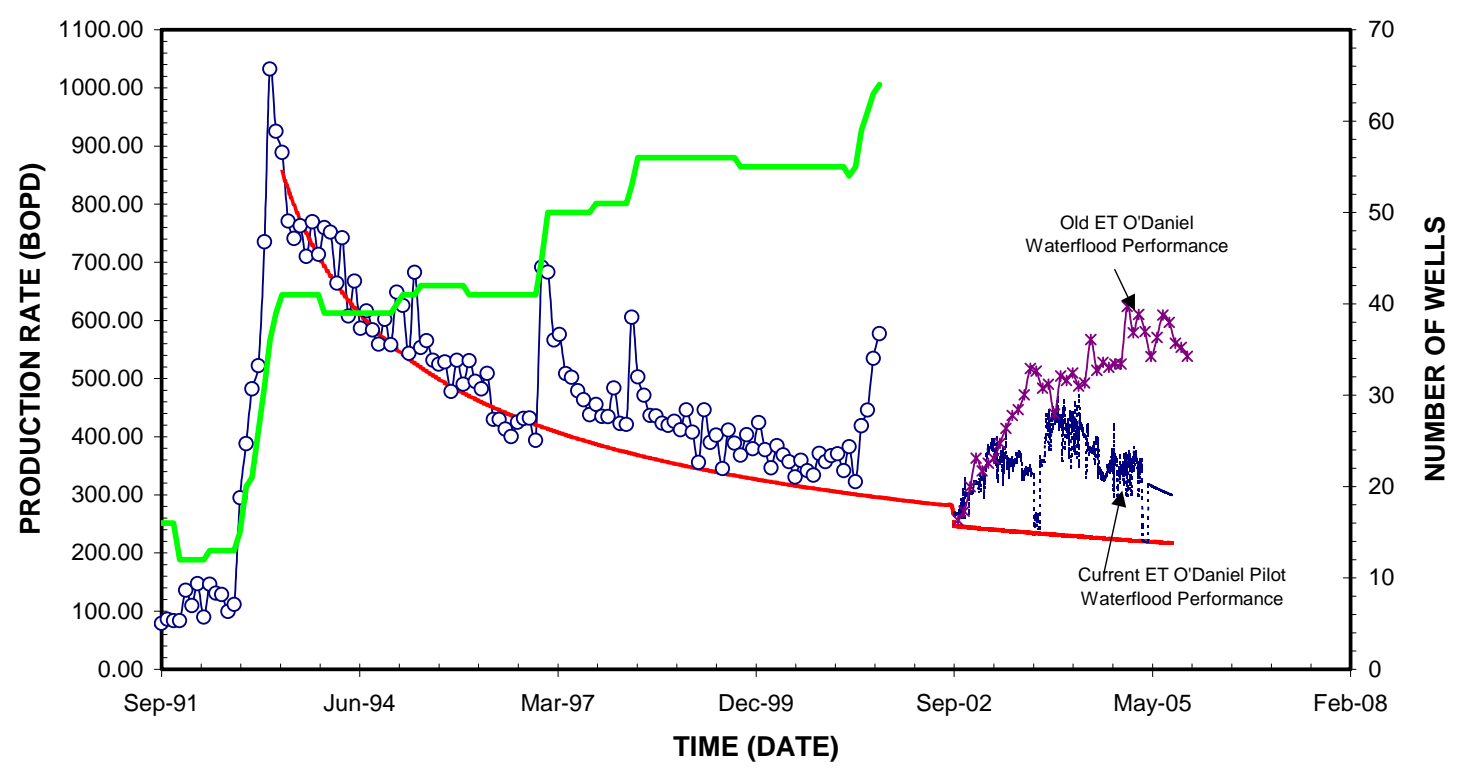

Fig. 1.2.10 - Oil production history and decline curve analysis.

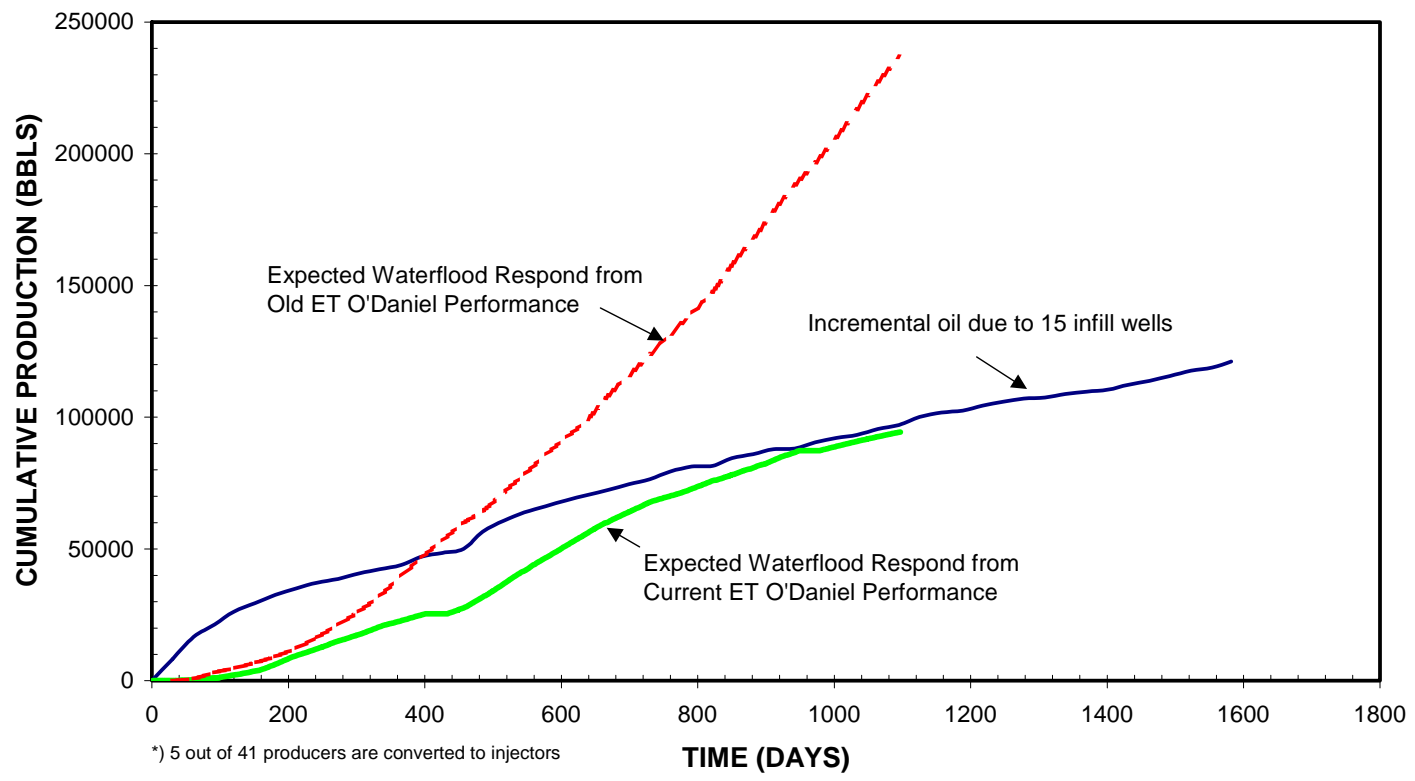

—Infill Wells —Current ET O'Daniel Pilot Respond — —Old ET O'Daniel Respond

Fig. 1.2.11 - Comparison of cumulative production between infill wells and waterflood responses. 


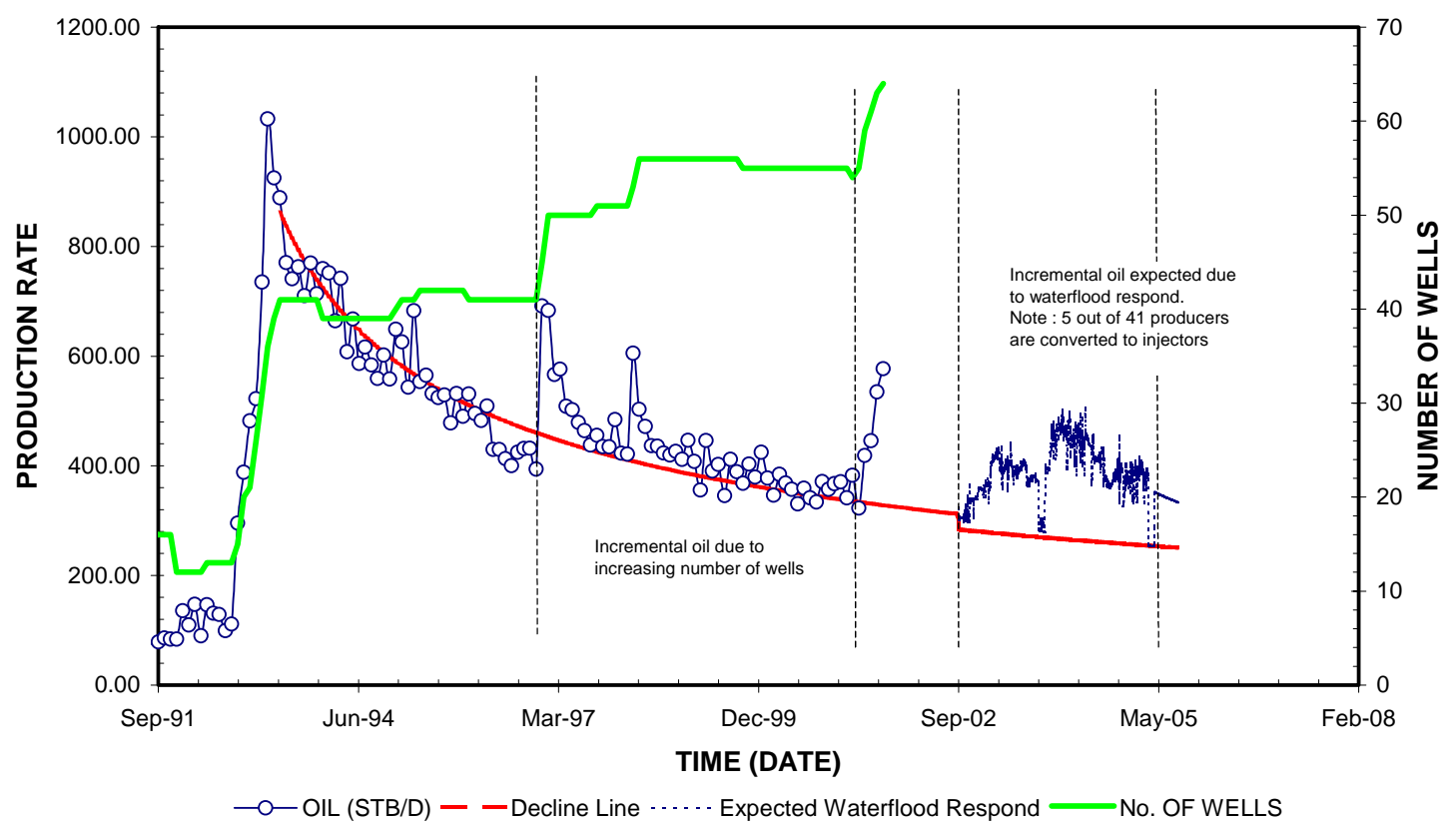

Fig. 1.2.12 - Decline curve analysis taken from all data points and continued with the forecasting incremental oil from current ET'O’Daniel waterflood performance.

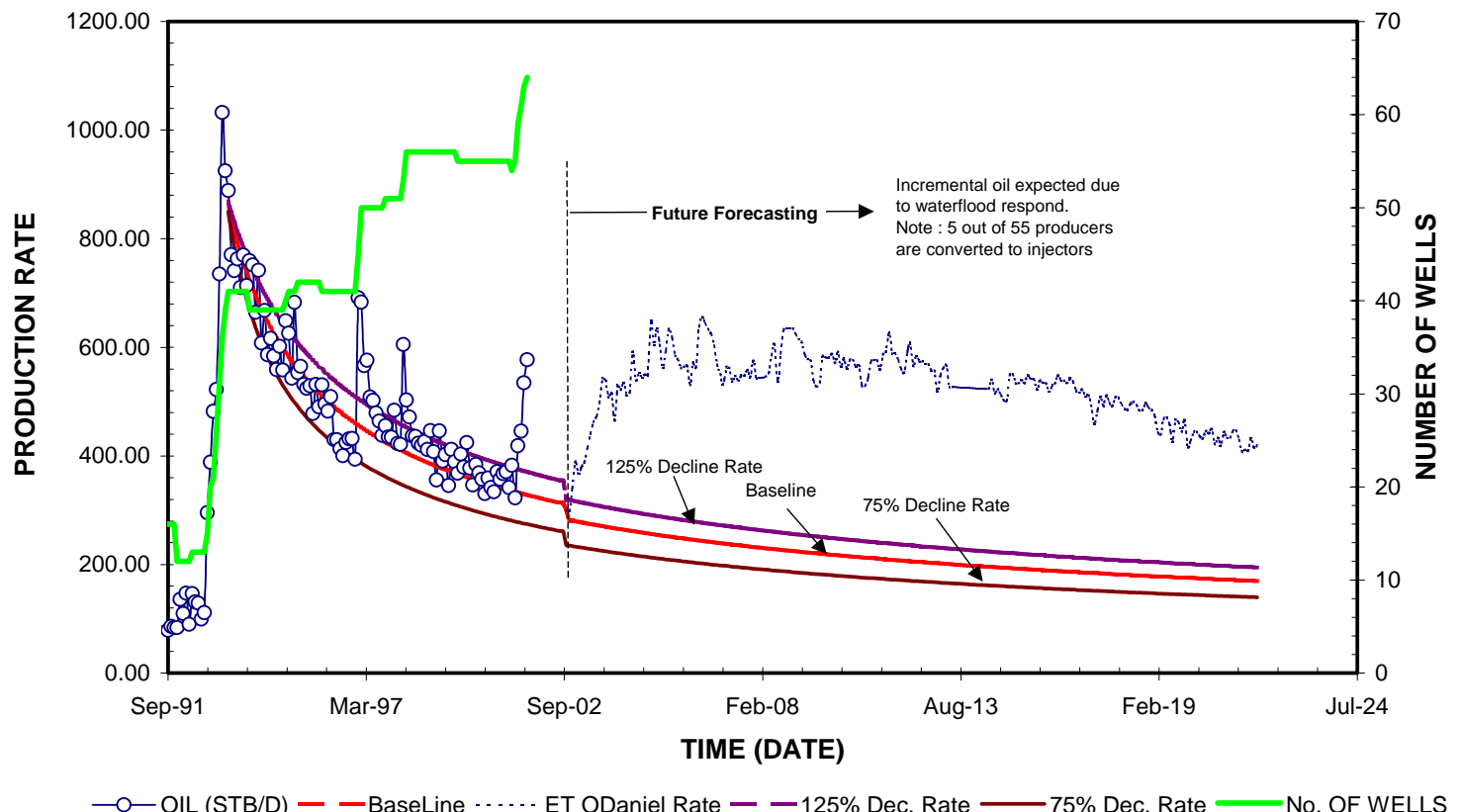

Fig. 1.2.13 - Decline curve analysis taken from all data points and continued with the forecasting incremental oil from old ET'O’Daniel waterflood performance. 


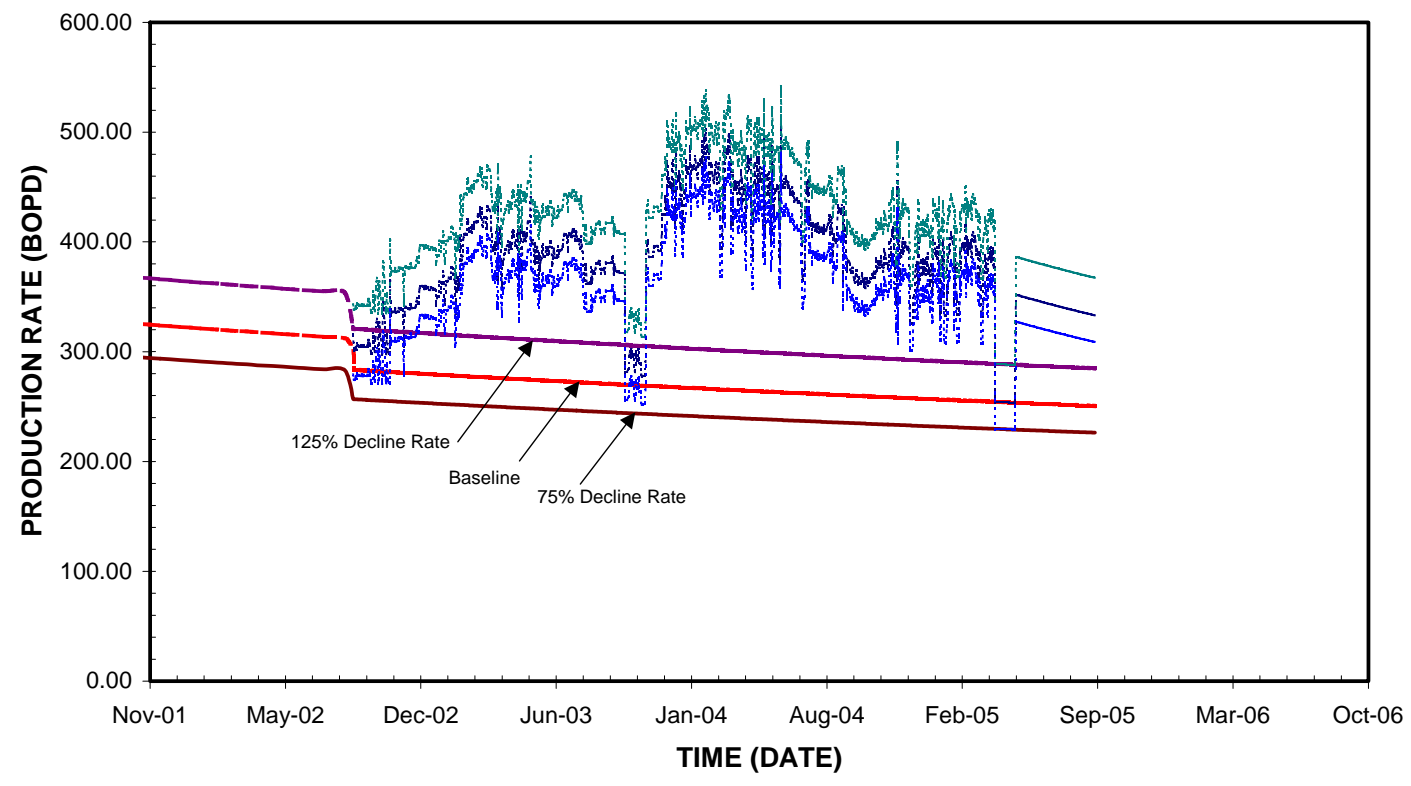

Fig. 1.2.14 - Nominal decline rate above and below base case decline curve for current ET’O’Daniel waterflood performance.

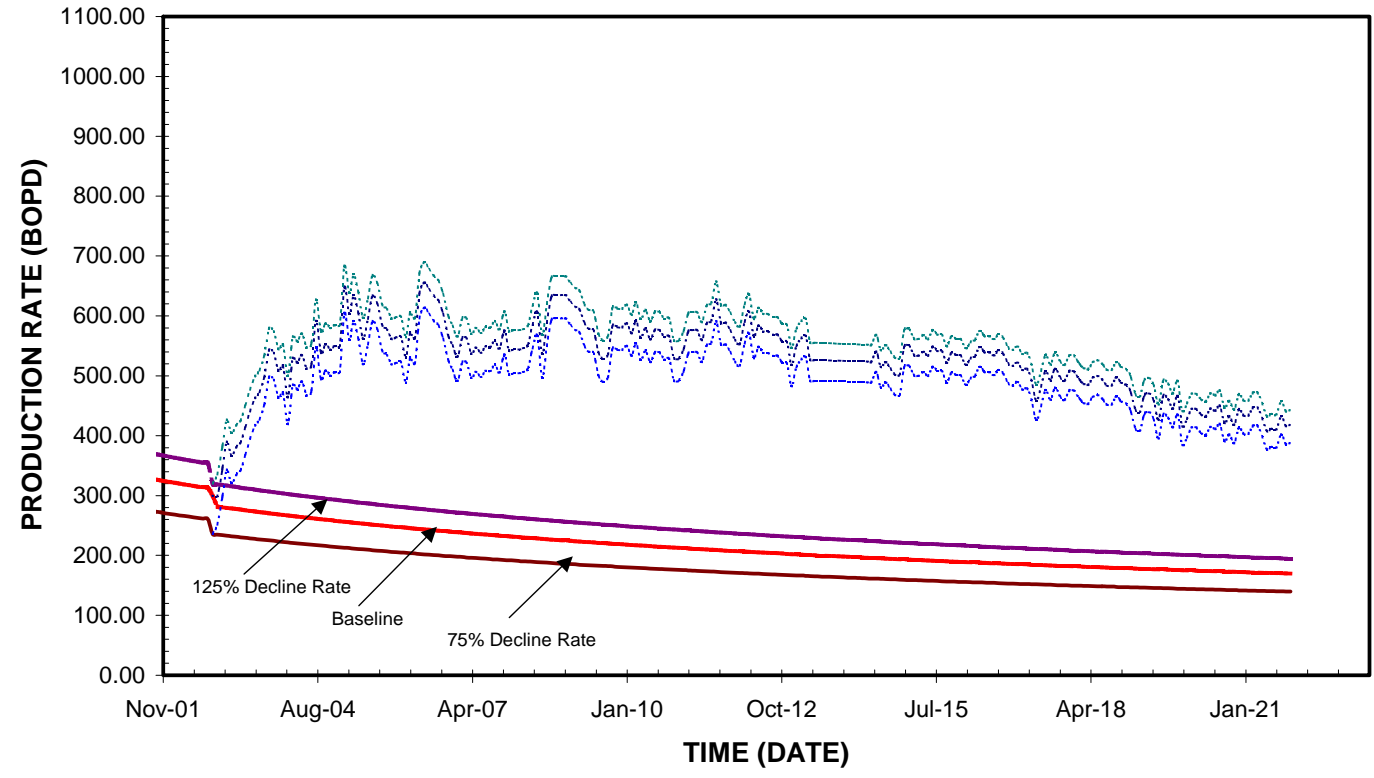

Fig. 1.2.15 - Nominal decline rate above and below base case decline curve for old ET’O’Daniel waterflood performance. 


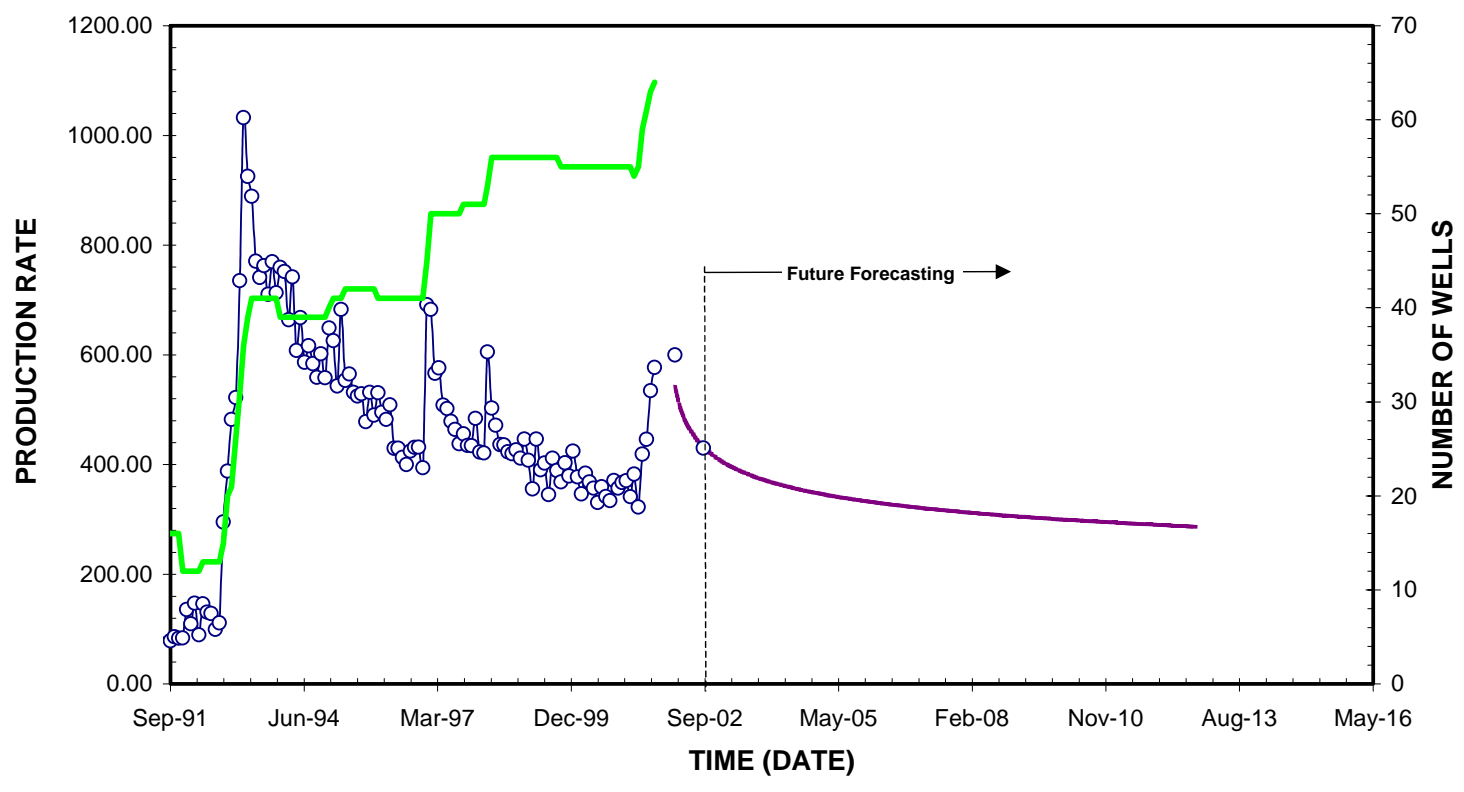

$\multimap$ OIL (STB/D) - —Without doing anything $\longrightarrow$ No. OF WELLS

Fig. 1.2.16 - Decline curve analysis taken from last data points.

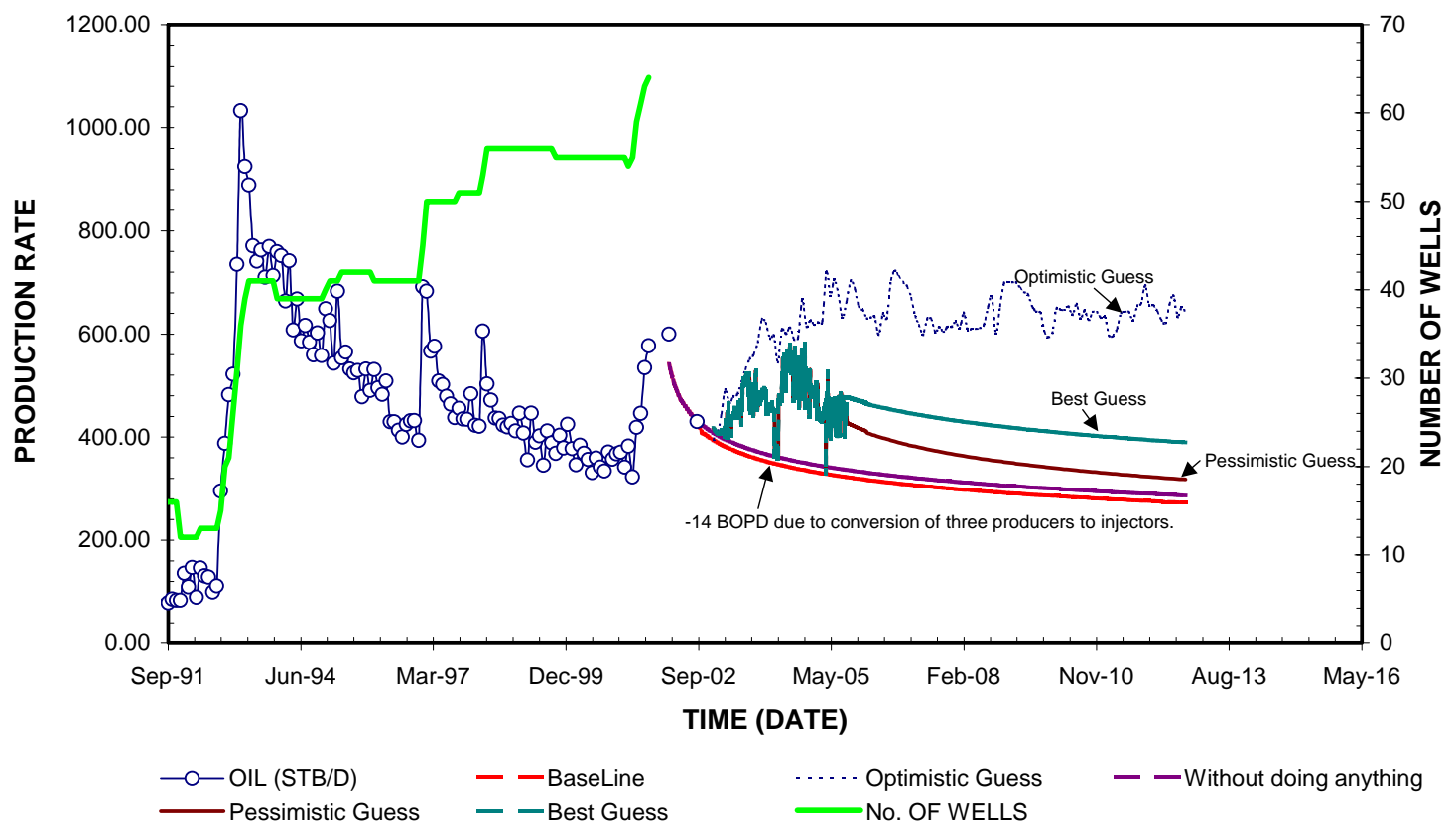

Fig. 1.2.17 - Decline curve analysis taken from last data points and continued with the forecasting incremental oil from old and current ET'O'Daniel waterflood performance. 


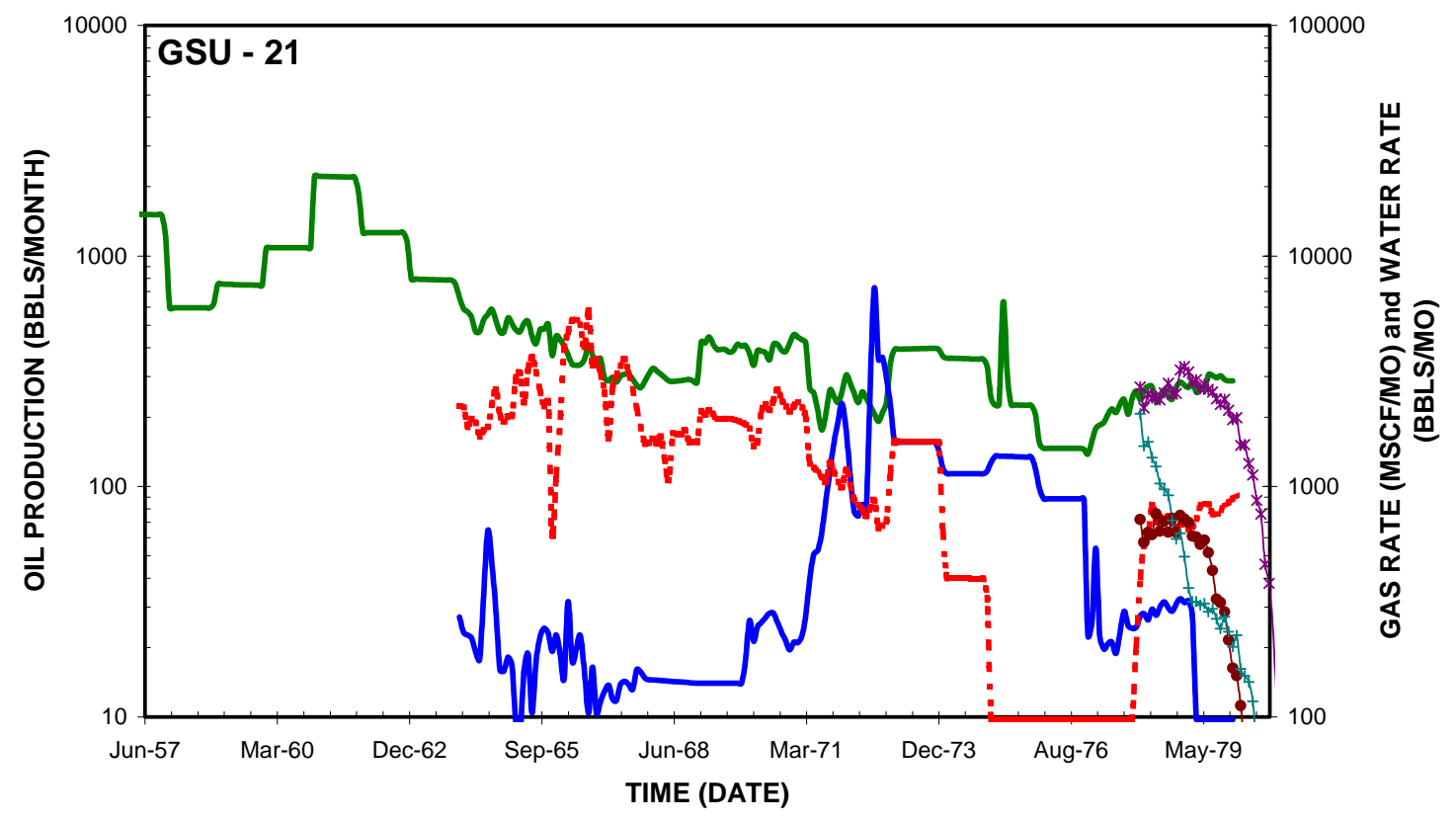

Fig. 1.2.18 - Production summary of GSU-21.

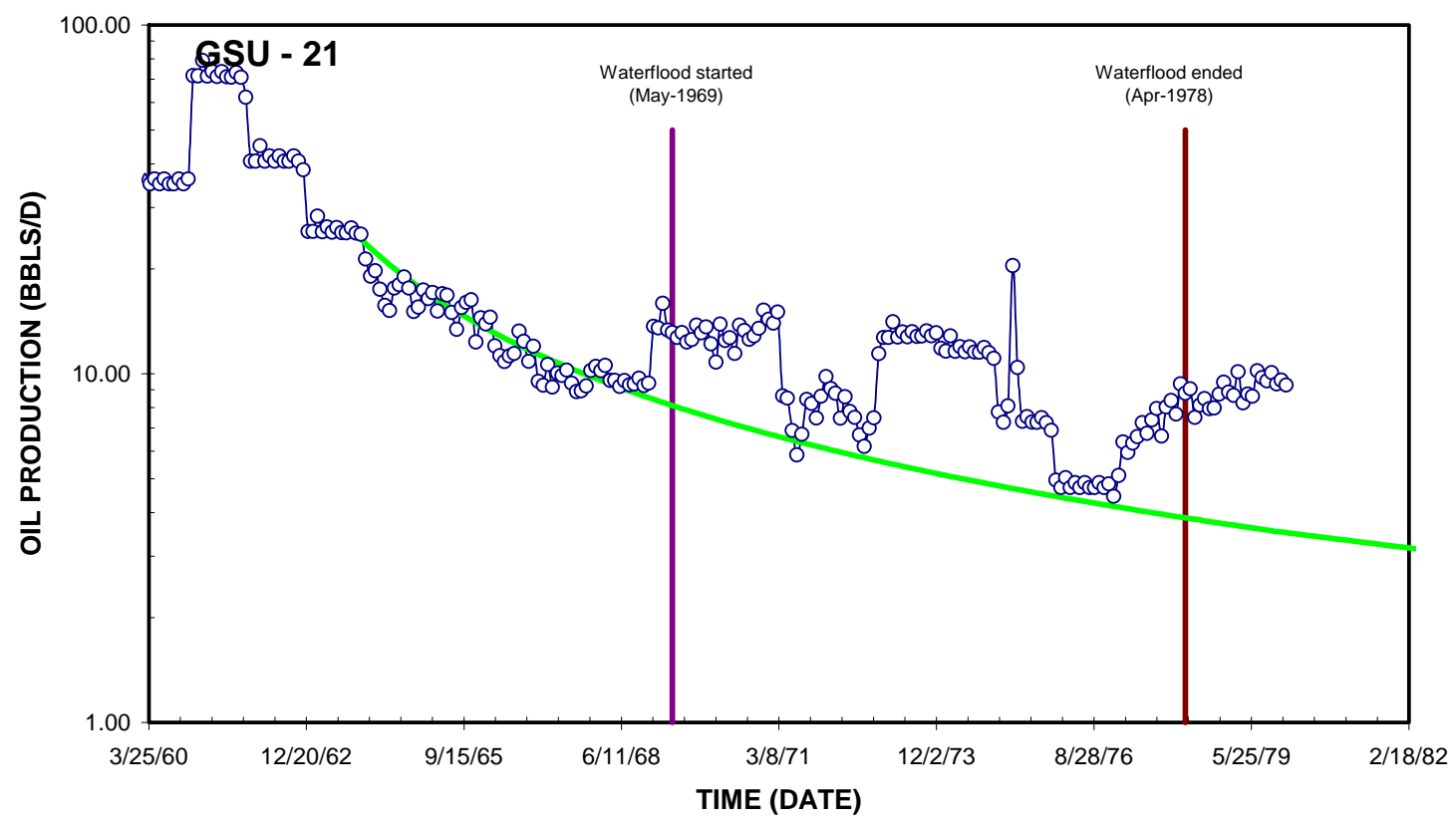

Fig. 1.2.19 - Decline curve analysis of GSU-21. 


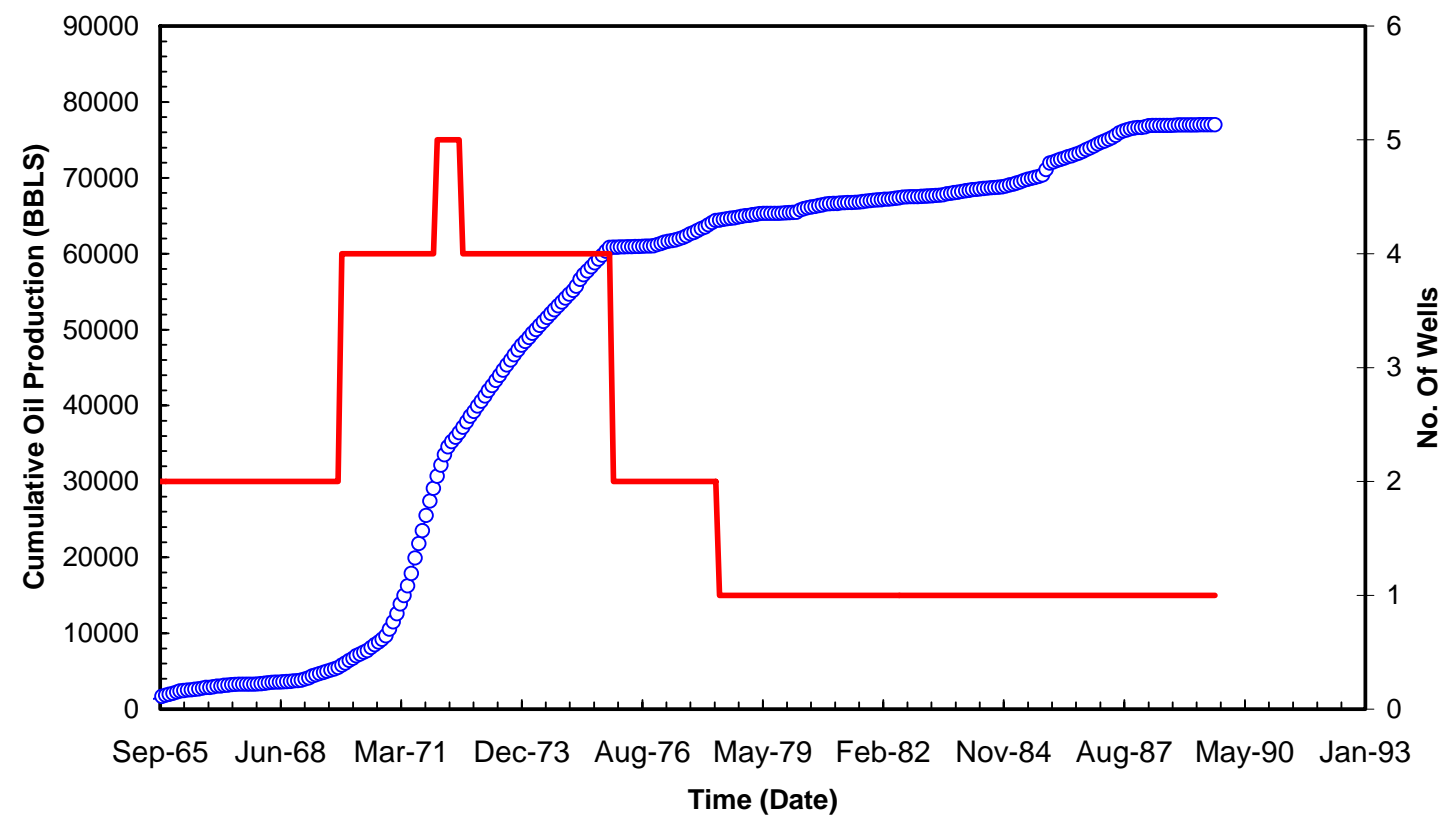

Fig. 1.2.20 - Cumulative oil recovery due to waterflood in the Germania unit. 
Appendix-A

Production Summary and Decline Analysis of Germania Wells 


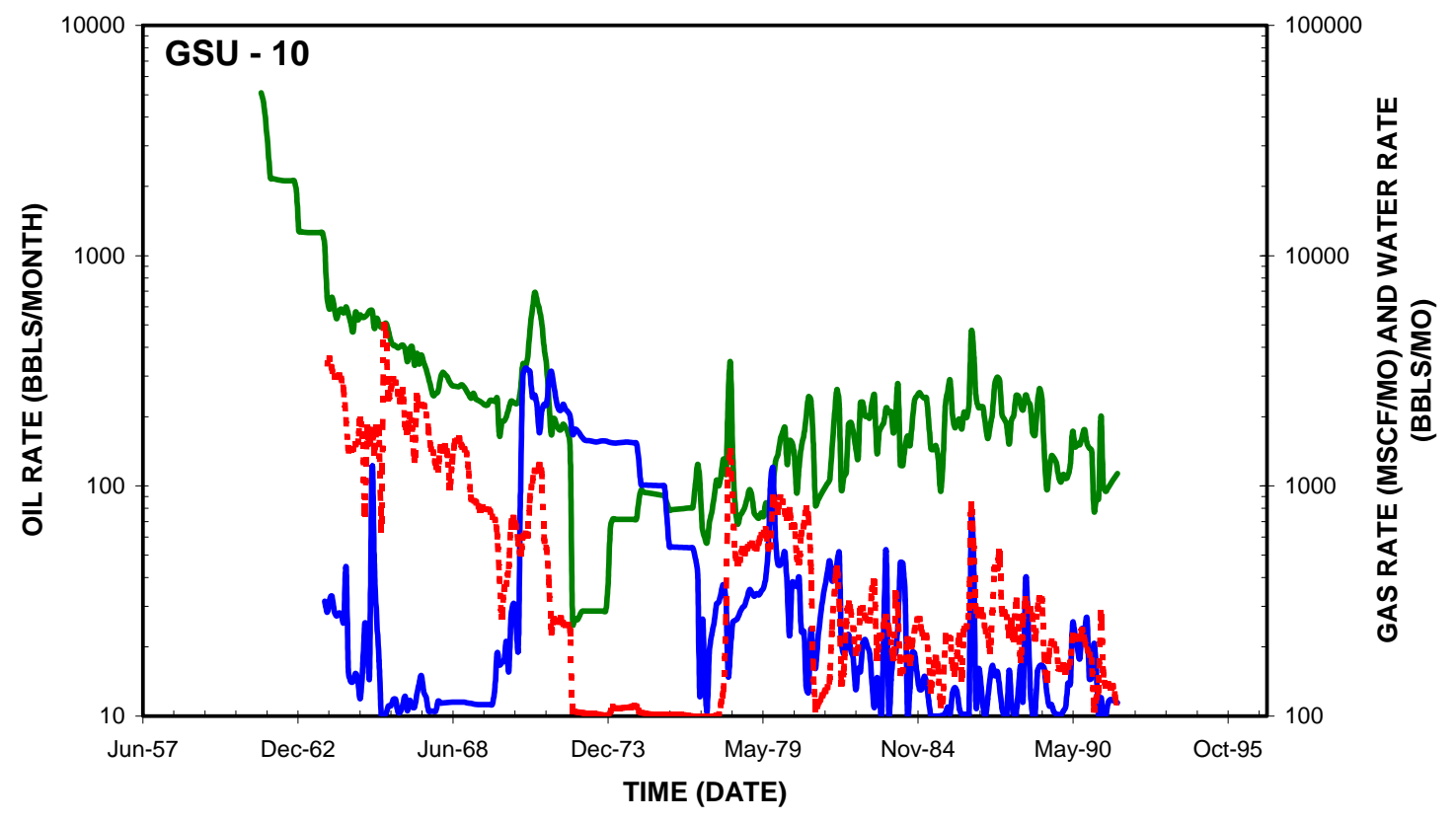

Fig. 1.2.1A - Production summary of GSU-10.

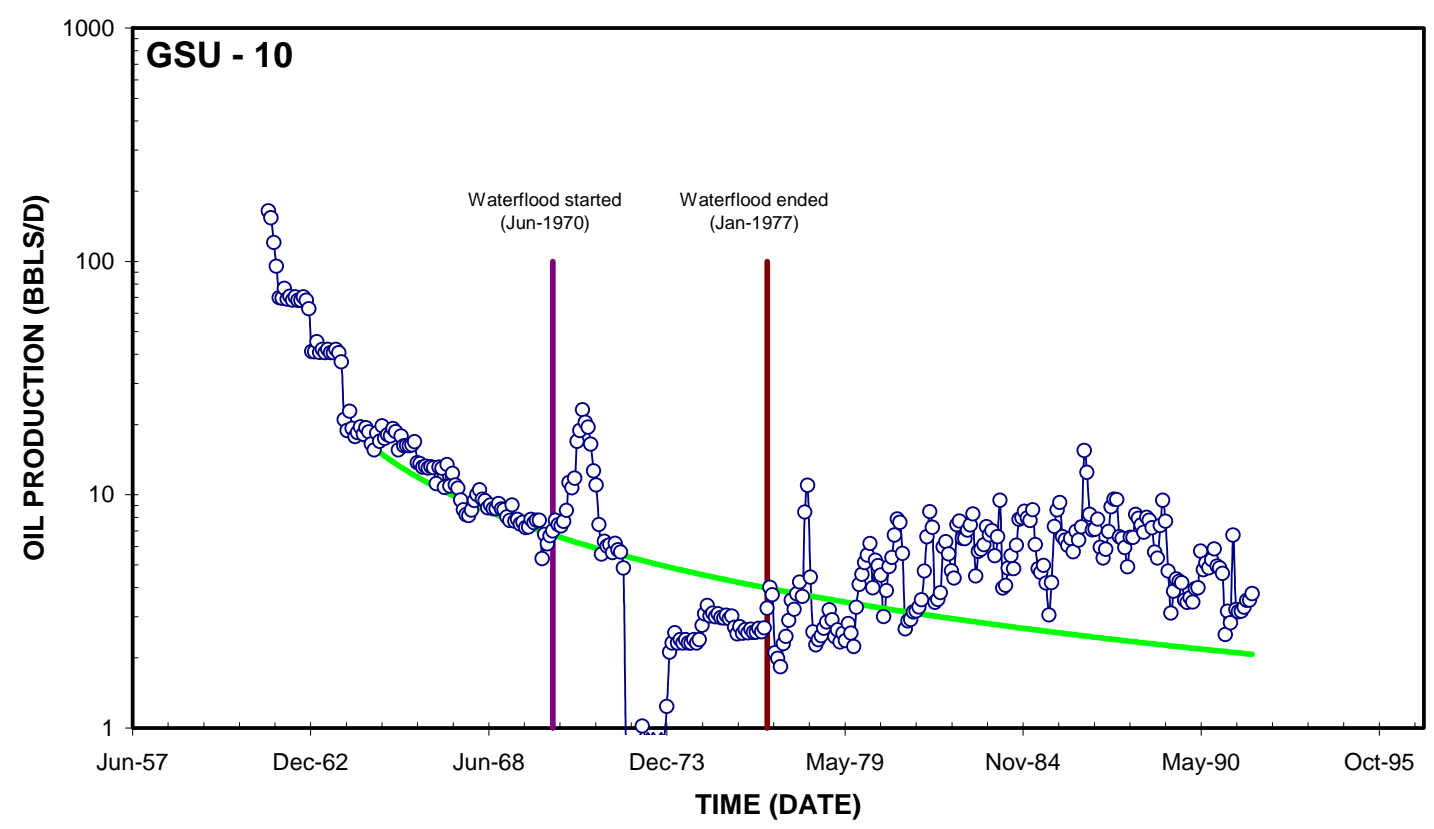

Fig. 1.2.2A - Decline curve analysis of GSU-10. 


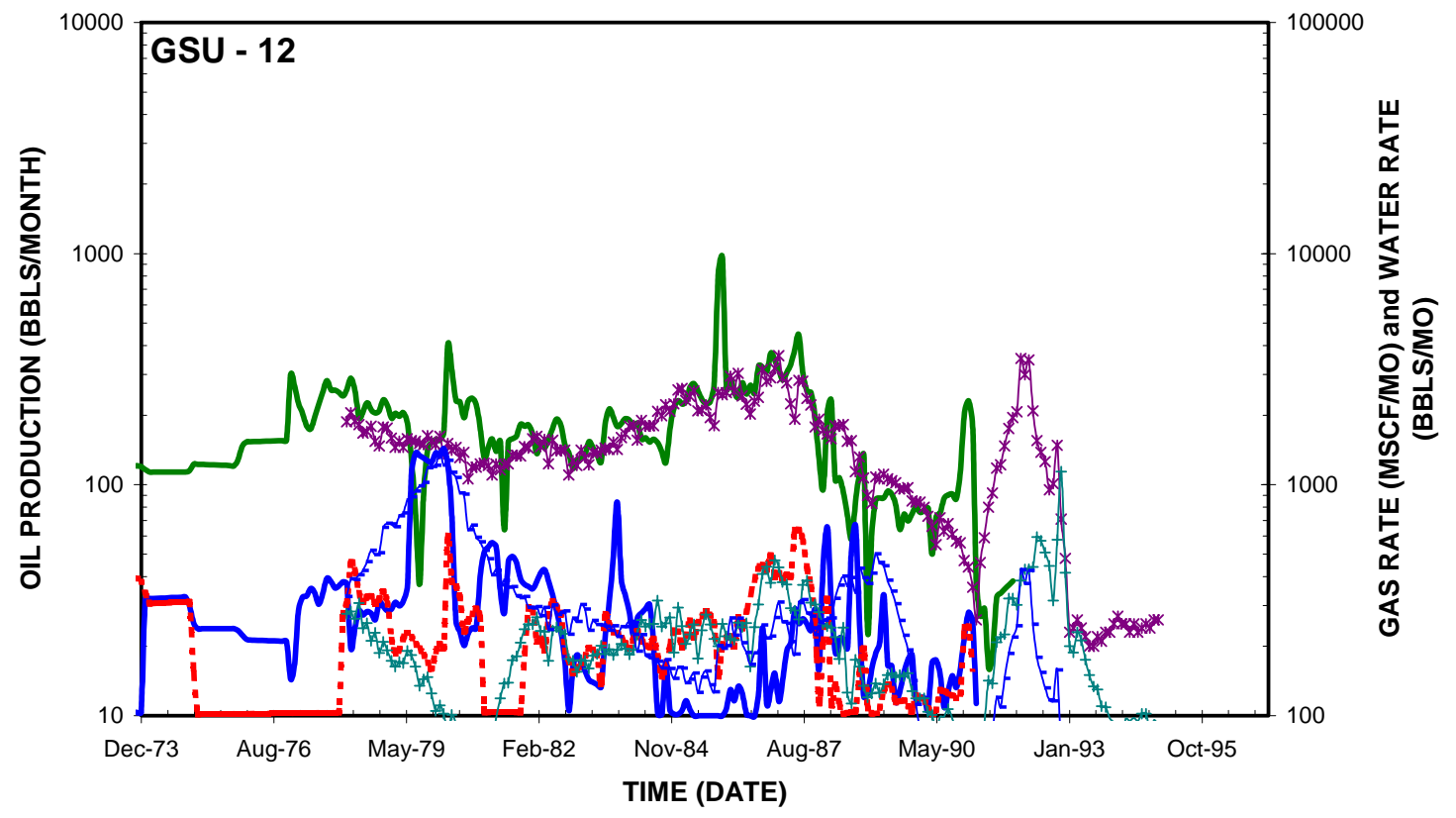

Fig. 1.2.3A - Production summary of GSU-12.

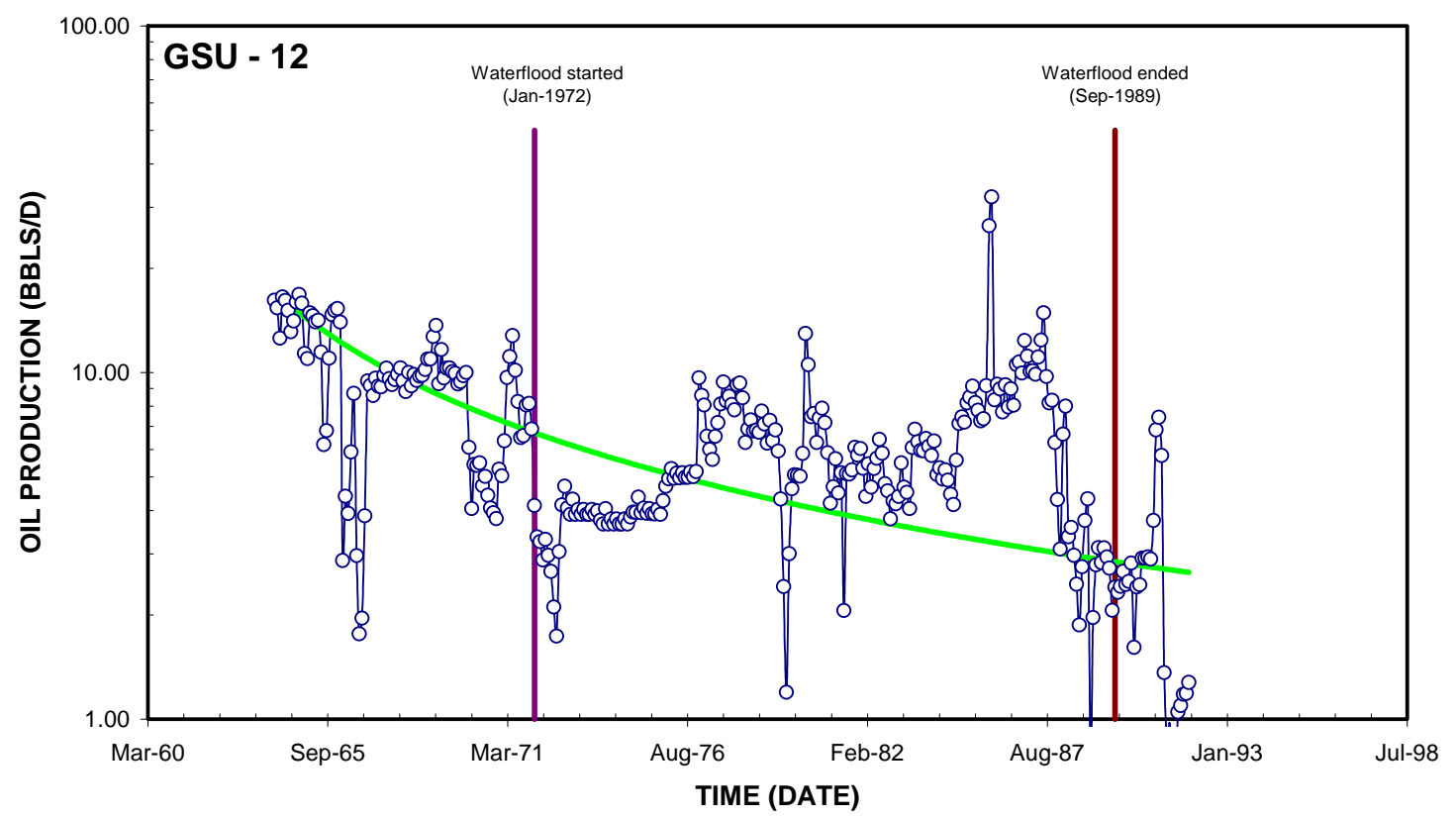

Fig. 1.2.4A - Decline curve analysis of GSU-12. 


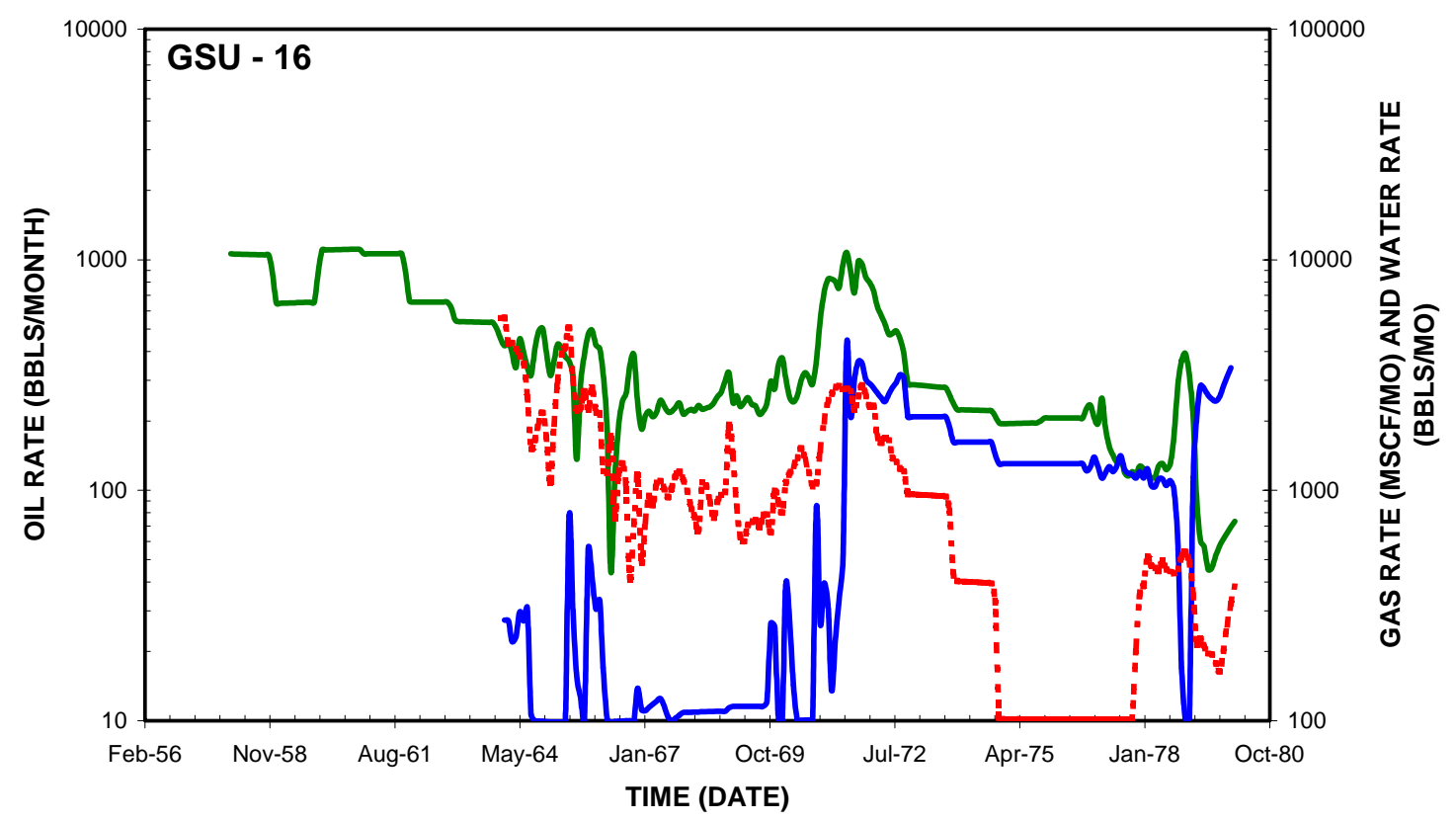

Fig. 1.2.5A - Production summary of GSU-16.

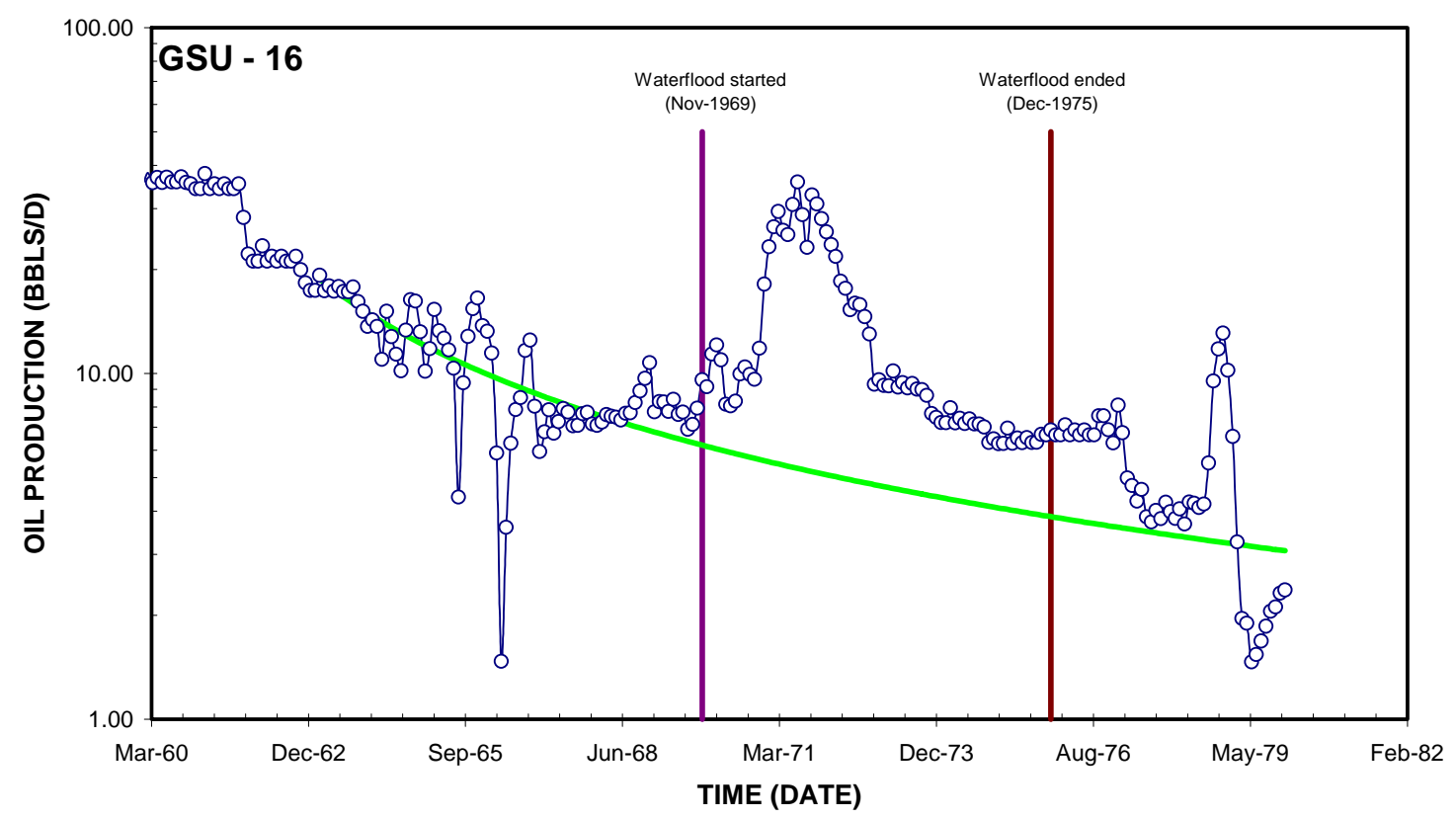

Fig. 1.2.6A - Decline curve analysis of GSU-16. 


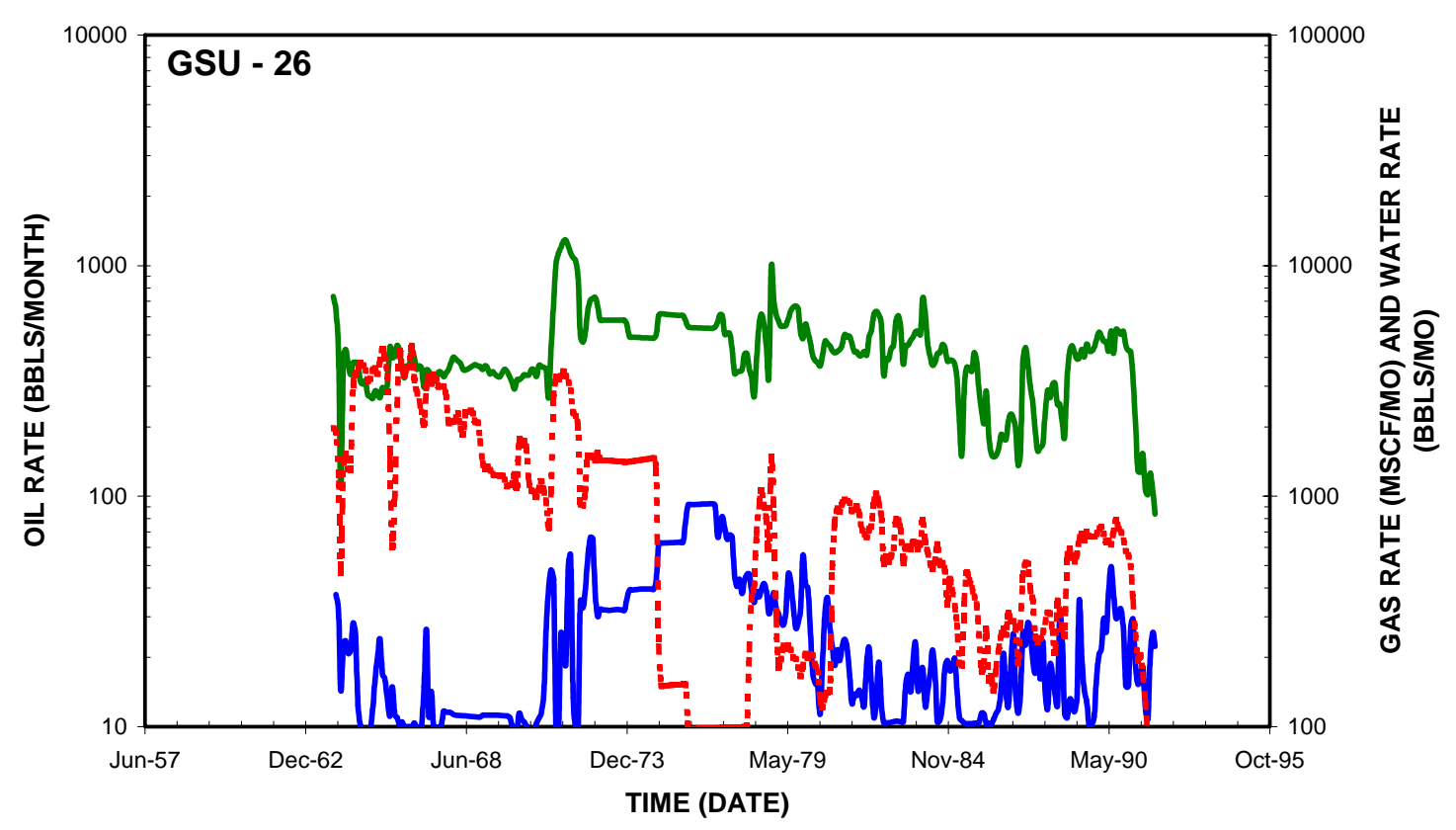

Fig. 1.2.7A - Production summary of GSU-26.

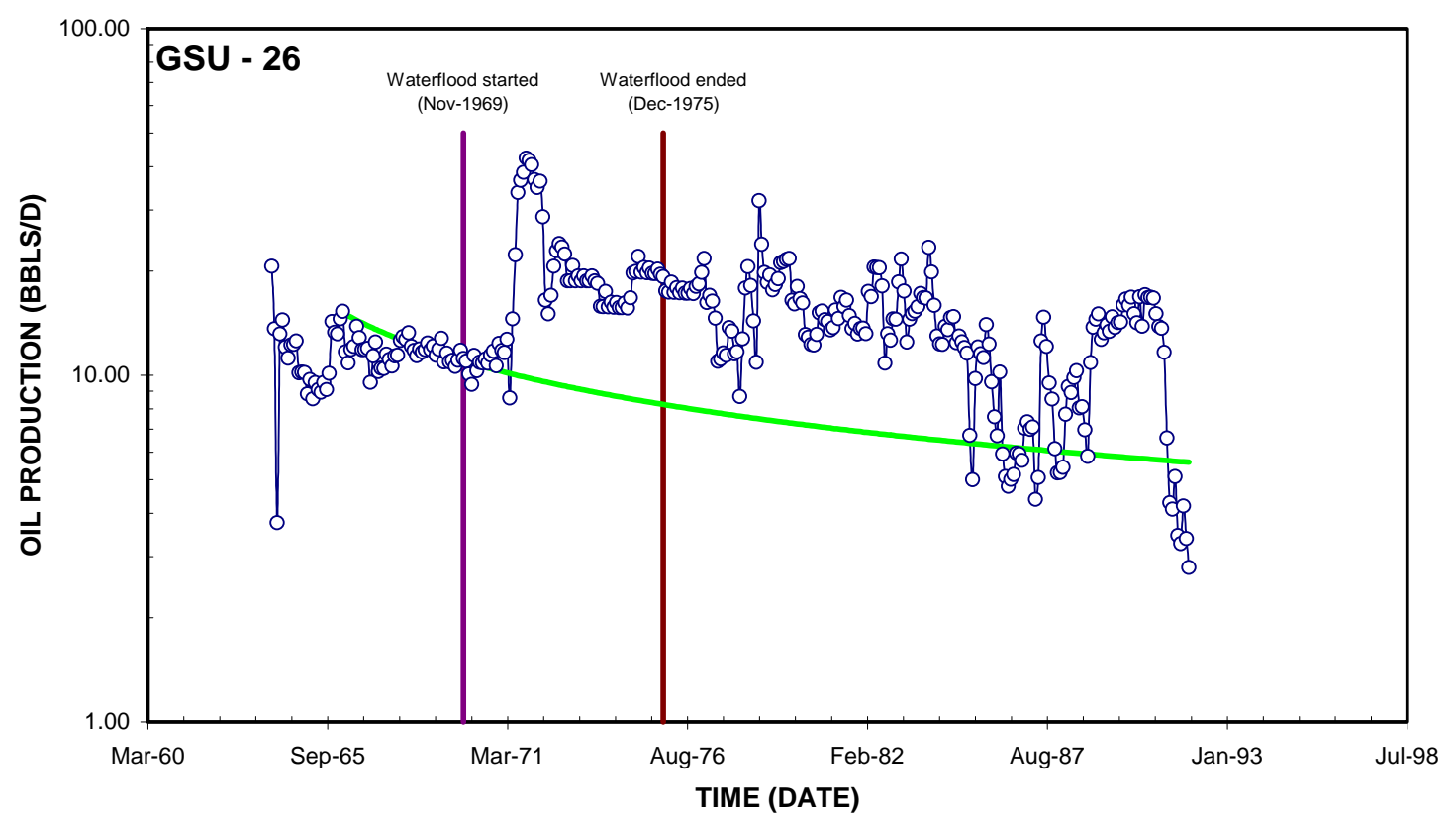

Fig. 1.2.8A - Decline curve analysis of GSU-26. 
Appendix - B

Production Summary and Decline Analysis of Old ET O’Daniel Unit 


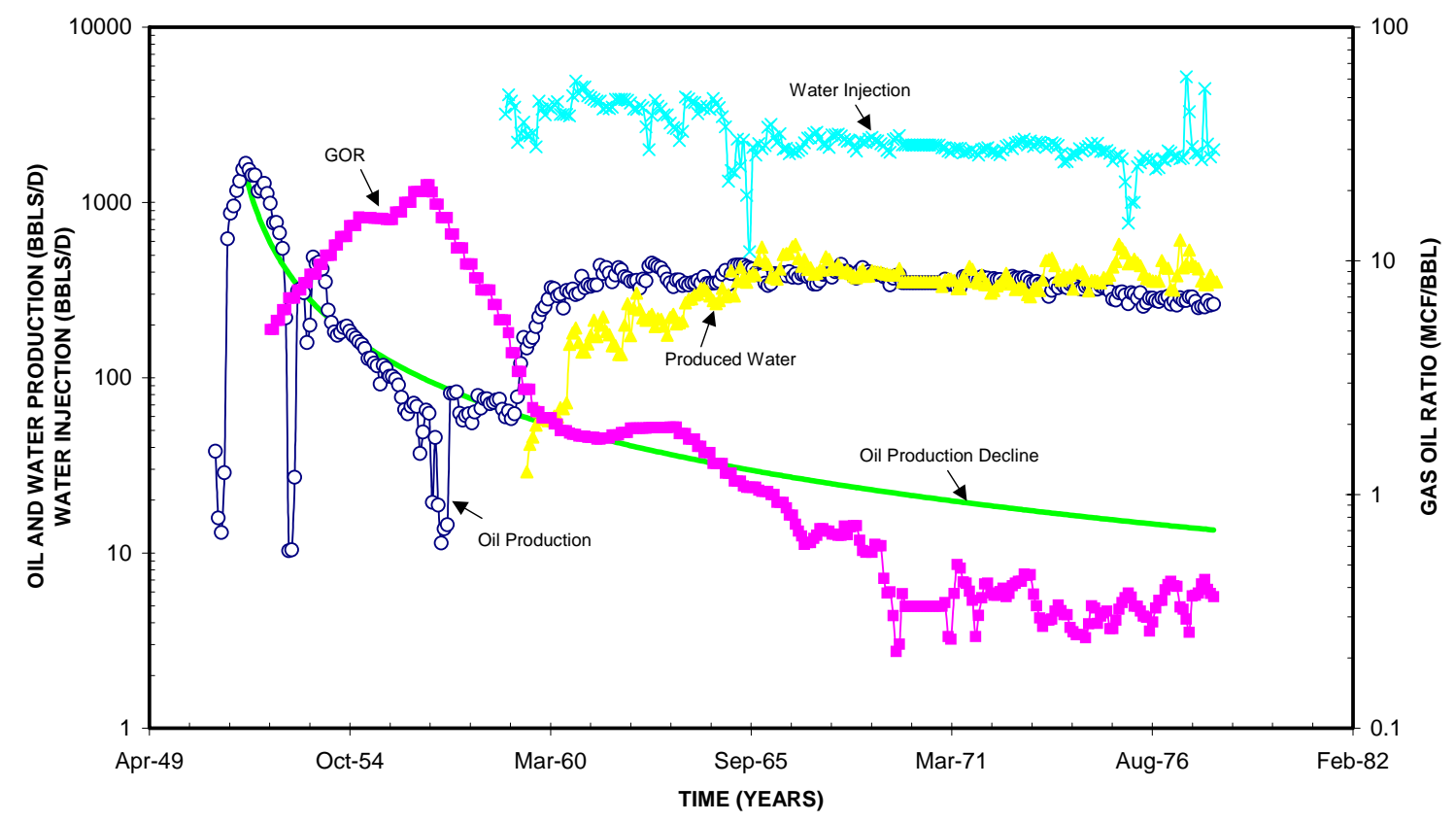

Fig. 1.2.1B - Production summary of old ET O’Daniel unit.

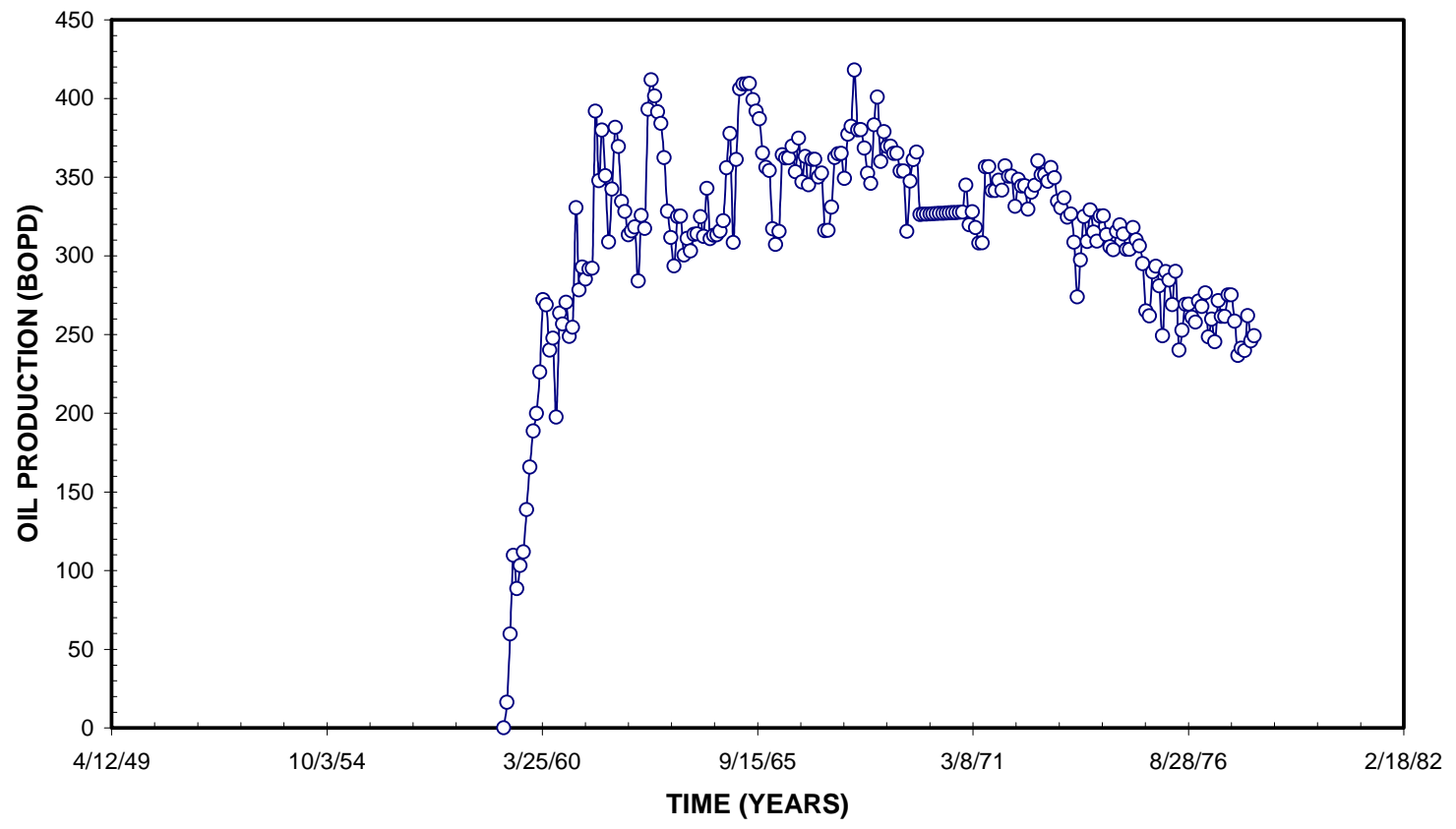

Fig. 1.2.2B - Incremental oil recovery of old ET O’Daniel unit. 


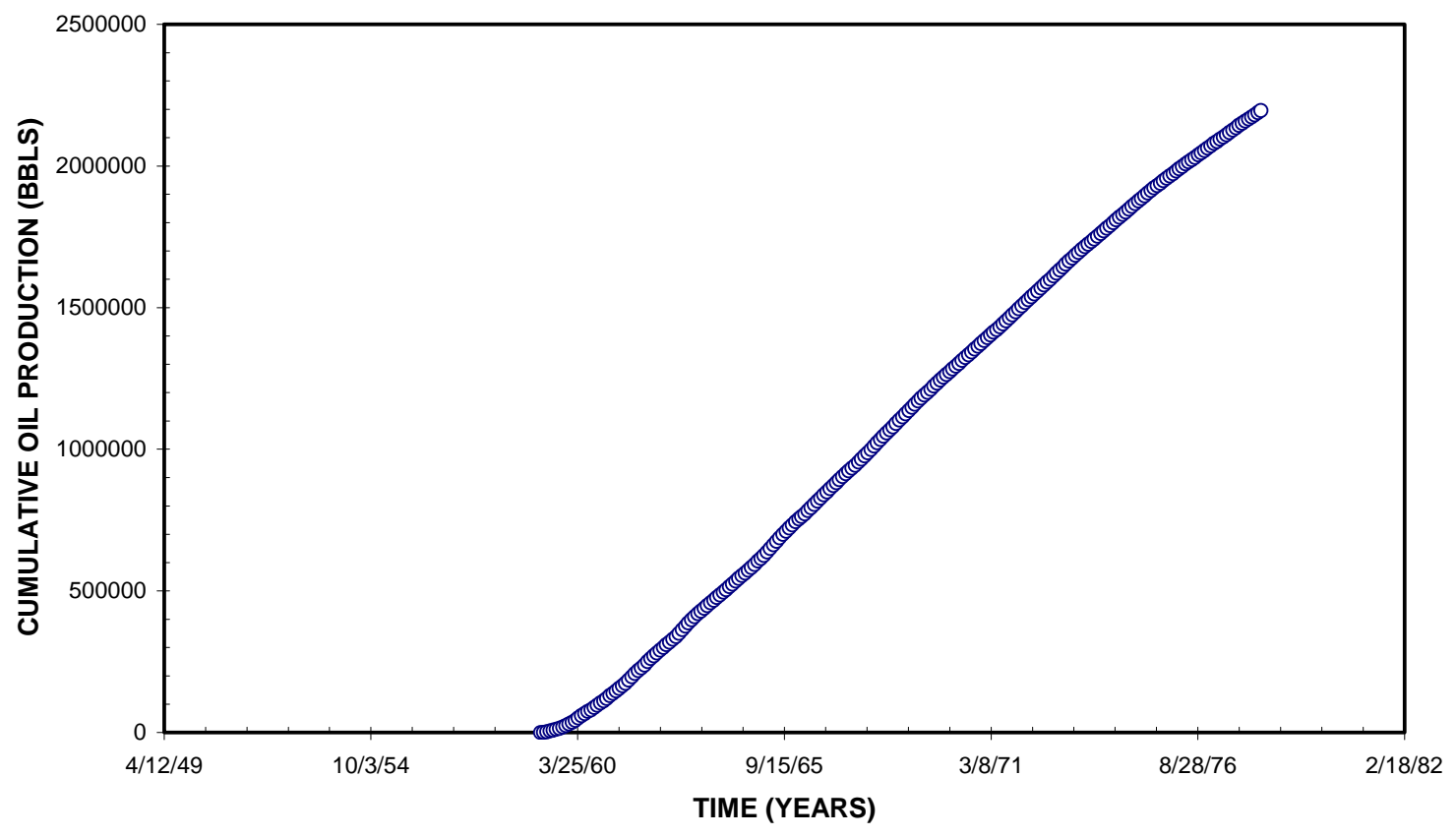

Fig. 1.2.3B - Cumulative oil production of old ET O’Daniel unit. 


\subsection{Characterization of the Germania Sparberry Unit from Analog Studies and Cased-Hole Neutron Log Data}

\section{Introduction}

\subsection{Project overview}

The Spraberry trend area is a unitized hydrocarbon production Basin in the heart of west Texas. The major production comes from fine grained, low permeability siltstones and sandstones, enhanced by an intricate network of natural fractures. Carbonate and siliclastic (submarine fans) depositional episodes during the Permian era make up the lithofacies of the Spraberry unit.

Up to date production from the Basin is estimated at about 800 million barrels of oil and 3 trillion cubic $\mathrm{ft}$ of gas from over 8000 active wells ${ }^{1}$, this figure could range between 8 $12 \%$ of the projected OHIP.

Of particular interest is the ET O'Daniel and Germania Spraberry units, two of eleven units operated by Pioneer Natural Resources. Extensive reservoir characterization work has been carried out in the ET O'Daniel based on recent core and log data acquisition, production data and simulation studies. The Germania Spraberry unit on the other hand lacks core and modern log data, and has not been characterized beyond pulse and tracer tests to analyze fracture trends and performance.

A preliminary step in the implementation of an enhanced recovery process within the unit is the characterization of the reservoir (petrophysics and fracture properties and fracture network).

This study is concerned with the log based characterization of the Germania unit and will focus on the petrophysical evaluation of the upper Spraberry unit, particularly the productive $1 \mathrm{U}$ and $5 \mathrm{U}$ intervals.

A database of 85 log suites, primarily consisting of gamma ray and old cased hole neutron logs are available for this study. Core based relationships developed in the ET O'Daniel unit are borrowed upon to aid the characterization of this field, and will generally suffice due to the similar depositional environment and proximity of the units from one another (6.2 miles).

Established criteria for predicting rock type and pay zones in the ET O'Daniel will be applied if found applicable to Germania and will guide subsequent characterization efforts in the unit. 


\subsection{Area of interest}

The Spraberry trend area spreads over an area of approximately half-a-million acres and is trapped by complex updip pinchouts and facies changes within the thick upper Spraberry producing interval. A few fields are simple anticlinal structures like Benedum and Pegasus. The regional fracture patterns are enhanced by anti-clinal folds producing a locally commercial reservoir at Pegasus ${ }^{2,3}$ (see Fig. 1.3.A11 in Appendix A).

The E.T. O'Daniel unit and the Germania unit are adjacent units at the north end of the Spraberry trend area. These fields are 2 of 11 fields operated by the Pioneer Natural Resources (PNR) and are located in the Midland County area of west Texas.

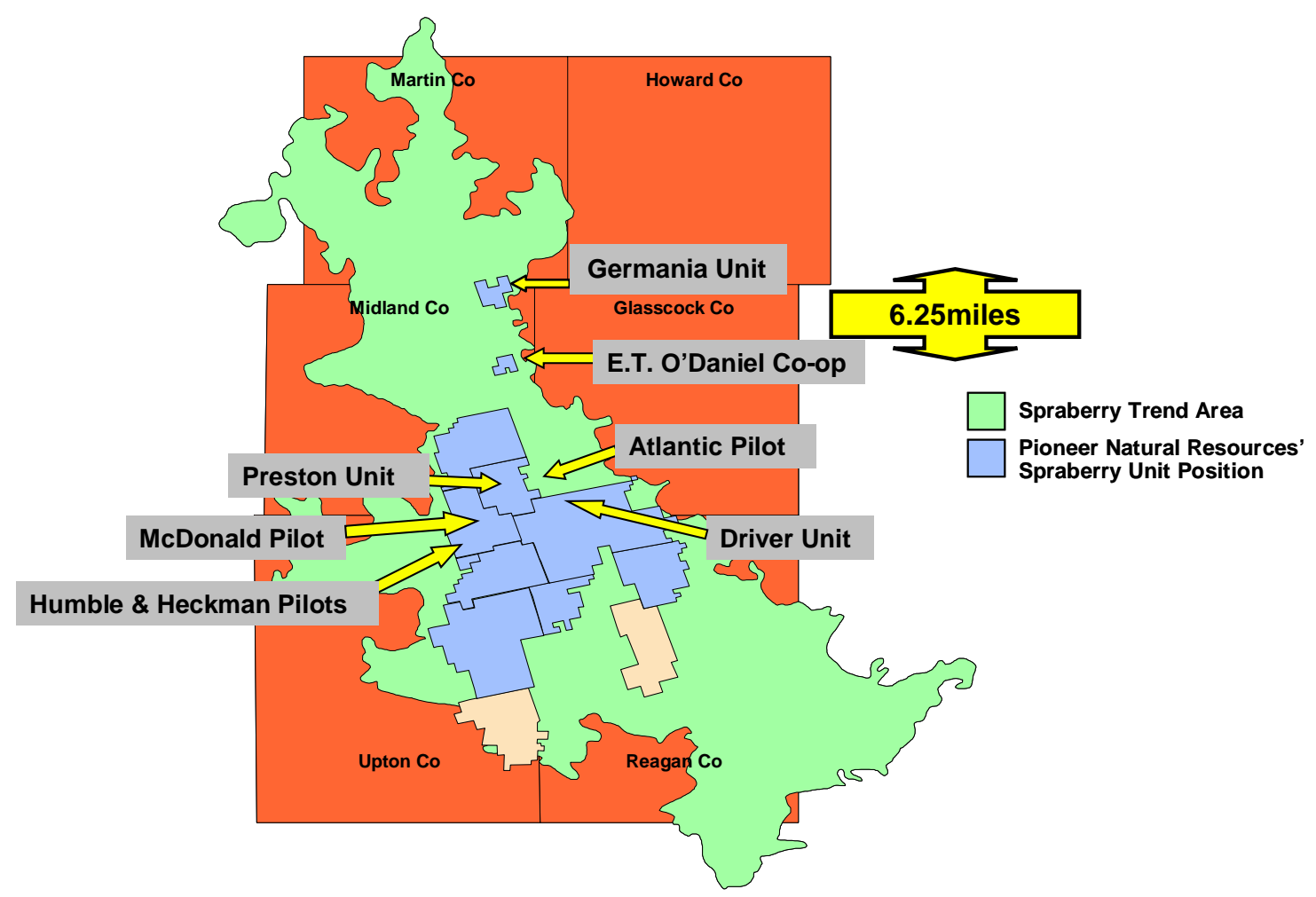

Fig. 1.3.1 - Unit locations within the Spraberry trend area.

The distance between the two fields is estimated to be about 6.2 miles based on inter-well distance measured from boundary wells (see Fig 1.3.1).

Whereas the ET O'Daniel has been the subject of major studies regarding fracture patterns $^{4-6}$, log - core analysis ${ }^{7-11}$ and waterflood and $\mathrm{CO}_{2}$ injection pilot projects ${ }^{1,12-14}$, no major investigation of the lithofacies or fracture characteristics of the Germania unit has been performed. Fracture trends on a gross scale by way of pulse and tracer tests is the basis of predicting flow behavior within the Germania unit. 
Due to the proximity of the ET O’Daniel unit to the Germania unit as well as the depositional environment within the four County area ${ }^{15,16}$ (Midland, Glasscock, Upton and Reagan) it becomes logical to superimpose the conclusions drawn from the petrophysical evaluation of the ET O’Daniel unit upon the Germania unit.

Bearing this in mind, further discussions on the characterization work regarding this area will be focused on the ET O’Daniel unit.

\subsection{Rock-log model}

Gamma ray and old cased hole neutron logs form the bulk of the electric logging data available within the ET O'Daniel. More recently, array induction, density and neutron porosity data have been acquired in pilot areas within the unit. This acquisition is localized and hence the older neutron logs are an indispensable source for wide scale characterization of the field.

A log based rock model ${ }^{10,11}$ was developed for the trend area using shale content (gamma ray) and porosity as discriminatory criteria for rock type. In this model, classification is made for 3 rock types $-\mathrm{A}, \mathrm{B}$ and $\mathrm{C}$.

Table 1.3.1 summarizes the identifiers for the rock model within the upper Spraberry operational units based on effective porosity and shale content ${ }^{17}$.

Table 1.3.1 - Criteria for pay identification in the ET O’Daniel unit.

\begin{tabular}{|c|c|c|c|c|c|c|c|}
\hline Formation & Rock Type & Shale Volume & PHIE & Facies & Fluorescence & Pay & Unit \\
\hline \multirow{3}{*}{ Upper Spraberry } & $\mathrm{A}$ & \multirow{2}{*}{$<15 \%$} & $>7 \%$ & SS & Strong & yes & $1 U, 5 U$ \\
\hline & $\mathrm{B}$ & & $<7 \%$ & $\mathrm{DS}+\mathrm{SS}$ & Weak & \multirow{2}{*}{ no } & $2 U, 3 U, 4 U$ \\
\hline & $\mathrm{C}$ & $>15 \%$ & & $\mathrm{SH}+\mathrm{DS}+\mathrm{SS}$ & None & & muddy zones \\
\hline
\end{tabular}

More recently, 'Thin section' analysis of core samples within the upper Spraberry were point count analyzed to establish framework, cement mineralogy and diagenetic features of the rock ${ }^{8}$. Especially useful in identifying and classifying samples was $\mathrm{x}$-ray diffraction (XRD) and scanning electron microscope (SEM) analysis to determine clay mineralogy and proportions of clay minerals within the various rock types. Prior to the results of the study, a direct relationship was assumed between porosity and gamma ray response and permeability and gamma ray response, which for the most part is true. What Schechter and Banik ${ }^{9}$ also showed was that clay content is a significant factor in predicting overall permeability. Sands with low clay content have a high overall permeability within the $1 \mathrm{U}$.

Rock type $A$ is the only reservoir quality rock identified within the upper Spraberry, types $\mathrm{B}$ and $\mathrm{C}$ are non-reservoir quality rock. A crossplot of the shale volume and the effective porosity provides an easy method of rock identification (Fig. 1.3.2). 
Rock Type A - Massive, clean siltstone, low clay and dolomite content. Strongly fluorescent with low water saturation.

Rock Type B - Low clay, low dolomitic content with weak or no fluorescence and high water saturation.

Rock Type C - Muddy clay rich zones that do not fluoresce.

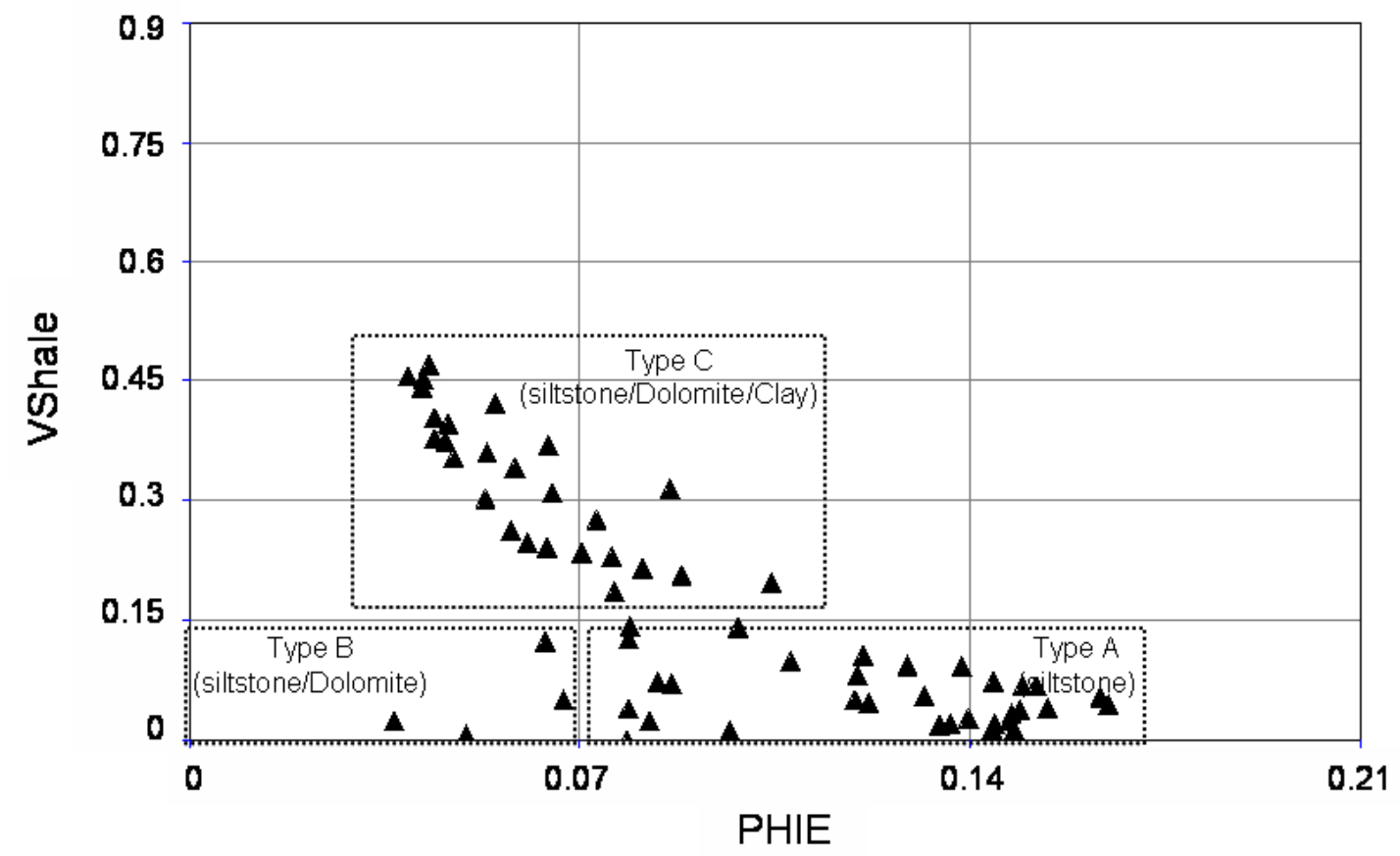

Fig. 1.3.2 - Crossplot of shale volume and porosity for well ET 47, $1 \mathrm{U}$ sand.

\subsection{Lithofacies based model}

Eight separate lithofacies ${ }^{18}$ are defined based on sedimentological, compositional and textural features of core samples. These are broadly divided into those with reservoir potential, and non-reservoir potential deposits.

Potential reservoir deposits consist of:

Type 1 - Massive siltstones and very fine grained sandstones

Type 2 - Thin bedded siltstones and very fine grained sandstones exhibiting basal intervals of massive sandstone grading vertically into parallel or cross laminated sandstone and siltstone.

Type 3 - Thin bedded, graded, cross laminated siltstones and very fine grained sandstones, interbedded with dark grey shales

Non-reservoir lithofacies consist of: 
Type 1 - Massive silty dolostone and dolomite-cemented siltstone

Type 2 - Black shales containing phosphatic nodules and abundant pyrite

Type 3 - Thin bedded argillaceous siltstone showing abundant soft sediment deformation Type 4 - Bioturbated argillaceous siltstone in which scattered silt-size grains of quartz and feldspar float in a groundmass of detrital clays

Type 5 - Parallel and finely laminated siltstone and silty shale.

\subsection{Non-depositional model}

A more generic classification of the rock types of the upper Spraberry that relate better with rock quality based on non-depositional factors is developed using petrographic analysis, petrophysical analysis and compositional information ${ }^{18}$.

To avoid confusion, the log based rock model will be referred to as the secondary classification, and the generic model as primary.

The core based primary model shows that the upper Spraberry can be divided into six distinct rock types:

Type 1 - Coarse siltstones and very fine grained sandstones (A)

Type 2 - Laminated or patchy siltstones and very fine grained sandstones (B)

Type 3 - Silty dolomite mudstones (C)

Type 4 - Very patchy dolomitic siltstones (D)

Type 5 - Shale and silty shale(E)

Type 6 - Highly laminated siltstones (F)

Type 1 is the only rock type with reservoir potential.

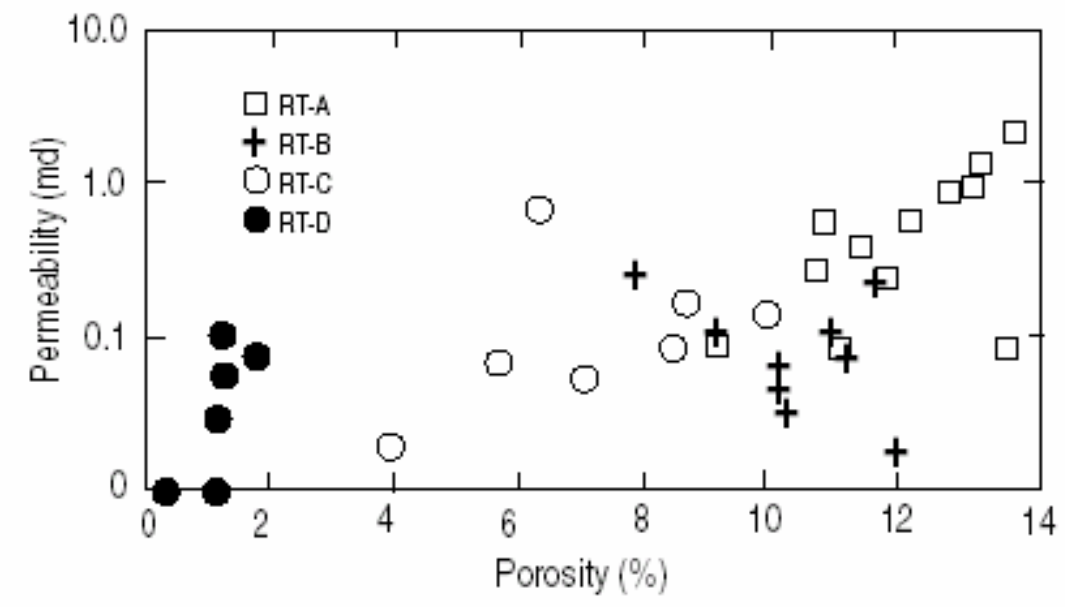

Fig. 1.3.3 - Porosity - permeability crossplot for the primary rock types identified ${ }^{18}$. 
The rock log model has proved consistent in the preliminary identification of pay and non-pay reservoirs. Deflections from gamma ray corresponding to lower values were used to define probable reservoir quality sandstones in the past, with typical cutoffs ranging from $45-50$ API units. With these cutoffs, individual zones within the $1 \mathrm{U}, 2 \mathrm{U}$, $3 \mathrm{U}, 4 \mathrm{U}$ and $5 \mathrm{U}$ intervals were thought to be possible pay zones in the ET O'Daniel wells. Core data has however shown that only the $1 \mathrm{U}$ and $5 \mathrm{U}$ exhibit any fluorescence, moreover, the intermediate intervals $2 \mathrm{U}$ to $4 \mathrm{U}$, despite showing gamma ray values in the 30 - 50 API range, had porosities under 7\% and much higher concentrations of dolomite cement $^{9}$. Core data indicate that shale volume can be determined from the gamma ray log, effective porosity from the density neutron log crossplots or from bulk density log data or from sonic transit time.

A typical playback using these criteria is shown in Fig. 1.3.4, in track 1 gamma ray and calculated shale volume using the Larionov non-linear model ${ }^{19}$, track 2 shows the shallow-medium-deep induction log, track 3 shows the effective porosity (shale volume corrected) and the core derived porosity values, track 4 shows the core derived permeability values and the calculated permeability values from log data using conventional regression techniques, track 5 shows pay and non - pay intervals using the log based rock model.

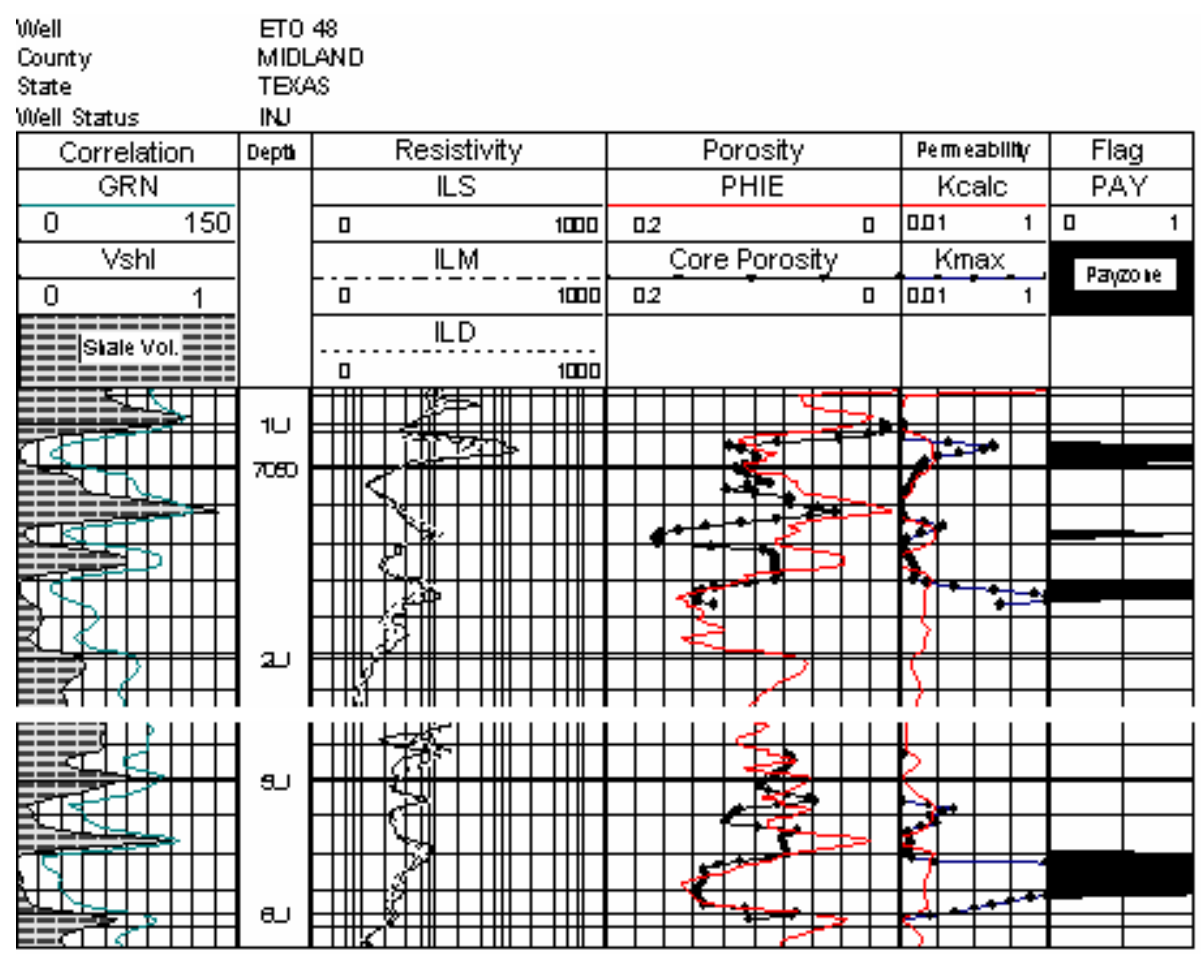

Fig. 1.3.4 - Identification of pay based on shale volume and effective porosity cutoffs. 


\subsection{Permeability estimation techniques}

An important parameter which is key to the rock model is the estimation of permeability from log data. Conventional methods estimate $\mathrm{k}$ from e-logs and consist of building models between regularly spaced core plug measurements and logs, without paying much attention to the various scales of core plug sampling ${ }^{20}$.

In uncored intervals $\mathrm{k}$ are usually estimated from well test, production or log data. Early attempts used porosity (unsatisfactorily), which is not unexpected as the permeability is related to pore throat size rather than pore volume. More recently, a host of relationships have been investigated between permeability and other rock attributes. Permeability as a function of porosity and irreducible water saturation ${ }^{21}$, or bulk density, neutron porosity, interval transit time and gamma ray ${ }^{22}$. A multi-dimensional histogram approach, deriving permeability based on bulk density, interval transit time and gamma ray was investigated successfully by Schlumberger. Some other functional relationships investigated are related to formation resistivity, normalized spontaneous potential and borehole Stoneley waves $^{22,23,24,25}$.

In analyzing permeability dependency on single or multiple variable, regression as well as discriminant analysis are the most widely used techniques of evaluation.

These techniques can be classified into two broad groups: Explicit probabilistic methods and implicit irobabilistic methods.

\subsubsection{Explicit probabilistic techniques}

Regression Analysis - This is a crossplot of 2 dimensions, used to predict values in intervals without core data and wells without core data. This method assumes the functional form of the relationship between the prediction and response variable is unknown. The drawbacks of this method is that it over-simplifies reality and tends to smooth out real variations or trends in the data, because more often than not, other independent factors influence the prediction, therefore making a two dimensional prediction inadequate for reliability. Sub-dividing the data into logically coherent groups in geologically correlated zones often improves the overall correlation.

Multiple Regression analysis includes additional variables or non-linear regression techniques.

The 'ACE” algorithm originally proposed by Friedman and Breiman ${ }^{26}$ provides a method for estimating optimal transformations for multiple regression that result in a maximum correlation between a dependent (response) random variable and multiple independent (predictor) random variables. Xue et. al. ${ }^{27}$, went further to develop a non-parametric approach that optimizes based on no predetermined functional form, derived solely based on the data set.

Discriminant analysis - This is a multi variate technique designed to separate samples into groups based on relationships found in a training set of data. The relationship must 
be such that they can be defined explicitly and must be linear combinations of functions of the predictor variables.

\subsubsection{Implicit probabilistic techniques}

Probabilistic or database methods are intrinsic (or implicit) relationships of data compiled in a multi dimensional database. A value of $\mathrm{y}$ is read from a database corresponding to a value of $\mathrm{x}$. In this way the implicit relationship between the data are preserved.

$\mathrm{N}$-Dimensional histogram - When the $\mathrm{x}$ corresponding to $\mathrm{y}$ concept is expanded to include additional variables, the approach becomes an 'n-dimensional histogram', and the discrimination of the dependent variable is generally improved. This method has the following advantages over regression techniques in that it has the ability to preserve the subtle relationships between variables, it fully utilizes the shape characteristics of the data and it has the ability to incorporate soft data such as facies type into the database to define the categories of qualitative histograms.

Cluster analysis - This is a multi-variate technique for classification of samples into groups based on little or no prior knowledge of that grouping. Simple cluster analysis does not use the information on facies known from the cored interval, but instead attempts to find natural groupings, called clusters based on the estimator variable.

\subsection{Porosity estimation}

Porosity is determined from 3 basic log types that measure porosity directly (neutron) or indirectly (density and sonic). Where a neutron count based porosity value is known from the older neutron logs, a conversion algorithm ${ }^{28}$, may be used to convert counts per second or any CPS derived unit (environmental units, API cps, etc) which exhibits a logarithmic scale of porosity to porosity values on a linear scale.

Where the density, neutron porosity and photoelectric effect curves are available, porosity measurements based on shale corrected lithology model can be reliable and consistent over a wide range of rock types ${ }^{29}$. No matrix parameters are required for this model unless light hydrocarbons are present. Shale corrected density and neutron data are used as input in this model and results depend on shale volume calculations and density and neutron shale properties selected for the model. Therefore porosity should be compared to core data and corrected accordingly till a suitable match is obtained between both data sets.

Where limited suite of porosity logs are available, a model based on the shale corrected density, shale corrected neutron or shale corrected sonic is used ${ }^{29}$. 


\subsubsection{Gamma ray}

The Gamma Ray log is a continuous recording of the intensity of the natural gamma radiations emanating from the formations penetrated by the borehole vs. depth.

In sedimentary formations, since the radioactivity can be attributed mainly to the clay minerals, the gamma ray log can be used to distinguish between shale and non-shale formations and to estimate the clay content of shaly formations.

\subsubsection{Clay content}

Clay or shale content can be quantified using a shale index from values given by the gamma ray log. Different models are available for quantifying this index:

General linear form

$V_{\text {shl }}=\frac{X_{\text {raw }}-G R_{\text {clean }}}{G R_{\text {shl }}-G R_{\text {clean }}}$

Other models used to modify the index to account for various degrees of non-linearity between the gamma ray response and the clay content are available:

Larionov's model for tertiary rocks

$V_{\text {shl_modified }}=0.083\left(2^{3.7 V_{\text {shl }}}-1\right)$

Larionovs' model for older rocks

$V_{\text {shl_modified }}=0.33\left(2^{2.0 V_{\text {shl }}}-1\right)$

Stiebers' model

$V_{\text {shl_modified }}=\frac{V_{\text {shl }}}{3-2 V_{\text {shl }}}$

Claviers' model

$V_{\text {shl_modified }}=1.7-\left(3.38-\left(V_{\text {shl }}+0.7\right)^{2}\right)^{0.5}$ 


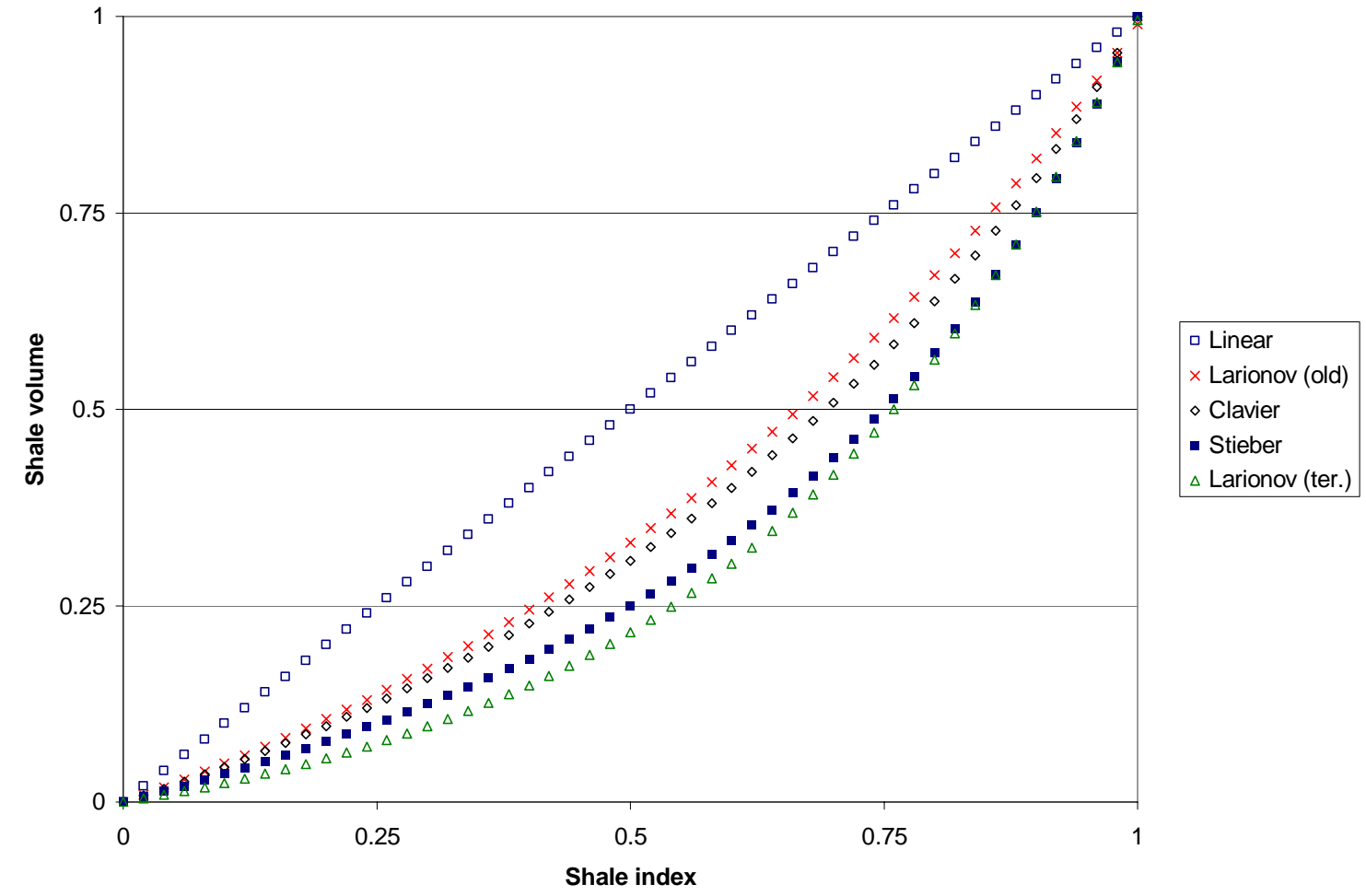

Fig. 1.3.5 - Non-linearity of the different models for estimating clay content using gamma ray.

\subsection{Log normalization}

Well log normalization is a fundamental part of well log analysis, and is one of the necessary steps for arriving at accurate rock quality descriptors. The foundation of the integrated log analysis process is the core, well test and log database ${ }^{30}$. The short comings in the foundations of the analysis ultimately influence the quality of the final estimations of permeability, the interdependence of the descriptors are shown in Fig. 1.3.6.

Errors in the database will trickle up to affect shaliness, porosity and water saturation calculations. Also errors in shaliness calculation will cause additional errors in porosity and water saturation because these calculations also depend on shaliness. When everything is done correctly useful values of permeability and effective permeability can be obtained from integrated studies.

In excess of fifty percent of all well logs are erroneous, Neinast and $\mathrm{Knox}^{31}$ base this percentage on an analysis of 1986 suites of well logs containing more than 34 million curve feet. The basic sources of error are tool malfunction, incorrect tool design, inconsistent shop and field calibration, and operator error. All but ten percent of the incorrect logs may be corrected and the data used quantitatively. 


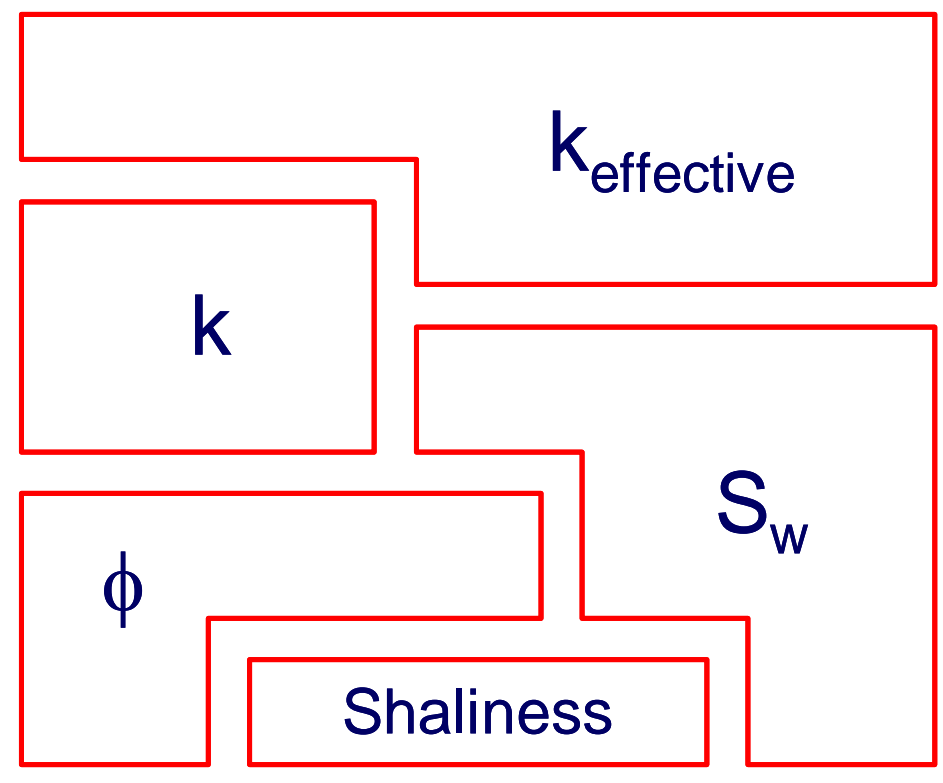

\section{Core, welltest \& Log data}

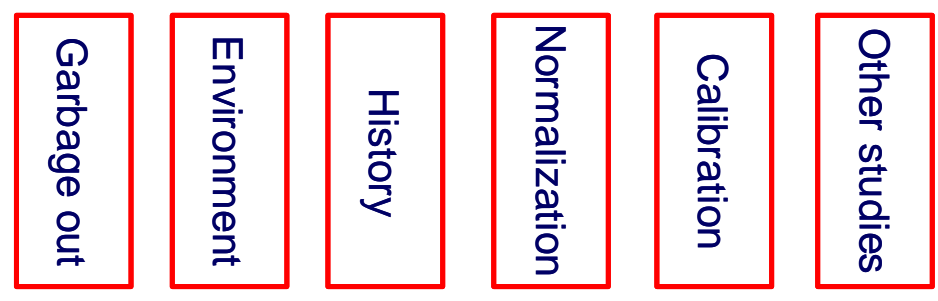

Fig. 1.3.6 - The log analysis process ${ }^{30}$.

Hunt $^{30}$ in his analysis, suggests that about $65-70 \%$ of gamma rays logs, $50 \%$ of density logs, $40 \%$ - $50 \%$ of neutron logs and $5 \%$ - $10 \%$ of sonic logs require some normalization to correct for variances in field calibrations of logging tools. After normalization well log data can be effectively integrated, correlated and calibrated with core data. The resulting correlations can be extended vertically to include layers that were not cored, and laterally to wells across the study area. The difference in scale of measurement of the two data sets must be taken into account. Core data have a scale of cubic inches, while log data have a scale of cubic meters and well test data have a scale of acre-feet.

The normalization procedure to correct log data requires the following ${ }^{31}$ :

Digitize the well log data

Select corresponding lithologic intervals

Accumulate and present data in appropriate form (Histograms, etc)

Compute porosity and water saturation

Compare with core analysis 
Map to reveal anomalies

Digitization - The individual curves are depth matched either prior to data capture in ASCII format or if post processing software is available to correct depth anomalies. All heading data is combined with the log values to allow pertinent corrections to be made in subsequent calculations.

Interval selection - Correlation of stratigraphic intervals is of extreme importance. The earth changes radically in a vertical direction and gradually in a lateral direction. Appropriate corresponding lithological sections must be chosen so that comparison of similar intervals may be accomplished. Every effort should be made to eliminate pay zones or other zones of interest as data for correlation prior to normalization.

Data presentation - Data must be accumulated in a form that allows rapid and concise corrections. Variations in thickness of explicit sections is eliminated by presenting the information in statistical format. The basic concept is the formation of patterns that the analyst recognizes and compares to make the proper corrections. Three methods of data presentation are histograms, crossplots and overlay.

\subsubsection{Histogram}

The histogram is made by plotting the percent frequency of occurrence of data on the abscissa against the log unit value on the ordinate. The mean and standard deviation are calculated along with the mode, maximum and minimum values, and the net and gross number of samples used in the histogram. Histograms of discriminated or complete log values may be prepared for specific correlated intervals. The individual frequency histograms may be compared with similar histograms from other wells, with core derived histograms, or with mass histograms or entire regions or fields.

\subsubsection{Crossplots}

Crossplot techniques for lithology and porosity determination have been in use for several years. Additional advantage of dual porosity device data may be taken if the lithology is known or assumed. Errors in individual tools may be detected when the crossplotted data falls outside the range delineated by constant mineral lines. When three porosity logs are available, the data can be used to develop an $\mathrm{M}-\mathrm{N}$ plot and allow corrections to properly compute porosity. The procedure is to first histogram and normalize individual logs, then verify and refine the normalization with crossplots.

\subsubsection{Overlays}

This is a simple process of correlating and overlaying similar type logs and noting the difference. 
Computation and comparison - After the data has been normalized, the water saturation, porosity, lithology, permeability, etc. are computed on a foot by foot basis and compared to the weighted average core data to determine the degree of compatibility

Mapping - Contour maps are generated on selected intervals. Generally porosity and water saturation are the parameters used to confirm normalization. All drastic changes and abrupt highs and lows are rigorously verified as to validity.

\section{Data Review (E.T. O'DANIEL)}

\subsection{Core sampling}

Cores samples were recently taken from six (6) wells within the ET O'Daniel unit, location of the wells are within the waterflood pilot area in the south east part of the lease (see Fig. 1.3.7). Though these samples are localized, they provide useful data towards verifying the established rock log model. Core data statistics are given in Table 1.3.2.

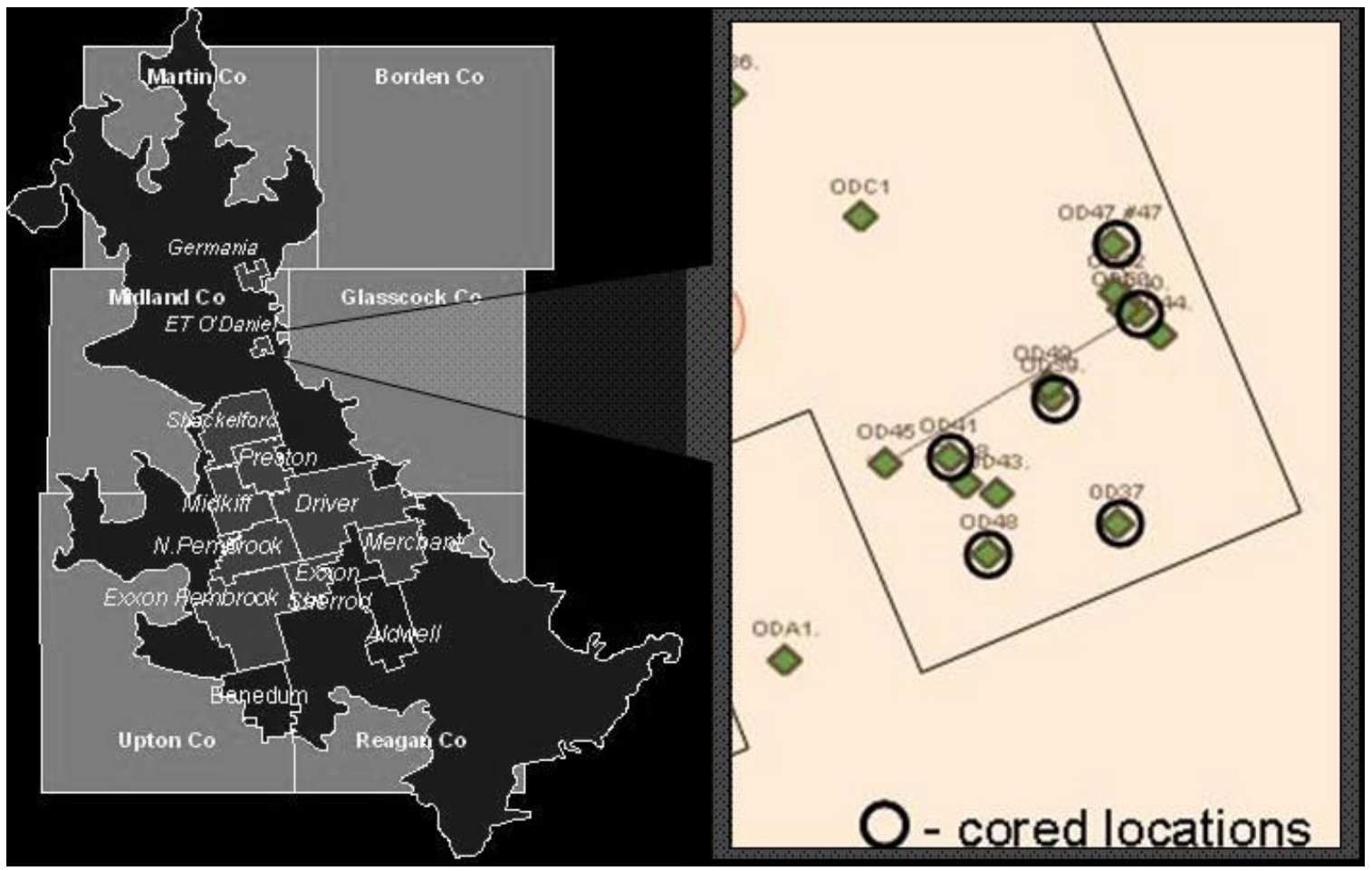

Fig. 1.3.7 - Map of cored well locations in the ET O’Daniel pilot area.

The analysis was carried out by Reservoirs Incorporated. Procedure for estimating core parameters involved firstly correlating core to log depth by gamma ray scan of the cores, water and oil saturations were determined using the Dean-Stark extraction method and 
ultraviolet photographs taken. Sponge oil volumes adjacent to the core samples were reported as \% pore volume and horizontal - vertical permeability to air was measured using a Hassler sleeve permeameter.

The core values obtained are integrated into the log database using the Geographix software, and depth matched using the log and core porosity response as a guide. This ensures that all data sampled from the logs with reference to the core data are for the same interval.

Table 1.3.2 - Summary of rock properties and saturations for ET O’Daniel wells.

\begin{tabular}{|c|c|c|c|c|c|c|c|c|c|}
\hline Well & Flow Unit & Interval, ft & Sw, v/v & So, v/v & Por \% & kmax, md & Gr. Den, g/cc & Bulk Den, g/cc & Fluorescence \\
\hline \multirow[t]{2}{*}{ ETO 37} & $1 U$ & $7040-7066$ & 0.52 & 0.12 & 8.14 & N/A & 2.71 & $\mathrm{~N} / \mathrm{A}$ & trace -100 \\
\hline & $5 U$ & $7216-7240$ & 0.53 & 0.16 & 8.73 & N/A & 2.68 & N/A & trace -100 \\
\hline \multirow[t]{2}{*}{ ETO 38} & $1 U$ & $7060-7087$ & 0.41 & 0.06 & 10.07 & 0.57 & 2.65 & N/A & $25-100$ \\
\hline & $5 U$ & $7210-7237$ & 0.34 & 0.08 & 10.39 & 1.36 & 2.65 & N/A & no - 100 \\
\hline ETO 39 & \multicolumn{9}{|c|}{ Core values previously integrated into LAS files } \\
\hline \multirow[t]{2}{*}{ ETO 40} & 10 & $7088-7115$ & 0.28 & 0.17 & 10.20 & 6.95 & 2.68 & $N / A$ & no -100 \\
\hline & $5 U$ & $7236-7264$ & 0.34 & 0.15 & 9.06 & N/A & 2.67 & N/A & no -100 \\
\hline \multirow[t]{2}{*}{ ETO 47} & $1 U$ & $7086-7108$ & 0.47 & 0.08 & 8.38 & 0.14 & 2.70 & 2.48 & N/A \\
\hline & $5 U$ & $7240-7267$ & 0.48 & 0.11 & 8.59 & 0.19 & 2.69 & 2.46 & N/A \\
\hline ETO 48 & \multicolumn{9}{|c|}{ Core values previously integrated into LAS files } \\
\hline
\end{tabular}

\subsection{Depth matched core-log playback}

A depth matched playback for a cored well ET O'Daniel 37 displays the core derived parameters in tracks 2,3 and 4 (core porosity / permeability, saturations and fluorescence). 


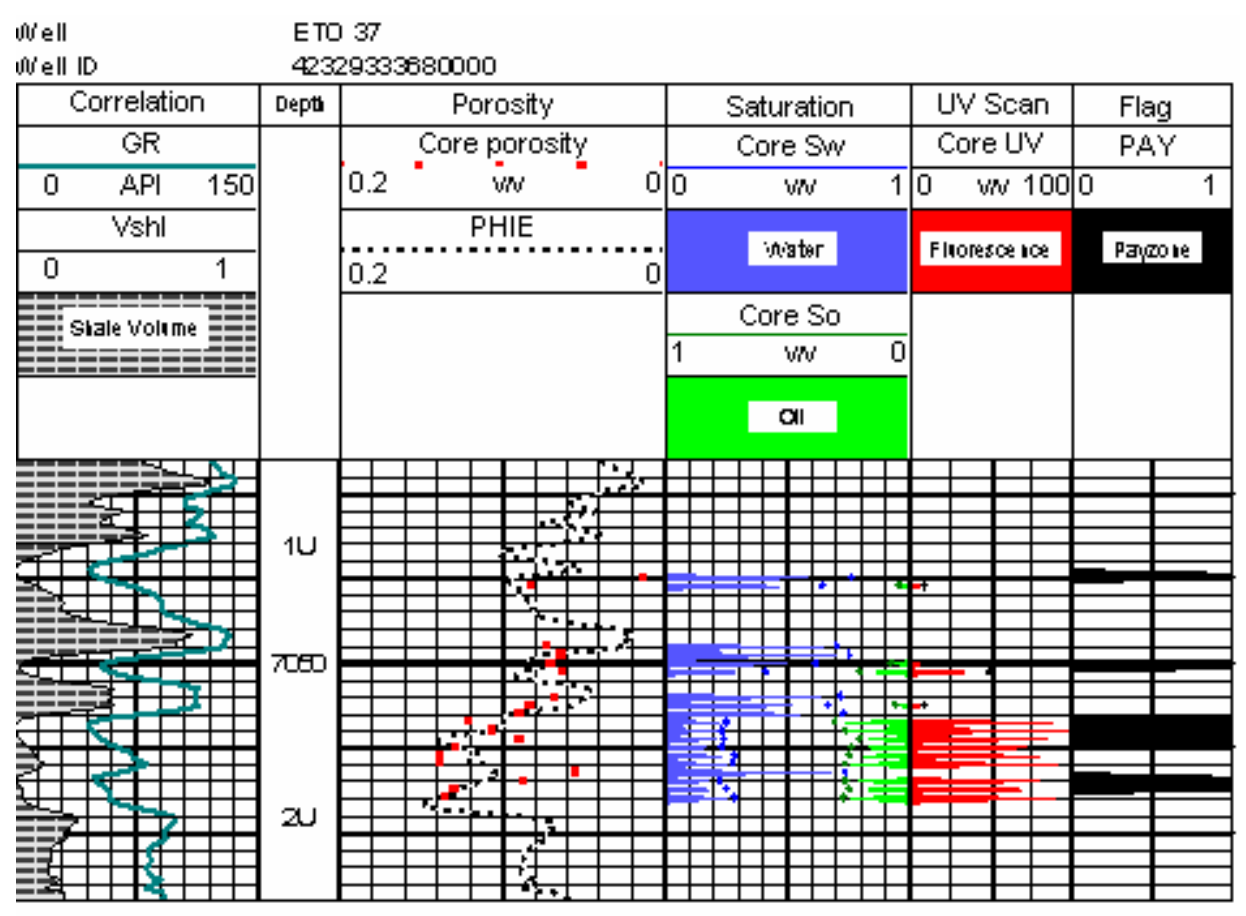

Fig. 1.3.8 - Typical core - log playback in the $1 \mathrm{U}$ interval.

Gamma ray and shale volume playback in track 1 are based on normalized data, track two displays porosity values from core and log data and permeability from core analysis.

The agreement between core and log porosity is fairly good (track 2), but of more importance is the pay flag in track 5 based on the rock model. This pay flags strongly correlate with the fluorescing interval in track 4.

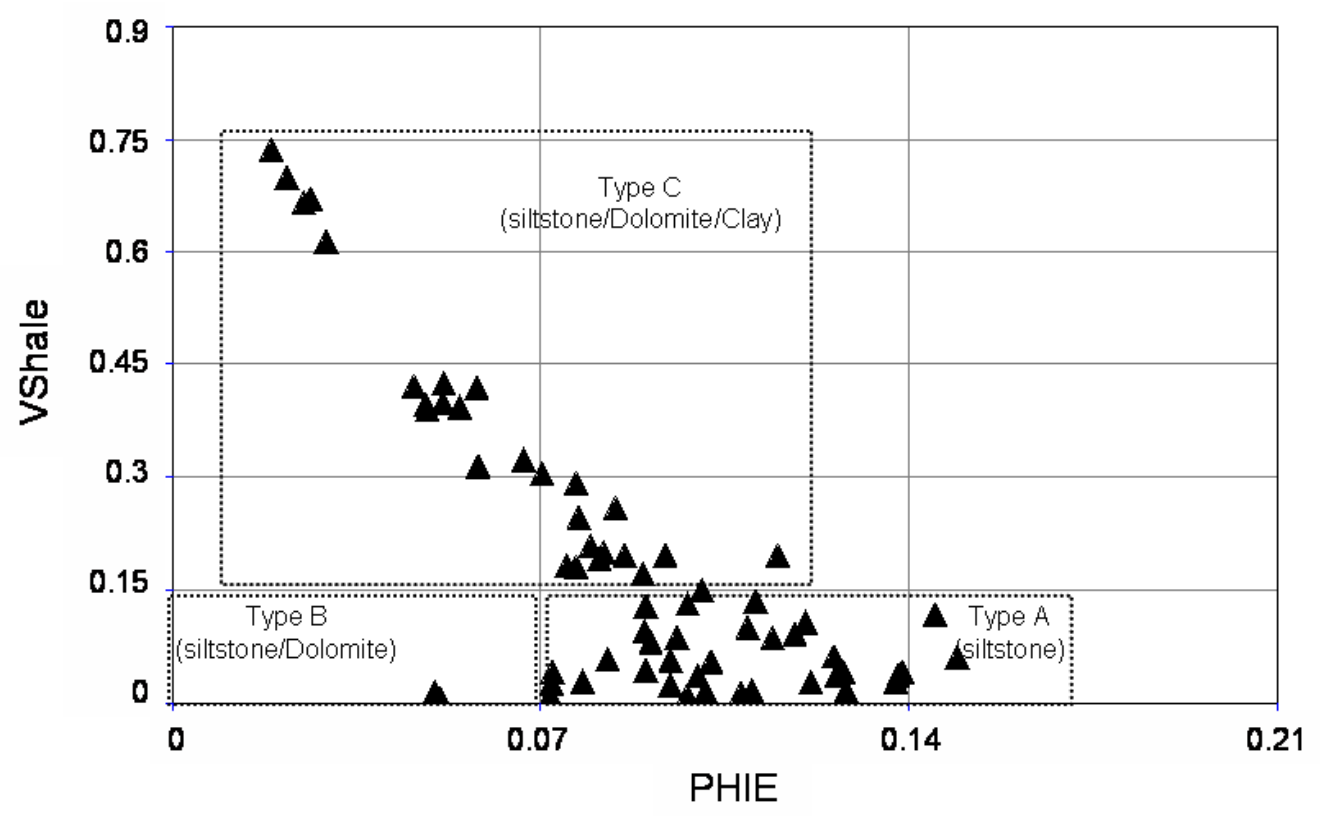

Fig. 1.3.9 - Crossplot of shale volume and porosity for ET 37, $1 \mathrm{U}$ sand. 
The shale volume - porosity crossplot gives a quantitative indication of the rock types within the analyzed interval. In Fig. 1.3.9 the amount of rock type B is minimal, while type A and C are evenly distributed in the $1 \mathrm{U}$ interval for well ETO37.

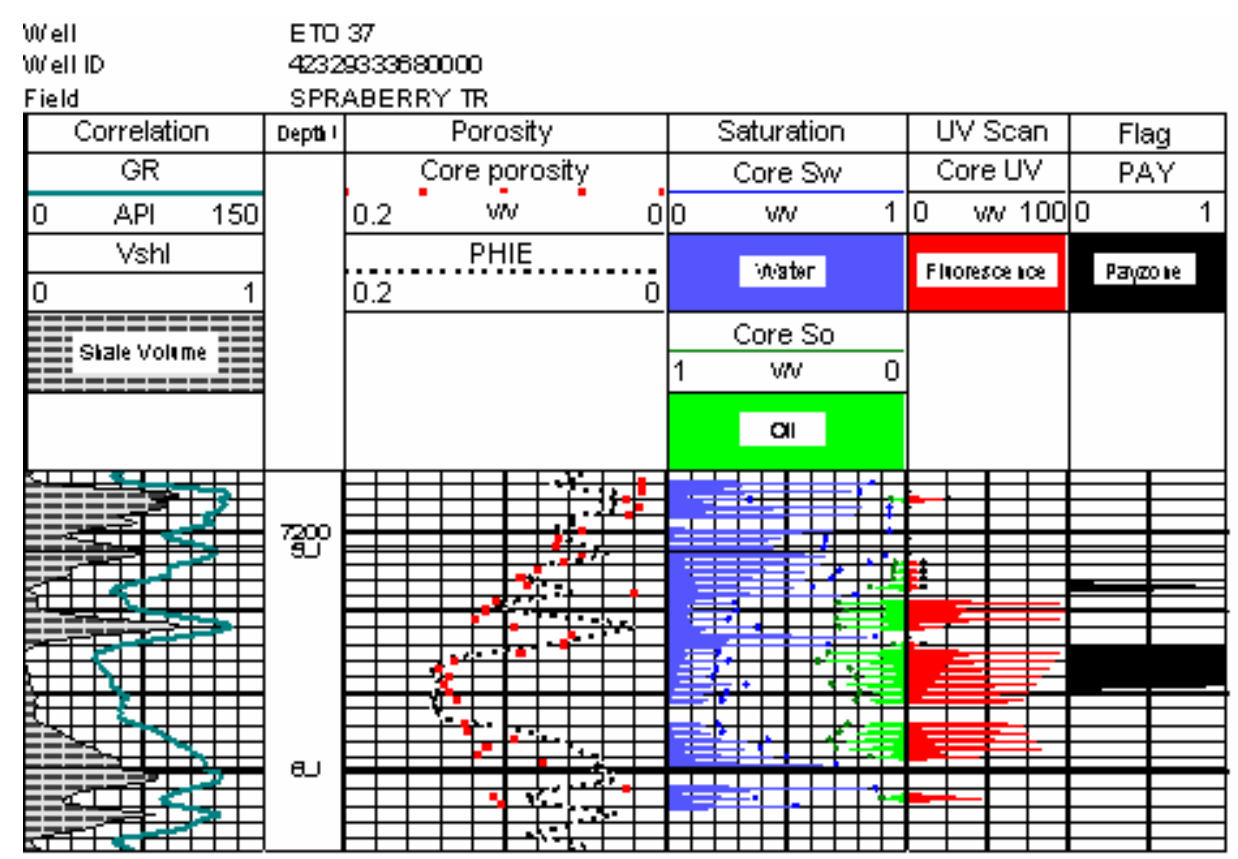

Fig. 1.3.10 - Typical core - log playback in the 5U interval.

The payflag and fluorescing interval for this unit (5U) correlate well after a depth shift based on core porosity and log porosity matching. The playback resulting from this optimal correlation is shown in Fig. 1.3.8

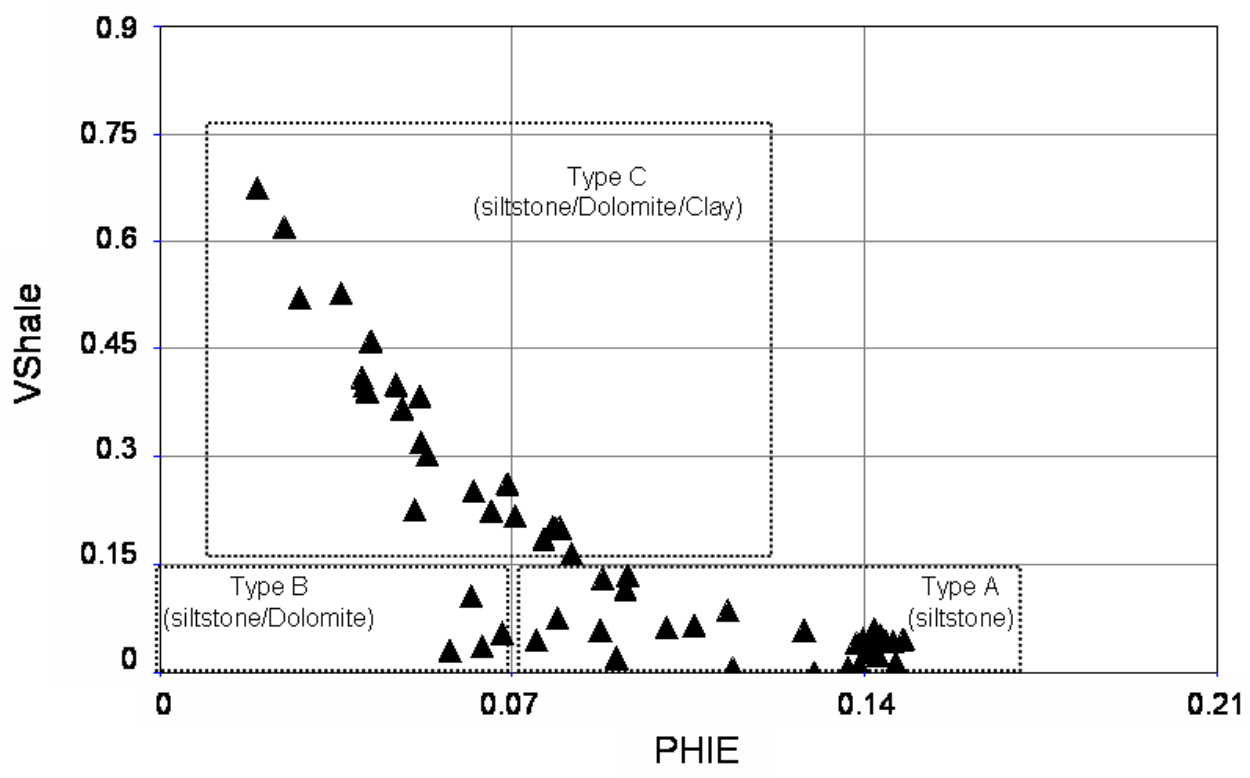

Fig. 1.3.11 - Crossplot of shale volume and porosity for well ET 37, 5U sand. 
A number of observations are evident from figs. 1.3.10 to 1.3.11, these are that the low shale intervals of the $1 \mathrm{U}$ and $5 \mathrm{U}$ units are almost exclusively payzones or type A rocks, while the higher shale intervals are exclusively non-hydrocarbon bearing sands. Also a estimate of the productive intervals (net pay) account for about $50 \%$ of the gross sand in the $1 \mathrm{U}$ unit and about $40 \%$ of the gross sand in the $5 \mathrm{U}$ intervals. Other well analyzed also displayed similar trends as observed in wells ETO 37.

The $1 \mathrm{U}$ and $5 \mathrm{U}$ pay zones are easily identified by integrating whole core analysis and open hole logs into a calibrated shaly-sand model. The $2 \mathrm{U}, 3 \mathrm{U}$ and $4 \mathrm{U}$ zones are not consistent with this model ${ }^{5}$, this is due to the large concentration of dolomitic cements, thus rendering low gamma ray (low shale content) sands in this region as non-pay.

\subsection{Lithology}

The density-neutron crossplots among other uses are invaluable as indicators for lithology and rock types. Figs. 1.3.12a - b, show the results of crossplots of neutron density in the wells in which they are available.

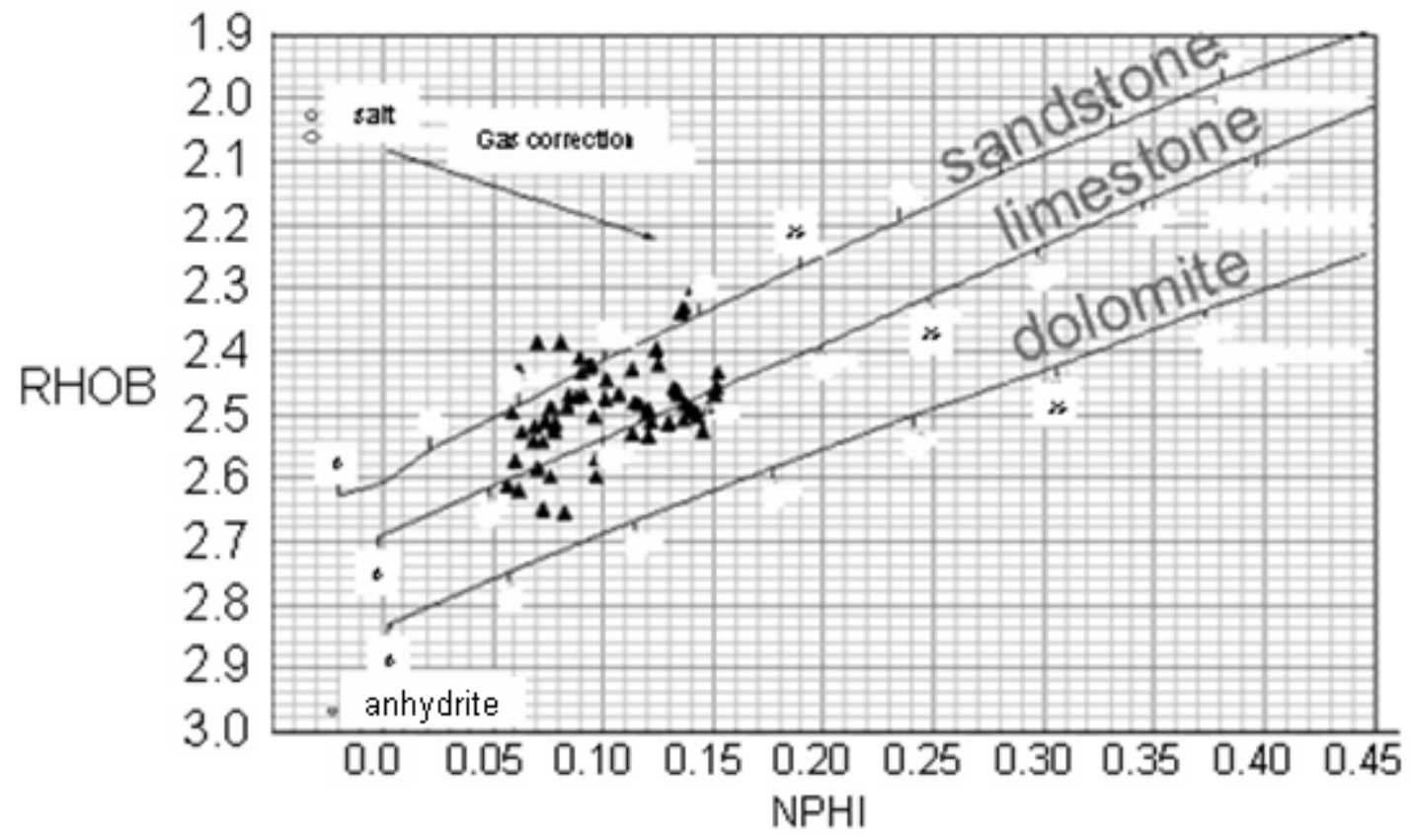

Fig. 1.3.12a - Crossplot for lithology identification in 1U sand for well ET 37. 


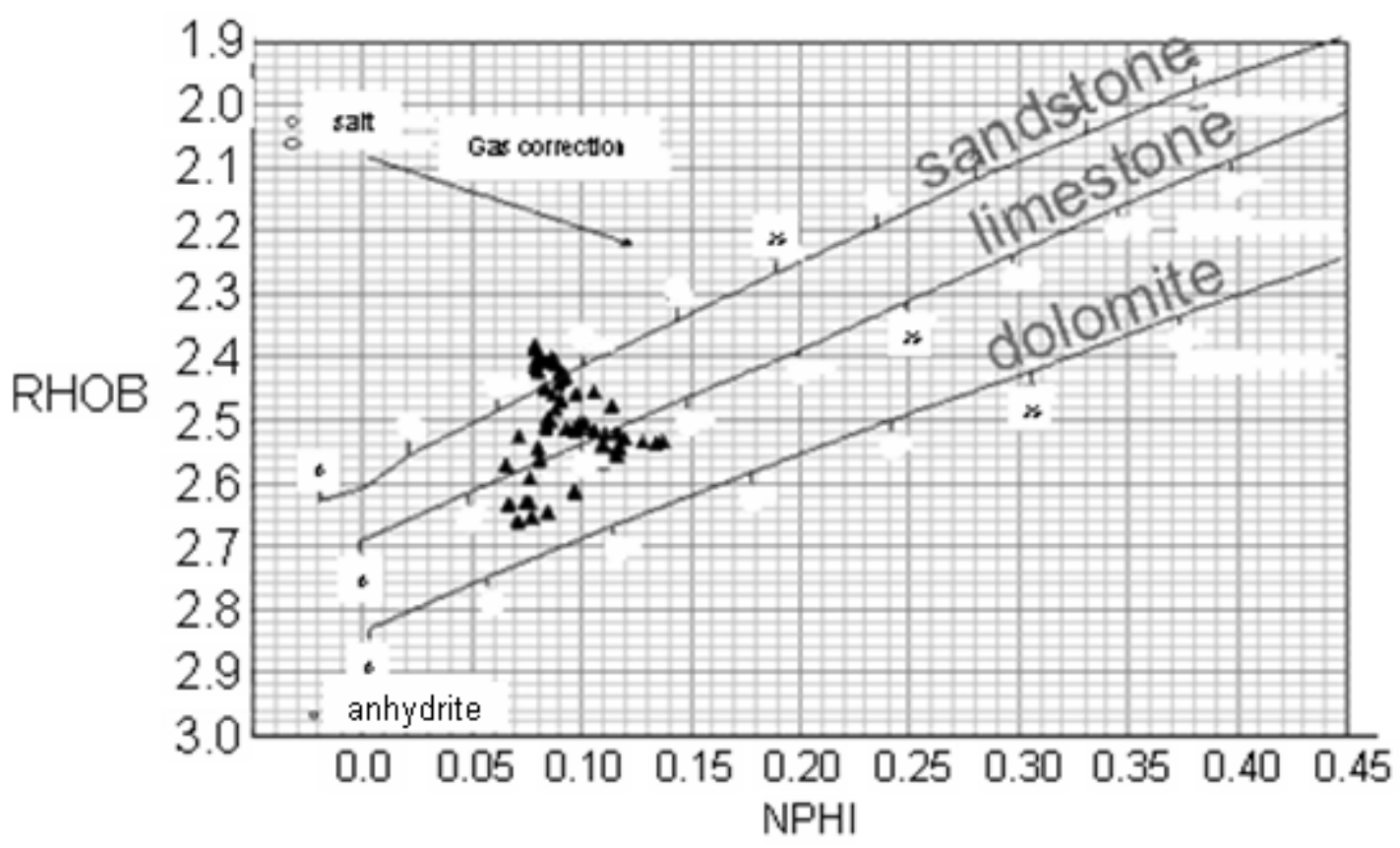

Fig. 1.3.12b - Crossplot for lithology identification in 5U sand for well ET 37.

The gas correction if applied will tend to shift the data down and right i.e. reduce density porosity and increase neutron porosity. Shale correction will depend on the type of shale (structural, laminated or dispersed).

\section{Data Analysis}

\subsection{Log conversions and normalization}

26 logs are available within the ET O’Daniel unit, including log data for the cored wells. The wells are a variety of observation wells, injectors and producers.

59 well logs are available within the Germania Spraberry unit. Most of the logs are neutron logs taken as far back as 1950, with a few recent porosity and resistivity logs.

Log normalizations are performed on both log data sets prior to any transformations or inferences as to the significance of the log analysis. Illustrative procedures are shown for ET O’Daniel in the proceeding sections. 


\subsubsection{Gamma ray}

This log forms the basis of pay identification within the Spraberry rock model. It is therefore important that the gamma ray is scaled appropriately to enable a consistent shale volume calculation from well to well.

Gamma ray curves for all the logs within the ET O’Daniel database were analyzed, and it was discovered that no two logs gave the same values at any chosen marker. Though this is expected, the wide variance in the response across these markers indicate the necessity for normalization of the gamma ray logs. More so, due to the fact that for a multi-well analysis, the Shale volume calculations will need to be revised for every well log if this process is not carried out.

\section{$\underline{\text { 3.1.1.1 Gamma ray maps }}$}

Often mapping techniques are used to discern trends of gamma ray values. These gamma ray values may sometimes show systematic variation that may often be mistaken as tool or calibration errors, we therefore need to map the lower and upper limits of the gamma ray values to verify whether or not trends exist rather than assume the need for normalization of gamma ray values.

Figs. 1.3.13 and 1.3.14 show maps of minimum and maximum raw gamma ray values obtained within $1 \mathrm{U}$ sand interval. Values are obtained by taking lowest and highest gamma ray observations within the interval of interest from digitized data. 


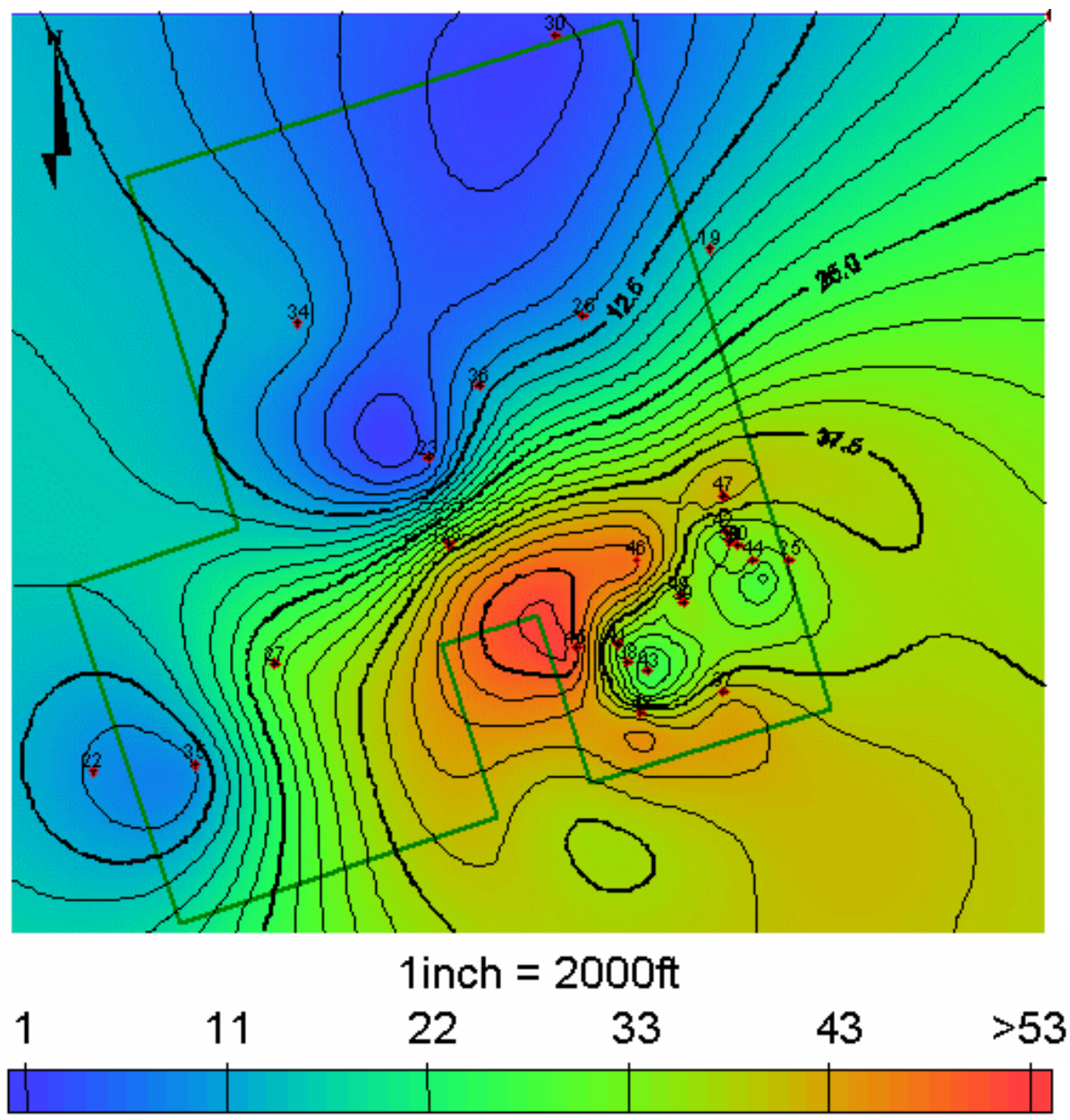

Fig. 1.3.13 - Minimum gamma ray values for ET O’Daniel unit in the $1 \mathrm{U}$ sand interval.

Often a bulls eye pattern on a contour map will give away the fact that the data points are random or lack any systematic variation in space. From the figure above we see that the NW section of the area is consistently low and the SW is consistently high, this might indicate a systematic trend. From the maximum gamma ray values in the $1 \mathrm{U}$ interval (Fig. 1.3.14) we do not see this trend, instead we see bullseye patterns, this will suggest that the trend in the $1 \mathrm{U}$ lacks consistency and hence indicate that normalization is required. 


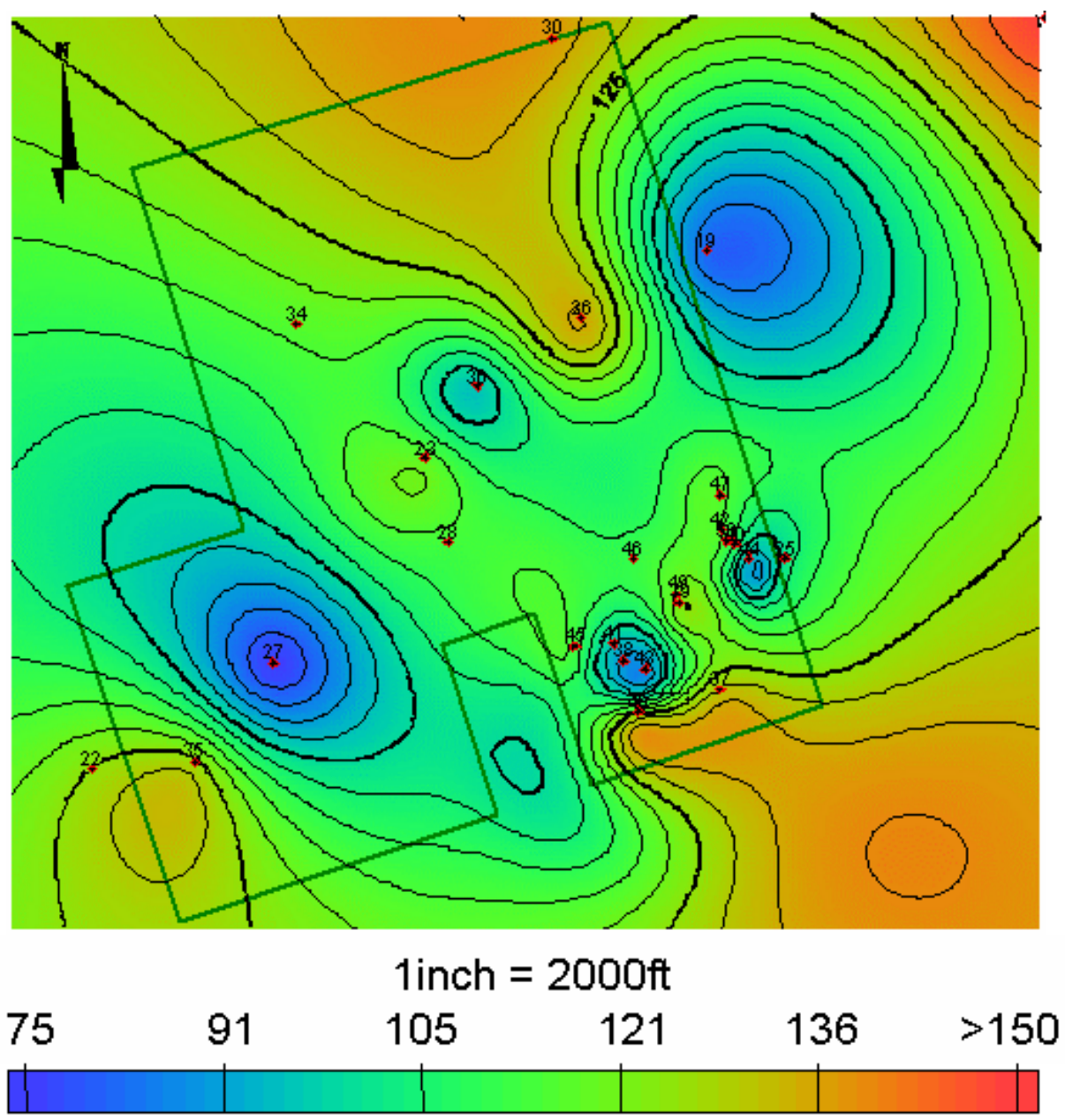

Fig. 1.3.14 - Maximum gamma ray values for ET O’Daniel unit in the 1U sand interval.

The same plots were generated for the $5 \mathrm{U}$ interval and the same conclusion was drawn based on the seemingly random distribution of the gamma ray values on both the minimum and maximum value distributions (see appendix B for figures). 


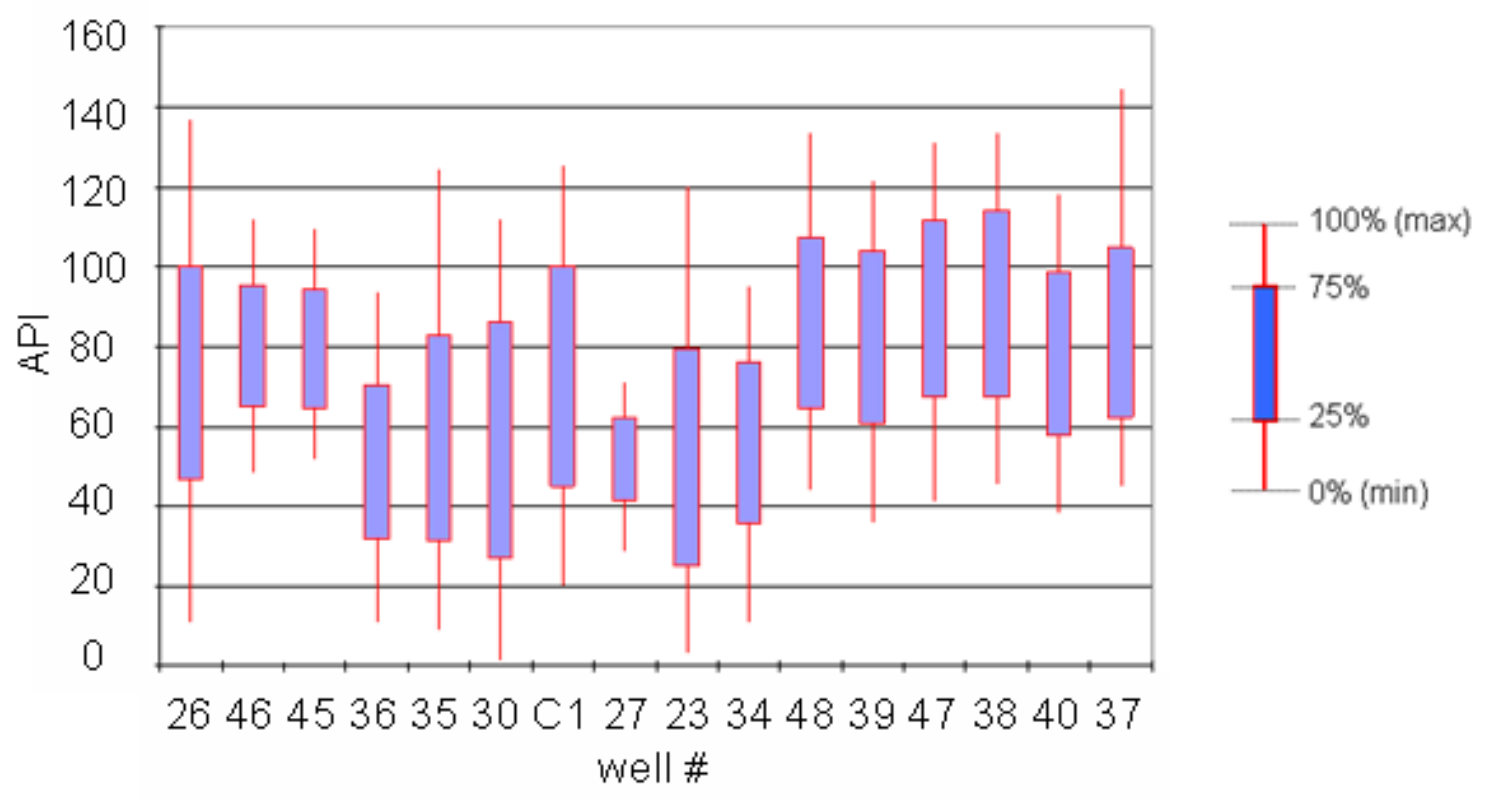

Fig. 1.3.15 - Variations in response from the gamma ray curves in ET O’Daniel.

\subsubsection{Gamma ray normalization}

It can be inferred that the field standard based on the limited database is the range with the highest number of occurrence. Wells 37, 38, 39, 40, 47, 48, C1 and 26 are spread across the $20-140$ API range and are the group exhibiting similar ranges.

A histogram for a type well representing the field standard is used to adjust all other wells deemed to require normalization. Fig. 1.3.16 shows the histogram and cumulative density functions for well 36 before and after normalization using well 26 as the standard. 


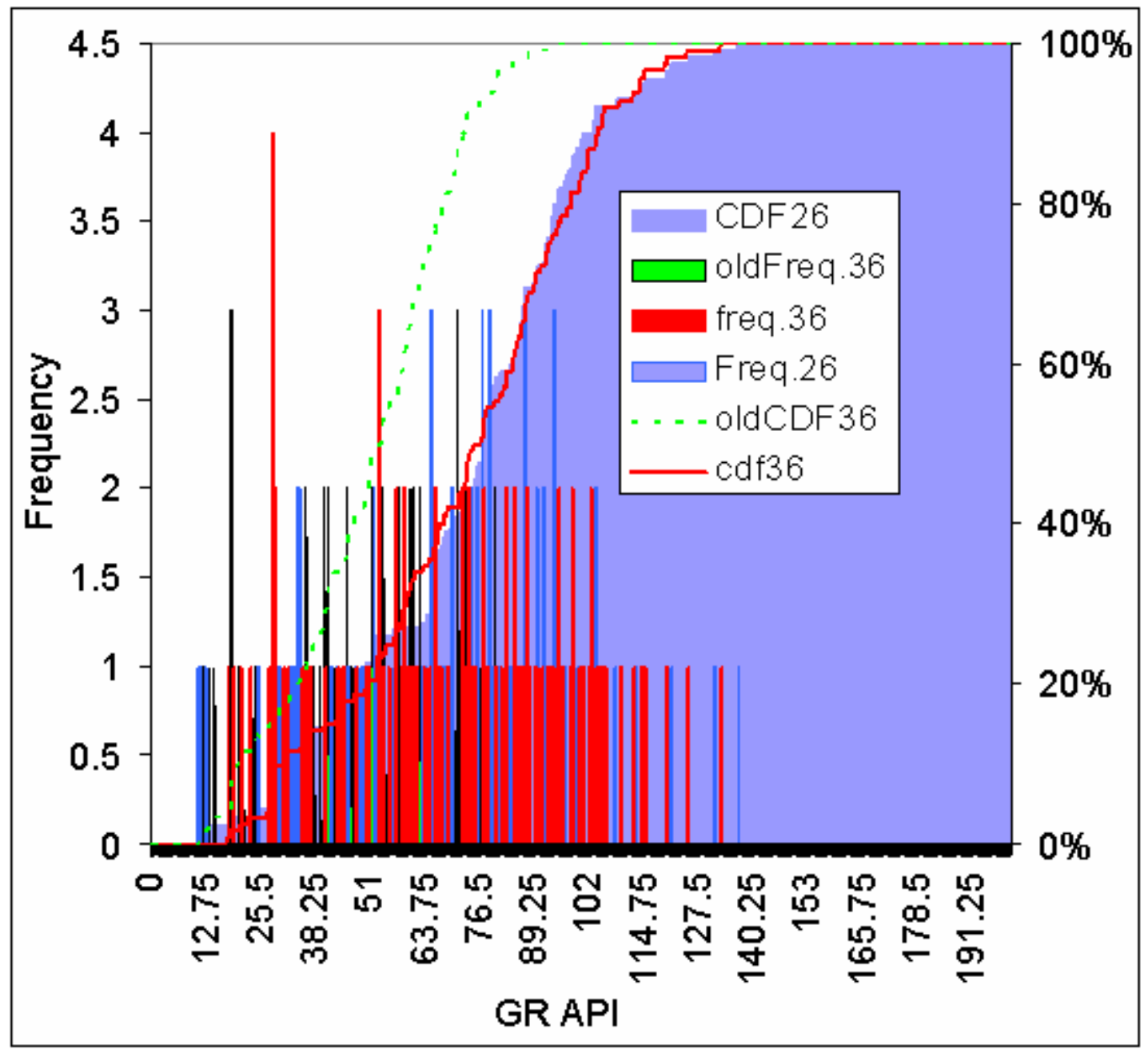

Fig. 1.3.16 - Histogram and CDF for wells 36, before and after normalizing against well 26.

A single equation for applying linear adjustments to log data is given by Shier $^{28}$.

$$
X_{\text {norm }}=R_{\text {low }}+\frac{\left(R_{\text {high }}-R_{\text {low }}\right)\left(X_{\text {raw }}-W_{\text {low }}\right)}{\left(W_{\text {high }}-W_{\text {low }}\right)}
$$

A different method used to adjust well log data involves the adjustment of each data point by a constant value such that the mean of the sample data equals the mean of the type log data. Thereafter, an 'Affine' correction is then applied to the sample data such that the variance of the sample equals the variance of the type log data. A computer program may be used to solve for the appropriate shift and correction factor required to match the mean and variance of the type log data.

Affine Correction ${ }^{32}$.

$$
X_{\text {norm }}=\sqrt{\text { s. } f}\left(X_{\text {raw }}-\mu\right)+\mu
$$




$\begin{array}{lll}\mathrm{X}_{\text {norm }} & - & \text { Normalized well value } \\ \mathrm{X}_{\text {raw }} & - & \text { Actual well value } \\ \mathrm{R}_{\text {low }} & - & \text { Regional low normalization value } \\ \mathrm{R}_{\text {high }} & - & \text { Regional high normalization value } \\ \mathrm{W}_{\text {low }} & - & \text { Wells lithological low value } \\ \mathrm{W}_{\text {high }} & - & \text { Wells lithological high value } \\ \text { s.f } & - & \text { Correction factor } \\ \mu & - & \text { Population mean }\end{array}$

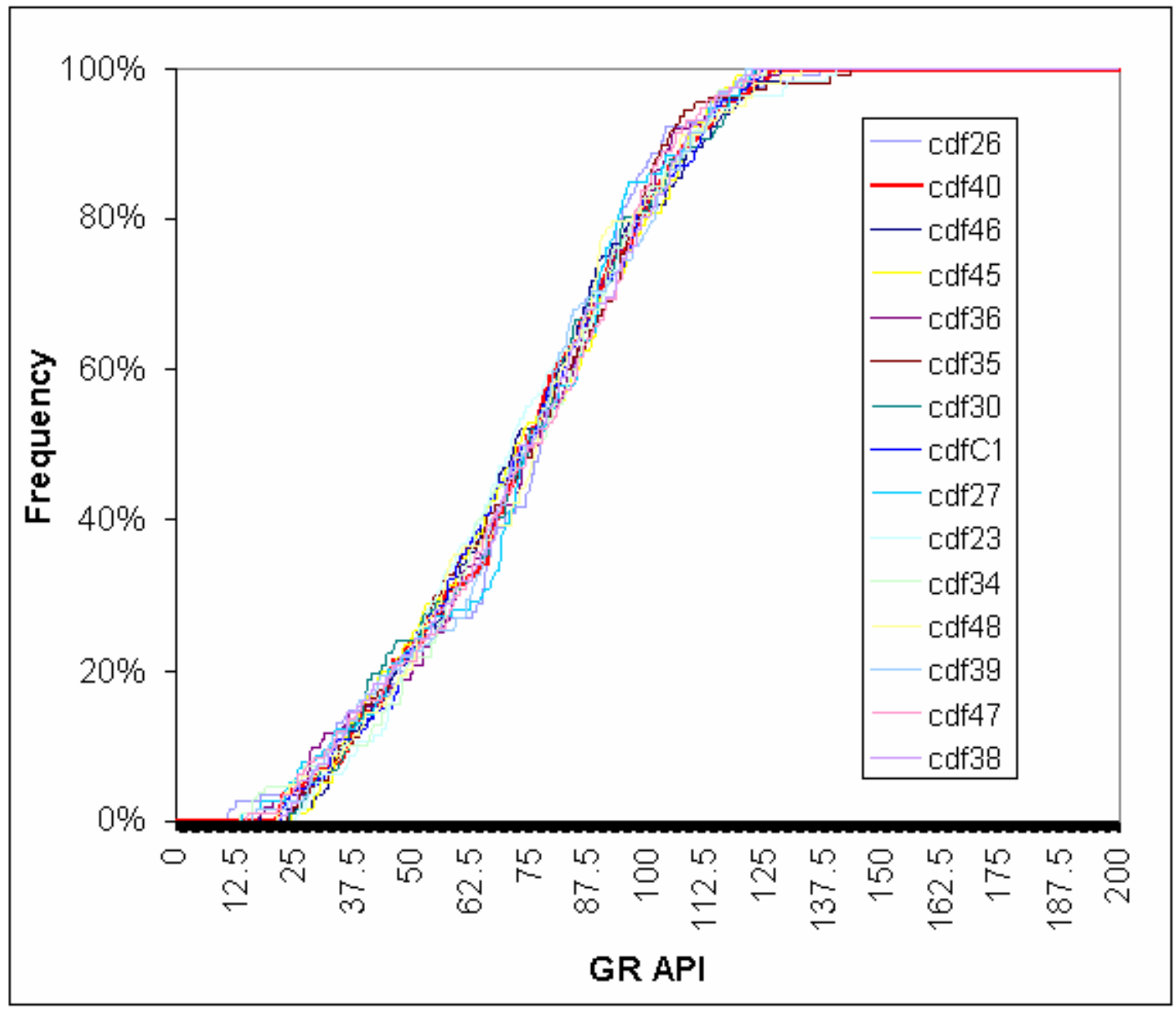

Fig. 1.3.17 - Corrected gamma ray distribution for ETO’Daniel wells. 


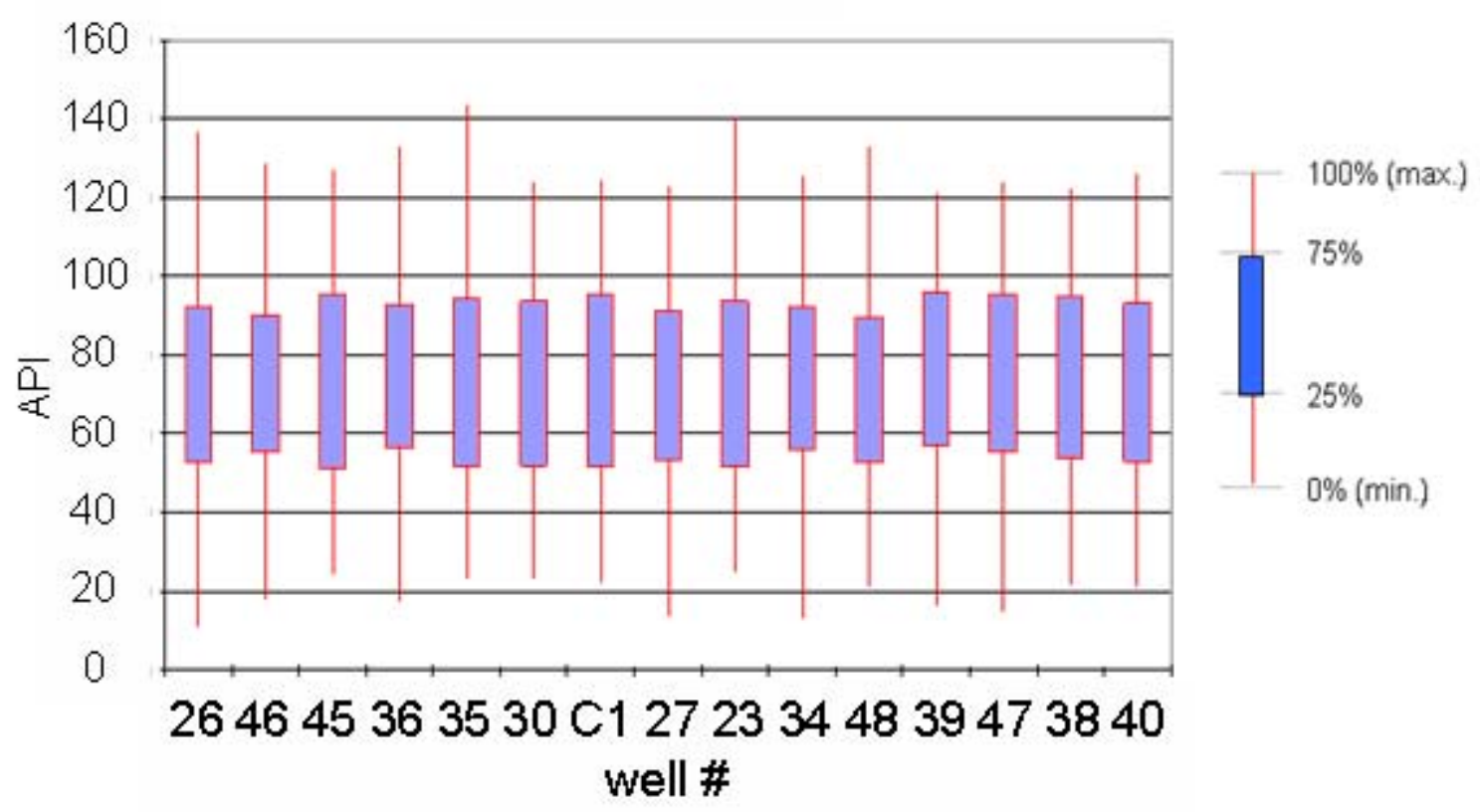

Fig. 1.3.18 - Normalized gamma ray values in $1 \mathrm{U}$ and $5 \mathrm{U}$ regions of upper Spraberry, ET O’Daniel unit.

\subsubsection{Neutron logs}

\subsubsection{Standardization of neutron $\log$ units}

The most common measure of porosity within the GSU log database is counts per second (cps) and is a measure of the amount of neutrons detected after bombarding the formation with energetic neutrons at the rate of several millions per second.

The neutron density decreases almost logarithmically with hydrogen richness, which is why porosity is a logarithmic function of neutron deflections.

The API RP33 recommends a system of neutron unit of calibration in the standardizationwell-logging pit of the University of Houston.

One API neutron unit is defined as $1 / 1000$ of the difference between instrument zero (tool response to zero radiation) and log deflection opposite a $6 \mathrm{ft}$ zone of Indiana limestone of $19 \%$ porosity. 


\subsubsection{Conversion from neutron units to linear porosity units}

A useful equation for converting a linear scale with respect to counts per second (logarithmic with respect to porosity), to a linear scale with respect to porosity is given by Shier ${ }^{28}$. This method is also known as the two - point method.

$$
X_{\text {norm }}=10^{\frac{y}{\left(W_{\text {nigh_cps }}-W_{\text {low_cps }}\right)}}
$$

where $y=X_{\text {raw }}\left(\log R_{\text {high } \phi}-\log R_{\text {low } \phi}\right)+\left(W_{\text {high_cps }}\right)\left(\log R_{\text {low } \phi}\right)-\left(W_{\text {low_cps }}\right)\left(\log R_{\text {high } \phi}\right)$

$\mathrm{X}_{\text {norm }} \quad-\quad$ Normalized well value (porosity, v/v)

$\mathrm{X}_{\text {raw }} \quad-\quad$ Actual well value (cps, API, EU)

$\mathrm{R}_{\text {high } \phi} \quad$ - $\quad$ Value for high porosity location from core or reliable $\log$ data (known for a particular region, unit $-\mathrm{v} / \mathrm{v}$ ).

$\mathrm{R}_{\text {low }} \quad$ - $\quad$ Known value for low porosity location from core or reliable log data (known for a particular region, unit - v/v).

$W_{\text {high_cps }} \quad$ - $\quad$ Well value at $\mathrm{R}_{\text {high }}$ location (cps, API, EU)

$\mathrm{W}_{\text {low_cps }} \quad$ - Well value at $\mathrm{R}_{\text {low }}$ location (cps, API, EU)

(Note: $\mathrm{R}$ in this case is not resistivity, but is used to denote regional value of parameter)

This equation is valid for all neutron curves measuring neutron counts irrespective of units.

The normalization equation requires the input of two lithologies from both a "type" well and the well being normalized. One lithology input is from a log interval that produces a high log reading and the other is from an interval that produces a low log reading. These lithology intervals that bound the normalization process are known as normalization zones. Normalization zones should have a well log response that is consistent from well to well (as is the case of lithology intervals consisting purely of salt and anhydrite). If such zones are unavailable, the analyst chooses zones whose behavioral changes are understood from location to location. This implies that for any one field, many normalization zones may have to be selected in order to properly limit the high and low readings of the different curve types being adjusted.

After identifying lithology intervals that will be used for normalization, the characteristic values of Rlow and Rhigh in these zones must be determined. This is accomplished by picking a "type" well (or wells) containing normalization zones considered by the analysts to have the correct well log response. This "type" well (or wells) is then defined as the standard to which all other curves will be adjusted. 


\subsection{Porosity}

Various logs are available that give a direct indication of porosity or matrix density. The database has mostly cased neutron logs that require conversion from API, cps or EU units to porosity units (Eq. 3.3). A few other wells have neutron porosity (NPHI, TNPH, TPHI) or density or acoustic (DT), the later two do not directly measure porosity.

When matrix lithology is known, shale free, and filled with water, all three porosity logs give the same values of porosity. These conditions are rarely encountered and therefore adjustments must be made for each of the different logs based on characteristic response in hydrocarbons and water.

The density log overestimates porosity in hydrocarbons, neutron logs underestimate porosity in hydrocarbons, and the acoustic log overestimates porosity in hydrocarbons ${ }^{30}$. To balance these anomalies out, an average porosity is often taken of the density and neutron logs, in the absence of the density log either the neutron or sonic is used to estimate porosity.

PHIA $=($ DPHI + NPHI $) / 2$

\subsubsection{Corrections for porosity}

Porosity as earlier mentioned in chapter I, can be obtained from a combination of the different porosity logs. The preferred log suite will be the density porosity and the neutron porosity or sonic porosity, unfortunately few wells have the desired combination and porosity is often resolved from a one dimensional analysis of the available log (mostly neutron porosity). The playbacks used for analysis are chosen based on availability of porosity curves for the particular well. 


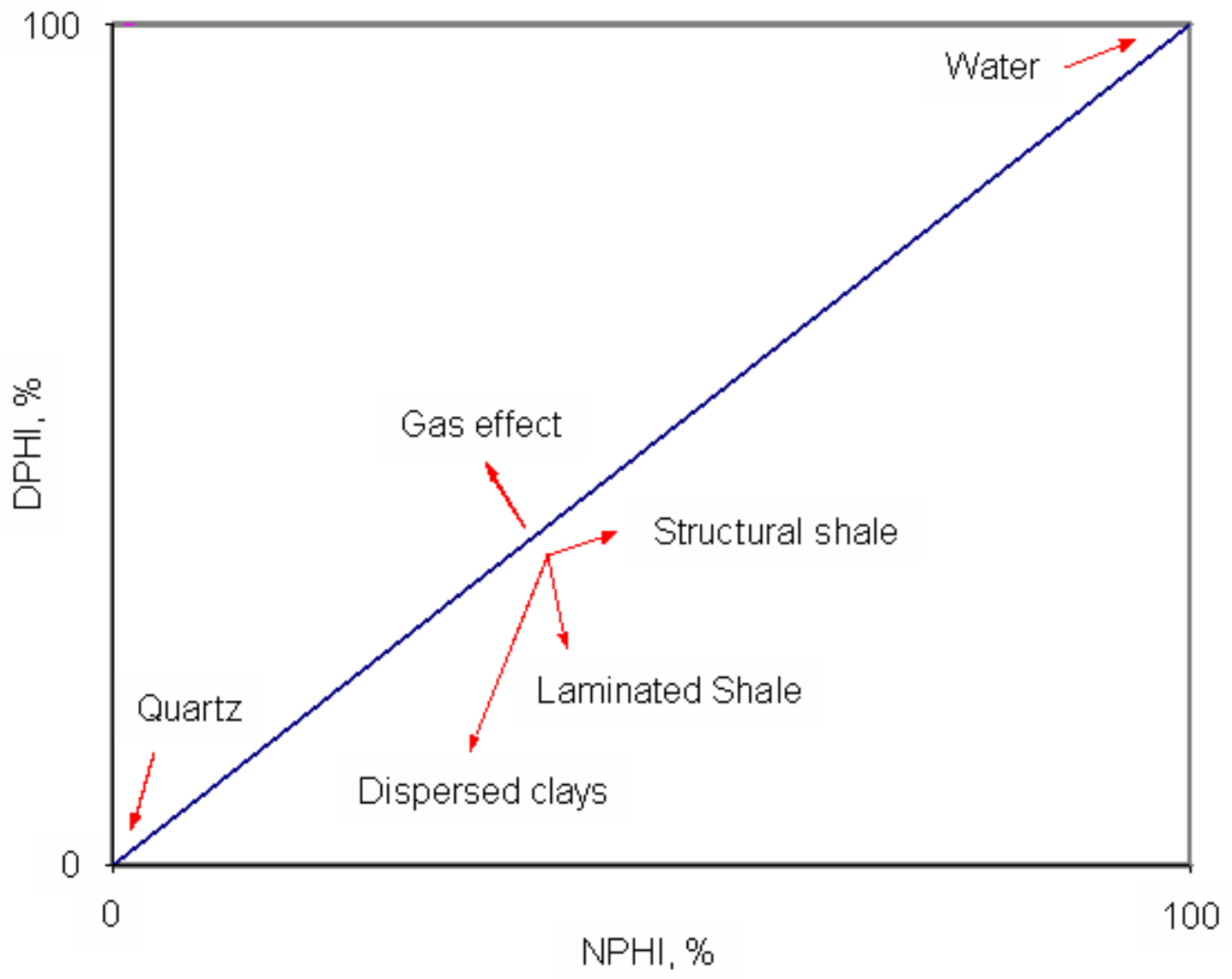

Fig. 1.3.19 - Effects on quality of porosity data from the density and neutron porosity tools.

Shale corrections are highly dependent on mode of shale features within the formation ${ }^{33}$. Shalines affects the porosity log response in proportion to the amount, type and distribution of shale. This distribution may be structural laminated or dispersed shale.

In the $1 \mathrm{U}$ and $5 \mathrm{U}$ sand units the shale distribution is in the form of laminae. Fig. 1.3.19 shows the effects of shaliness and gas on porosity values obtained from neutron and porosity logs.

Effective Porosity - The Effective porosity is less than the total or log measured porosity. This is due to the residual porosity within the unconnected pore spaces particularly within the clay minerals. Effective porosity (PHIE) can often be estimated by correcting for the presence of shale, given by:

PHIE = PHIA (1-Vshl) 


\subsection{ET O’Daniel log-core model}

\subsubsection{Log porosity - core porosity $x$-plots}

The core and log porosity crossplots indicate the level of agreement between core data and log data. If there is sufficient agreement between both porosities or a relationship between both data sets can be consistently established, further analysis can be confidently carried out on the basis of log porosity.

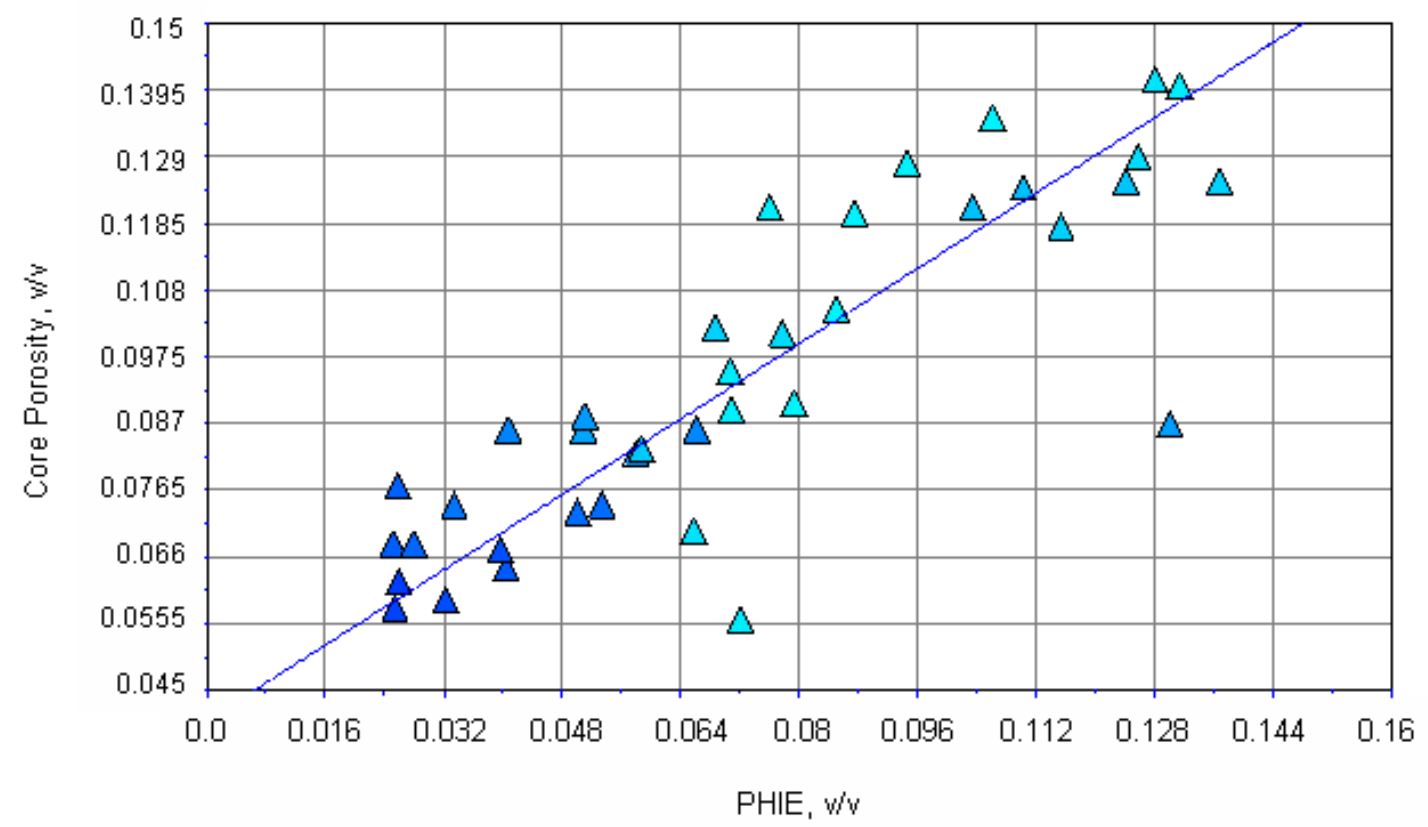

Fig. 1.3.20 - Crossplot core porosity vs. log porosity for ETO’Daniel 39, 1U sand. Light to dark markers represent a 3rd dimension of increasing shale content on a scale of 0 to 1 .

A depth match is performed prior to a crossplot of both porosity values (core and log). The depth match may be improved by analyzing the degree of correlation obtained for crossplots based on depth shifting the core data. This is done if a 'clear' relationship cannot be established just by visual analysis.

From regression analysis a best fit equation for the $\mathrm{x}$-plot in $1 \mathrm{U}$ was found to be:

$\mathrm{Y}=0.050342+0.539983 \mathrm{X}$ and $\mathrm{R}^{2}=0.677$

And for 5U:

$\mathrm{Y}=0.05810+0.560472 \mathrm{X}$ and $\mathrm{R}^{2}=0.620651$ 


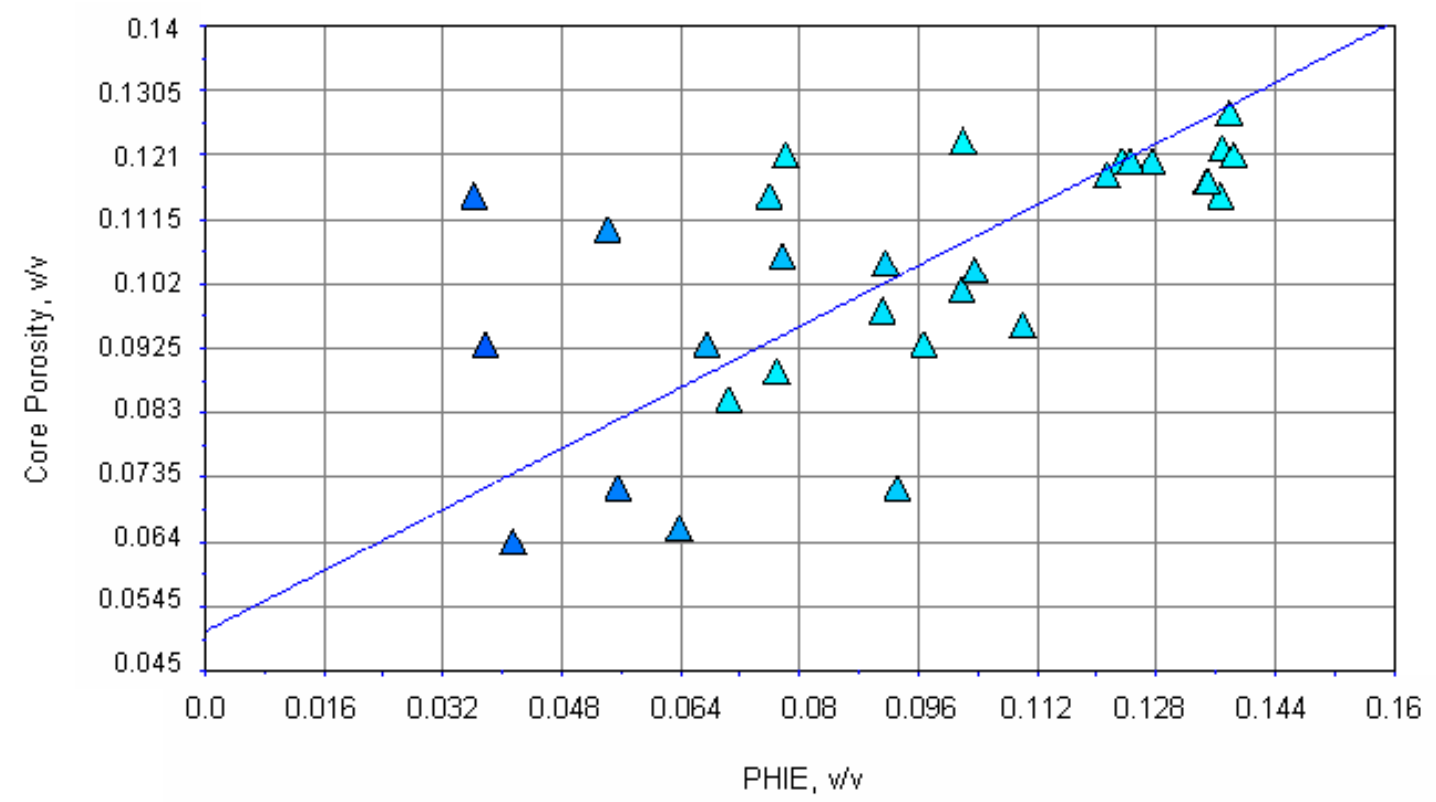

Fig. 1.3.21 - Crossplot core porosity and log porosity for ET 39, 5U sand.

The ET O'Daniel 39 well gave the most consistent core to log relationship of all the cored wells analyzed, more so within the $1 \mathrm{U}$ interval. Table 1.3.3 shows the summary of regression results obtained from the crossplots of cored wells in the ET O'Daniel field. 


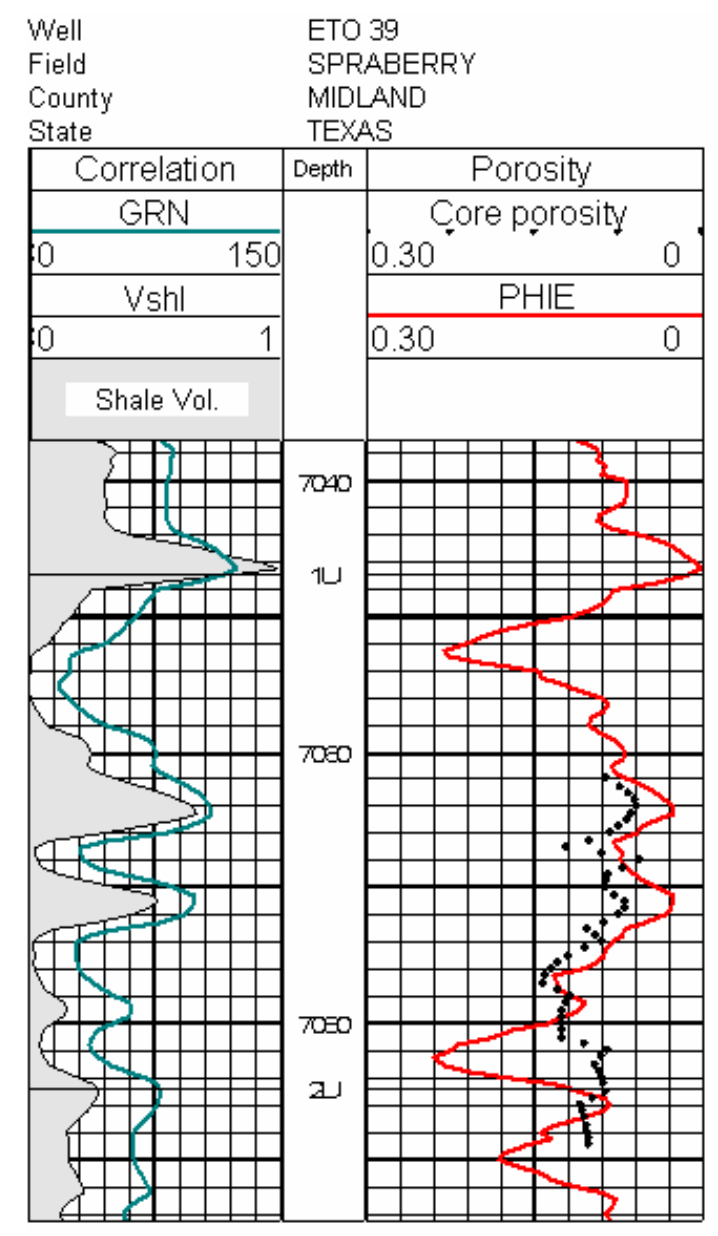

Fig. 1.3.22 - Log playback of log and core porosity in ET 39, $1 \mathrm{U}$ sand

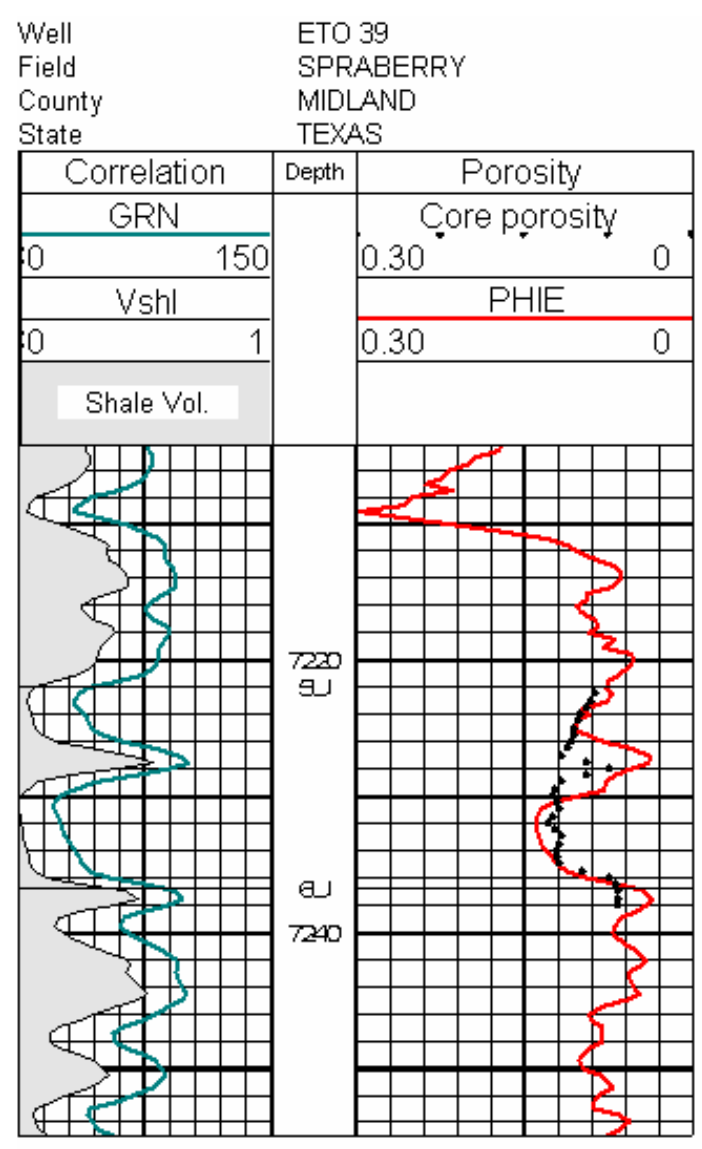

Fig. 1.3.23 - Log playback of log and core porosity in ET 39, $5 \mathrm{U}$ sand

Table 1.3.3 - Regression from crossplots of core - log porosity for cored ET O’Daniel wells.

(Best results are obtained in ETO\#39 well).

\begin{tabular}{|c|c|c|c|c|}
\hline \multicolumn{3}{|c|}{1 U } & \multicolumn{2}{c|}{ UU } \\
\hline Well & Least Sq. Regression & R2 & Least Sq. Regression & R2 \\
\hline 37 & $y=-0.018494+1.12948 x$ & 0.542 & $y=-0.024442+0.949273 x$ & 0.106 \\
\hline 38 & $y=-0.024815+1.049007 x$ & 0.230 & & 0.621 \\
\hline 39 & $y=0.050342+0.539983 x$ & 0.677 & $y=0.050810+0.560472 x$ & 0.396 \\
\hline 40 & $y=0.050857+0.520833 x$ & 0.509 & $y=0.028280+0.662358 x$ & 0.073 \\
\hline 47 & $y=0.017356+0.560368 x$ & 0.073 & $y=-0.021898+1.243577 x$ & 0.776 \\
\hline 48 & $y=0.014259+1.042477 x$ & 0.519 & $y=0.034999+0.748994 x$ & \\
\hline$y=$ core porosity, $x=\log$ porosity & & & \\
\hline
\end{tabular}


Core - log porosity discrepancies are observed from the log playbacks and regression correlations, this is often due to bound water contained in the clays. This is also referred to as residual porosity earlier mentioned, this results in porosity estimates from logs exceeding that determined from cores.

\subsection{Variables influencing permeability}

\subsubsection{Regression analysis}

Various variables often influence the flow capacity of the rock, such as clay mineral content (shale), distribution, pore throat size / capillary pressure, connate water saturation, porosity etc. Crossplots of these variables and permeability will often reveal underlying relationships, furthermore, regression analysis may produce a functional mathematical model to represent this relationship.

Readily available are porosity and shale volume data obtained from the neutron, density or acoustic logs and gamma ray logs respectively. Porosity values (PHIE) are verified against core porosity data (Figs. 1.3.22 and 1.3.23).

Figure 1.3.24 a - b show the shale volume - permeability relationship for wells 39 and 47. The trend is consistent for all the wells investigated i.e. permeability decreasing with increasing shale content. 


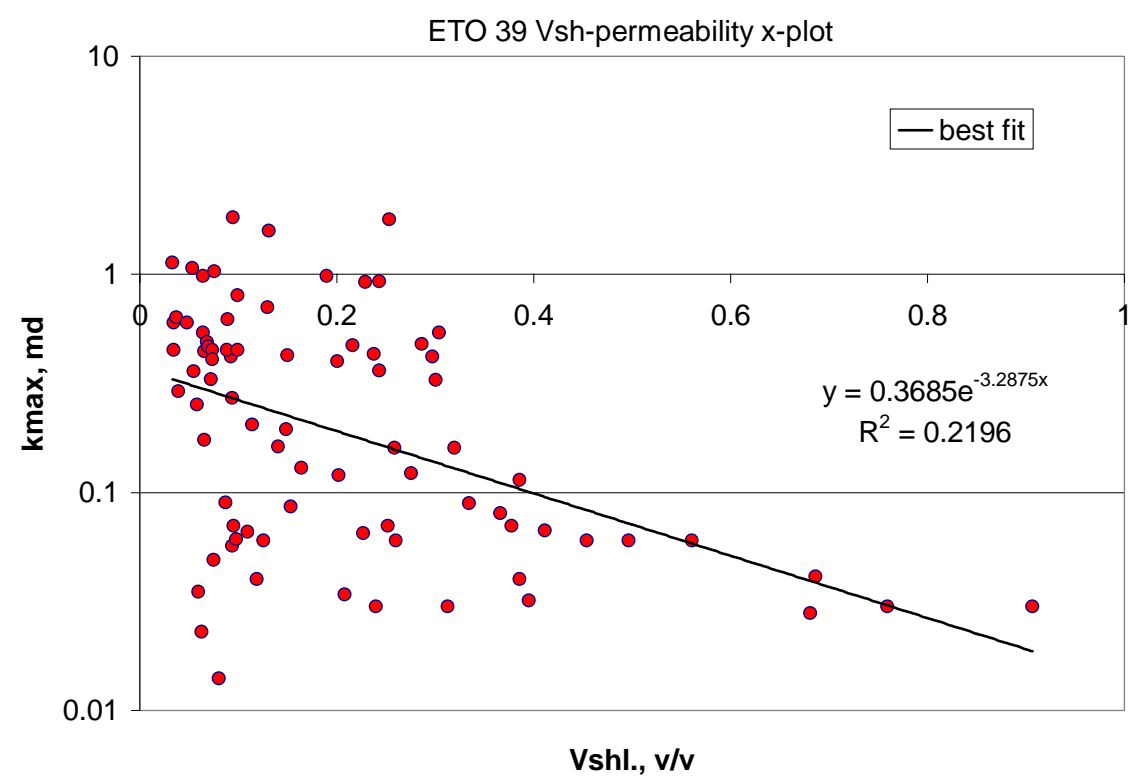

Fig. 1.3.24a - ET 39 crossplot for shale volume and permeability.

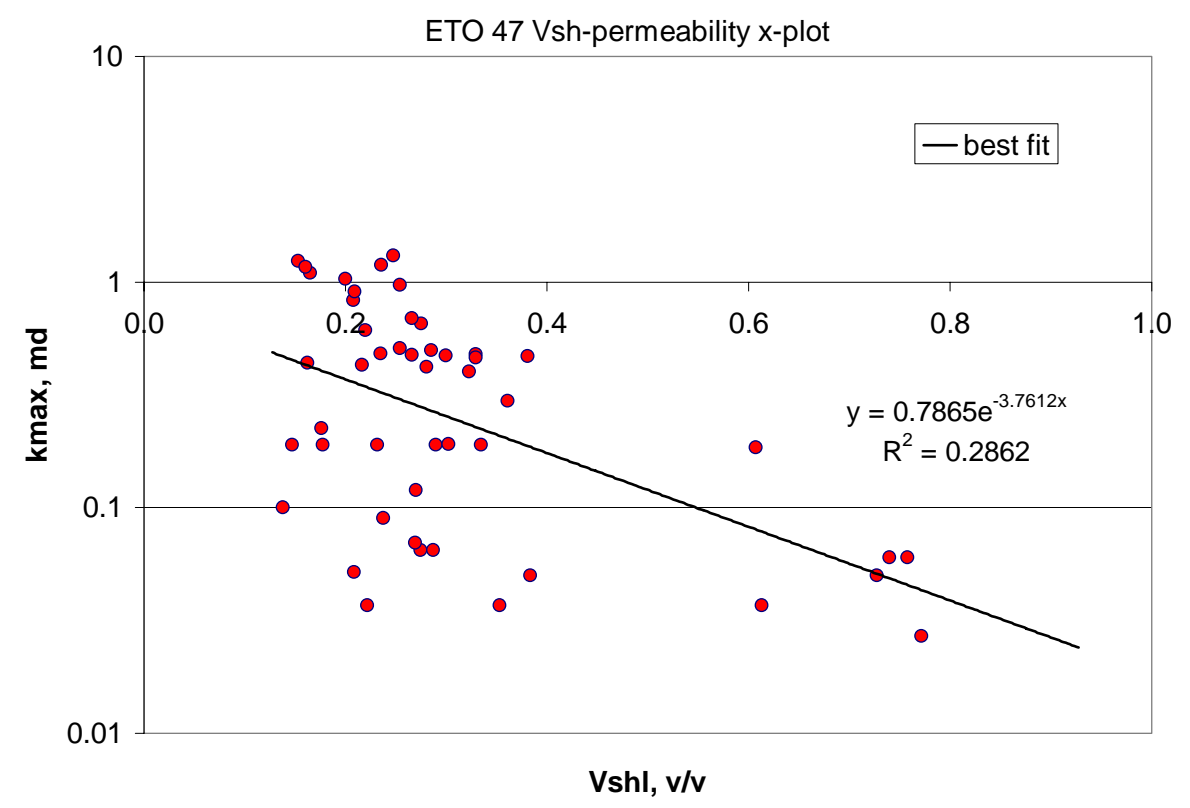

Fig. 1.3.24b - ET 47 crossplot for shale volume and permeability.

\subsubsection{Shale effects on porosity and permeability}

Figs. 1.3.24a and b show the relationship between shale volume and core permeability values. Within the shaly Spraberry sands, shale is in the form of laminae and therefore we 
expect a significant effect on the density and neutron porosity log values. Gas effects are negligible in the Spraberry payzones due to the absence of a gas cap, therefore any corrections to be made are for shaliness.

Shale corrections are applied to the neutron porosity data as given in Eq. 3.5.

Figs. 1.3.25a and b show the relationship between the uncorrected neutron porosity data and permeability. From established correlations for porosity and permeability ${ }^{28}$, we expect to observe an increase in permeability associated with an increase in porosity, but in the figures below, this trend is masked by the effects of the shale laminae in the logged intervals.

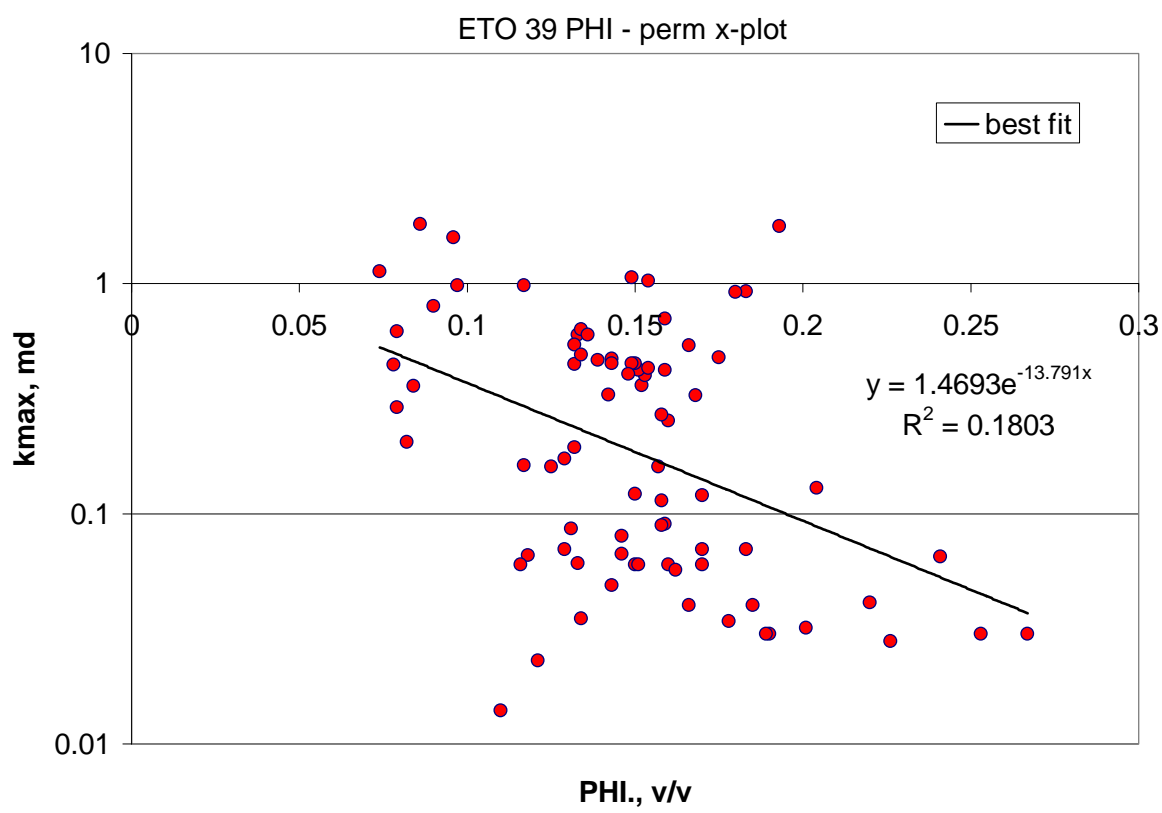

Fig. 1.3.25 a - ET 39 crossplot for log porosity and permeability. 


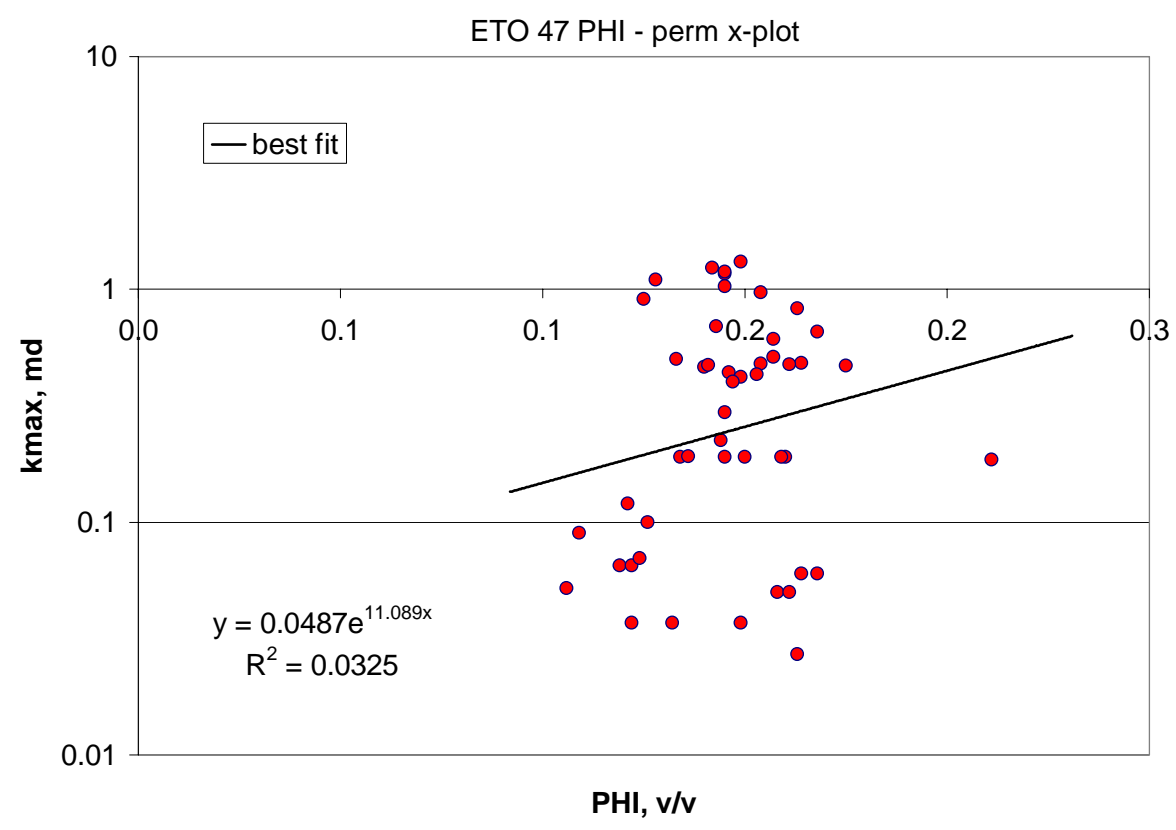

Fig. 1.3.25 b - ET 47 crossplot for log porosity and permeability.

From Figs. 1.3.24, we can see that the shale volume clearly influences the permeability, therefore we must apply corrections to the log porosity to obtain a useable model for predicting permeability.

After correcting for shaliness, plots generated for porosity-permeability (Figs. 1.3.26a and $b$ ) show the functional relationships for predicting permeability based on porosity.

The porosity values from the neutron log are used for this prediction exercise as this is the available porosity log type within the database, with the exception of only a few wells which may have both or the three porosity types. 


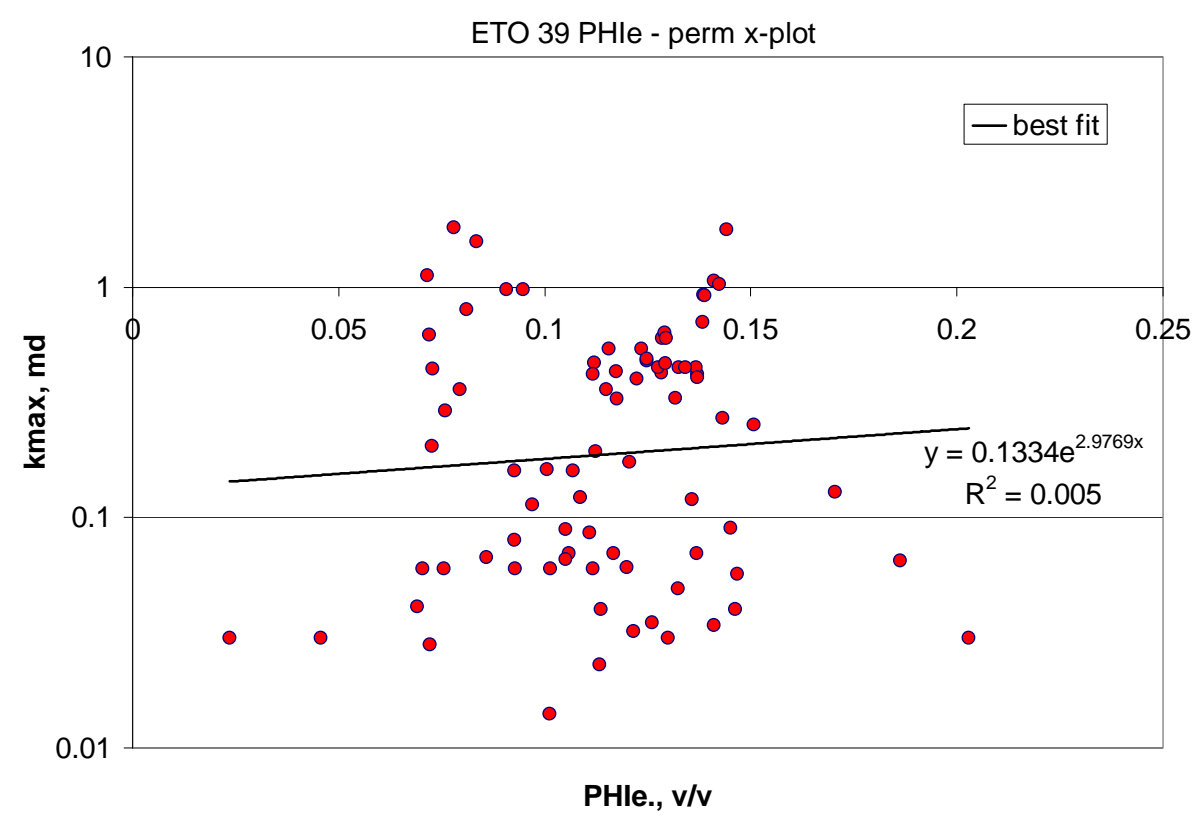

Fig. 1.3.26a - ET 39 crossplot for shale corrected porosity and permeability.

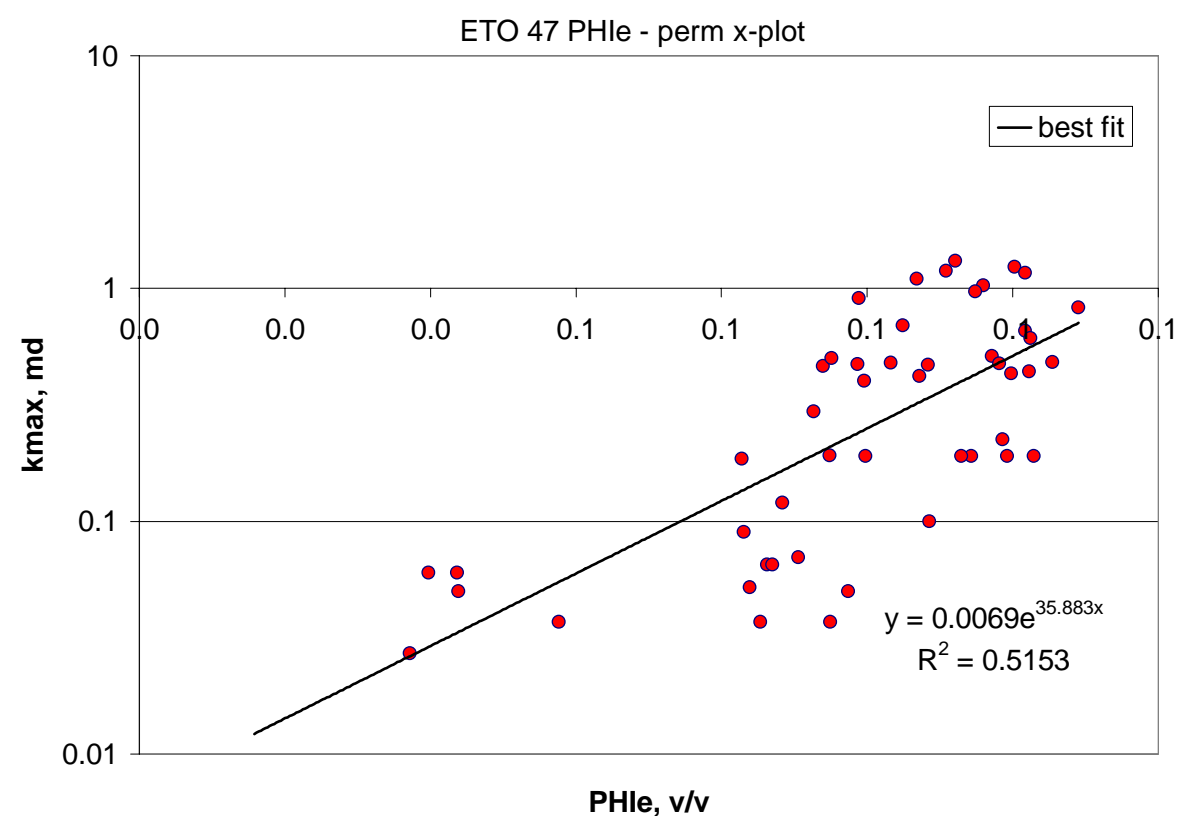

Fig. 1.3.26b - ET 47 crossplot for shale corrected porosity and permeability.

Figures above show the crossplots of 2 of the control wells used to establish a porosity permeability relationship. The porosity is corrected for shale (PHIE), and this corrected porosity is regressed against corresponding core permeability values for each of the wells analyzed. Well ETO 47 gives the best correlation, the resulting relationship between porosity and permeability is given as: 
$\mathrm{Y}=0.0069 \mathrm{e}^{35.883 \mathrm{X}}$ and $\mathrm{R}^{2}=0.515$

where $\mathrm{y}=$ permeability and $\mathrm{x}=$ effective porosity for a given well in the zones of investigation (1U and $5 \mathrm{U}$ in this case).

\subsubsection{Data conditioning ('ACE')}

Besides porosity, rock type, clay content and lithology, initial water saturation and pore throat size most probably have an influence on effective permeability. The limitation of any log derived permeability is in the fact that these variables are static volumetric terms, whereas permeability is a measure of the movement of fluid through rock (Hunt, Pursell, 1997). Any permeability correlation between porosity and or water saturation will not likely have a wide geographic or geologic application. The only way to obtain a robust permeability distribution is by acquiring field wide core and well test data.

Correlating permeability in the Germania unit is hampered due to an absence of core data, production data is available, and is beyond the scope of this project. Therefore we are limited to methods which use static properties to correlate the permeability, specifically the ‘Alternating Conditional Expectation' (ACE) method.

From the established rock model (Table 1.3.1), the upper Spraberry has been classified into 3 rock types based on shale content and porosity. As the rock type is classified based on porosity and shale volume it will not be used as a variable in the estimation of the permeability transform. Therefore, 2 independent variables will be used: shale volume and porosity in calculating the dependent variable, permeability.

\subsubsection{The 'ACE' transformations}

The optimized multi-variate regression was performed to determine the optimal transformation for porosity type data (density or neutron porosity).

The resulting playback for the $1 \mathrm{U}$ and $5 \mathrm{U}$ intervals (Fig. 1.3.27) shows the match from conventional regression from cored wells and from the ACE algorithm.

The following transformations were used to obtain the ACE model:

$\mathrm{NPHI}_{\text {transform }}=-303.86 \phi^{2}+125.24 \phi-11.738$

Vshl $_{\text {transform }}=-3.8536 \mathrm{~V}^{2}-0.63206 \mathrm{~V}+0.66875$

$\mathrm{k}_{\mathrm{ACE}}=0.40339 \mathrm{~A} \Sigma_{\text {transform }}^{2}+0.64404 \Sigma_{\text {transform }}+0.018403$

where $\Sigma_{\text {transform }}=\mathrm{NPHI}_{\text {transform }}+\mathrm{Vshl}_{\text {transform }}$ and $\mathrm{R}^{2}=0.77$ 
The correlation coefficient obtained using the ACE algorithm is higher than that from conventional regression, but it is obvious from the playback in fig. 1.3.27 that both methods do not adequately model the permeability using porosity and clay content. In a separate study ${ }^{34}$, NMR core analysis was performed on two samples from wells within the ET O'Daniel unit to develop an empirical NMR permeability model for the upper Spraberry sandstones. NMR permeability was derived for reference using $K=4.6 \mathrm{~T}_{2 \mathrm{ml}}{ }^{2} \phi^{4}$, where $T_{2 m l}$ is the logarithmic $T_{2}$ of the $T_{2}$ distribution curve. Such a study emphasizes the complexity of modeling permeability based on primary reservoir properties, albeit, no NMR data is available in the database to enable a comparison of the ACE model and the NMR model.
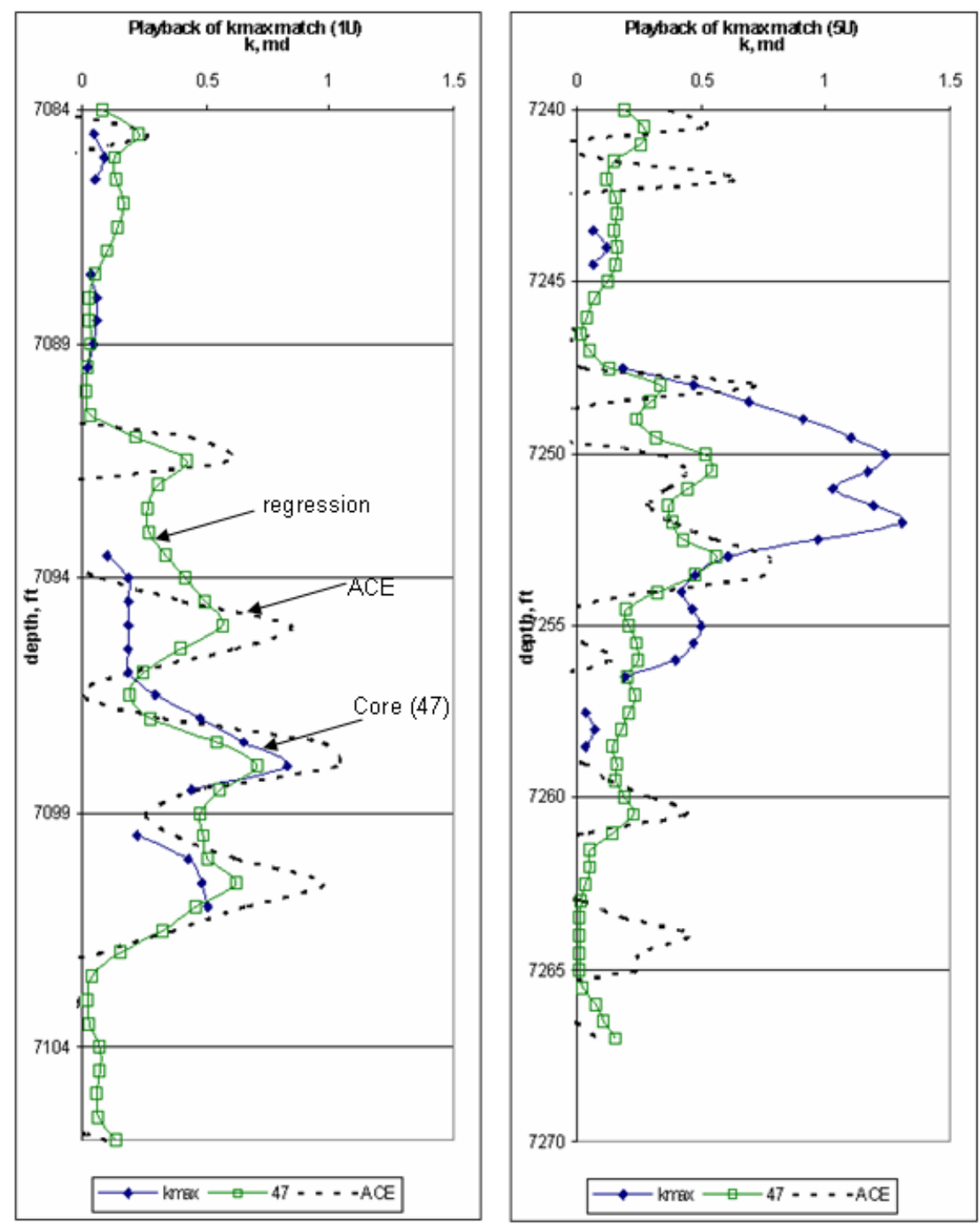

Fig. 1.3.27 - Playback of results from conventional regression and ACE regression. 


\subsection{Water saturation}

Table of values for Archie parameter values for use in the quantitative analysis of Spraberry sands have been published ${ }^{11}$ based on log data analysis in the Spraberry sands by Schlumberger. Table 1.3.4 gives expected range of values for the Spraberry.

The Archie equation has been used extensively in the Spraberry ${ }^{5,11}$ to successfully estimate saturations within the upper Spraberry interval. A match of the saturation profile of the Shackelford I-38 was made with 1, 1.66 and 1.46 for a, $\mathrm{m}$ and $\mathrm{n}$ respectively. These values agree with the averages proposed in Table 1.3.4.

The tortuosity exponent (a), usually varies from 0.62 to 1.2 but 1 is often used as it has a narrow range of variation and is not related exponentially to the formation factor, $\mathrm{F}$.

Ro $=F^{*} R w$, where $F=a / \phi^{m}=R o / R w$

Cementation factor (m) may vary from 1 to as much 4, rocks with fractures or fissures may have low cementation values often close to 1 . The saturation exponent (n) is usually 2 , for shaly sands this value is less than 2 .

Table 1.3.4 - Archie parameters used in determining saturation in the upper Spraberry.

\begin{tabular}{|c|c|c|c|c|}
\hline & Min & $\operatorname{Max}$ & Average & Comment \\
\hline Rw & 0.03 ohm-m & 0.04 ohm-m & 0.35 & Measured at 130F \\
\hline Ro & 0.7 ohm-m & 3 ohm-m & 1.3 & \multirow{2}{*}{$\begin{array}{l}\text { Min and max values are for porosity ranging from } 8 \text { to } 20 \% \\
\text { Average value for porosity of } 12 \%\end{array}$} \\
\hline & & & & \\
\hline $\mathrm{m}$ & & 1.8 & & Possibly lower for clean sands \\
\hline $\mathrm{F}$ & 20 & 100 & & \\
\hline$\phi$ & $8 \%$ & $20 \%$ & $12 \%$ & Average for upper spraberry \\
\hline $\mathrm{n}$ & 1.5 & 1.9 & & Usually less than 2.0 for shaly sands \\
\hline
\end{tabular}

The generalized form of the Archie equation is $S w^{n}=a R w / \phi^{m} R t$

This equation is applied in the wells that have resistivity log values over the $1 \mathrm{U}$ and $5 \mathrm{U}$ sand intervals to estimate average saturations. In applying Archies equation, certain parameters will be varied so as to match the measured core saturations. Going by Table 1.3.4, the Rw, a, and n values are fixed at $0.035 \mathrm{ohm}-\mathrm{m}, 1$ and 1.7 respectively, while Rt true resistivity is obtained from the laterolog or induction log. The parameter whose sensitivity will determine the match based on measured core saturation will be $\mathrm{m}$, the cementation exponent.

Figure 1.3.28 (saturation track) shows the match between the Archie calculated water saturation and the core derived saturation. A cementation exponent of 1.7 gave a good match on most of the cored wells (see Figs. 1.3.28 and 1.3.29). A crossplot of Core and Archie derived saturation values was used to evaluate the optimal match by choosing a cementation exponent value $(\mathrm{m})$ that results in the best correlation coefficient for the compared wells. In some wells the match was not optimal i.e well \#47 and \# 40 (1U), but all wells considered, $\mathrm{m}$ of 1.7 gave a good fit. 
From depth averaged Archie calculated saturation values, Table 1.3.5 was developed for wells with core and / or resistivity data.

Table 1.3.5 - Interval averaged water saturations for well with resistivity curves.

\begin{tabular}{|c|c|c|c|c|}
\hline Well & Int. & Avg. SwA & Avg. Core Sw & Date Logged \\
\hline 37 & $1 U$ & N/A & $48.04 \%$ & $10 / 19 / 1995$ \\
\hline & $5 U$ & N/A & $63.60 \%$ & $10 / 19 / 1995$ \\
\hline 38 & $1 U$ & $28.16 \%$ & $29.07 \%$ & $8 / 14 / 1998$ \\
\hline & $5 U$ & N/A & $22.91 \%$ & $8 / 14 / 1998$ \\
\hline 39 & $1 U$ & $42.05 \%$ & N/A & $7 / 5 / 1998$ \\
\hline & $5 U$ & $42.00 \%$ & N/A & $7 / 5 / 1998$ \\
\hline 40 & $1 U$ & $30.18 \%$ & $22.76 \%$ & $9 / 4 / 1998$ \\
\hline & $5 U$ & $31.05 \%$ & $31.15 \%$ & $9 / 4 / 1998$ \\
\hline 47 & $1 U$ & $33.16 \%$ & $52.66 \%$ & $7 / 22 / 1998$ \\
\hline & $5 U$ & $N / A$ & $48.65 \%$ & $7 / 22 / 1998$ \\
\hline 48 & $1 U$ & $29.04 \%$ & N/A & $9 / 24 / 1998$ \\
\hline & $5 U$ & $36.00 \%$ & N/A & $9 / 24 / 1998$ \\
\hline 49 & $1 U$ & $36.82 \%$ & N/A & $2 / 15 / 2001$ \\
\hline & $5 U$ & $35.37 \%$ & N/A & $2 / 15 / 2001$ \\
\hline 50 & $1 U$ & $50.86 \%$ & N/A & $2 / 15 / 2001$ \\
\hline & $5 U$ & $36.37 \%$ & N/A & $2 / 15 / 2001$ \\
\hline
\end{tabular}




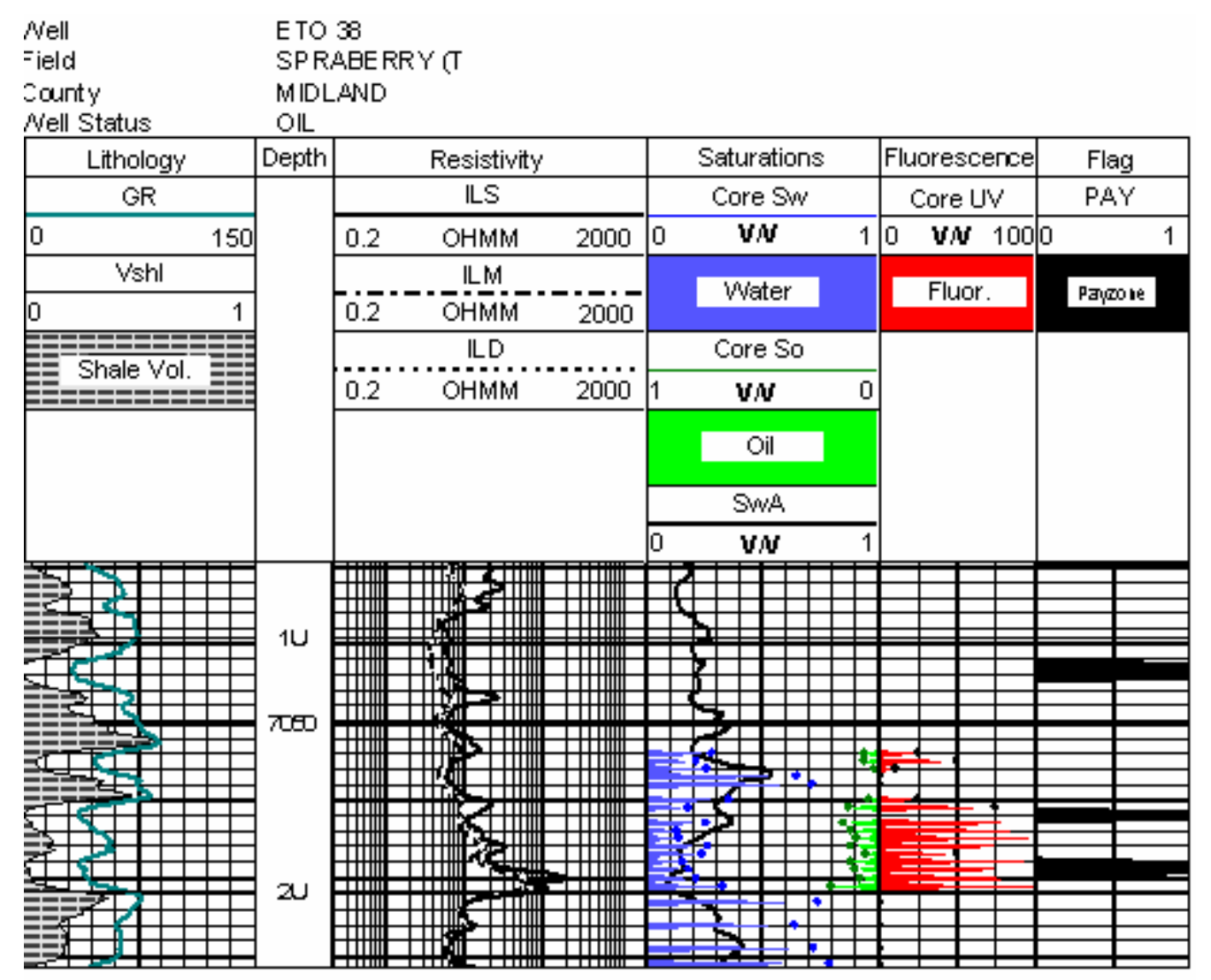

Fig. 1.3.28 - Saturation profile matched for ET 38, $1 \mathrm{U}$ using Rw 0.035 ohm-m and m 1.7.

Determining $\mathrm{Sw}$ in a fractured reservoir using the Archie equations is complicated because the cementation exponent, $\mathrm{m}$, may be as low as 1 . Rasmus ${ }^{35}$, proposes an equation for calculating $\mathrm{m}$ in fractured reservoirs.

$$
m=\frac{\log \left[\phi_{s}^{3}+\phi_{s}^{2}\left(1-\phi_{t}\right)+\left(\phi_{t}-\phi_{s}\right)\right]}{\log \phi_{t}}
$$

where

$\mathrm{m}=$ Archie cementation exponent

$\phi_{\mathrm{s}}=$ matrix porosity calculated from Sonic log

$\phi_{\mathrm{t}}=$ total porosity from neutron or density logs

From well \#47, a single well average of log values for $\phi_{\mathrm{s}}$ and $\phi_{\mathrm{t}}$ are 0.1236 and 0.1512 , after evaluating for $\mathrm{m}$ using Eq. 3.6, the resulting value of $\mathrm{m}$ equal to 1.667 which is in the range of the optimal value previously determined from core Sw and SwA comparison. 


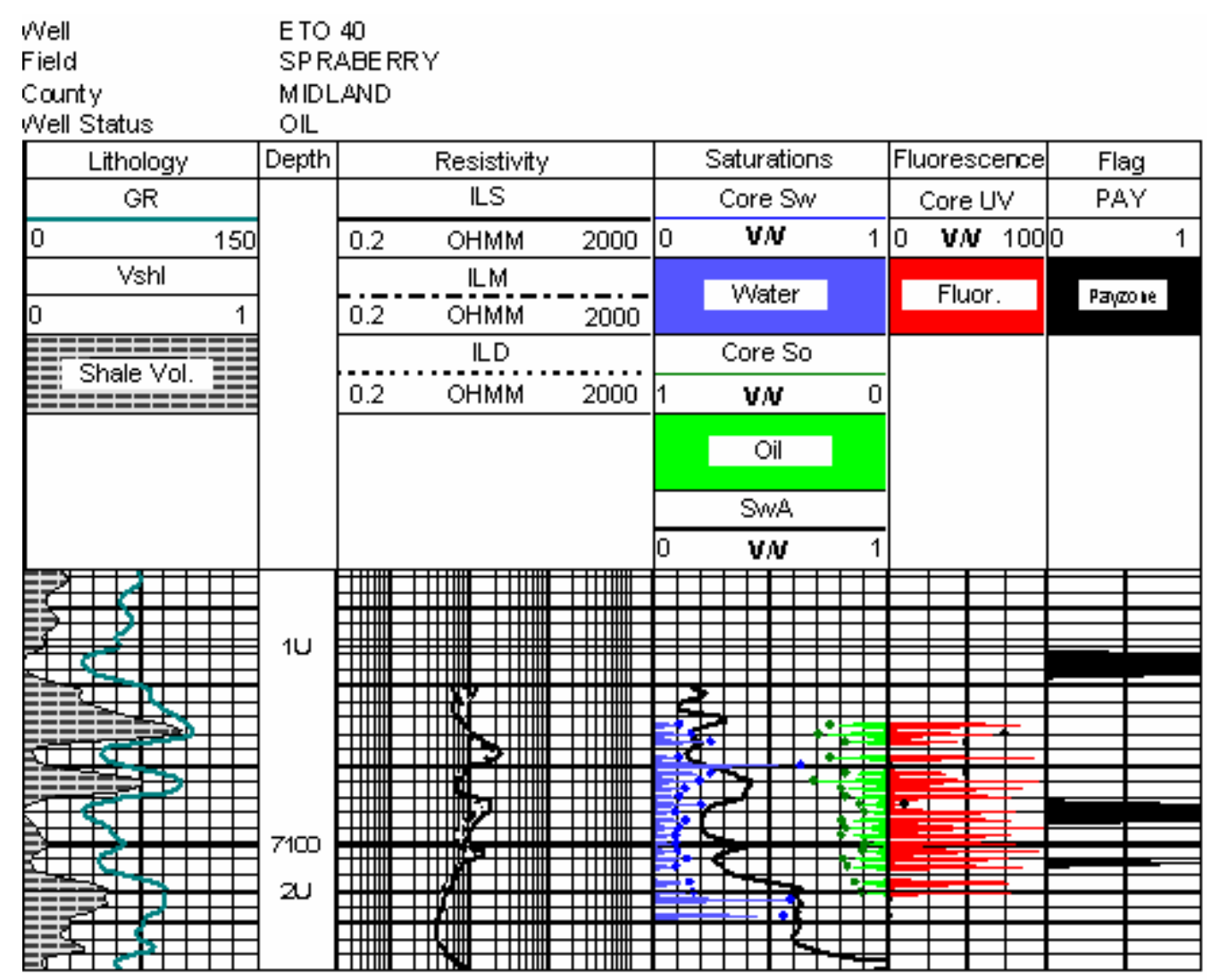

Fig. 1.3.29 - Saturation profile matched for ET 40, $1 \mathrm{U}$ using Rw 0.035 ohm-m and m 1.7. 


\section{ET O’Daniel and Germania Analogy}

On the basis of available log data, shale volume determined from gamma ray logs and porosity response will form the basis of comparison of the two units. Permeability of the matrix in the Spraberry unit is low in general and flow capacity is enhanced as a result of the interconnected natural fractures, for this reason, it can be established at this early stage that one of the three major indices (matrix permeability) for comparing the two fields show sufficiently similar response, although there is no core permeability data in the Germania unit to correlate.

\subsection{Shale volume}

Figs. 1.3.30 and 1.3.31 show the distribution of average shale volume fraction within the $1 \mathrm{U}$ and 5U intervals in the ET O'Daniel unit. The values are averaged every 0.5 feet of depth and these values used are based on the normalized values determined in chapter III.

The shale volume indices clearly follow a normal distribution and summary statistics for each interval are as shown alongside the distribution.

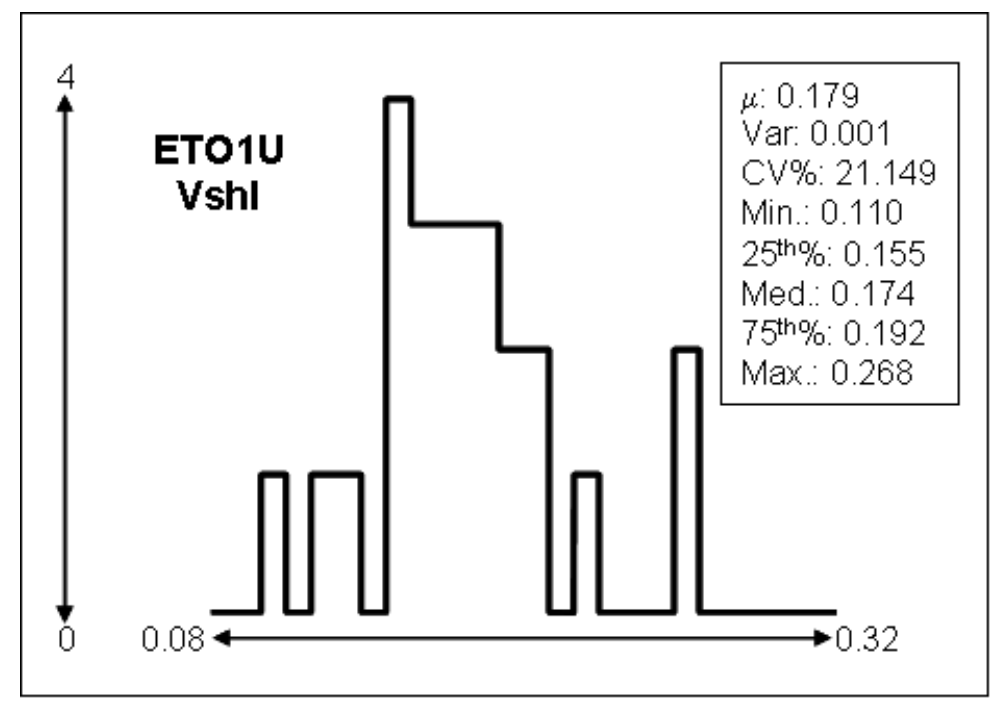

Fig. 1.3.30 - Statistics of Vsh values for ET O’Daniel, 1U sand. 


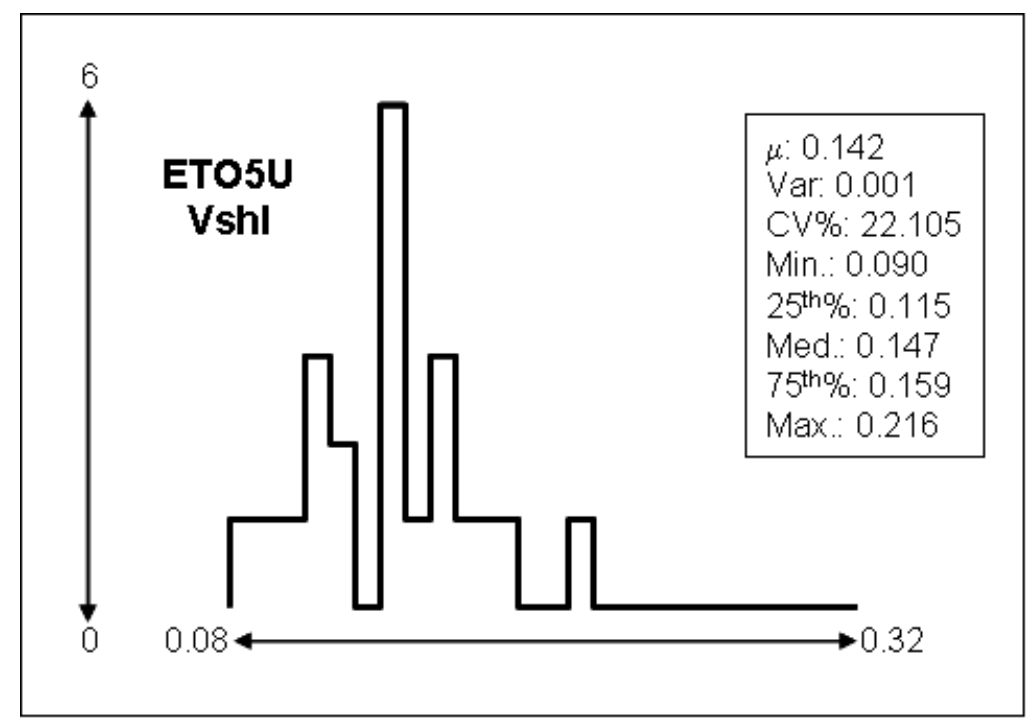

Fig. 1.3.31 - Statistics of Vsh values for ET O’Daniel, 5U sand.

Figs. 1.3.32 and 1.3.33 similarly show shale volumes in the Germania unit, and like the distribution follows a normal distribution.

When comparison of the two fields are made based on the shale volumes, we observe similarities in the mean and Inter-Quartile range (IQR) for both the $1 \mathrm{U}$ and $5 \mathrm{U}$ units.

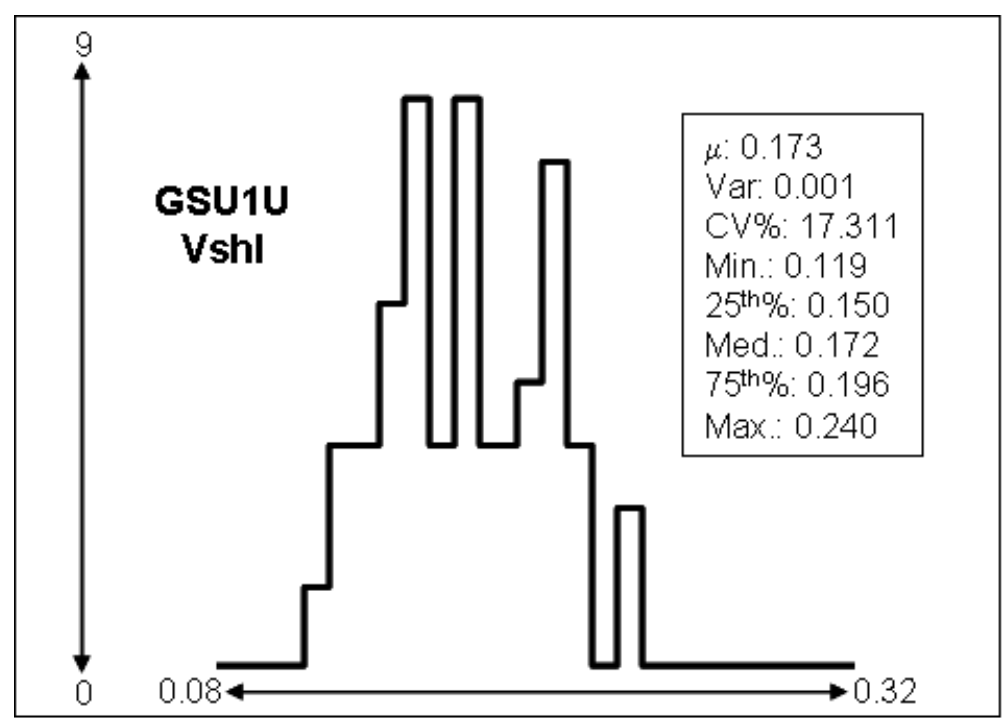

Fig. 1.3.32 - Statistics of Vsh values for Germania, 1U sand. 


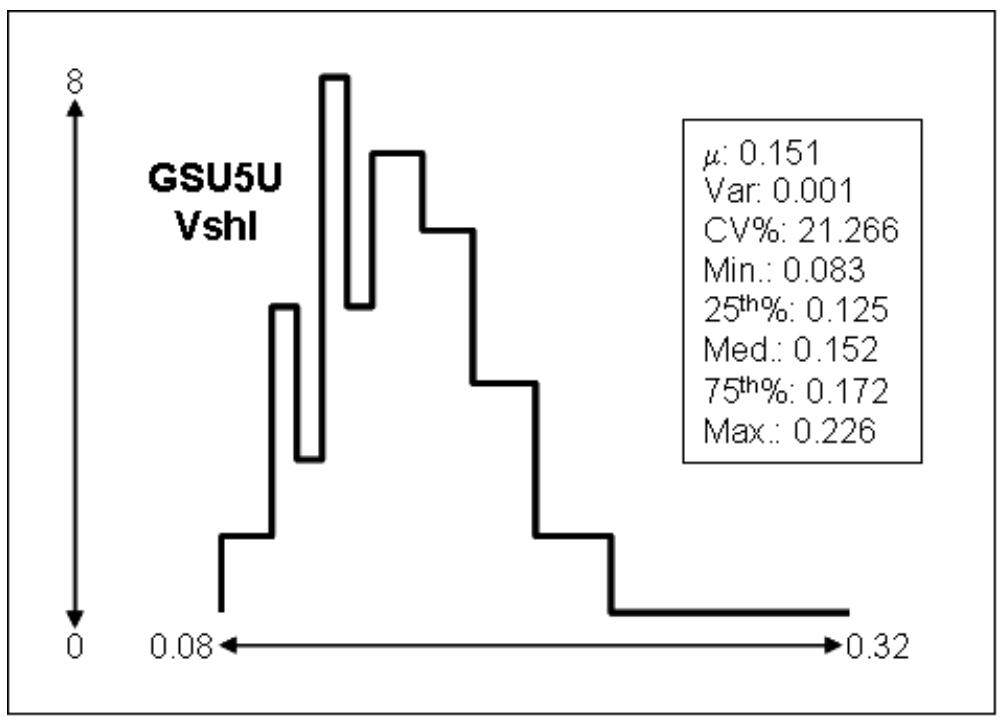

Fig. 1.3.33 - Statistics of Vsh values for Germania, 5U sand.

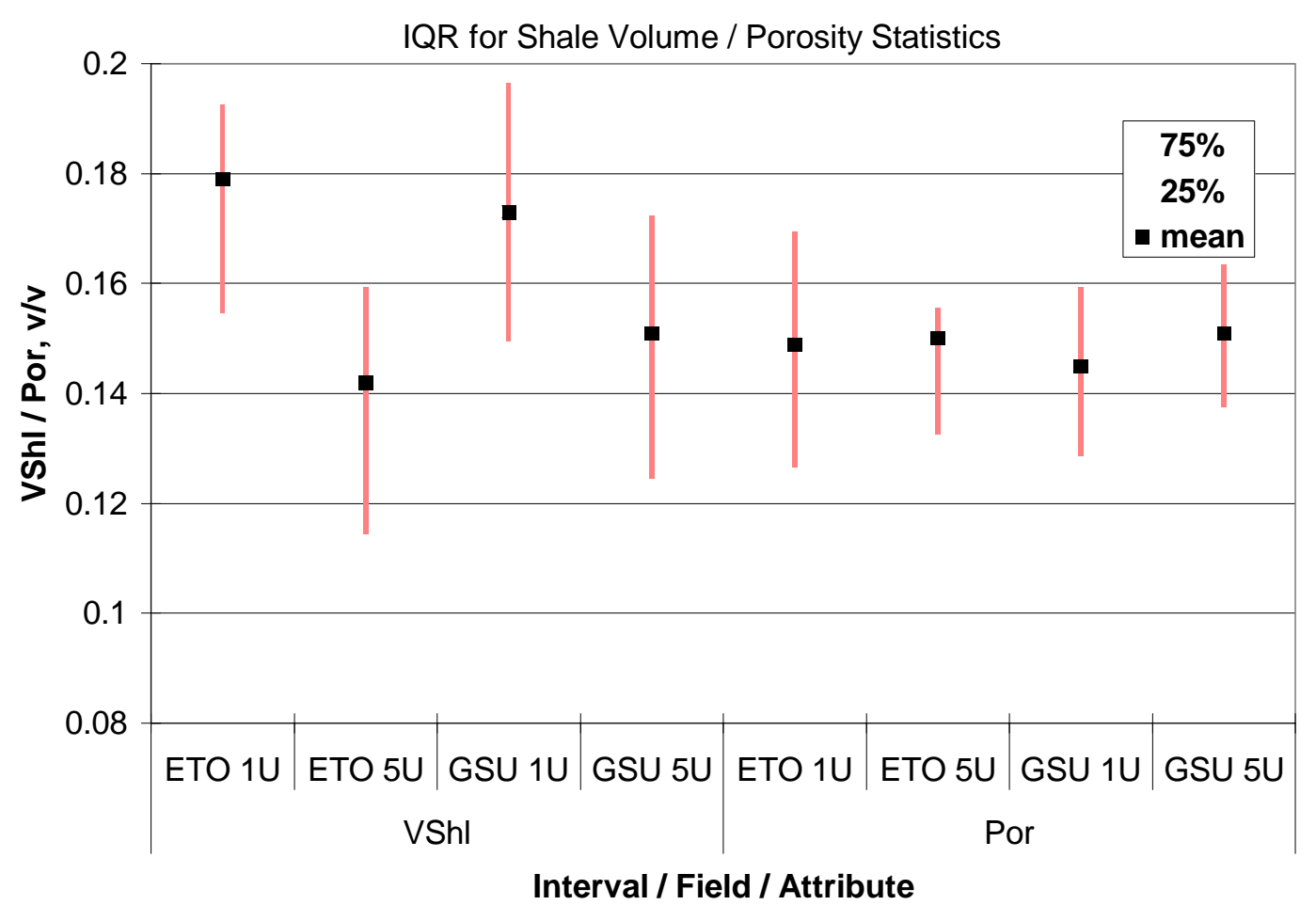

Fig. 1.3.34 - IQR and mean values of shale volume and porosity for ET O’Daniel and Germania units.

Fig. 1.3.34 compares the mean and IQR for the shale volume and porosity between sand members in each unit. The $1 \mathrm{U}$ in the ET O’Daniel and Spraberry are almost identical in 
mean, quartile distribution and most other statistical measures for the shale volumes distribution.

The $5 \mathrm{U}$ also shows similarities in most measures, but generally exhibits a lower range of porosities, and a slightly lower mean with respect to the $1 \mathrm{U}$ interval.

\subsection{Porosity}

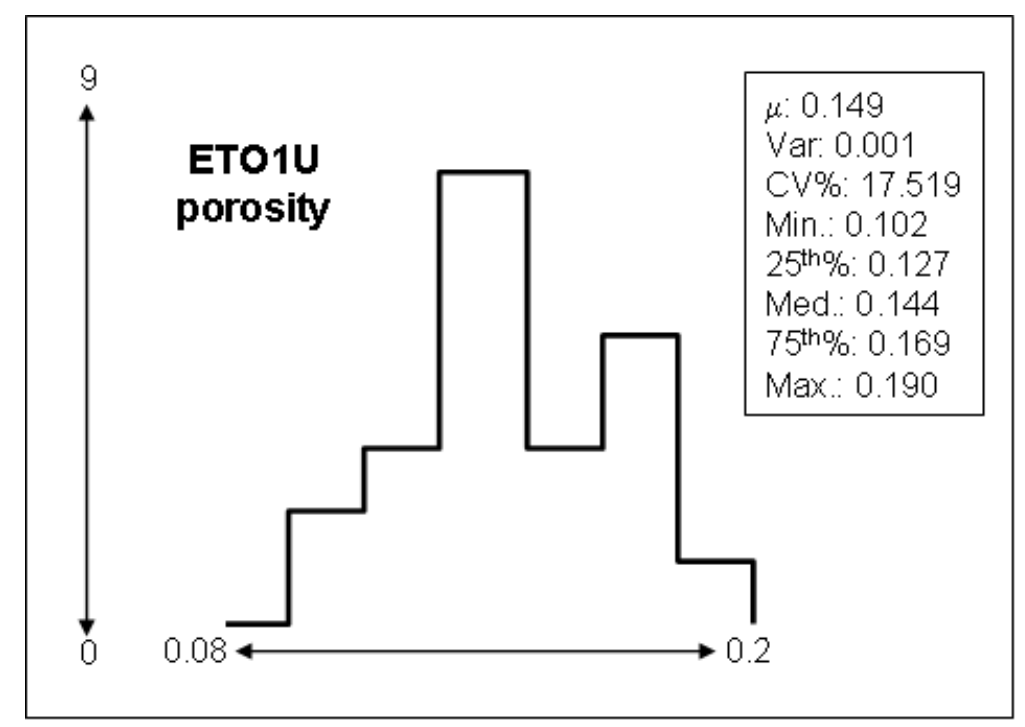

Fig. 1.3.35 - Statistics of porosity values for ET O’Daniel, 1U sand.

Porosity values are similar within the $1 \mathrm{U}$ interval in both units as seen in figs. 1.3.35 to 1.3.36, with a slight skew observed in the Germania $1 \mathrm{U}$ and $5 \mathrm{U}$ interval. Besides the skew, the mean and IQR indicate that the sands (1U and $5 \mathrm{U})$ have similar range of values. The sands are generally of low porosity and permeability, and shale is laminated, however, on the average shale tends be relatively low as observed in the Shale volumes obtained from well averages in the sand intervals. 


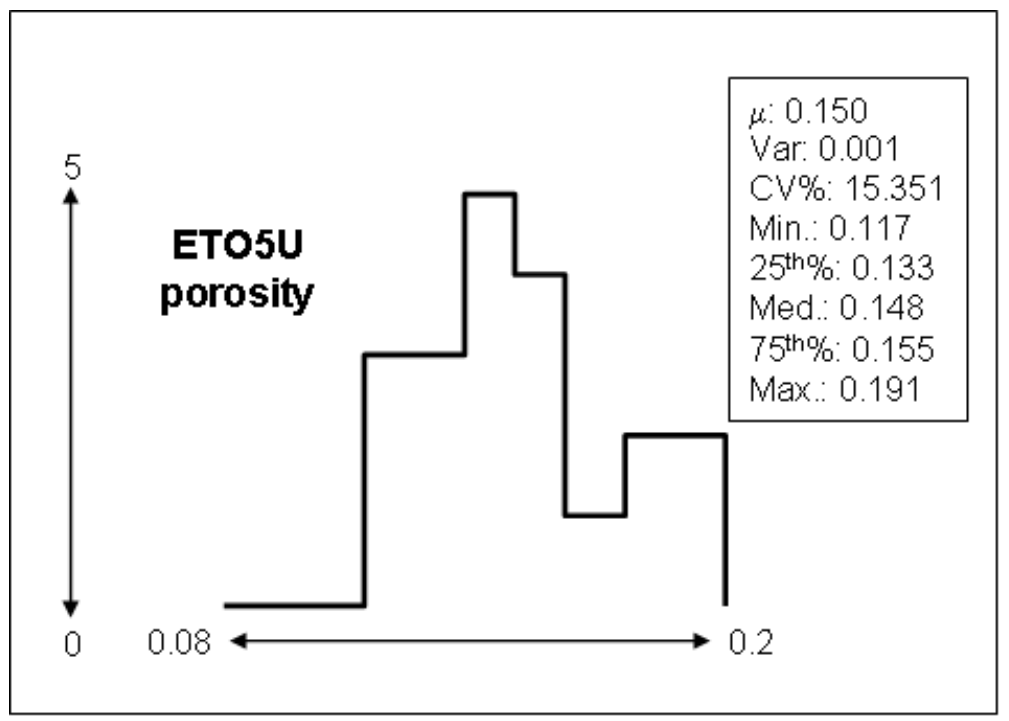

Fig. 1.3.36 - Statistics of porosity values for ET O’Daniel, 5U sand.

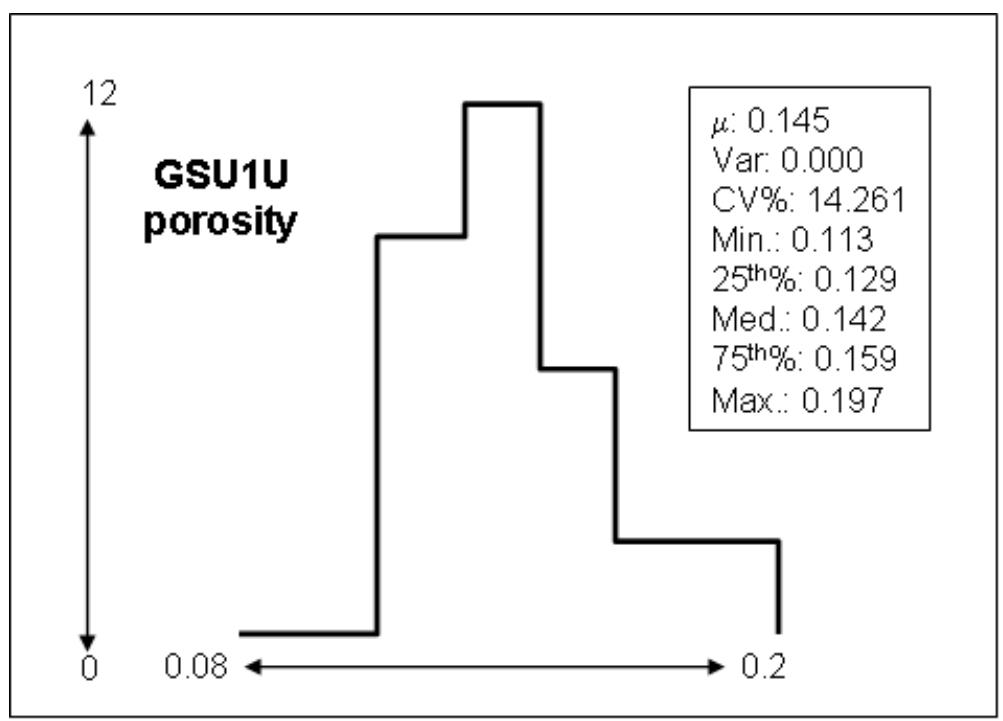

Fig. 1.3.37 - Statistics of porosity values for Germania, 1U sand. 


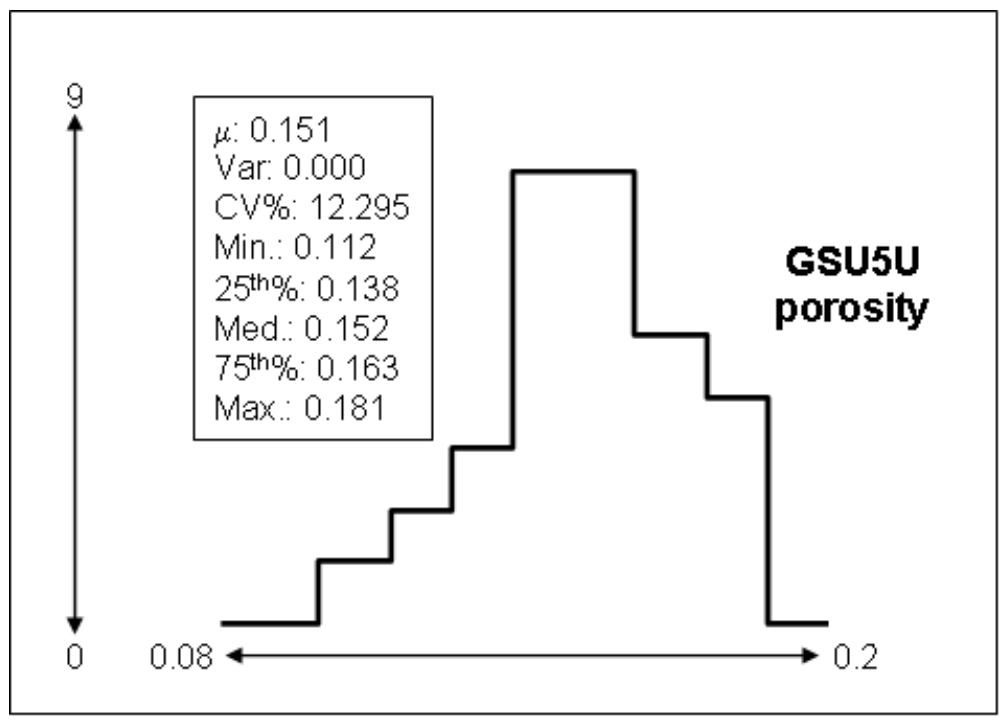

Fig. 1.3.38 - Statistics of porosity values for Germania, 5U sand.

\subsection{Kolmogorov - Smirnov test for porosity and shale volume}

This test is based on measuring the maximum vertical separation between two empirical CDF's ${ }^{36}$ given as $d_{\text {max }}$. This method makes it possible to compare entire distributions rather than any single statistical measure.

For a one sided test at the $5 \%$ confidence level, the value of $1.36\left(\mathrm{I}_{1} \mathrm{I}_{2}\right)^{0.5}\left(\mathrm{I}_{1}+\mathrm{I}_{2}\right)^{0.5}$ must exceed $I_{1} I_{2} d_{\text {max }}$ for the two empirical distribution forms to be considered the same.

Sample size for data set $1, \mathrm{I}_{1}=31$

Sample size for data set $2, I_{2}=22$

Critical value not to be exceeded is given by $\mathrm{I}_{1} \mathrm{I}_{2} \mathrm{~d}_{\text {max }}$, and the test value is given by 1.36 $\left(\mathrm{I}_{1} \mathrm{I}_{2}\right)^{0.5}\left(\mathrm{I}_{1}+\mathrm{I}_{2}\right)^{0.5}$. From Fig. 1.3.39, the value of $\mathrm{d}_{\max }$ is given by the maximum vertical distance between the two functions.

Resulting values are 47.06 and 258 respectively, this indicates that the distributions are somewhat different. A limitation ${ }^{37}$ of this test is that it is more sensitive close to the center of the distribution, than at the tail, evidenced by the Fig. 1.3 .39 where $d_{\max }$ occurs about the center. 


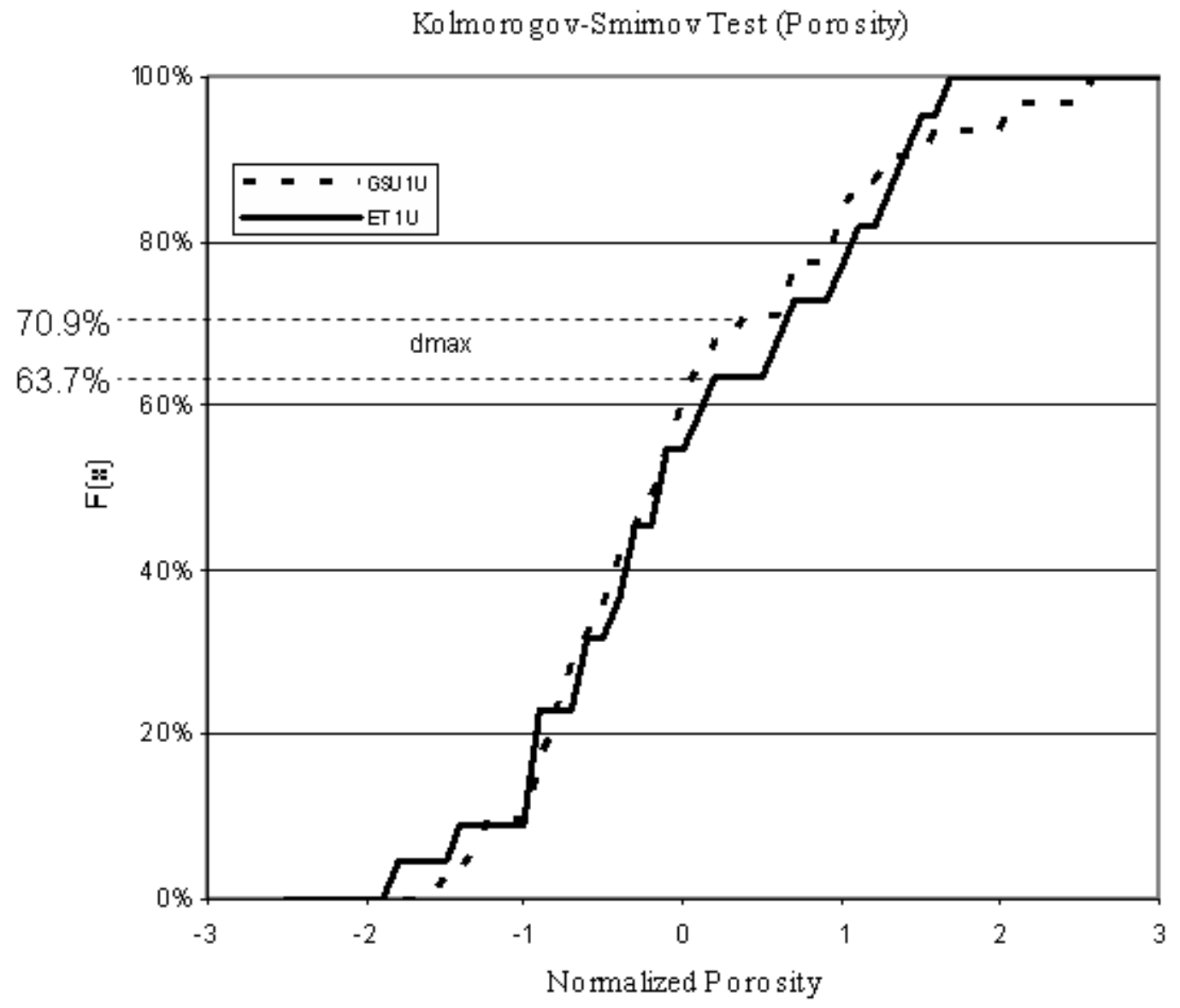

Fig. 1.3.39 - Kolmogorov - Smirnov test on porosity function, 1U sand.

Results of other comparisons between the units of ET O'Daniel and Germania for porosity and shale volume are summarized in Table 1.3.6 below.

A further limitation in the value of any inference as a result of a comparative analysis of Germania and ET O’Daniel lies in the sample size. The ET O’Daniel dataset is about half the size of the Germania dataset for shale volume, it is therefore a possibility that improvement in correlations will be made as the database is expanded. See appendix B11 for illustration of effect of varying sample sizes on the Kolmogorov - Smirnov test for a normal distribution.

Table 1.3.6 - Kolmogorov-Smirnov test for porosity and shale volume.

\begin{tabular}{|c|c|c|c|c|c|}
\cline { 2 - 6 } \multicolumn{1}{c|}{} & $\mathbf{I}_{\mathbf{1}}$ & $\mathbf{I}_{\mathbf{2}}$ & $\mathbf{d}_{\max }$ & Critical & Test \\
\hline Porosity 1U & 31 & 22 & $6.90 \%$ & 47.06 & 258.56 \\
\hline Porosity 5U & 31 & 20 & $10.60 \%$ & 65.72 & 241.84 \\
\hline Vshl 1U & 50 & 23 & $7.10 \%$ & 81.65 & 394.05 \\
\hline VshI 5U & 50 & 21 & $6.00 \%$ & 63.00 & 371.33 \\
\hline
\end{tabular}




\subsection{Litho- stratigraphic section}

Another factor that lends itself to the verification of the ET O'Daniel and Germania units being analogous to one another is the depositional continuity over the two fields.

The $1 \mathrm{U}$ and $5 \mathrm{U}$ sands as well as the over and underlying intervals are well defined in both fields, and are at approximately the same depth horizons, and most of all, are picked by all the wells analyzed. Similar depositional characteristics are also observed i.e. prominent shale markers and fining/coarsening sand trends.

Figs. 1.3.40 and 1.3.41 show gross thickness map of both fields with line of section (AA') indicated and the litho-stratigraphic section displaying the 5 sand units in the upper Spraberry. 

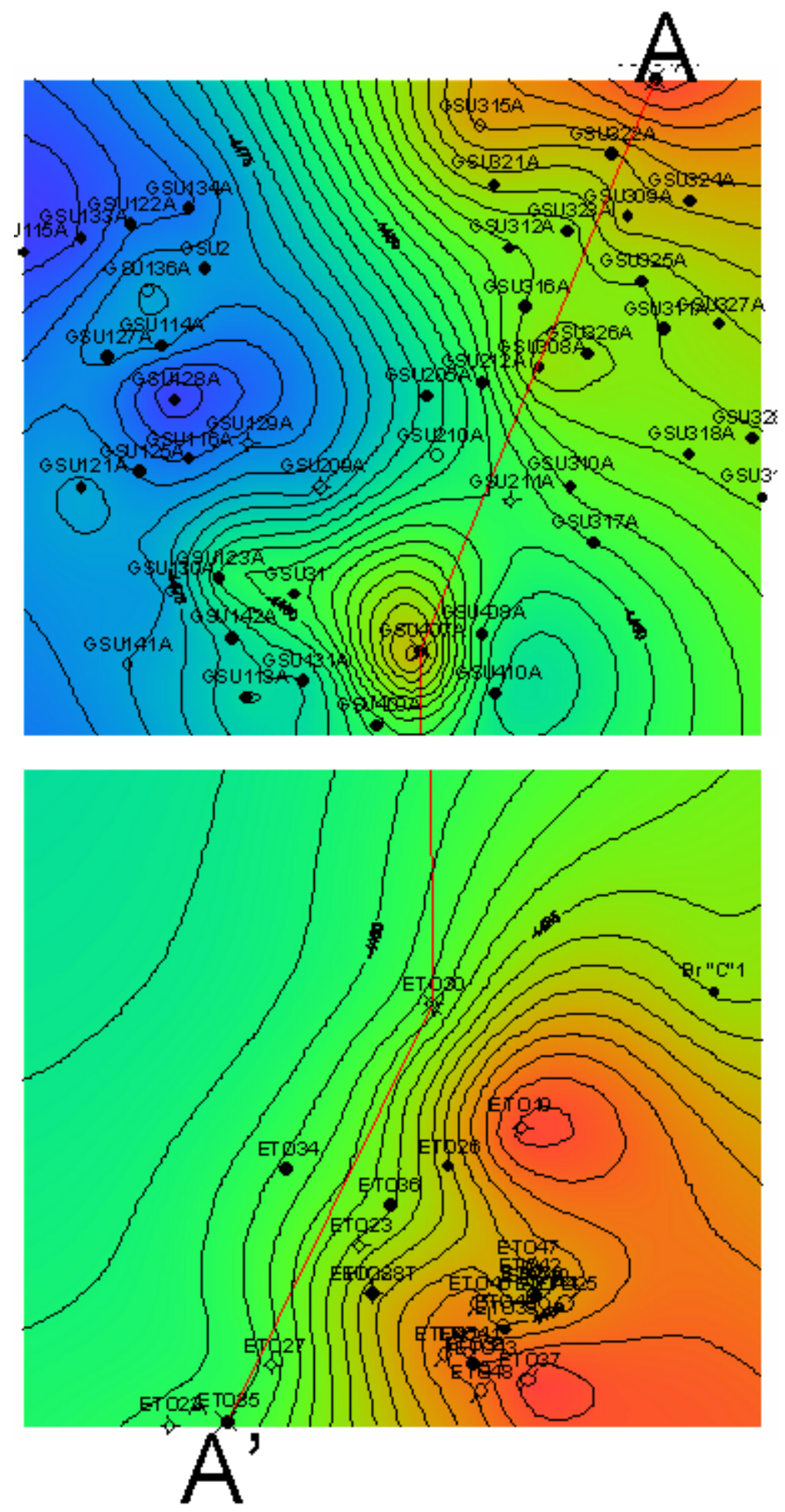

Fig. 1.3.40 - Gross thickness map of the $1 \mathrm{U}$ sand.

(Section A-A' along the north-south dip wise axis of the Spraberry trend area). 
Line of section A-A' shows a section taken in the paleodip direction of the Spraberry. In the previous literature ${ }^{2,16}$, it is suggested that deposition of terrigenous clastic sediments were southward thinning forming elongate fan shaped sand wedges.

Wells GSU\#314, GSU\#407, ETO\#30 and ETO\#35 are used for this section and are in sequence from North-East to South-West. Interwell distances are large, but intermediate wells exhibit same sand sequence and as such, wells spanning across both units were chosen to emphasize the lateral continuity of the $1 \mathrm{U}, 5 \mathrm{U}$ and intermediate sand intervals.

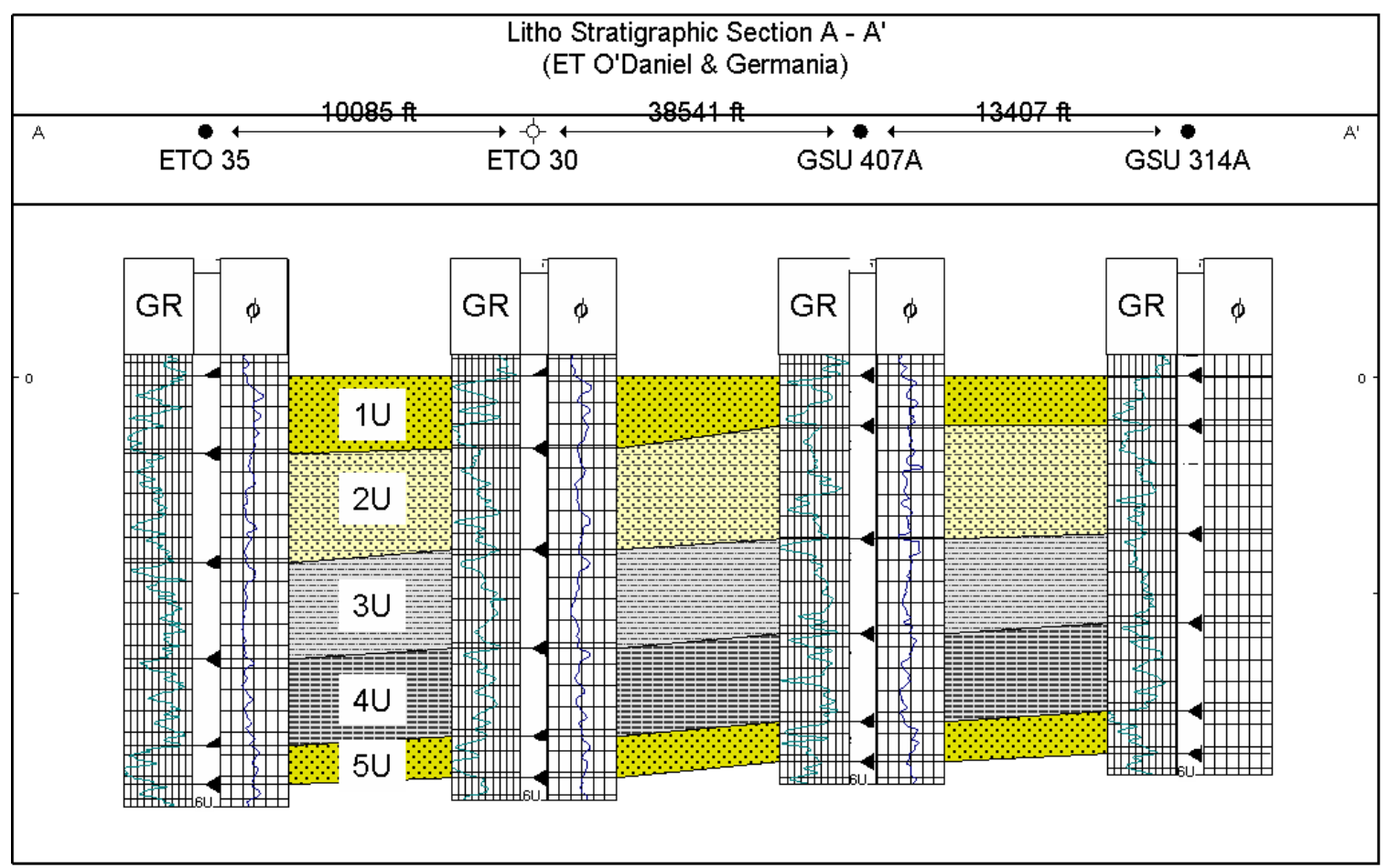

Fig. 1.3.41 - Lithostratigraphic section A-A' with datum at top of $1 \mathrm{U}$ interval. 


\section{Model Application in Germania Unit}

\subsection{Germania}

\subsubsection{Picks and interval properties}

The Germania unit shows through the gamma ray logs, the characteristic response observed in the ET O'Daniel unit within the five reservoir units (1U thru 5U). The formation markers were generally distinguishable and continuous over the lateral space between both units. Application of the log based rock model to distinguish reservoir and non-reservoir quality rock through porosity and shale indicators will form the basis of the reservoir description process and the generation of structure and isopach maps that describe this unit.

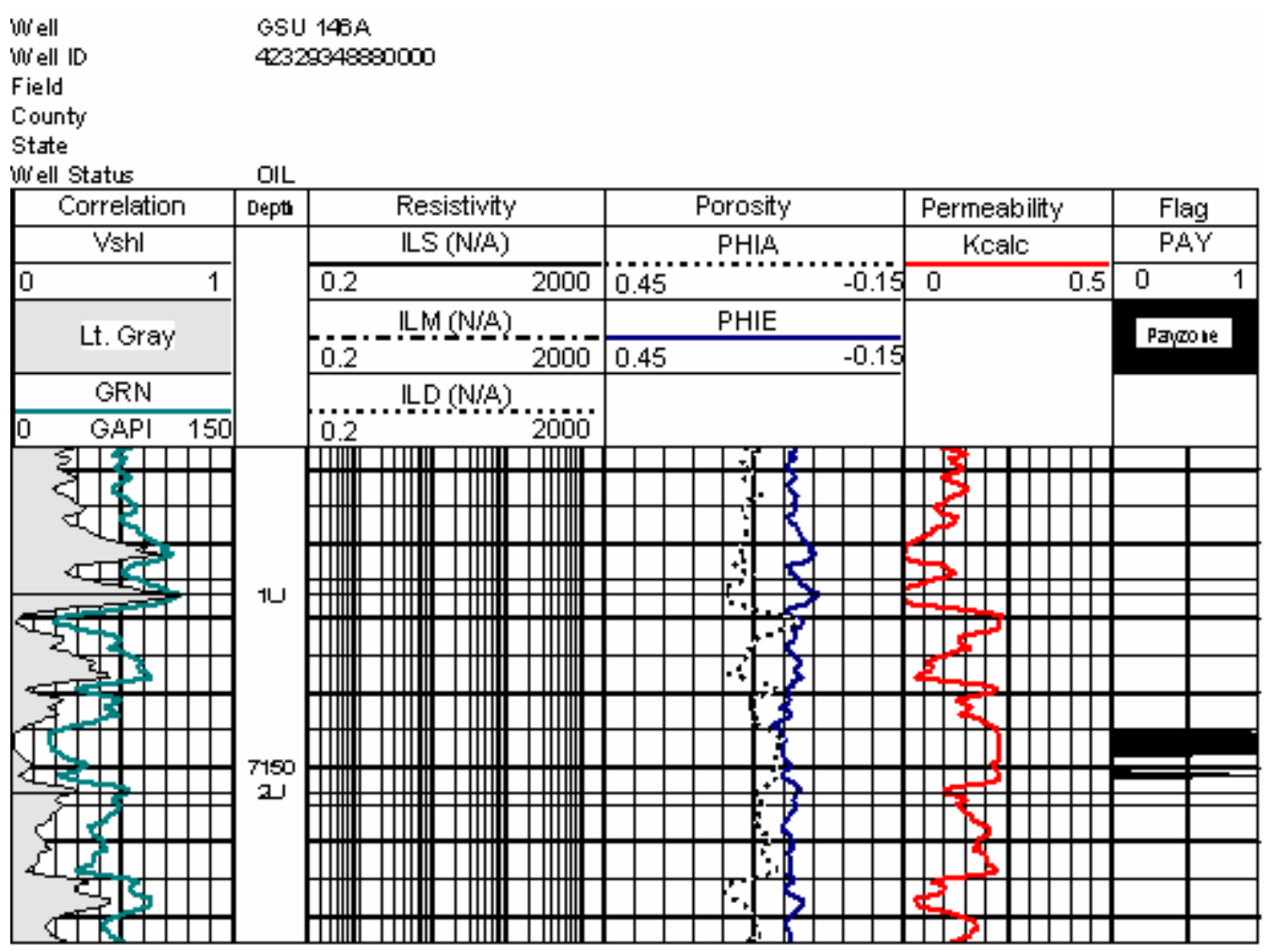

Fig. 1.3.42 - Payzone prediction based on rock model for GSU146A, 1U sand. 


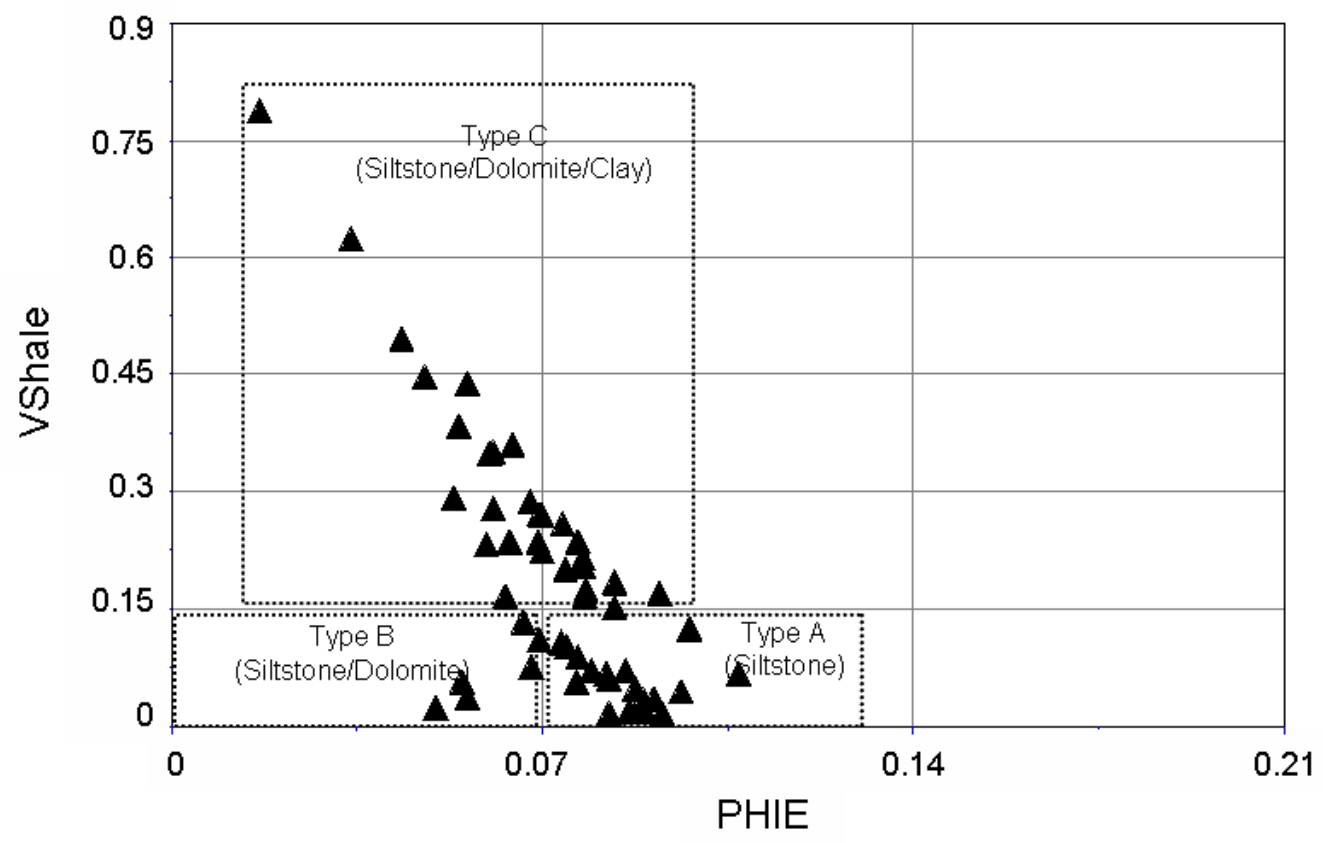

Fig. 1.3.43 - Estimate of rock types in GSU146A, 1U sand from shale volume - porosity crossplot.

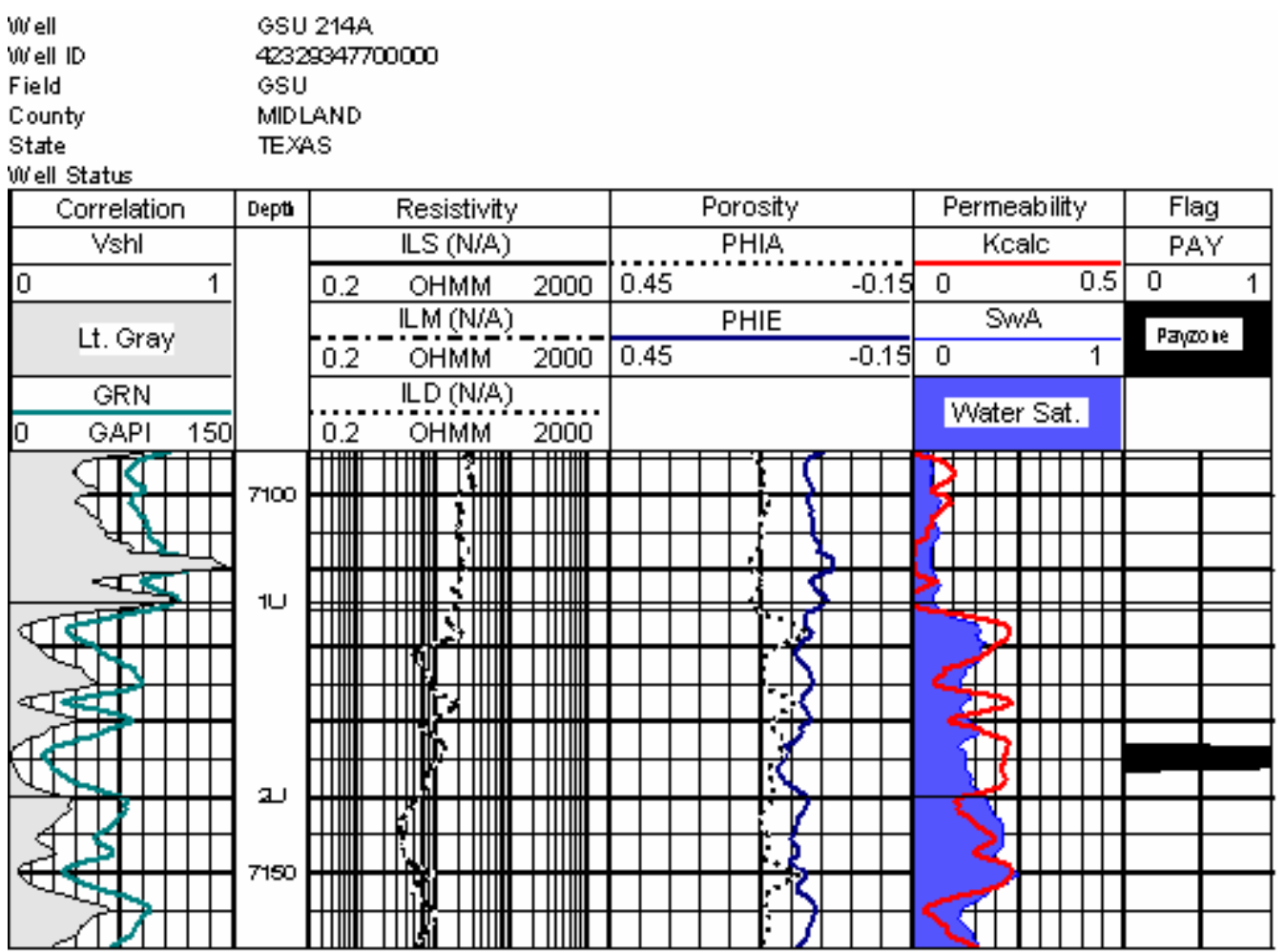

Fig. 1.3.44 - Payzone prediction based on rock model for GSU 214A, 1U sand. 


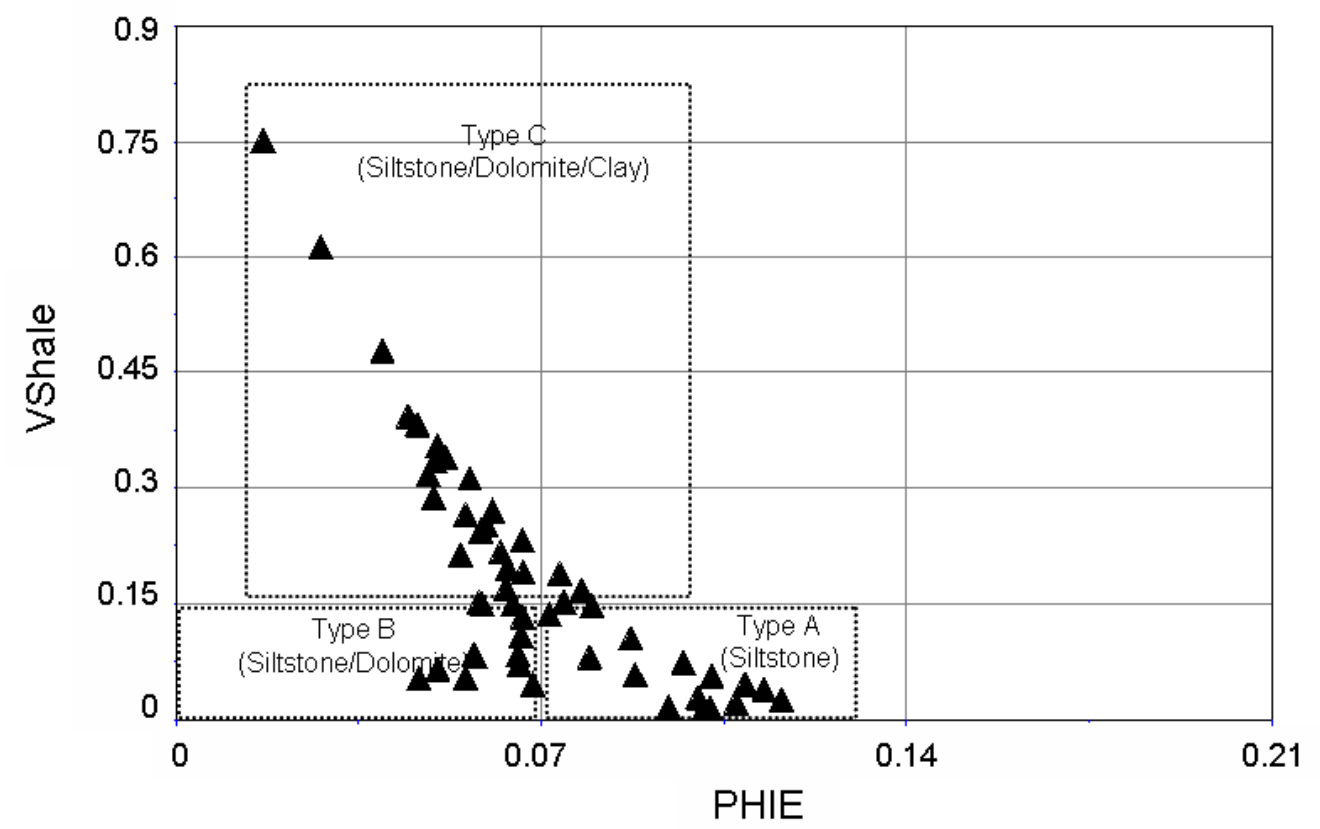

Fig. 1.3.45 - Vshale crossplot for GSU 214A, 1 U sand.

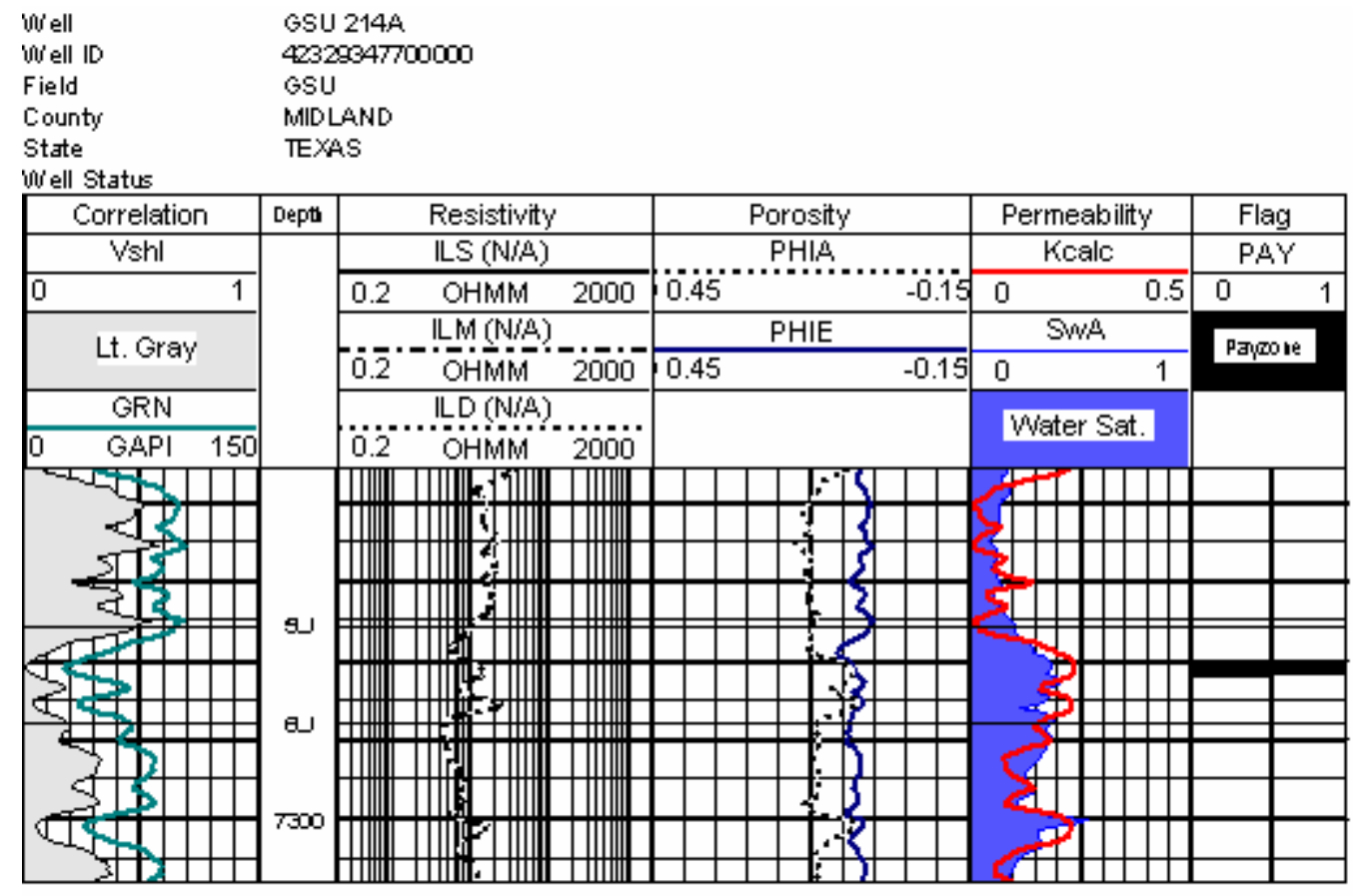

Fig 1.3.46 - Payzone prediction for GSU214A, 5U sand. 


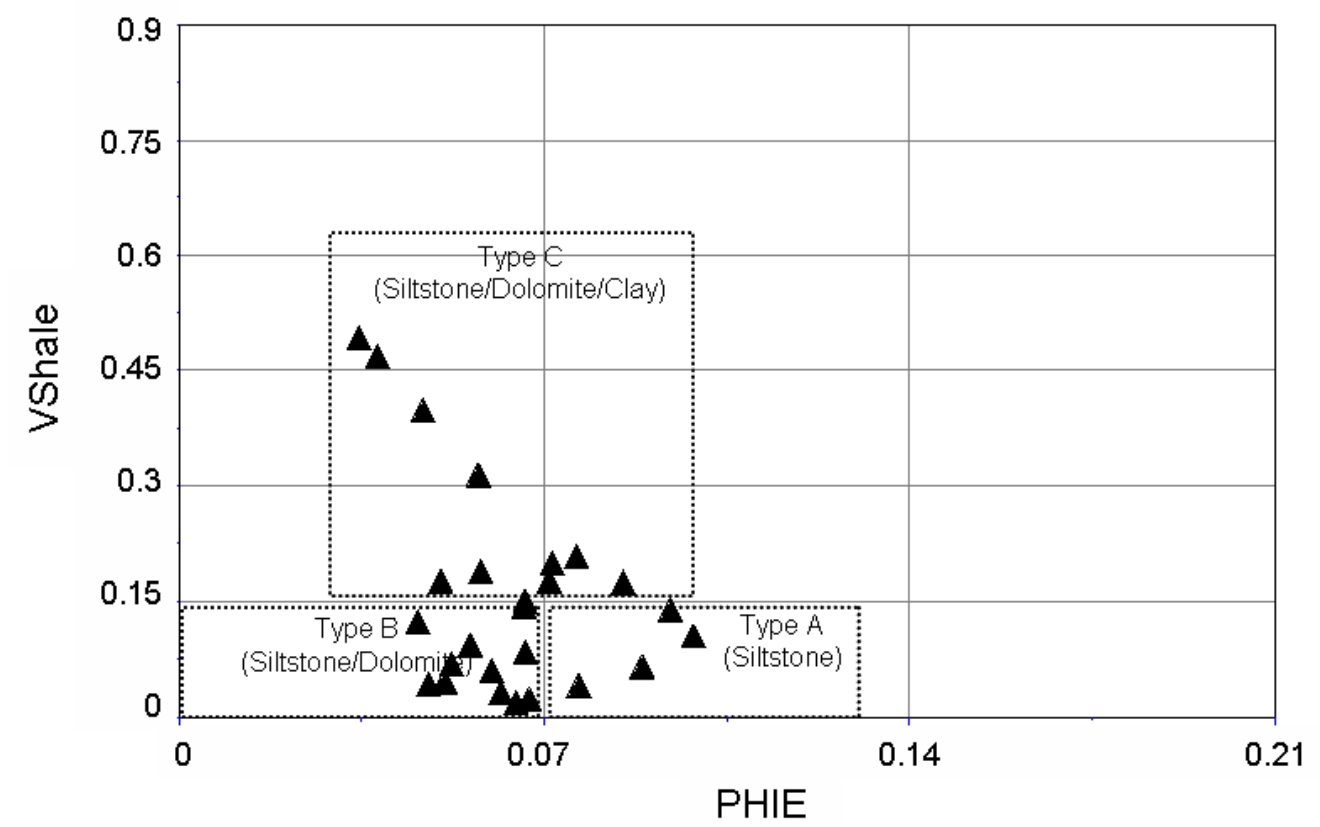

Fig. 1.3.47 - Vshale crossplot for GSU 214A, 5U sand.

The log based rock model developed from log analysis and petrographic studies in the ET O’Daniel unit, was applied with partial success in the Germania Spraberry unit. From observation within the GSU log playback, the rock model consistently underestimated the pay interval compared with the average ET O’Daniel interval.

Figs. 1.3.42 to 1.3.47 provide a visual means of quantifying the net sand with respect to the gross sand thickness. It is evident that the net to gross sand ratio drastically reduces based on the rock - log model estimates in the Germania unit with respect to the ET O’Daniel prediction estimates.

The $1 \mathrm{U}$ sand shows a net to gross ratio of about $30 \%$ as compared to $50 \%$ in the ET O’Daniel unit. A dissimilar trend is also observed, in that the low clay sands are not exclusively rock type A, but are mostly type B - dolomitic siltstone. In the 5U unit, the net to gross ratio observed here is about $20 \%$ compared to the $40 \%$ observed in the equivalent ET O’Daniel unit.

On the other hand, the more recent neutron porosity logs did yield consistent results with the application of the rock - log model in the Germania Spraberry unit. The logged well pay estimates ranged within the $50 \%$ and $40 \%$ values for the $1 \mathrm{U}$ and $5 \mathrm{U}$ units as in ET O’Daniel. 


\subsection{Uncertainties}

\subsubsection{Porosity}

Porosity derived from neutron logs are susceptible to errors when gas is present near enough to the wellbore. The normalization and conversion process does not eliminate these errors completely, especially due to the lack of unit standardization and well-bore environmental corrections. Hence due to the large number of neutron logs converted to porosity values in comparison to the 'more reliable' neutron porosity logs in the database used to perform the study, inferences based on the resulting porosity values are prone to errors. Quantification of the errors is not estimated in this study.

\subsubsection{Permeability}

The flow capacity of the rock may be dependent on many factors such as depositional porosity, pore throat size, irreducible water saturation, clay content, and in the case of diagenetically modified rock, alteration by mineralization or dissolution. Investigating each of these factors on permeability is often hampered by the difficulty in acquiring quality data, hence permeability is often reduced to log data, pressure transient tests, and conventional core data analysis. There are no direct measurements of producibility from logs.

Shale volume and porosity were the only variables used to investigate permeability and, therefore, limited the reliability of the permeability predictions.

\subsubsection{Water saturation}

An inverse approach was used in determining saturations, and as is often the case, there is non-uniqueness in the defining parameters. In this case, the Archie parameters have preestablished tolerances as shown in chapter II, but nevertheless different combination of Archie parameters within this tolerance may still yield consistent matches from well to well.

\subsubsection{Net pay}

Due to the limitations in the porosity predictions earlier mentioned, the rock log model which is based on porosity and shale volume will equally be subject to errors. Therefore, even though tried and tested, the rock log model may fail to adequately predict the location and thickness of productive sub units. 


\section{Conclusion}

Electrofacies patterns within the ET O'Daniel are easily recognizable in the Germania Spraberry unit and this correlation is evident across the units of the upper Spraberry. The general reservoir structure is consistent across both unit and thus becomes the essence of the Germania unit characterization.

Studies in the analog unit - ET O'Daniel, provided valuable input towards the characterization effort of the Germania for a variety of reasons, the most fundamental being the log based rock model used for rock type discrimination.

The simplicity of the model makes it easy to use as a criteria for determining pay quality within the upper Spraberry, once the correlatable units are identified. Secondly, the core analysis performed by Reservoirs Incorporated enabled the development of porosity and permeability relationships that may be applied in the GSU, the result of which are porosity and permeability maps to guide further development and simulation studies. The conclusions can be summarized as follows:

The Isopach maps indicate channel deposits and interdistributary flat features that are consistent with the depositional evaluation proposed by Handford et al. The facies indicate detrital clasts of both depositional episodes as evident in the lithology charts in chapter II which indicate that the $1 \mathrm{U}$ and $5 \mathrm{U}$ intervals are made up of terrigenous sandstones as well as dolomitic carbonate facies.

Particularly evident in the ET O'Daniel Isopach maps are the north - south thinning of the sandstone intervals, this is indicative of an energy source north of the Spraberry.

The Germania unit isopach (net thickness) is conservative in its estimation of net pay thickness, this is can be attributed to the limitations of the log based rock model in predicting payzones based on normalized cased hole neutron logs. Neutron logs alone do not lend themselves to corrections (environmental etc). Porosity correction techniques require a multi-dimensional array of porosity data i.e. density and neutron or sonic. Ideally, the density and neutron porosity logging devices should be run together so as to allow for necessary compensatory corrections in log data.

Optimal non-parametric regression techniques did not clearly improve the estimations of permeability based on shale volume and porosity. The method in itself is efficient, but alternative or more appropriate relationships specific to the Spraberry units need to be investigated. Additional studies on pore throat distribution based on core analysis may offer opportunities to improve the predictions.

Techniques for determining water saturation in the spraberry or shaly sands do not recommend using the Archie's equation. The Archie model is simplistic in its approach and is only considered a means of 'estimating' water saturation for this study. 
Acquisition of core data within the Germania unit is not an option. To enable further evaluation of the GSU, core derived parameters must be available to guide simulation studies and inform fracture characterization to yield an integrated reservoir model. 


\section{REFERENCES}

1. Schechter, D.S., McDonald, P., Sheffield, T., and Baker, R.: "Reservoir Characterization and $\mathrm{CO}_{2}$ pilot Design in the Naturally Fractured Spraberry Trend” paper SPE 35469 presented at the 1996 Permian Basin Oil and Gas Conference, Midland, 27-29 March.

2. Handford, C.R.: "Sedimentology and Genetic Stratigraphy of Dean and Spraberry Formations (Permian), Midland Basin, Texas” AAPG Bulletin (1981), 65, no. 9, 1602.

3. Barfield, E.C., Jordan, J.K., and Moore, W.D.: "An Analysis of Large-Scale Flooding in the Fractured Spraberry Trend Area Reservoir” JPT (1959), 11, 15.

4. Schechter, D.S.: “Advanced Reservoir Characterization and Evaluation of $\mathrm{CO}_{2}$ Gravity Drainage in the Naturally Fractured Spraberry Trend Area” 2nd Annual Technical Progress Report, US DOE Contract No. DE-FC22-95BC14942, Socorro, New Mexico (August 1997).

5. Schechter, D.S.: “Advanced Reservoir Characterization and Evaluation of $\mathrm{CO}_{2}$ Gravity Drainage in the Naturally Fractured Spraberry Trend Area” 1st Annual Technical Progress Report, US DOE Contract No. DE-FC22-95BC14942, Socorro, New Mexico (August 1996).

6. McDonald, P., Lorenz, J.C., Sizemore C., Schechter, D.S., and Sheffield, T.: "Fracture Characterization Based on Oriented Core from the Spraberry Trend Reservoir: A Case Study” paper SPE 38664 presented at the 1997 SPE Annual Technical Conference and Exhibition, San Antonio, 5-8 October.

7. Reservoirs Inc.: "Geological and Petrophysical Analysis of the Upper Spraberry Formation, Midland County, Texas” Report prepared for Parker \& Parsley Development Co., Midland, Texas (1997), 1 \& 2.

8. Schechter, D.S., and Banik; A.S.: "Characterization of the Naturally Fractured Spraberry Trend Shaly Sands Based on Core and Log Data” paper SPE 35224 presented at the 1996 Permian Basin Oil and Gas Conference, Midland, 27-29 March. 
9. Schechter, D.S., and Banik; A.S.: “Integration of Petrophysical and Geologic Data with Open-Hole Logs for Identification of the Naturally Fractured Spraberry Payzones” paper SPE 38913 presented at the 1997 SPE Annual Technical Conference and Exhibition, San Antonio, 5-8 October.

10. Mardock, E.S., and Myers, J.P.: "Radioactivity Logs Define Lithology in the Spraberry Formation” Oil and Gas Journal (Nov. 1951), 90.

11. Lyttle, W.J., and Rieke, R.R.: "Well Logging in the Spraberry” Oil and Gas Journal (Dec. 51), 92

12. Schechter, D.S., and Putra, E.: “ $\mathrm{CO}_{2}$ Pilot Design and Water Injection Performance in the Naturally Fractured Spraberry Trend Area, West Texas” paper SPE 71605 presented at the 2001 SPE Annual Technical Conference and Exhibition, New Orleans, Sept. 30-Oct. 3.

13. Putra, E.: "Reservoir Simulation of Waterflood Pilot in Naturally Fractured Spraberry Trend” paper SPE 54336 presented at the 1999 Asia Pacific Oil and Gas Conference and Exhibition, Jakarta, 20-22 April.

14. Schechter, D.S., and Buoyun, Guo .: “An Integrated Investigation for Design of a $\mathrm{CO}_{2}$ Pilot in the Naturally Fractured Spraberry Trend Area, West Texas” paper SPE 39881 presented at the 1998 International Petroleum Conference and Exhibition, Villahermosa, 3-5 March.

15. Wilkinson, W.M.: "Fracturing in Spraberry Reservoir, West Texas" AAPG Bulletin, (Feb 1953), 37, no. 2, 253.

16. Schmitt, G.T.: "Genesis and Depositional History of Spraberry Formation, Midland Texas” AAPG Bulletin (Sept. 1954), 38, no. 9, 1957

17. Tyler, N., and Gholston, J.C.: "Heterogeneous Deep Sea Fan Reservoirs, Shackelford and Preston Waterflood Units, Spraberry Trend West Texas” University of Texas at Austin Bureau of Economic Geology, Report of Investigations (1998), 171, 38

18. Montgomery, S.L., Schechter, D.S., and Lorenz, J.: “Advanced Reservoir Characterization to Evaluate $\mathrm{CO}_{2}$ flooding, Spraberry Trend, Midland Basin, Texas” AAPG Bulletin (2000), 84, no. 9, 1247. 
19. Asquith, G.B.: Log Analysis of Shaly Sandstone Reservoirs: A Practical Guide, AAPG (1992), Tulsa Oklahoma

20. Greder, H.N., Mus, E.A., Veillerette, A.M., Pellerin, and F.M.: "Derivation of a Core Permeability Log and Extrapolation to Uncored Intervals” Proc. 36th Society of Professional Well Log Analyst Symposium (1995), 12p.

21. Balan, B., Mohaghegh, S., Ameri, S.: "State-of-the-Art in Permeability Determination from Well Log Data: Part I- A Comparative Study Model Development” paper SPE 30978 presented at the 1995 Eastern Regional Conference and Exhibition, Morgantown, 17-21 September.

22. Allen, J.R.: "Prediction of Permeability from Logs by Multiple Regression" Trans. 36th SPWLA Symposium (1995)

23. Van Den Bosch, R., Fulop, A., and, Pistre, V.: "Permeability Prediction from Well Logs - A Case Study in German Rotliegend Sandstones” Trans. 36th SPWLA Symposium (1995), 12p.

24. Hook, J.R., Nieto, J.A., Kalkomey, C.T., and Ellis, D.: "Facies and Permeability Prediction from Wireline Logs and Core - A North Sea Case Study” Trans. 35th SPWLA Symposium (1994), AAA1.

25. Saxena, V.: "Hydrocarbon Evaluation through Modulus Decomposition of Sonic Velocities in Shaly Sands” Trans. 37th SPWLA Symposium (1996), 14p.

26. Breiman, L., and Friedman, J.H.: "Estimating Optimal Transformations for Multiple Regression and Correlations” Journal of the American Statistical Association (1985), 80, 580.

27. Guoping Xue, Datta-Gupta, A., Valko, P., and Blasingame, T.: “Optimal Transformations for Multiple Regression: Application to Permeability Estimation from Well Logs” paper SPE 35412 presented at the 1996 Improved Oil Recovery Symposium, Tulsa, 21 April.

28. Shier, D.E., Course Notes for Well Log Normalization Work Shop, Published privately by Energy Data Services, Golden, Colorado (1991).

29. Crain, E.R., Garrido, M.:"'Qualitative Analysis of Older Rocks for Porosity and Permeability - Lake Maracaibo, Western Flank Reservoirs, Venezuela” Petroleos de Venezuela (PDVSA) 
30. Hunt, E., Aly, A., and Pursell, D.: "Fundamentals of Log Analysis, Part 6, Determining Basic Log Analysis Parameters - a, m, n, and Rw” World Oil, (Oct. 1996), 217, no.12, 59

31. Neinast, G. S., and Knox, C. C.: "Normalization of Well Log Data” Trans. 14th SPWLA Symposium (1973), I1.

32. Isaaks, E.H., and Srivastava, R.M.: An Introduction to Applied Geostatistics, Oxford University Press (1989).

33. Hunt, E., and Pursell, D.: "Fundamentals of Log Analysis, Part 7, Determining Shaliness from Logs” World Oil, (1996), 218, no.3, 55

34. Reservoirs Inc.: “NMR Core Analysis: Upper Spraberry Formation, ET O’Daniel Lease Area, Midland County, Texas” Report prepared for Parker and Parsley Development Co., Midland, Texas (1997)

35. Rasmus J.C.: "Variable Cementation Exponent, m, for Fractured Carbonates” The Log Analyst (1983), 41.

36. Jensen J.L., Lake L.W., Corbett P.W., and Goggin D.J.: Statistics for Petroleum Engineers and Geoscientists, Prentice Hall (1997).

37. NIST/SEMATECH e-Handbook of Statistical Methods, http://www.itl.nist.gov/div898/handbook/

38. Gibson, G.: "The Relation of Fractures to the Accumulation of Oil in the Spraberry Formation” Proc. 3rd Oil Recovery Conference, West Texas Petroleum Research Committee, Bulletin 15 (1951), 16.

39. Ogden, V., and Locke, J.: “Core Data Discussions” ibid., Bulletin 15, 57.

40. Marshall, J.W.: "Spraberry Reservoirs of West Texas” Geological Notes (1952), 2189.

41. Lang, W.H., Jr.: “Porosity Log Calibrations” The Log Analyst (1980), 21, no. 2, 14.

42. Shier, D.E.: “A Comparison of Log Response between Logging Companies and Different Vintages of Tools” The Log Analyst (1997), 38, no. 3, 47.

43. Shier, D.E., and Sponable, D.M.: “Correcting Drift in GNT Neutron Log Data from West Texas” The Log Analyst (1997), 38, no. 3, 16. 
44. Hardle, W.: Applied Non-parametric Regression, Cambridge University Press, Cambridge (1990)

45. Reservoir Inc.: "Geological and Petrophysical Analysis of the Upper Spraberry Formation in Midland County, Texas” Report prepared for Parker and Parsley Development Co., Midland, Texas (1996)

46. Jensen, J.L., and Lake, L.W.: “Optimization of Regression Based Porosity Permeability Predictions” presented at the 1985 10th Canadian Well Logging Society, Calgary

47. Boardman, D.W. et al.: "Use of Effective Properties for Characterization of a Naturally Fractured Reservoir in East Venezuela” paper SPE 53881 presented at the 1999 Latin American and Caribbean Petroleum Engineering Conference, Caracas, 21-23 April.

48. Guevara, E.H.: “Geologic Characterization of Permian Submarine Fan Reservoirs of the Driver Waterflood Unit, Spraberry Trend, Midland Basin, Texas” University of Texas at Austin Bureau of Economic Geology, Report of Investigations (1988), no. 172, 44p.

49. Asquith, G.B.: “The Importance of Determining Pore Type from Petrophysical Logs in the Evaluation of a Permian Wolfcamp Reentry, Northern Midland Basin” The Log Analyst (1997), 38, no. 3, 37.

50. Ingerman V.G.: "Statistical Integration of Log Data for Lithology Determination and Formation Evaluation” The Log Analyst (1995), 36, 38.

51. Katahara, K.W.: “Gamma Ray Log Response in Shaly Sands” The Log Analyst (1995), 36, 50.

52. Gamboa, P., Reis, J., and Morris, S.: “Development of a Log Based Petrophysical Model for a Complex Dolomite Reservoir” The Log Analyst (1994), 35, 47.

53. Whatler, P.: “Core Data Quality Issues” The Log Analyst (1994), 35, 43.

54. Nelson, P.H.: "Porosity - Permeability Relationships in Sedimentary Rocks” The Log Analyst (1994), 35, 38. 


\section{APPENDIX A \\ BACKGROUND GEOLOGY AND STRATIGRAPHY}

\section{Geology/Geophysical Background}

The Spraberry trend area spans an area about 150 miles in length and 50 miles at the broadest width, and has been found to be productive in an area approximately 500,000 acres $^{16}$. The trend is estimated to originally contain over 8.5 billion barrels of oil of which only $8-12 \%$ have been recovered thus far. Structural contours show that the Spraberry trend lies updip and to the east of current day Basinal axis, which exists adjacent to the central Basin platform (Figure 1.3.A1).

\section{Regional Stratigraphy and Lithofacies}

The Midland Basin is known to be composed of largely of shallow - marine shelf to shelf margin carbonates, as well as deep Basin deposits ${ }^{2}$. Early opinions were that the Dean and Spraberry formations were wolfcampian in age, Silver and $\operatorname{Todd}^{2}$ reported based on biostratigraphic and physical stratigraphic evidence that the formations are Leonardian in age.

Stratigraphic sections analyzed by Jeary ${ }^{2}$ suggest that the Spraberry formation Leonardian in age and is coeval with the upper and middle Clearfork and Glorieta formations (Figures 1.3.A2 and 1.3.A3), these correlations become less obvious in other areas of the Basin due to discontinuity of clastic strata across shelf margins and well control reasons.

The Spraberry formation is approximately $1000 \mathrm{ft}$ thick and is generally composed of $852 \mathrm{ft}$ of black shale and silty shale, $131 \mathrm{ft}$ of siltstones and $5 \mathrm{ft}$ of thin bedded limestones or dolomites. This formation belongs to the lower Leonard and rests conformably on the Wolfcamp. The black fissile shales and thin dolomite beds of the Clearfork group which directly overlies the Spraberry are fractured similar to the Spraberry rocks and with the same lithologic appearance.

The mass of the Spraberry can be separated into 3 distinct and correlative units, which are classified as the upper, middle and lower Spraberry with each unit of approximately the same thickness. The texture and mineralogical character of the major constituents can be described based on rock types as follows:

Siltstones $\sim$ Major percentage of grains fall within the silt size range $(<1 / 16 \mathrm{~mm})$, with about $60 \%$ between the grade limits of $0.03 \mathrm{~mm}-0.06 \mathrm{~mm}$. Grains range from angular 


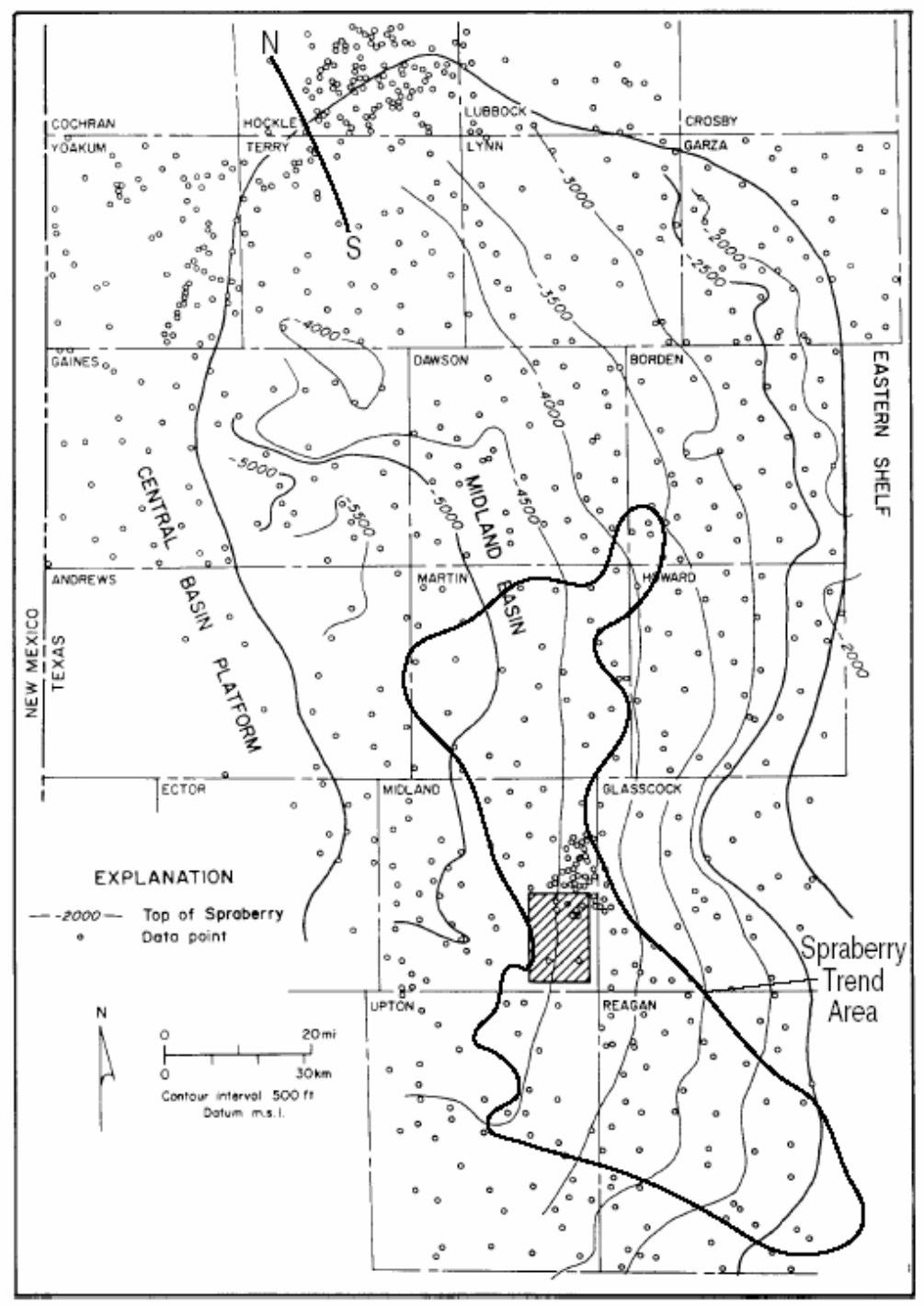

Fig. 1.3.A1 - Structure contour data, top of Spraberry sandstone, Midland Basin, west Texas ${ }^{18}$. 


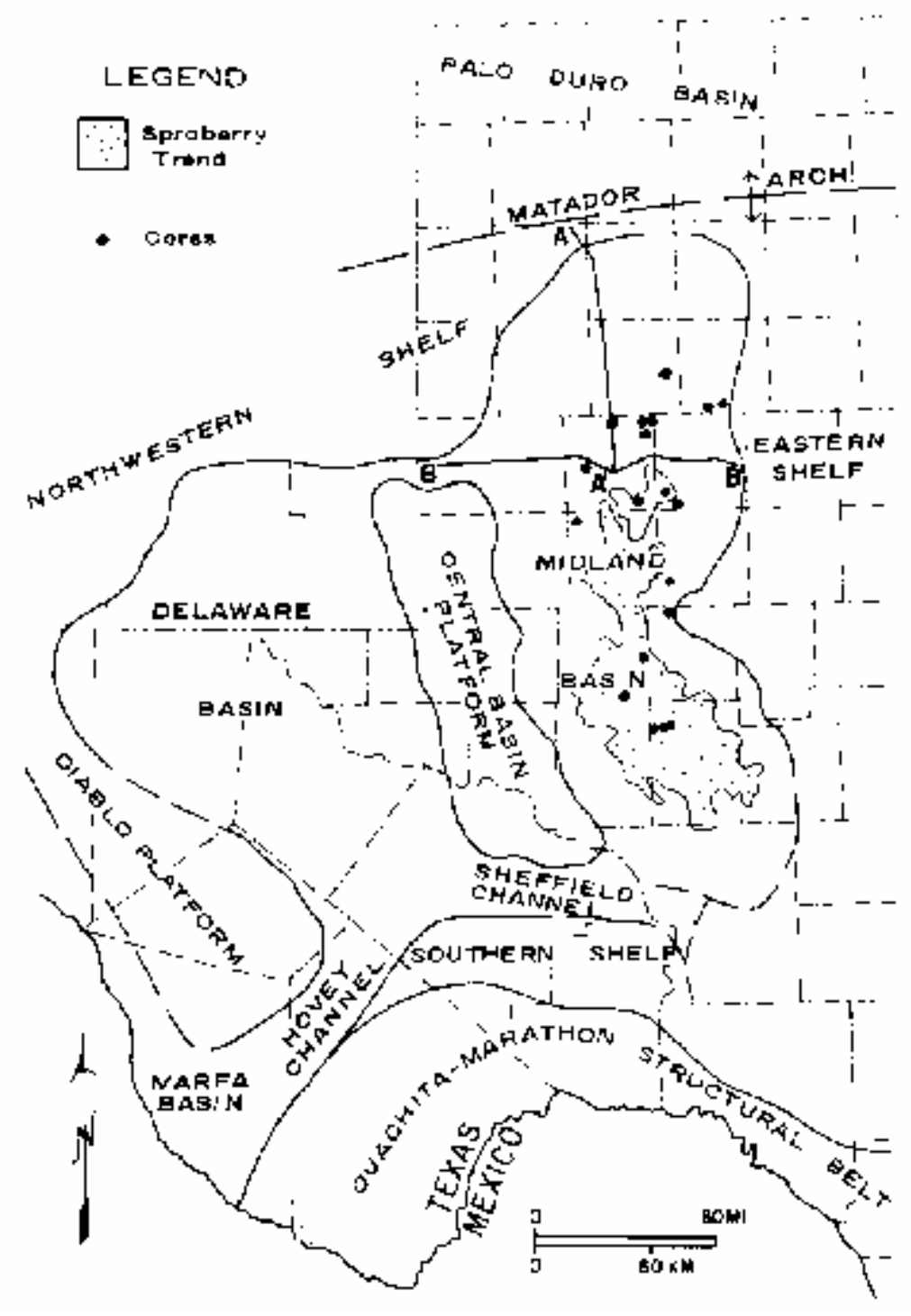

Fig. 1.3.A2 - Regional geologic setting of Permian Basin, west Texas².

to very angular and the sorting is from fair to poor. Primarily, the cementing agent is dolomite with some silica.

Dolomites $\sim$ Vary in texture from fine to crystalline, the fine crystals being primary.

Shales Several types of shales have been encountered, massive blocky as well as the commonly found fissile brittle type. Most of the shales have been classified as carbonaceous, but in all petrographic slides examined, the shales were found to be silty and ferruginous. 

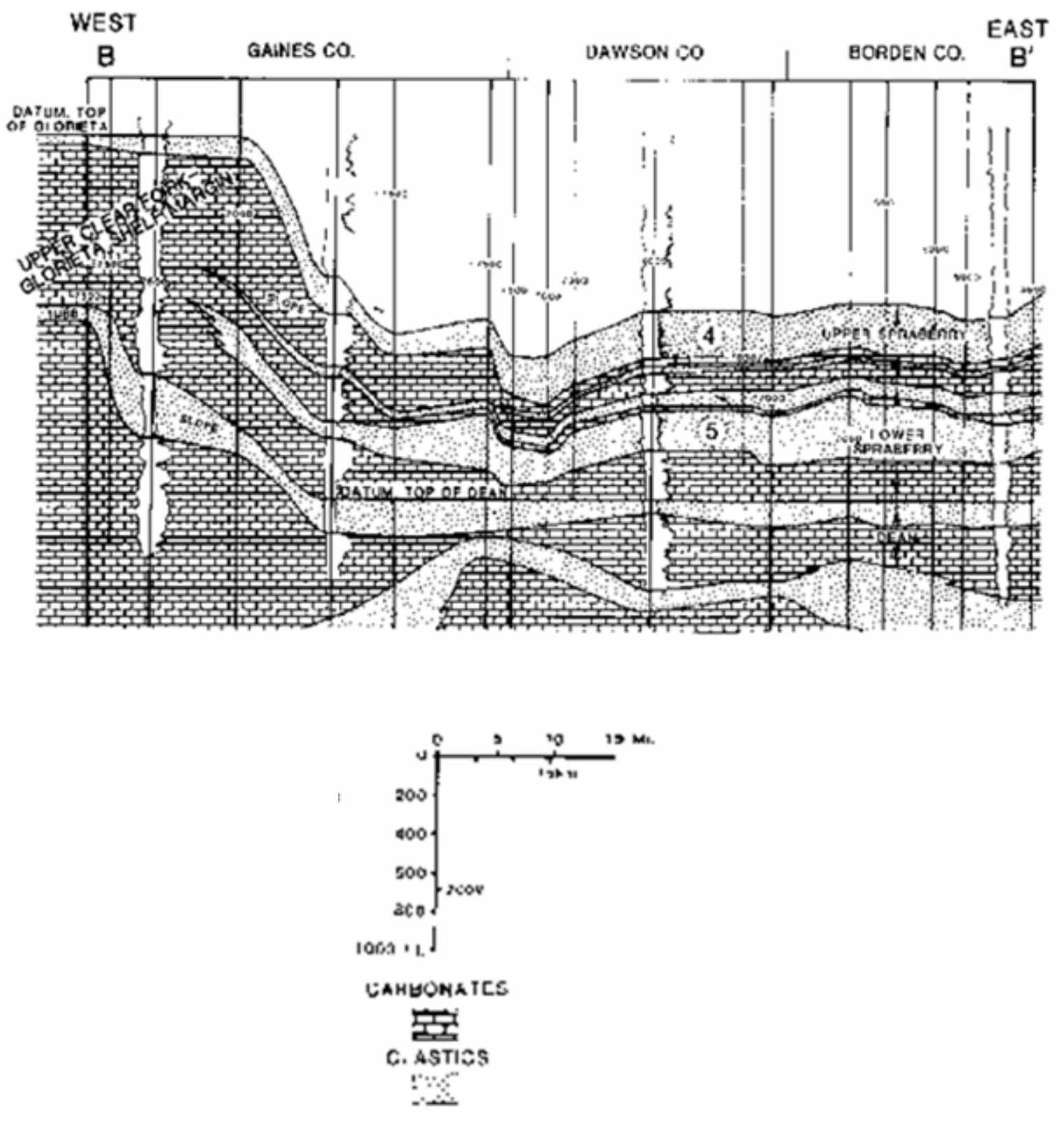

Fig. 1.3.A3 - West - east stratigraphic cross section BB'2

(Note shelf-to-Basin correlations and informally identified stratigraphic units 4 and 5. See Fig.

\subsection{A2 for location).}

Characteristics of the lithofacies are found to be areally and vertically consistent as observed from all the wells examined. Distinctions can be made from electric logs between the upper, middle, and lower Spraberry, however there is no easily apparent characteristic that differentiates the clastic content in the three units from one another, 
when observed by petrographic microscope. This provides a useful means of recognizing three sandstone-siltstone lithofacies based on bedding characteristics:

- Massive to Parallel and cross laminated sandstone and siltstone.

- Laminated Sandstone and siltstone

- Bioturbated sandstone and siltstone

Gamma-Ray logs can often distinguish lithofacies. Laminated and Bioturbated siltstones having a greater shale content than parallel and cross laminated sandstones and siltstones respond with a more radioactive curve.

A Lithofacies map (Fig. 1.3.A4) of the four County area (Upton, Reagan, Midland and Glasscock) substantiates the geological features at the close of the Wolfcamp time. The northeast of the Spraberry trend non clastics are dominant with a gradual facies change toward the productive area, where approximately $87 \%$ of the Spraberry is shale. West of the producing area a rather abrupt shale to limestone facies change occurs, with several wells on the west side of the present day structural highs containing $85 \%$ of non-clastics. The interpretation being that during the time of Spraberry deposition, the west side of the Midland Basin was elevated sufficiently to provide an environment of warm shallow waters, in which carbonates were precipitated and deposited. Superimposed on the lithofacies map are isopach contours representing the thickness of Spraberry type rocks after total deposition and after accounting for all structural movement. 


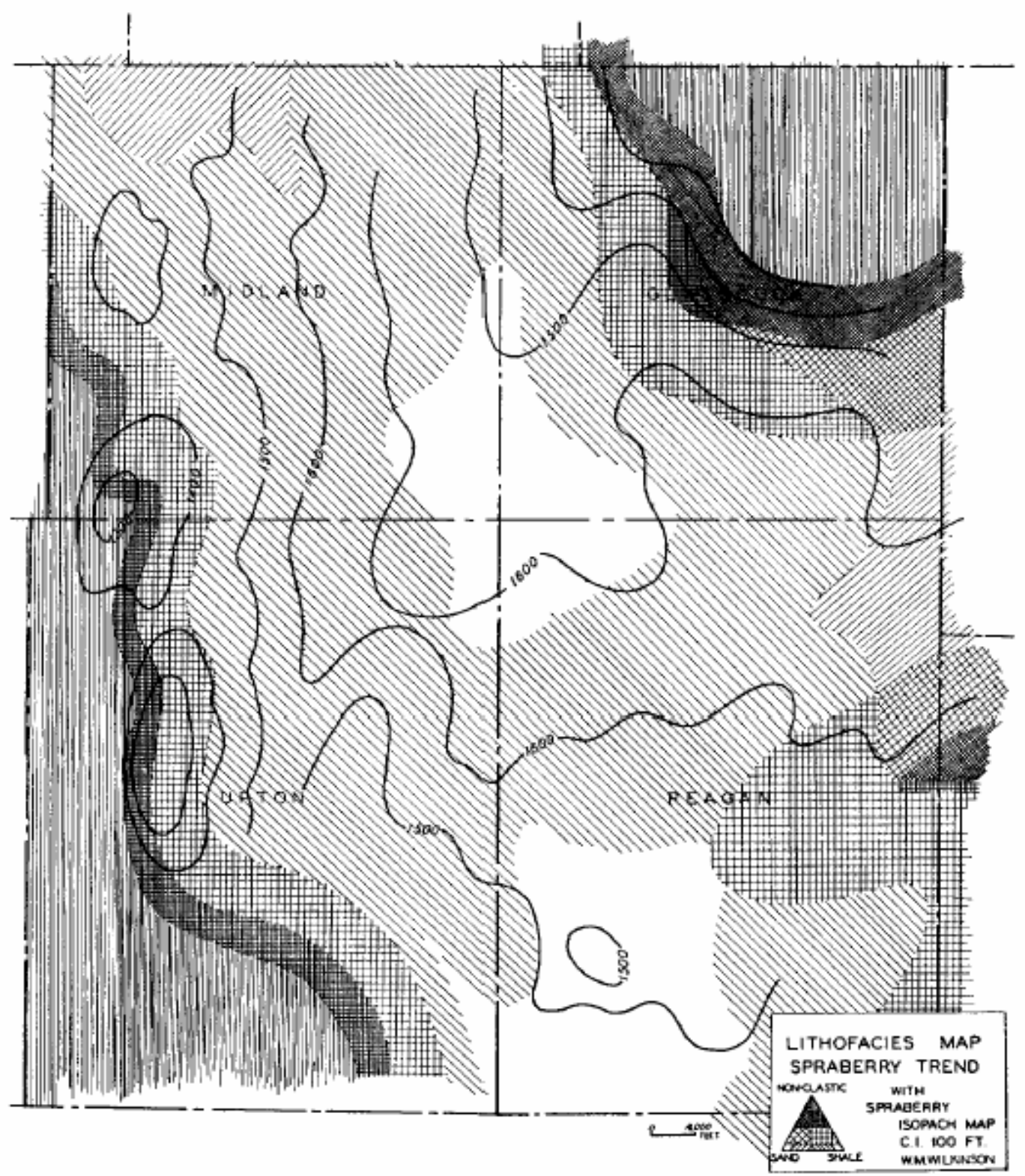

Fig. 1.3.A4 - Lithofacies map, four County area with Spraberry isopach contours ${ }^{15}$.

\section{Depositional Model}

The Spraberry formation was deposited by debris flow, turbidity currents, saline density currents and suspension settling. Some sediments were later altered by slumping, soft sediment faulting, and fluidization. Deep Basin carbonate members were largely deposited by debris flow, turbidity, saline and density currents, while deposition of terrigenous clastics was dominated by turbidity, saline and density currents, and suspension settling. Thus clastic and carbonate members shared the same deep-marine setting and most depositional processes. However, the exclusive occurrence of saline 
density-current deposits within the terrigenous clastic facies indicate that large frequently tapped reservoirs of dense saline waters were stored in shelf lagoons or salt pans near the shelf margin, but only during times of clastic deposition on the shelf.

The fundamental part of any Spraberry model (Figs. 1.3.A5 - A7) must recognize major alternating periods of carbonate and clastic deposition, incorporate the principal attributes of the carbonate and clastic patterns, and show how styles interacted to form the observed stratigraphic framework ${ }^{2}$.

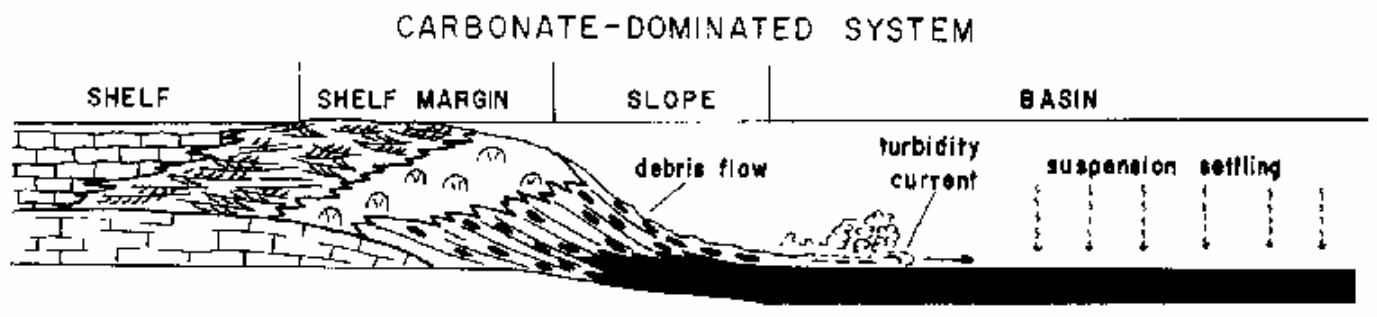

$$
\text { CLASTIC-DOMINATED SYSTEM }
$$

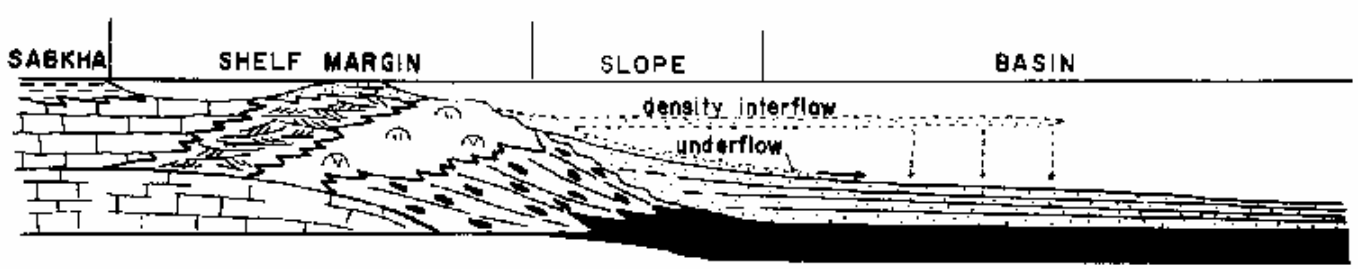

Fig. 1.3.A5 - Facies model of clastic and carbonate dominated shelf margin systems ${ }^{2}$.

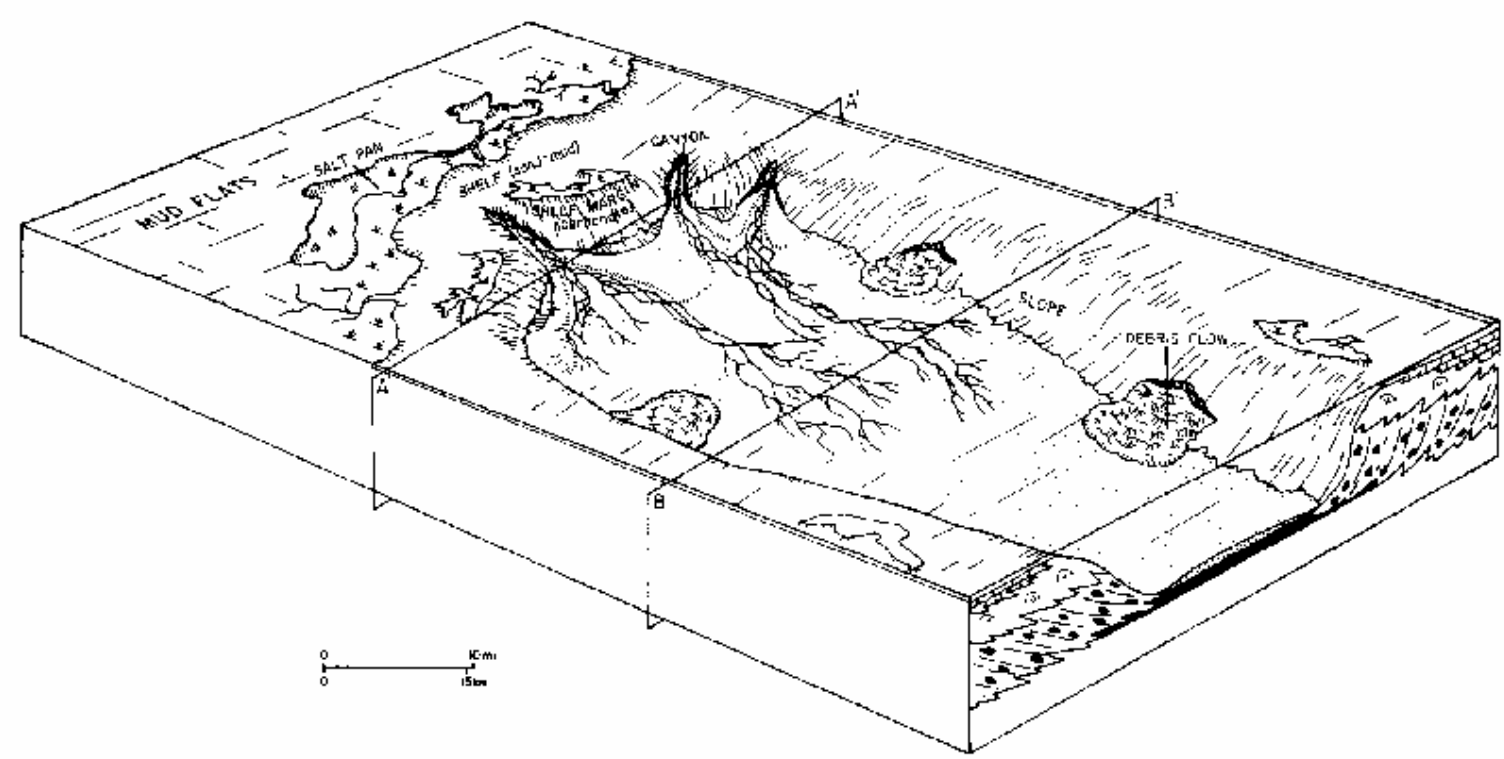

Fig. 1.3.A6 - Distribution of environments, geometry of deposits, and channel patterns ${ }^{2}$. 


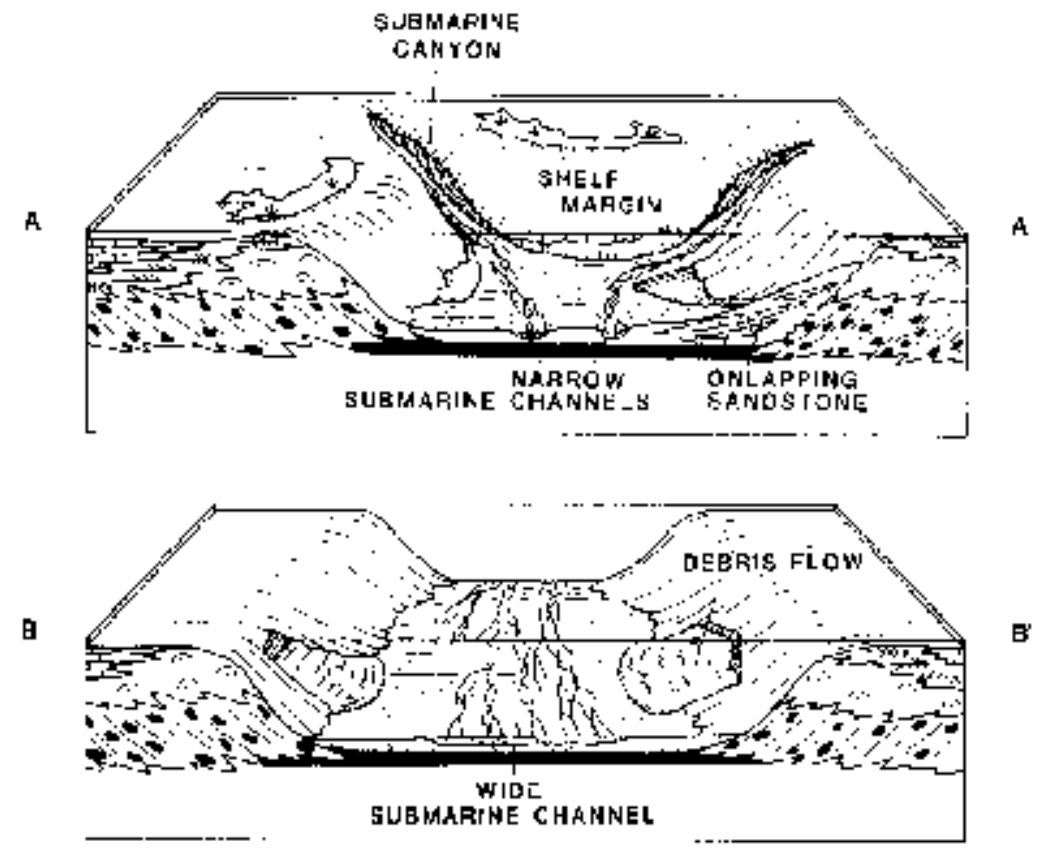

Fig. 1.3.A7 - Sections of onlapping clastics and downdip widening of channels ${ }^{2}$.

(Sections AA' and BB' from fig. 1.3.A6)

During carbonate depositional episodes (Fig. 1.3.A5) which characterized the northern shelf margin in early and late Clear Fork time, calcareous organisms flourished. A massive carbonate shelf margin $(1,700-2,000 \mathrm{ft})$ above the floor of the adjacent midland Basin, prograded Basinward over resedimented carbonate debris forming the slope. Slumps debris flow and turbidity currents were the principal mechanisms by which carbonate sediments were moved downslope into the Basin.

During Tubb and Glorieta times, large influx of silt and mud caused mud rich sabkhas and salt pan environments to prograde rapidly across the southern Palo Duro Basin towards the shelf margin of the Midland Basin, giving rise to clastic depositional episodes (Fig. 1.3.A5). Sub tidal to inter tidal sand sheets accumulated at the shelf margin, from which silt and sand were periodically carried into the Basin by saline density currents that drained hypersaline shelf lagoons and salt pans.

Deposition of terrigenous-clastics sediments formed southward thinning, elongate fan shaped wedges (Fig. 1.3.A6). Sandstone and siltstone were deposited principally from density underflow and interflow currents. Deposition from bottom hugging currents formed elongate patterns which branch and rejoin in updip regions and bifurcate in downdip regions. 


\section{Clastic Patterns}

The total sand and silt found in the upper Spraberry decreases from north to south. During upper Spraberry time, areas in the northeastern part of the Midland Basin subsided more rapidly and received a thicker sequence of sand and silt. Around the edges of the Basin a gradual decrease is noted in the total net thickness of the sand and silt in the direction of the shelf areas. The rate of decrease is more rapid in the northern part of the Basin where there is thicker development of the coarse clastics (Fig. 1.3.A8). Within the four County area (Midland, Glasscock, Upton and Reagan), the thickness is nearly constant. 


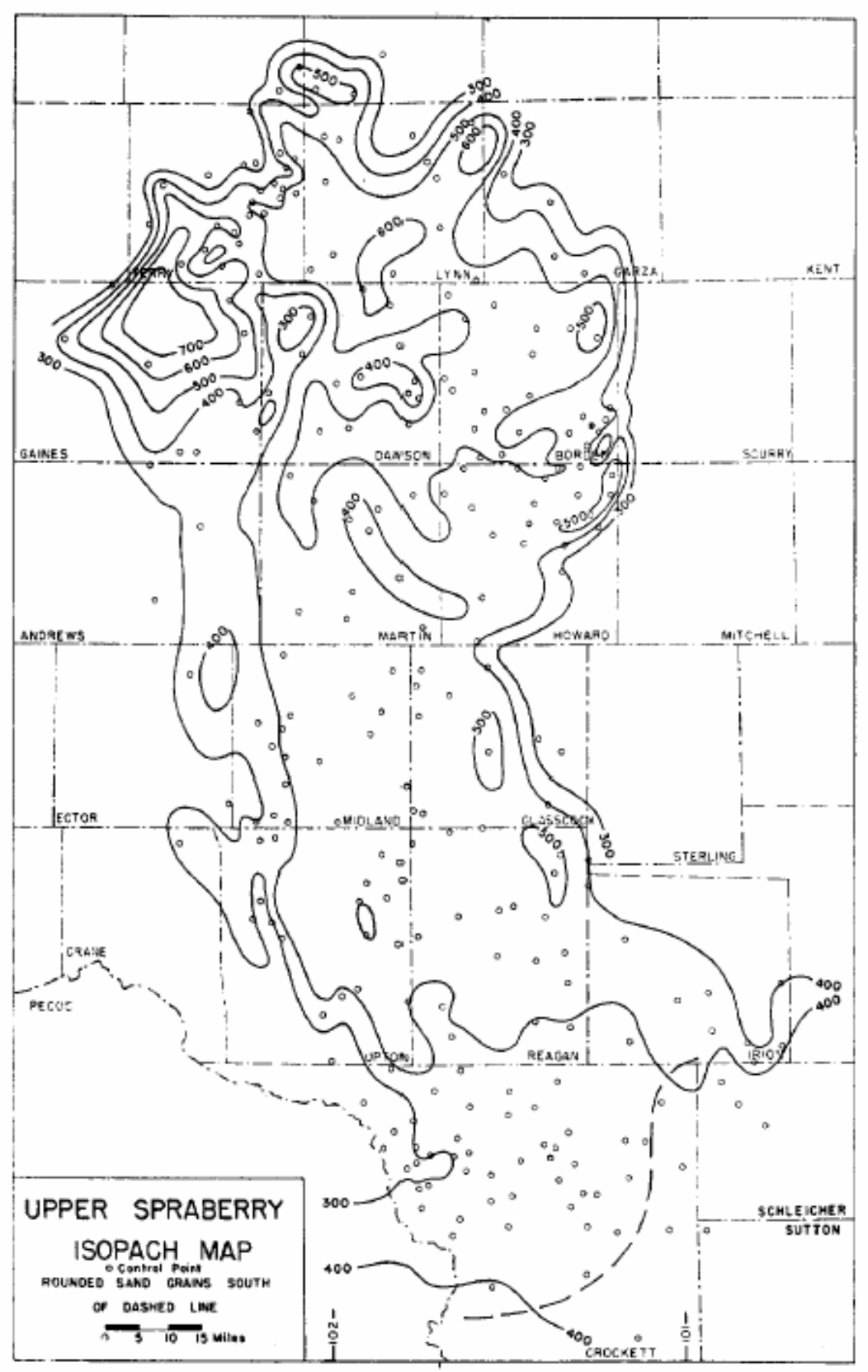

Fig. 1.3.A8 - Isopach map of upper Spraberry unit in Midland County ${ }^{16}$.

Clastic ratio - Determined by the total thickness of the clastics (sand, shale, silt) at any control point divided by the total thickness of non-clastics (limestone, dolomite). Data is based on sample and electric log study (Fig. 1.3.A9). Over a wide area in the central part of the Basin, the clastic ratio is greater than 8 (more than $88 \%$ of the section is composed of clastics). The width of this zone decreases north and south. Another slight decrease in the clastic ratio occurs around the edge of the more negative area developed in northeastern Gaines County. Where the section is thinner there may have been a better 
development of carbonates. Possibly carbonates were deposited as rapidly around the edges as in the central part, but the central part subsided more rapidly and received a thicker sequence of clastics. Along the northeastern edge the decrease in clastics was, in part, due to the thick Pennsylvanian and Wolfcampian reefs ${ }^{16}$.

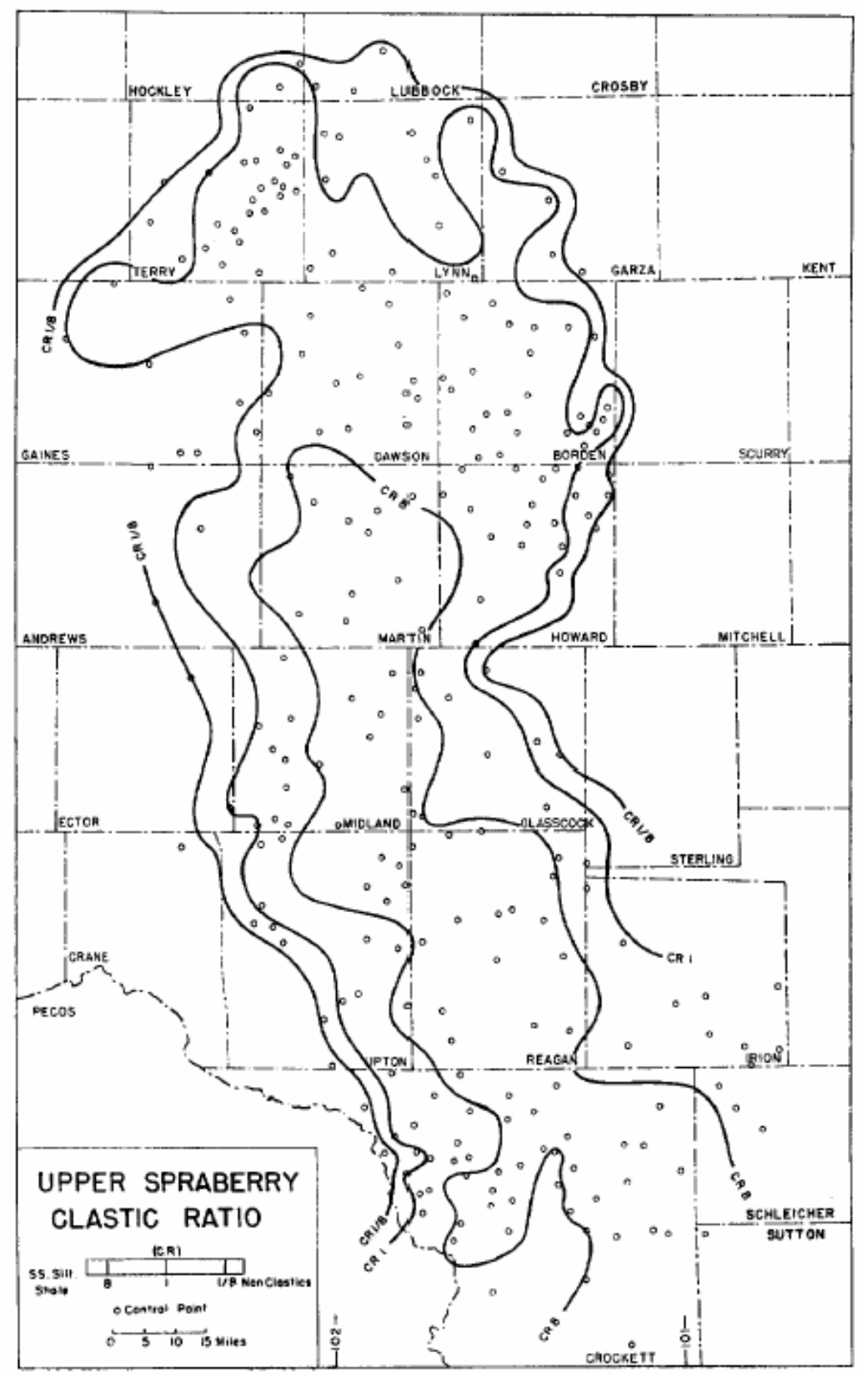

Fig. 1.3.A9 - Clastic ratio map of upper Spraberry unit in Midland County ${ }^{16}$.

\section{Flow Units}

The Spraberry is divided into 3 main intervals (Upper, Middle and Lower), Tyler \& Gholston further sub-divided the Spraberry into distinct episodes or operational units. Of 
the six units found in the upper Spraberry, only two (1U and $5 \mathrm{U}$ ) are reservoir quality rock, while in the lower Spraberry only two operational units are identified. Little is known about the lower Spraberry formation, as focus is mostly on the upper Spraberry.

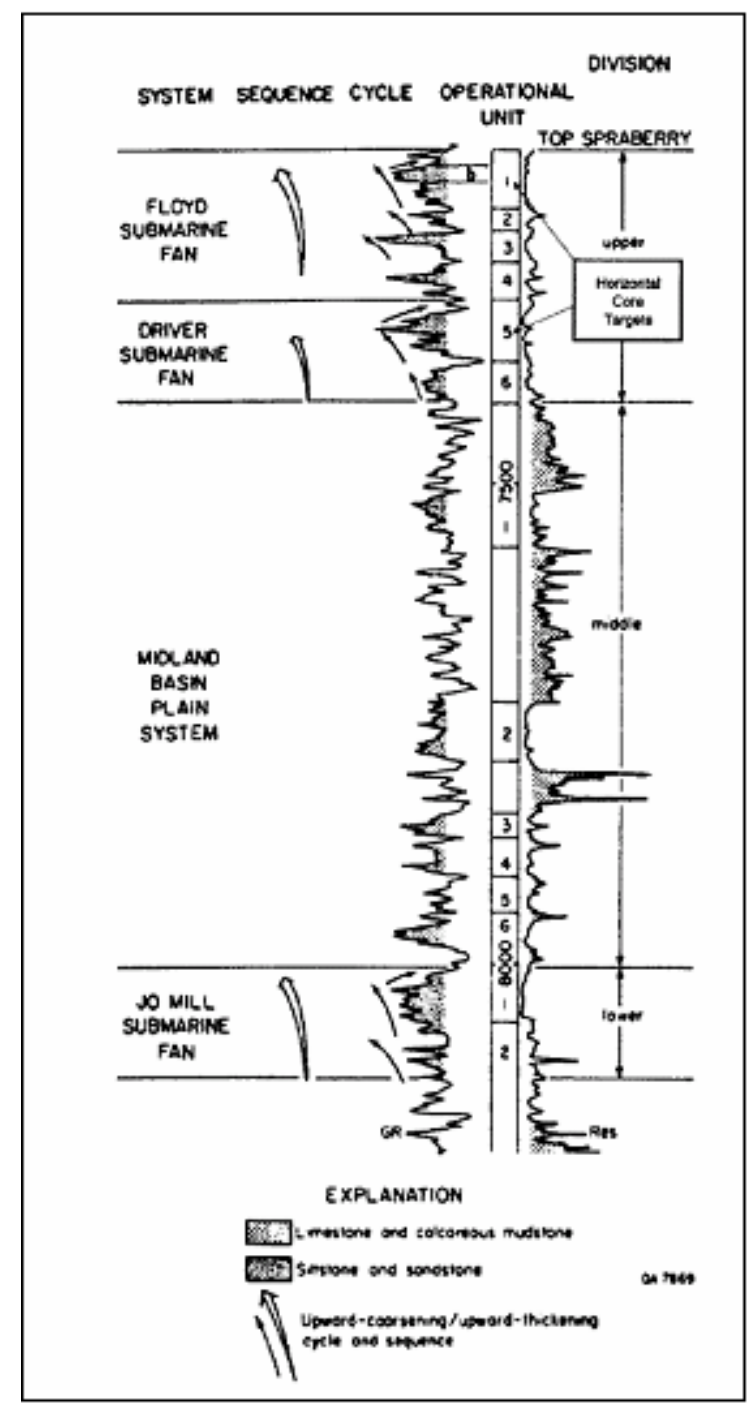

Fig. 1.3.A10 - Operational units within the upper Spraberry formation.

(TXL Fee 'B' \#1 well located in the central trend area).

In the siltstones the average intergranular siltstone porosity is less than ten percent ${ }^{38}$, and the permeability varies from 0.002 to 2.5 millidarcys ${ }^{39}$.

Fractures constitute the major void space in the reservoir rock. Core evidence shows that vertical fractures are well developed in the intervening shales and argillaceous limestones ${ }^{4}$ and apparently less pronounced in the massive siltstone members.

The fracture type reservoirs of the Spraberry can be divided into two classes: a) fields associated with local pre- Leonard structures; b) fields not associated with other than a broad west-dipping monocline. Class ' $b$ ' belongs to an almost continuous belt of 
production from the Pembrook field of Upton County north through the Germania field of Midland County (Fig. 1.3.A11).

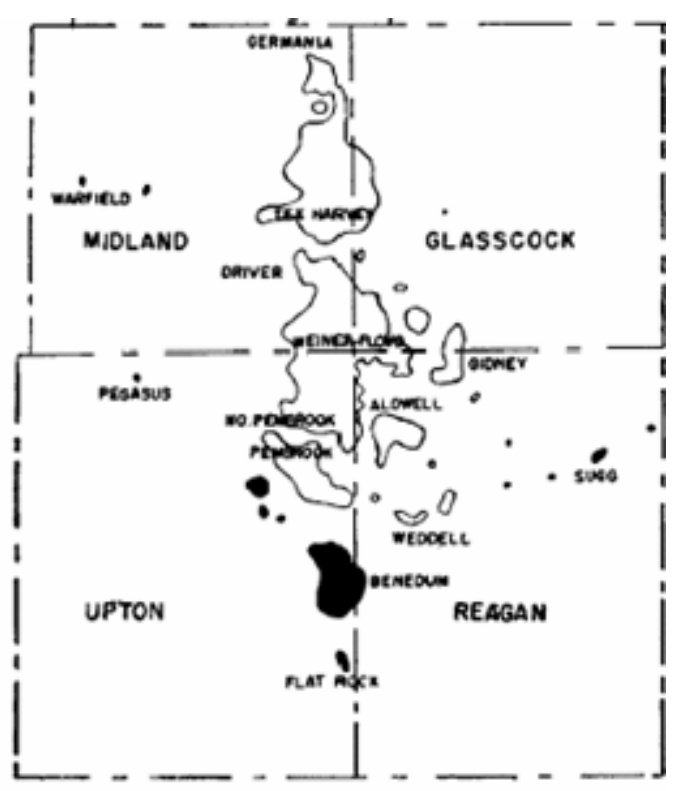

Fig. 1.3.A11 - Location of class A \& B Spraberry reservoirs of west Texas.

(class 'A' reservoirs are shown bold, and class 'B' reservoirs open). 


\section{APPENDIX B}

MAPS FOR ET O'DANIEL AND GERMANIA

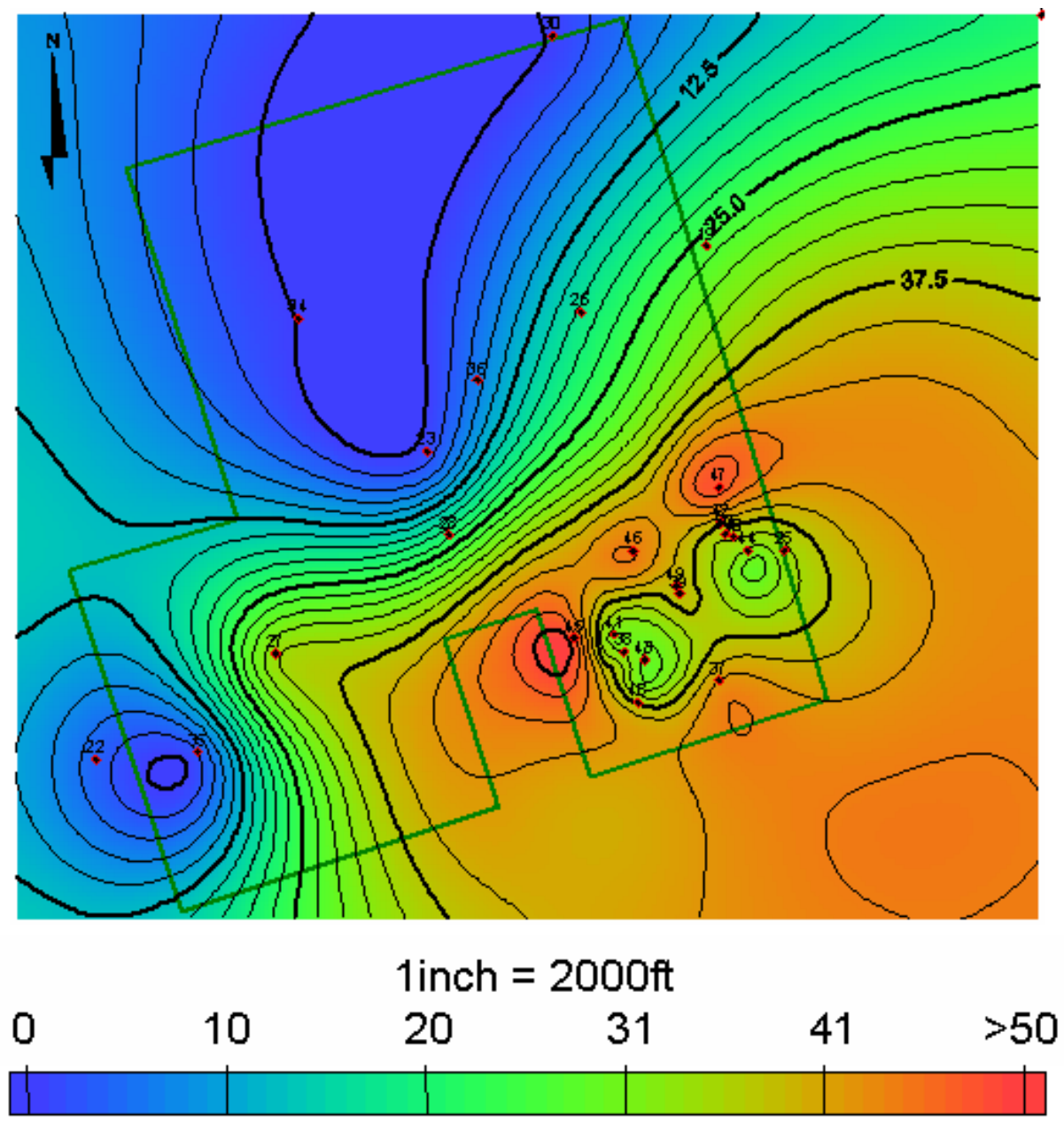

Fig. 1.3.B1 - Minimum gamma ray map of ET O’Daniel, 5U sand. 


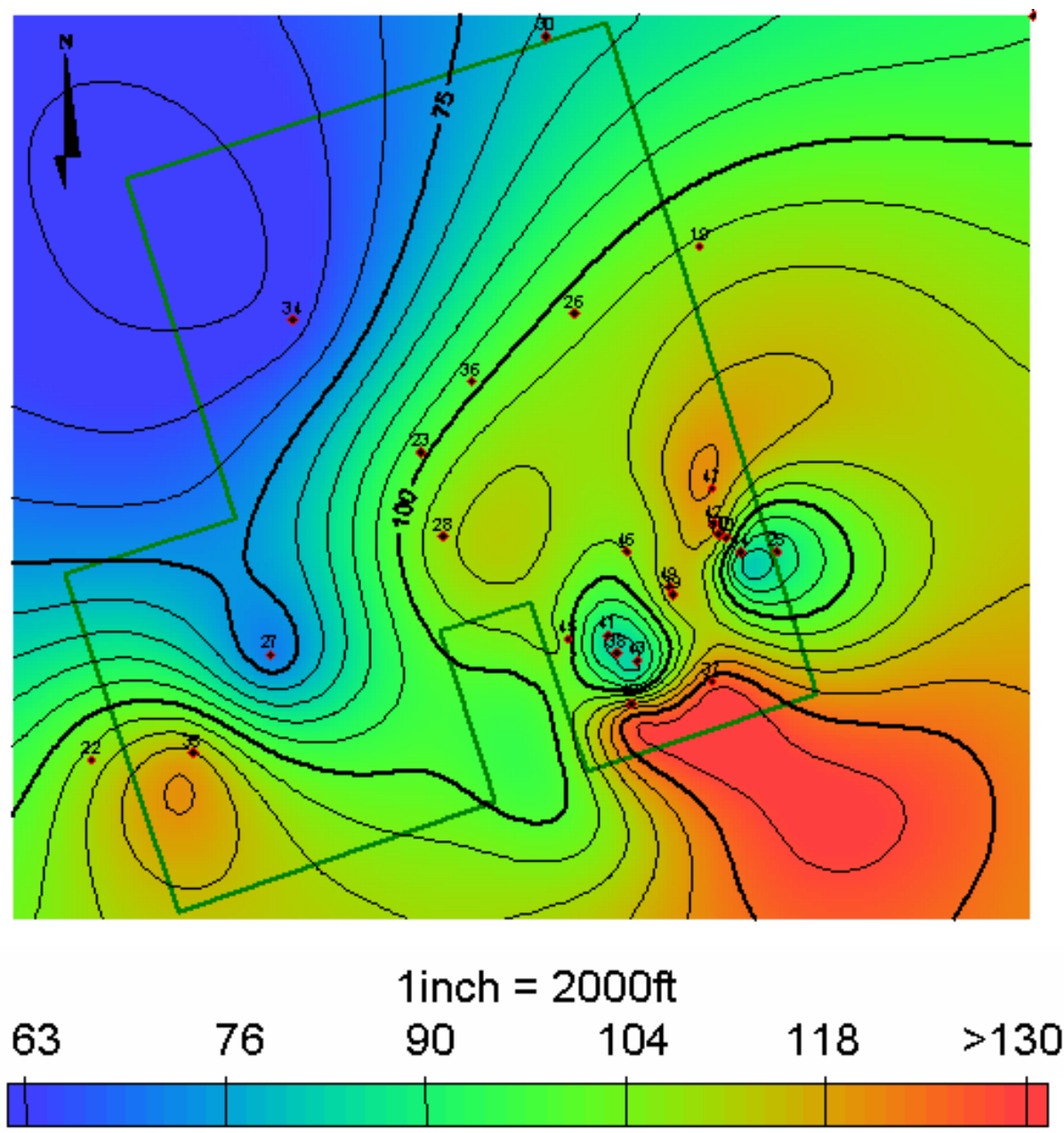

Fig. 1.3.B2 - Maximum gamma ray map of ET O’Daniel, 5U sand. 


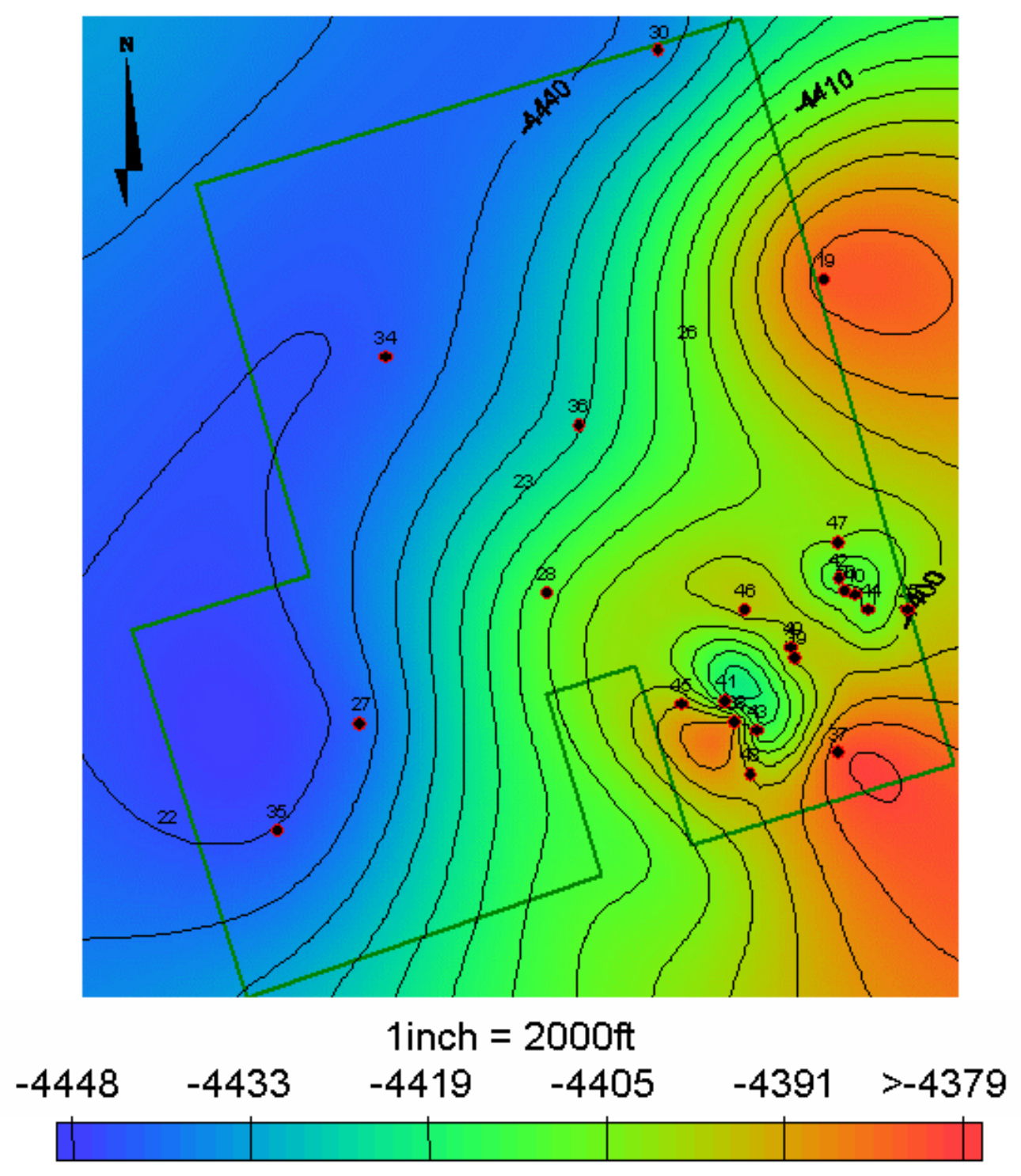

Fig. 1.3.B3 - Paleo-structure map of ET O’Daniel, 1U sand. 

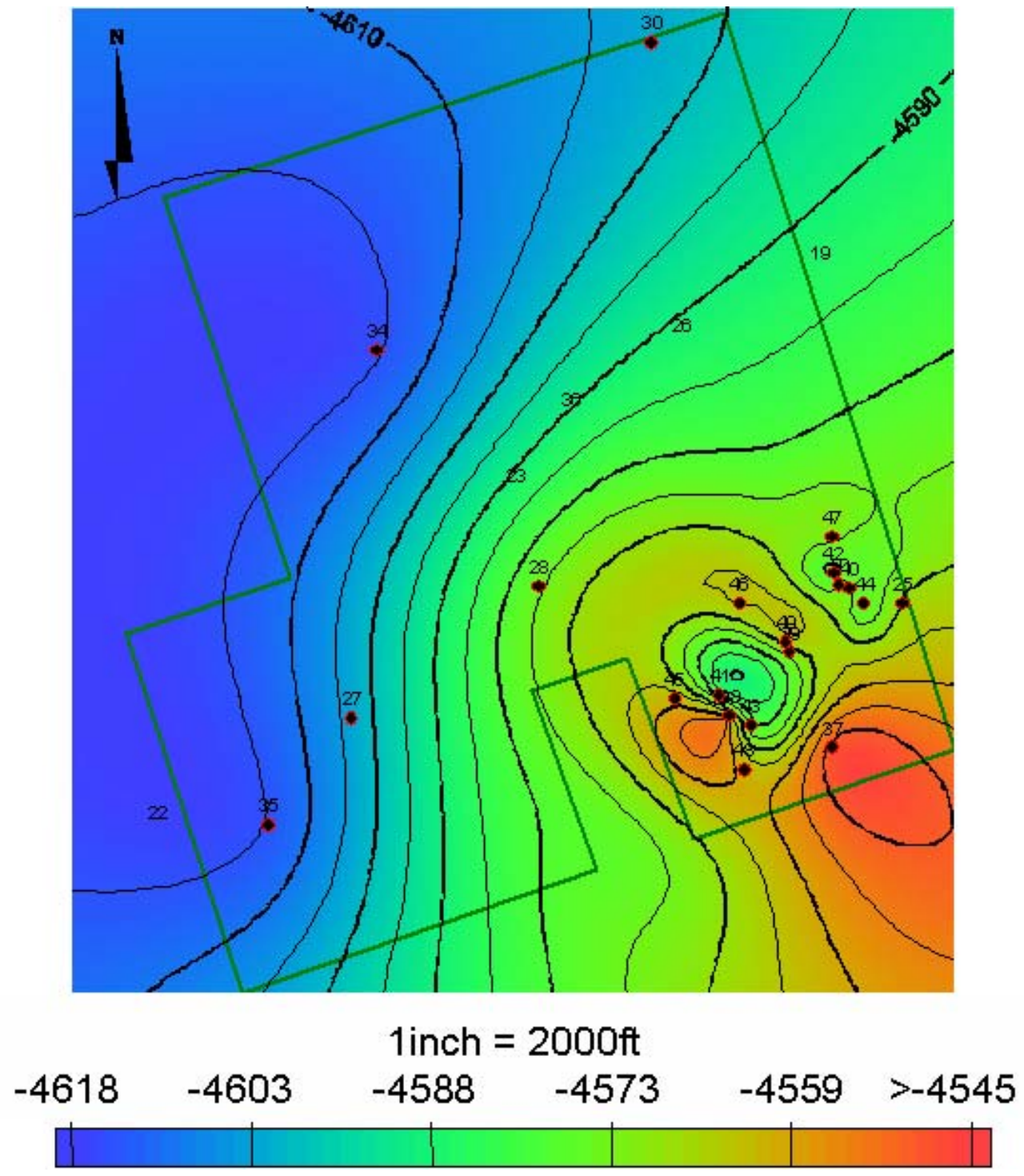

Fig. 1.3.B4 - Paleo-structure map of ET O’Daniel, 5U sand. 

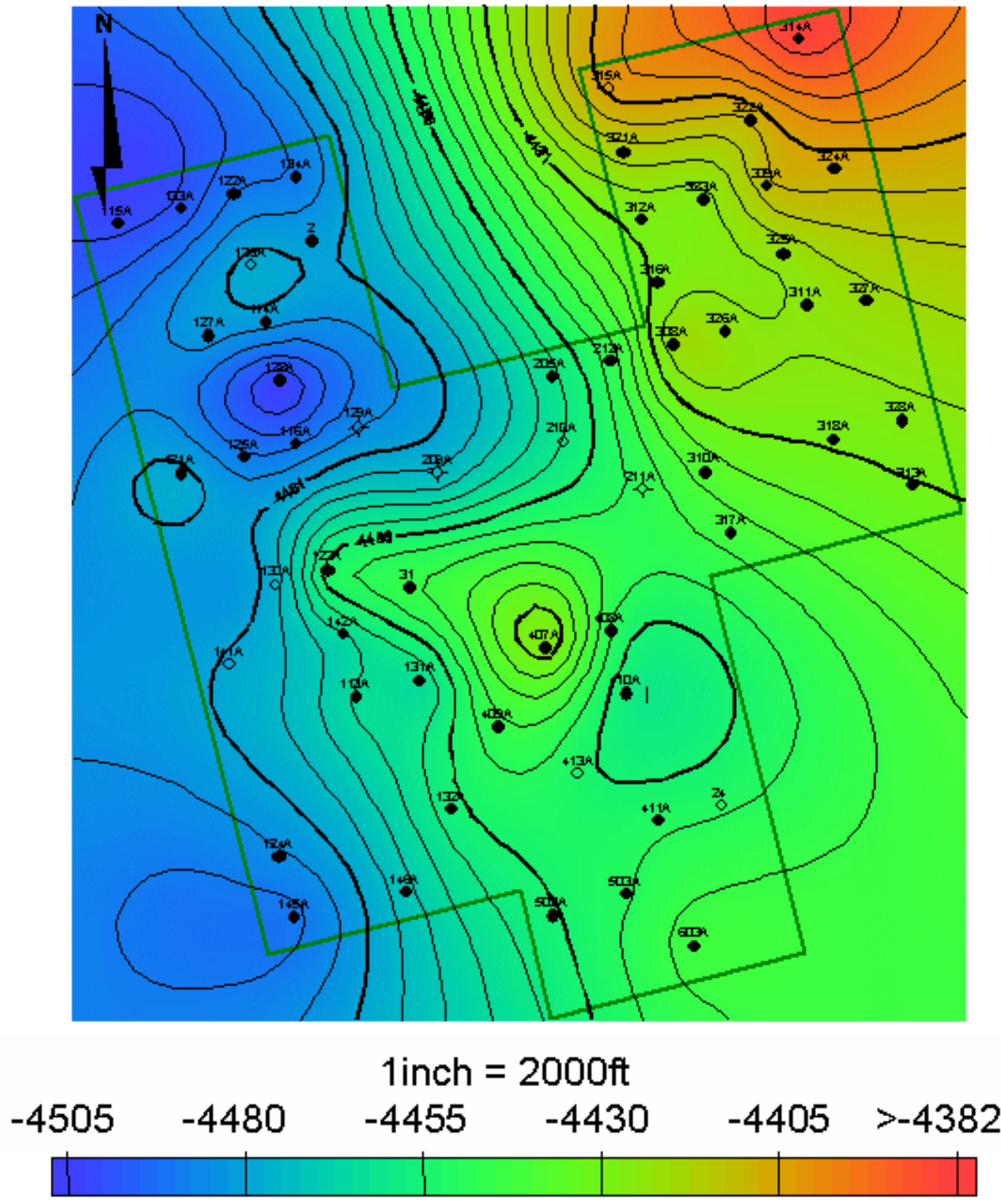

Fig. 1.3.B5 - Paleo-structure map of Germania, 1U sand. 


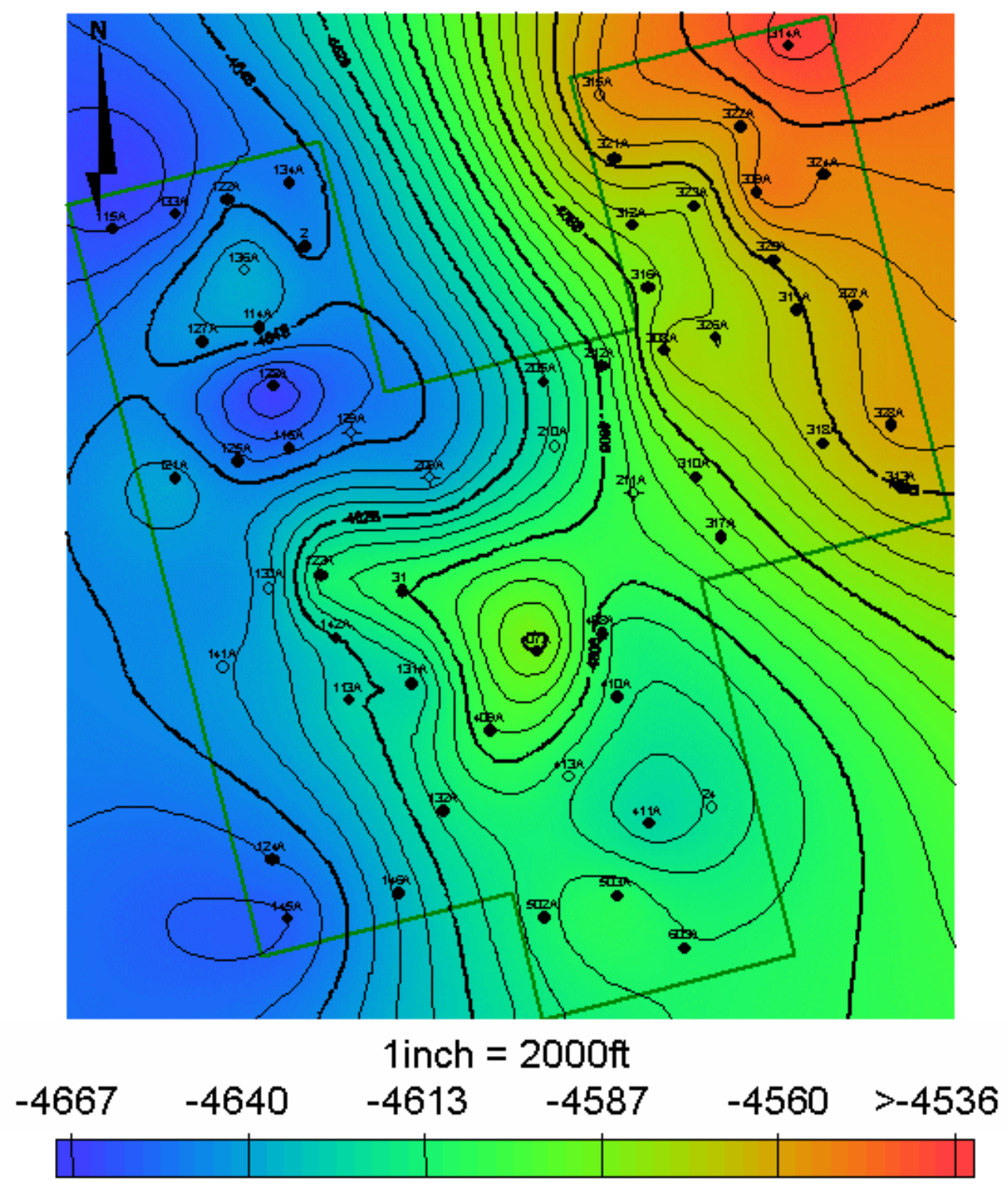

Fig. 1.3.B6 - Paleo-structure map of Germania, 5U sand. 


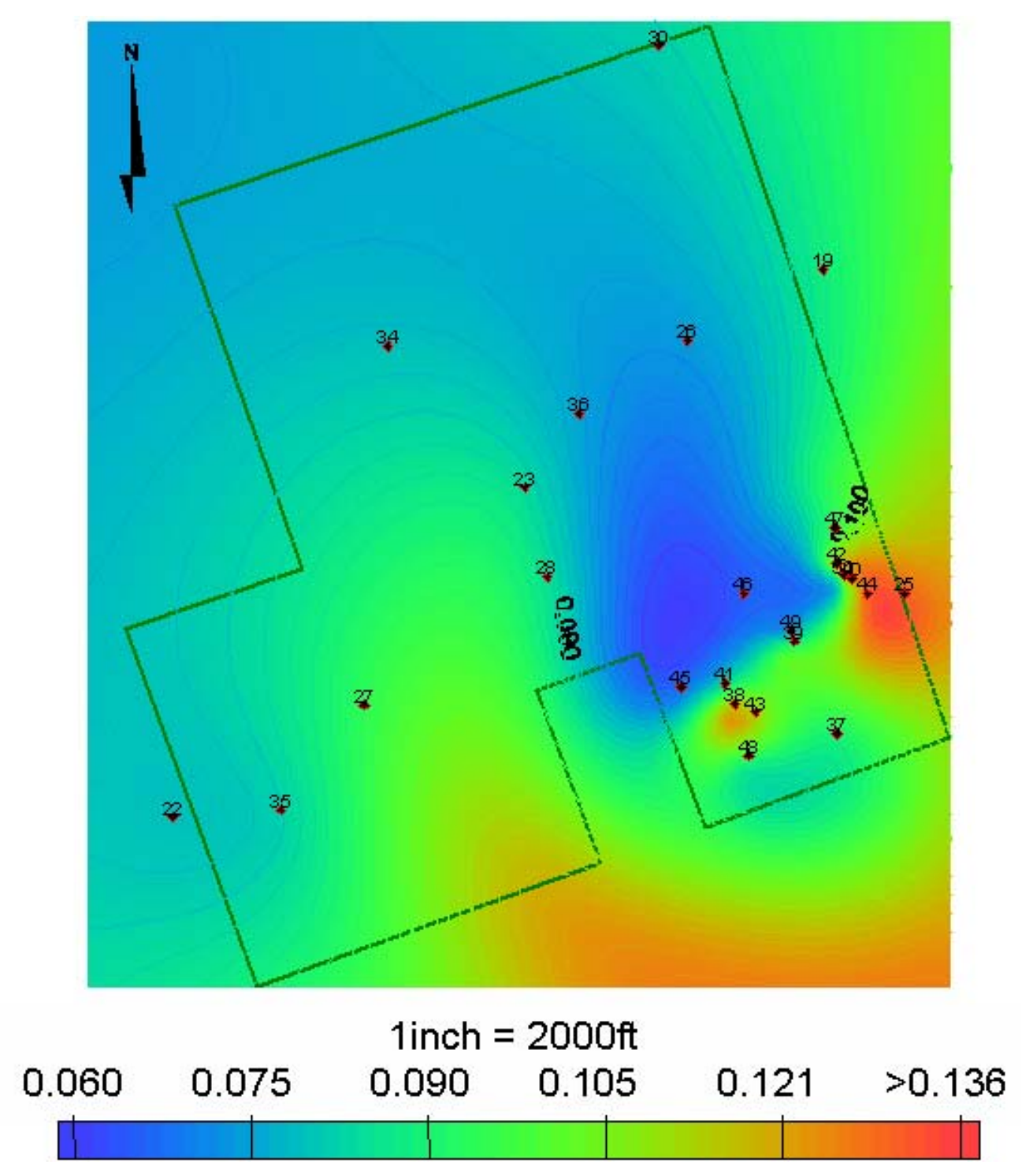

Fig. 1.3.B7 - Porosity distribution map of ET O’Daniel, 1U sand. 


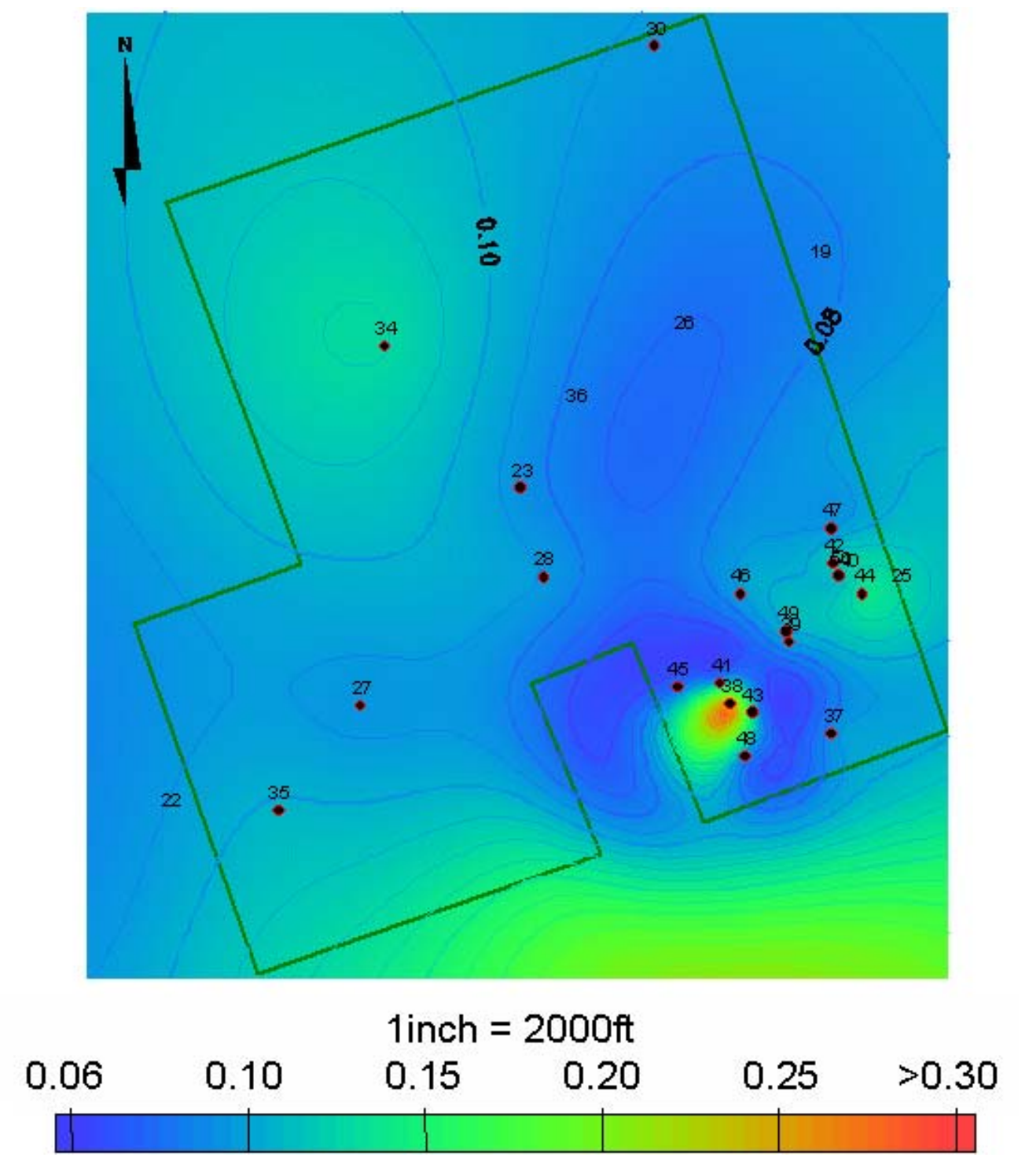

Fig. 1.3.B8 - Porosity distribution map of ET O’Daniel, 5U sand. 

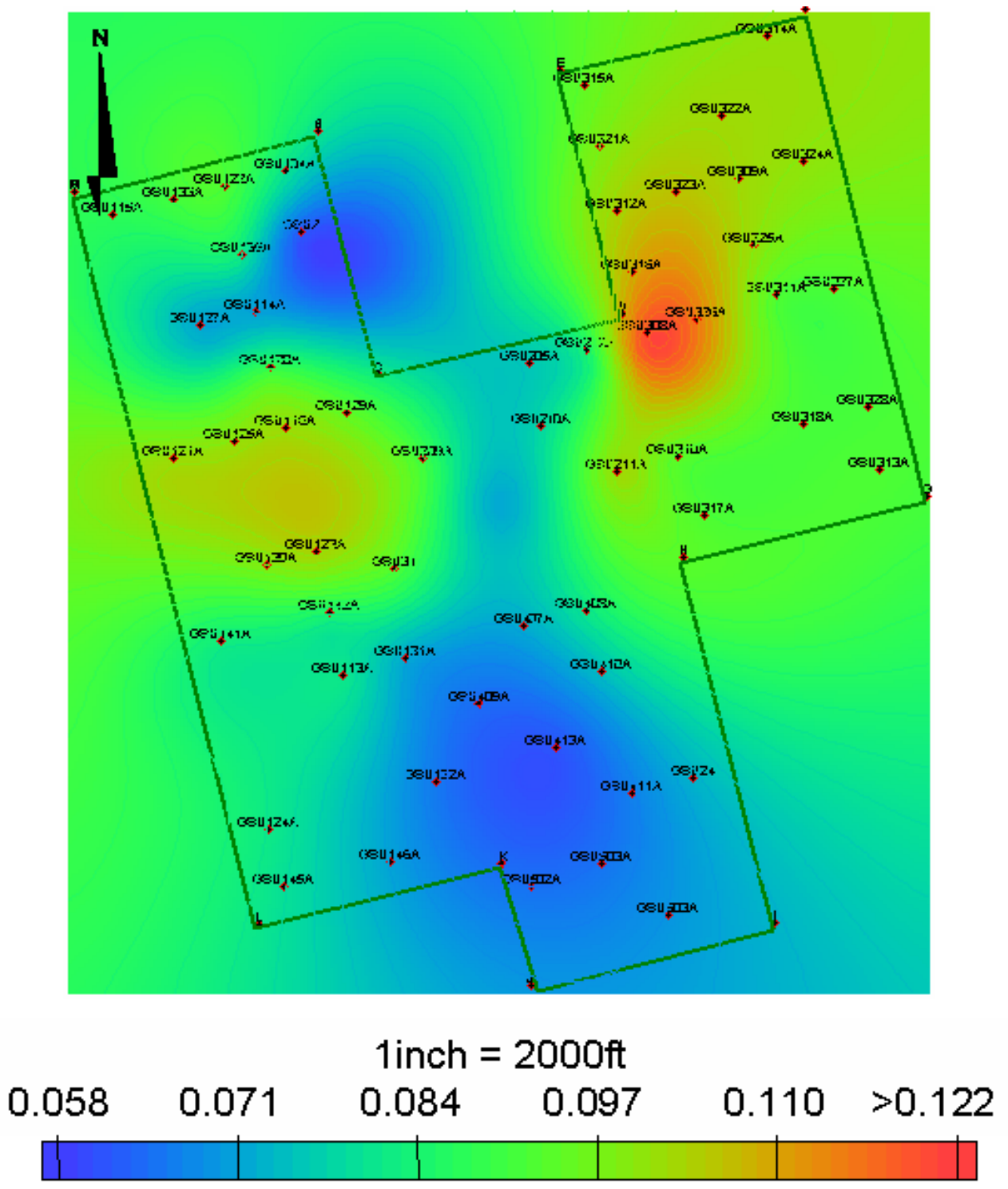

Fig. 1.3.B9 - Porosity distribution map of Germania, 1U sand. 

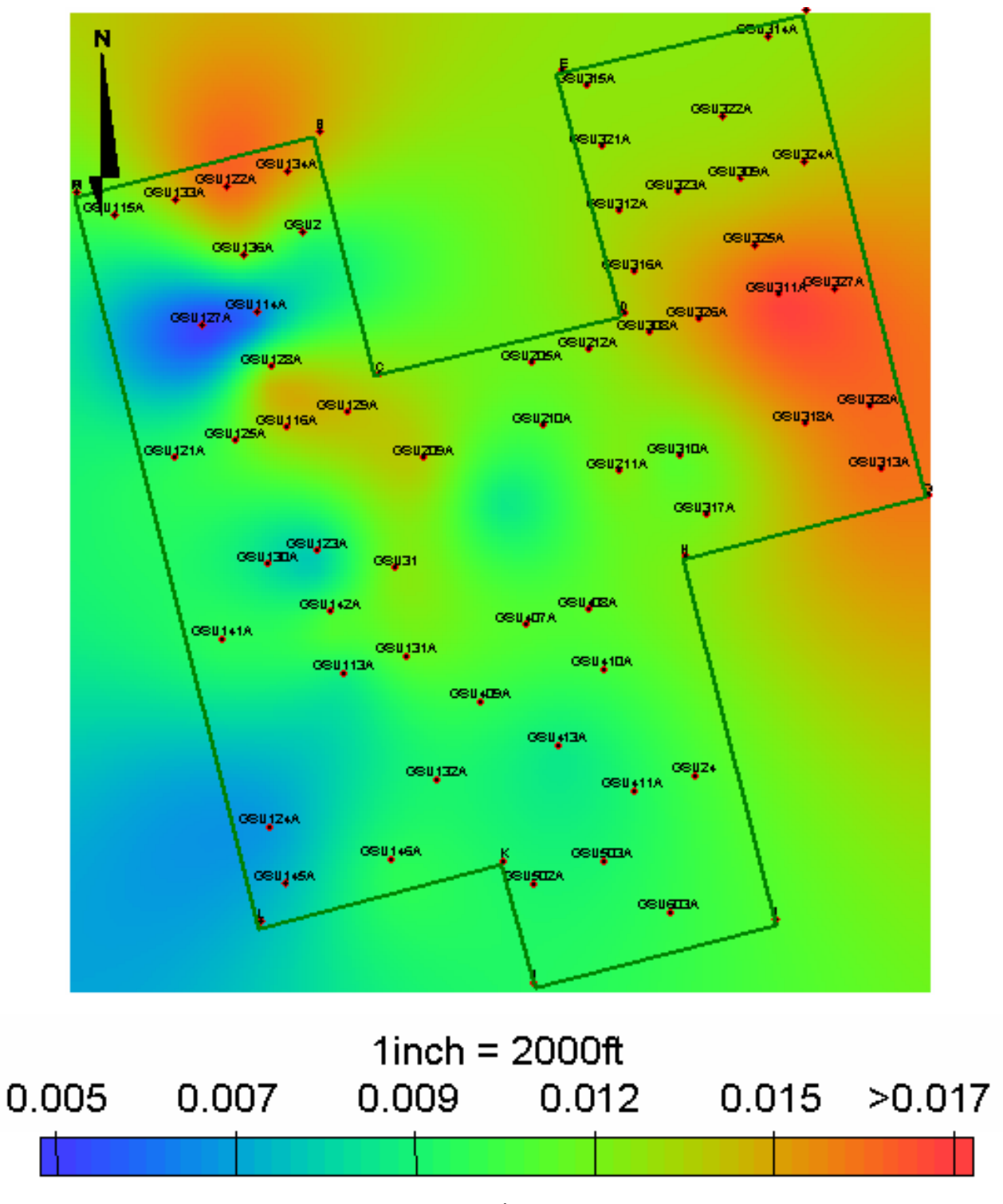

Fig. 1.3.B10 - Porosity distribution map of Germania, 5U sand. 
Theoretical Normal Distributions with varying sample sizes

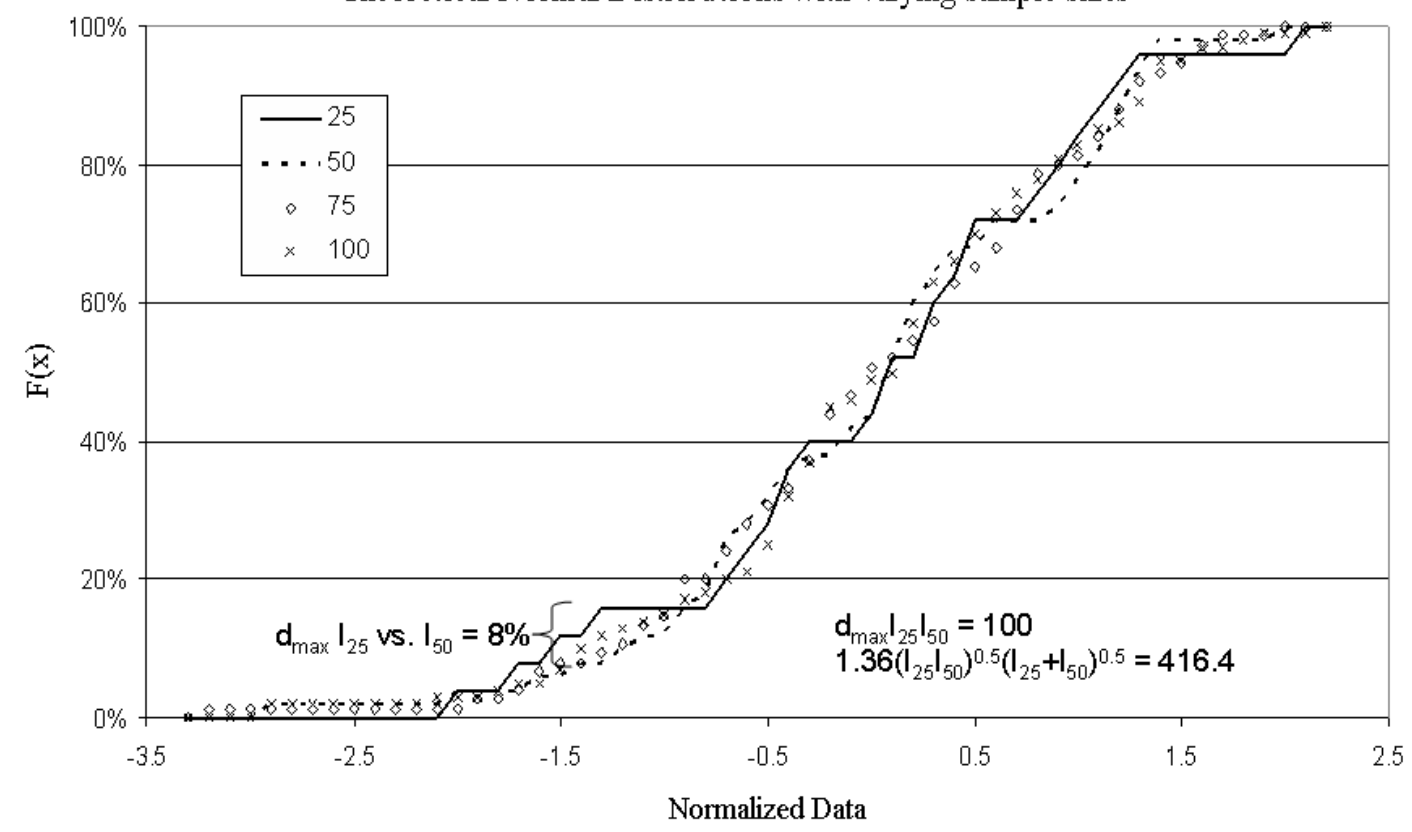

Fig. 1.3.B11 - Effect of sample sizes on Kolmogorov -Smirnov distribution test. 


\section{I.4 Evaluation of Waterflooding Performance in Germania Spraberry Unit}

\section{Introduction}

The Germania Unit is located in Midland County, 12 miles east of Midland, Texas (Fig.1.4.1) and covers and area of approximately 4900 acres. It is part of the Spraberry Formation in the Midland Basin which is one of the largest known oil reservoirs in the world bearing between 8.9 billion barrels (Handford, 1981) and 10.5 billion barrels (Guevara, 1988) of oil originally in place (OOIP). Of this, 740 million barrels have been produced since its discovery in 1949. The Spraberry formation has been affected by postdepositional tectonic activity creating a network of secondary porosity. The field is considered geologically complex since it comprises typically low porosity, low permeability fine sandstones and siltstones that are interbedded with shaly non-reservoir rocks and natural fractures existing over a regional area that have long been known to dominate all aspects of performance in the Spraberry Trend Area.

Germania Unit has been waterflooded using the conventional techniques applied in naturally fractured reservoirs in the Spraberry area, where all injection wells were aligned parallel along the major fracture trend to force the oil to flow towards a line of production wells. Many wells have been abandoned in The Germania Unit as a result of either casing failures or low productivity. In this area, conventional waterflooding techniques have often led to economic failures in the attempt to recover additional oil, because the injected water tends to channel through the high permeability fracture system leaving the rock matrix, where the additional oil resides , virtually unaffected by the waterflood process, and thus understanding the mechanics and interaction between the fracture system, matrix, wells and the past performance of the waterflooding may lead to more effective oil production and therefore to a significant increasing in the recovery factor.

Germania Unit was discovered in 1957. During the first 8 years under primary recovery, the reservoir was poorly developed due to low well productivity and well spacing. During this primary stage, the unit produced under solution gas drive. The total cumulative oil production corresponding to this period was 0.55 million barrels of oil at an average oil rate of 188 BOPD. In 1965 a waterflooding program was initiated and continued until 1990. The purpose of this waterflooding program was to improve the recovery by sweeping the oil from the injectors located in the middle part of the structure, towards the producers located throughout the reservoir. The water was injected through 5 wells located in different positions of the reservoir. The cumulative water injected under this period was 2.44 million barrels and the cumulative water production was 0.95 million barrels. In May 1990 the water injection was suspended when the average water cut in the producer wells increased up to 0.75, two infill drilling campaign took place increasing the numbers of producer wells from 20 to 98 in a period of 10 years and increasing the number of active wells up to 66 .Oil production reached its maximum peak at 956 BOPD in 1992. The reservoir continued producing under this 
condition (water injection equal to zero) from 1990 to 2002. The cumulative oil production and injection as of June 2003 were 2.24 million barrels and 2.44 million barrels respectively. In February 2003 the operator began a new water injection project (under a new injection pattern) from six injector wells by converting three wells to water injectors, returning two wells to water injectors and drilling a new injector well (Fig.1.4.2). Each one of the six injector wells is injecting 270 BWPD. Since this program was initiated, some producers have shown favorable response to the injection (they have increased the oil rate respect to the rate they had before the new injection process took place). Currently the production level is 470 BOPD through 64 active wells and the cumulative oil production is 2.242 million barrels.

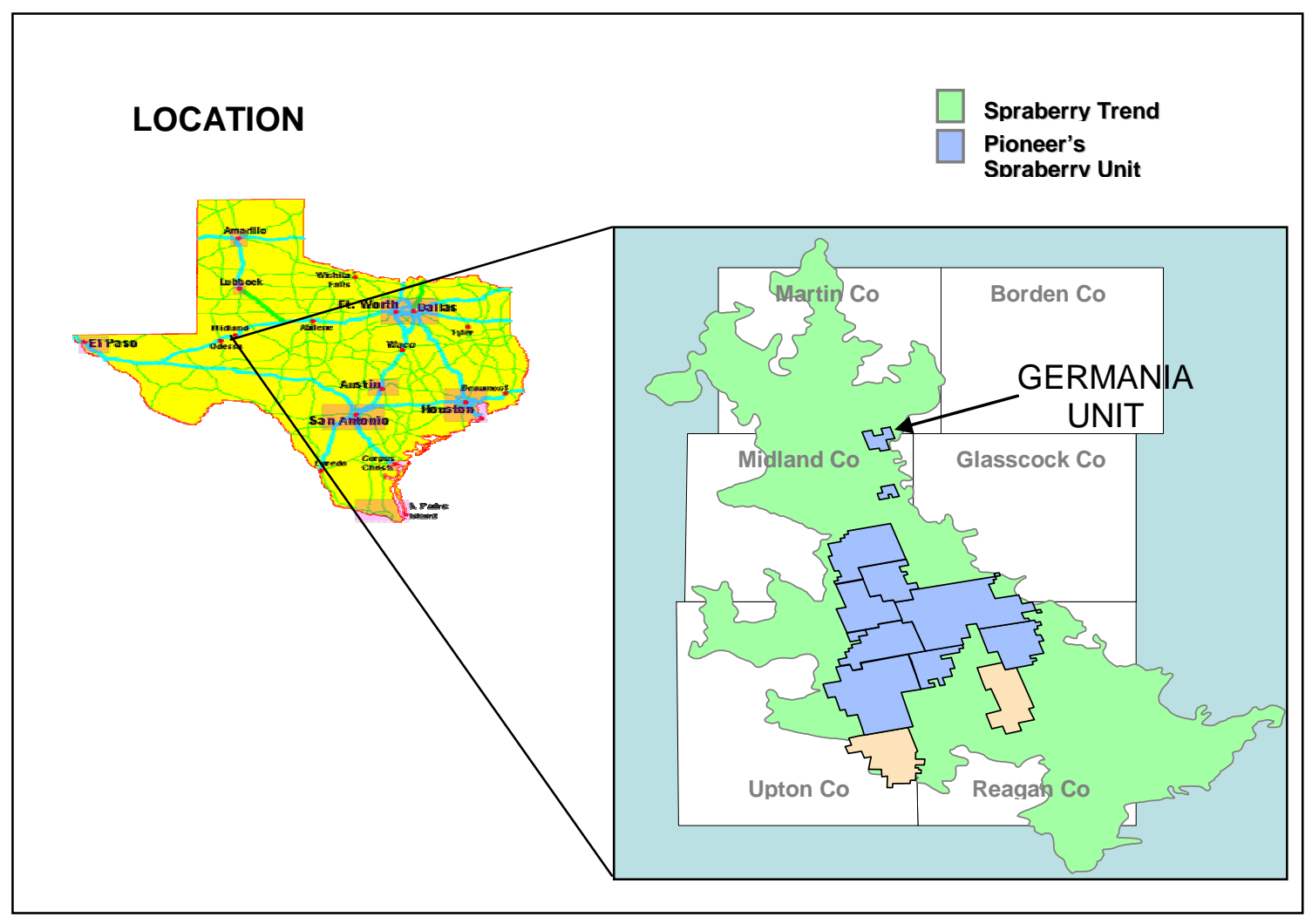

Fig.1.4. 1- Location of Germania Spraberry Unit. 


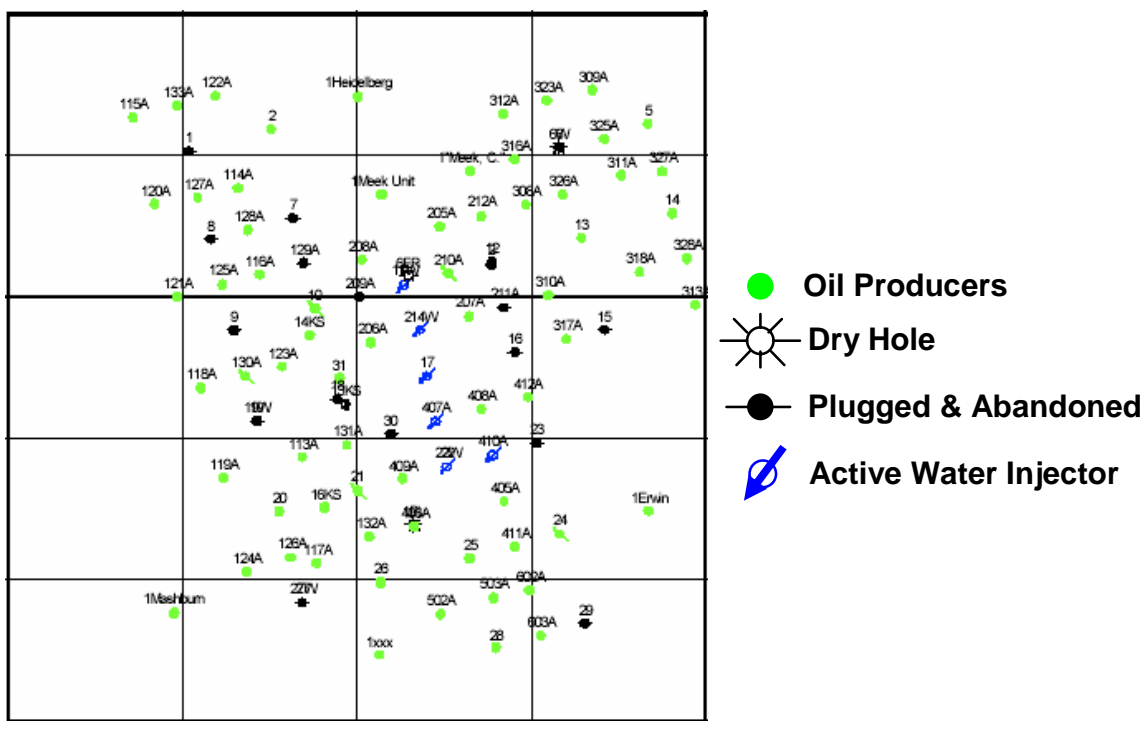

Fig.1.4. 2- Location of New Water Injectors Wells in Germania Spraberry Unit.

\subsection{Description of the Problem}

The Germania unit as well as other units in the Spraberry Area has been waterflooded using the conventional waterflood techniques applied in natural fractured reservoirs, where all injection wells are aligned parallel along major fracture trend to force the oil to flow perpendicular to the fracture trend towards a line of producer wells.

In the past, several studies have been conducted to propose different waterflooding techniques and development plans for Germania Unit; however none of the previous studies, have addressed the reservoir characterization and monitoring of the waterflooding project and propose alternatives of development taking into account the current and future conditions of the reservoir.

Consequently, this project will be addressed to provide a significant reservoir characterization and evaluate the performance of the waterflooding to provide facts, information and knowledge to obtain the maximum economic recovery from this reservoir. Thus, attempts are made to describe the reservoir, understand the performance of the reservoir under the current waterflooding project, and controlled surveillance will be carried out to improve field performance.

\subsection{Objectives of the Research}

The main objectives of this study are:

1. Integrate the production and injection data to characterize the reservoir. During the primary and secondary performance, wells indicating high cumulative production may indicate high permeability zone and porosity. On the other hand, wells with relative low cumulative production may indicate very low permeability and porosity or poor mechanical condition, skin damage , or isolated pay intervals. 
2. Evaluate development opportunities with emphasis toward preventing trapped oil, and maximizing recovery. These development opportunities may comprise perforating additional intervals in some wells.

3. Identify bypassed oil and flood front to locate infill wells and look for further development opportunities by selecting areas with high oil saturation remaining and showing in pictorial displays the location of various flood fronts showing visual differentiation between areas of the reservoir that have and have not been swept by the water.

4. Provide possible fracture orientation and its effect on the production based on past performance of the waterflood. The analysis of the on-trend and off-trend well production will help to support the theory of northeast-southwest trend. The on-trend and off-trend wells will be chosen based on their location with regards to the injectors.

5. Identify problems in some wells by using the concept of water-oil ratio and its derivative to differentiate whether the wells are experiencing coning problems, layer breakthrough or near wellbore channeling.

6. Estimate the remaining reserves associated to the drainage radius of every well by performing decline curve analysis of individual wells completed in the reservoir. Present the results in pictorial displays showing the areas of the reservoir with the most remaining reserves. In this stage different scenarios will be analyzed to forecast the reserves and make extrapolations in the future to evaluate the benefits of waterflooding in Germania Unit area and predict the future performance of the field under different producing and injection schemes.

7. Analyze the historical relationship between reservoir withdrawals and the water injection rate in different areas of the unit to optimize the performance of the waterflood.

\subsection{Research Methodology}

The following methodology was used to achieve the objectives of the project:

1. The data needed was collected, reviewed, and validated and data base constructed using the software Oil Field Manager (OFM), which is a powerful surveillance software application that provides an array of modules and tools for managing and analyzing static and dynamic data. Since the data was obtained from different related sources, it was reviewed, re-organized, and finally reduced to a format manageable in OFM. The data collected comprises: production and injection, coordinates, dates and events, wellbore, limits of leasing, logs, PVT analyses, etc. The calculations and processes were done using the main modules of the program (Decline Curve Analysis, System Functions, Calculated Variables, Plots, Reports, Bubble Maps, Grid Maps and Scatter Plots) and the interrelation among them, was also considered.

2. The study was approached by considering the overall performance of the Germania Unit as well as the performance and experiences obtained in others areas of Spraberry Unit. Under a full field scope surveillance system, the different modules of OFM were used and statistical analyses for different wells were also considered. 
3. The final step in this waterflooding surveillance and reservoir characterization study was reporting the results achieved, derived conclusions, as wells as recommendation for future field operation and developing plan.

\section{Reservoir Performance}

\subsection{Primary Performance in Germania Spraberry Unit}

The Germania Spraberry Unit is located in Midland county, 12 miles east of Midland, Texas and began its primary production in 1957. After the discovery, the unit was developed in a 160 acre-spacing and by the end of this stage (primary performance) in 1965 a total of 11 wells were drilled and some of them temporarily abandoned or shut-in due to different reasons ( low productivity, high water cut, and casing failures). The total cumulative oil production corresponding to this period was 0.55 million barrels of oil at an average oil rate of 188 BOPD and the production reached a maximum peak of 480 BOPD in 1961 and the water cut by the end of the stage averaged 20 percent (Fig. 1.4.3). The production of liquid per well averaged 37 BLPD and the average production of oil per day per well was 37 BOPD (Fig.1.4.4). The oil produced during this stage ( 0.55 million barrels) represents only 1.7 percent of the total produced by the unit (Germania Spraberry) as of June 2002.

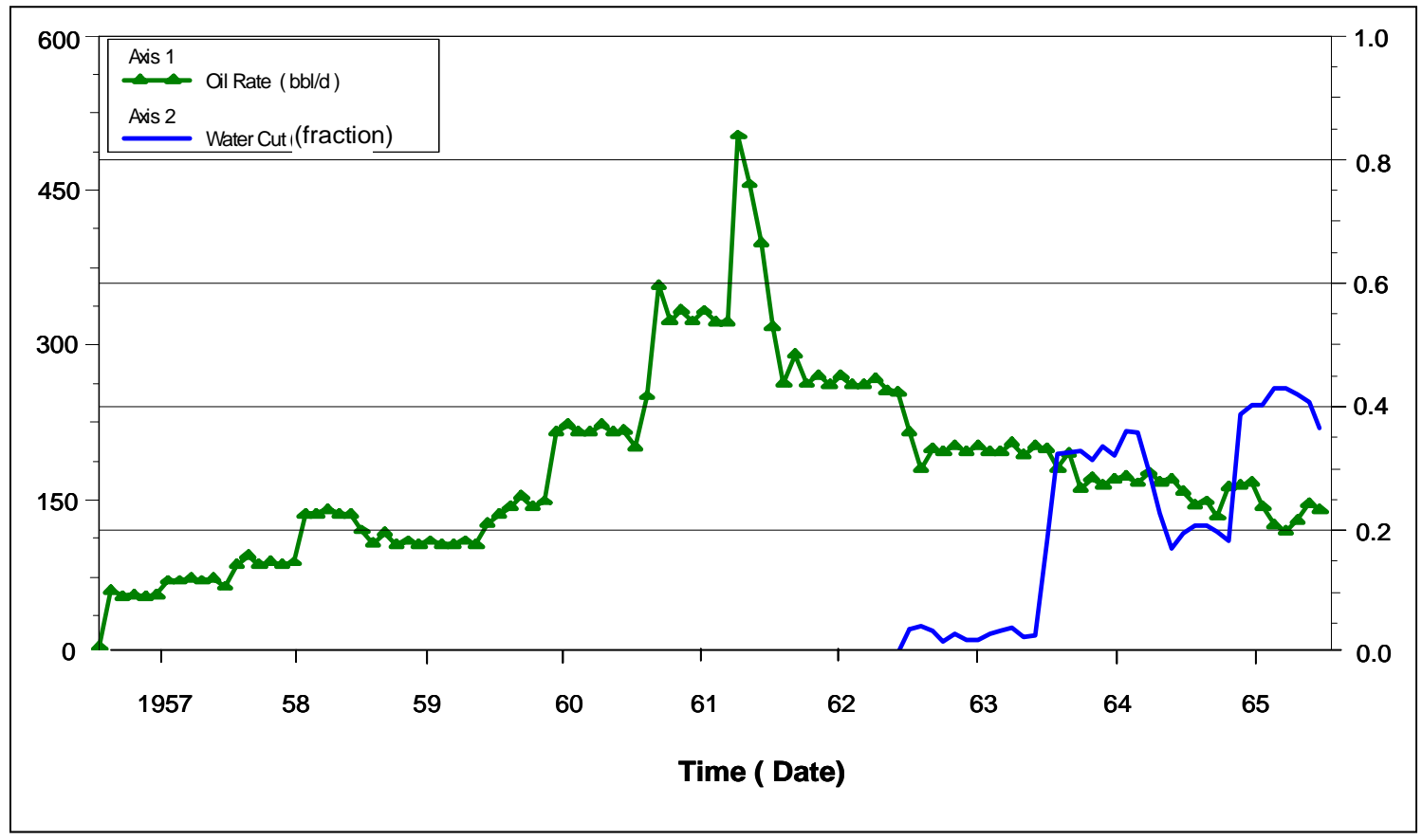

Fig. 1.4.3-Oil Rate and Water Cut during Primary Depletion of Germania Spraberry Unit. 


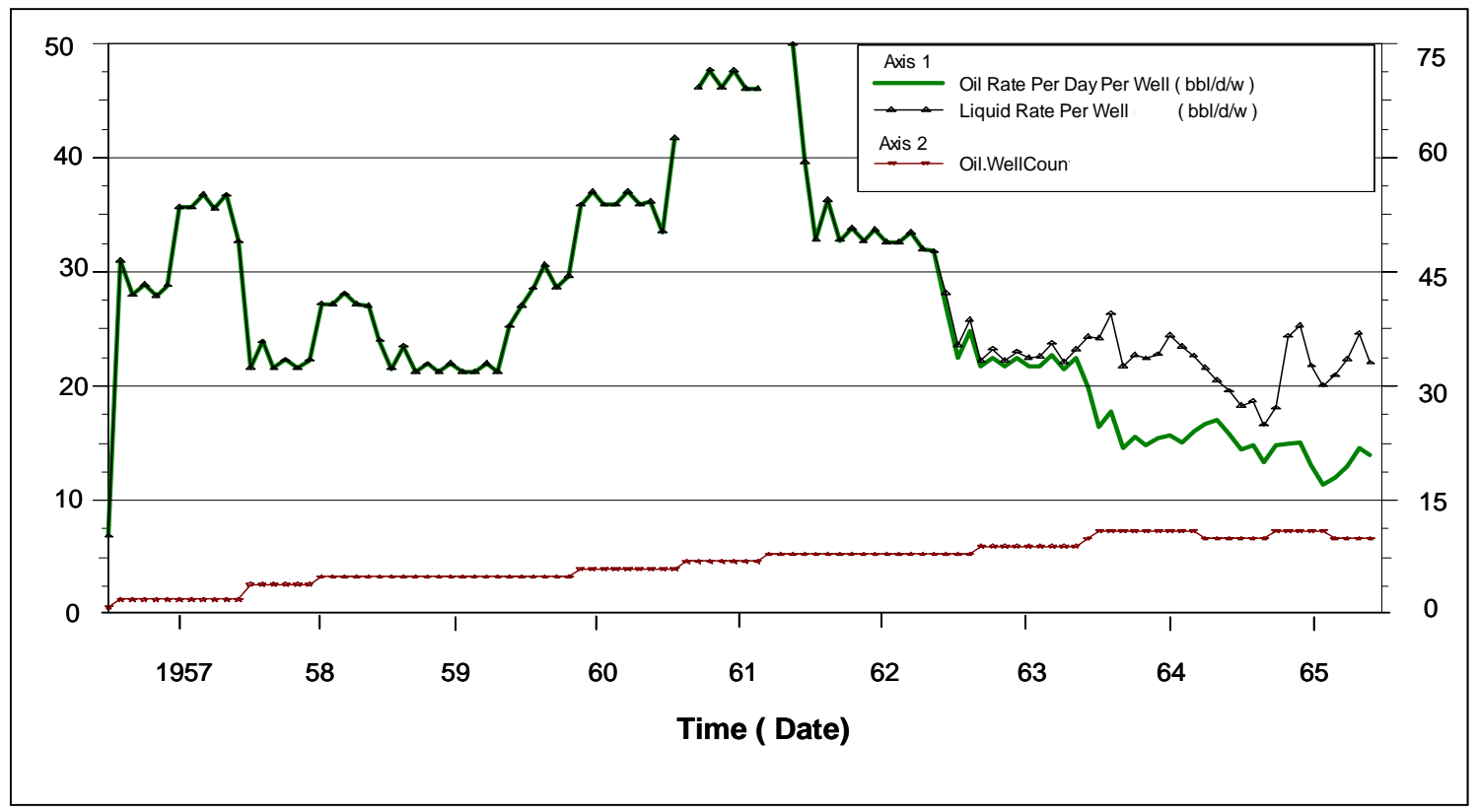

Fig. 1.4.4-Oil Rate per well, Liquid Rate per well and Active wells during Primary Depletion for Germania Spraberry Unit.

\subsection{Secondary Performance in Germania Spraberry Unit (Waterflooding).}

In 1965 a waterflooding program was initiated and continued until 1990. The purpose of this waterflooding program was to improve the recovery by sweeping the oil from the injectors located in the middle part of the structure towards the producers located throughout the reservoir. The water was injected through 5 wells (wells: $11 \mathrm{~W}$, 19W, 22W, 17W, and 6W) located in different positions of the reservoir (Fig.1.4.5). The cumulative water injection under this period was 2.44 million barrels, the average water injection rate per well was 688 BWPD (Fig. 1.4.6), and the cumulative water production was 0.95 million barrels of oil. 


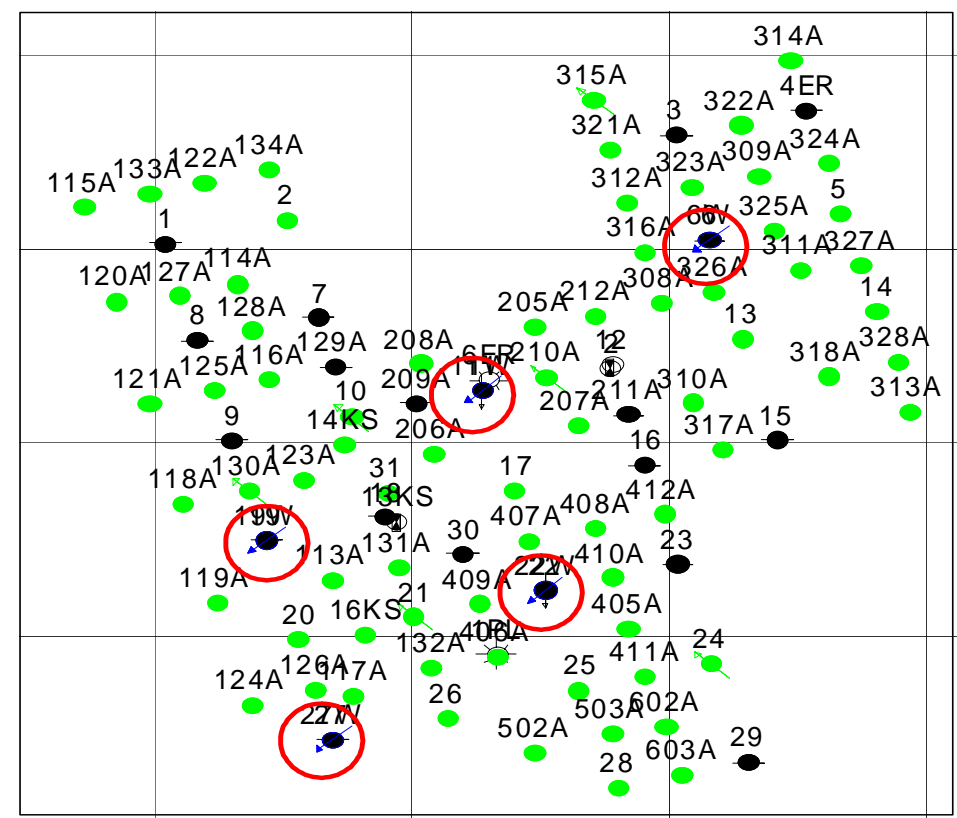

Fig.1.4.5 -Base Map of Germania Spraberry Unit showing the wells injecting water from 1965 to 1990.

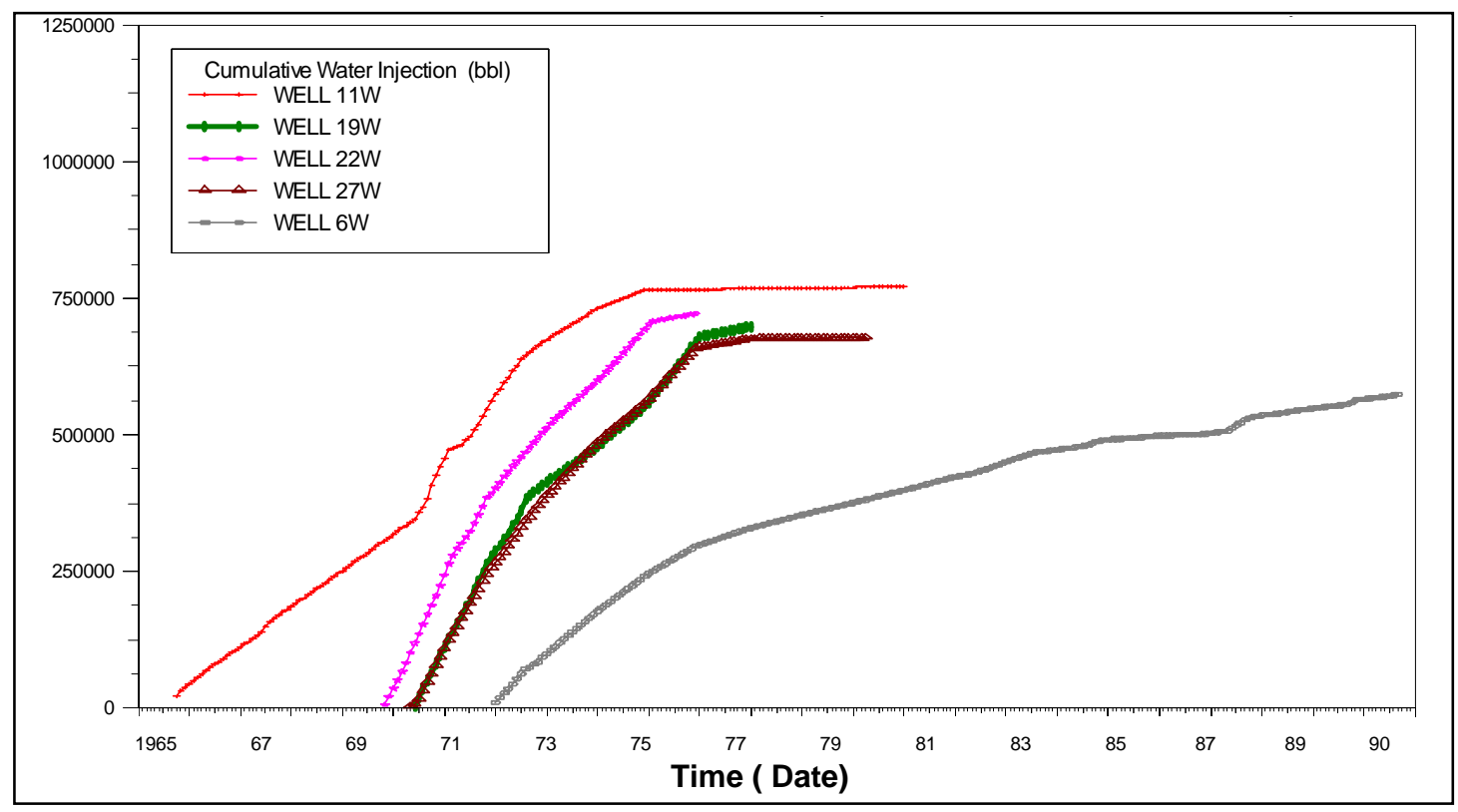

Fig.1.4.6 -Cumulative Water Injection for the five wells injecting from 1965 to 1990.

In May 1990 the water injection was suspended when the average water cut in the producer wells increased up to 0.75 , two infill drilling campaign took place increasing 
the numbers of producer wells from 20 to 98 in a period of 10 years, increasing the number of active wells up to 66 and developing the reservoir under a 40 acre-spacing. Oil production rate reached its maximum peak at 956 BOPD in 1992. The reservoir continued producing under this condition (water injection equal to zero) from 1990 to 2002 (Fig.1.4.7).

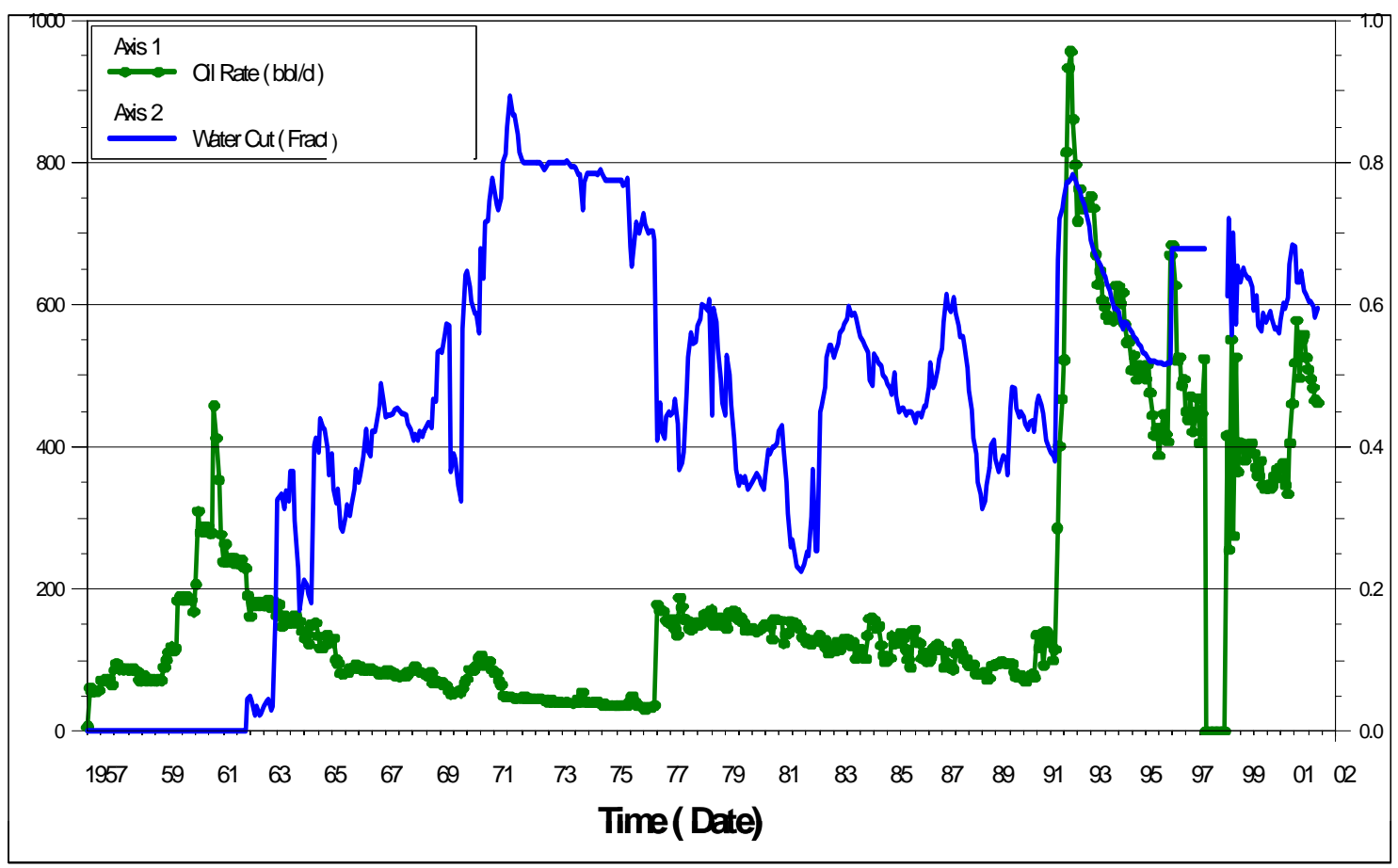

Fig.1.4.7-Oil Rate and Water Cut during Primary and Secondary Depletion of Germania Spraberry Unit.

The cumulative water production and injection as of June 2003 were 2.24 and 2.44 million barrels respectively. In February 2003 the operator began a new water injection project (under a new injection pattern) through six injector wells by converting three wells to water injectors, returning two wells to injectors and drilling a new injector well (Fig.1.4.8). Each one of the six injectors is currently injecting 270 BWPD. Since this program was initiated, some producers have shown favorable response to the injection. Currently the production rate is 470 BOPD through 64 active wells and the cumulative oil production is 2.242 million barrels. During the secondary performance the average oil production per well was 12 BOPD, average liquid production per well was 40 BLPD, and the numbers of active wells was increased significantly by infill drilling and controlling the operations in the field (Fig.1.4.9). 


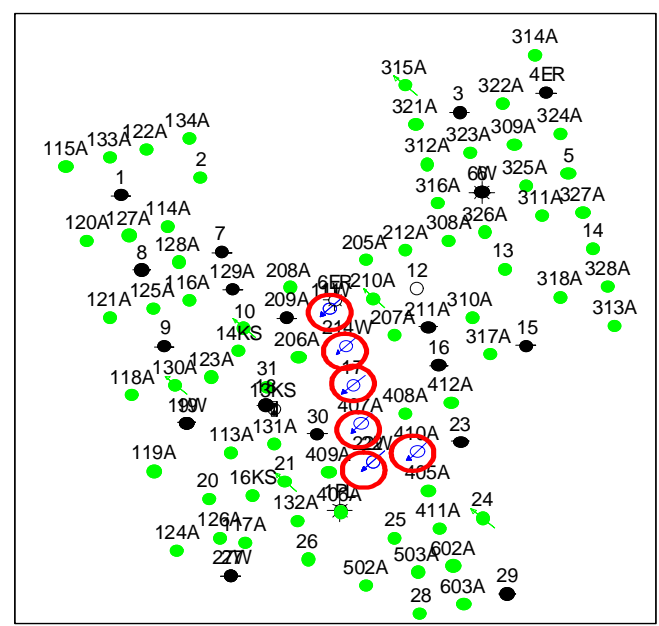

Fig.1.4.8 -Base Map of Germania Spraberry Unit showing wells injecting water under the new Injection Pattern

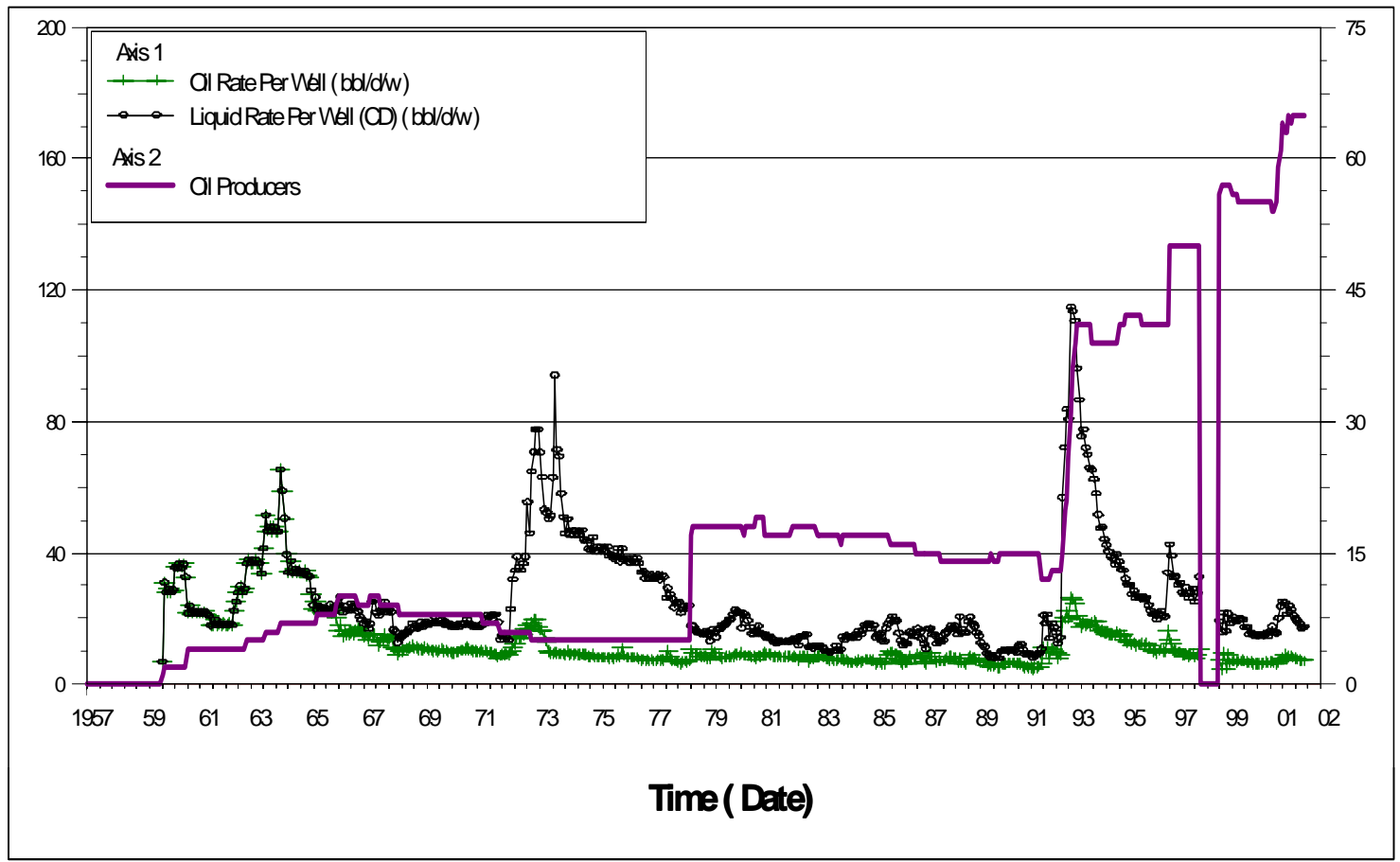

Fig.1.4.9-Oil Rate per well, Liquid Rate per well and Active wells during Primary and Secondary Depletion for Germania Spraberry Unit.

\section{Reservoir Monitoring and Surveillance System}

This chapter describes a reservoir management approach to waterflood Surveillance in Germania Spraberry Unit considering interrelated parts of the system. The primary function of this surveillance system is to provide facts, information, and 
knowledge necessary to control operations in the field and maximize the recovery from the unit.

Sometimes the actual performance of most fields may not agree with the expected performance of it. In the case of Germania Spraberry Unit, the differences between its performance and the performance of others units in Spraberry may be due to inadequate geological description, well completion problems, etc. The reasons for its low productivity and disappointing waterflood performance have remained unexplained until now. Various hypotheses have been proposed to explain the poor performance of the unit. These hypotheses include: lack of pattern confinement and injection well density, poor waterflood pattern development, complex fracture networks, fracture mineralization, wettability effects, lack of understanding of the imbibition transfer mechanism and stress-sensitive permeability.

In this chapter we have tried to identify the key parameters that have significant effect on the actual waterflood performance and some possible explanations of this behavior, and recommendations to improve the performance of the unit. Thus, attempts would be made to monitor the performance of the field and improve its recovery.

For this, we developed a data base using the software Oil Filed Manager (OFM) which is a powerful surveillance software application that provides an array of modules and tools for managing and analyzing static and dynamic data. Since the data was obtained from different related sources, it was reviewed, re-organized, and finally reduced to a format manageable in OFM. The data collected comprises: production and injection for 103 wells, coordinates, dates and events, wellbore, limits of leasing, logs, PVT analyses, etc. The calculations and processes were performed using the main modules of the program (Decline Curve Analysis, System Functions, Calculated Variables, Plots, Reports, Bubble Maps, Grid Maps and Scatter Plots) and the interrelation among them, was also considered.

\subsection{Production Heterogeneity Indexing}

In this part we describe a surveillance tool for production data referred to as Production Heterogeneity Index which quantifies and qualifies well performance anomalies for the purpose of assessing completion efficiency and determining the most successful practices in the unit as well as a surveillance tool for the waterflooding performance. The assessment of the Production Heterogeneity Index is also a valuable tool to production and reservoir engineers for selecting workover or stimulation candidates and determining the best completion practices in Germania Spraberry Unit in their efforts to improve the performance of the field. To properly apply the Production Heterogeneity Index and assure the validity of this analysis method, the following assumptions ${ }^{3}$ were made:

- All wells being analyzed are completed and producing in the same formation ( in some cases it is possible to obtain meaningful empirical correlations from commingled formations)

- The complete monthly well production history is available back to the beginning of life of each well. 
- No artificial rates restrictions or constraints are placed on the wells being analyzed.

- All wells are producing with an equivalent type artificial lift system.

- All wells are producing under similar reservoir pressure conditions (It maybe possible to make corrections for large variations in reservoir pressure if pressure data is available for the wells in question).

- Sufficient numbers of wells area available to perform meaningful normalization of the data.

To estimate the Production Heterogeneity Index for the oil rate in every well, we applied the equation given by:

$$
\text { HI Oil Rate }=\frac{\text { Oil Rate }}{\text { Average OilRate }}-1
$$

Where:

- HI Oil Rate = Production Heterogeneity Index for the oil rate, Dimensionless.

- Oil Rate = oil production rate for the well, $\mathrm{BOPD}$

- Average OilRate $=$ average oil rate of all wells being analyzed, BOPD.

Similarly, we applied the Production Heterogeneity Index for the water rate. Given by the following equation:

$$
\text { HI Water Rate }=\frac{\text { Water Rate }}{\text { Average WaterRate }}-1
$$

Where:

- HI Water Rate = Production Heterogeneity Index for the water rate, Dimensionless.

- Water Rate = water production rate for the well, BWPD.

- Average WaterRate = average oil rate of all wells being analyzed, BWPD

For the case of Germania Spraberry Unit, we analyzed a total of 64 active wells (using the oil and water rate at the last date available in the database (June 2003)), by applying the equation (3.1) and equation (3.2) for every well.

According to the equation (3.1) wells showing Production Heterogeneity Index for the oil rate greater than zero have a current oil rate greater than the average oil rate of the reservoir (in this case Germania Spraberry Unit); whereas, wells with Heterogeneity Index for the oil rate less than zero have a current oil rate less than the average oil rate of the entire reservoir.

On the other hand, wells showing Production Heterogeneity Index for the water rate greater than zero mean they have a current water rate greater than the average water rate of the reservoir; whereas, wells with Heterogeneity Index for the water rate less than zero mean they have a current water rate less than the average oil rate. This is according to equation (3.2).

Combining the Production Heterogeneity Index for both rates oil and water, we can subdivide the wells into 4 different groups, as follows: 
- Wells with Production Heterogeneity Index for both oil and water greater than zero (oil rate and water rate above the average).

- Wells with Production Heterogeneity Index for both oil and water less than zero (oil rate and water rate below the average).

- Wells with Production Heterogeneity Index for oil greater than zero and Production Heterogeneity Index for water less than zero (oil rate above the average and water rate below the average).

- Wells with Production Heterogeneity Index for oil less than zero and Production Heterogeneity Index for water greater than zero (oil rate below the average and water rate above the average).

Based on the four categories of wells mentioned above, we created the cross-plot in Fig. 1.4.10 showing the Production Heterogeneity Index for oil and water in 64 active wells of Germania Spraberry Unit. We can also plot the geographic location for each one of the wells analyzed (Fig. 1.4.11) and study its behavior with respect to the position in the reservoir as well as its position with respect to injectors and the fracture orientation (Fig.1.4.12).

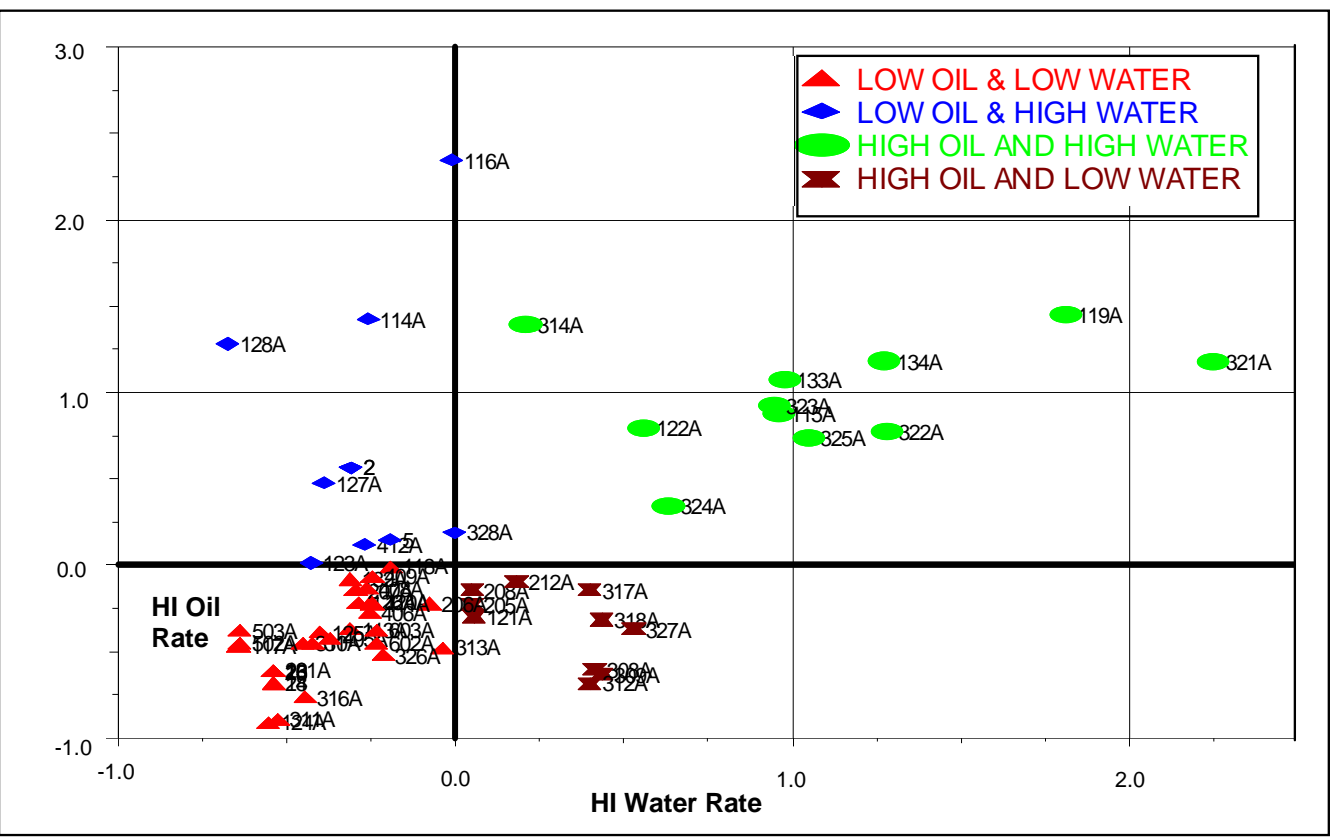

Fig.1.4.10-Cross-Plot showing the Production Heterogeneity Index for Oil and Water in 64 active wells of Germania Spraberry Unit. 


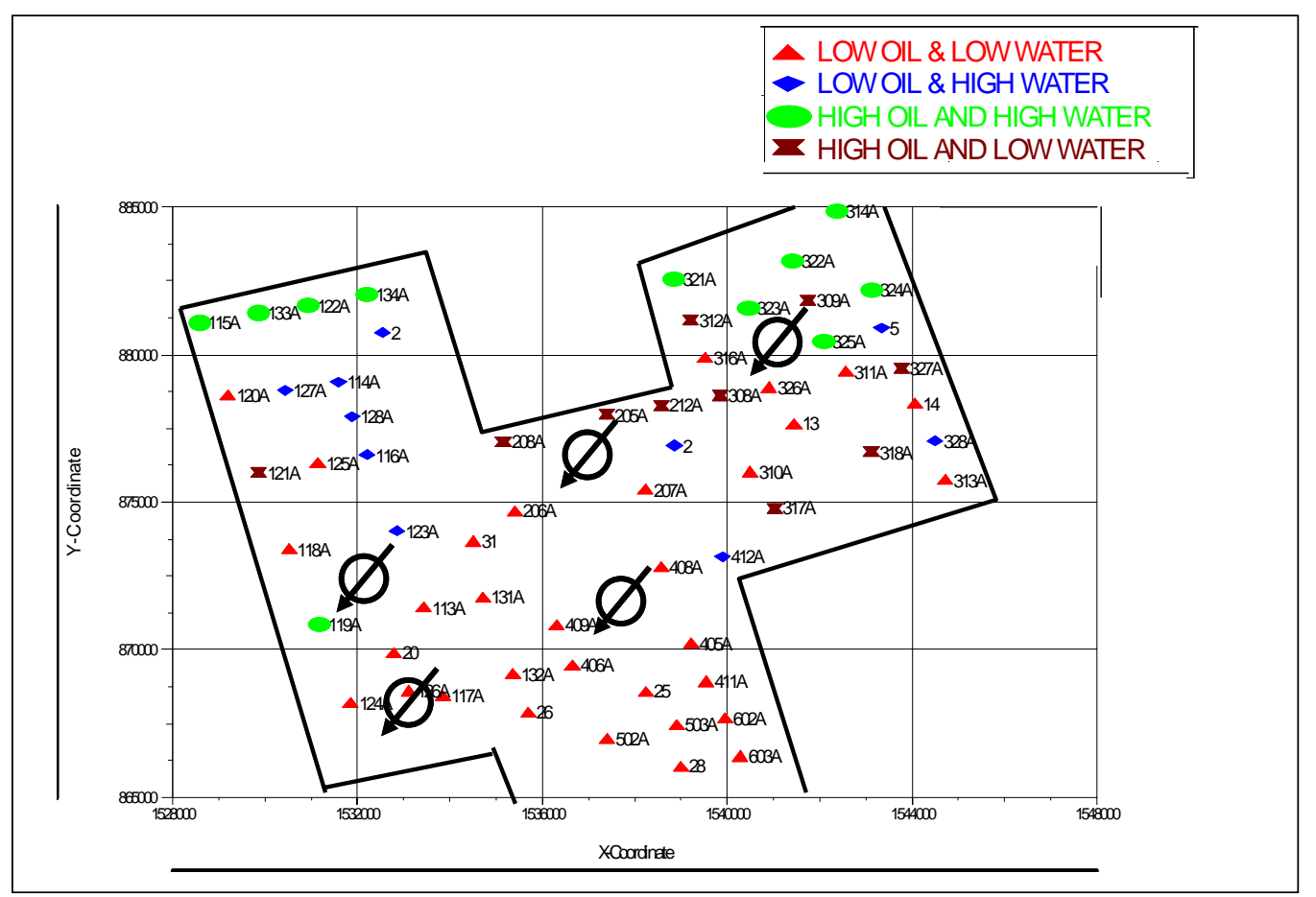

Fig.1.4.11-Base Map showing the 64 Active Wells in Germania Spraberry Unit and its position with regards to the injectors (old injection pattern).

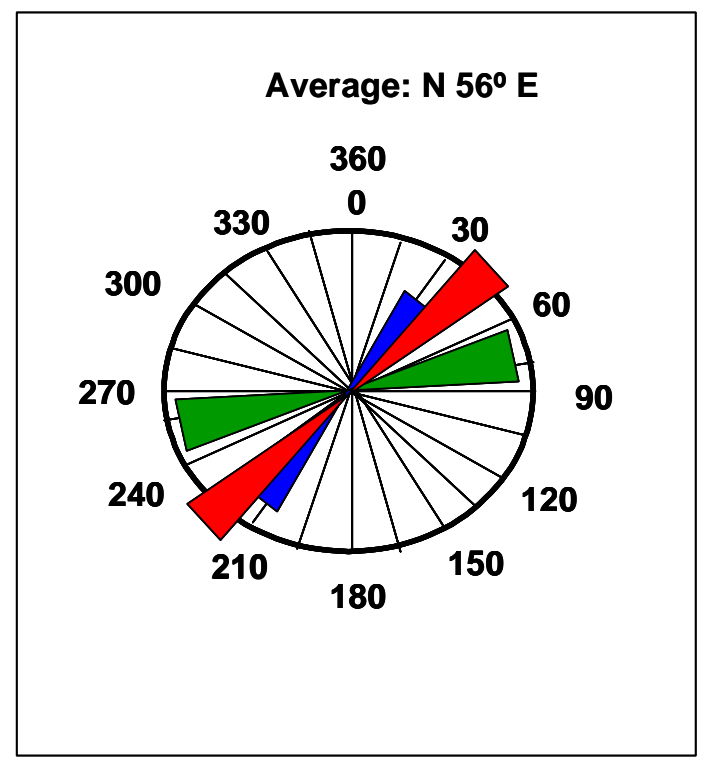

Fig.1.4.12-Fracture Orientation from Core Analysis.

In general, the distribution of the different category of wells in the reservoir is an indication of the high degree of heterogeneity of the fracture system. 
Wells with both water rate and oil rate below the average are distributed throughout the reservoir not following a trend; they represent good candidates for workover, stimulation or recompletion.

Wells with water rate below the average and oil rate above the average are located in a line forming a line oriented northeast-southeast which is in accordance with the major fracture orientation ( this is also in agreement with the dominant tracer response observed in some wells in the area ( in O'Daniel Spraberry Unit)).

Wells with both water rate and oil rate above the average, tends to follow a line with the same orientation of the major fracture trend. However, since they are located far away from the injectors, close to the upper limit of the lease, their behavior is probably affected by the operation and production taking place beyond the limits of Germania Spraberry Unit. Wells with water rate above the average and oil rate below the average clearly follow a line with an orientation parallel to the line of well injecting water (new injection pattern); those are wells candidates to conformance technology or remedial work to reduce the water rate. These results are summarized in Table 1.4.1.

Table 1.4.1- Category of active wells based on the current Production Performance

\begin{tabular}{|c|c|c|c|}
\hline CATEGORY & WELLS & Production Remarks & $\begin{array}{l}\text { Location } \\
\text { Remarks }\end{array}$ \\
\hline $\begin{array}{l}\text { High oil rate \& } \\
\text { High water Rate }\end{array}$ & $\begin{array}{l}\text { 115A,133A,122A,134A,119A,3 } \\
\text { 21A,314A,322A, 325A }\end{array}$ & $\begin{array}{l}\text { Could be influenced } \\
\text { by operations beyond } \\
\text { the limits of Germania } \\
\text { or by communication } \\
\text { problems. }\end{array}$ & $\begin{array}{l}\text { Located far } \\
\text { away from the } \\
\text { injectors. }\end{array}$ \\
\hline $\begin{array}{l}\text { High oil rate \& } \\
\text { Low Water rate }\end{array}$ & $\begin{array}{l}\text { 121A, 208A,205A,212A, } \\
\text { 312A,308A,317A,309A,318A,3 } \\
\text { 27A }\end{array}$ & Good Producers & $\begin{array}{l}\text { Follow the } \\
\text { same direction } \\
\text { of major } \\
\text { fracture trend }\end{array}$ \\
\hline $\begin{array}{l}\text { Low oil rate \& Low } \\
\text { water rate }\end{array}$ & $\begin{array}{l}\text { 120A,125A,118A,206A,31,113 } \\
\text { A,131A,20,124A,126A,117A,1 } \\
\text { 32A,26,207A,409A,408A,406A } \\
\text {,405A,25,411A,502A,503A,602 } \\
\text { A,28,603A,316A,326A,13,310 } \\
\text { A,311A,14,313A }\end{array}$ & $\begin{array}{l}\text { Candidates for } \\
\text { workover and /or } \\
\text { stimulation }\end{array}$ & $\begin{array}{l}\text { Scattered } \\
\text { throughout the } \\
\text { unit }\end{array}$ \\
\hline $\begin{array}{l}\text { Low oil rate \& } \\
\text { High water Rate }\end{array}$ & $\begin{array}{l}\text { 2,127A,114A,128A, } \\
\text { 116A,123A,412A,328A,5 }\end{array}$ & $\begin{array}{l}\text { Candidates for } \\
\text { Conformance control }\end{array}$ & $\begin{array}{l}\text { Form a line } \\
\text { parallel to the } \\
\text { new injection } \\
\text { pattern }\end{array}$ \\
\hline
\end{tabular}




\subsection{Injection Withdrawal}

This waterflood surveillance incorporates analyses of production/injection data for Germania Spraberry Unit to monitor the relationship between reservoir withdrawals and the water injection rate. This relationship was monitored by evaluating the Voidage Replacement Ratio (VRR) given by:

$$
V R R=\frac{q W i}{q_{O} B_{o}+q W B_{W}+q_{o}(G O R-R s) B g}
$$

Where:

$$
\begin{array}{ll}
V R R & =\text { Voidage Replacement Ratio, Dimensionless. } \\
q W i & =\text { water injection rate, STB/D. } \\
q o & =\text { oil production rate, STB/D. } \\
B o & =\text { oil formation volume factor, RB/STB. } \\
q W & =\text { water production rate, STB/D. } \\
B_{W} & =\text { water formation volume factor, RB/STB. } \\
G O R & =\text { producing gas-oil ratio, scf/STB. } \\
R s & =\text { solution gas-oil ratio, scf/STB. } \\
B g & =\text { gas formation volume factor, } \mathrm{RB} / \mathrm{scf} .
\end{array}
$$

The Voidage Replacement Ratio (VRR) was analyzed during two different periods: from 1965 to 1989 (first injection period) and from January 2003 to August 2003 which correspond to the second injection period (under the new injection pattern). The first period exhibited an overall VRR greater than 1 suggesting that the volume being injected exceed the total volume being produced (Fig. 1.4.13). From 1969 to 1975 the average value of VRR was 20, indicating that 20 barrels of water were injected per 1 barrel of fluid produced (oil, water, and gas). This may explain the high water cut and rapid breakthrough observed in some wells (especially those surrounding the injectors) and is perhaps one of the most responsible factors for the poor performance of the unit during this period. The second period exhibits an overall VRR of 1 (Fig.1.4.14), thus indicating that the water injection rate is matching the fluid production rate and therefore the water injection rate is optimum (currently 1600 BWPD ), this also may indicate that the waterflooding project ( under the new pattern of injection) is likely to be successful. 


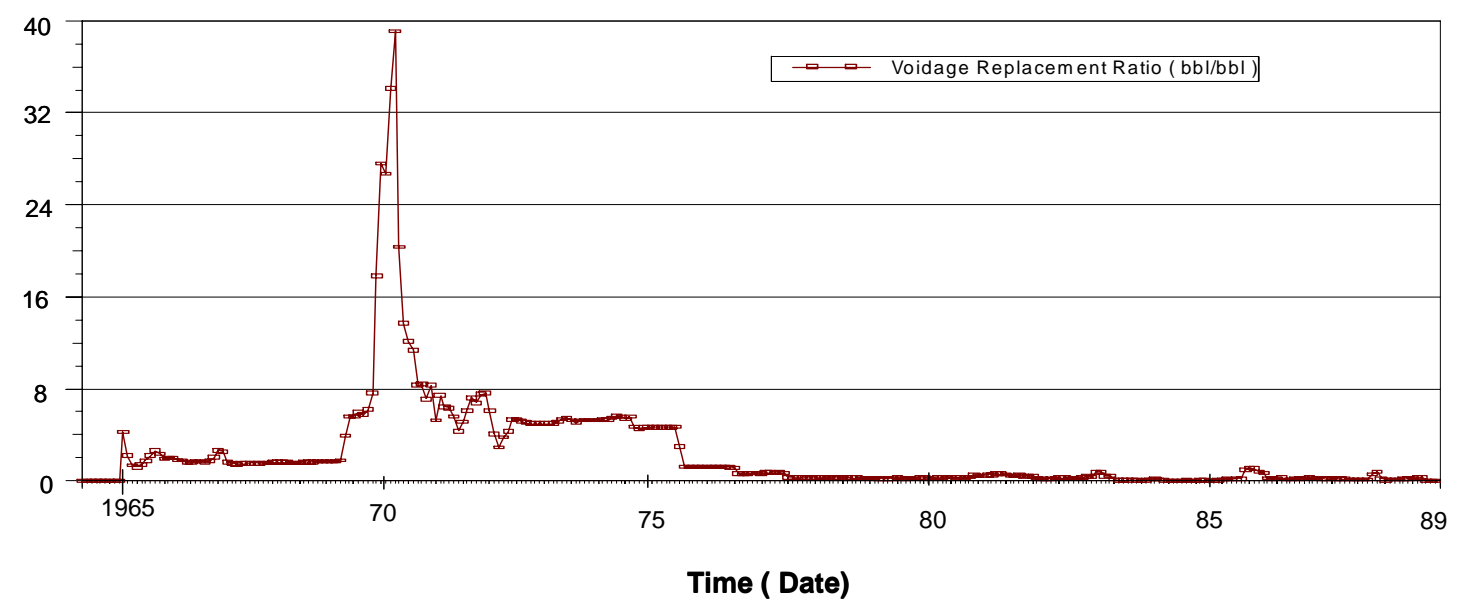

Fig.1.4.13-Voidage Replacement Ratio for the First Period of Injection

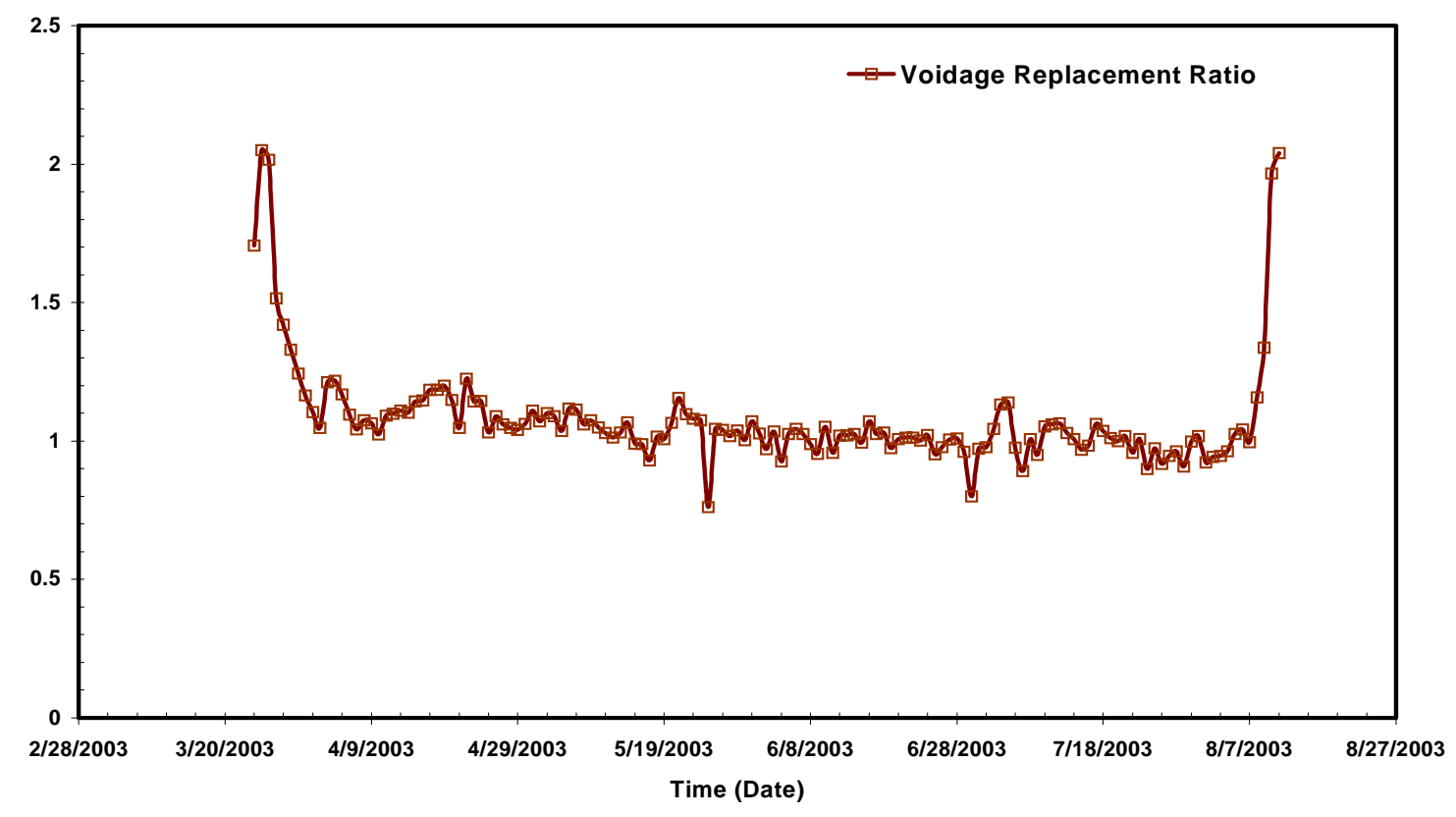

Fig.1.4.14-Voidage Replacement Ratio for the Second Period of Injection

\subsection{On-trend and Off-trend wells}

A major objective of this part of the study was to corroborate fracture orientation and identify waterflood response based on the performance of on-trend and off-trend wells. In this part of the study, production plots were generated to illustrate the differences in behavior and tendencies of both on-trend and off-trend wells. 
Traditionally the fracture orientation in the Spraberry formations is known to be approximately 50 degrees east of north ( $\mathrm{N} 50^{\circ} \mathrm{E}$ ). Through the use of production plots and bubble maps we tried to establish the behavior of the production and support this trend . The definition of on-trend and off-trend is with respect to the major fracture orientation trend; on-set wells follow the same orientation as the major fracture orientation (parallel to the fractures); whereas off-trend wells follow a direction different as the fracture orientation line. The on-trend and off-trend studied are shown in Fig.1.4.15

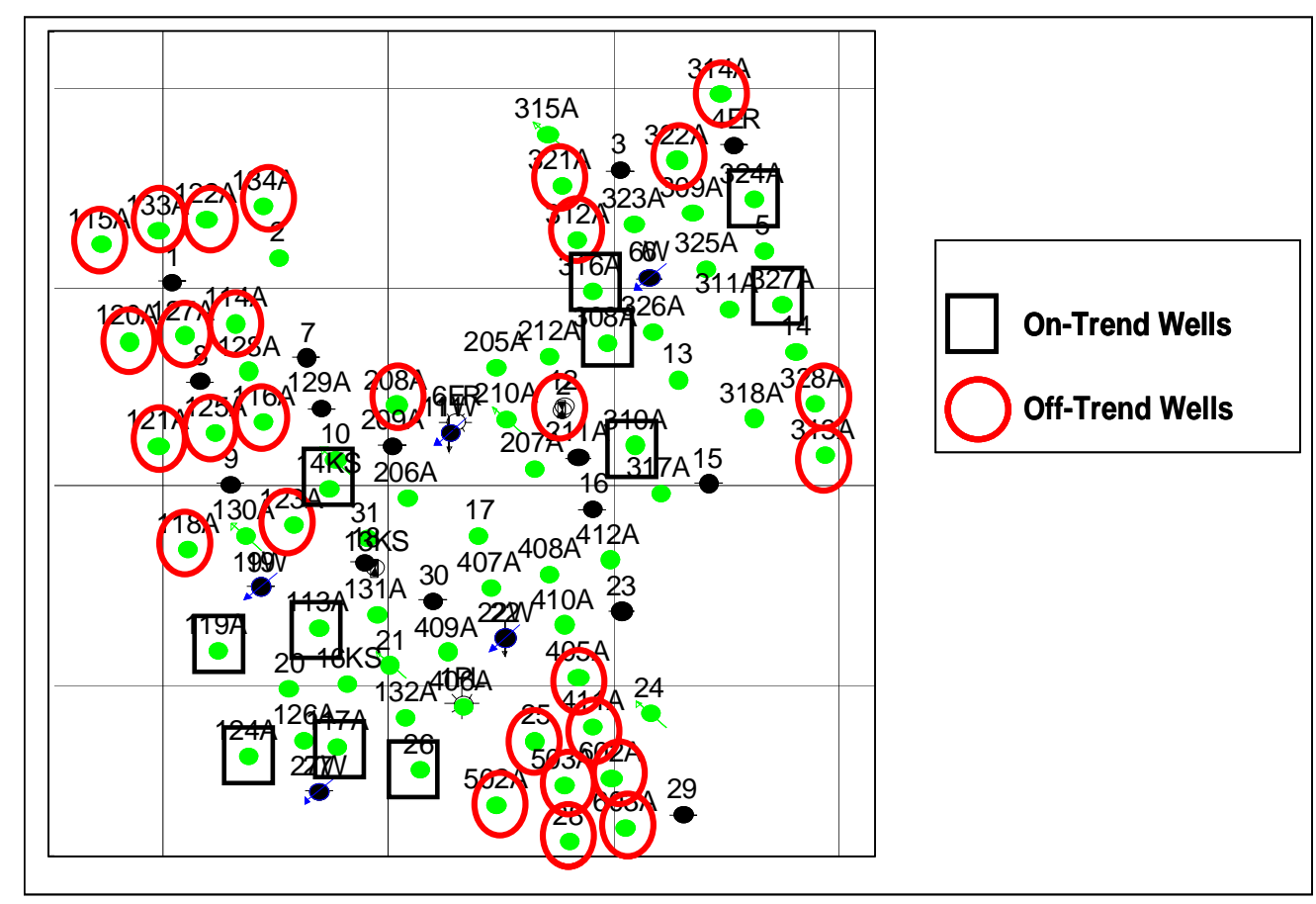

Fig.1.4.15-Base Map Showing the On-trend and Off-trend wells.

Fig.1.4.16 shows the same peak in the average oil rate per well for both on-trend and off-trend producers. The oil peak illustrates the flushing out of the fracture system by the flooding water. The peaks are also followed by a somewhat hyperbolic type decline in the oil rate as the imbibition process progresses. The decline rate is about the same for both on-trends and off-trends. In early production time, the on-trends tends to have a slightly greater oil rate compared to the off-trend wells; but after a while both tend to have the same rate (in other words, the on-trends seems to have a faster response). On the other hand Fig.1.4.17 shows that the water-oil ratio tends to increase in the off-trend shortly after the injection process was initiated (in 1965) and exhibit a higher water-cut than the on-trend wells most of the time until they both tend to reach the same value of water-oil rate.

The explanation for this behavior is based on wettability effects. Since the reservoir is weakly-water wet, the rock tends to imbibe the water being injected pushing the fluid (movable oil and water) towards the off-trend wells. The water being injected is 
moving much slower into the fractures. This performance suggests that this reservoir is water-wet (this is in agreement with the results obtained through core analyses performed in the area) indicating that the performance is greatly influenced by the wettability of the rock. This also corroborates that the fracture orientation is 56 degrees east of north $\left(\mathrm{N} 56^{\circ} \mathrm{E}\right)$. The performance of both on-trends and off-trends has showed oil bank followed by sharp breakthrough of the water front.

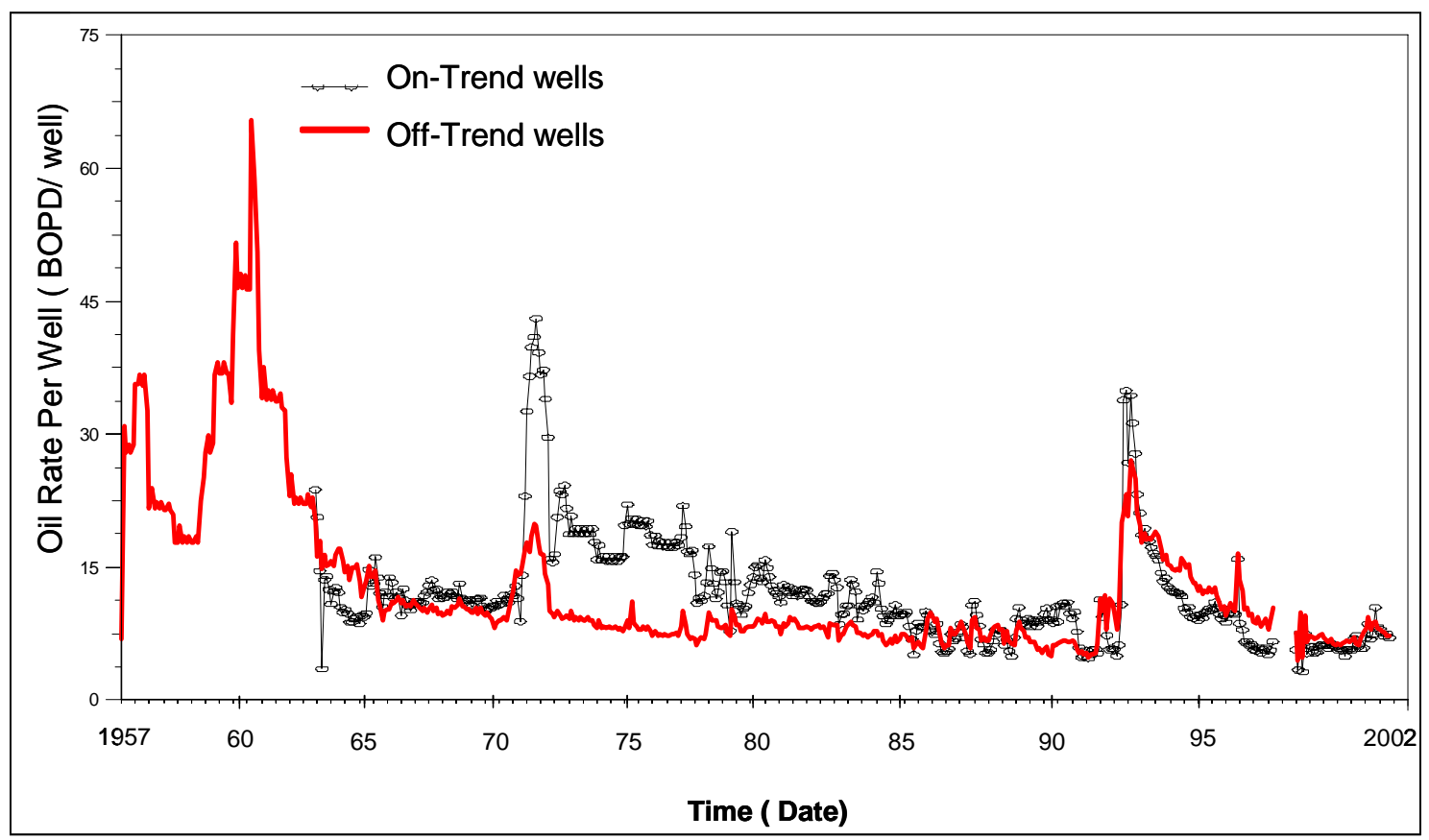

Fig. 1.4.16-Oil Rate per well for On-trend and Off-trend wells. 


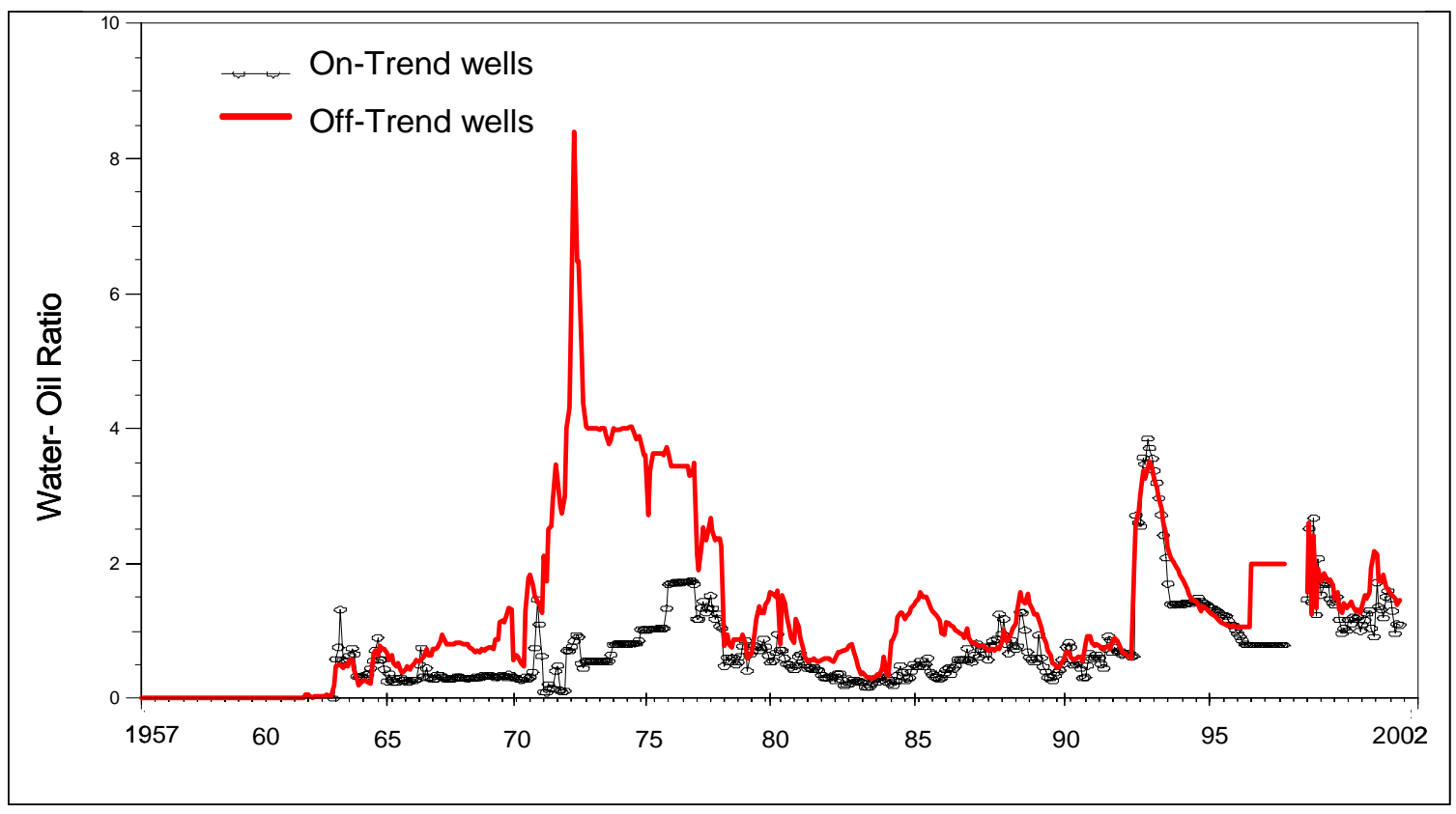

Fig. 1.4.17-Average water-Oil ratio for On-trend and Off-trend wells.

\subsection{Vintaging Wells}

To be able to compare the performance of wells drilled in different time of the unit development, it was necessary to determine the date of first production for each well. The wells were sorted according to their age and assigned to groups (vintages) for specific purposes. This is very important to evaluate the individual performance of the different vintages and select the best practices and operations utilized for each group as well as evaluating the impact of them on the recovery. Fig. 1.4.18, shows the different vintages or drilling campaigns used by the operator to develop the unit. 


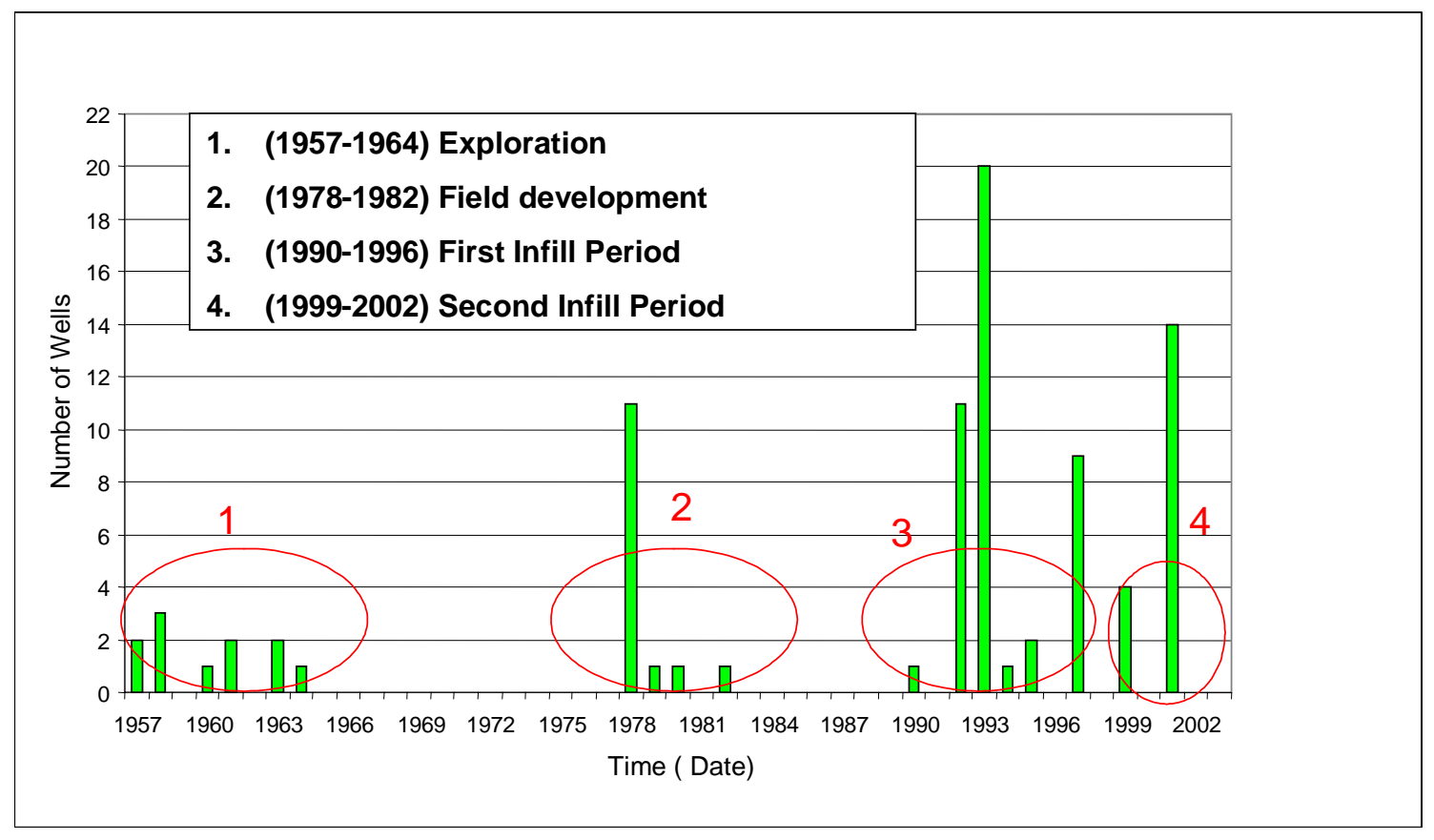

Fig.1.4.18-Different Drilling Campaigns for Development of Germania Spraberry Unit.

\subsubsection{Vintage 1957-1963.}

A total of 11 wells were drilled and produced from 1957 to 1964 to explore and develop the field. They were drilled in different locations of the unit. The purpose of this group of wells was to develop the reservoir when the field was under primary production. Fig.3.1.4.19 shows the location of the wells drilled from 1957 to 1963. Of this 11 wells, a total of three (GSU-11, GSU-17, and GSU-22) were converted into water injectors in January 2003 when the injection pattern was changed and are currently injecting 800 BWPD; two are still active (GSU-12 and GSU-26); two are temporarily plugged and abandoned, and four were abandoned. Wells drilled and produced during this period showed medium initial oil rate of 48 BOPD as shown in Fig.1.4.20 and Table 1.4.2. 


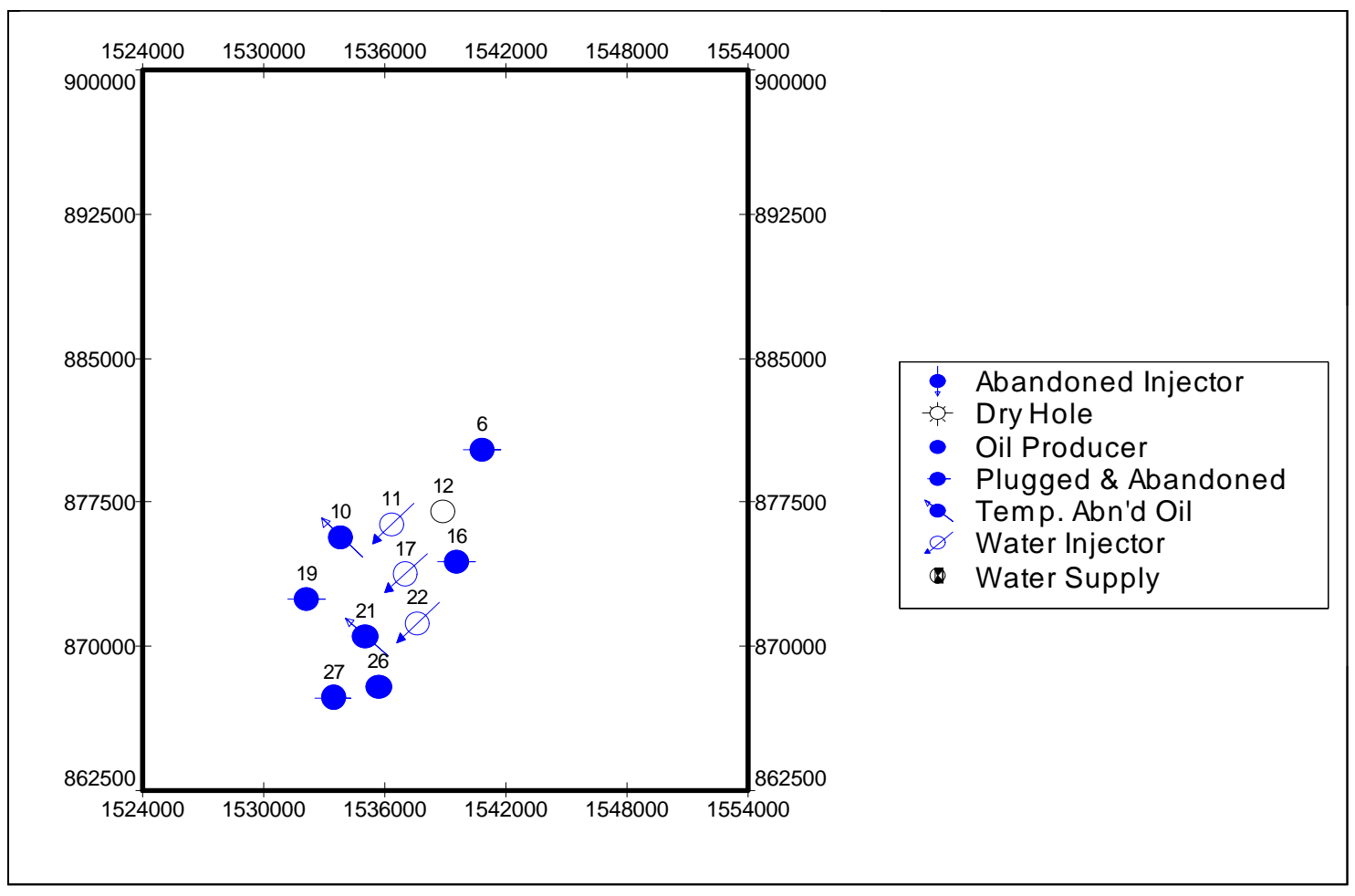

Fig.1.4.19-Base Map showing the Location of Wells Drilled from 1957 to 1963.

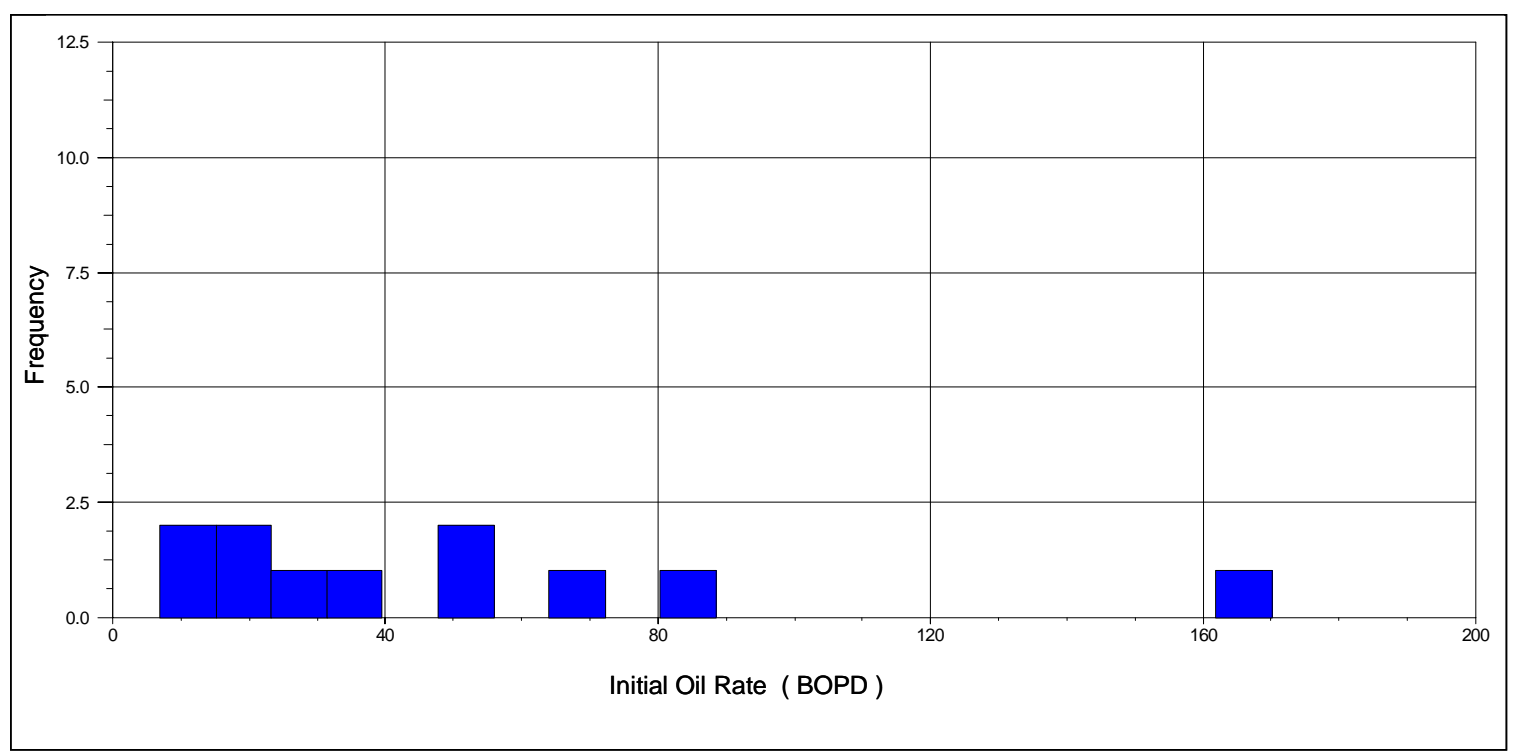

Fig.1.4.20-Histogram of Initial Oil Rate for Wells Drilled from 1957 to 1963. 
Table 1.4. 2-Statistical Analysis for Wells Drilled from 1957 to 1963.

\begin{tabular}{|rr|}
\hline First 0il rate ( BNPD) & \\
Samples: & 11 \\
Minimum: & 6.8972 \\
Maximum: & 170.1338 \\
Range: & 163.2366 \\
Medium: & 88.5155 \\
Sum: & 533.5898 \\
Arithmetic Average: & 48.5082 \\
Geometric Average: & 31.5498 \\
Variance: & 2061.6347 \\
Abs Deviation: & 33.6007 \\
Sample Std Deviation: & 47.6214 \\
Pop. Std Deviation: & 45.4052 \\
\hline
\end{tabular}

\subsubsection{Vintage 1978-1982}

A total of 14 wells were drilled during the second drilling campaign (from 1978 to 1982) to develop the field. They were drilled in different locations of the unit and in a 160 acre-spacing. The purpose of this group of wells was to develop the reservoir when the field was already under secondary production (the campaign began 13 years after the initiation of the waterflooding process). Fig.1.4.21 shows the location of the wells drilled from 1978 to 1982. Of this 14 wells, a total of seven (GSU-2, GSU-13, GSU-14, GSU-20, GSU-25, GSU-28, and GSU-31) are currently active and seven are plugged and abandoned (GSU-1, GSU-9, GSU-23, GSU-29, GSU-18, GSU-3, and GSU-7) due to either low productivity or high water curt (average was 80 percent) that they experienced shortly after they began producing. Wells drilled and produced during this period showed a medium initial oil rate of only 11 BOPD as shown in Fig.1.4.22 and Table 1.4.3. 


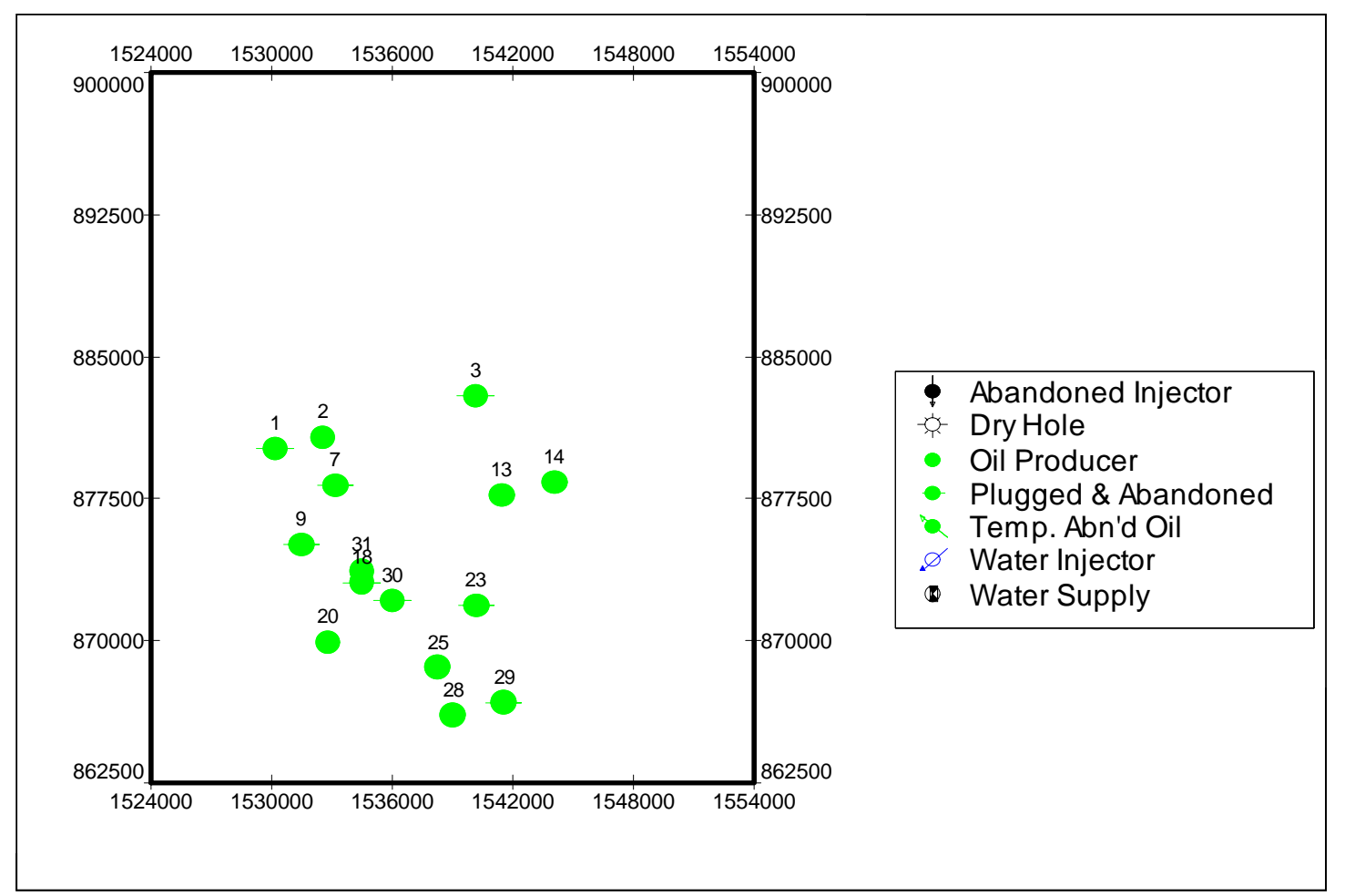

Fig.1.4.21-Base Map showing the Location of Wells Drilled from 1978 to 1982.

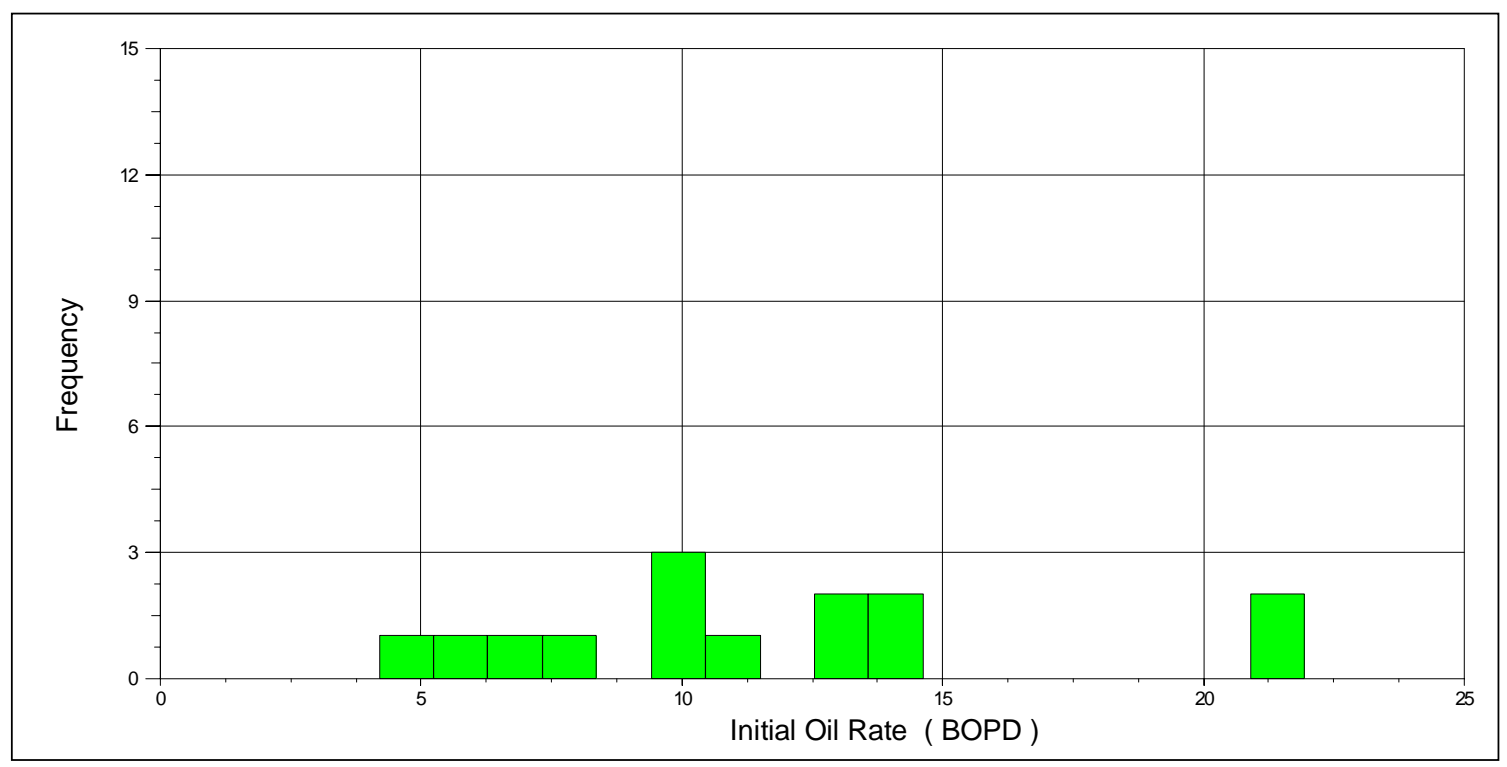

Fig.1.4.22 -Histogram of Initial Oil Rate for Wells Drilled from 1978 to 1982. 
Table 1.4.3 Statistical Analysis for Wells Drilled from 1978 to 1982.

\begin{tabular}{|rr|}
\hline First 0il rate ( BNPD ) & \\
Samples: & 14 \\
Minimum: & 1.0645 \\
Maximum: & 21.9355 \\
Range: & 20.8710 \\
Medium: & 11.5000 \\
Sum: & 162.6757 \\
Arithmetic Average: & 11.6197 \\
Geometric Average: & 10.6261 \\
Variance: & 24.6767 \\
Abs Deviation: & 3.9514 \\
Sample Std Deviation: & 5.1551 \\
Pop. Std Deviation: & 4.9676 \\
\hline
\end{tabular}

\subsubsection{Vintage 1990-1996}

A total of 44 wells were drilled during this infill-drilling campaign (from 1990 to 1996) to develop the field. They were drilled to reduce the spacing to 80 acres. The purpose of this group of wells was to develop the reservoir when the field was already under secondary production (this campaign began 25 years after the initiation of the waterflooding process). Fig.1.4.23 shows the location of the wells drilled from 1990 to 1996. Of this 44 wells, a total of 37 are currently active, which represents more than 50 percent of the active wells in the unit; 3 are temporarily plugged and abandoned due to either low productivity or the high water curt (average was 80 percent) that they experienced shortly after they began producing, and two (GSU-407 and GSU-410) were converted to water injectors in January 2003 having a water injection rate of about 540 BWPD. Wells drilled during this period experienced a medium initial oil rate of 44 BOPD as shown in Fig.1.4.24 and Table 1.4.4. 


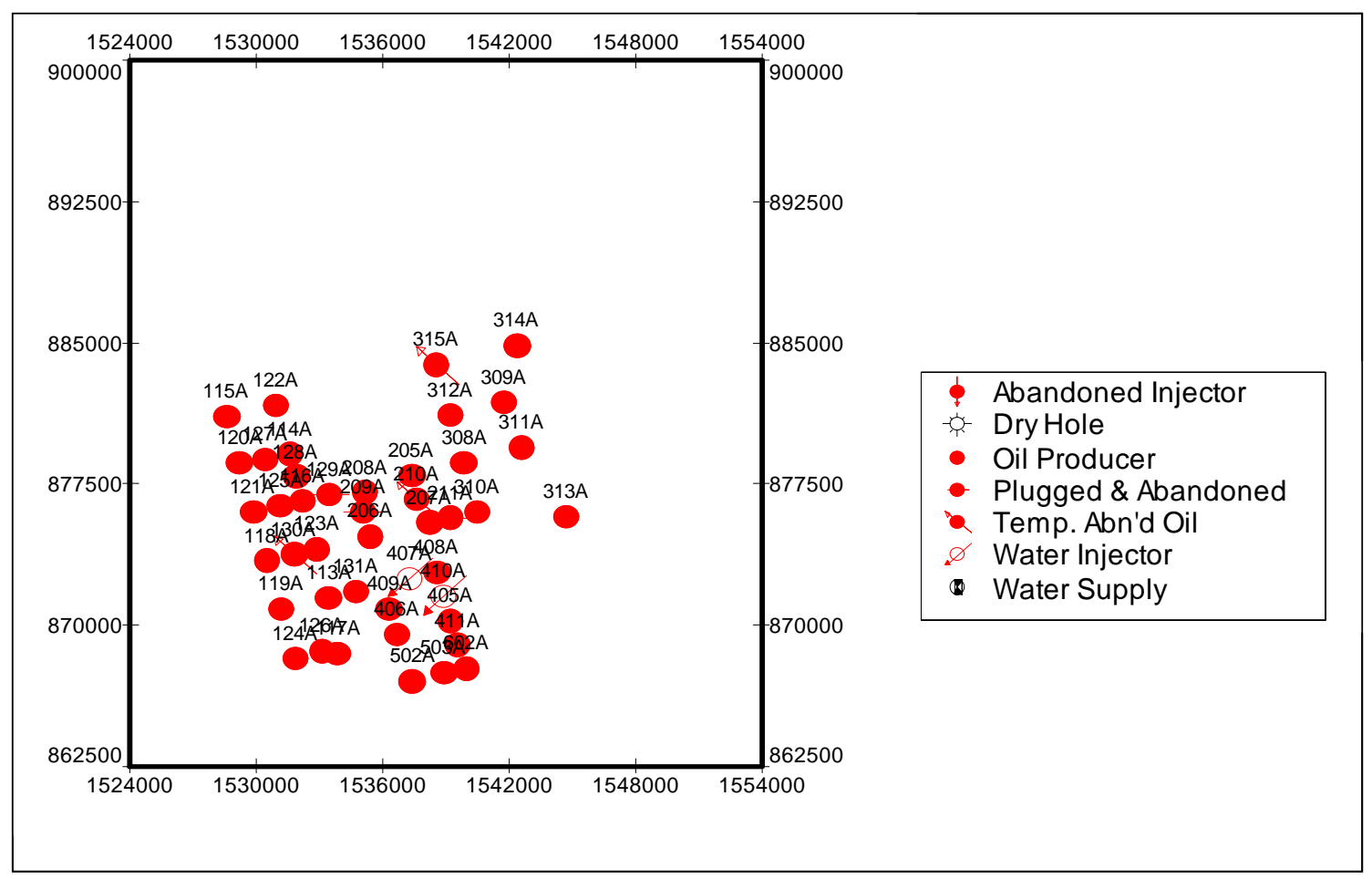

Fig.1.4.23-Base Map showing the Location of Wells Drilled from 1990 to 1996.

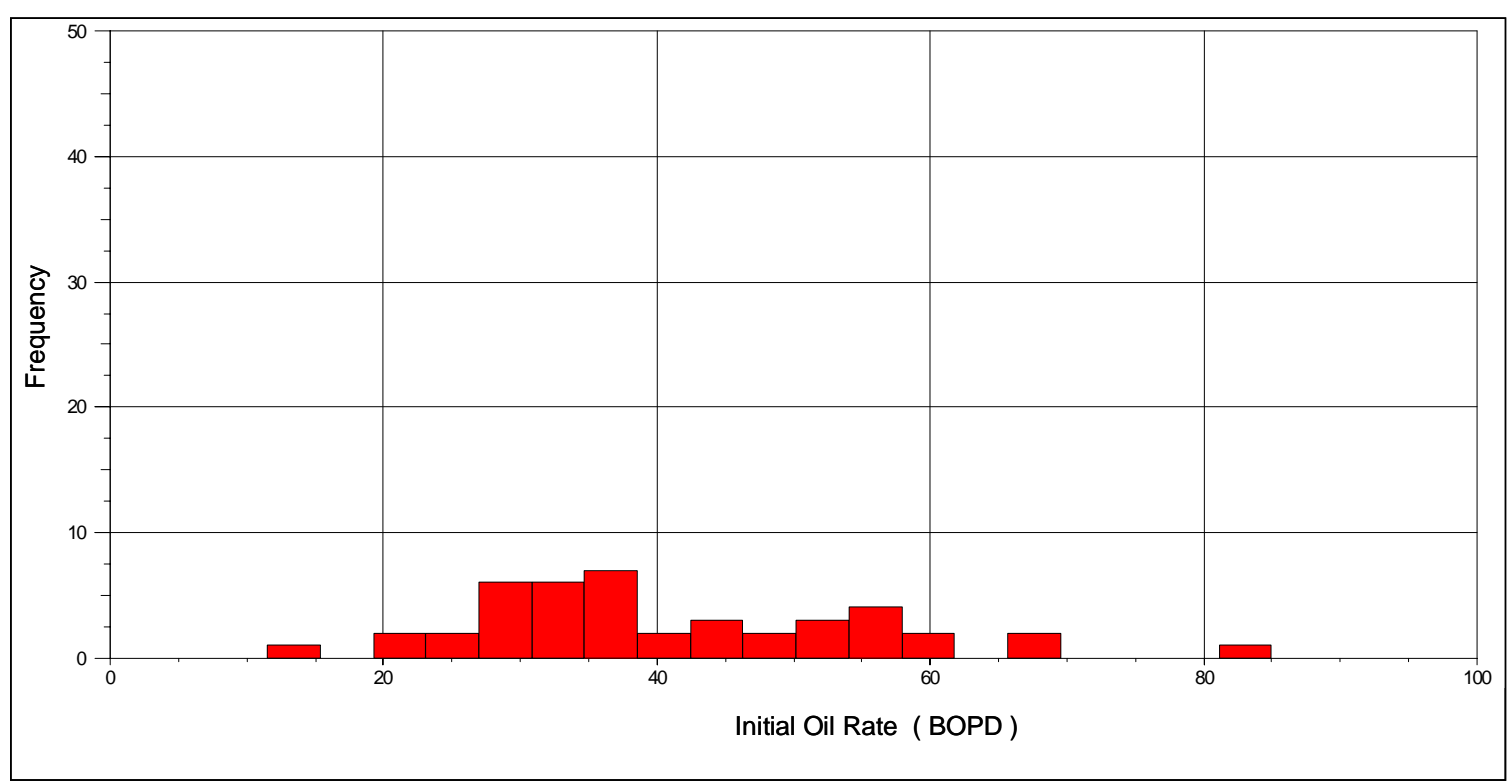

Fig.1.4.24-Histogram of Initial Oil Rate for Wells Drilled from 1990 to 1996. 
Table 1.4.4 Statistical Analysis for Wells Drilled from 1990 to 1996.

\begin{tabular}{rr|} 
Statistical Analysis & \\
First oil rate ( BNPD) & 42 \\
Samples: & 7.6393 \\
Minimum: & 81.2000 \\
Maximum: & 73.5607 \\
Range: & 44.4196 \\
Medium: & 1667.0433 \\
Sum: & 39.6915 \\
Arithmetic Average: & 37.4718 \\
Geometric Average: & 169.2272 \\
Variance: & 10.8822 \\
Abs Deviation: & 13.1664 \\
Sample Std Deviation: & 13.0087 \\
Std Deviation: &
\end{tabular}

\subsubsection{Vintage 1999-2002}

A total of 18 wells were drilled during this infill drilling campaign (from 1999 to 2002) to develop the field. They were drilled to reduce the spacing to 40 acres. The purpose of this group of wells was to develop the reservoir when the field was already under secondary production (this campaign began 42 years after the initiation of the waterflooding process). Fig.1.4.25, shows the location of the wells drilled from 1999 to 2002. All wells drilled during this period are currently active, producing with a moderate average water cut. Wells drilled during this period experienced medium initial oil rate of only 15 BOPD as shown in Fig.1.4.26 and Table 1.4.5. 


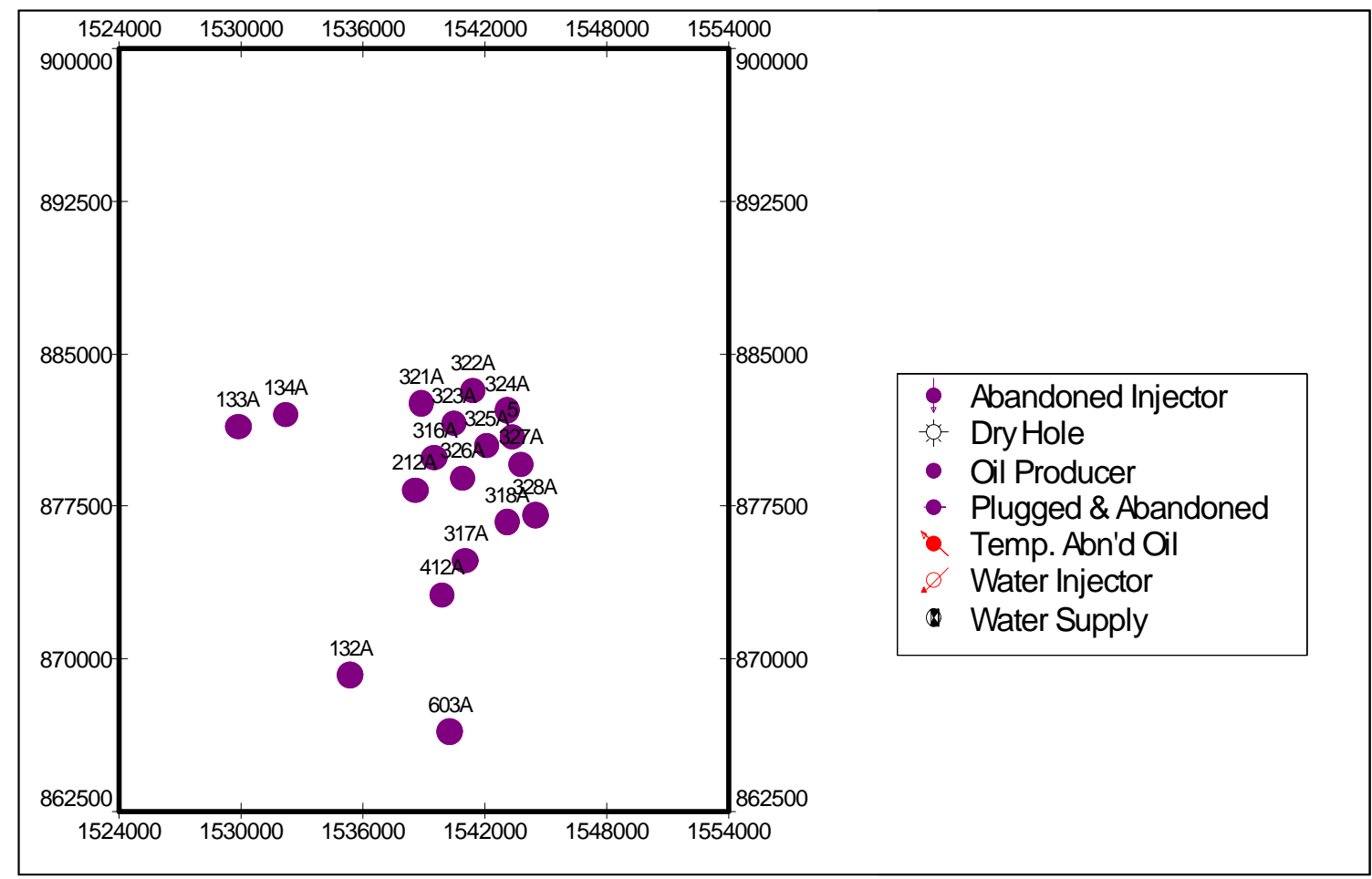

Fig.1.4.25-Base Map showing the Location of Wells Drilled from 1999 to 2002.

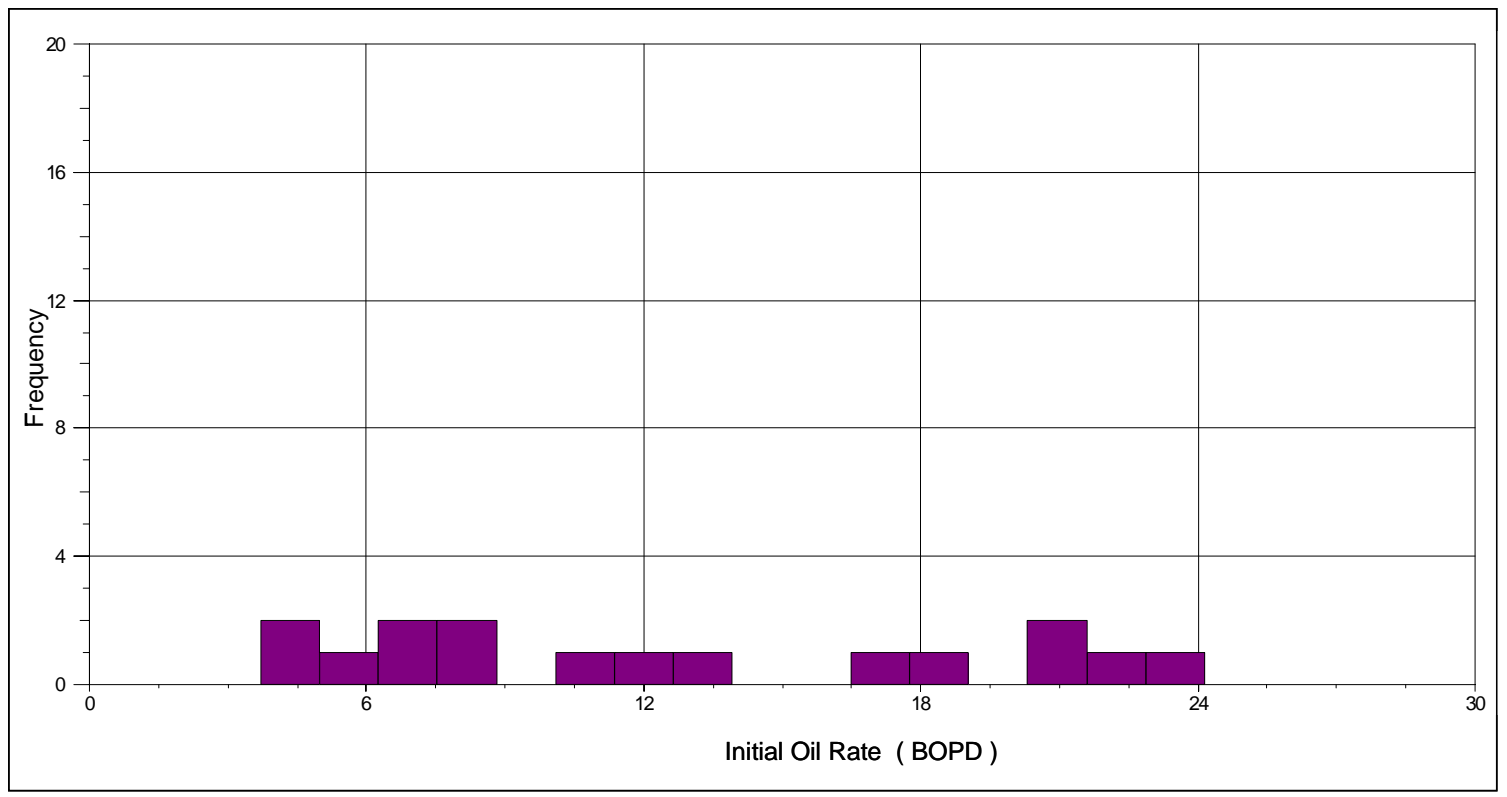

Fig. 1.4.26-Histogram of Initial Oil Rate for Wells Drilled from 1999 to 2002. 
Table 1.4. 5 Statistical Analysis for Wells Drilled from 1999 to 2002.

\begin{tabular}{rr|}
\hline Statistical Analysis & \\
First Oil rate ( BNPD) & 16 \\
Samples: & 2.4235 \\
Minimum: & 27.9835 \\
Maximum: & 25.5600 \\
Range: & 15.2035 \\
Medium: & 201.7435 \\
Sum: & 12.6090 \\
Arithmetic Average: & 10.8083 \\
Geometric Average: & 42.6894 \\
Variance: & 5.8581 \\
Abs Deviation: & 6.7480 \\
Sample Std Deviation: & 6.5337 \\
Std Deviation: &
\end{tabular}

\subsubsection{Comparative Analysis for Vintages}

According to Fig. 1.4.27, the second drilling campaign (1996 to 1996) is the one that exhibits the highest current production rate because is the one with the most wells drilled (44 wells).

Fig. 1.4.28, shows that all wells belonging to the four different campaigns, exhibit about the same decline rate. In this plot, we can also observe that the vintage that exhibit the highest average initial oil rate per well is the campaign of wells drilled between 1957 and 1964 (48 BOPD). It is because they were drilled when the reservoir had original pressure and initial oil water saturation.

Wells drilled between 1978 and 1982, had the lowest average initial oil rate ( 11 BOPD) even though they were drilled in the second campaign, when the water saturation and the cumulative water injected were lower than the existing in the reservoir when the third and four campaigns took over. However, after 6,000 days in production the oil rate of this group of wells (campaign 1978 to 1982) is greater than its initial rate; this is an indication of the response of the injection in this set (normally most of the floods take a long time to increase oil production as a result of large distances between the injectors and the producers; especially if the permeability of the formation is low). This response is also seen in the first drilling campaign (1957 to 1964) after 750 days in production and in the third drilling campaign (1990 to 1996) after 1,000 days in production as shown in Fig. 1.4.28. The wells drilled between 1999 and 2002 have shown little or no response to the water injection. The effect showed by the different group of wells, are due to the 
reduction of the well spacing which enhances the injection/production profile and connectivity.

Fig. 1.4.29, shows that wells drilled between 1957 and 1964 exhibit the highest initial water-oil ratio. However; as the rest of the wells were drilled, the different campaigns tended to reach the same value of water-oil ratio, averaging a current value of 2.

Historically; wells drilled during the third campaign (1990 to 1996), and the fourth campaign (1999 to 2002) have an initial oil rate higher than the remaining two campaigns. This is because in the third and fourth campaigns, the wells accessed an area previously unflooded by the wells in the first and second campaigns.

Fig. 1.4.30; shows the cumulative oil production reached by the wells of the four different drilling campaigns. The wells drilled in the first campaign exhibit the highest oil cumulative (1.4 million barrels) because they have been in production through the entire life of production of the unit.

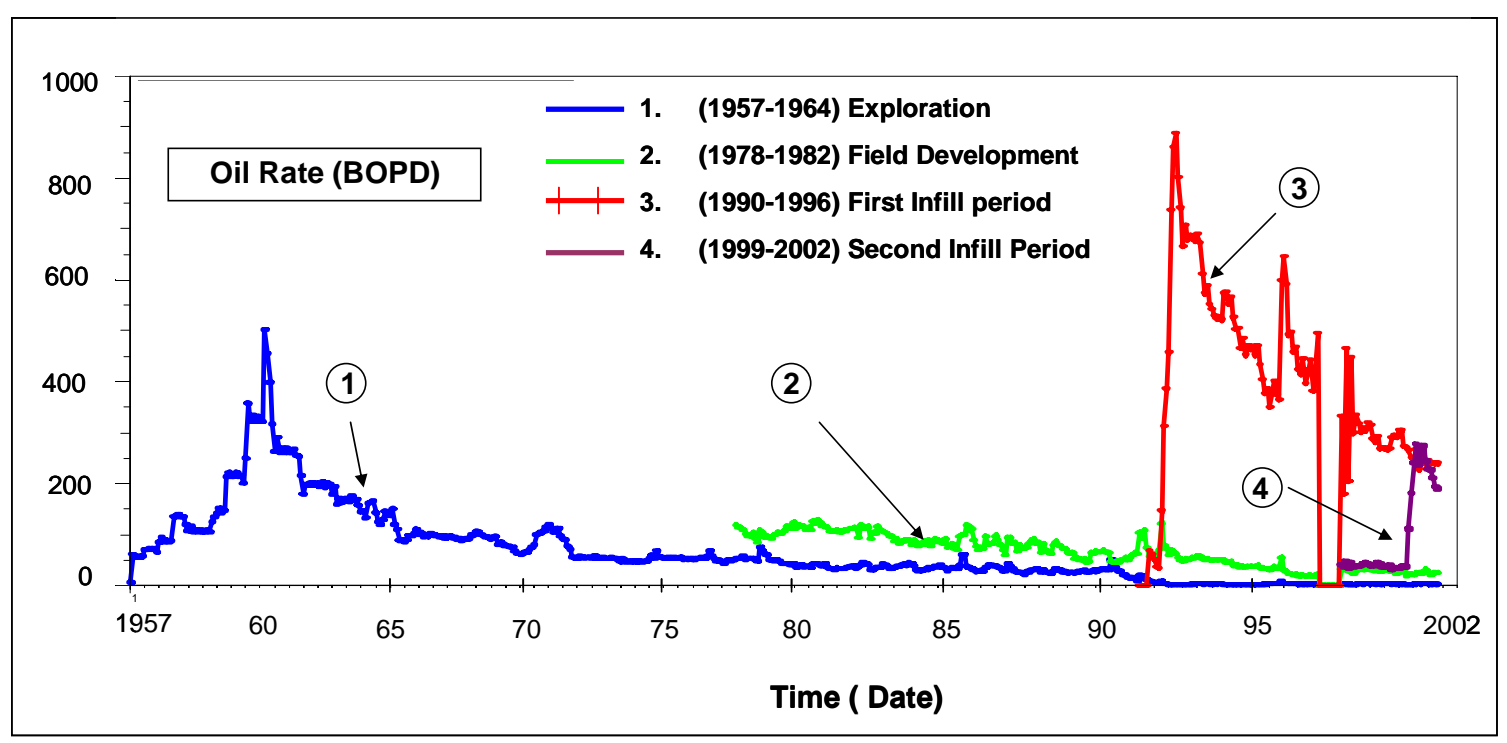

Fig.1.4.27-Histogram of Initial Oil Rate for Wells Drilled from 1999 to 2002. 


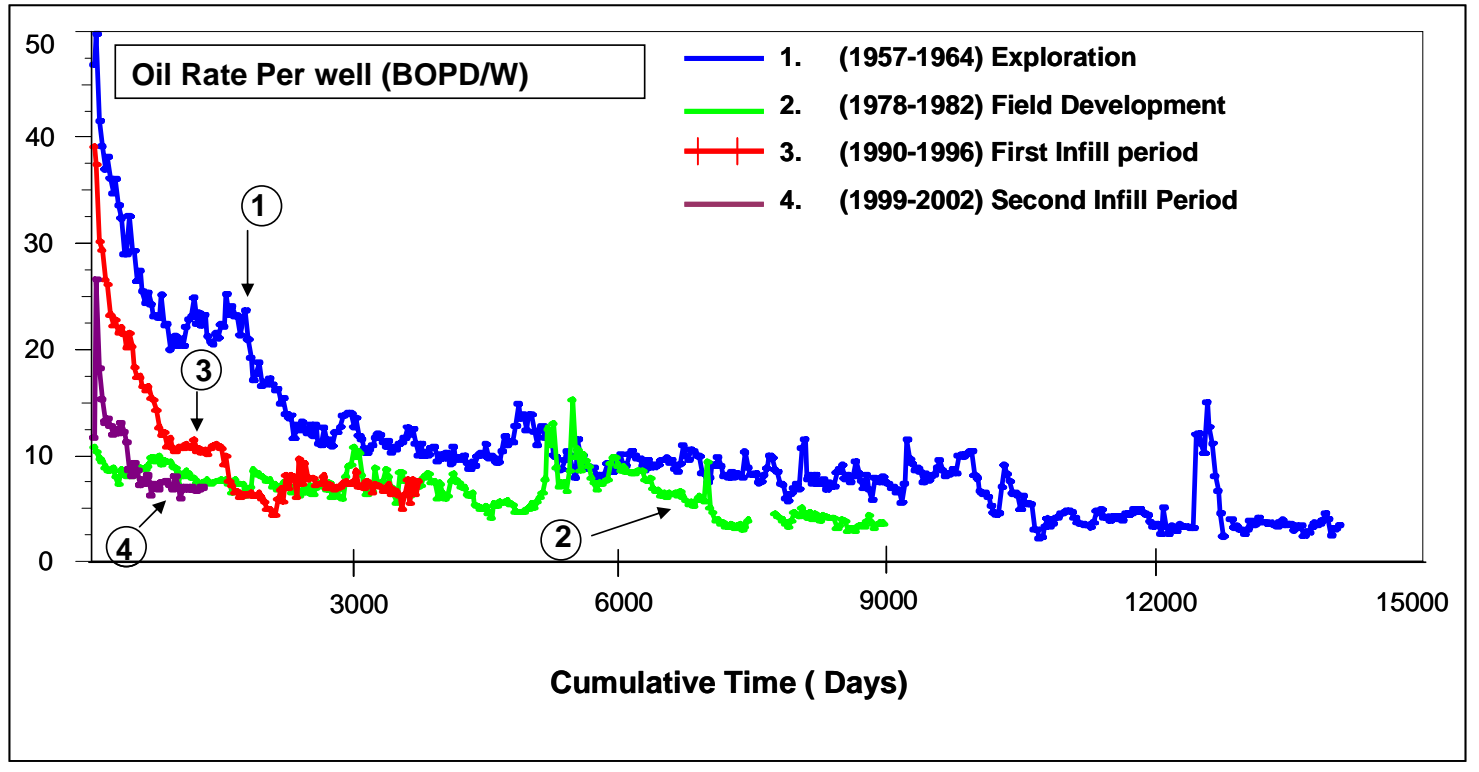

Fig. 1.4.28-Historical Oil Rate per well for Different Campaigns of wells during the Development of Germania Spraberry Unit.

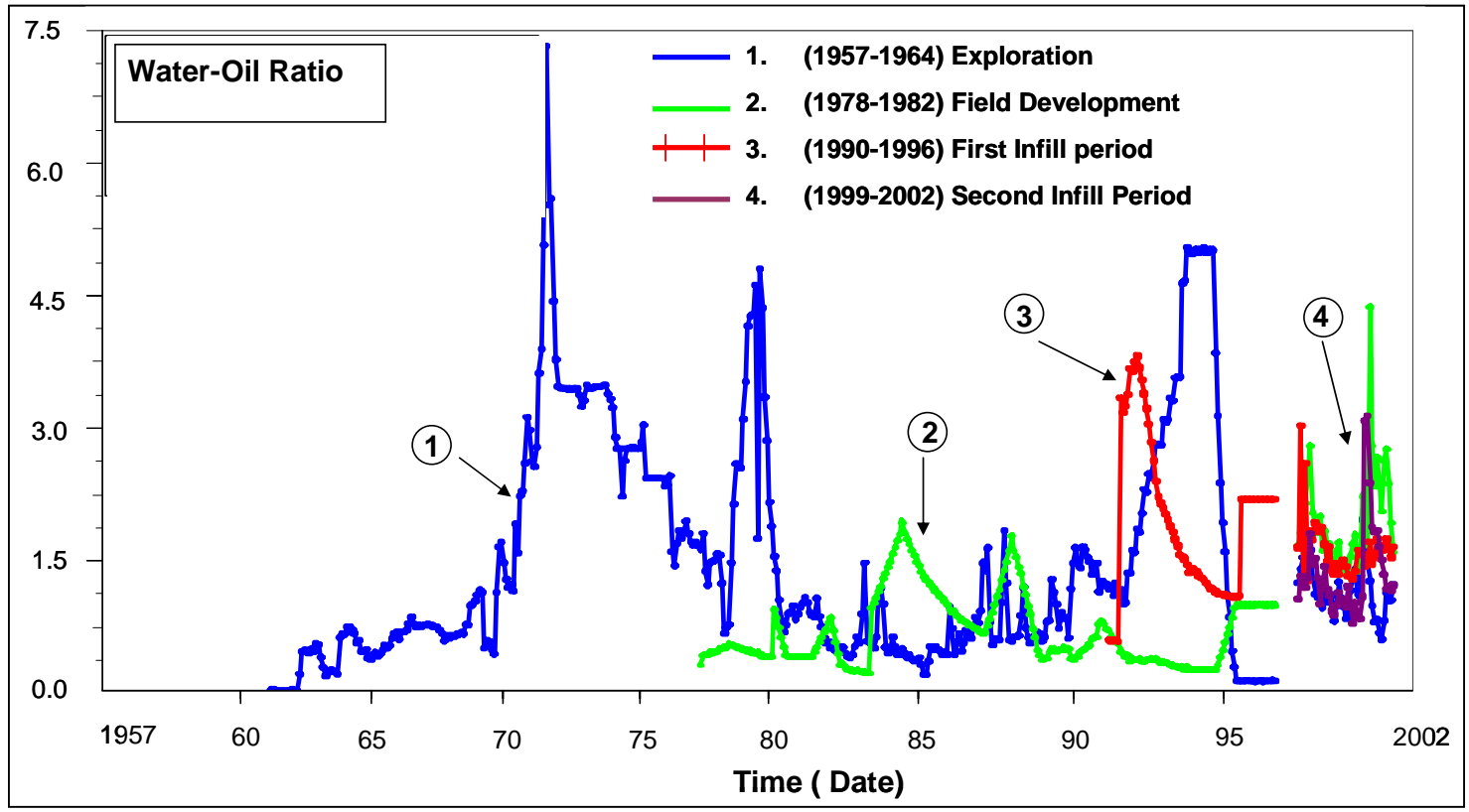

Fig. 1.4.29-Historical Water-Oil Ratio for Different Campaigns of wells during the Development of Germania Spraberry Unit. 


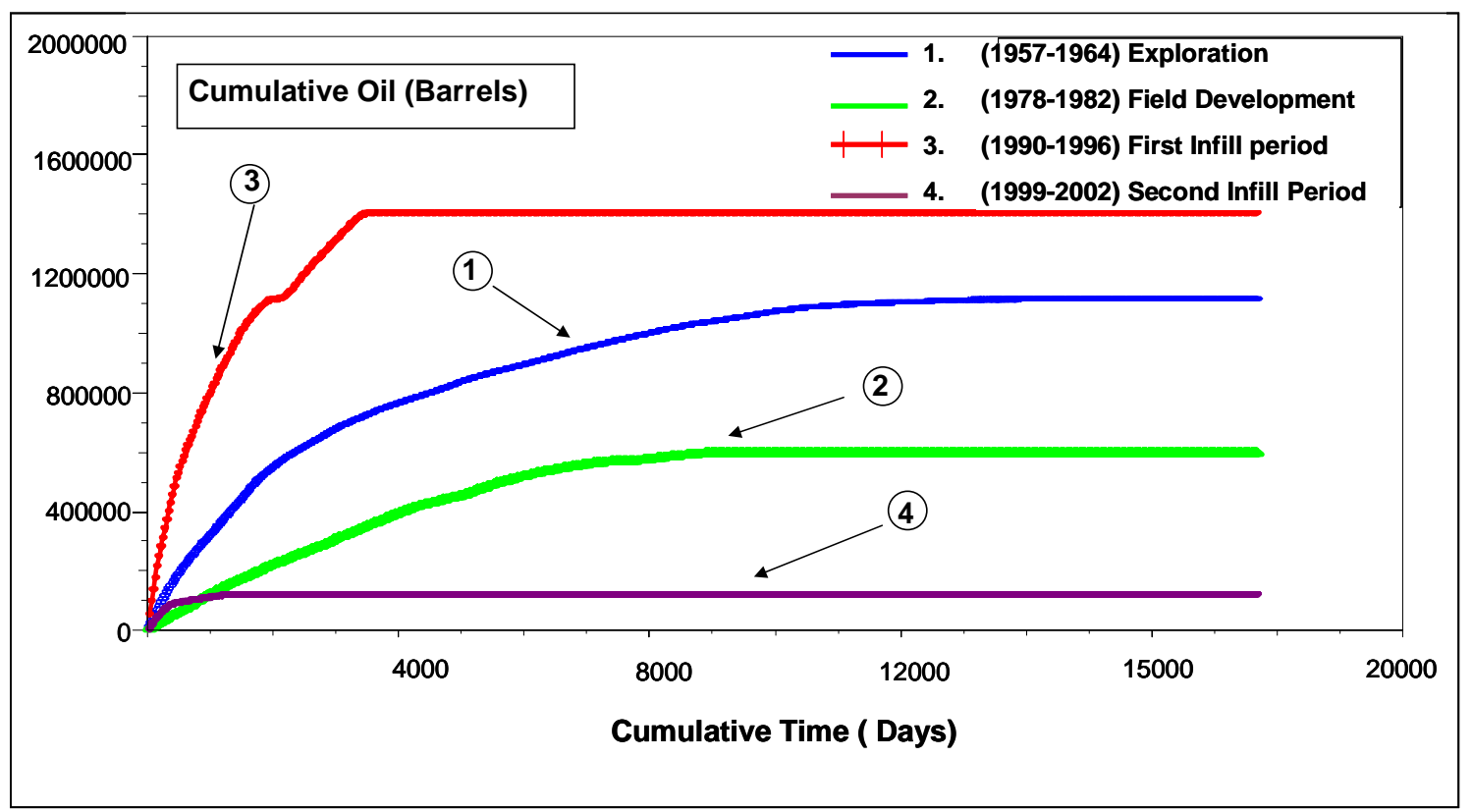

Fig. 1.4.30-Cumulative Oil Production for Different Campaigns of wells during the Development of Germania Spraberry Unit.

\subsection{Individual Tracts.}

The Germania Spraberry Unit, have been subdivided into 6 different areas (tracts). Each individual area was study and further comparisons among the different areas were made in this study. Fig. 1.4.31 shows the location and definition of the six different areas in Germania Spraberry Unit. 


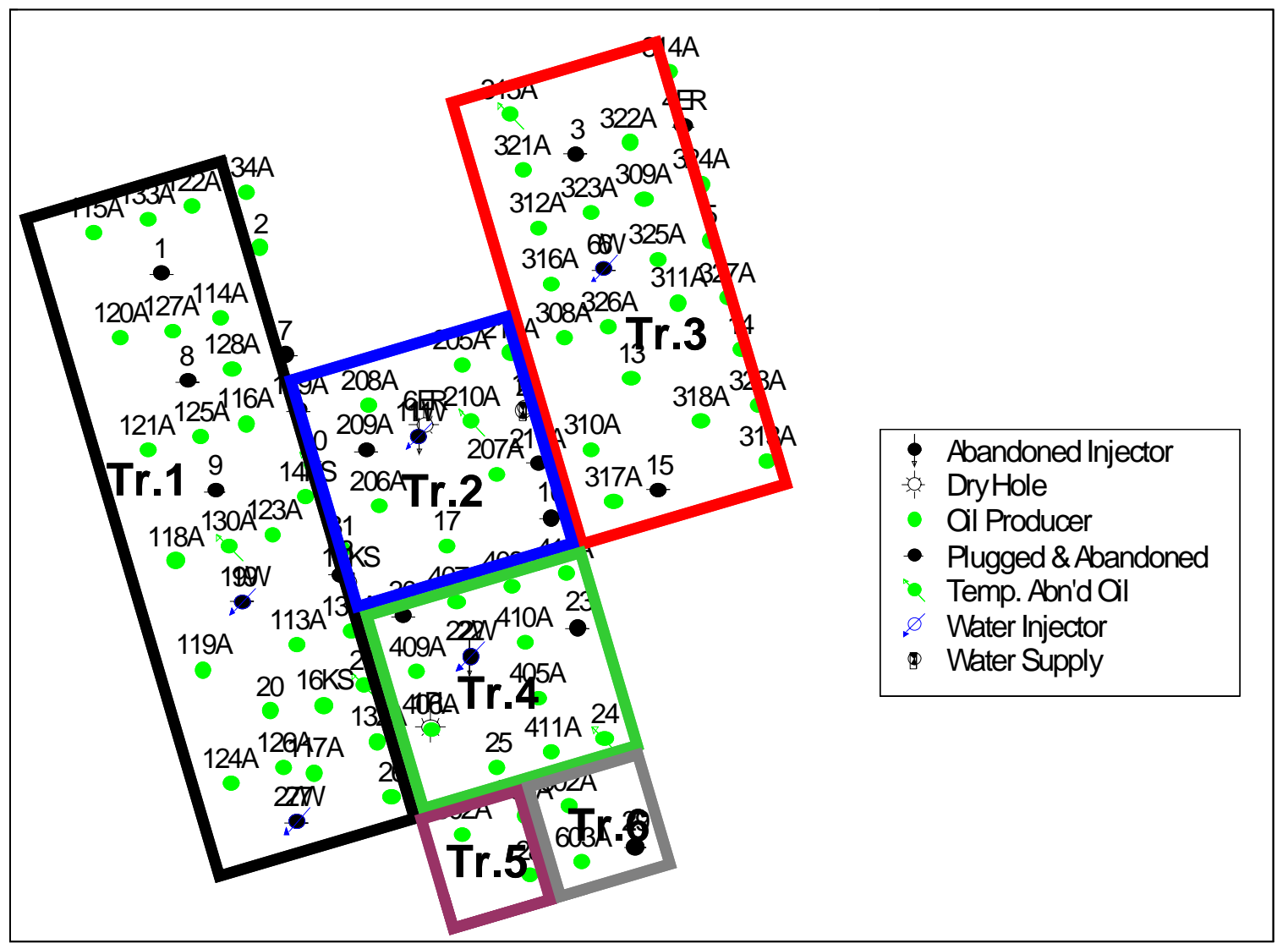

Fig.1.4.31-Location of Individual Tracts in Germania Spraberry Unit.

\subsubsection{Tract. 1}

Tract 1 comprises the largest area present in Germania Spraberry Unit. It has an area of 1874 acres and has been developed since the discovery of the unit in 1957. It is also the tract with the most producer wells (33). Water breakthrough in this tract occurred in 1963 (6 years after the initiation of the development of the field) and the water cut continued to grow up to 90 percent in 1992 because of the water injection response showed by some wells located in this area ( water injectors GSU-19 and GSU27 were located in this tract). As shown in Fig. 1.4.32, the production in this tract reached a maximum peak at 400 BOPD in 1993 and the average water cut have been 60 percent. As shown in Fig. 1.4.33, the development of this part of the reservoir has been mostly based on the increment of the number of producers through the 4 drilling campaigns. This area has a total of 33 wells 24 of which are currently active with a total oil production of 170 BOPD (37 percent of the oil currently being produced in the entire unit).

3 of the 5 largest producers of the unit are located in this area (well GSU-10, GSU-21, and GSU-26 which exhibit a cumulative oil production of 126,979; 159,771; 
and 159,157 respectively and have been active for a long period of time. As of June 2003, this area has a cumulative oil production of 1.425 million barrels which represents 43.25 percent of the total produced by the entire unit.

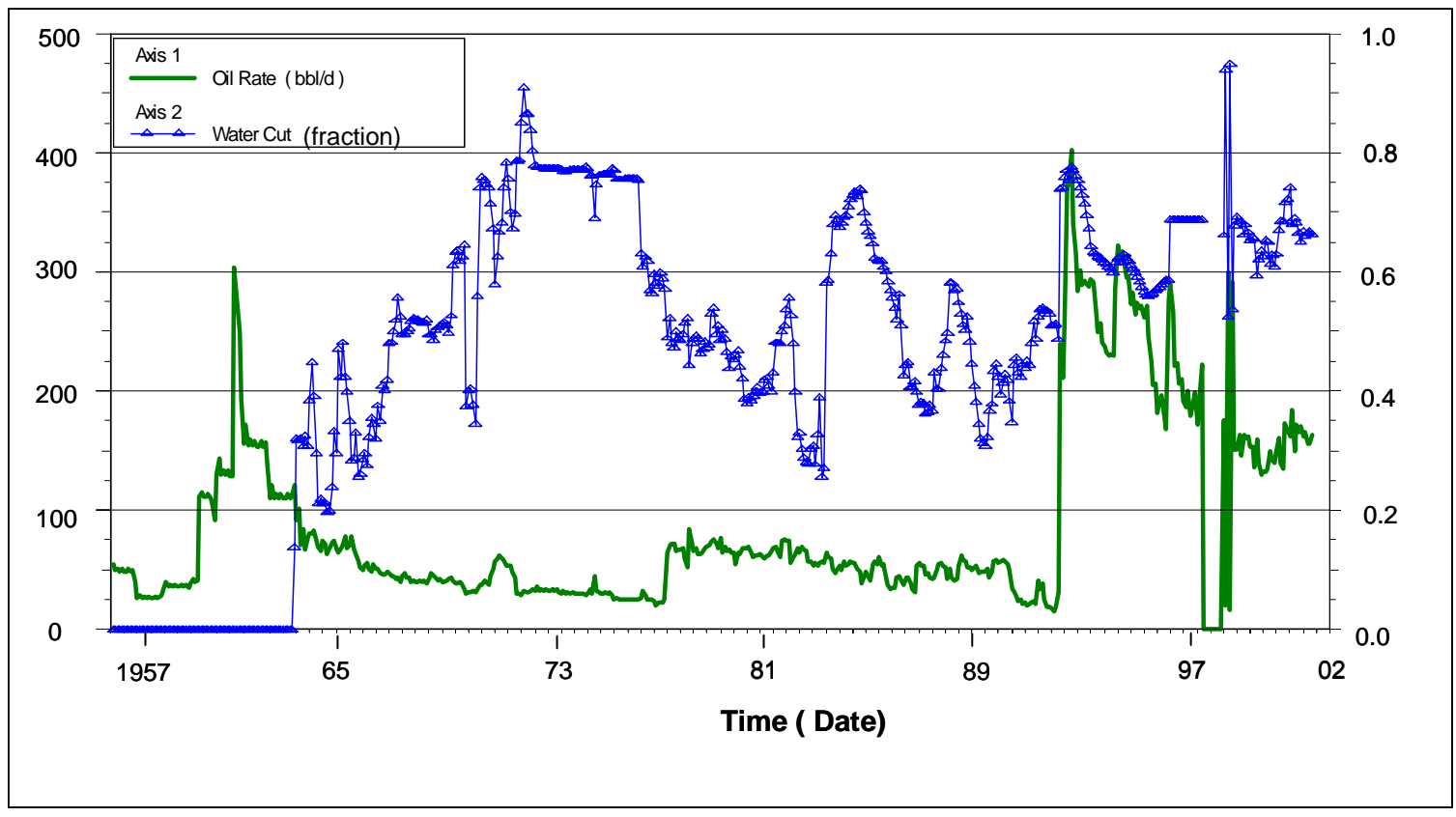

Fig.1.4.32-Oil Rate and Water Cut for Tract 1. (Germania Spraberry Unit.)

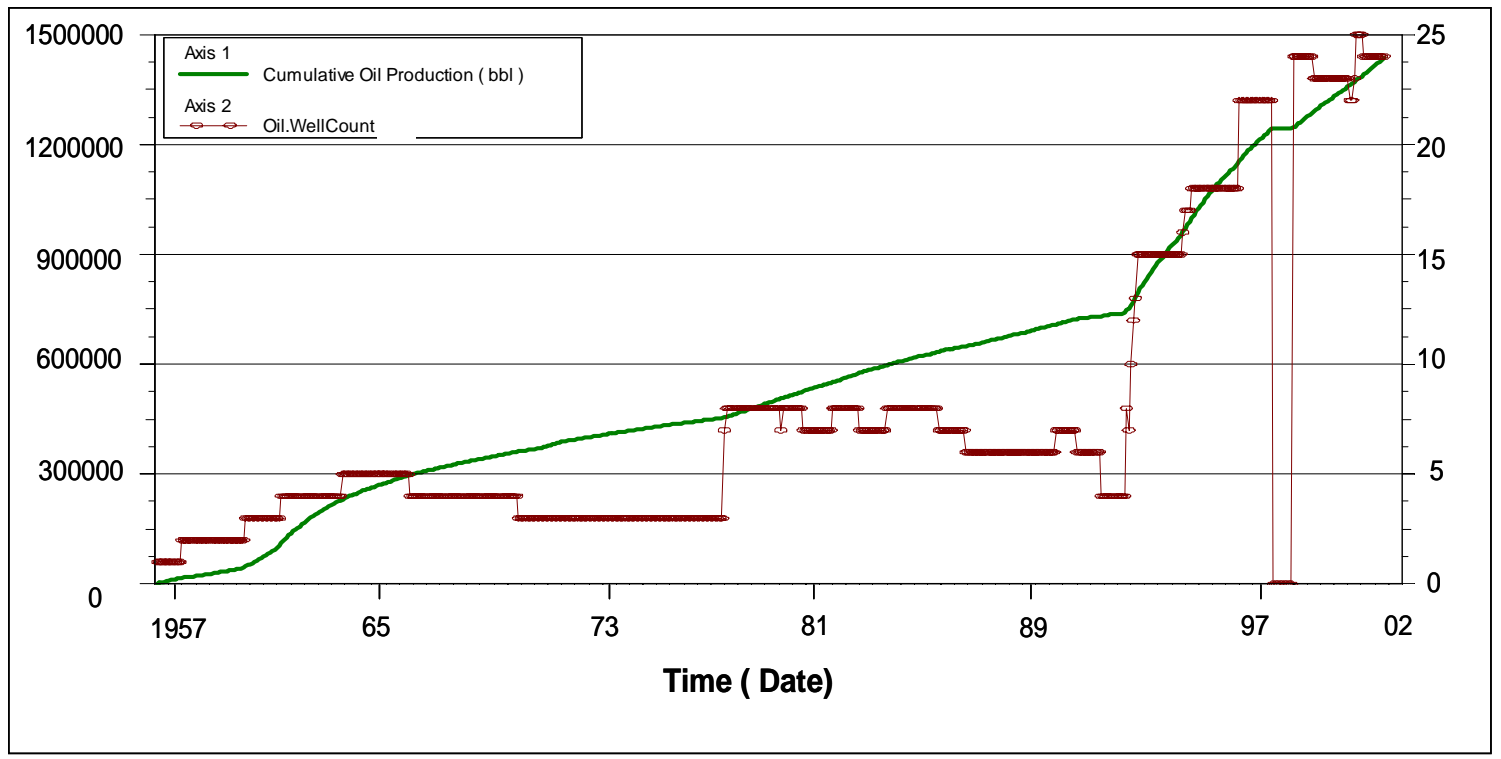

Fig.1.4.33-Cumulative Oil Production and Active wells for Tract 1. (Germania Spraberry Unit). 


\subsubsection{Tract. 2}

The tract 2 comprises an area of 663 acres and has been developed since the discovery of the unit in 1957. Water breakthrough in this tract occurred in 1963 (6 years after the initiation of the development of the field) and the water cut continued to grow up to 90 percent in 1971 because of the water injection response showed by some wells located in this area (water injector GSU-11 was located in the center of this tract). As shown in Fig. 1.4.34, the production in this tract reached a maximum peak at 170 BOPD in 1961 (before the waterflooding project was implemented) and the average water cut have been 60 percent. As shown in Fig. 1.4.35, the development of this part of the reservoir has been mostly based on the increment of the number of producers through the 4 drilling campaigns. This area has a total of 5 wells producing, with a total oil production rate of 38 BOPD (this represents only 7.8 percent of the oil currently being produced in the entire unit).

2 of the 5 largest producers of the unit are located in this area (wells GSU-16 and GSU-17 which exhibit a cumulative oil production of 117,414 and 177,119 respectively and have been active for a long period of time). As of June 2003, this area has a cumulative oil production of 0.622 million barrels which represents 19 percent of the total produced by the entire unit.

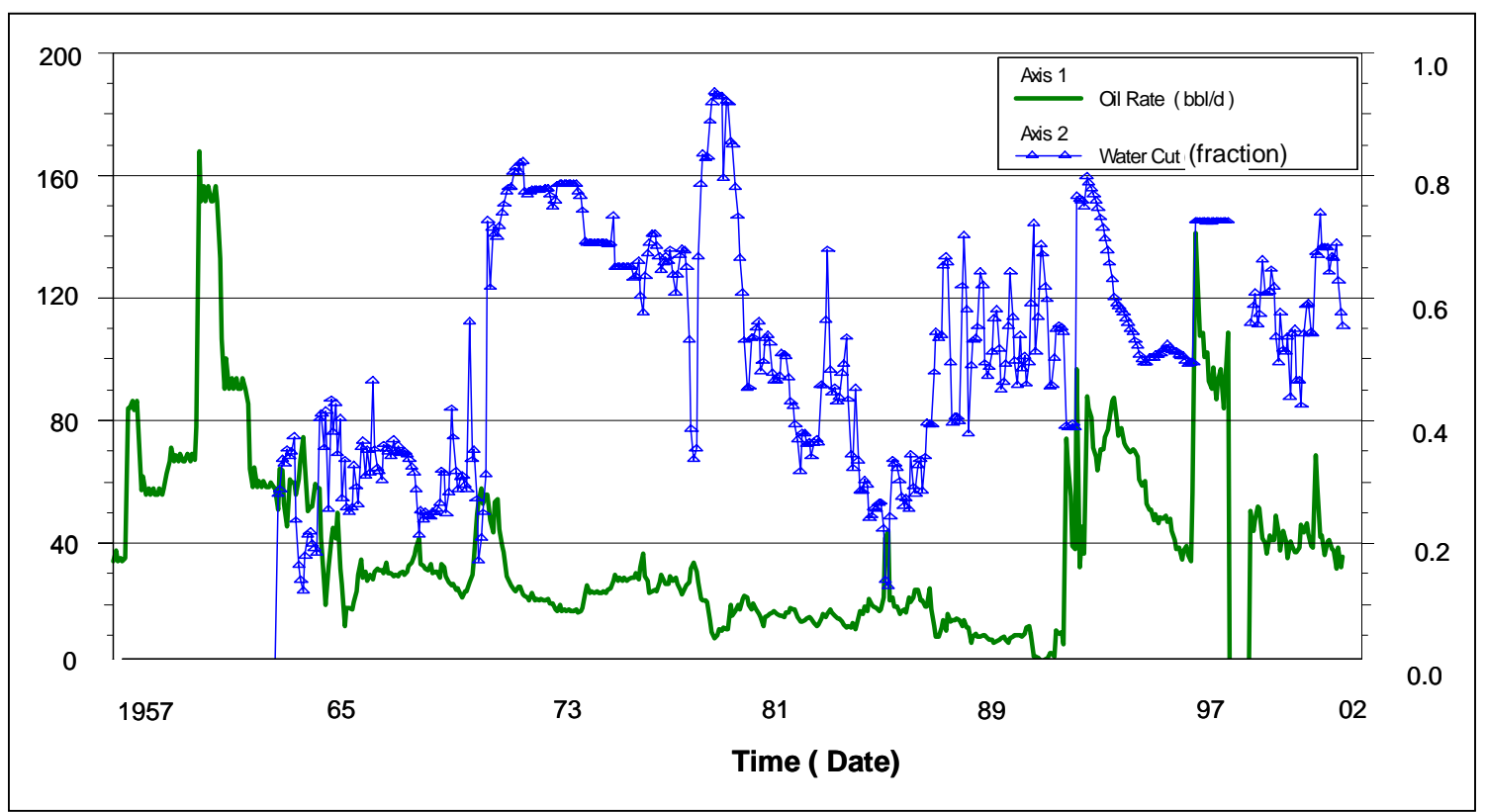

Fig.1.4.34-Oil Rate and Water Cut for Tract 2. (Germania Spraberry Unit.) 


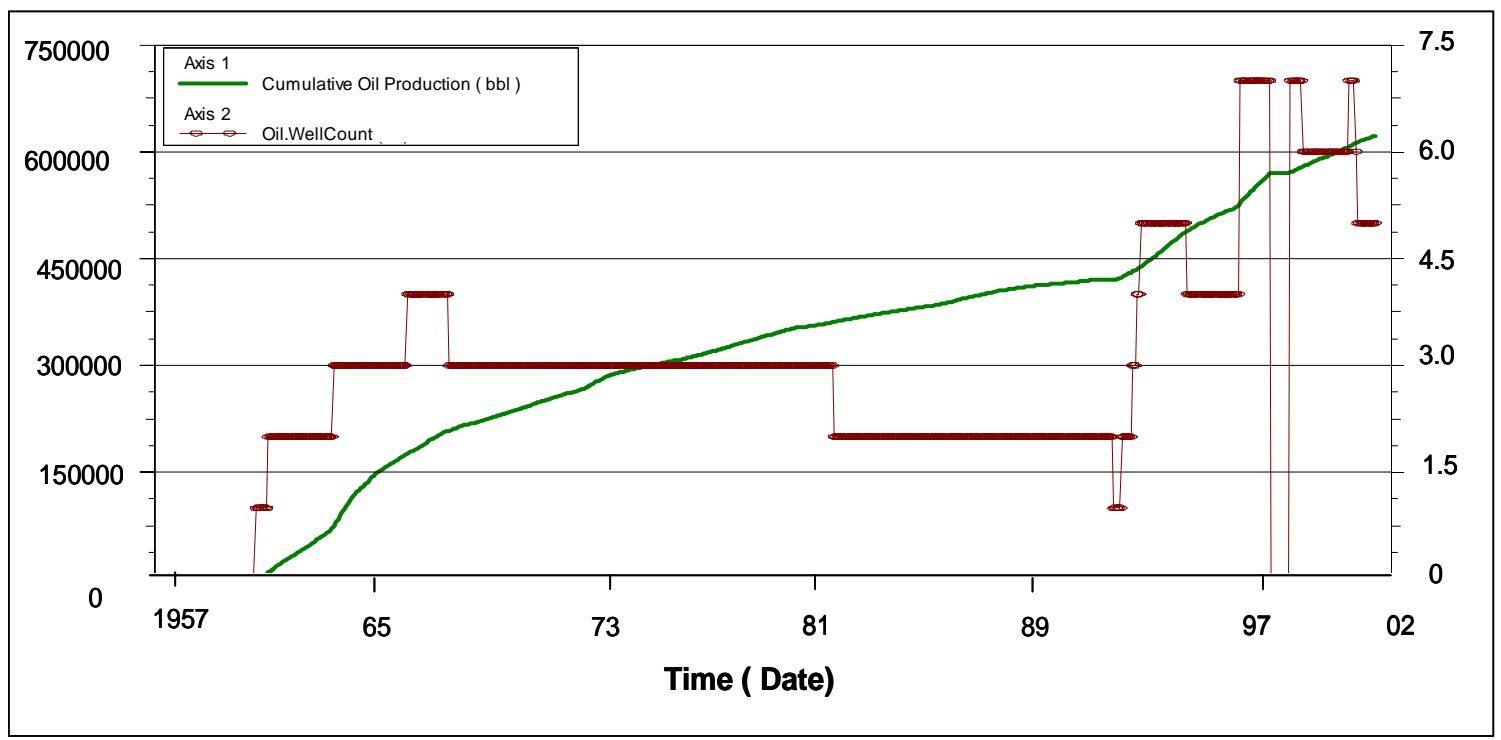

Fig.1.4.35-Cumulative Oil Production and Active wells for Tract 2. (Germania Spraberry Unit).

\subsubsection{Tract. 3}

The tract 3 comprises an area of 1345 acres and has been developed since the 1963 (6 years after the discovery of the unit). Water breakthrough in this tract occurred in 1963 and the water cut continued to increase up to 99 percent in 1971. The well responsible for the high water cut was the well GSU-6 located in the center of the tract (the only active well in tract 3 at that time). This well was later converted to water injector in 1971. As shown in Fig. 1.4.36, the production in this tract is currently about 195 BOPD (41.4\% of the total being produced in the entire unit) and the average water cut is 50 percent. As shown in Fig. 1.4.37, the development of this part of the reservoir has been mostly based on the increment of the number of producers through 3 drilling campaigns. This area has a total of 22 wells producing, with a total cumulative oil production of 0.579 million barrels (this represents 17.8 percent of the total produced in the entire unit).

Currently the central part of this tract is invaded by the water injected through the well GSU-6 (625,000 barrels of water injected) and the well GSU-11 located in tract 2 (760,000 barrels of water). 


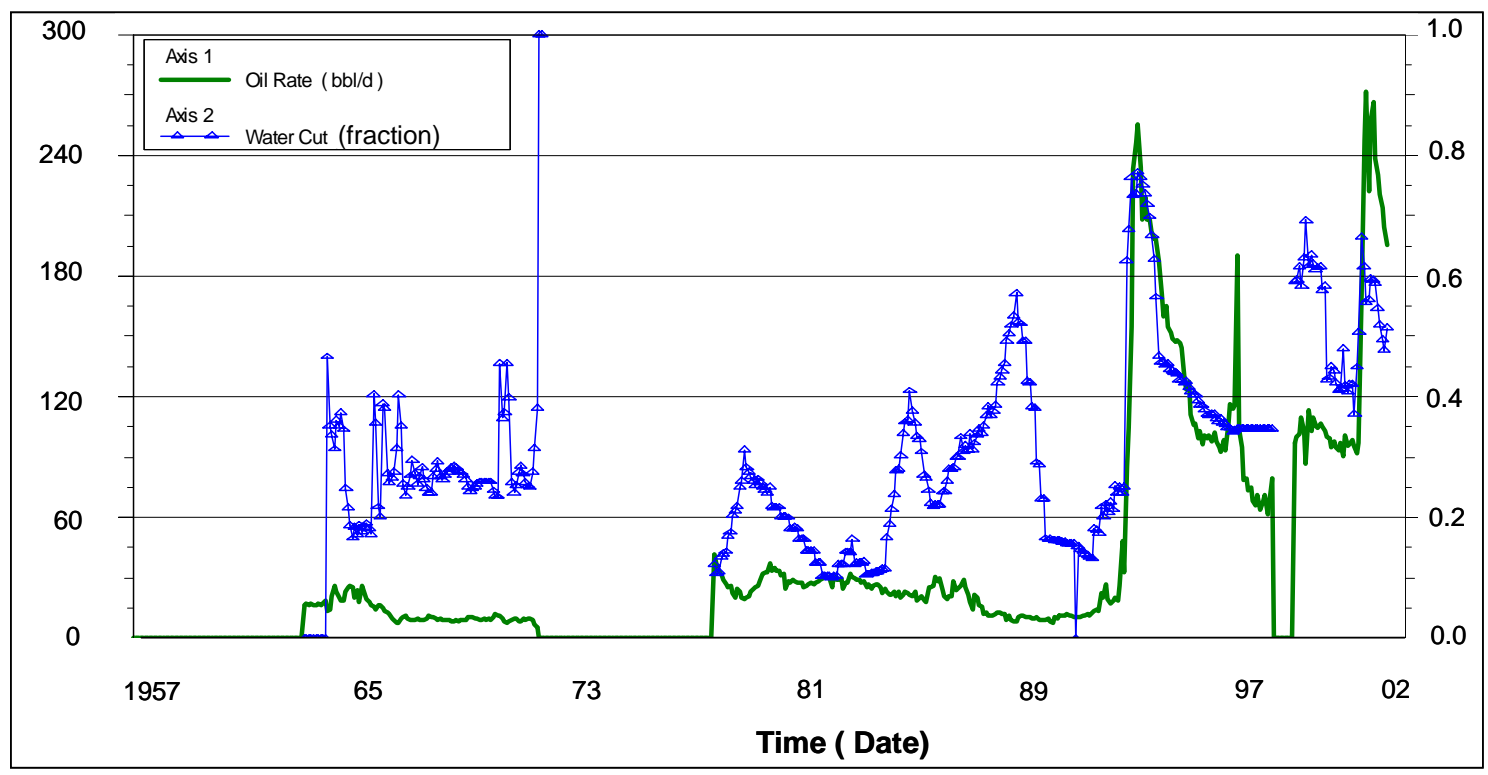

Fig.1.4.36-Oil Rate and Water Cut for Tract 2. (Germania Spraberry Unit.)

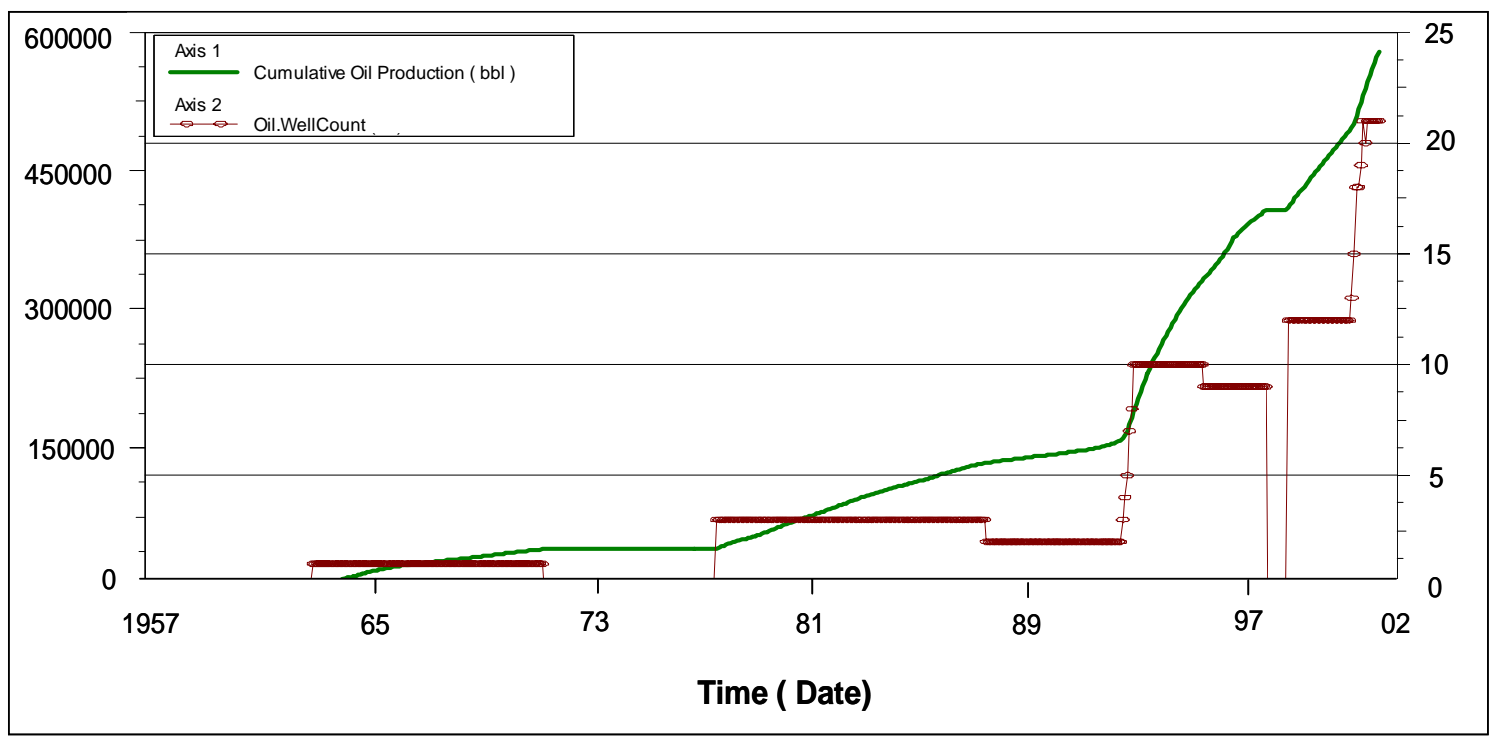

Fig.1.4.37-Cumulative Oil Production and Active wells for Tract 2. (Germania Spraberry Unit).

\subsubsection{Tract. 4}

The tract 4 comprises an area of 663 acres and has been developed since the discovery of the unit in 1957. Water breakthrough in this tract occurred in 1962 (5 years after the initiation of the development of the field) and the water cut continued to increase up to 99 percent in 1969. The well responsible for the high water cut was the well GSU-22 located in the upper corner of the tract. This well was converted to water 
injector in November 1971 and is still injecting water as part of the new injection pattern acting in the reservoir. As shown in Fig. 1.4.38, the production in this tract is currently about 50 BOPD (through 9 active wells) and the average water cut is 65 percent. As shown in Fig. 1.4.39, the development of this part of the reservoir has been mostly based on the increment of the number of producers through 4 drilling campaigns. This area has a total of 9 wells producing (out of a total of 14), with a total cumulative oil production of 0.446 million barrels (this represents 12.75 percent of the total produced in the entire unit).

Currently the central part of this tract is invaded by the water injected through the well GSU-22 (722,182 barrels of water injected).

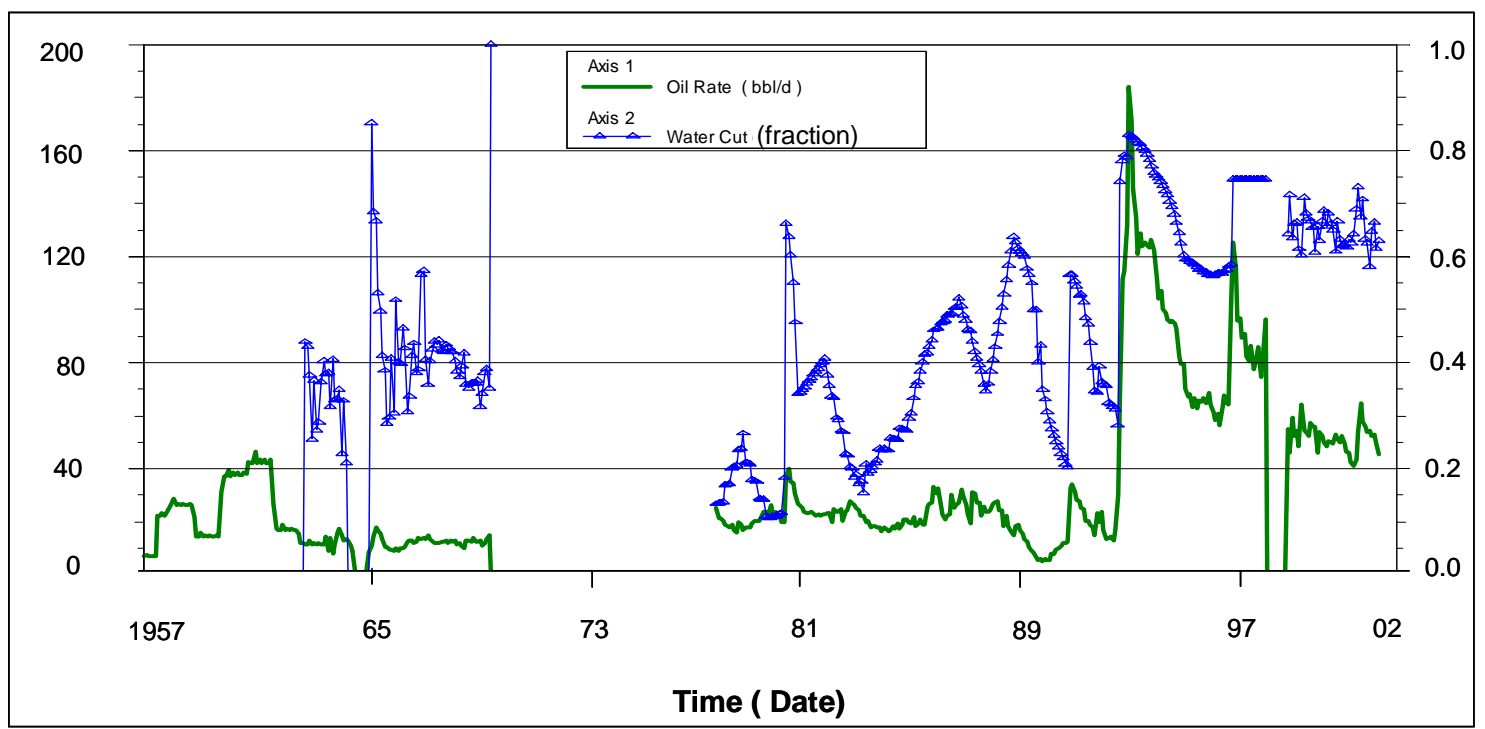

Fig.1.4.38-Oil Rate and Water Cut for Tract 3 (Germania Spraberry Unit).

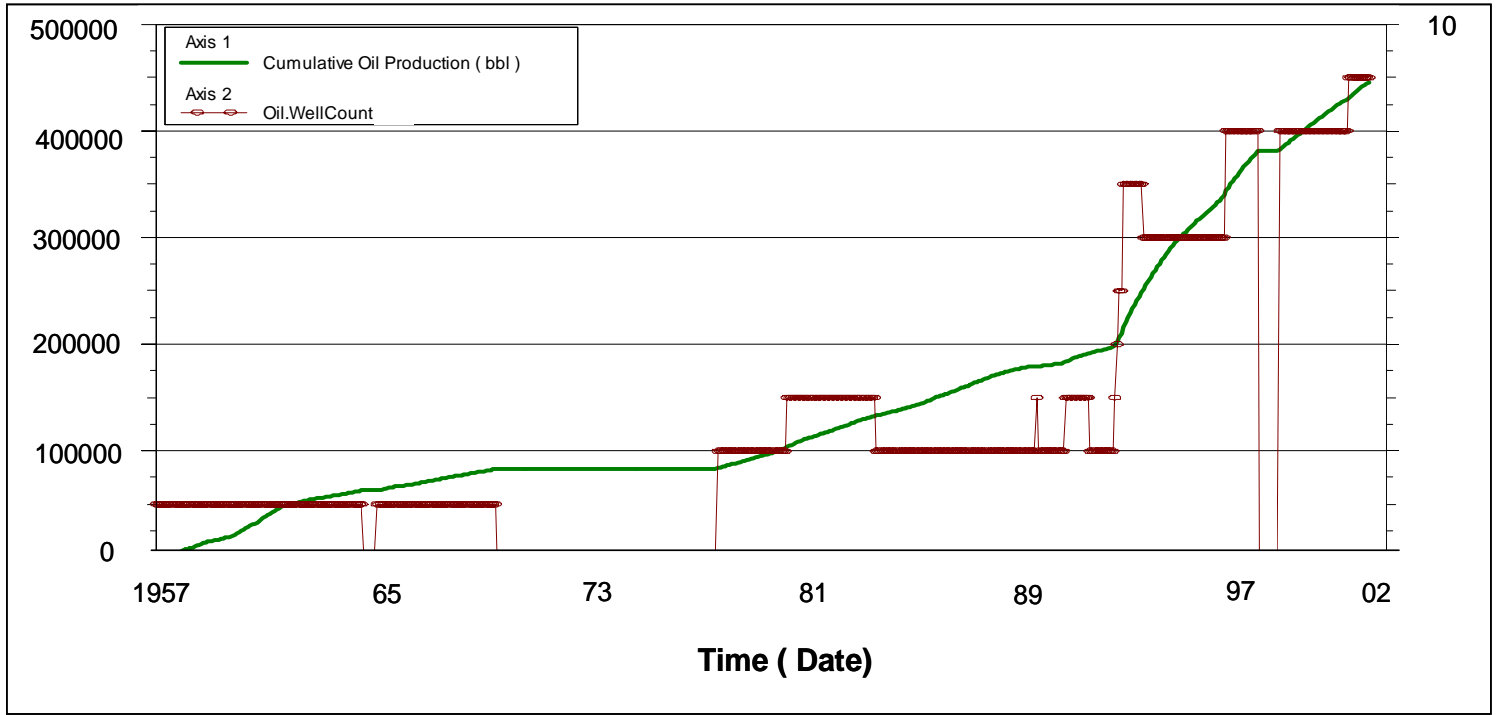

Fig.1.4.39-Cumulative Oil Production and Active wells for Tract 3. (Germania Spraberry Unit). 


\subsubsection{Tract. 5}

The tract 5 comprises an area of 166 acres and has been developed since the second drilling campaign in 1978. Water breakthrough in this tract occurred in 1985 and the water cut continued to increase up to 70 percent in 1988. As shown in Fig. 1.4.40, the production in this tract is currently about 12 BOPD (through 3 active wells) and the historical average water cut has been 55 percent. As shown in Fig. 1.4.41, the development of this part of the reservoir has been mostly based on the increment of the number of producers through 2 drilling campaigns. This area has only 3 wells producing and a total cumulative oil production of 0.098 million barrels (this represents only 3 percent of the total produced in the entire unit).

This tract has been developed only during the secondary stage of depletion and most of the water associated to the production of its well has been the result of the water injected in the tract 4 through the well GSU-22 (722,182 barrels of water injected).

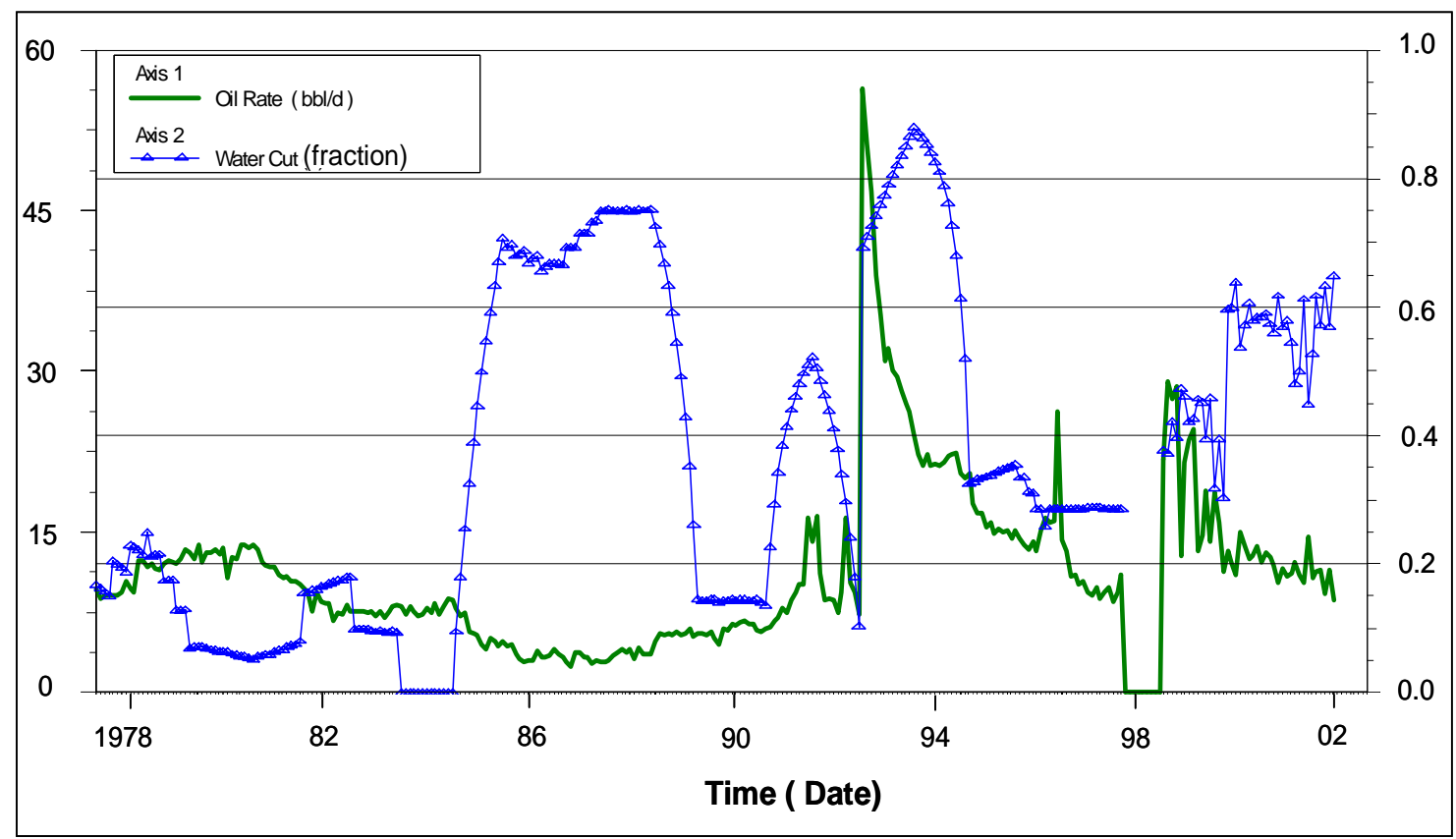

Fig.1.4.40-Oil Rate and Water Cut for Tract 5 (Germania Spraberry Unit). 


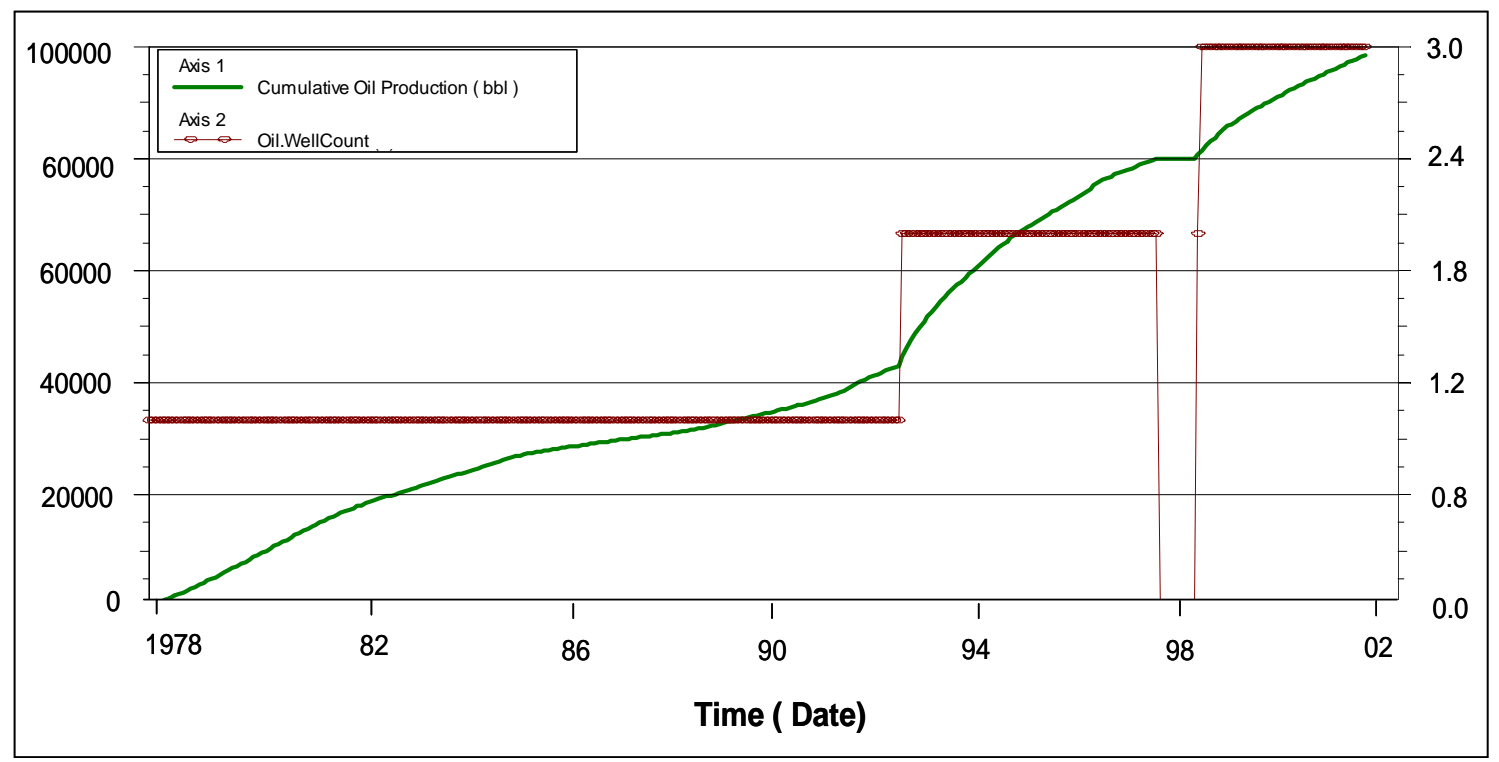

Fig.1.4.41-Cumulative Oil Production and Active wells for Tract 5 (Germania Spraberry Unit).

\subsubsection{Tract. 6}

The tract 6 comprises an area of 166 acres and has been developed since the second drilling campaign in 1978. Water breakthrough in this tract occurred in 1984 and the water cut continued to increase to 70 percent in 1987. As shown in Fig. 1.4.42, the production in this tract is currently 11 BOPD (through 2 active wells) and the historical average water cut has been 58 percent. As shown in Fig. 1.4.43, the development of this part of the reservoir has been mostly based on the increment of the number of producers through 2 different drilling campaigns. This area has only 2 wells producing and a total cumulative oil production of 0.062 million barrels (this represents only 1.9 percent of the total produced in the entire unit).

This tract has been developed only during the secondary stage of depletion and most of the water associated to the production of its well has been the result of the water injected in the tract 4 through the well GSU-22 (722,182 barrels of water injected).

The well GSU-29 has been the most responsible for the production in this tract (produced for 14 years) and then the wells GSU-602 and GSU-603 were completed to continue developing the tract. 


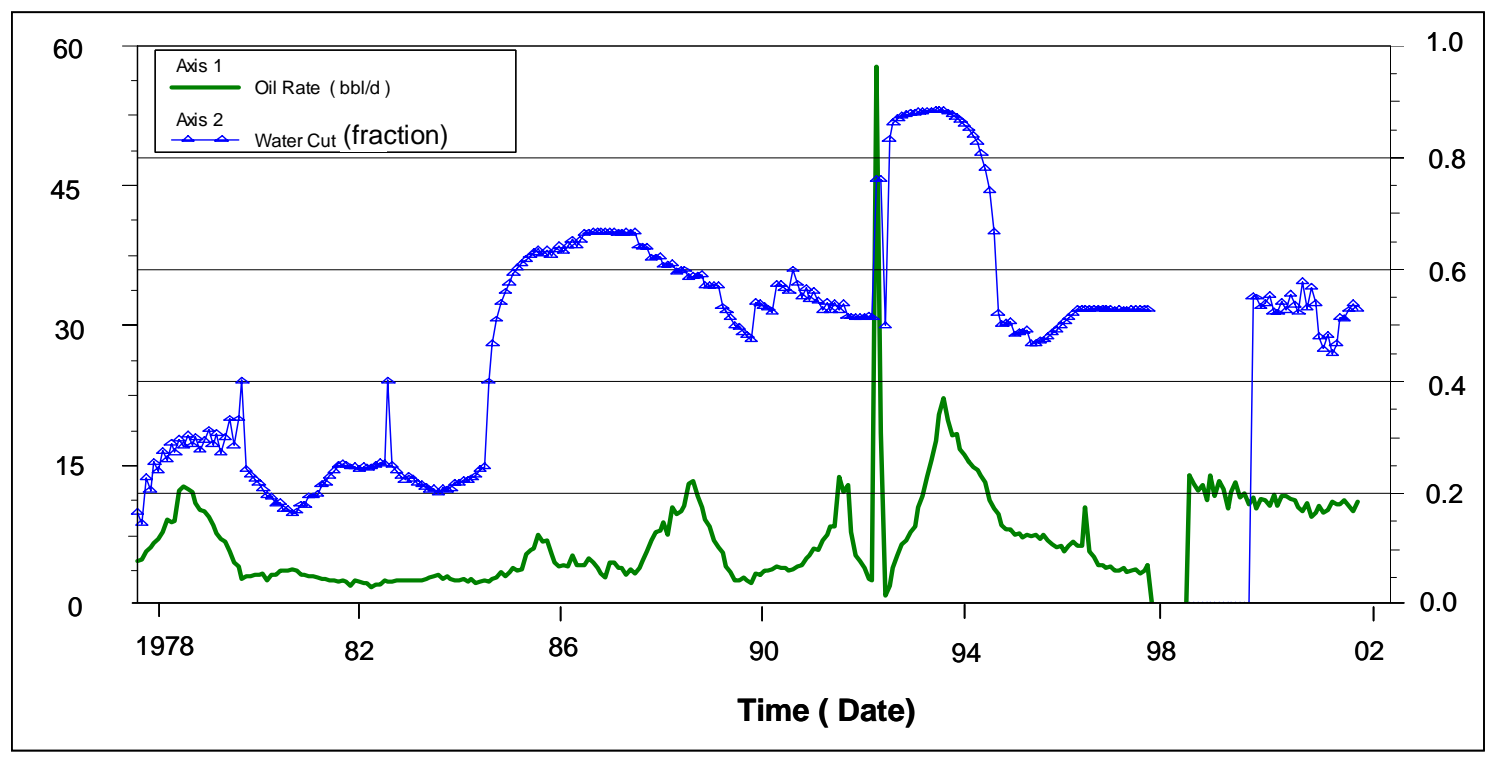

Fig.1.4.42-Oil Rate and Water Cut for Tract 6 (Germania Spraberry Unit).

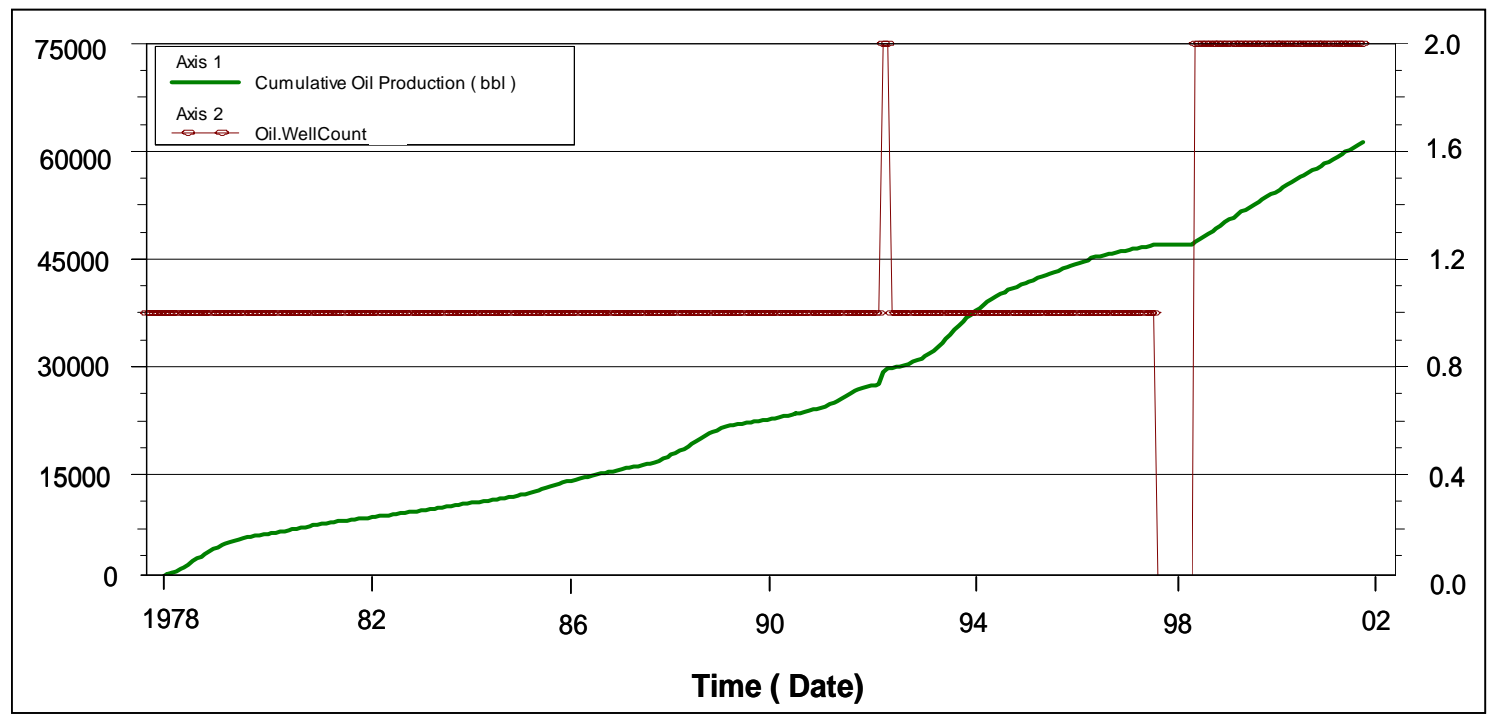

Fig.1.4.43-Cumulative Oil Production and Active wells for Tract 6 (Germania Spraberry Unit).

\subsubsection{Comparative Analysis for Tracts.}

Fig. 1.4.44 shows that tract 1 has the highest historical average oil rate (100 BOPD), and also has the most wells completed (a total of 40 producers have been completed in this tract.). As shown in Fig. 1.4.45, the average oil rate per well have been very similar in all tracts, being the tract 3 the one with the highest value of oil rate per well at last date $(9.2 \mathrm{BOPD} / \mathrm{W})$. All tracts have also shown the same rate of decline throughout the entire history of production of the unit. 
As shown in Fig. 1.4.46, in 1992 (when the injection was suspended), there was a considerable increment in the oil rate in all tracts (average rate of increment per tract was 280 BOPD), this is due to the third drilling campaign (first infill drilling period) performed in all tracts.

Table 1.4.6 indicates that tract 2 has exhibited the best performance in terms of cumulative oil produced per acre (938 barrels per acre); because of the response of the waterflood in this area. This also suggests that is the most drained area of the unit. Under waeterflooding period, the average cumulative produced per acre is 110 barrels. In the entire unit, the average cumulative oil produced per acre is 664 barrels. This is a very poor performance compared to the average of Spraberry (463 barrels of oil produced per acre) and is perhaps and indication of the potential opportunity to increase the recovery in Germania Spraberry Unit.

As shown in Fig. 1.4.47, the water-oil ratio, showed a value of 15 in tract 2 in 1979, as a consequence of the response of the water injected through well 6W (located in tract.3).The water-oil ratio, also showed a high value (19) in tract 1 in 1999 when the average water cut in this tract was 90 percent and the numbers of active wells increased from zero to 23. Tracts 3, 4, 5, and 6 have shown an historical average water-oil ratio of 3 , indicating a uniform drainage in all these tracts.

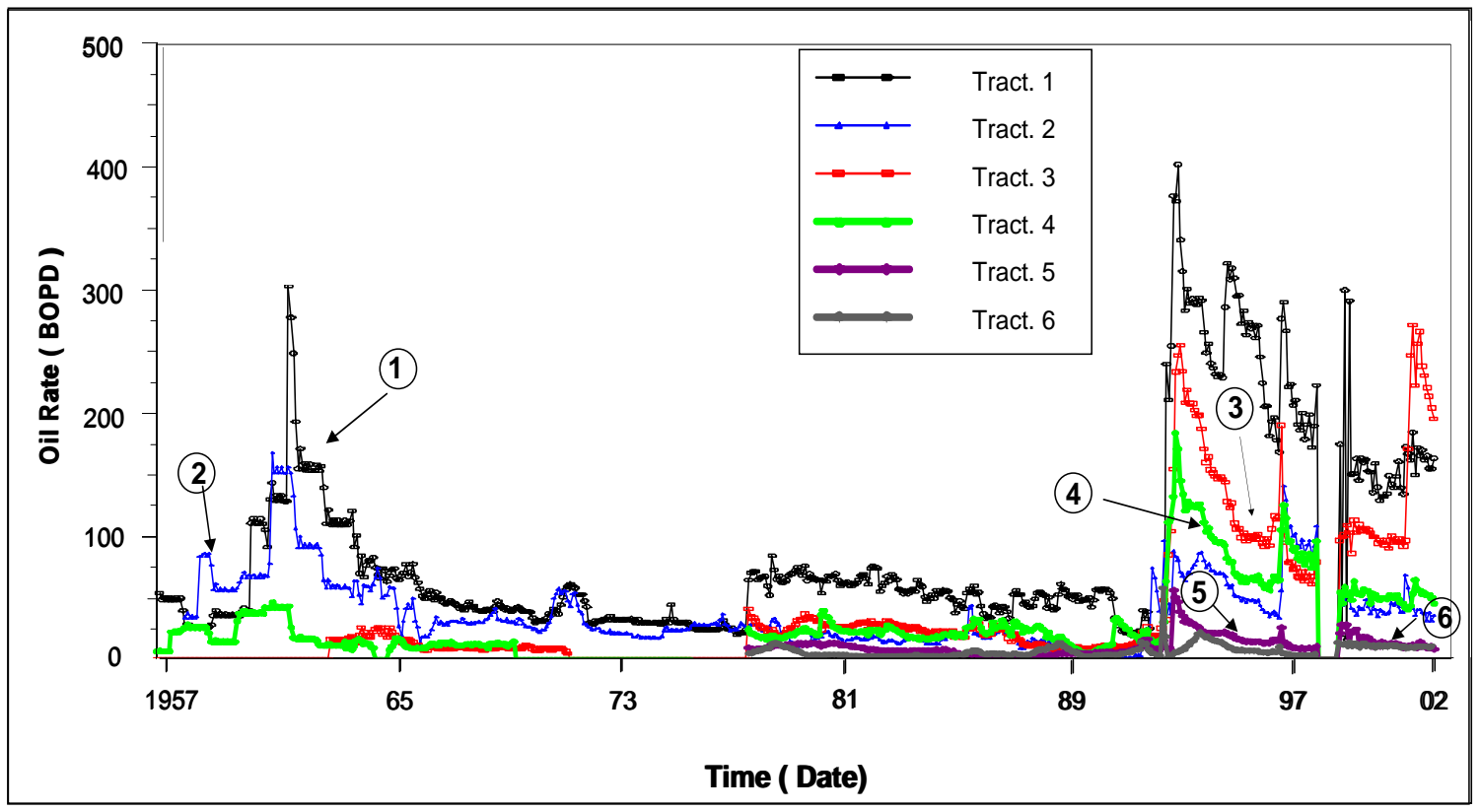

Fig.1.4.44-Historical Oil Rate for Different Tracts of Germania Spraberry Unit. 


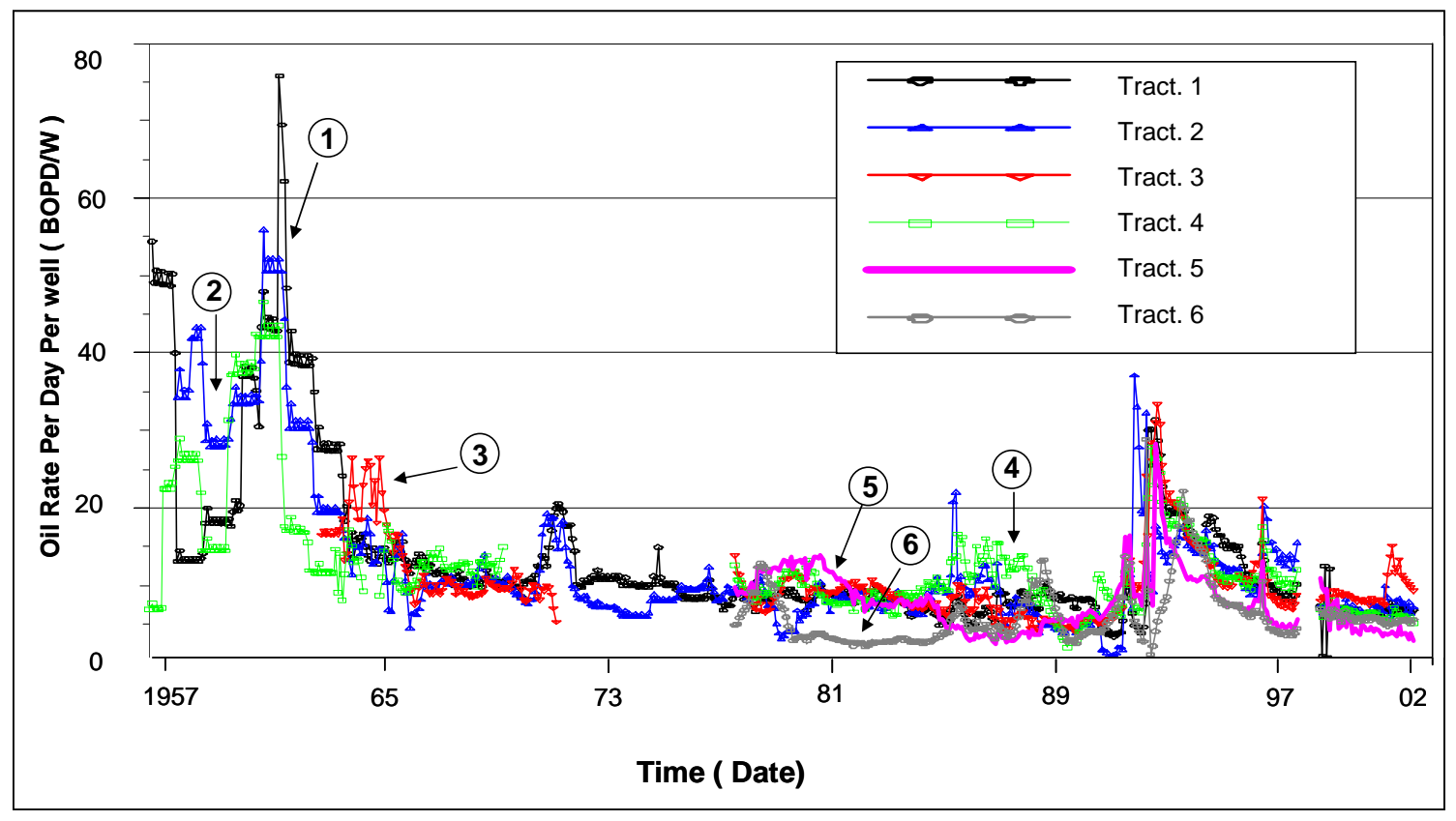

Fig.1.4.45-Historical Oil Rate per well for Different Tracts of Germania Spraberry Unit.

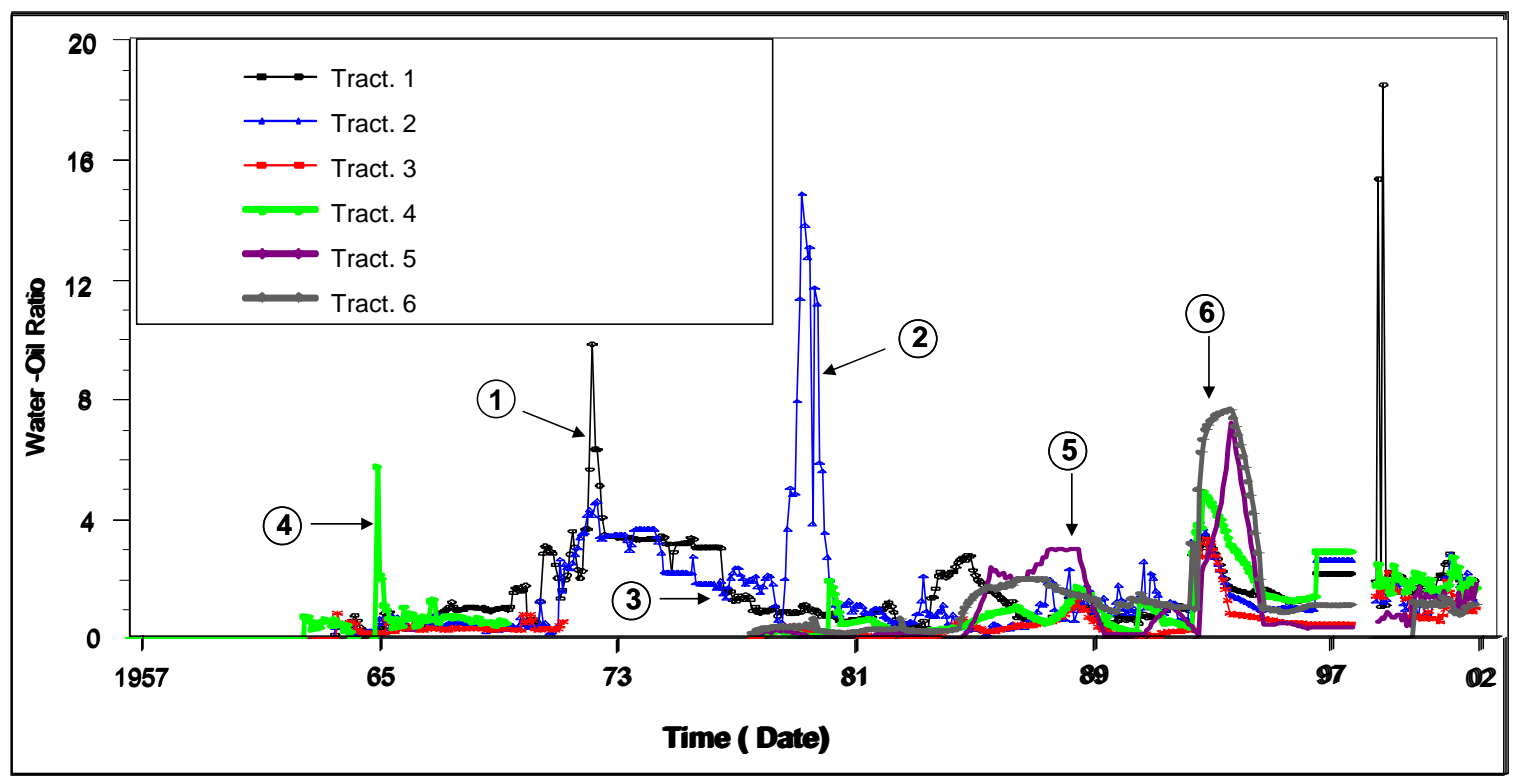

Fig.1.4.46-Historical Water-Oil Ratio for Different Tracts of Germania Spraberry Unit. 
Table 1.4. 6 Oil Recovery for Different Tracts of Germania Spraberry Unit.

\begin{tabular}{|c|r|c|c|c|c|c|}
\hline Tract. & $\begin{array}{c}\text { Area } \\
\text { (Acres) }\end{array}$ & $\begin{array}{c}\text { No. of Wells } \\
\text { (Producers) }\end{array}$ & $\begin{array}{c}\text { Cum. Oil Production } \\
\text { (Before Waterflooding) } \\
\text { (MMBbls) }\end{array}$ & $\begin{array}{c}\text { Cum. Oil Production } \\
\text { (After Waterflooding) } \\
\text { (MMBbls) }\end{array}$ & $\begin{array}{c}\text { Total Oil Cum. } \\
\text { (MMBbls) }\end{array}$ & Cum. Oil Per Acre \\
(Bbls)
\end{tabular}

\subsection{Well Performance Monitoring System.}

The monitoring system was designed to systematically develop a comprehensive picture of how each well is performing. Several tools are used and combined to understand the performance of the wells in the unit for evaluating trends and identifying anomalies in some of them. The performance plots are generated for each well then analyzed individually and as a group to develop a complete picture of each performance. After a potential problem is identified, the potential increase in production through remedial action is estimated. Wells that do not show signs of anomalies should be left to produce uninterrupted, but continue to be monitored on a monthly basis using the type of plots shown in this study. These are customized plots developed for routine performance monitoring of oil wells and can be used by operation personnel responsible for the day to day operation and maintenance of Germania Spraberry Unit.

This study presents a methodology which can be used to quickly evaluate and diagnose mechanisms and represents an effective tool for the selection of water control treatment and workover candidates. It mainly uses plots generated from available production history data.These plots can be automatically generated using the database and variables constructed in Oil Field Manager (OFM) for Germania Spraberry Unit.

A description of each type of plot constructed is given below.

\subsubsection{Water Control Diagnostic Plots}

Based on numerical simulation studies on reservoir water coning and channeling, it was discovery that log-log plots of water-oil ratio vs. time show different characteristic trends for different mechanisms. The time derivatives of WOR were found to be capable of differentiating whether the well is experiencing water coning, high permeability layer breakthrough or near wellbore channeling ${ }^{3}$. The desire to define different type of excessive water production problems has always been an important issue in Germania Spraberry Unit because in this area many wells have been pre-maturely abandoned as a result of very high water production (due to normal displacement of the water being injected) or casing failures (due to the corrosive nature of San Andreas water). In 
general, there are three basic classifications of the problems. Water coning, multilayer channeling and near wellbore problems are the most noticeable among others ${ }^{6}$.

Very often, a near wellbore problem could suddenly occur during a normal displacement and production ${ }^{6}$. Figs. 1.4.47-54, show the typical behavior for wells experiencing near wellbore water channeling. In all these wells, the initial WOR was constant and above 1.The WOR rapidly increased and followed a linear slope after the implementation of the waterflood. Then, the WOR increased and the slope went above 100.

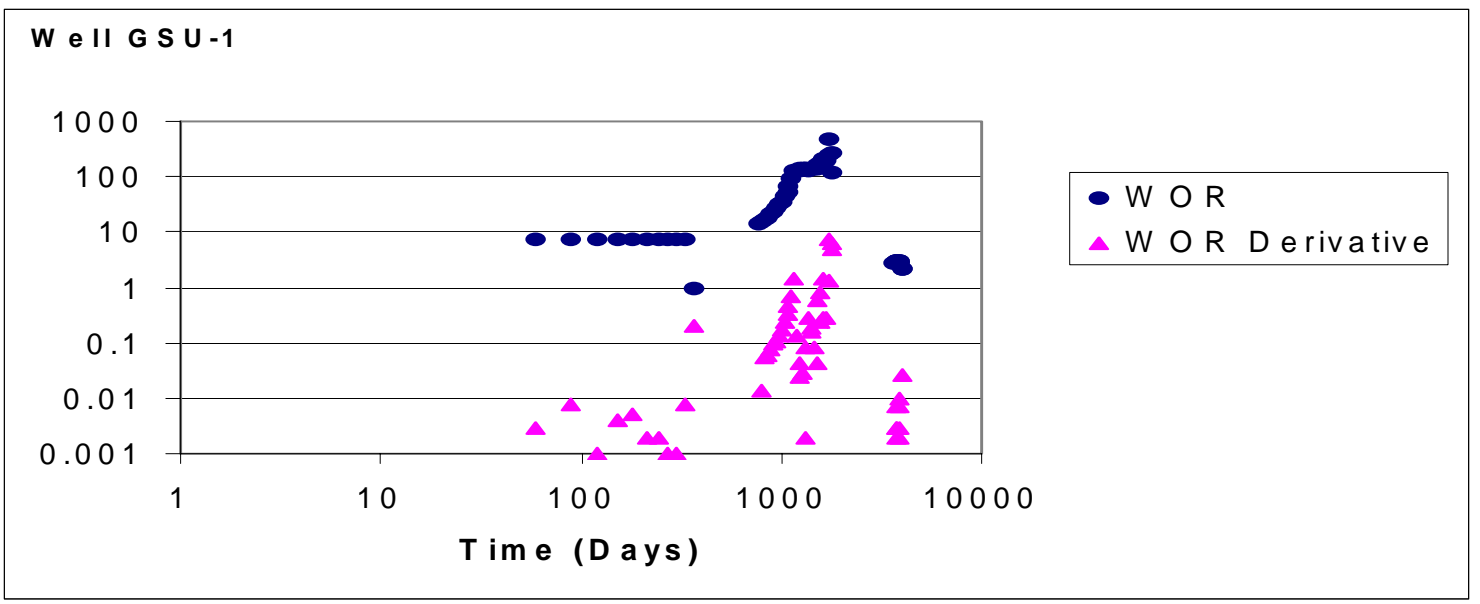

Fig.1.4.47-WOR and WOR Derivative for well GSU-1: Experiencing Near Wellbore Water Channeling.

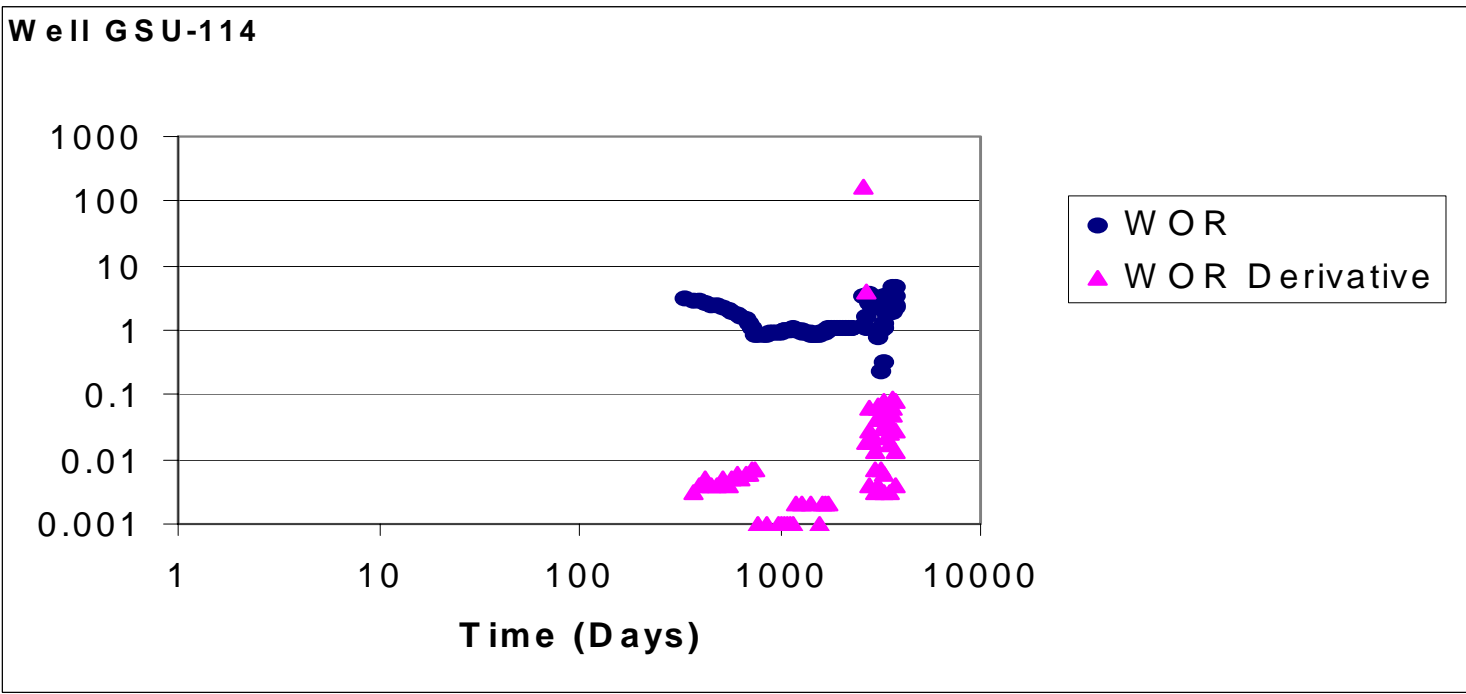

Fig. 1.4.48-WOR and WOR Derivative for well GSU-114: Experiencing Near Wellbore Water Channeling. 


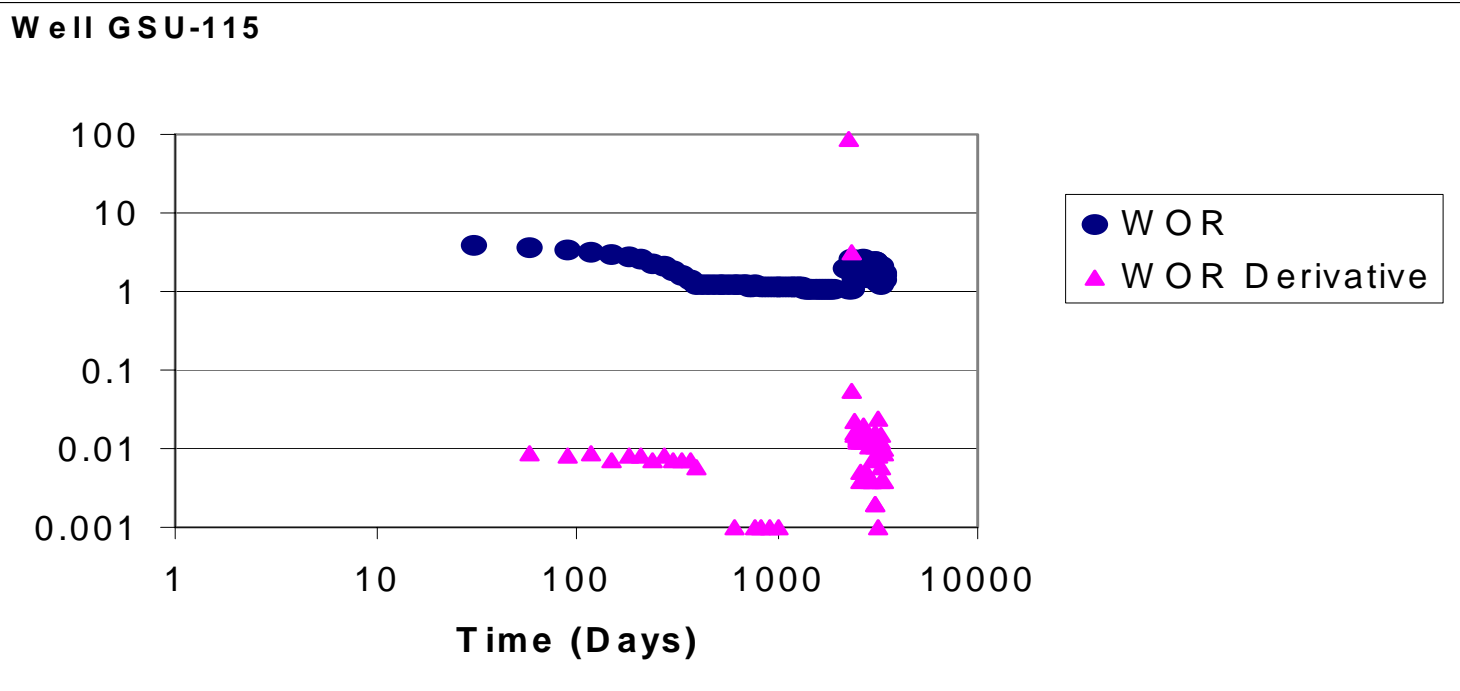

Fig. 1.4.49-WOR and WOR Derivative for well GSU-115: Experiencing Near Wellbore Water Channeling.

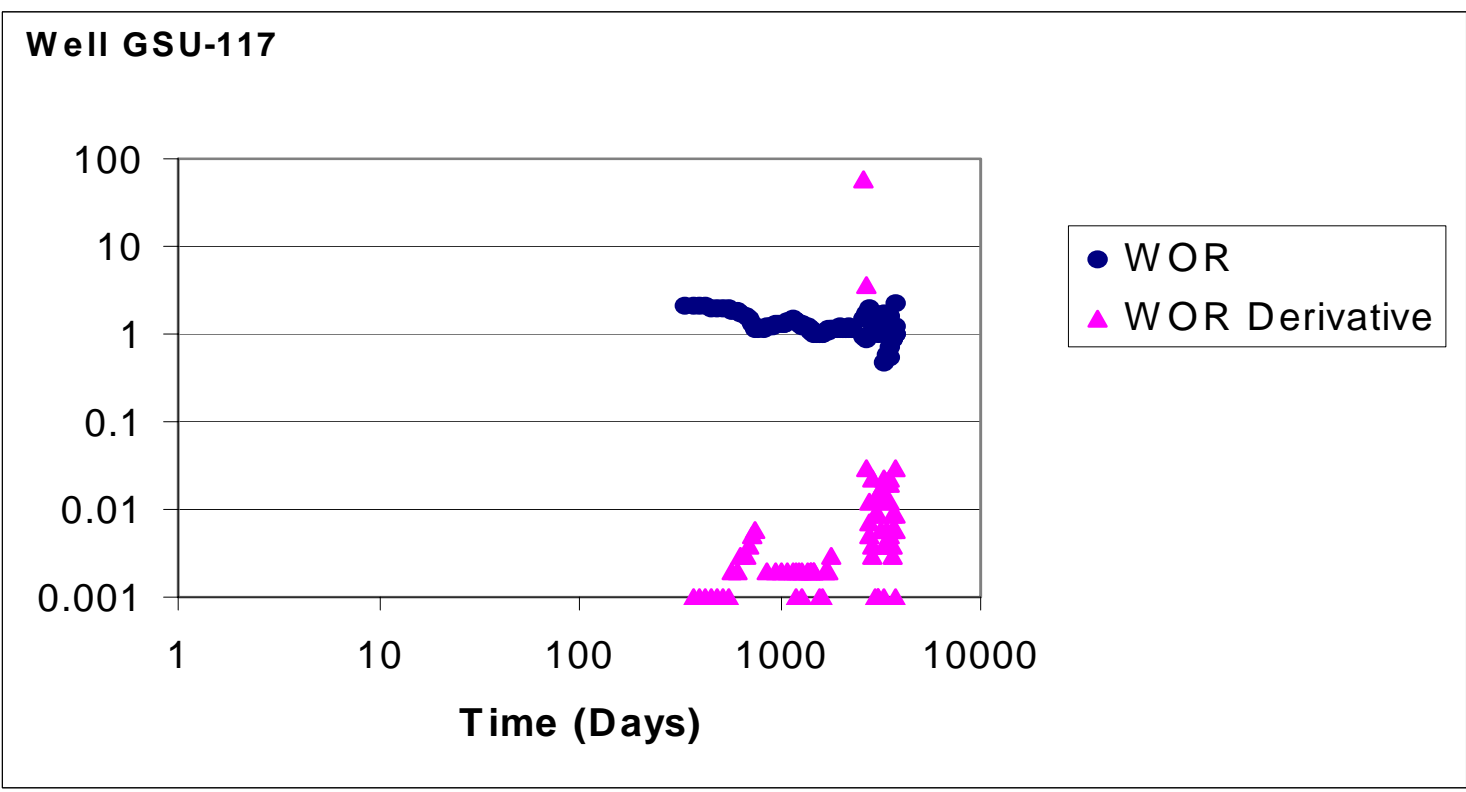

Fig. 1.4.50-WOR and WOR Derivative for well GSU-117: Experiencing Near Wellbore Water Channeling. 


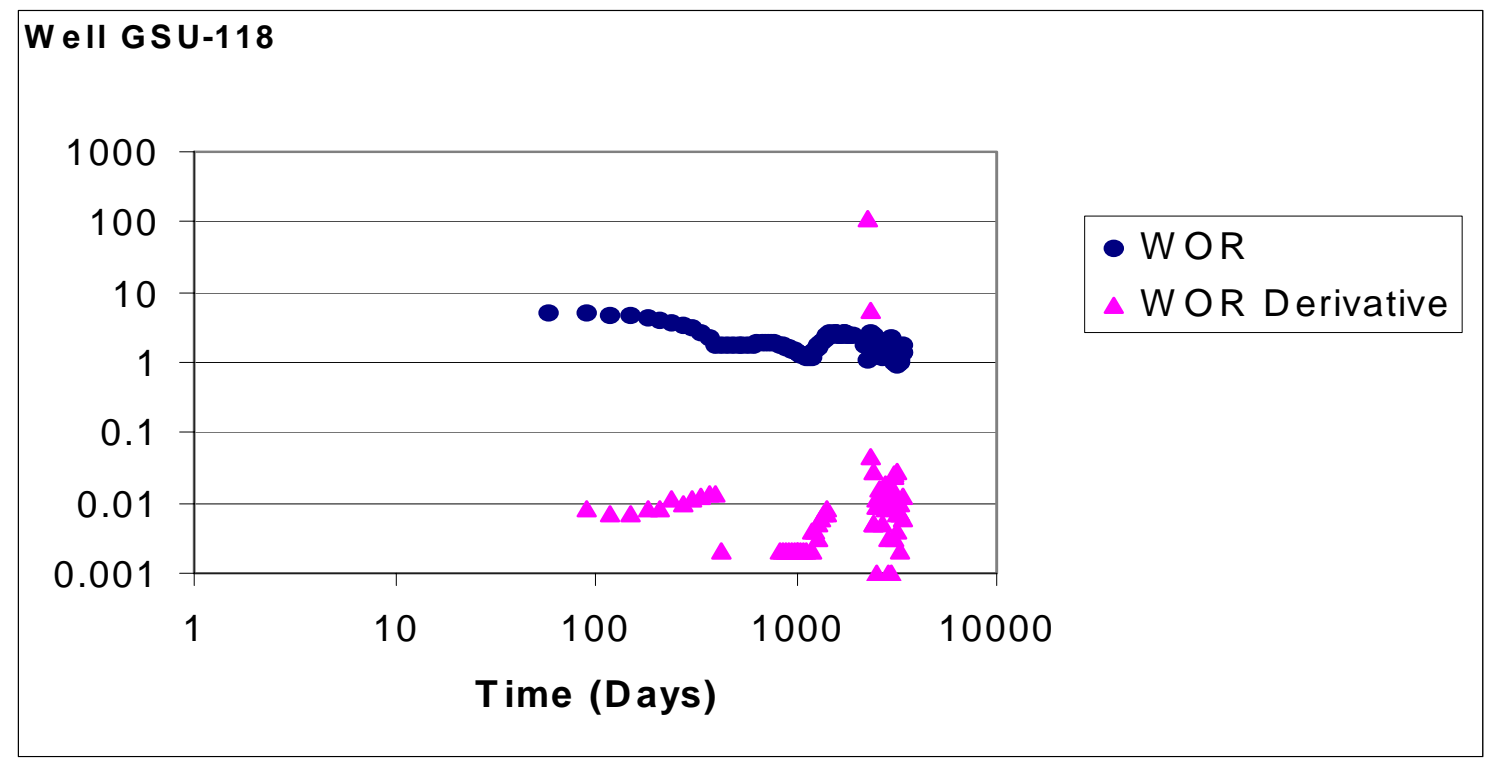

Fig. 1.4.51-WOR and WOR Derivative for well GSU-118: Experiencing Near Wellbore Water Channeling.

Well GSU-119

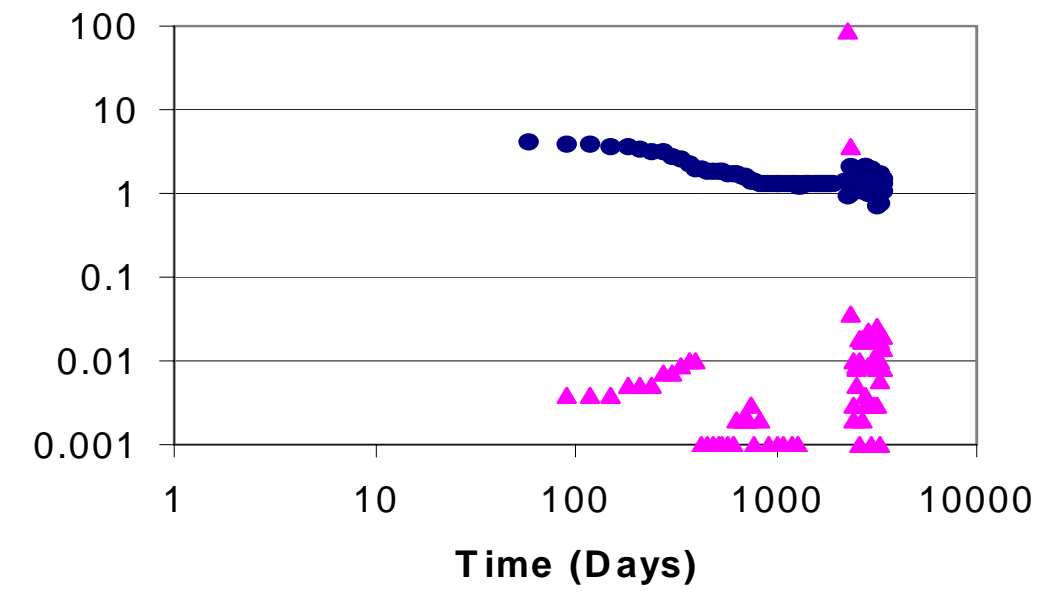

- W OR

$\triangle$ W OR Derivative

Fig. 1.4.52-WOR and WOR Derivative for well GSU-119: Experiencing Near Wellbore Water Channeling 


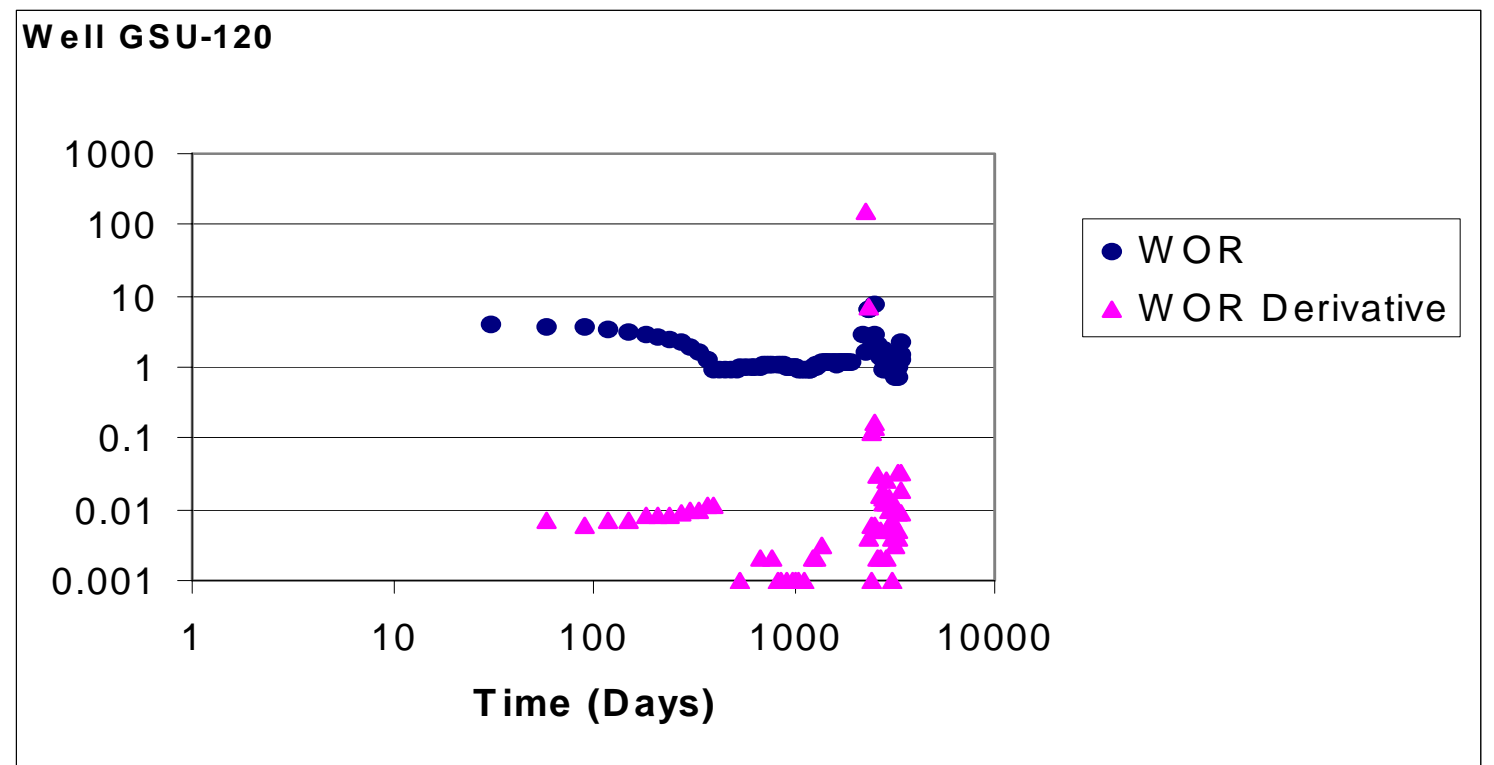

Fig. 1.4.53-WOR and WOR Derivative for well GSU-120: Experiencing Near Wellbore Water Channeling

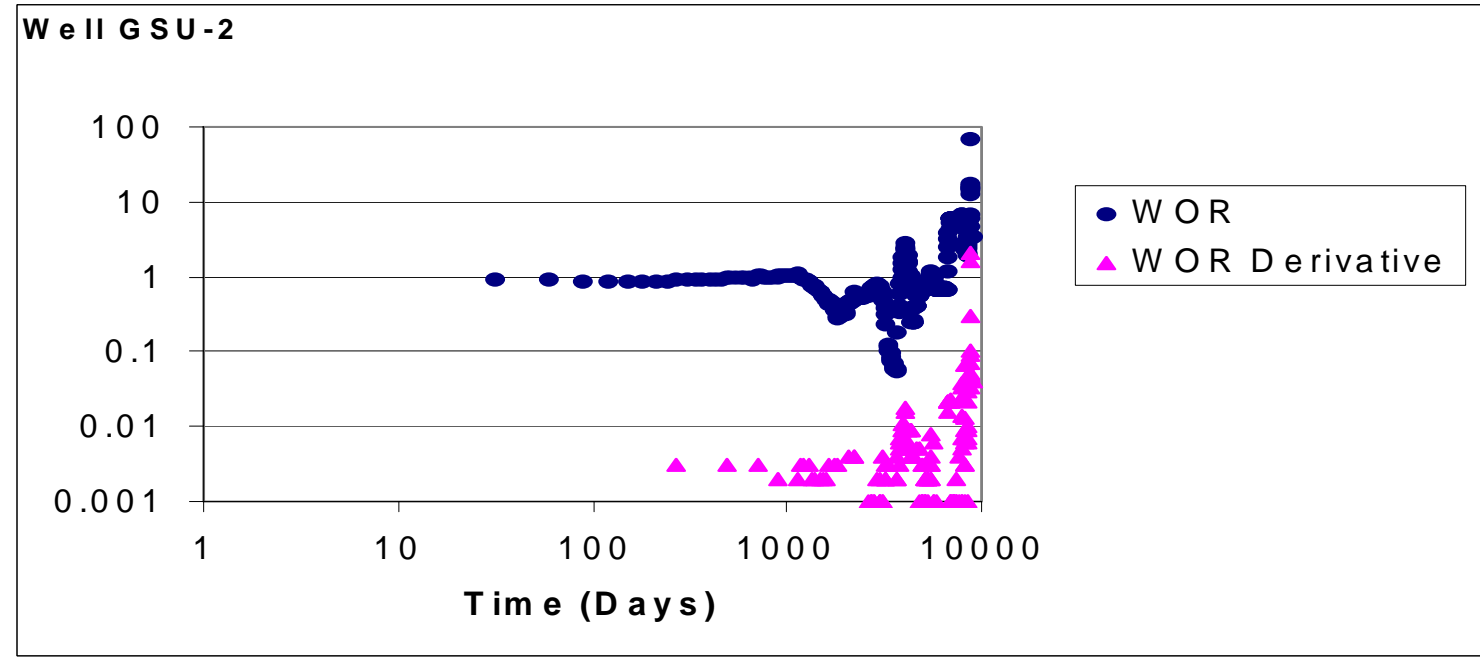

Fig. 1.4.54-WOR and WOR Derivative for well GSU-2: Experiencing Near Wellbore Water Channeling.

Figs.1.4.55 and 1.4.56 show the typical behavior for wells experiencing bottom water coning with late time channeling behavior. In all these wells, the WOR shows a nearly constant positive slope and WOR Derivative change its slope from negative to positive. 


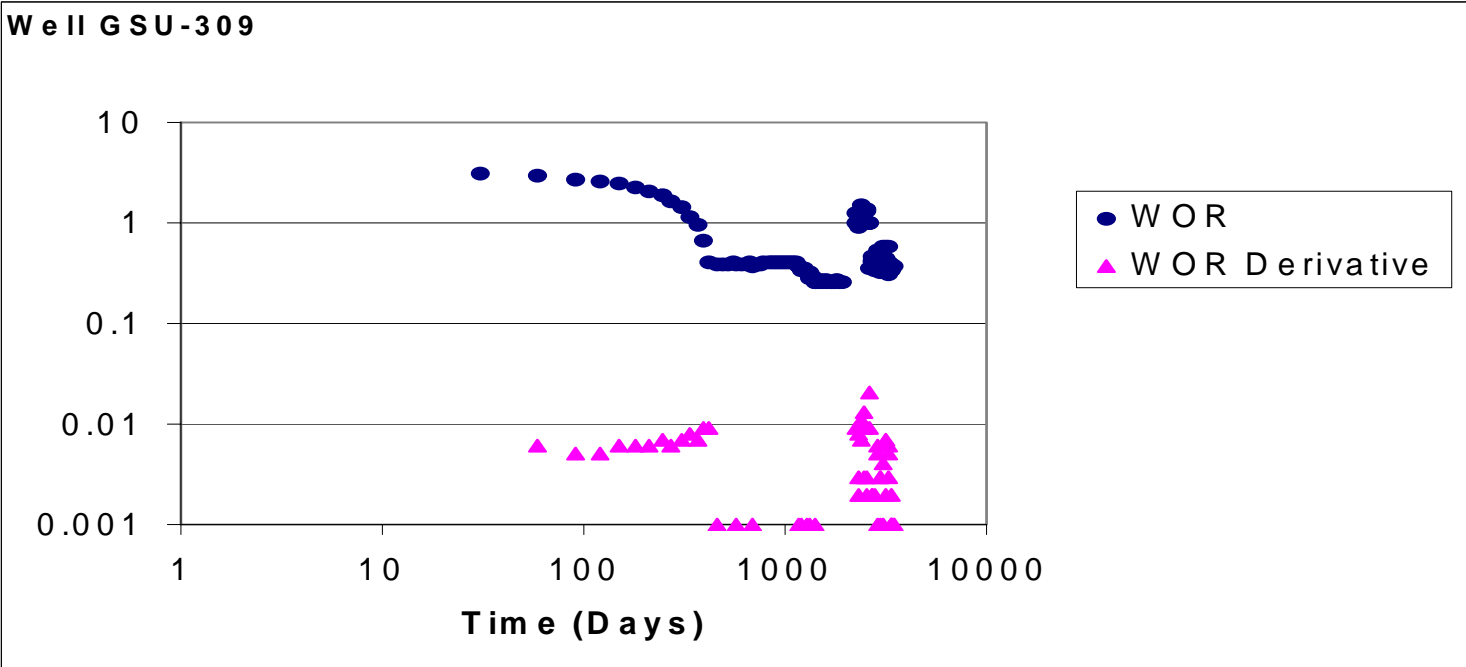

Fig. 1.4.55-WOR and WOR Derivative for well GSU-309: Experiencing Bottom Water Coning with Late Time Channeling.

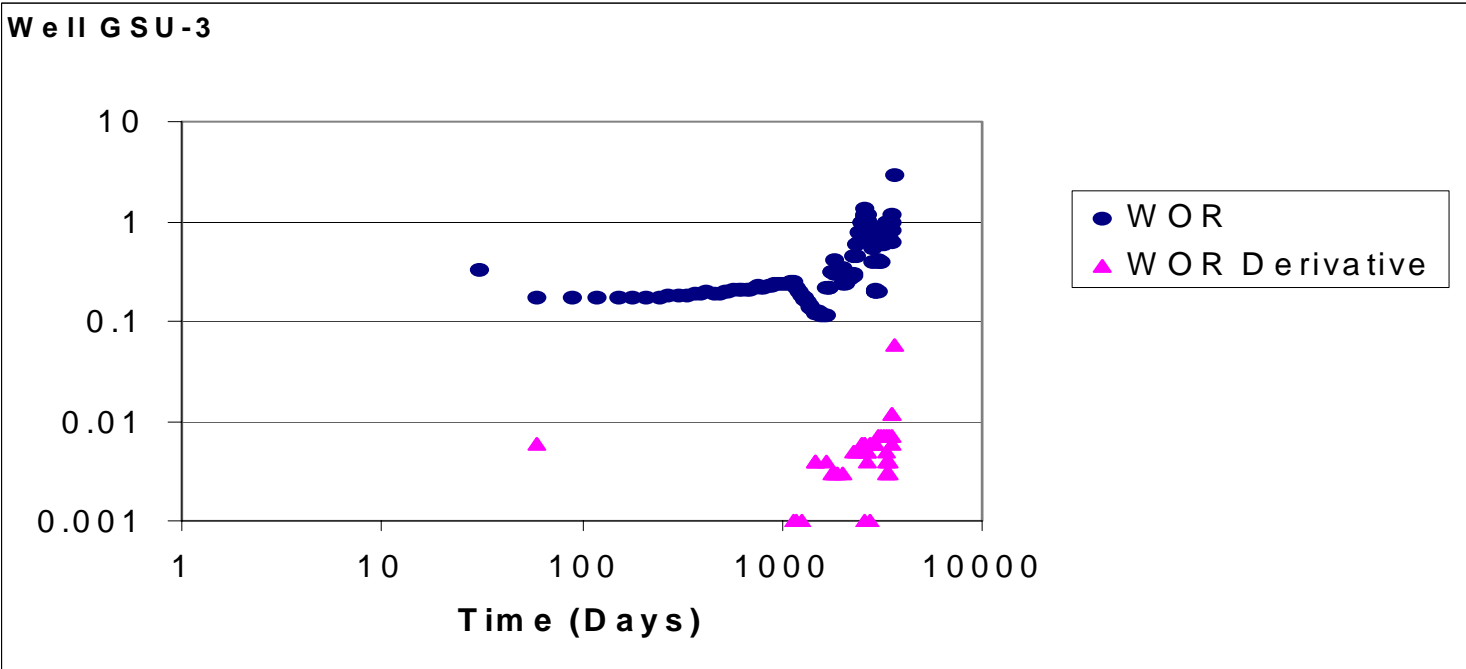

Fig.1.4.56-WOR and WOR Derivative for well GSU-3: Experiencing Bottom Water Coning with Late Time Channeling.

Figs.1.4.57-63 show the typical behavior for wells experiencing rapid channeling (perhaps associated to high permeability channels or fractures). In all these wells, both the WOR and its derivative show a drastic increment from the very beginning of the production life. 


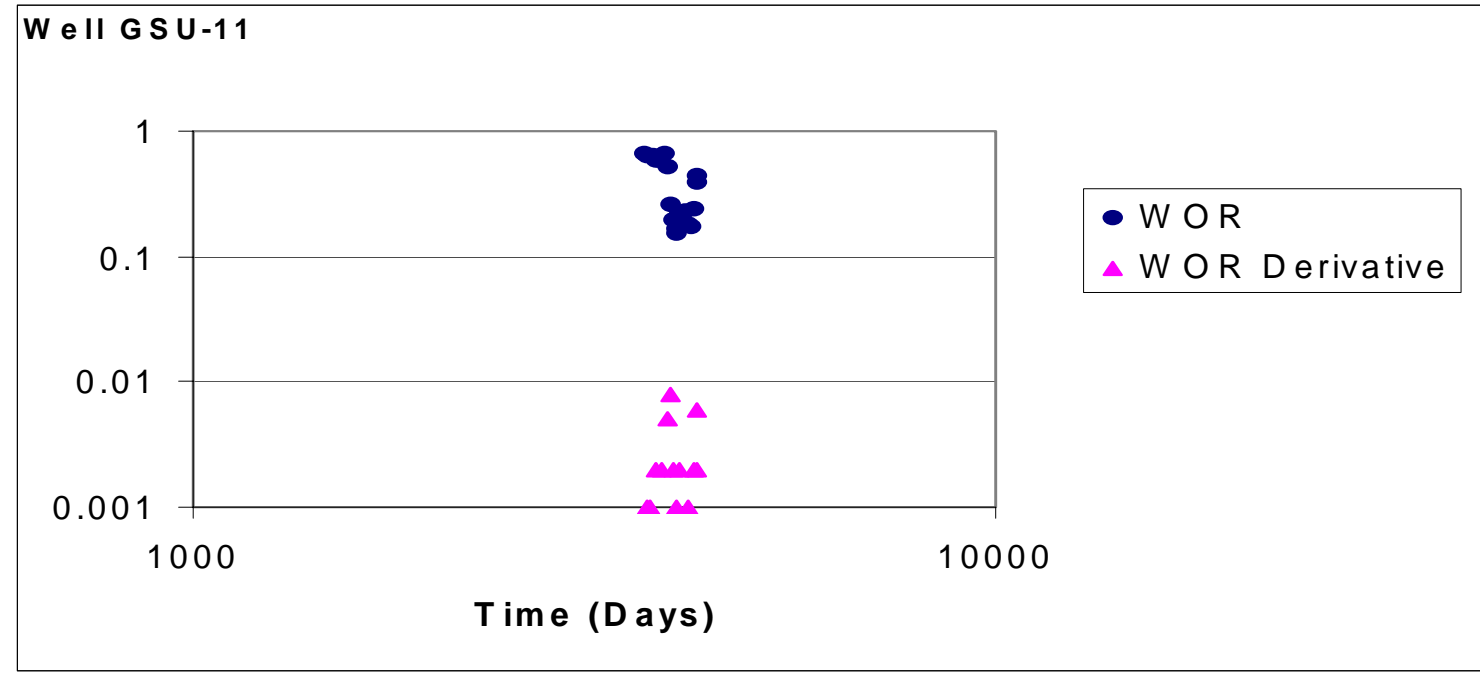

Fig. 1.4.57-WOR and WOR Derivative for well GSU-11: Experiencing Rapid Channeling.

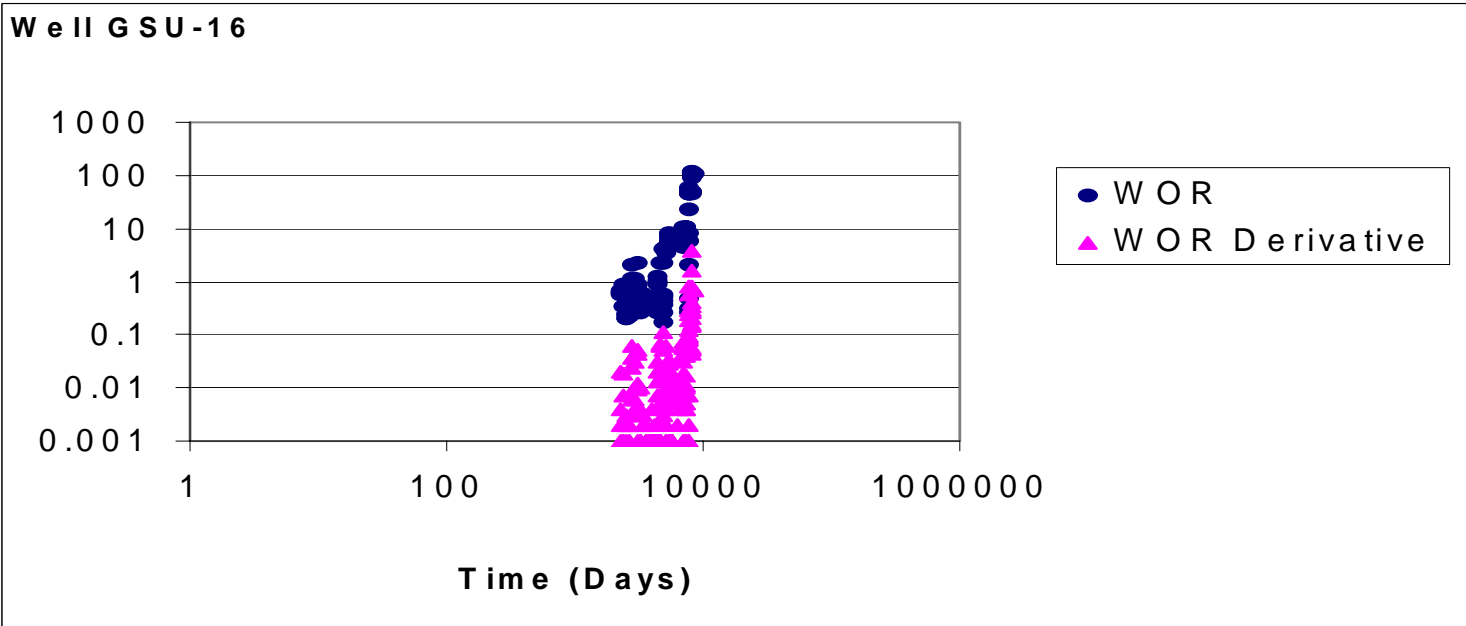

Fig. 1.4.58-WOR and WOR Derivative for well GSU-16: Experiencing Rapid Channeling. 


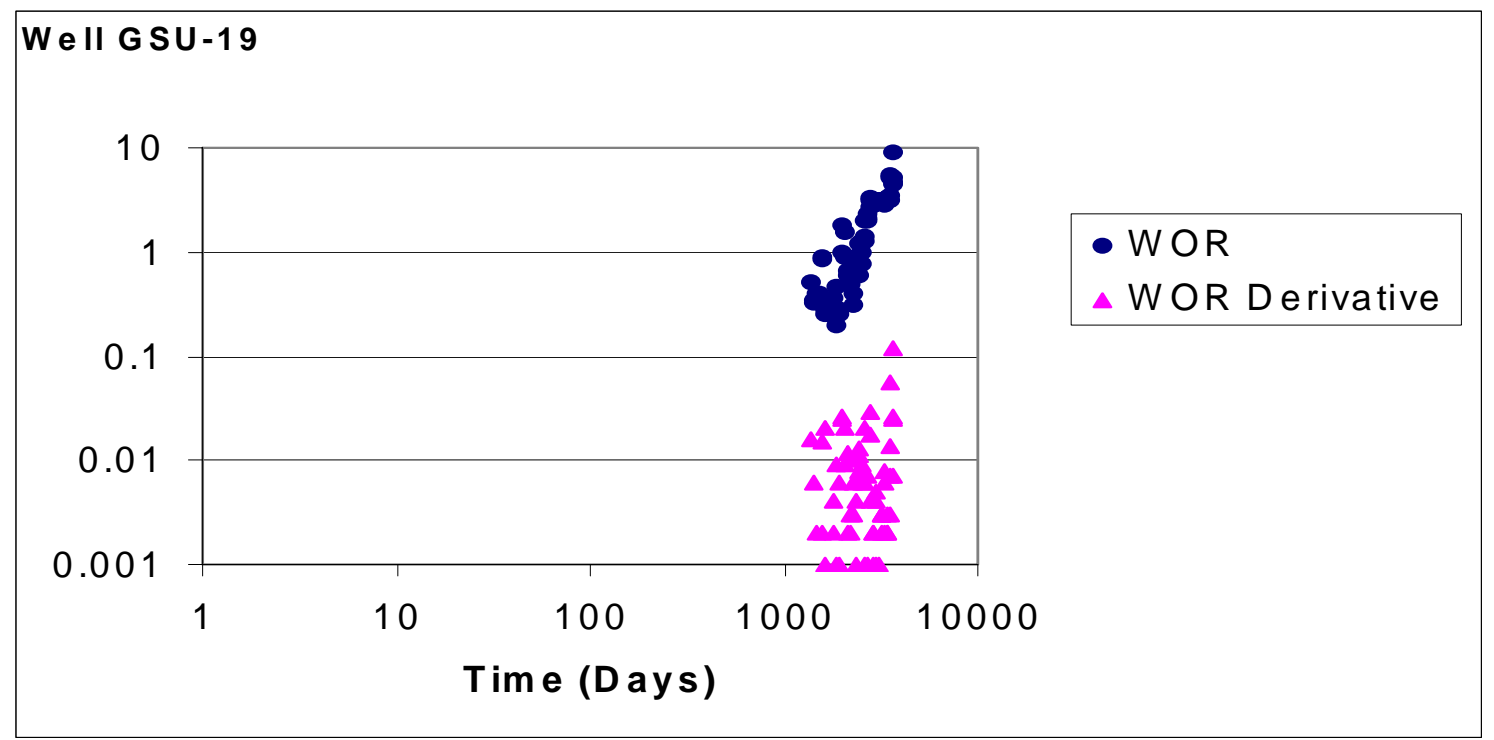

Fig. 1.4.59-WOR and WOR Derivative for well GSU-16: Experiencing Rapid Channeling.

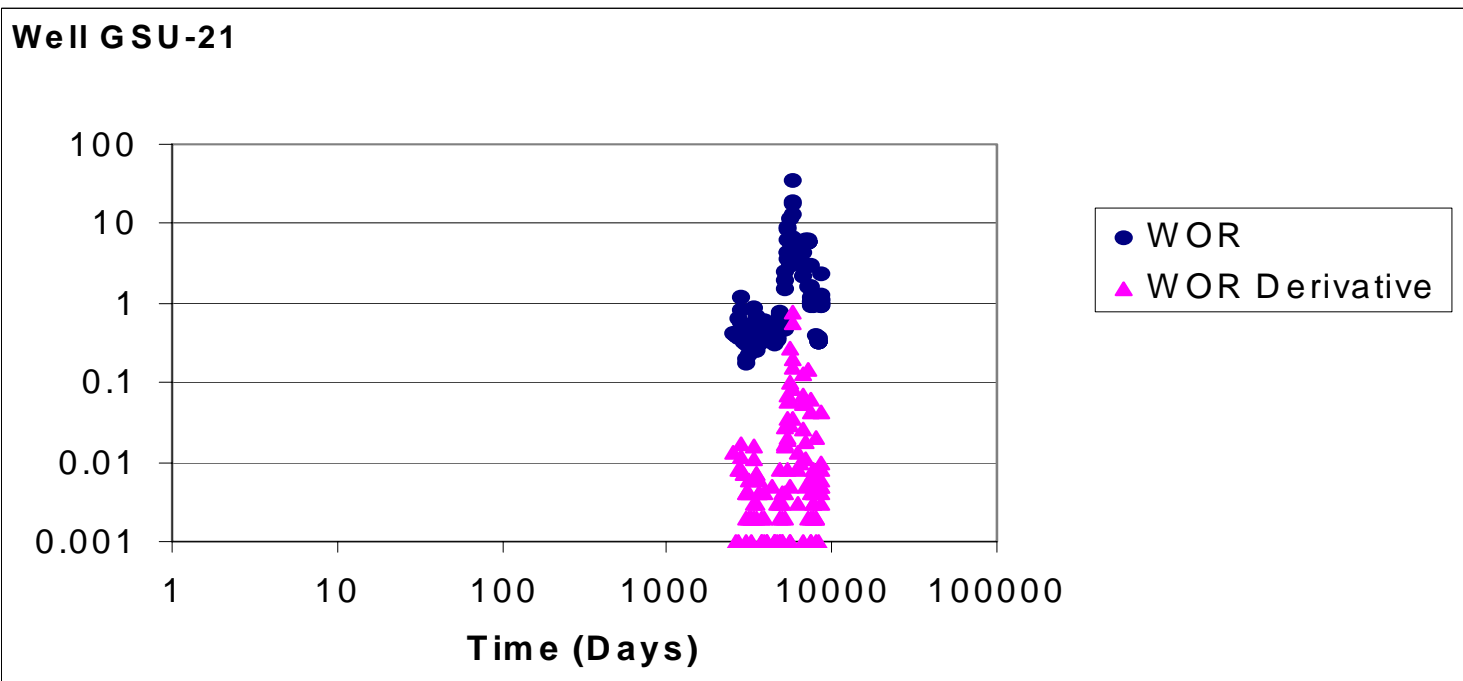

Fig. 1.4.60-WOR and WOR Derivative for well GSU-21: Experiencing Rapid Channeling. 


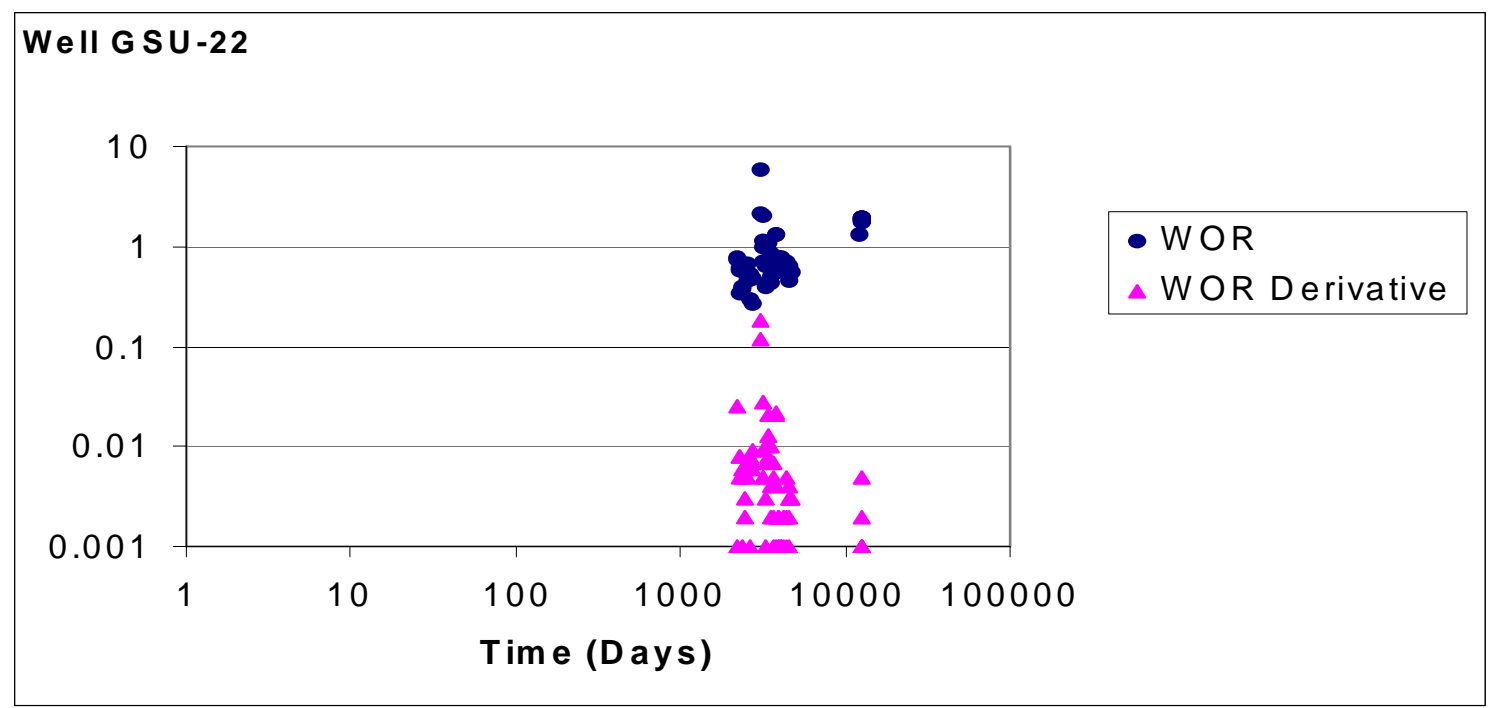

Fig. 1.4.61-WOR and WOR Derivative for well GSU-22: Experiencing Rapid Channeling

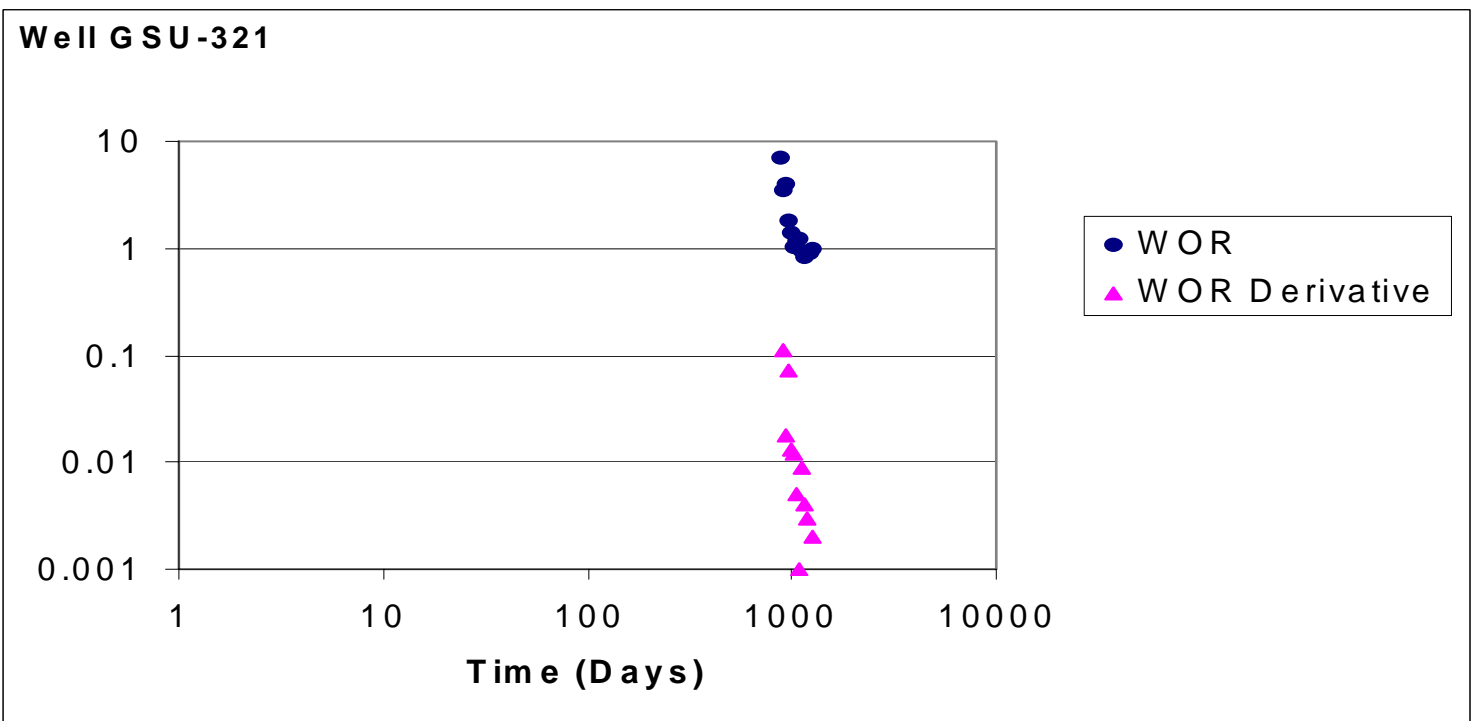

Fig. 1.4.62-WOR and WOR Derivative for well GSU-321: Experiencing Rapid Channeling. 


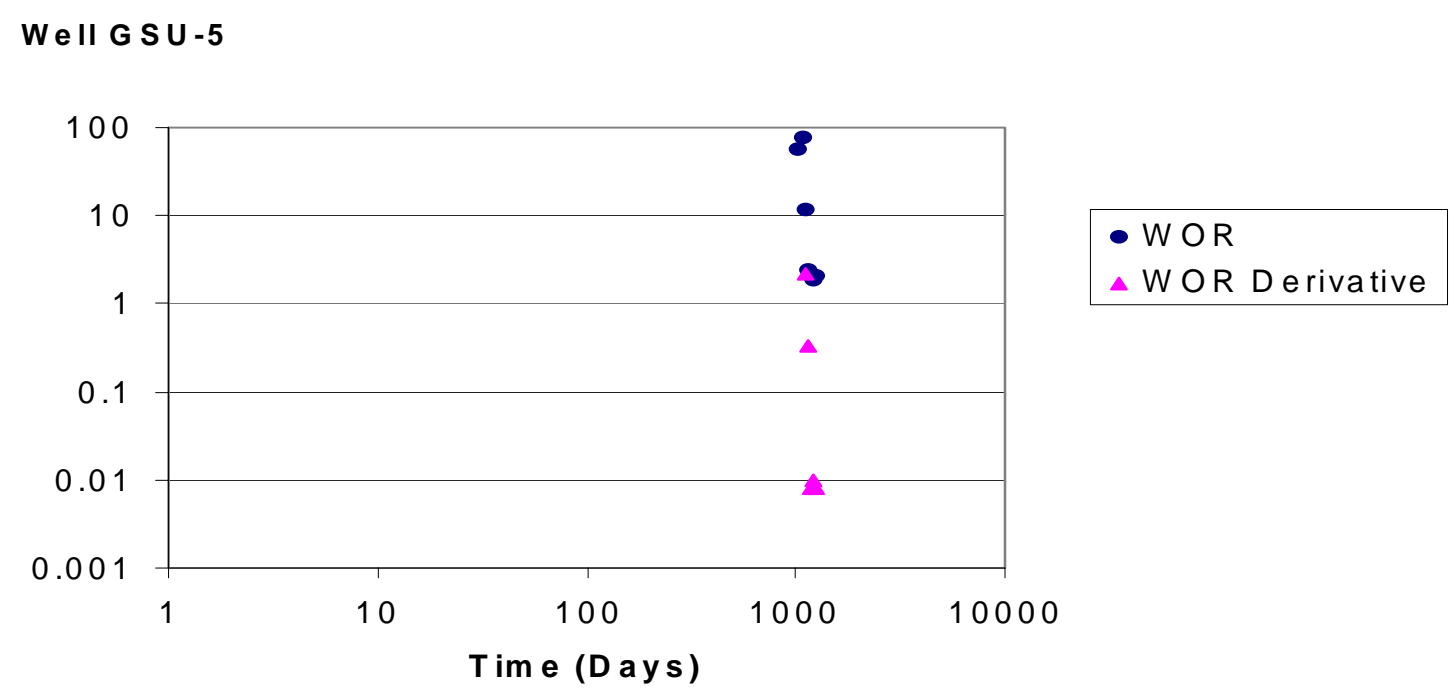

Fig. 1.4.63-WOR and WOR Derivative for well GSU-5: Experiencing Rapid Channeling.

Figs.1.4.64 - 71 show the pattern for wells experiencing normal displacement with high WOR. In all these wells, both the WOR and the WOR derivative change their slope and are mostly scattered throughout the production life.

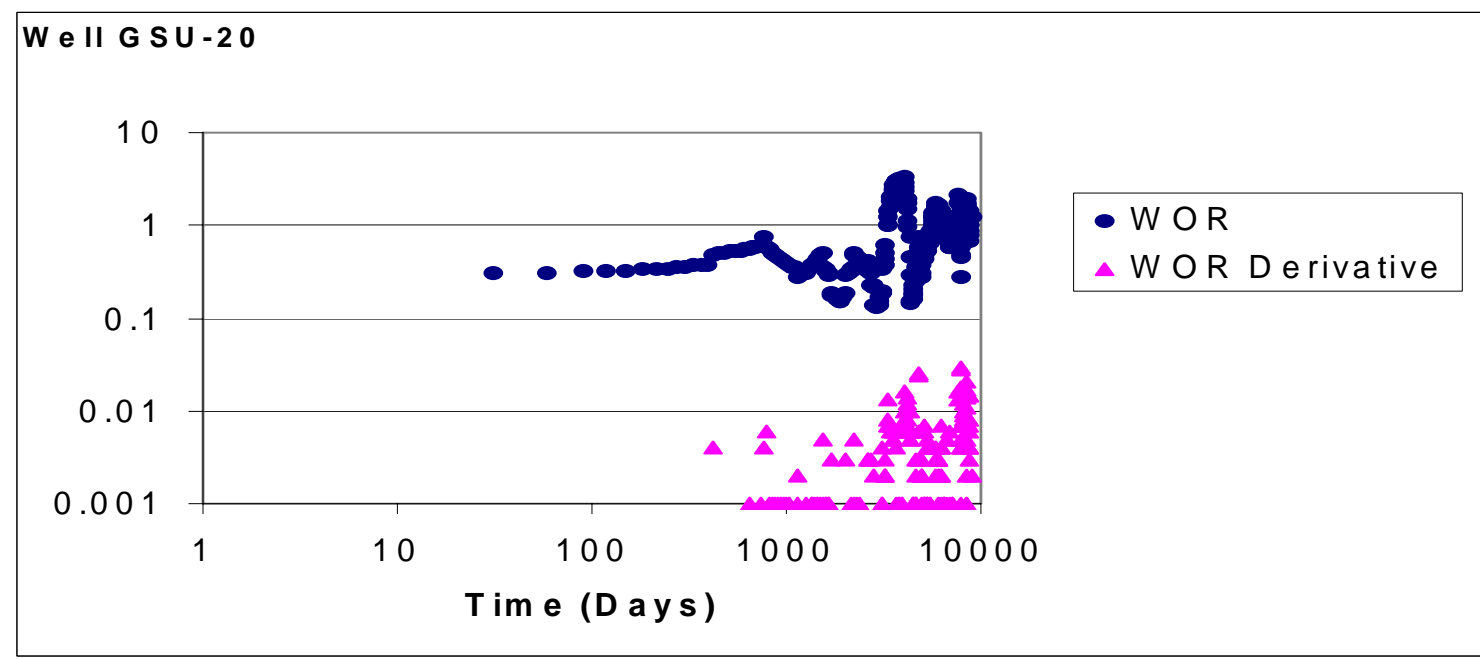

Fig. 1.4.64-WOR and WOR Derivative for well GSU-20: Experiencing Normal Displacement with High WOR. 


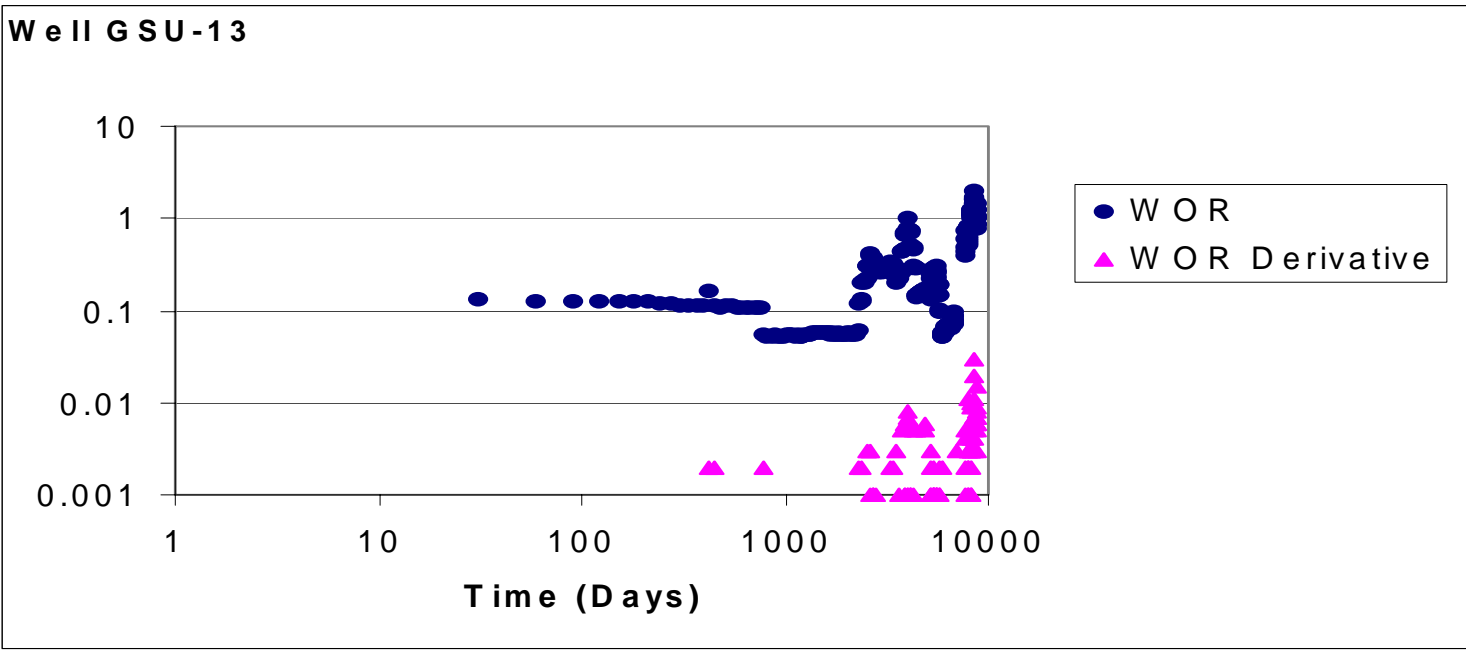

Fig. 1.4.65-WOR and WOR Derivative for well GSU-13: Experiencing Normal Displacement with High WOR.

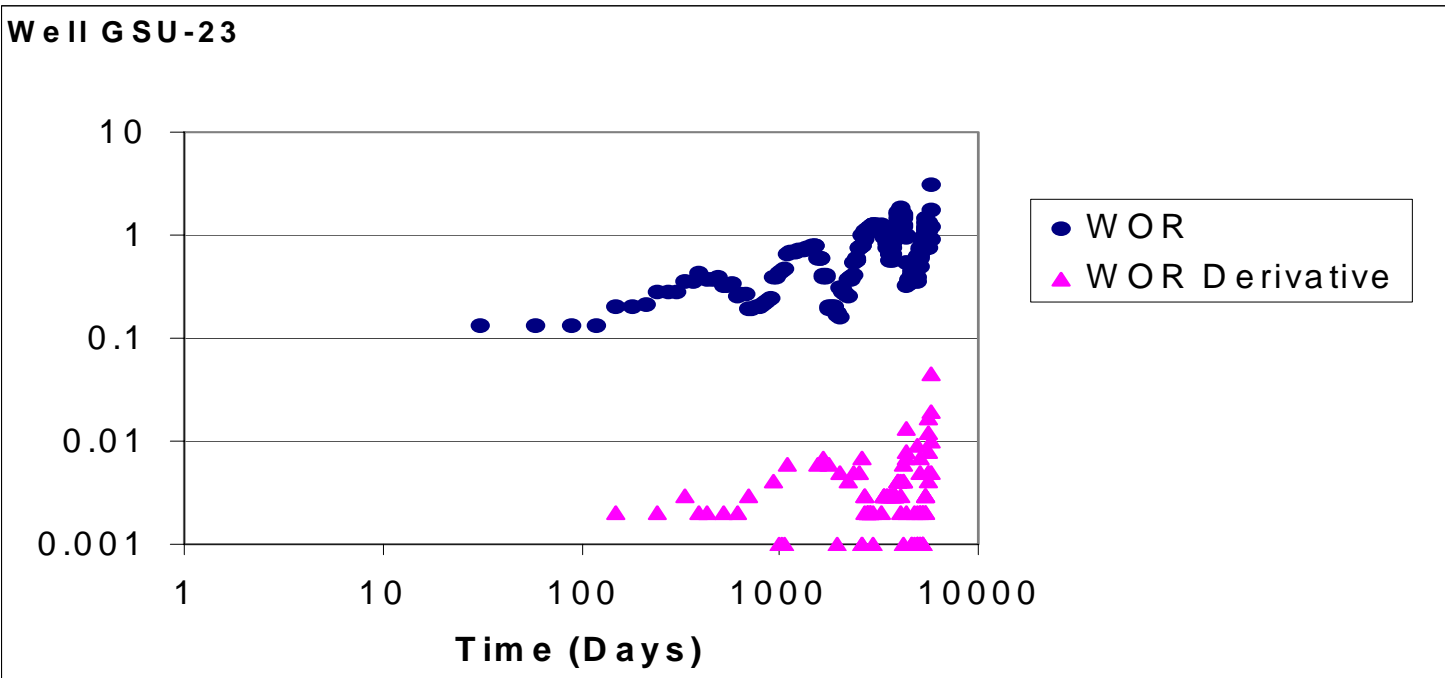

Fig. 1.4.66-WOR and WOR Derivative for well GSU-23: Experiencing Normal Displacement with High WOR. 


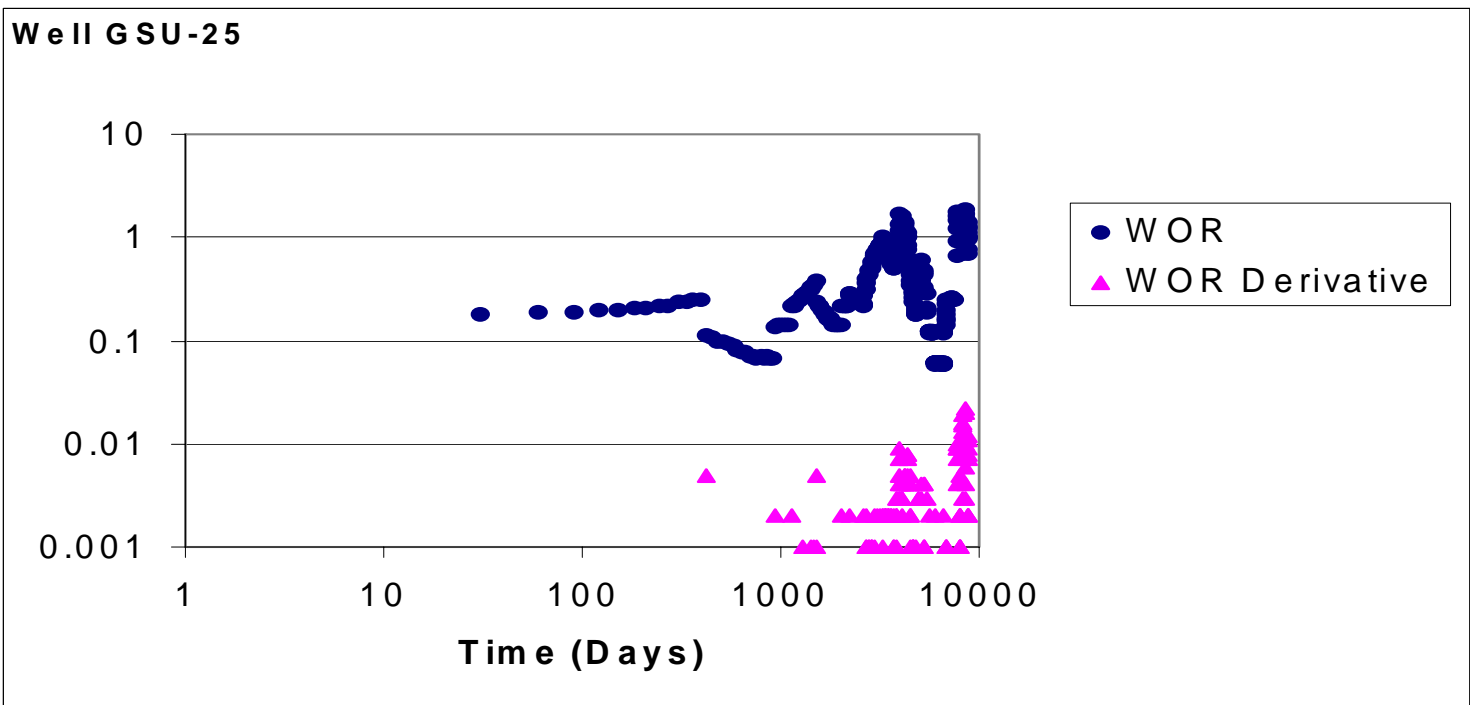

Fig. 1.4.67-WOR and WOR Derivative for well GSU-25: Experiencing Normal Displacement with High WOR.

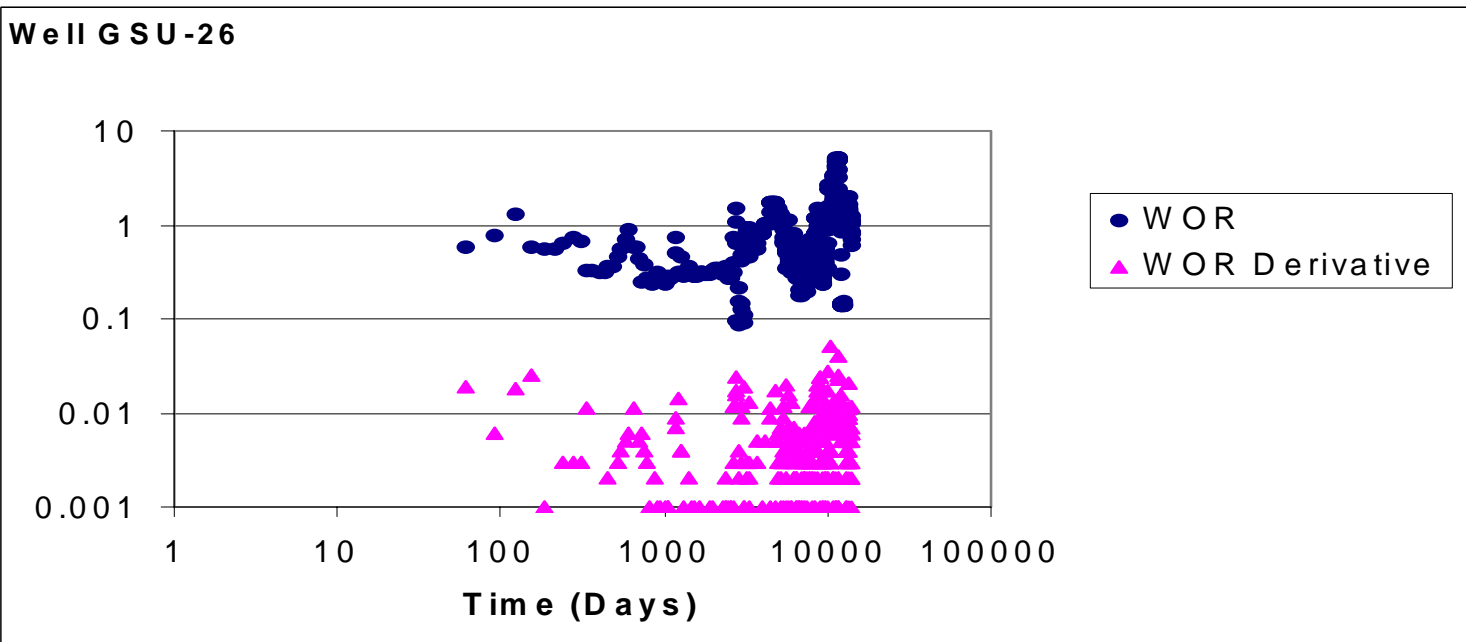

Fig. 1.4.68-WOR and WOR Derivative for well GSU-26: Experiencing Normal Displacement with High WOR. 


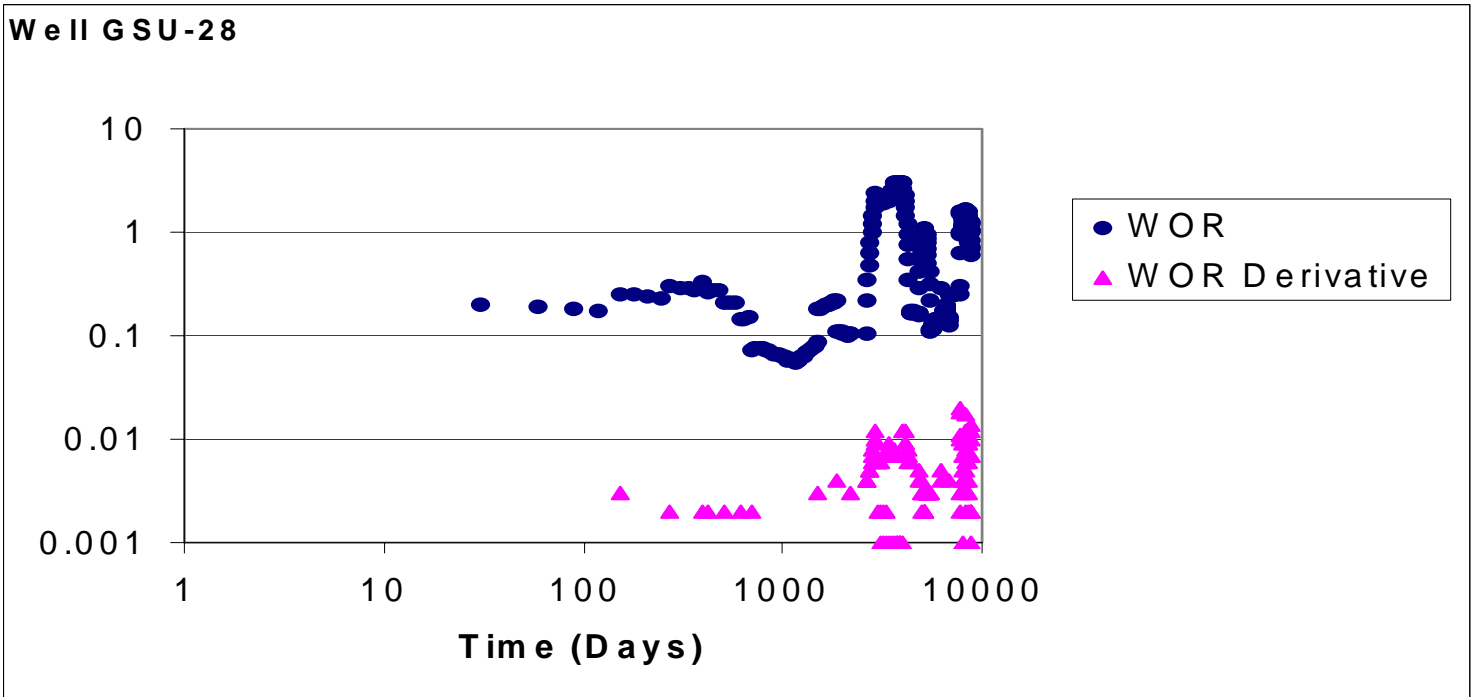

Fig. 1.4.69-WOR and WOR Derivative for well GSU-28: Experiencing Normal Displacement with High WOR.

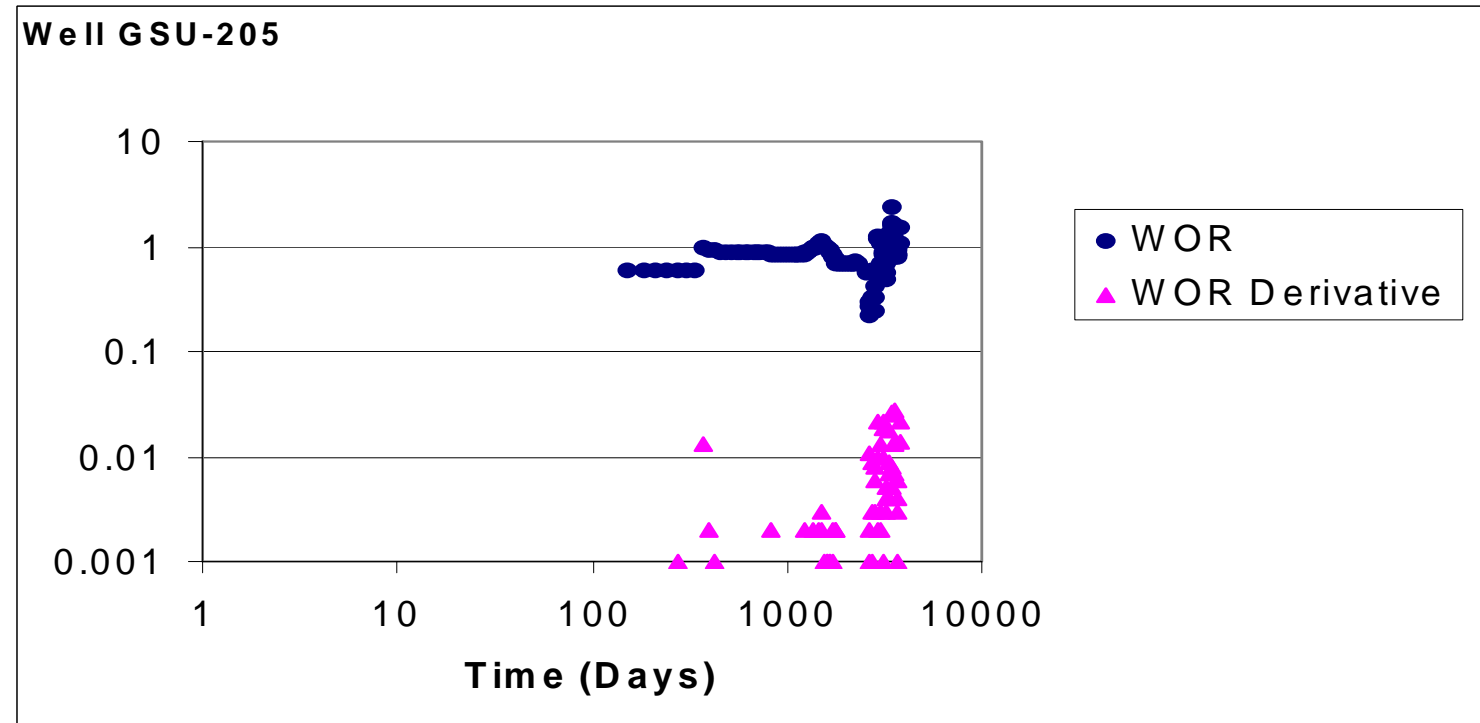

Fig. 1.4.70-WOR and WOR Derivative for well GSU-205: Experiencing Normal Displacement with High WOR. 


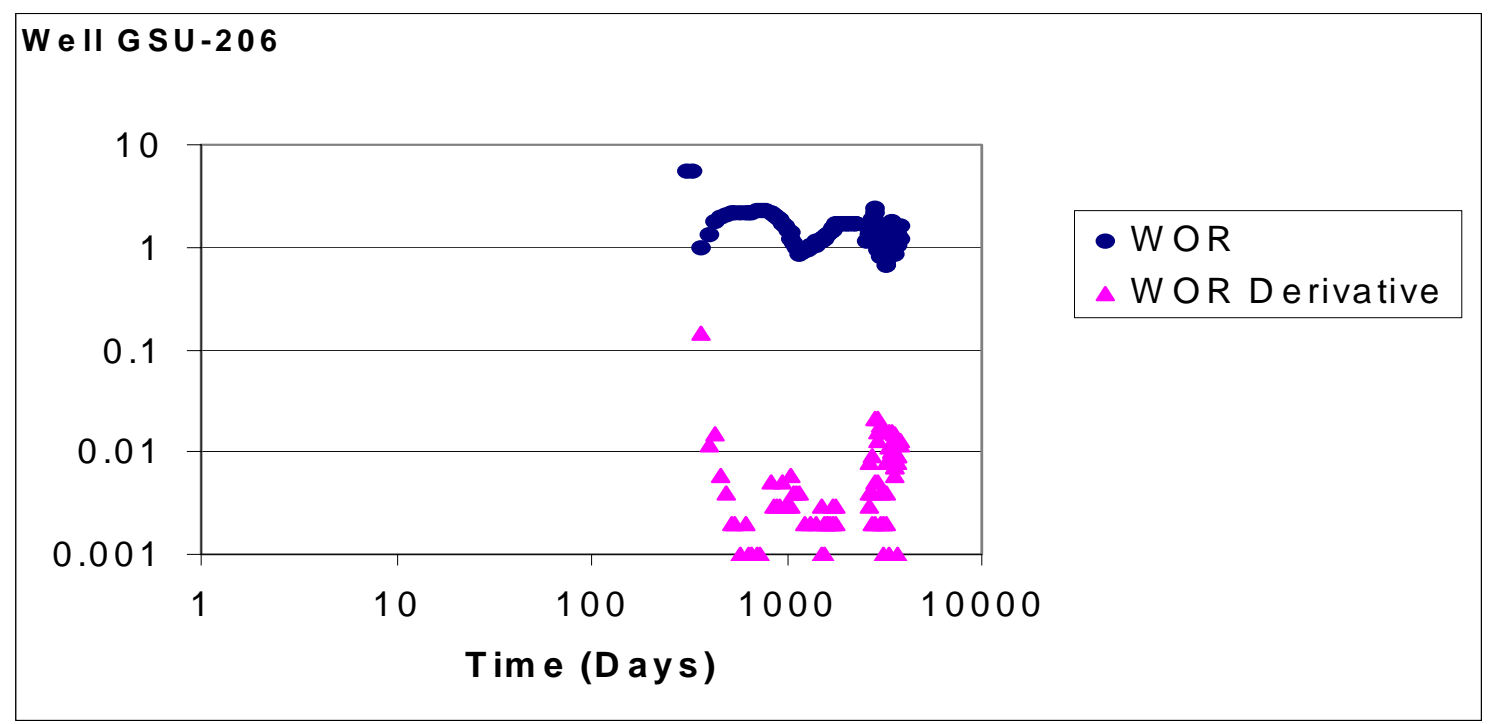

Fig. 1.4.71-WOR and WOR Derivative for well GSU-206: Experiencing Normal Displacement with High WOR.

Table 1.4.7 summarizes the results and the diagnostic of wells analyzed using Water Control Diagnostic Plots (log-log plots of WOR vs. time and WOR derivative vs. time).

Table 1.4. 7 Summary from Water Control Diagnostic Plots for wells in Germania Spraberry Unit.

\begin{tabular}{|l|l|l|}
\hline \multicolumn{1}{|c|}{ Wells } & \multicolumn{1}{|c|}{ Diagnostic } & \multicolumn{1}{c|}{ Remarks } \\
\hline $\begin{array}{l}\text { GSU-1, GSU-114, GSU-115, } \\
\text { GSU-117, GSU-118, GSU-119, } \\
\text { GSU-120, GSU-10 }\end{array}$ & Near Wellbore Channeling & Well GSU-1 may have casing leak \\
\hline GSU-309, GSU-3 & $\begin{array}{l}\text { Botton Water coning with } \\
\text { late time channeling }\end{array}$ & $\begin{array}{l}\text { Well GSU-3 Plugged and Abandoned } \\
\text { Well GSU-309 Active }\end{array}$ \\
\hline $\begin{array}{l}\text { GSU-11, GSU-16, GSU-19, } \\
\text { GSU-21, GSU-22, GSU-321, } \\
\text { GSU-5 }\end{array}$ & Rapid Channeling & $\begin{array}{l}\text { Well GSU-5 may have casing leak } \\
\text { Wells may be associated to fractures }\end{array}$ \\
\hline $\begin{array}{l}\text { GSU-20, GSU-13, GSU-23, } \\
\text { GSU-25, GSU-26, GSU-28, } \\
\text { GSU-205, GSU-206 }\end{array}$ & $\begin{array}{l}\text { Normal displacement with } \\
\text { high WOR }\end{array}$ & $\begin{array}{l}\text { Wells located in areas with high } \\
\text { water saturation }\end{array}$ \\
\hline
\end{tabular}

\subsubsection{Scatter Plots}

Another type of plot used in this study for well performance monitoring system is a kind of plot called Scatter Plot. Scatter plot provides another tool available in Oil Field 
Manager (OFM) for analyzing multiple variables at the same time and their interactions over time. Besides being a mapping tool, Scatter Plot is also a plotting tool that has the capability of presenting any combination of variables on the two axes ${ }^{5}$.

For monitoring, we used this strong analytical tool by plotting the cumulative oil vs. the cumulative water for all active wells (64 wells) in Germania Spraberry Unit and following the track for every well to detect some deviations respect to the normal behavior.

Fig.1.4.72 shows the scatter plot for all active wells producing in Germania Spraberry Unit. Well GSU-26 has been an excellent well because has been the one with the most cumulative (159,000 barrels of oil and 106,000 barrels of water) and has always maintained the same slope. This is a good well to select the best practice of completion in the area. Well GSU- 2, was producing with an almost constant slope and then, after a cumulative oil production of 60,000 barrels of oil, the water production suddenly increased indicating that the breakthrough in this well occurred after 60,000 barrels of oil produced or the flood front reached the perforation of the well. Well GSU-409 has produced only 31,000 barrels of oil and 143,000 barrels; this is indicative of either channeling or highly drained area around this well. The water production could increase in this well because it is located in front of two injection wells (GSU-407 and GSU22).Well GSU-13 and 25 constitute two good wells because have maintained a very low slope in the plot ( this means they produce at a high rate of oil respect to the rate of water).

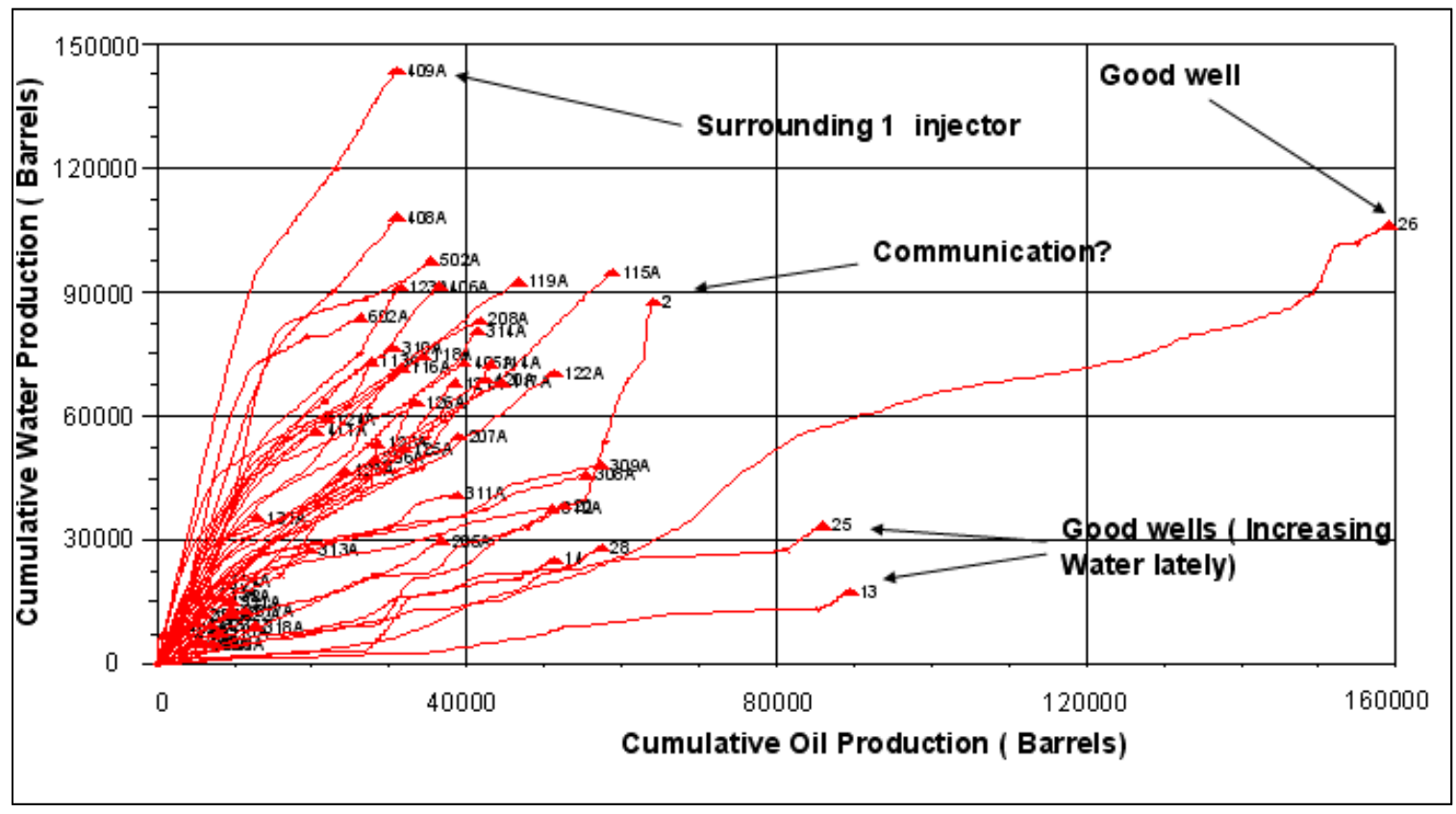

Fig.1.4.72-Scatter plot showing the performance of Cumulative Oil vs. Cumulative Water for active wells in Germania Spraberry Unit. 


\subsection{Flood Front Maps and Bypassed Oil.}

Flood front maps are a pictorial display showing the location of various food fronts. The maps, often called "bubble maps," allow visual differentiation between areas of the reservoir that have and have not been swept by injected water ${ }^{6}$ and were generated using the module GRID in Oil Field manager. These maps are very useful to identify areas with little or no water (bypassed oil).The generation of these maps is based on interpolation techniques (ordinary Kriging). In this study these kinds of maps were used with the aim of evaluating, the water, oil, and gas distribution and the fluid fronts as a function of time. Since this representation is a snap shot in time, this particular views allowed determination either visually or numerically of the cumulative fluids in a any part of the reservoir and therefore help to keep track of the flood fronts in the area.

Fig 1.4.73 shows bubble maps of cumulative oil for different times and stages of depletion of Germania Spraberry Unit. In the bubble maps, we can see that most of the production has taken place around the injectors (GSU-11, GSU-19, GSU-22, GSU-27 and GSU-6). The dark spots in the maps suggest areas with response to the injection and therefore the most drained areas of the unit. According to this bubble map, the central part of the unit is the most depleted.

Fig 1.4.74 shows bubble maps of cumulative oil at last date (2002). In the bubble maps, we can see that most of the production has taken place in the wells GSU-21, GSU26, GSU-16, GSU-10, and GSU-12. This map can be used as a reference to locate infill drilling wells in the areas with little or no oil production.

Areas in which wells have cumulative oil production (from 1957 to 2002) greater than 80,000 barrels (Fig 1.4.74) generally correspond to areas of greater net pay in the operational units $1 \mathrm{U}$ and $5 \mathrm{U}$. Areas of highest cumulative production ("sweet spots") are in the north-central part of the waterflood unit, where ten wells have each produced between 70,000 and 159,000 barrels of oil. This map also suggests an influence of reservoir stratigraphy and fracture trend on oil production. Areas having the best oilproducing wells ("sweet spots") and their adjacent water injection wells formed trends parallel to the main set of natural fractures ( $\mathrm{N} 56^{\circ} \mathrm{E}$ ) and are also correlative with axes of maximum net pay. 


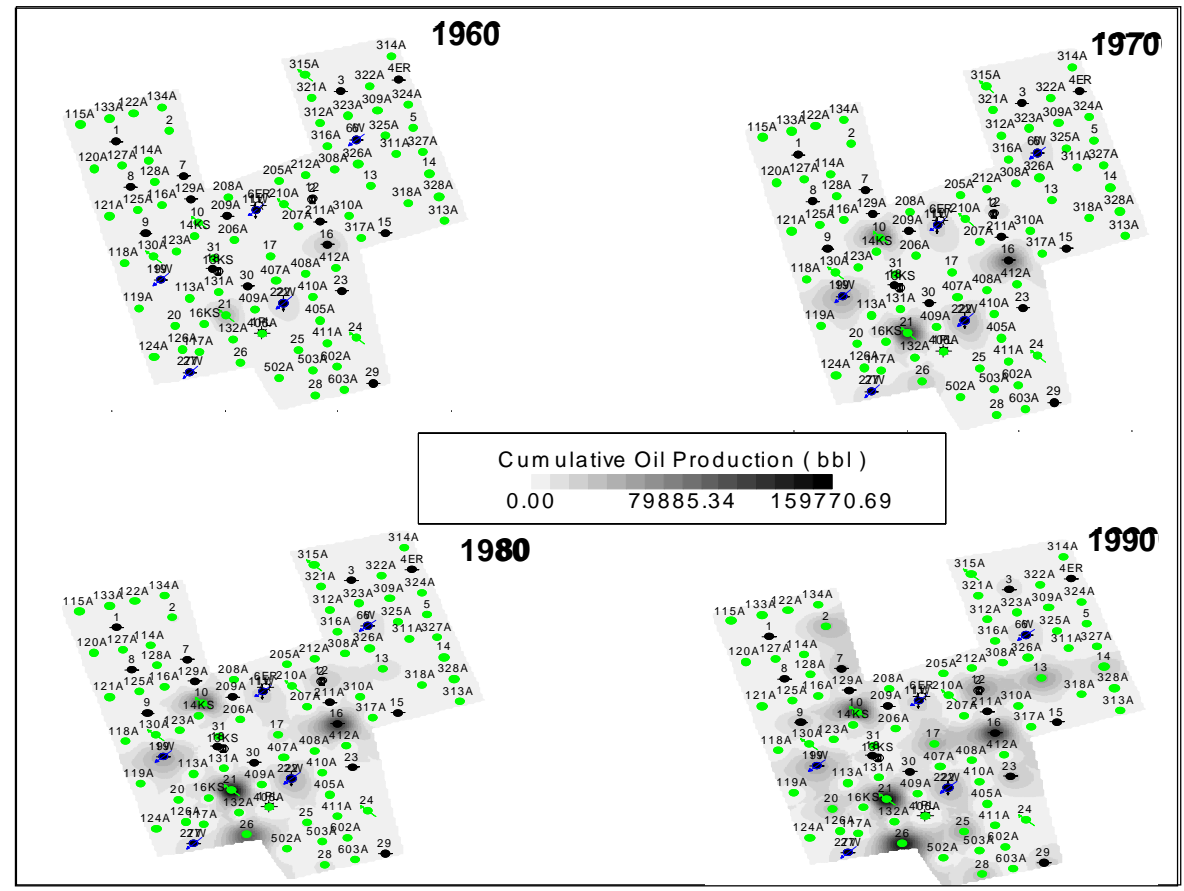

Fig.1.4.73-Bubble Maps for different periods of exploitation in Germania Spraberry

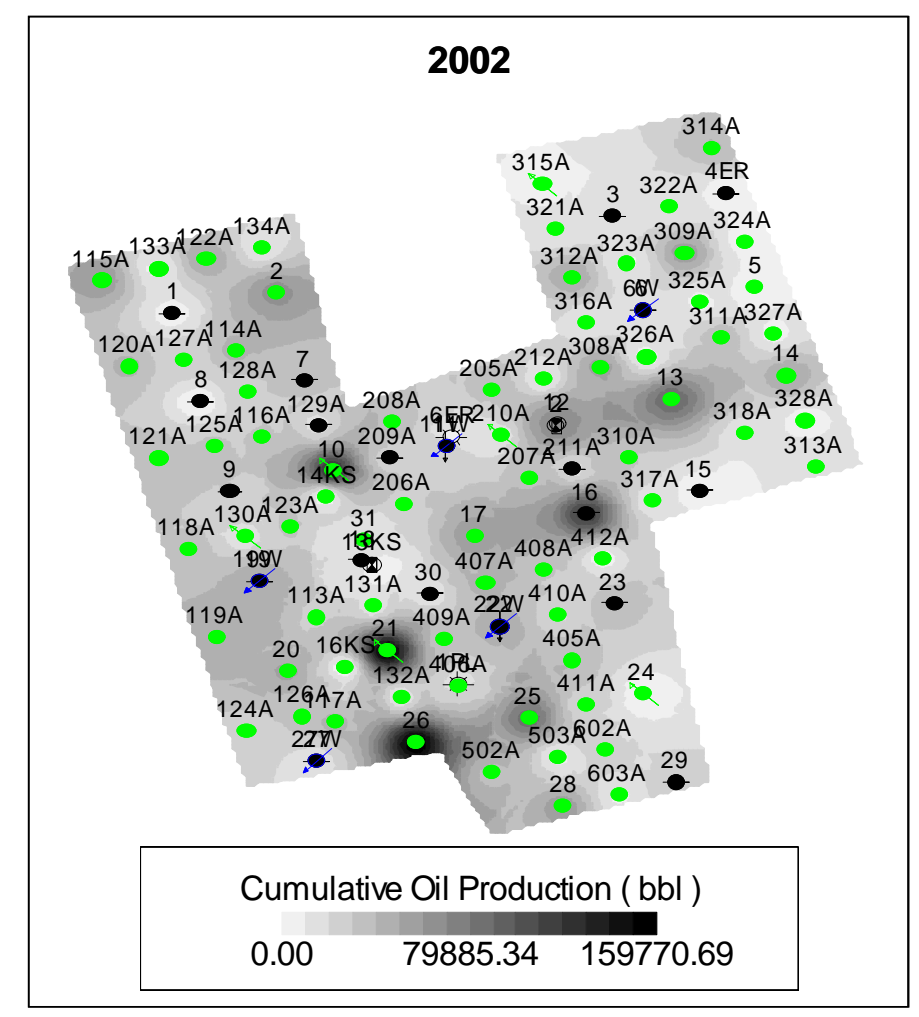

Fig.1.4.74-Bubble Maps of Cumulative Oil Production in Germania Spraberry. 
Fig 1.4.75 shows bubble maps of cumulative water production for different stages of depletion. In the bubble maps, we can see that most of the production (areas represented by dark spots) has taken place in the wells GSU-21, GSU-26, GSU-16, GSU-10, and GSU-12. This map can also be used as a reference to locate infill drilling wells in the areas with little or no water production (areas represented by the light spots in bubble map at last date (year 2002)). These maps also show correlation between the cumulative water production and the main fracture trend.

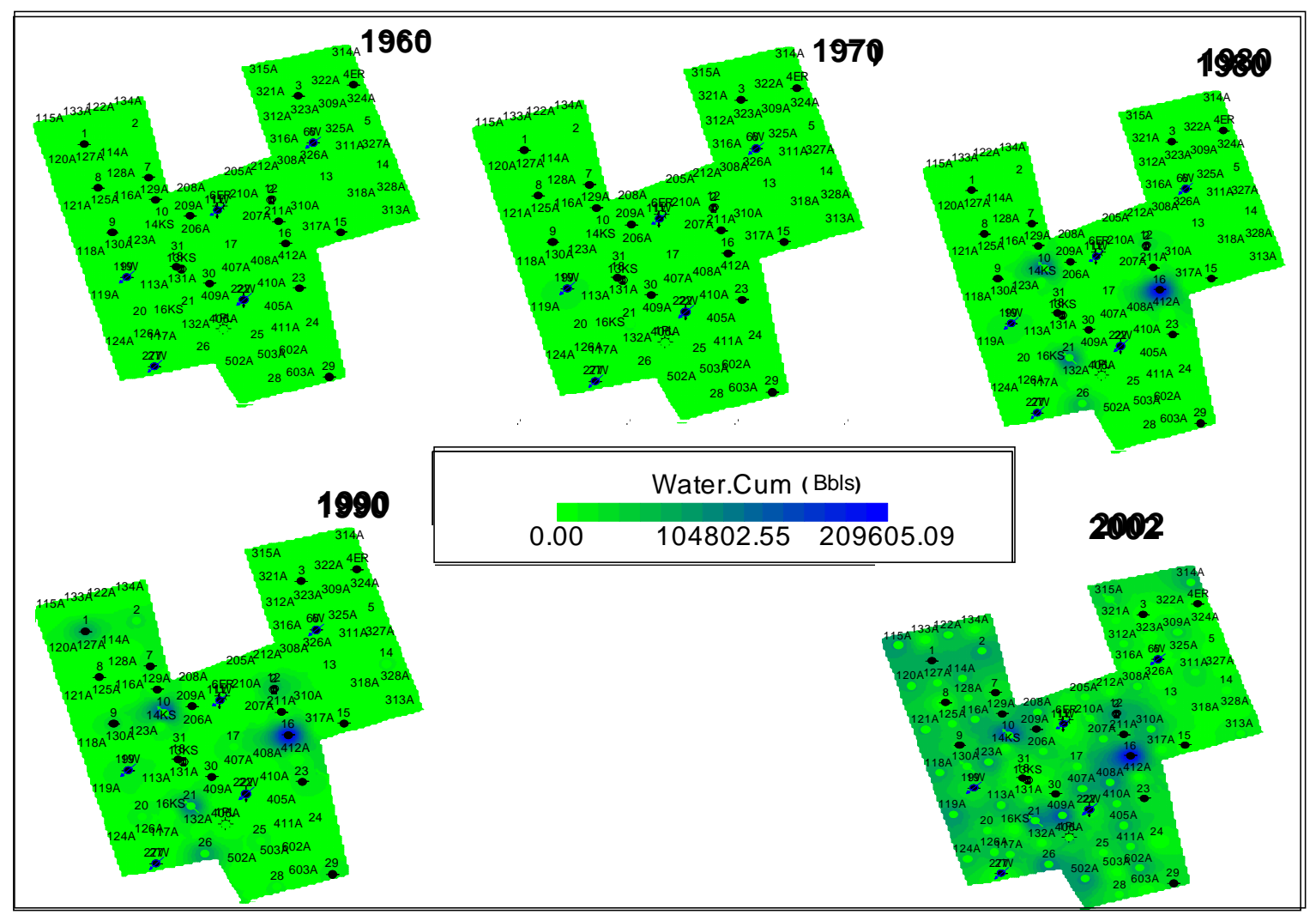

Fig.1.4.75 - Bubble Maps of Cumulative Water Production in Germania Spraberry.

Fig 1.4.76 shows bubble maps of cumulative gas production for different stages of depletion In the bubble maps, we can see that most of the production has taken place in the wells GSU-21, GSU-26, GSU-16, GSU-10, and GSU-12. These maps also show correlation between the cumulative water production and the main fracture orientation. 


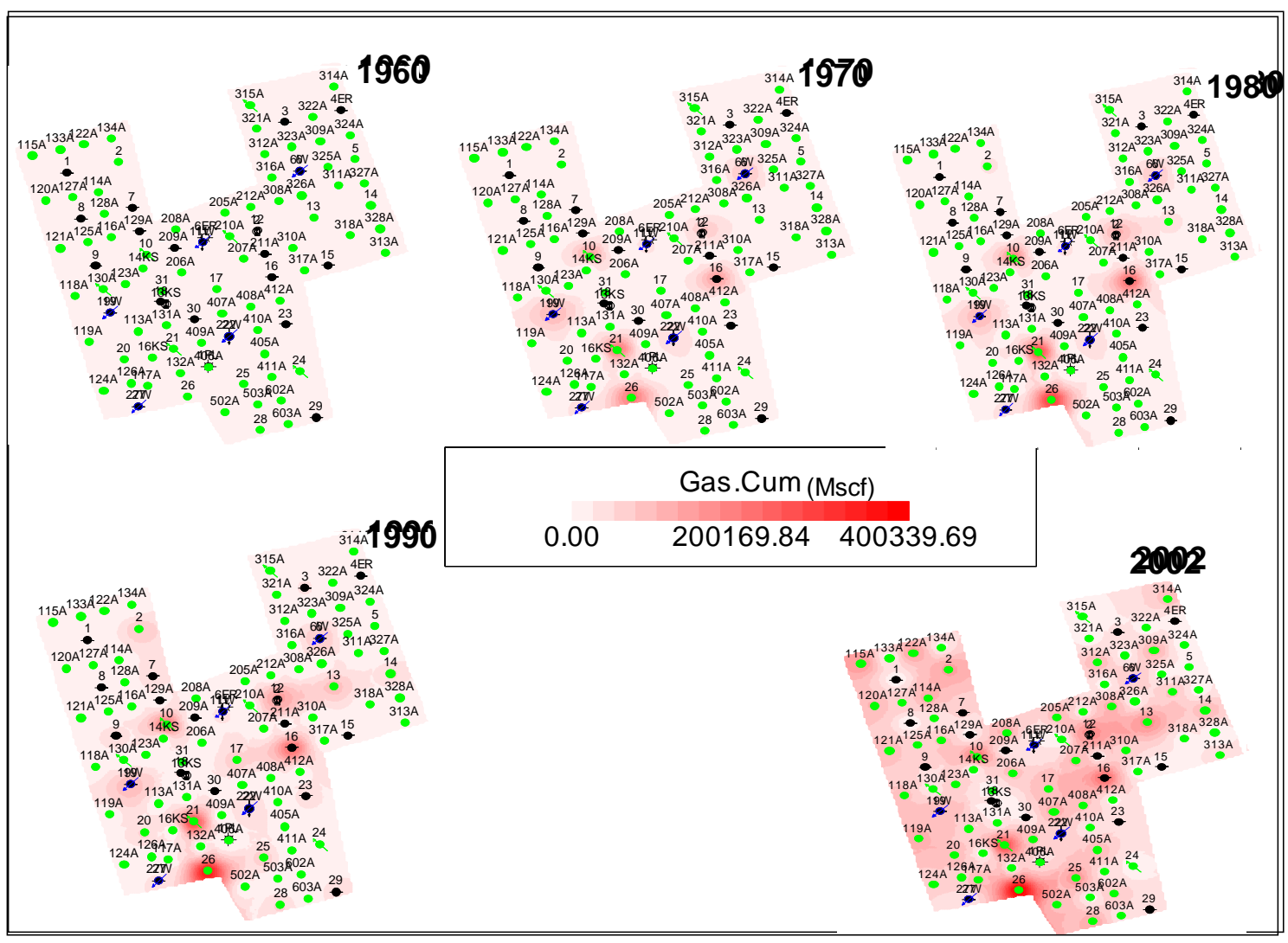

Fig.1.4.76 - Bubble Maps of Cumulative Gas Production in Germania Spraberry.

\section{Production Forecast and Reserve Estimation}

A major activity in this project was to estimate the remaining reserves and its distribution in the reservoir for monitoring and identification of further development opportunities. In this case, since we have sufficient production data, we applied the most widely used method of forecasting future production (Decline Curve Analysis) to estimate the remaining reserves associated to drainage radius of every well and extrapolate the performance of the reservoir in the future.

Due to the nature of oil production rate from naturally fractured reservoirs, a hyperbolic type decline curve was used to fit the production trend and forecast the future production rate. We performed and extrapolated the future performance starting from the last production point available (June 2003) for all 64 active wells in the reservoir and then displayed the reserves (remaining reserves and estimated ultimate recovery (EUR)) in a bubble map, this helped us to identify some opportunities by locating the areas with the most remaining reserves in the reservoir ("sweet spots").

The results show that under the current operation conditions (new injection pattern and water injection rate), the reservoir can produce 1.410 million barrels of oil additional (through the wells currently active) and increase the ultimate recovery up to 
4.652 million barrels in the next 20 years. The results, also suggest that the areas with the most remaining reserves are those located in the north-east part of the unit.

The decline curve analysis was performed under the following premises:

- Hyperbolic type decline

- Economic Limit: 1 BOPD

- Time Limit: 20 years

- $\quad$ Fractional power exponential decline (b) $=0.7$

- Starting Rate: Last oil rate in the data base for every well.

- Starting Date: Last Production Date (June 2003)

The equation used to perform the decline curve analysis in every active well is as follows:

$$
q_{t}=q_{i}\left(1+\left(a_{i} * b t\right)\right)^{-(1 / b)}
$$

Where:

$q_{t} \quad=$ producing rate at end of time $\mathrm{t}$, BOPD.

$q_{i} \quad=$ initial rate at time $\mathrm{t}=0$, BOPD

$a_{i}=$ constant of integration equal to the production decline rate as a fraction, fraction/year.

$b \quad=\quad$ exponent of hyperbolic decline, Dimensionless.

$t \quad=$ time from start of analysis period, Years.

To estimate the remaining reserves for every well over the next 20 years, we integrated the equation 4.4 to obtain the following equation:

$$
N p=\frac{q_{i}}{a_{i}(1-b)}\left(1-\left(1+\left(a_{i} b t\right)\right)^{(1-(1 / b))}\right) 365
$$

Where:

$N p \quad=$ Cumulative production from start of the analysis period to the end of year " $\mathrm{t}$ ", STB

$q_{i} \quad=$ initial rate at time $\mathrm{t}=0, \mathrm{BOPD}$

$a_{i} \quad=$ constant of integration equal to the production decline rate as a fraction, fraction/year.

$b \quad=$ exponent of hyperbolic decline, Dimensionless.

$t \quad=$ time from start of analysis period, Years.

Fig 1.4.77- 82 show the remaining reserves estimated with equation 4.5 for every well and its corresponding produced reserves ( as of June 2003) 


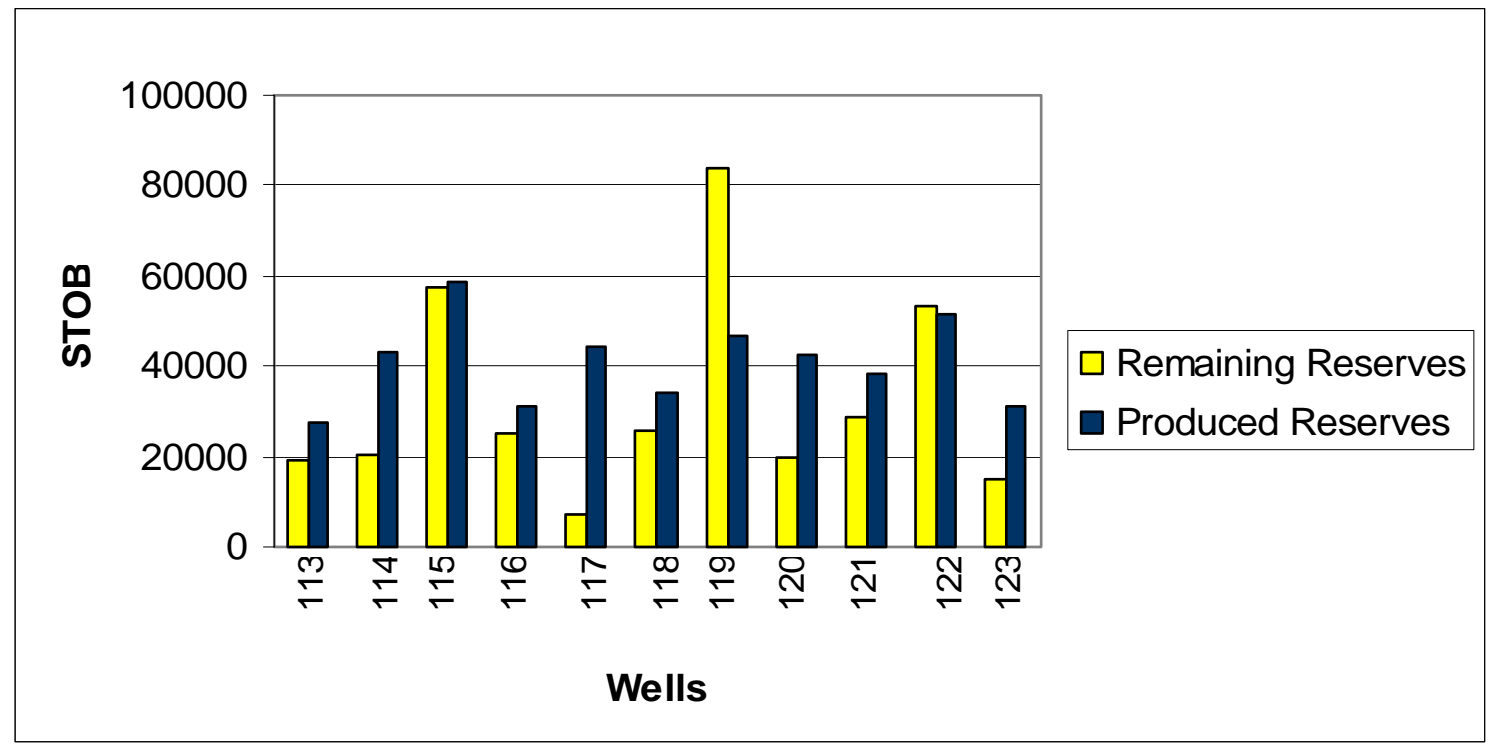

Fig. 1.4.77- Remaining and Produced Reserves for wells GSU-113,GSU-114,GSU-115,GSU116,GSU-117,GSU-118,GSU-119,GSU-120,GSU-121,GSU-122, and GSU-122.

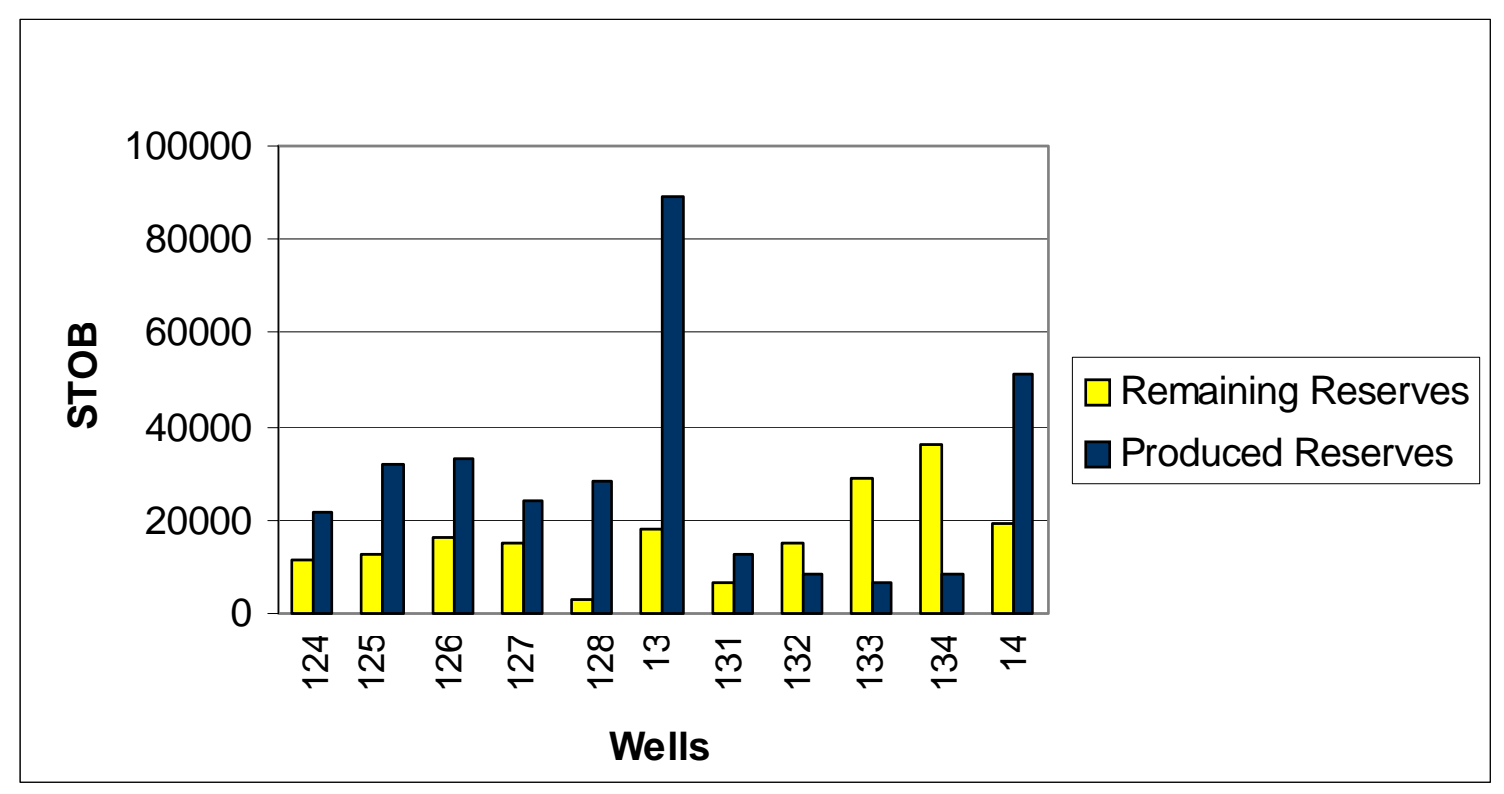

Fig. 1.4.78- Remaining and Produced Reserves for wells GSU-124,GSU-125,GSU-126,GSU127,GSU-128,GSU-13,GSU-131,GSU-132,GSU-133,GSU-134, and GSU-14. 


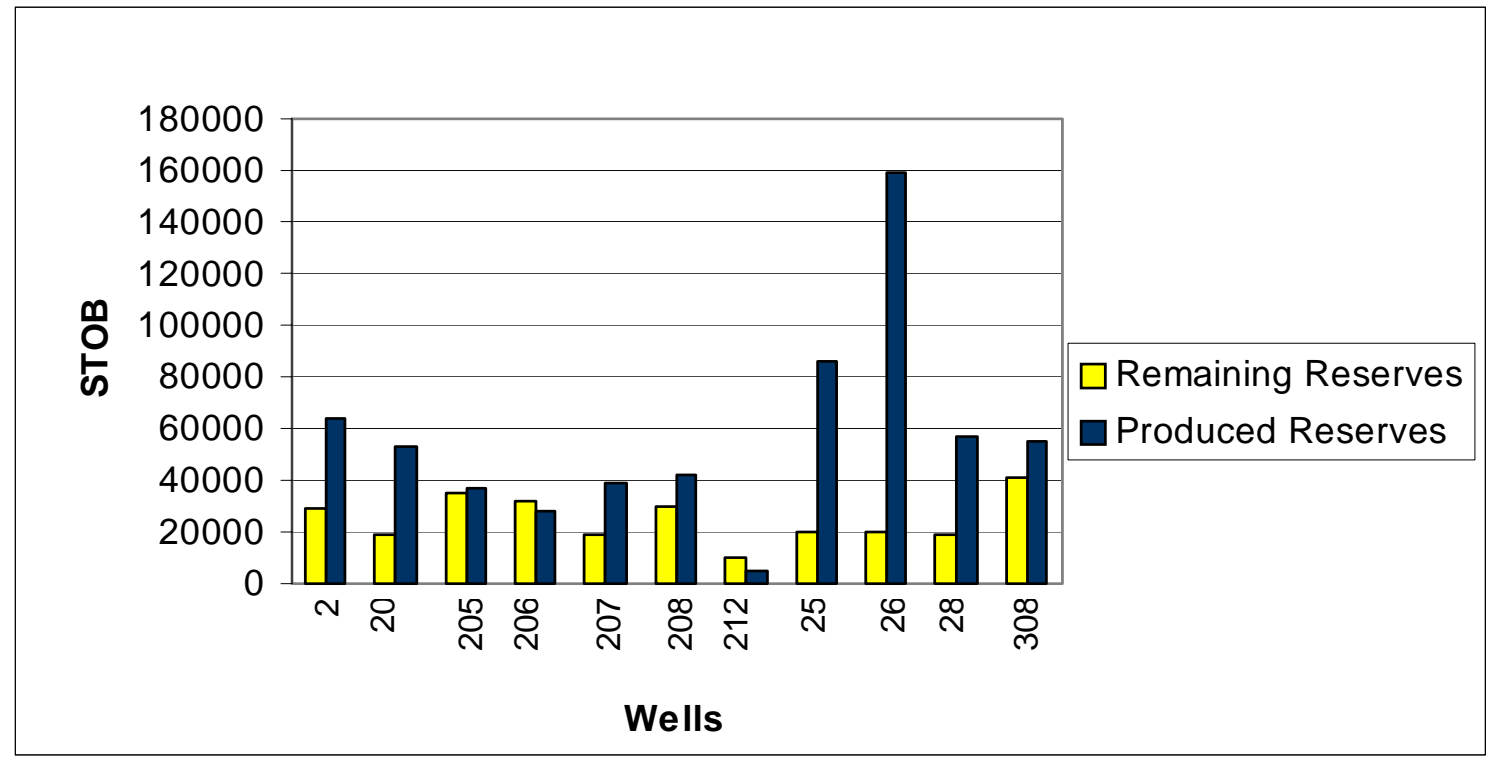

Fig. 1.4.79- Remaining and Produced Reserves for wells GSU-2,GSU-20,GSU-205,GSU206,GSU-207,GSU-208,GSU-212,GSU-25,GSU-26,GSU-28, and GSU-308.

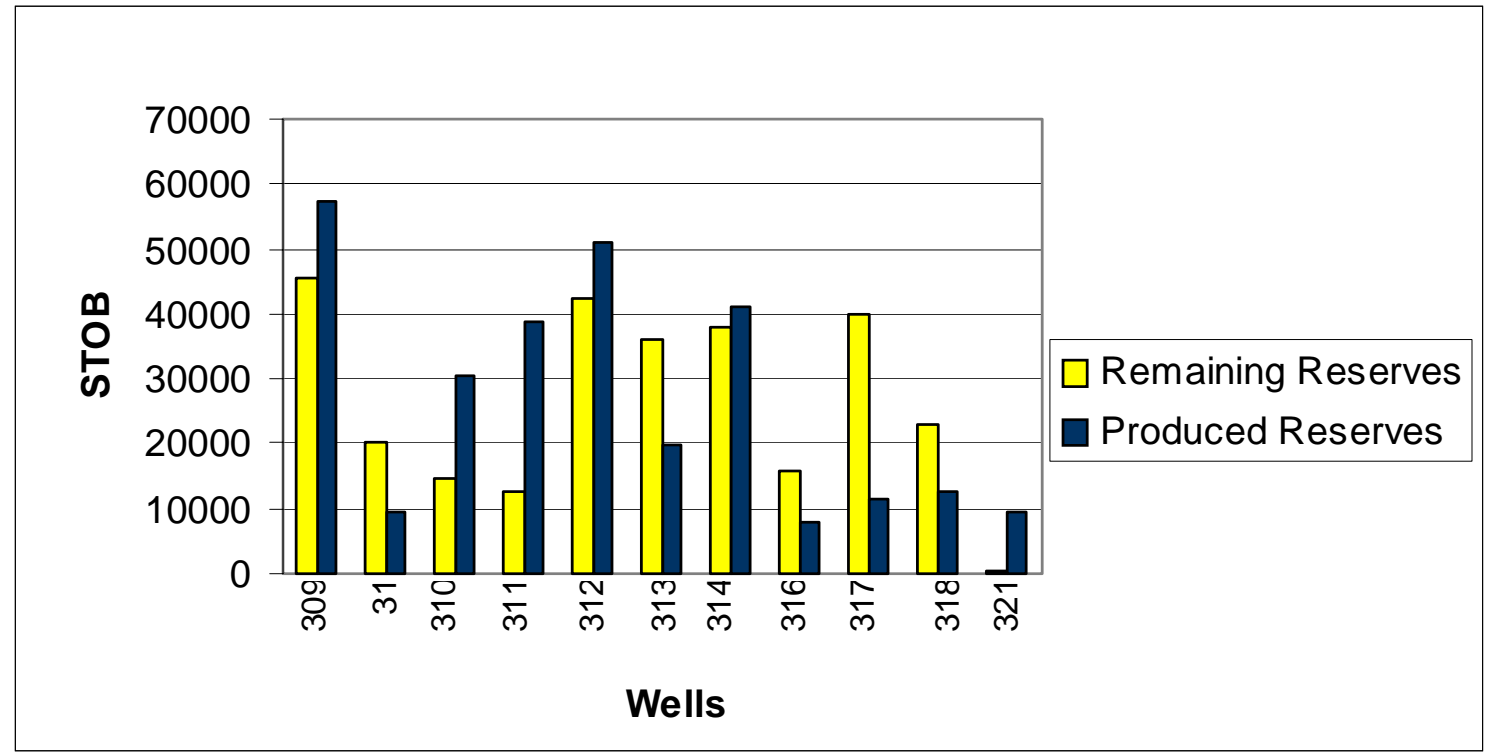

Fig. 1.4.80 - Remaining and Produced Reserves for wells GSU-309,GSU-31,GSU-310,GSU311,GSU-312,GSU-313,GSU-314,GSU-316,GSU-317,GSU-318, and GSU-321. 


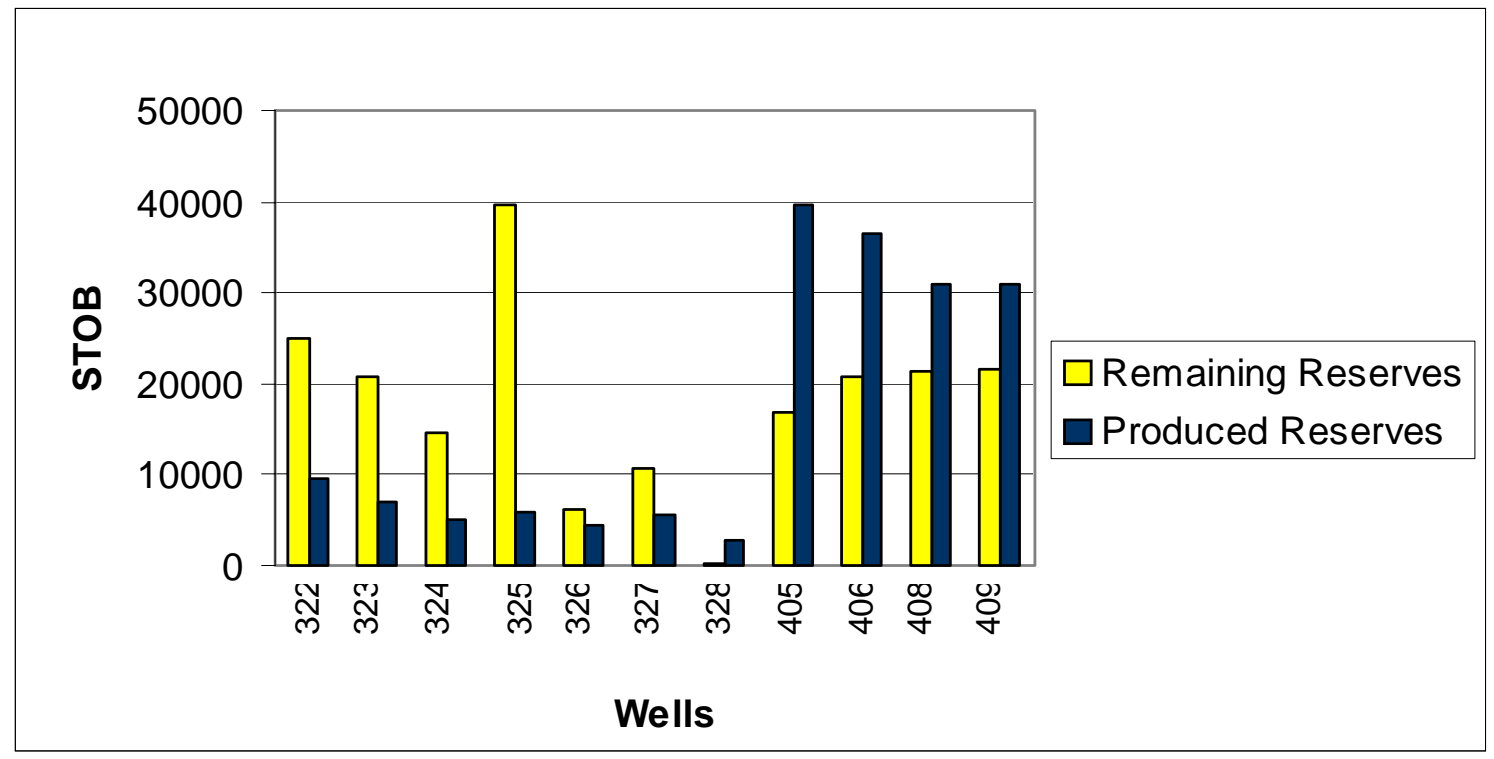

Fig. 1.4.81- Remaining and Produced Reserves for wells GSU-322, GSU-323, GSU-324, GSU325, GSU-326, GSU-327, GSU-328, GSU-405, GSU-408, and GSU-409.

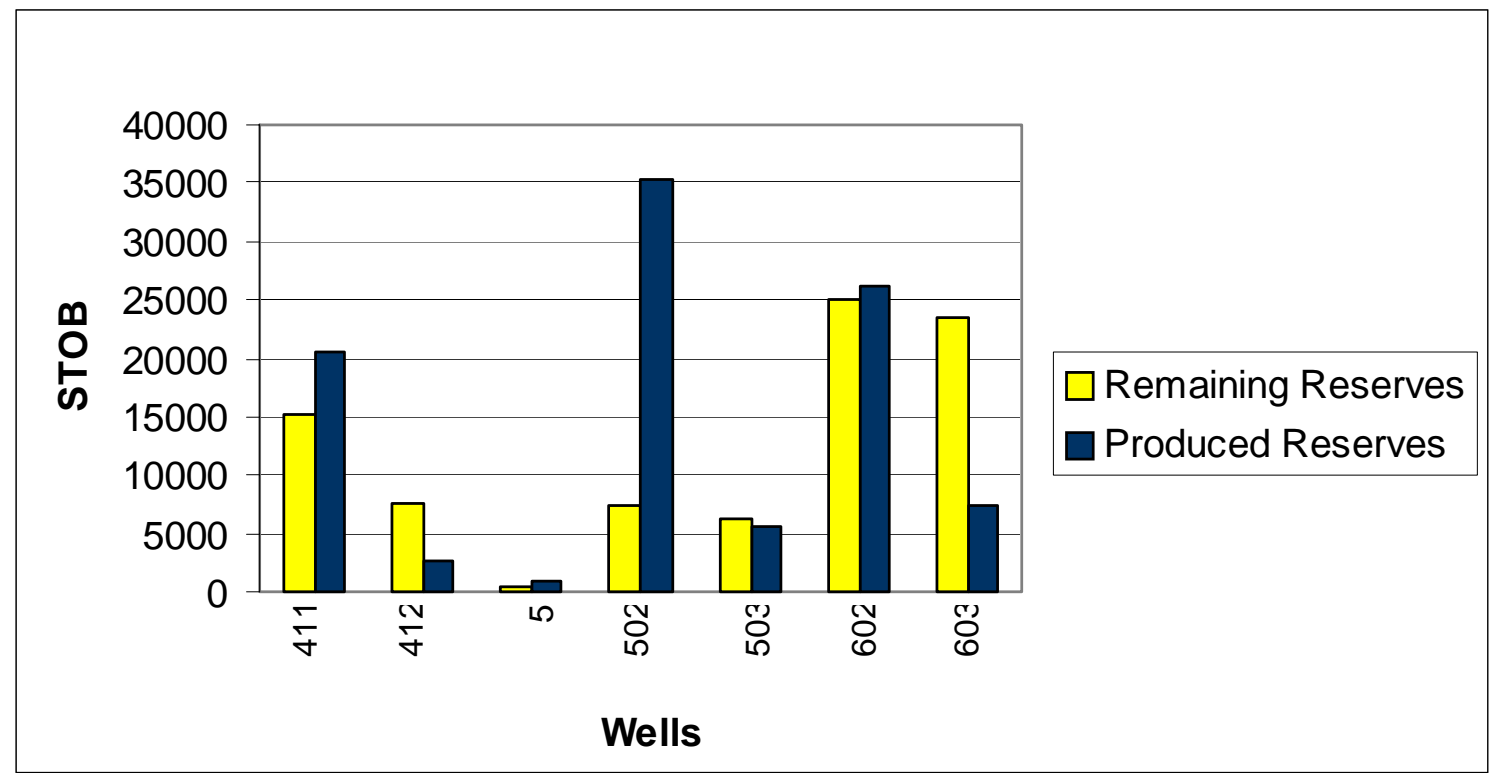

Fig. 1.4.82- Remaining and Produced Reserves for wells GSU-411,GSU-412,GSU-5,GSU502,GSU-503,GSU-602, and GSU-602.

We also plotted the results of both remaining reserves and estimated ultimate recovery for every active well in a bubble map. Fig. 1.4.83 and 1.4.84 depict the areal distribution of the remaining reserves and estimated ultimate recovery respectively. In both figures, we can identify prospective areas for the future development of the unit. 
According to these figures the areas with the most remaining reserves and therefore most opportunities are located in the north-east part of the unit.

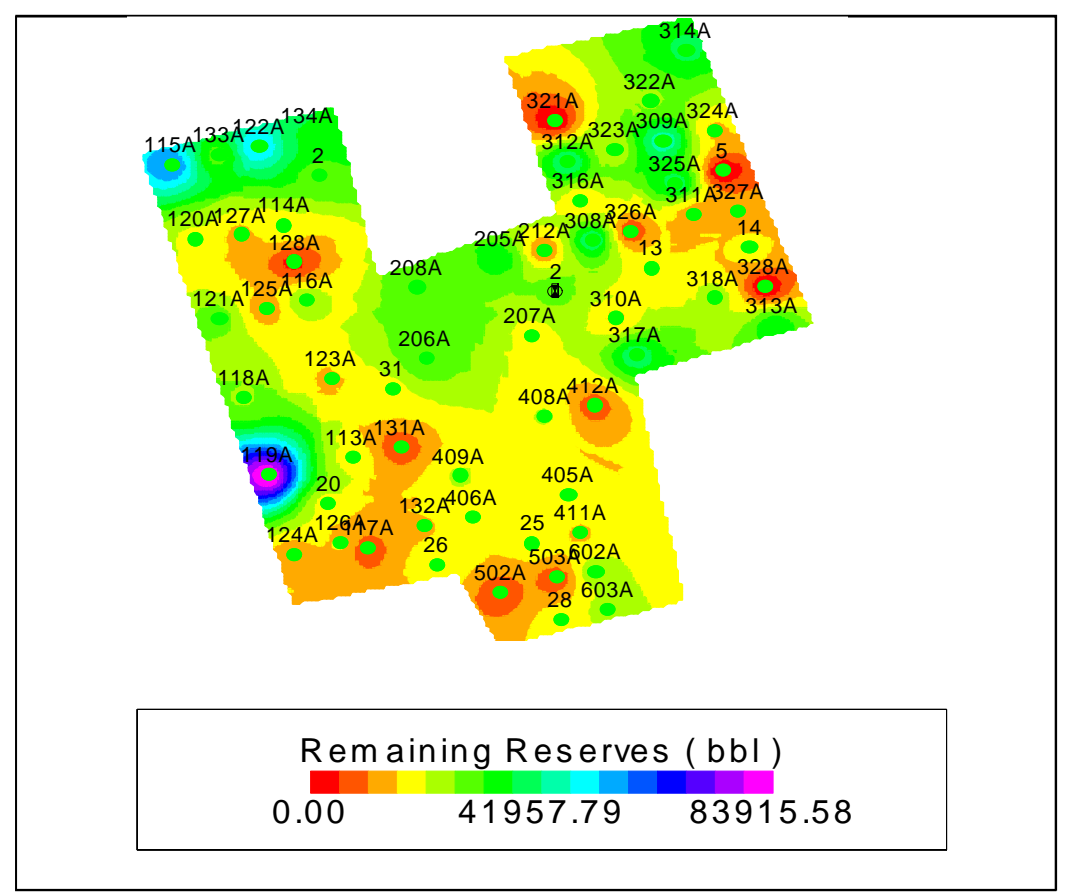

Fig.1.4.83- Bubble Maps of Remaining Reserves. 


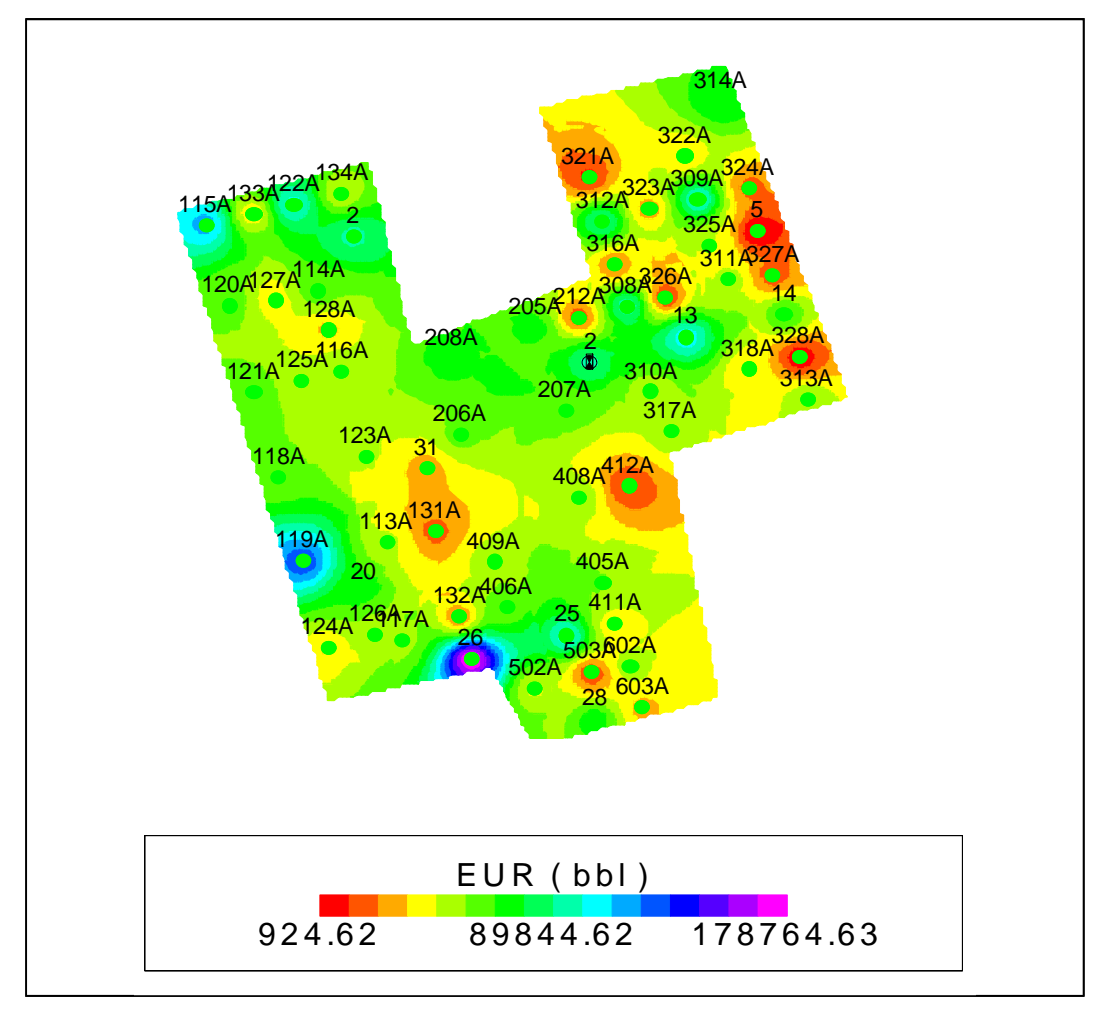

Fig. 1.4.84 - Bubble Maps of Estimated Ultimate Recovery (EUR).

Besides estimating the remaining reserves using hyperbolic-type decline, we also plotted the water-oil ratio vs. cumulative oil production for the entire unit. Fig. 1.4.85 illustrates this analysis. The estimated ultimate recovery is equal to the one obtained through the application of hyperbolic-decline type analysis (6.562 million barrels). The extrapolation (dash line is done from the current cumulative production of 2.12 million barrels until reaching economic limit of WOR equal to 50 ). These results suggest that the unit will be most likely producing and additional 1.410 million barrels through the well currently actives. The figure also illustrates the impact of the different drilling campaigns on the recovery. This analysis also suggests that a new infill drilling campaign (reducing the wells spacing) targeting the areas with the most remaining reserves "sweet spots" would have a great impact on the production and the recovery. 


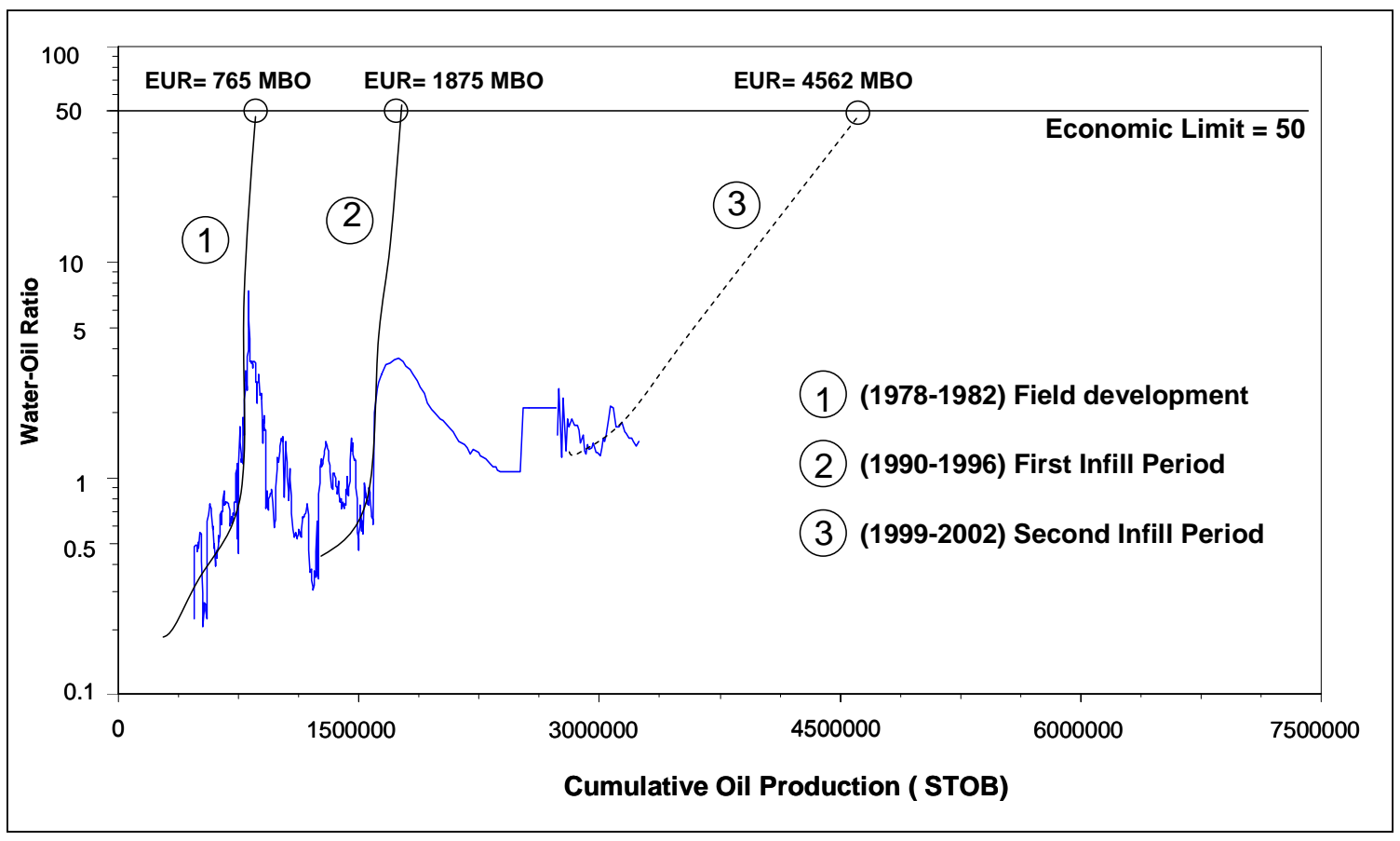

Fig. 1.4.85- WOR behavior and Cumulative Oil Production due to Infill drilling and Waterflooding in Germania Spraberry Unit.

\section{Development Opportunities}

There are some opportunities in Germania Spraberry Unit to increase areal efficiency through infill drilling to control reservoir heterogeneity and connectivity. Infill drilling has shown a significant impact on the waterflooding recovery in reservoir which characteristics are similar to those in Germania Spraberry Unit. Based upon an analysis of the performances of 24 reservoirs in West Texas, some studies have shown a certain correlation trend between the waterflood recovery and the well spacing. ${ }^{3}$ In the case of Germania Spraberry Unit, more than 80 infill drilling wells have been drilled as the unit have gone from primary on 160 acre spacing , through waterflooding on 80 acre spacing, to 40 acre spacing and oil reserves have been increased from 0.765 to 4.562 million barrels by the implementation of these programs. Based on that, we believe that reducing the well spacing to 20 acres in those areas of greater net thickness and higher percent of sandstone and siltstone along with the new injection pattern; constitute a great opportunity to increase the recovery factor in this unit.

Further use of horizontal drilling (targeting the areas with the most remaining reserves) to take advantage of the natural fractures provides an opportunity to increase well productivity and additional recovery over that of conventional or vertical wells.

Some wells have been completed only in either the unit $1 \mathrm{U}$ or in the unit $5 \mathrm{U}$ and therefore additional oil recovery could be obtained by well recompletions or by deepening wells currently bottomed in the upper unit (1U). These recompletion 
opportunities should be evaluated with the purpose of preventing or recovering trapped oil and maximizing sweep efficiency in future operations exposing more of the oil zone, or plugging back to reduce excessive water production. For example, in producing wells that offset, or are adjacent to injectors, some channeling of injected water may occur, resulting in high water cuts. Injection profile work, followed by the use of plugging material may mitigate this problem.

\section{Conclusion and Recommendations}

The methodology, analyses, and results described here can be used to improve the recovery and monitor the performance of Germania Spraberry unit, as wells as others waterflood units in Spraberry.

The following specific conclusions can be drawn based on our findings in the research work:

1. Germania Spraberry Unit can be successfully flooded with the new injection pattern and with injection rate of 1600 BWPD.

2. Under the current conditions, 1.414 million barrels can be recovered in the next 20 years through the wells currently active, especially in the north-east part of the unit.

3. Infill-drilling wells reducing the spacing to 20 acres represent an opportunity to increase the recovery factor in the unit.

4. The production performance in Germania Spraberry Unit is clearly dominated by the presence of natural fractures and the wettability of the rock.

5. The average Voidage Replacement observed from 1969 to 1975 indicates that the water injection rate was too high in proportion to the fluid production rate. This may explain the high water cut and rapid breakthrough observed in some wells and is perhaps one of the most responsible factors for the poor performance of unit.

6. The log-log plot of WOR and its derivative provide more insight and information for well performance evaluation and surveillance system. Using this surveillance technique, coning and channeling can be discerned and normal displacement, and breakthrough behavior can be differentiated. Results obtained with this type pf plots, indicate that wells GSU and GSU-5 may be experiencing casing leak.

7. Based on decline-curve analysis for active wells, a bubble map showing the areas with the most opportunities (most remaining reserves) was displayed. The map showed that the areas with the most remaining reserves are located towards the north-east part of the unit.

8. Heterogeneity Indexing is a useful surveillance tool for ranking and identifying specific wells with poor or superior performance in Germania Unit. It can also be used as a quick screening tool to identify opportunities in the area. The results of the application of this screening technique suggest that wells GSU-2, GSU-127, GSU114, GSU-128, GSU-116, GSU-123, GSU-412, GSU-328, and GSU-5 are good candidates for the application of water control techniques. 
9. Tract 2 has the best performance in terms of cumulative oil per acre (938 barrels per acre). This is consequence of the response of the injection in this area (one injector was located at the center of this tract and the rest surrounding the tract.).

10. Wells drilled in the first campaign ( from 1957 to 1964) have shown the highest value of average initial rate ( 48 BOPD) and the performance shown by wells drilled during the third campaign (from 1990 to 1996) demonstrate the importance and impact of infill drilling in this unit.

11. Areas having the best oil-producing wells (“sweet spots”) and their adjacent water injection wells formed trends parallel to the main set of natural fractures ( $\mathrm{N} 56^{\circ} \mathrm{E}$ ) and are also correlative with axes of maximum net pay.

\section{Recommendations for future work.}

1. Modern well logs and core data are necessary for the purpose of characterization to identify oil-saturated intervals to be completed for oil production and avoid water bearing intervals, evaluate primary cementing in order to prevent casing leaks from recompleted wells, and design an optimum hydrofracturing to minimize the possibility of inducing fractures that might connect oil-prospective intervals with water-bearing intervals.

2. Examine the feasibility of tertiary miscible flooding using $\mathrm{CO}_{2}$ to reduce the residual oil saturation and increase the recovery in the unit after cessation of the waterflooding project.

3. Examine the feasibility of conducting studies of economic evaluation involving risk and uncertainties in the data and economics conditions.

4. Examine the feasibility of conducting a numerical reservoir simulation in this unit to make sensitivities of different parameters (fracture spacing, matrix and fracture permeability, relative permeability, and capillary effects) and evaluate its effect on the recovery. 


\section{REFERENCES}

1. Guevara, E.H.: "Geological Characterization of Permian Basin submarine fan Reservoirs of the Driver Waterflood Unit, Spraberry Trend, Midland Basin, Texas, ” Report of Investigation No. 172.

2. Sterling, J.L.: "Fracture Generation and Fluids in the Spraberry Formation, Midland Basin, Texas,” MS Thesis, New Mexico Institute of Mining and Technology, Socorro, New Mexico, May 2000.

3. Reese, R.D.: "Completion Ranking Using Production Heterogeneity Indexing," paper SPE 36604 presented at the 1996 SPE Annual Technical Conference and Exhibition, Denver, Colorado, 6-9 October.

4. Chan, K.S.: "Water Control Diagnostic Plots," paper SPE 30775 presented at the 1995 SPE Annual Technical Conference and Exhibition, Dallas, Texas, 22-25 October.

5. Oil Filed Manager user’s Manual, Schlumberger, Houston, Texas (2002).

6. Thakur, G.C. and Satter, A.: Integrated Waterflood Asset Management, Penn Well Publishing Co., Tulsa, Oklahoma (1998).

7. Thakur, G.C.: "Waterflood Surveillance Techniques- A Reservoir Management Approach,” paper SPE 23471 available from SPE, Richardson, Texas (1991).

8. Putra, E. and Schechter, D.S.: "Reservoir Simulation of Waterflood Pilot in Naturally Fractured Spraberry Trend Area,” paper SPE 54336 presented at the 1999 SPE Asia Pacific Oil and Gas Conference and Exhibition, Jakarta, Indonesia, 20-22 April.

9. Beliveau, D. and Payne, D.A.: “Analysis of Waterflood Response of a Naturally Fractured Reservoir,” paper SPE 22946 presented at the 1991 SPE Annual Technical Conference and Exhibition, Dallas, Texas, 6-9 October.

10. Guidroz, G.M.: “E.T O’Daniel Project- A Successful Spraberry Flood,” paper SPE 1791 available from SPE, Richardson, Texas (1967).

11. Wade, J.M., et al.: "Case History of Oil Well Performance Monitoring and production Optimization in the Eldfisk and Ekofisk Fields, Norwegian North Sea," paper SPE 48847 presented at the 1998 SPE International Conference and Exhibition, Beijing, China, 2-6 November.

12. Fetkovich, M.J.: “Decline Curve Analysis Using Type Curves,” 1972, AIME, New York.

13. Towler, B.F.: Fundamental Principles of Reservoir Engineering, Textbook Series Vol. 8, SPE, Richardson, TX (2002), 170-172.

14. Seba, R.D.: Economics of Worldwide Petroleum Production, Gulf Publishing Co., Houston, Texas (2000) Chap. 2, 14-46.

15. Schechter, D.S.: “Advanced Reservoir Characterization and Evaluation of $\mathrm{CO}_{2}$ Gravity Drainage in the Naturally Fractured Spraberry Trend Area," The Second Annual Technical Progress Report for Contract No. DE-FC22-95BC14942. PRRC Report No. 97-29. 
16. Schechter, D.S.: "Preferred Waterflood Management Practices for the Spraberry Trend Area” Annual Technical Progress Report for Contract No. DE-FC26$01 \mathrm{BC} 15274$. 


\section{APPENDIX}

FORECAST ANALYSIS AND RESERVE ESTIMATION FOR ACTIVE WELLS IN GERMANIA SPRABERRY UNIT. 


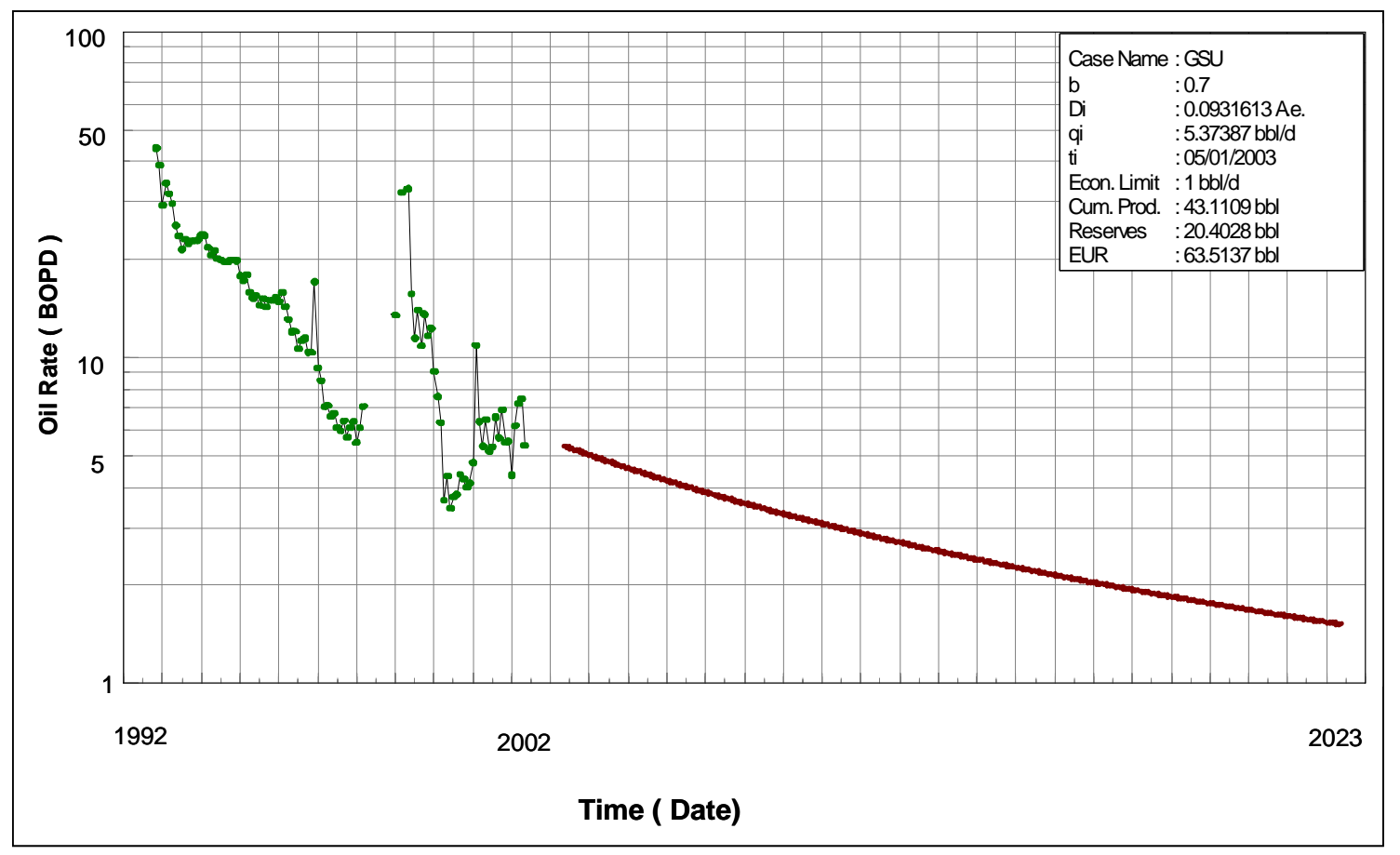

Fig. 1.4.A. 1- Decline Curve Analysis for Well GSU-114

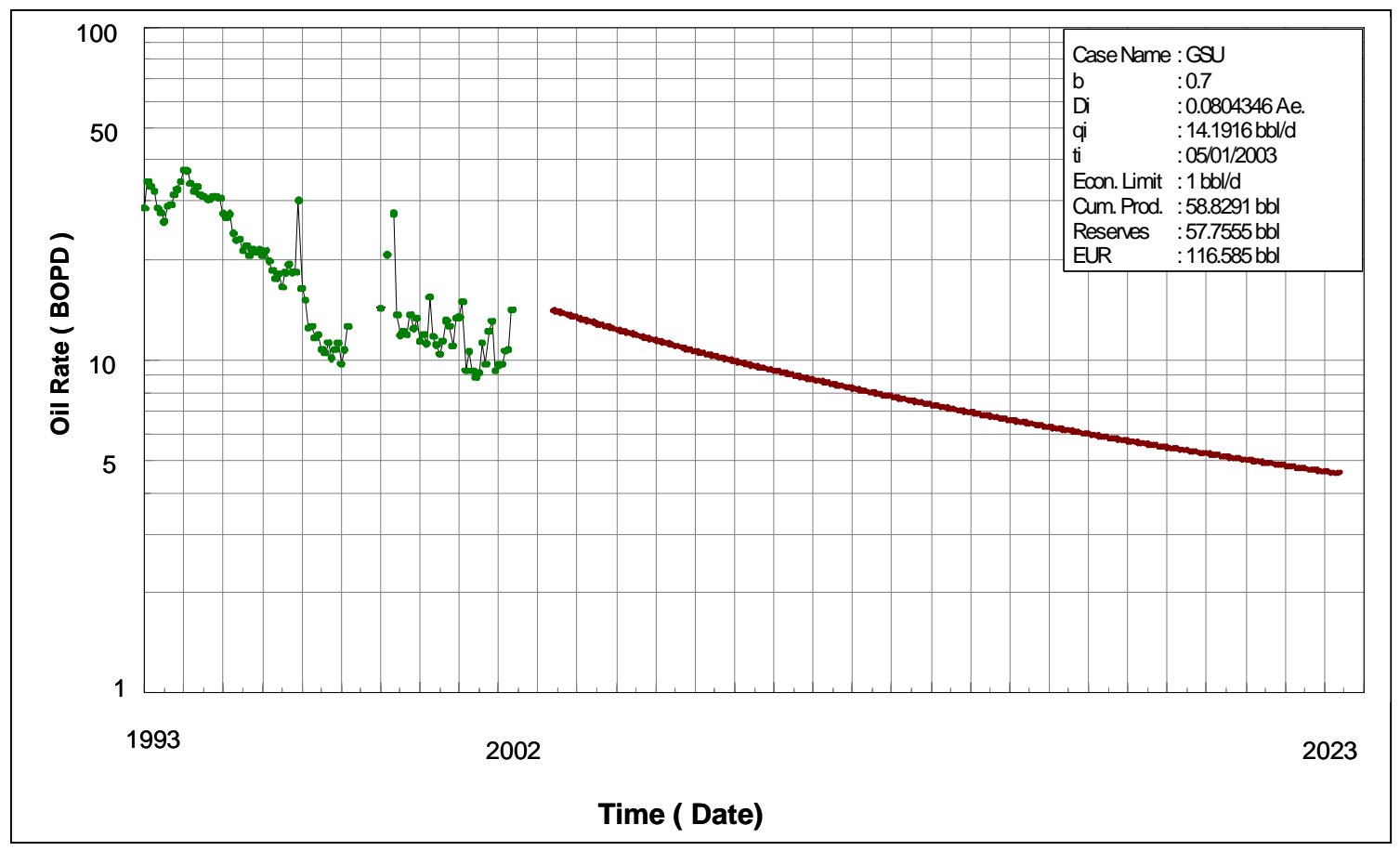

Fig. 1.4.A. 2-Decline Curve Analysis for Well GSU-115 


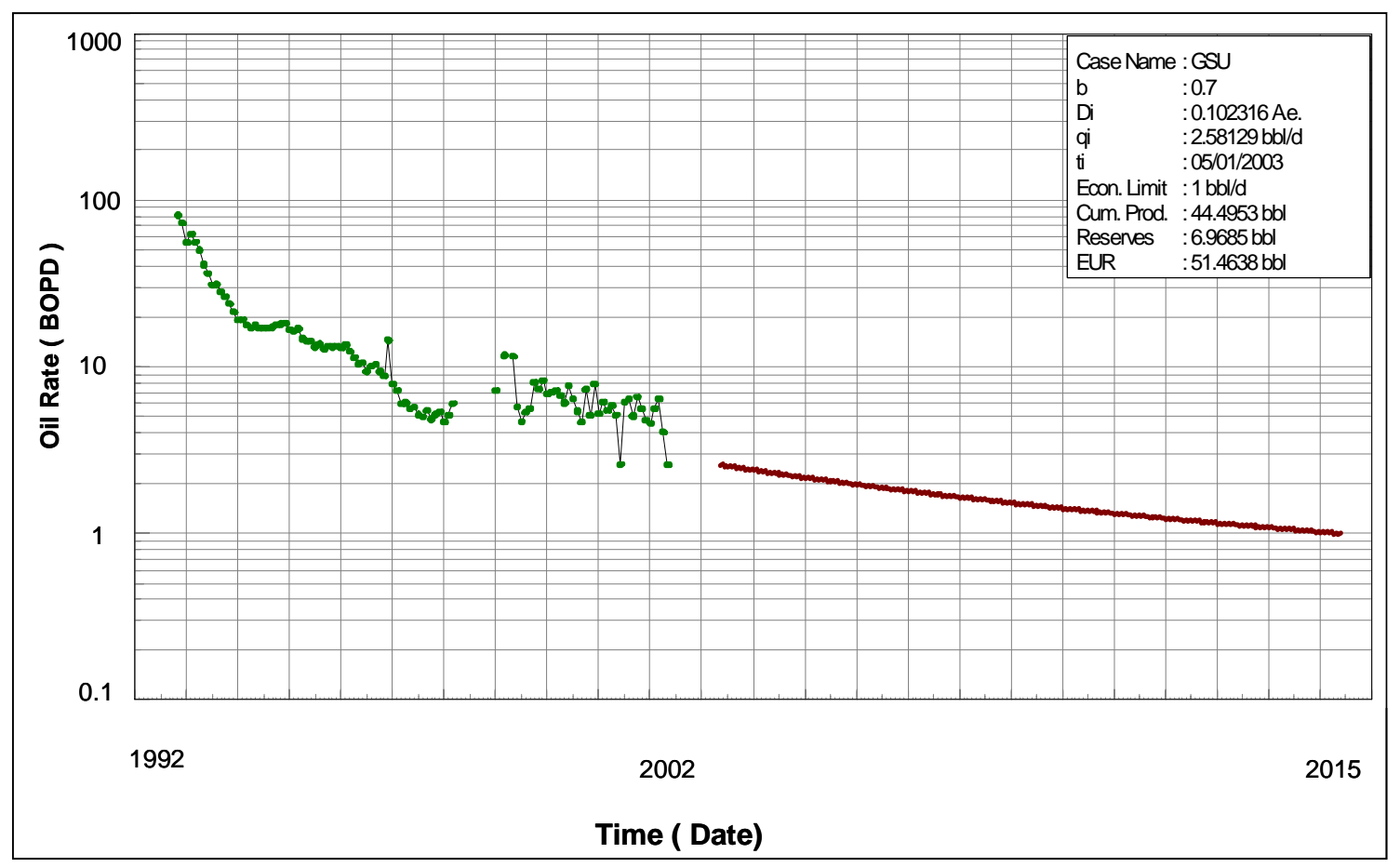

Fig. 1.4.A. 3- Decline Curve Analysis for Well GSU-117

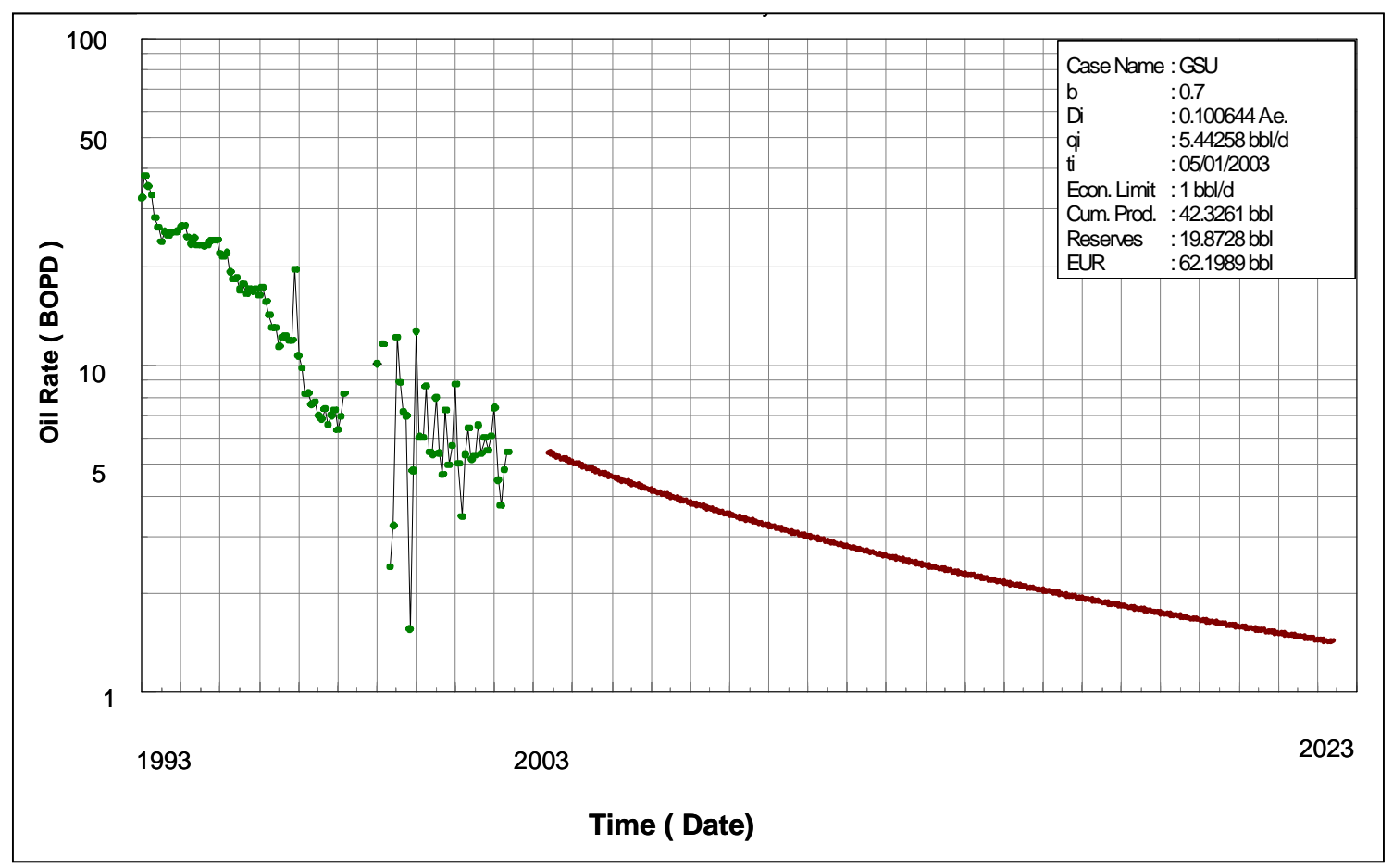

Fig. 1.4.A. 4- Decline Curve Analysis for Well GSU-120 


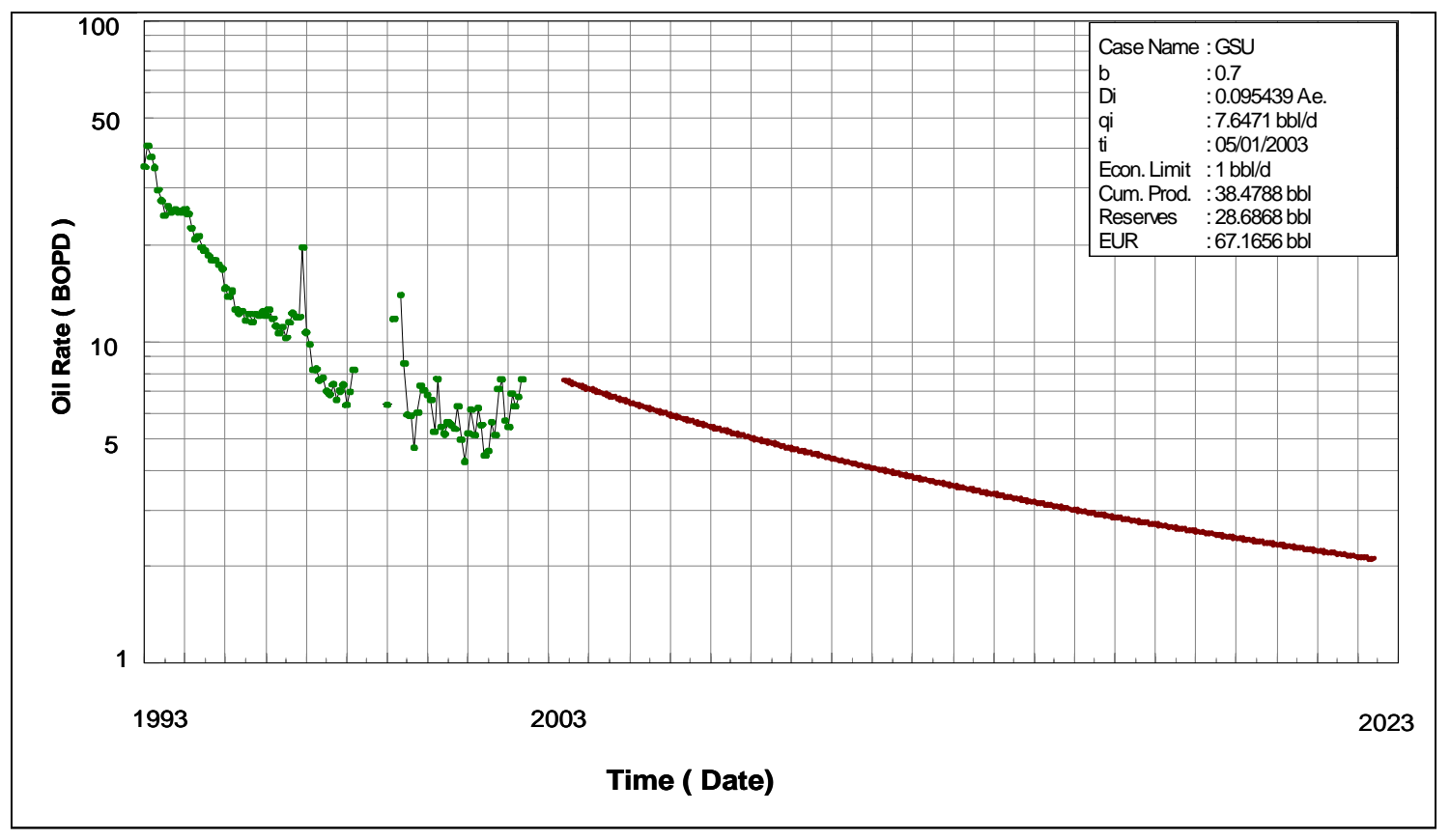

Fig. 1.4.A. 5- Decline Curve Analysis for Well GSU-121

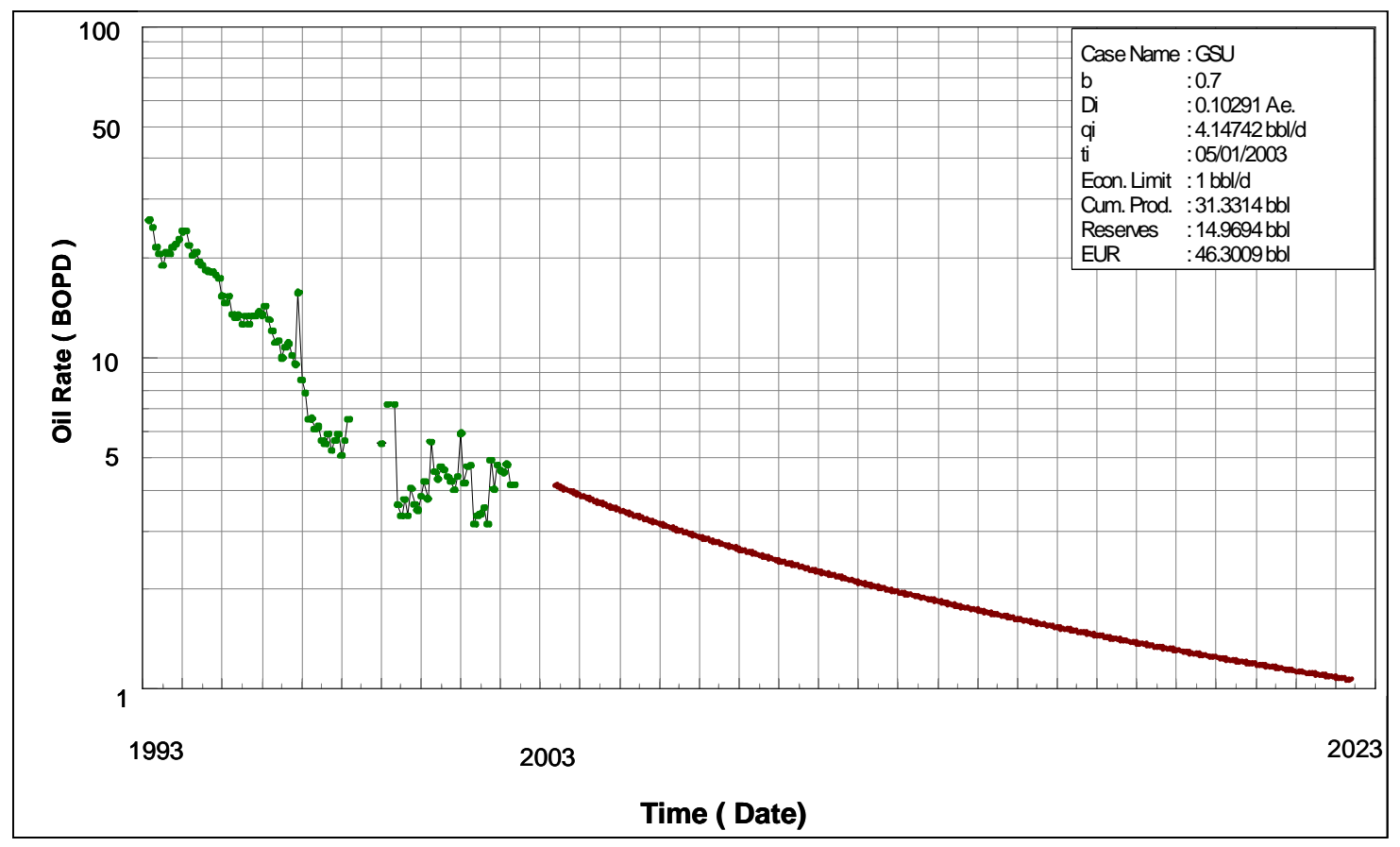

Fig. 1.4.A. 6- Decline Curve Analysis for Well GSU-123 


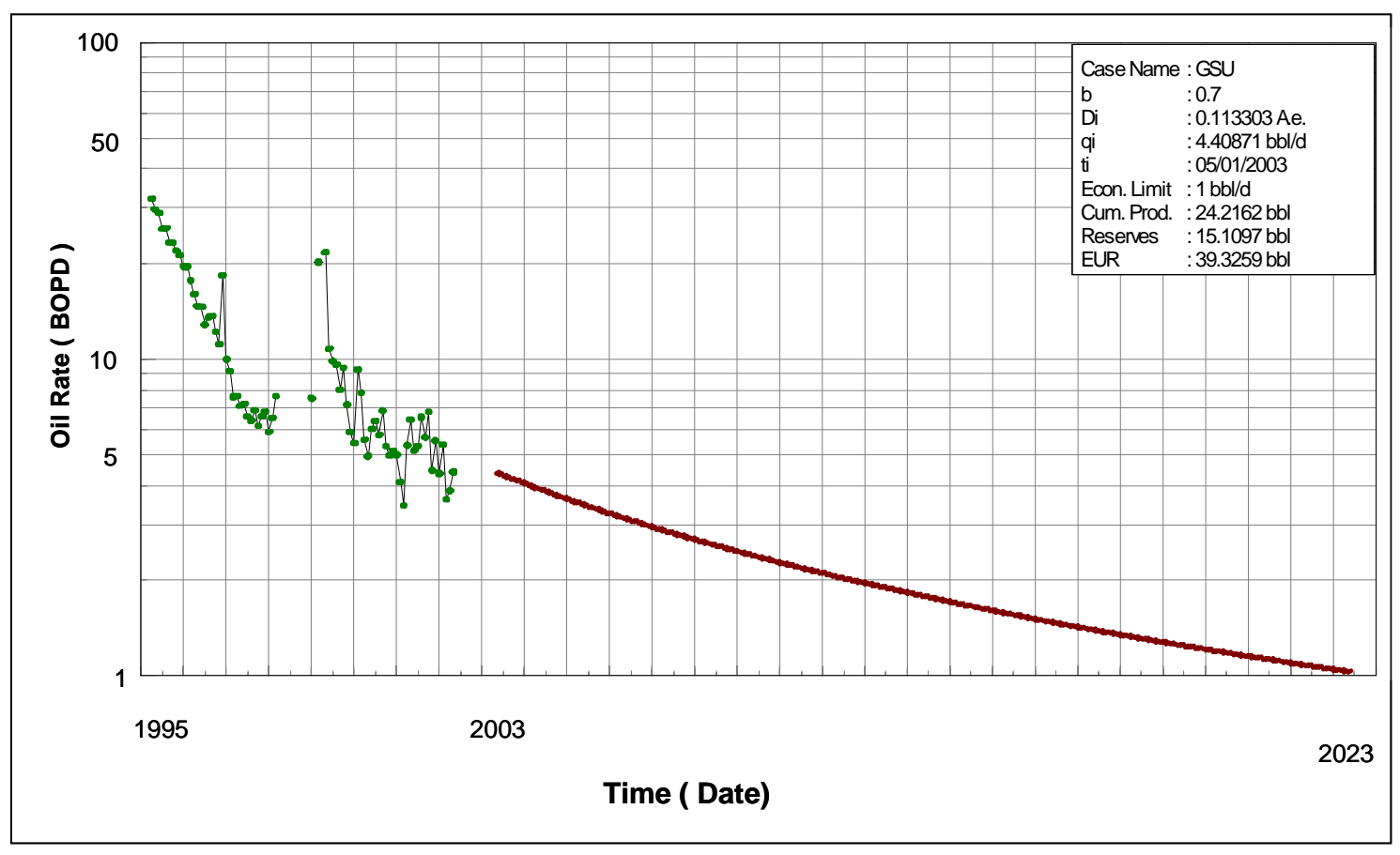

Fig. 1.4.A. 7- Decline Curve Analysis for Well GSU-127

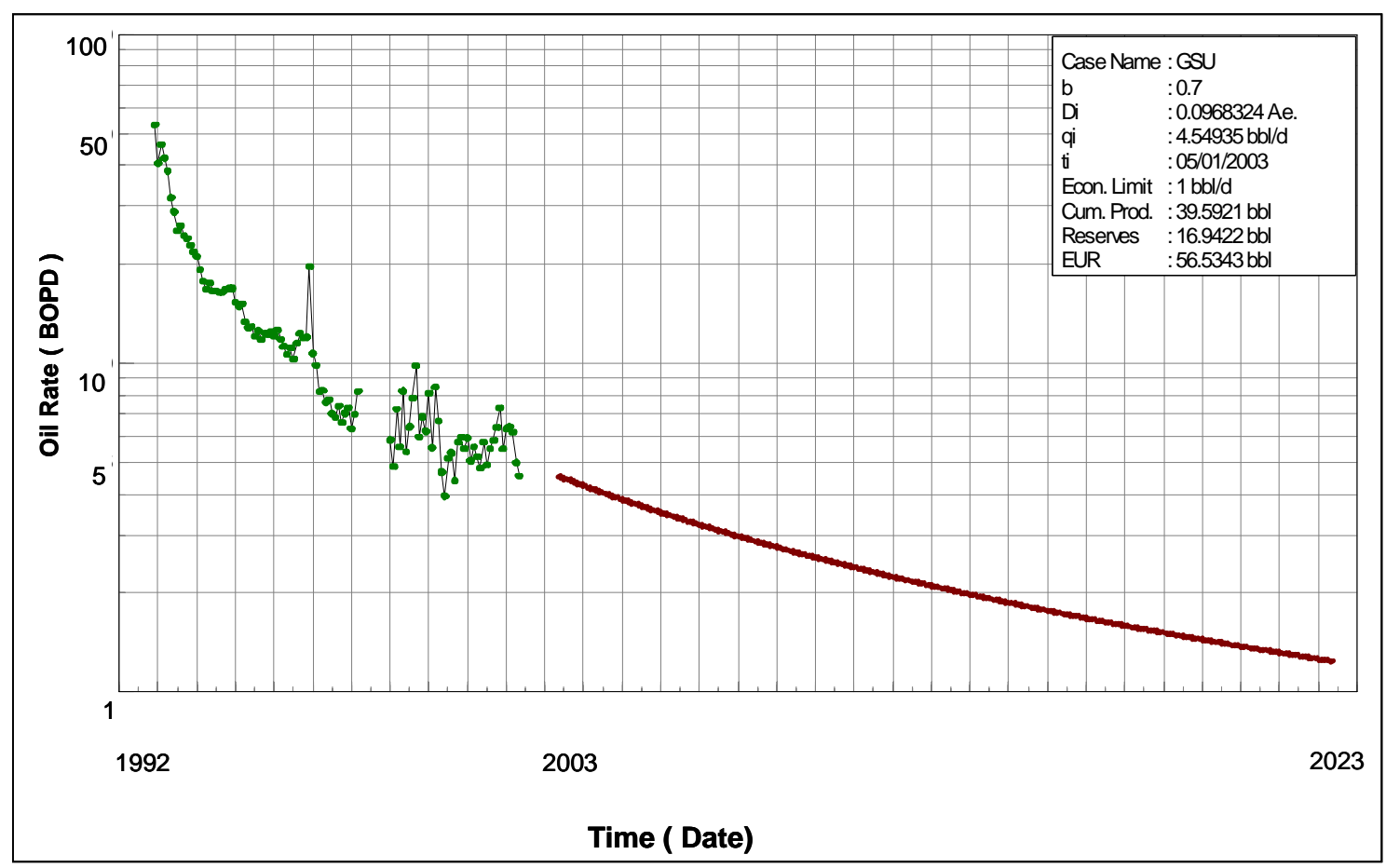

Fig. 1.4.A. 8- Decline Curve Analysis for Well GSU-405 


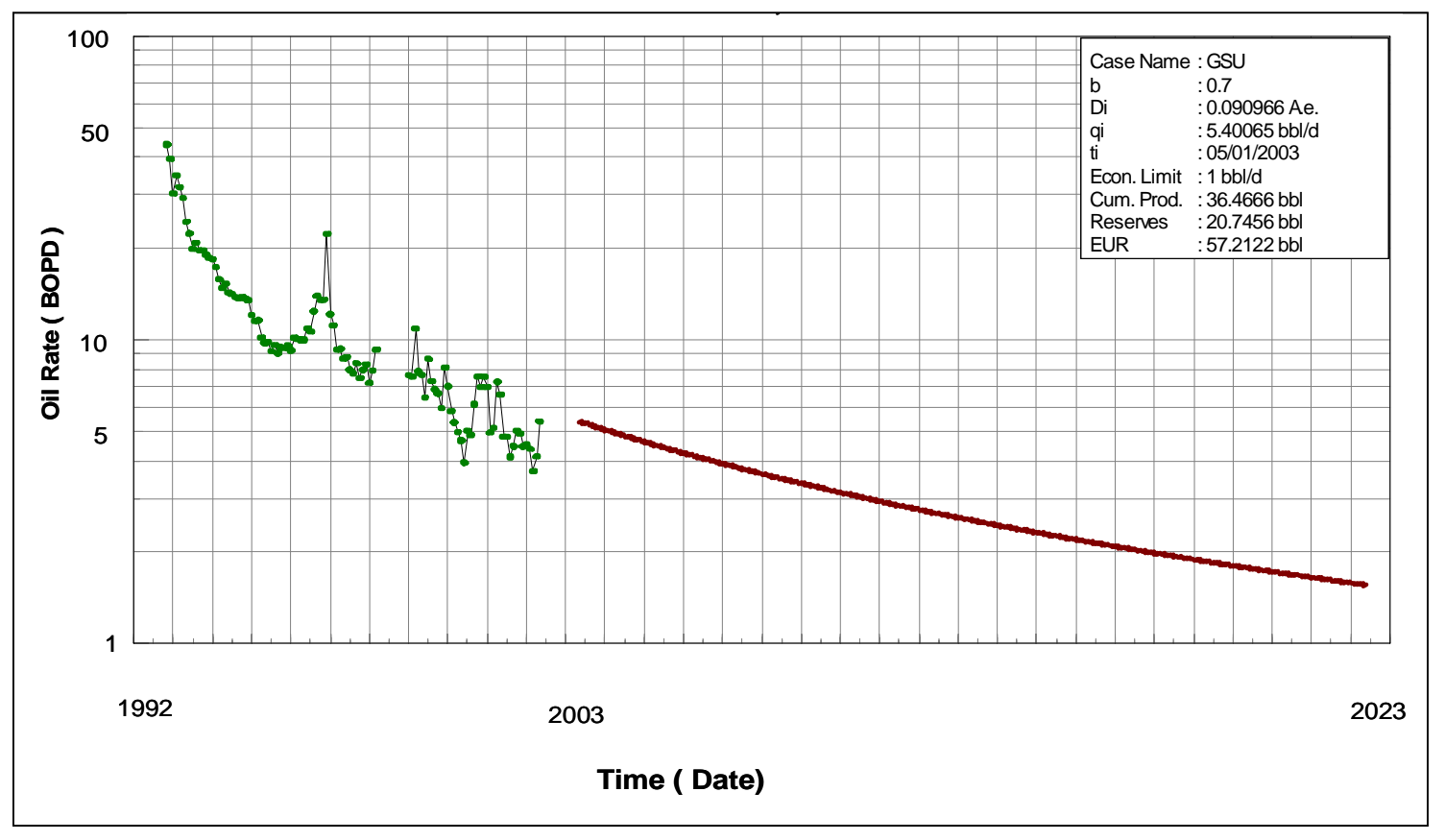

Fig. 1.4.A. 9- Decline Curve Analysis for Well GSU-406

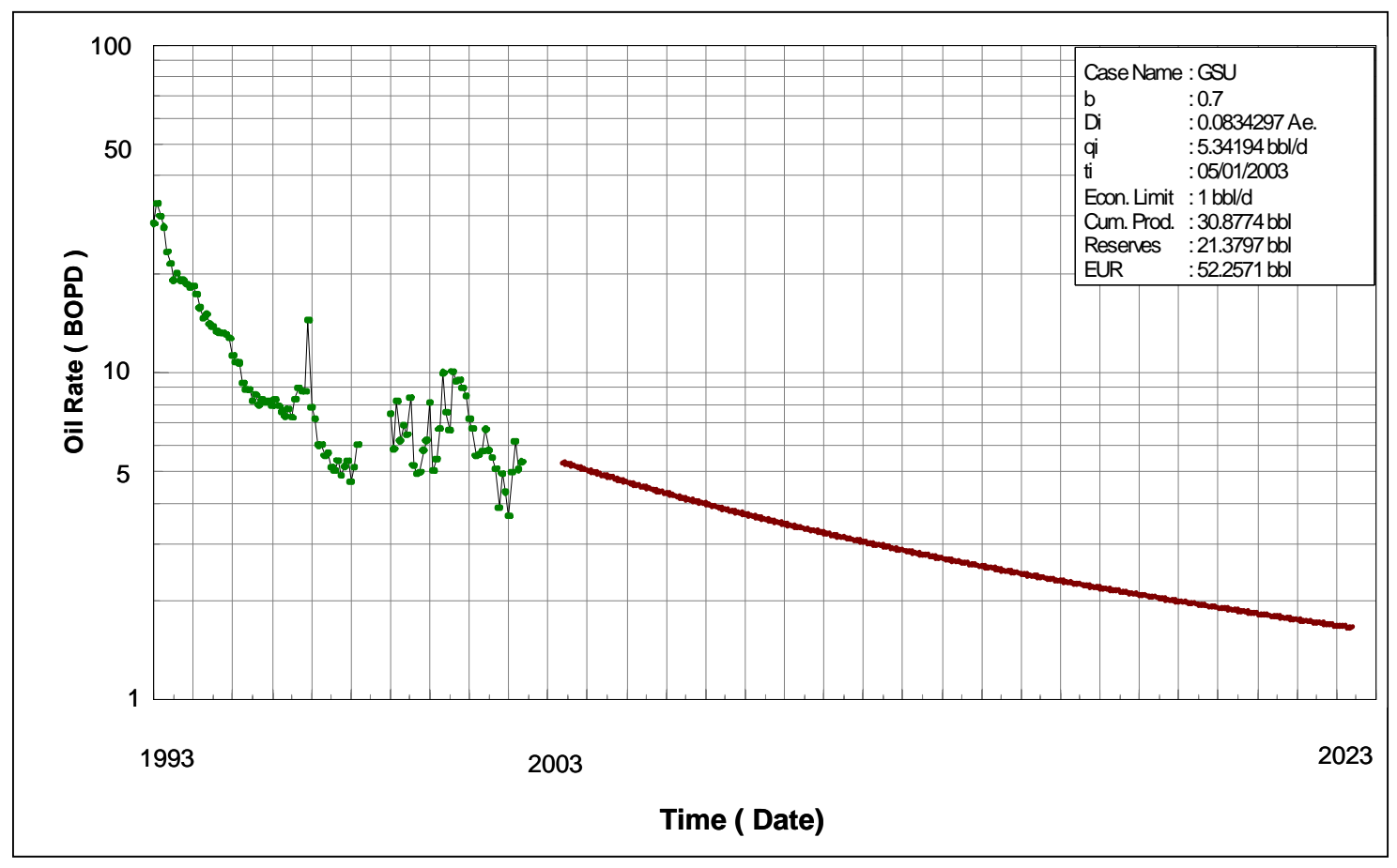

Fig. 1.4.A. 10- Decline Curve Analysis for Well GSU-408 


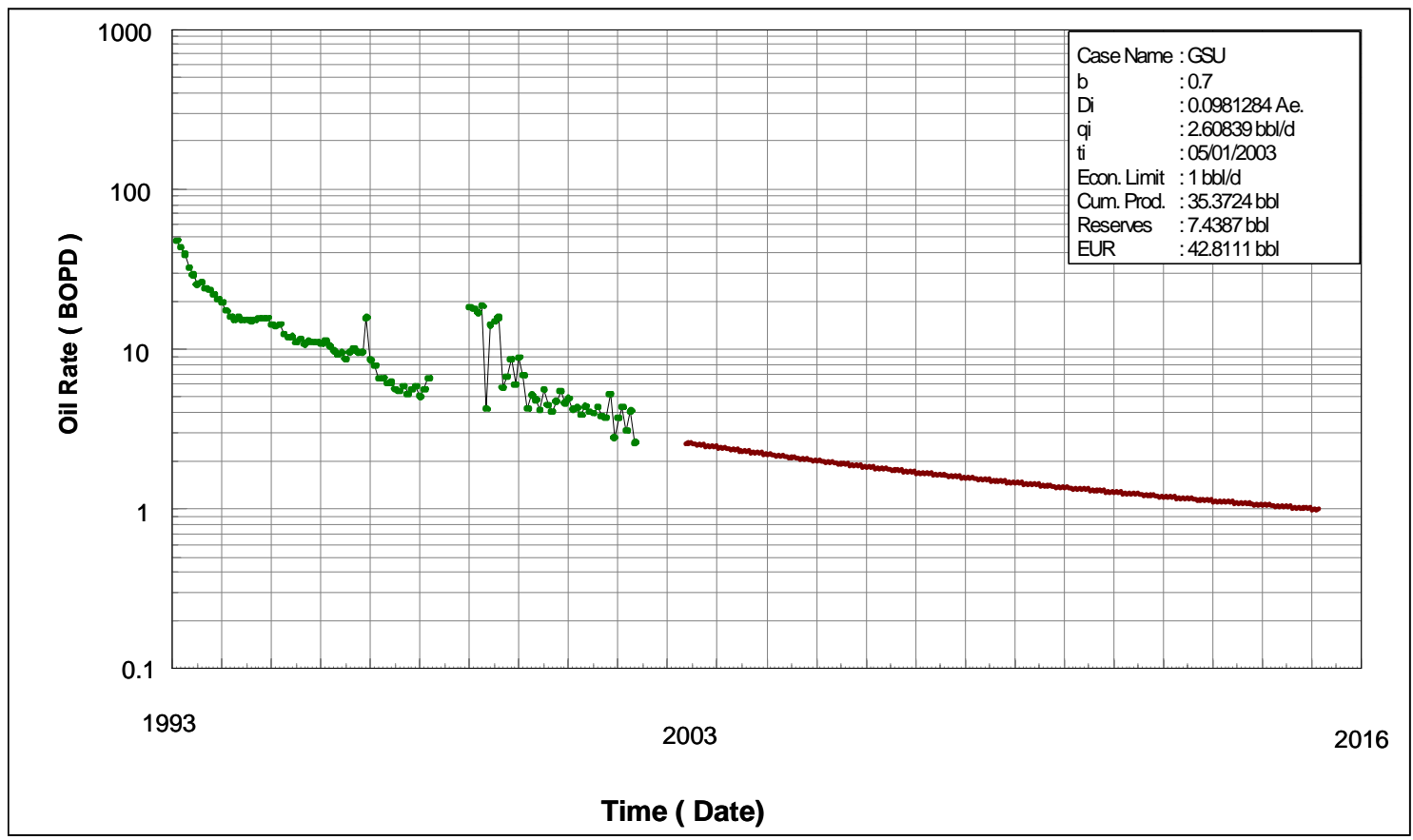

Fig. 1.4.A.11 - Decline Curve Analysis for Well GSU-502 
Table 1.4.A. 1-Remaining Reserves and Estimated Ultimate recovery for Active Wells.

\begin{tabular}{|c|c|c|c|}
\hline Well & $\begin{array}{l}\text { Remaining } \\
\text { Reserves } \\
\text { ( Barrels) }\end{array}$ & $\begin{array}{c}\text { Cumulative } \\
\text { Oil } \\
\text { (Barrels) } \\
\text { (As of 2003) }\end{array}$ & $\begin{array}{l}\text { Estimated } \\
\text { Ultimate } \\
\text { Recovery } \\
\text { (Barrels) }\end{array}$ \\
\hline $113 \mathrm{~A}$ & 19216 & 27587 & 46802 \\
\hline $114 \mathrm{~A}$ & 20403 & 43111 & 63514 \\
\hline $115 \mathrm{~A}$ & 57755 & 58829 & 116585 \\
\hline $116 \mathrm{~A}$ & 25131 & 31429 & 56560 \\
\hline $117 \mathrm{~A}$ & 6969 & 44495 & 51464 \\
\hline $118 \mathrm{~A}$ & 25742 & 34287 & 60030 \\
\hline $119 A$ & 83916 & 46613 & 130529 \\
\hline $120 \mathrm{~A}$ & 19873 & 42326 & 62199 \\
\hline $121 \mathrm{~A}$ & 28687 & 38479 & 67166 \\
\hline $122 \mathrm{~A}$ & 53172 & 51211 & 104383 \\
\hline $123 \mathrm{~A}$ & 14969 & 31331 & 46301 \\
\hline $124 \mathrm{~A}$ & 11488 & 21868 & 33356 \\
\hline $125 \mathrm{~A}$ & 12909 & 31863 & 44772 \\
\hline $126 \mathrm{~A}$ & 16149 & 33368 & 49517 \\
\hline $127 \mathrm{~A}$ & 15110 & 24216 & 39326 \\
\hline $128 \mathrm{~A}$ & 3169 & 28542 & 31711 \\
\hline 13 & 18372 & 89433 & 107805 \\
\hline $131 \mathrm{~A}$ & 6599 & 12883 & 19483 \\
\hline $132 \mathrm{~A}$ & 15084 & 8180 & 23263 \\
\hline $133 \mathrm{~A}$ & 28799 & 6655 & 35454 \\
\hline $134 \mathrm{~A}$ & 36255 & 8286 & 44541 \\
\hline 14 & 19352 & 51288 & 70640 \\
\hline 2 & 28723 & 64118 & 92841 \\
\hline 20 & 18643 & 52566 & 71209 \\
\hline $205 A$ & 34693 & 36747 & 71440 \\
\hline $206 \mathrm{~A}$ & 31784 & 28078 & 59862 \\
\hline $207 A$ & 19231 & 38885 & 58116 \\
\hline $208 \mathrm{~A}$ & 29909 & 41669 & 71578 \\
\hline $212 \mathrm{~A}$ & 9699 & 5481 & 15180 \\
\hline 25 & 19582 & 86032 & 105614 \\
\hline
\end{tabular}


Table A.1-Continued.

\begin{tabular}{|c|c|c|c|}
\hline Well & \begin{tabular}{|c|} 
Remaining \\
Reserves \\
( Barrels)
\end{tabular} & 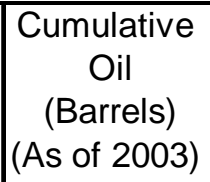 & $\begin{array}{l}\text { Estimated } \\
\text { Ultimate } \\
\text { Recovery } \\
\text { (Barrels) }\end{array}$ \\
\hline 26 & 19608 & 159157 & 178765 \\
\hline 28 & 18780 & 57354 & 76134 \\
\hline $308 \mathrm{~A}$ & 41480 & 55329 & 96809 \\
\hline $309 A$ & 45525 & 57294 & 102820 \\
\hline 31 & 20027 & 9602 & 29629 \\
\hline $310 \mathrm{~A}$ & 14684 & 30337 & 45021 \\
\hline $311 \mathrm{~A}$ & 12508 & 38629 & 51137 \\
\hline $312 \mathrm{~A}$ & 42395 & 51011 & 93406 \\
\hline $313 \mathrm{~A}$ & 35863 & 19642 & 55505 \\
\hline $314 \mathrm{~A}$ & 37824 & 41292 & 79116 \\
\hline $316 A$ & 15661 & 7880 & 23541 \\
\hline $317 A$ & 39891 & 11367 & 51259 \\
\hline $318 \mathrm{~A}$ & 23002 & 12622 & 35623 \\
\hline $321 \mathrm{~A}$ & 400 & 9514 & 9914 \\
\hline $322 \mathrm{~A}$ & 25111 & 9602 & 34713 \\
\hline $323 \mathrm{~A}$ & 20902 & 6938 & 27841 \\
\hline $324 \mathrm{~A}$ & 14648 & 5125 & 19774 \\
\hline $325 \mathrm{~A}$ & 39668 & 5829 & 45497 \\
\hline $326 \mathrm{~A}$ & 6061 & 4460 & 10521 \\
\hline $327 A$ & 10660 & 5712 & 16372 \\
\hline $328 \mathrm{~A}$ & 400 & 2933 & 3333 \\
\hline $405 \mathrm{~A}$ & 16942 & 39592 & 56534 \\
\hline $406 \mathrm{~A}$ & 20746 & 36467 & 57212 \\
\hline $408 \mathrm{~A}$ & 21380 & 30877 & 52257 \\
\hline $409 A$ & 21550 & 30963 & 52513 \\
\hline $411 \mathrm{~A}$ & 15306 & 20575 & 35881 \\
\hline $412 \mathrm{~A}$ & 7595 & 2715 & 10310 \\
\hline 5 & 400 & 925 & 1325 \\
\hline$\overline{502 A}$ & 7439 & 35372 & 42811 \\
\hline$\overline{503 A}$ & 6337 & 5668 & 12005 \\
\hline $602 \mathrm{~A}$ & 24970 & 26139 & 51109 \\
\hline $603 \mathrm{~A}$ & 23391 & 7391 & 30782 \\
\hline
\end{tabular}




\section{DEVELOPMENT OF RESERVOIR MANAGEMENT DATABASE SOFTWARE}

\section{Introduction}

Properly managing a reservoir that is so large and communicates, via the fractures, over great distances, poses a complicated technological and data management constraint. This problem acts as a deterrent for waterflood operations in Spraberry reservoirs. Reservoir engineering, by definition, requires precise injection, production and pressure data. Acquisition and control of this data has always been a constraint to providing the optimum method for water injection. The result is large volumes of oil that could have been recovered via water injection remain untapped. We believe by proper data acquisition and precise reservoir engineering techniques, any lack of confidence in waterflooding can be overcome.

In this final report, we have developed a general and more user friendly production database system. This database software was written using a visual basic-object oriented programming language. It has the capability to perform similar tasks as the OFM database system has, such as decline curve analysis, material balances, bubble map plot and in addition it has more unique features in grouping the wells, performing decline curve analysis for well by well and a group of wells, displaying the graph in $\mathrm{x}-\mathrm{y}$ plot, $2 \mathrm{D}$ and 3D map, and much more.

This program is applicable for any type of reservoir. This report is intended to show the use of this program for managing the data in the Germania Unit. Figure 3.1 shows the front page of the database software. The production and injection input data format follows the TOW data format for an easy access updating of the production data. Figure 3.2 shows the result of uploading the well location, well information, and production/injection data. Once we have uploaded the well data, we can zoom-in and zoom-out the picture to find specific information on a certain well in the cluster location (Fig. 3.3). The zoom-in result can be seen in Fig. 3.4. By pointing the cursor to a certain well, the menu bar in the right hand side shows some information on the selected well. Figure 3.4 shows the menu bars, File, Tool, Window and Help menus. File menu consists New Project, Open Project, Save Project, Save As Project and Exit menus.Tool menu consists Create Group, Load Group File, Interpolation options and Chart Container. Windows menu consists of Field Map, Data and Graph Viewer, Bubble Map, Decline Curve Analysis and Gas Material Balances and Help menu consists of Contents, Product Support, System Information and About menus. Figures 3.5 and 3.6 show example of the tabulated production history and oil production graph of well 207A and we can perform decline analysis with a user-defined range. There are three options for decline analysis: exponential, harmonic and hyperbolic declines. The program also allows user to select the best-fit option automatically based on extended Spivey algorithm (1986). We can also to deselect some data points that may be excluded of finding the decline trend. The example of decline curve analysis using hyperbolic option is presented in Fig. 3.7. 
There are many other features or applications of this software such as bubble map, isobaric map, PVT application, Material Balances that are presented in the Appendix as manual/tutorial.

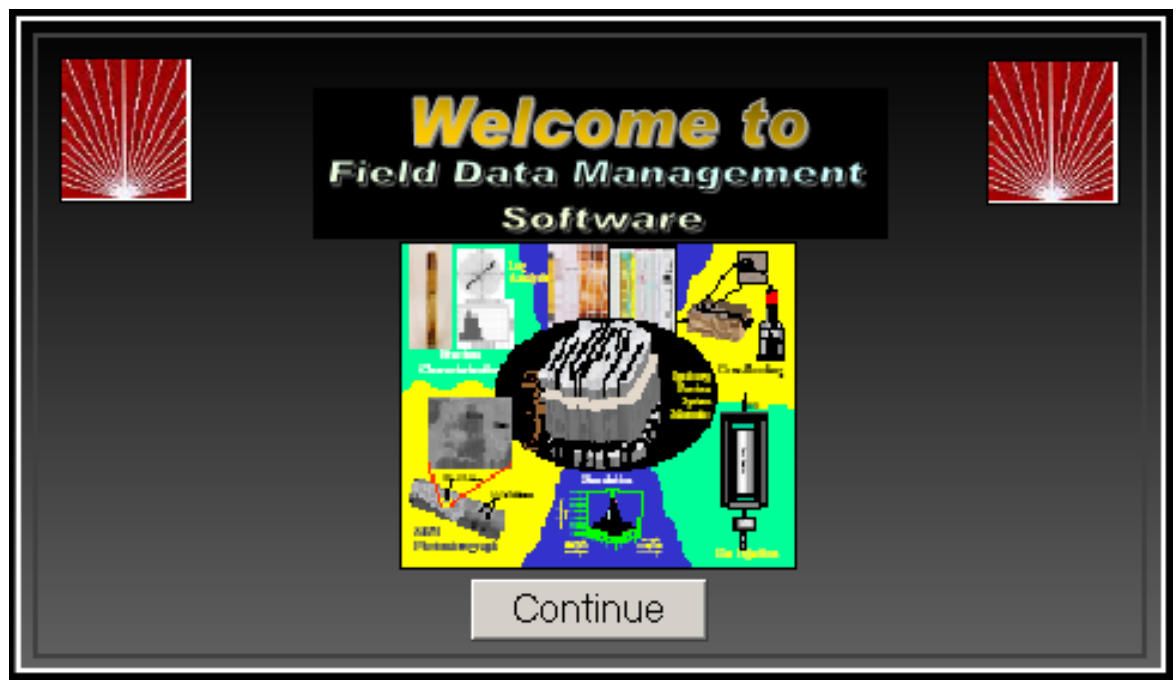

Fig. 2.1—Front Page of Field Data Management Software

\begin{tabular}{|c|c|c|c|c|c|c|c|c|c|}
\hline Well Data & & & & & & & & $-1 \square$ & 기 $x$ \\
\hline WELLID & NAME & Lattitude & Longitude & X Coord & Y Coord & T. Depth & Status & Drilled by & $\Delta$ \\
\hline 76 & $14 \mathrm{KS}$ & & & 1533644.3 & 874919 & 8050 & OIL & & \\
\hline 77 & 31 & & & 1534501.5 & 873684.9 & 4222 & OIL & & \\
\hline 78 & $16 \mathrm{KS}$ & & & 1534080.2 & 870030.3 & 8115 & OIL & & \\
\hline 79 & $6 \mathrm{ER}$ & & & 1536486.7 & 876605.3 & 8105 & DRY & & \\
\hline 80 & $205 A$ & & & 1537388.1 & 877975.8 & 8852 & OlL & & \\
\hline 81 & $407 \mathrm{~A}$ & & & 1537261.4 & 872457.9 & 8850 & WINJ & & \\
\hline 82 & $117 \mathrm{~A}$ & & & 1533847.4 & 868459.2 & $8850 \mid$ & OIL & & \\
\hline 83 & $206 \mathrm{~A}$ & & & 1535407.6 & 874702.3 & 8850 & OIL & & \\
\hline 84 & $116 \mathrm{~A}$ & & & 1532204.9 & 876615.8 & 8875 & OIL & & \\
\hline 85 & $602 \mathrm{~A}$ & & & 1539955.5 & 867681 & 8025 & OIL & & \\
\hline 86 & $406 \mathrm{~A}$ & & & 1536651.2 & 869482.6 & 8850 & OIL & & \\
\hline 87 & $308 \mathrm{~A}$ & & & 1539847.4 & 878619.6 & 8850 & OIL & & \\
\hline 88 & $113 \mathrm{~A}$ & & & 1533430.1 & 871450.8 & 8860 & OlL & & \\
\hline 89 & $405 \mathrm{~A}$ & & & 1539220.8 & 870215.9 & 7950 & OIL & & \\
\hline 90 & $114 \mathrm{~A}$ & & & 1531587.7 & 879080.2 & 7943 & OIL & & \\
\hline 91 & $309 \mathrm{~A}$ & & & 1541755.5 & 881850.6 & 8070 & OIL & & \\
\hline 92 & $207 \mathrm{~A}$ & & & 1538231 & 875443 & 7950 & OIL & & \\
\hline 93 & $118 \mathrm{~A}$ & & & 1530526.7 & 873421.1 & 7986 & OIL & & \\
\hline 94 & $115 \mathrm{~A}$ & & & 1528586 & 881ก8ค? & 8051 & กII & & $\nabla$ \\
\hline 11 & & & & & & & & 1 & \\
\hline \multicolumn{7}{|l|}{ 国 } & Close & View Map & \\
\hline
\end{tabular}

Fig. 2.2-Well Data Form 


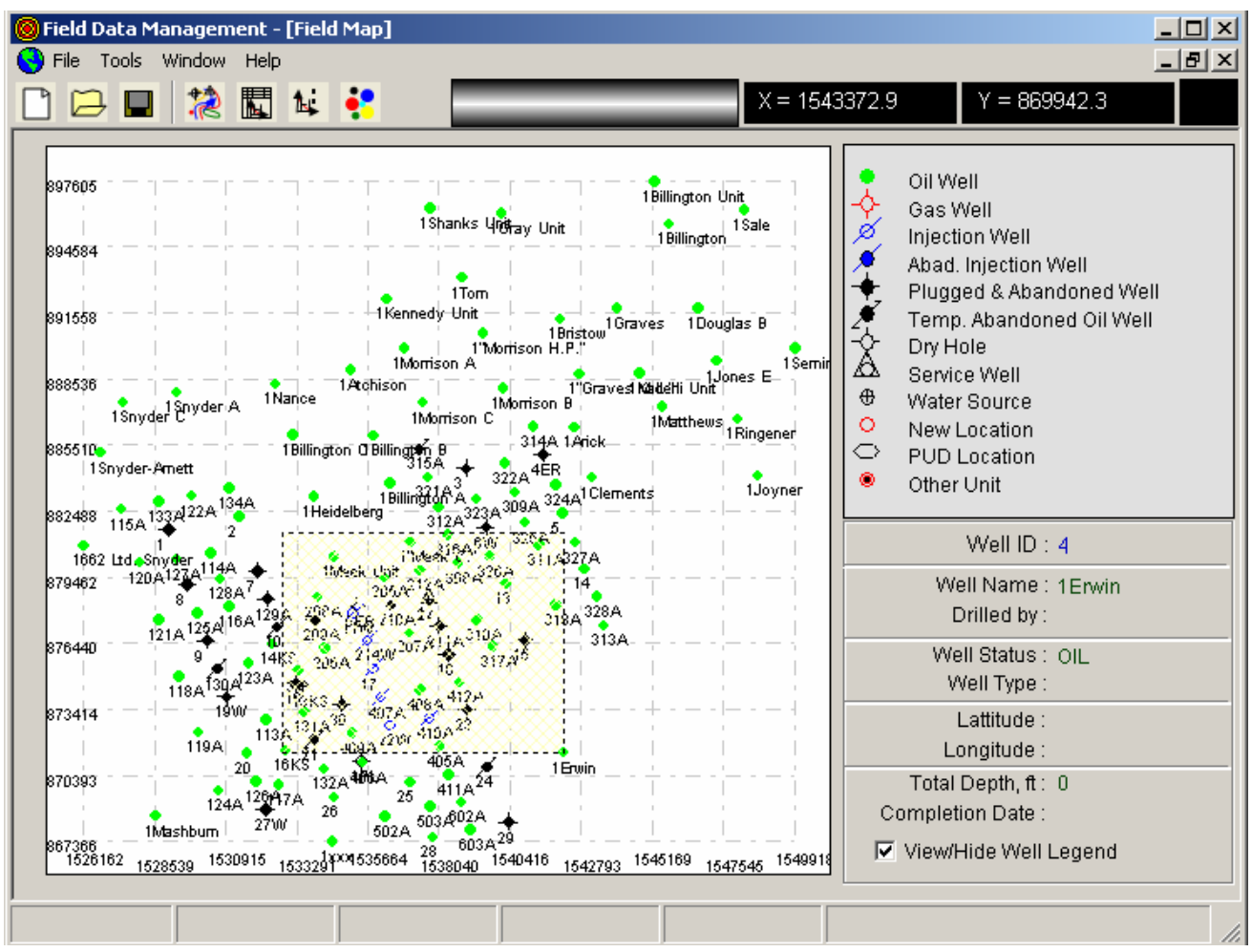

Fig. 2.3-Map of Germania Unit

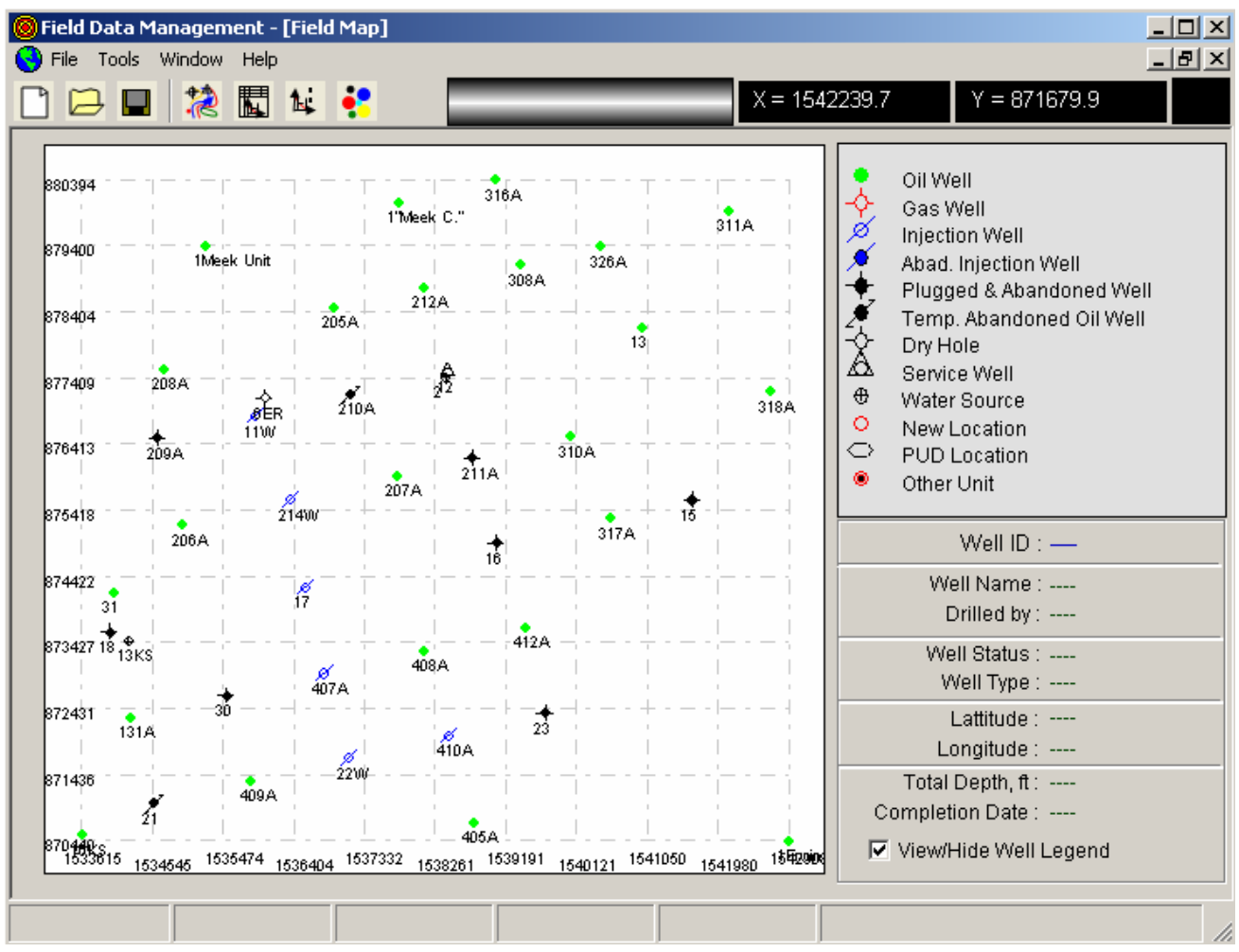

Fig.2.4-Zoom-in view of particular cluster 


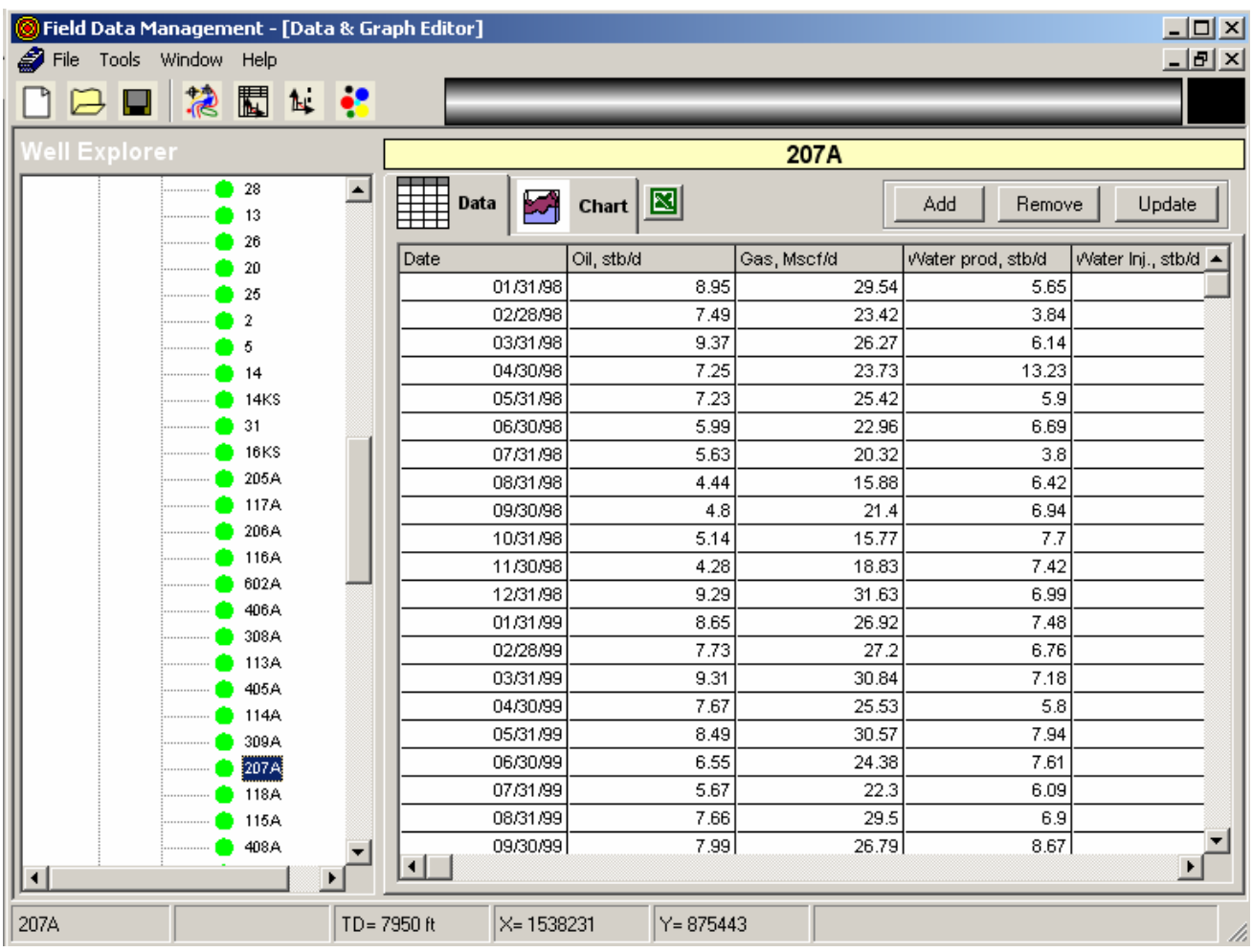

Fig. 2.5-Tabulated Production History Data of Well 207A

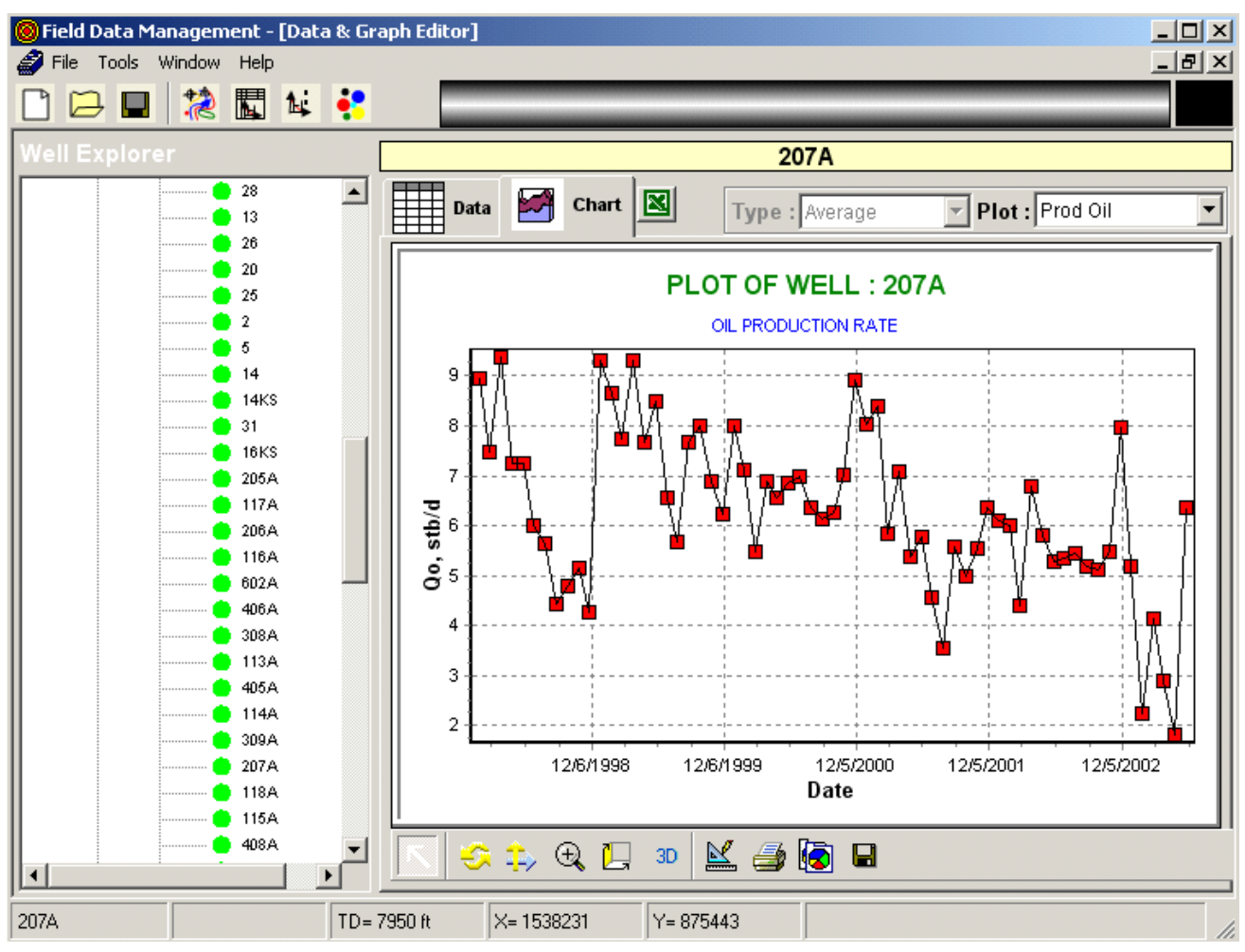

Fig. 2.6 — Graph of Oil Production of Well 207A 


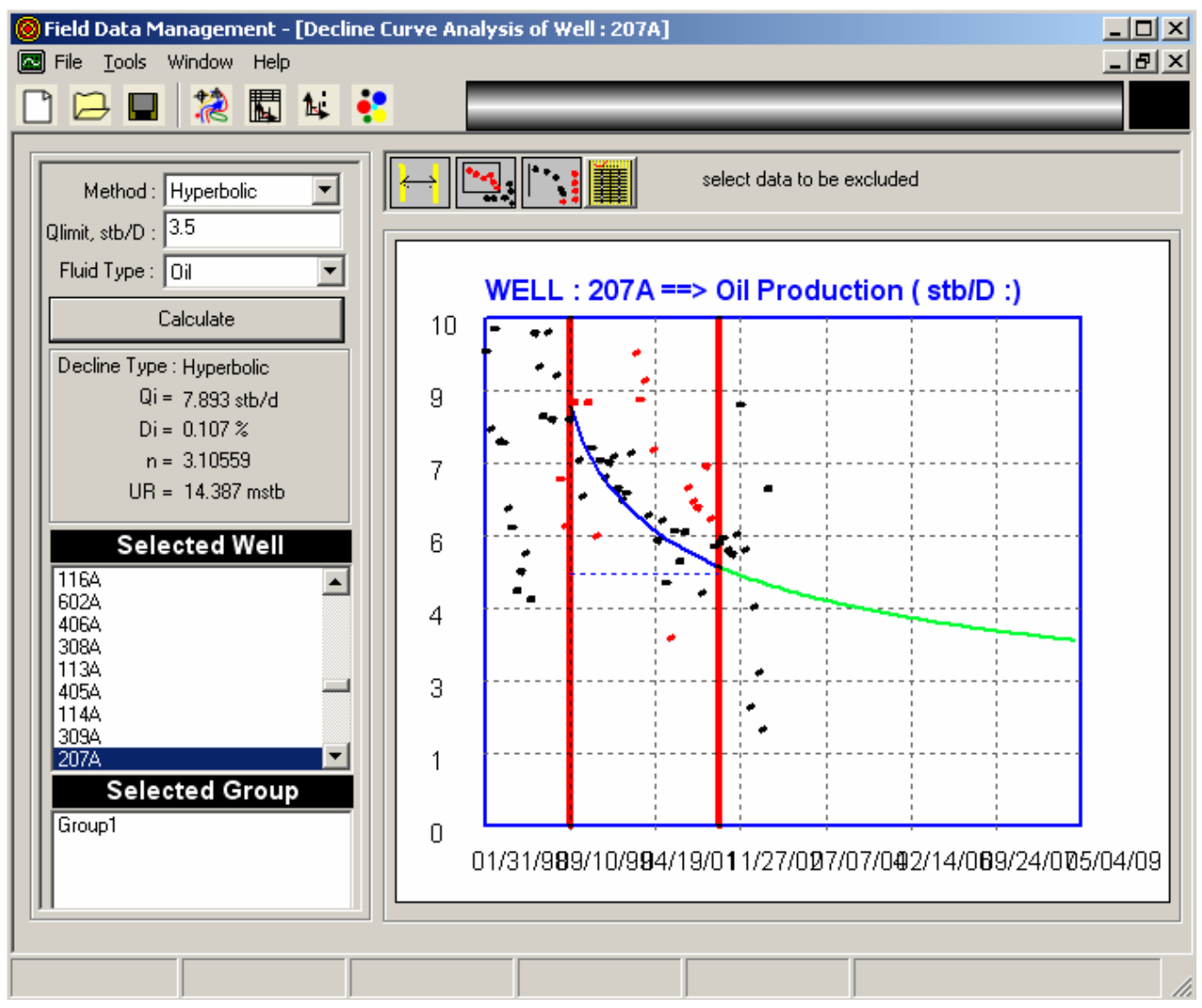

Fig.2.7-Hyperbolic Decline Curve of Well 207A

\section{References}

1. Schechter et al.: "Preferred Waterflood Management Practices for the Spraberry Trend Area," Semi-Annual Report (DOE Contract No.: DE-FC26-01BC15274), March - Sept 2002.

2. Spivey, J.P.: “A New Algorithm for Hyperbolic Decline Curve Fitting,” paper SPE 15293 presented at the Symposium on Petroleum Industry Application of Microcomputers, June 18-20, 1986. 
APPENDIX-A

FIELD DATA MANAGEMENT SOFTWARE

FEATURES

- FIELD MAP VI EWER

- DATA AND GRAPH VI EWER

- BUBBLE MAP / PIE CHARTS

- ISOBARIC MAPS

- GAS MATERI AL BALANCE 


\section{TUTORIAL}

\section{Problem description}

The example illustrates how to handle reservoir data (regular or sporadic) such as fluids production, injection data, the status of wells etc. The example will also familiarize you on how to create bubble maps, pie charts and production charts for single well or a group of wells.

\section{Data preparation}

- Create a working directory in a convenient place.

- Copy all data-files from the Tutorial directory, normally residing on /DSS/tutorials/example, to the current working directory.

- The data files for this software have an extension ".prj"

\section{Open new project}

To begin, start DSS FDM. You will need to double-click on the DSSFieldDM2.exe icon.

Select File | Open Project. The screen will look like the one shown below. 


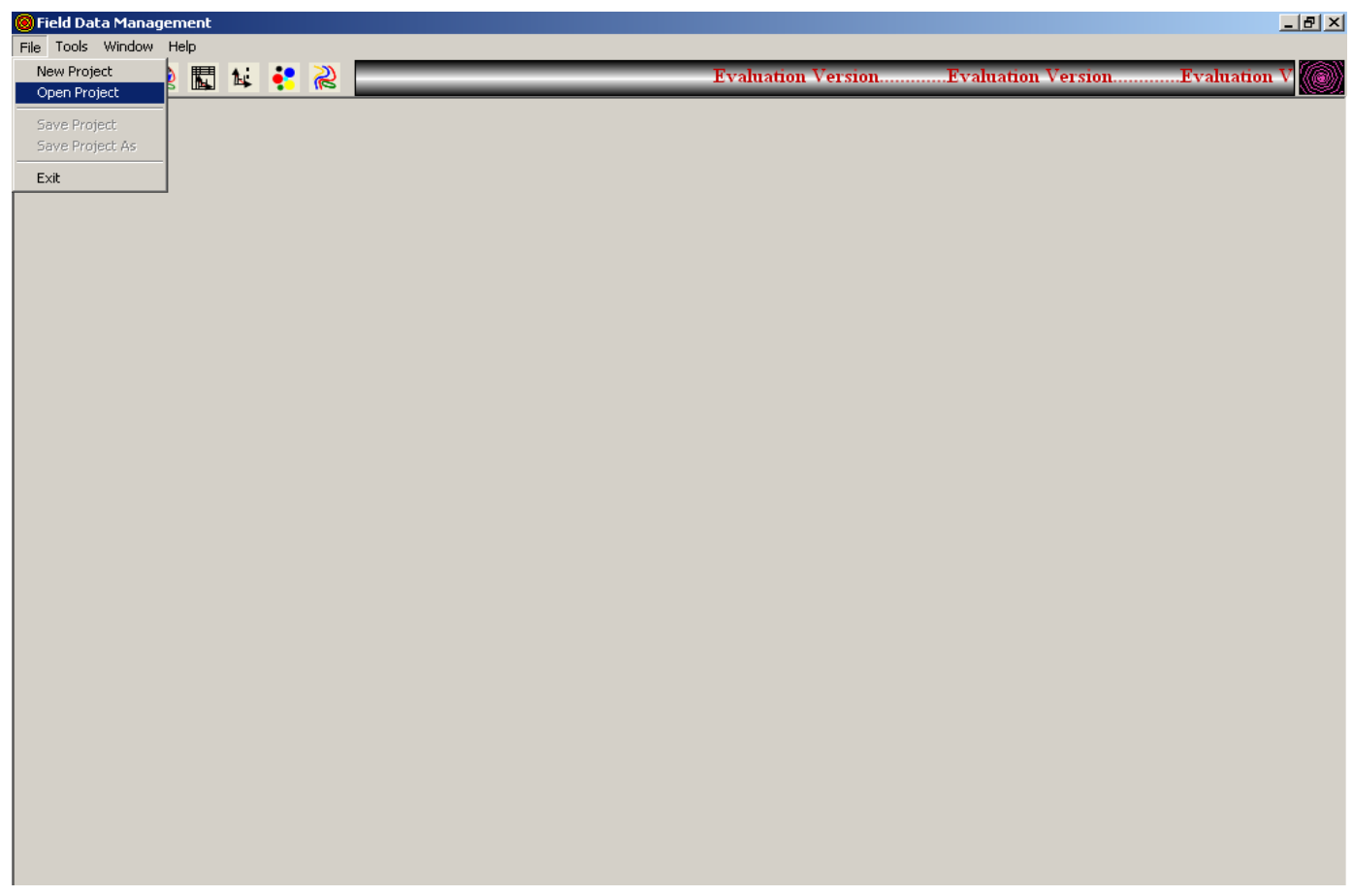

Call the project Example1. A screen will open up confirming that a correct data set has been entered. If an incorrect dataset is loaded an error message will prop up.

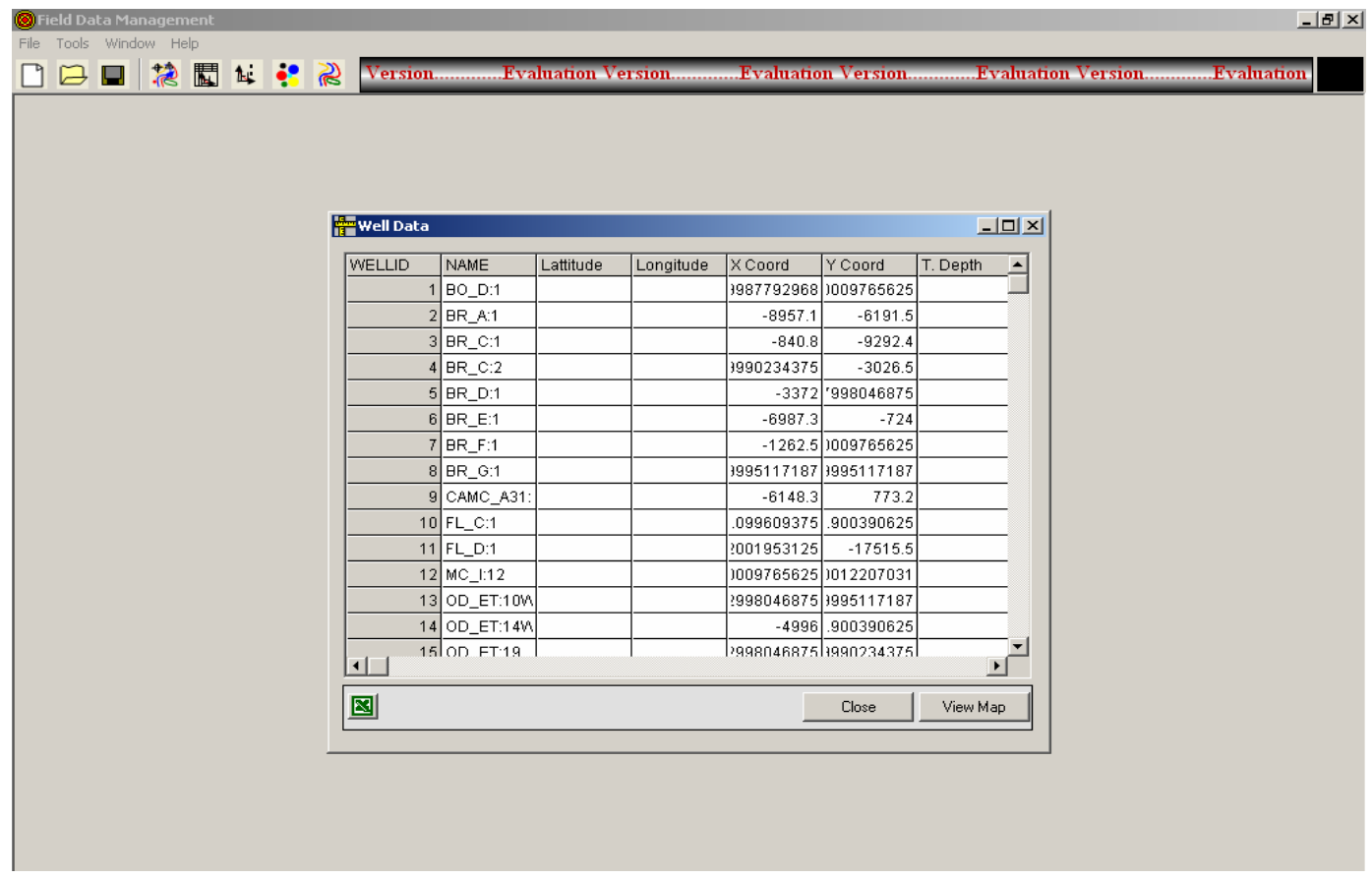

Click on View Map to view the map of this field. The legend on the right hand side describes the type of well and also gives the history of the well on moving the cursor to that particular well. 


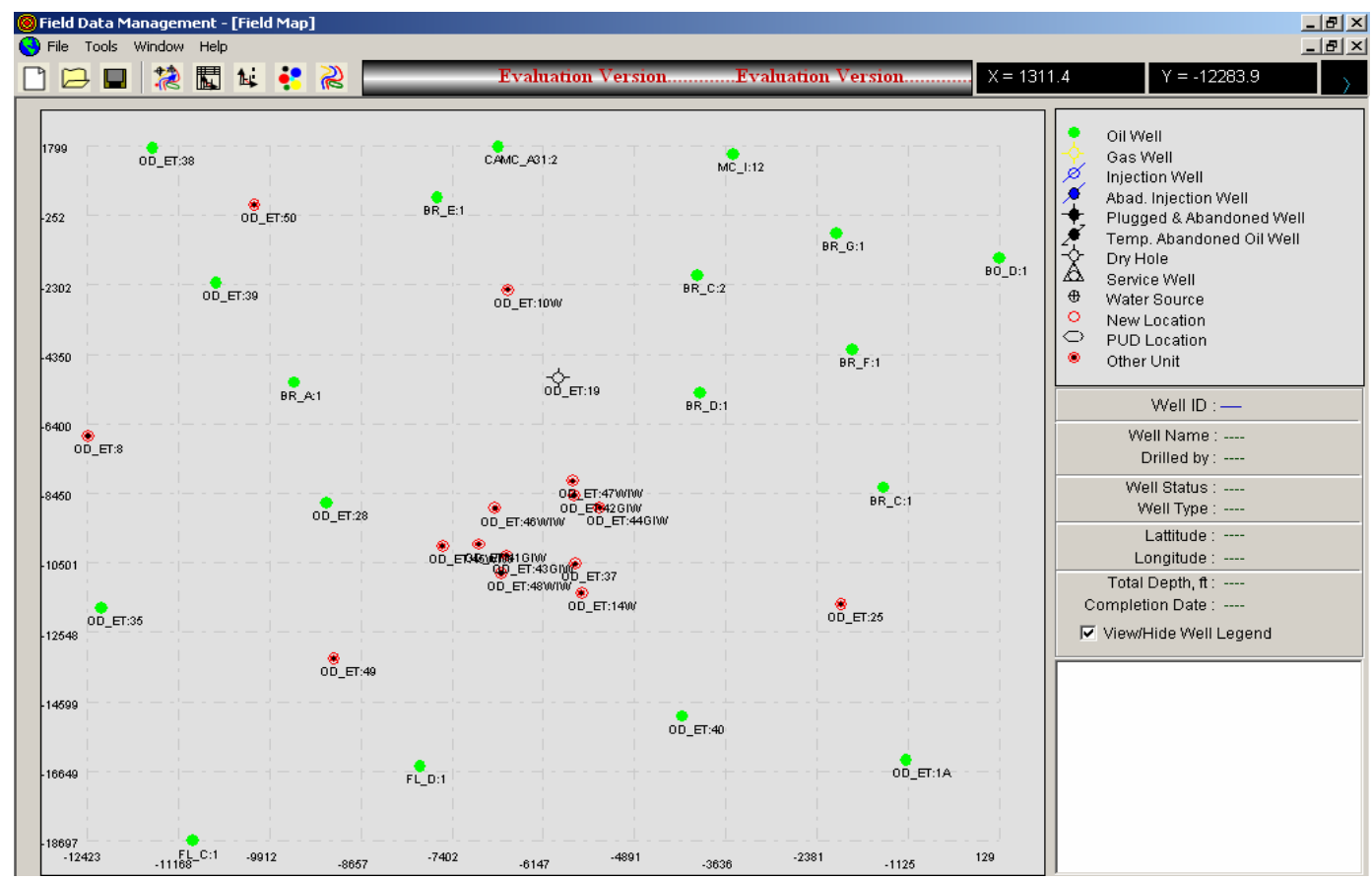

Since we have seen how to view the field map. Lets us explore the options available in the View field map mode.

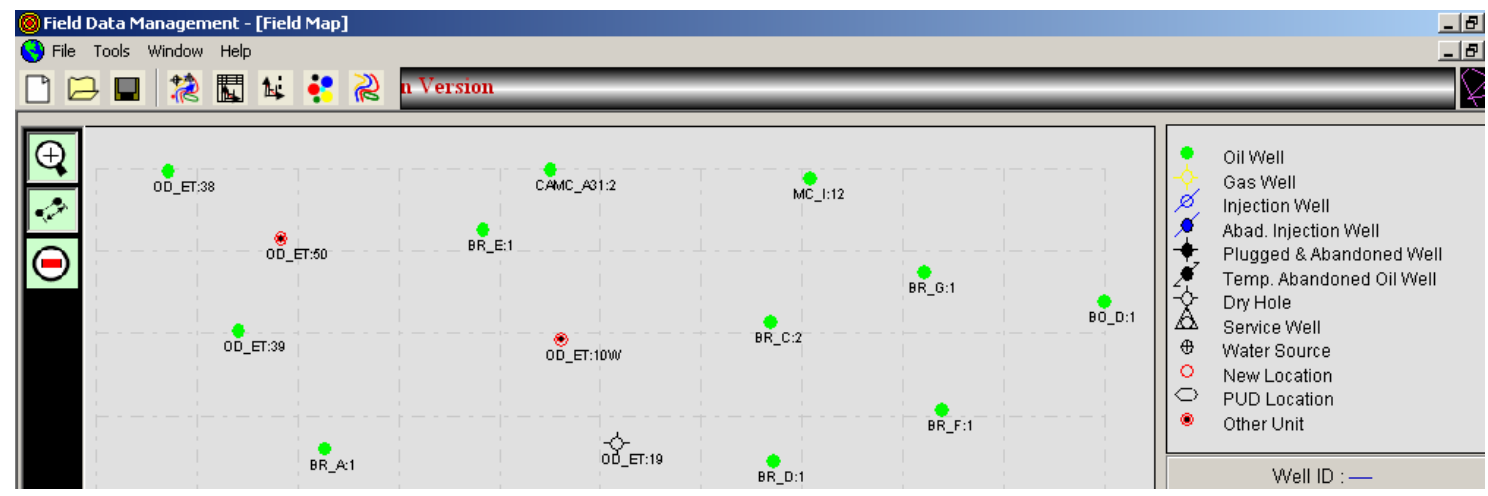

On moving the cursor to the far left side of the screen, three more icons show up.

$\bigoplus$ Clicking this icon enables the zoom option.

This option calculates the distance between any two points in the map. 


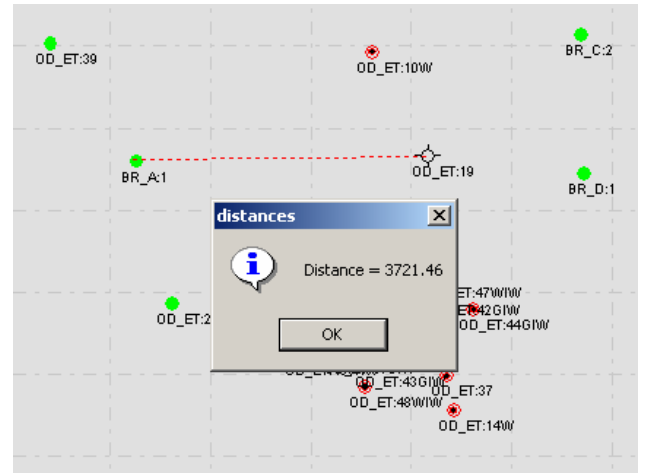

$\ominus$ Clicking this icon resets the map. 


\section{GROUPING WELLS}

Grouping selected wells is the very important in order to study a particular field or region. The following example helps you to understand how to create and load groups.

Suppose we want to group the following wells under the group name "Groupex1"

- BO_D:1

- BR_A:1

- BR_C:1

- FL_C:1

- MC_l:12

- OD_ET:8

Select Tools : Create Group and a Group Builder window props up.

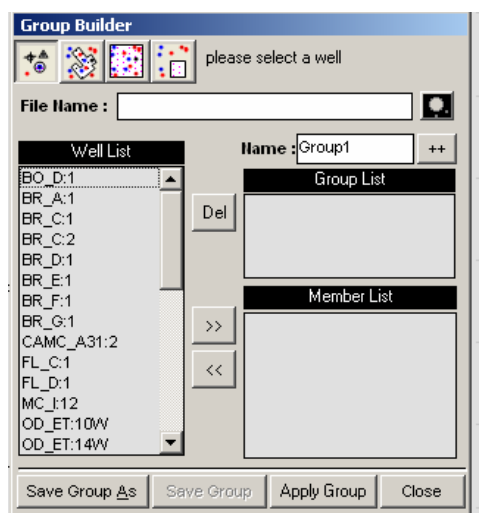

Enter the name of the group "Groupex1" in the Name window and press on "++". You would find the group name added under Group list. In order to select the wells under the member list you can either click on the well name in the field map or chose the well from the well list and transfer to the Member list through ">>" button. You can choose more than one well by pressing CTRL key. Similarly you can remove a well from the Member list through the "<<" button. Once the wells has been added the screen should be as shown in Figure below.

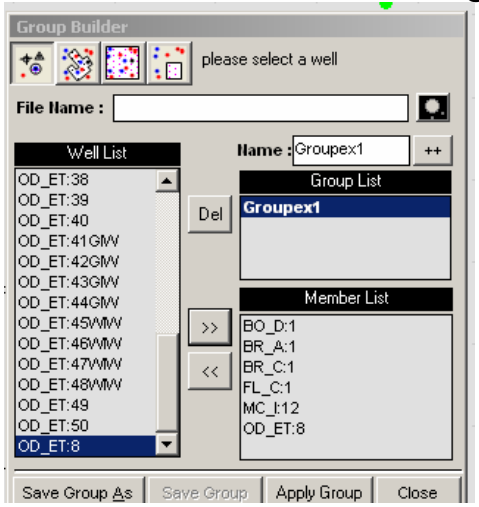


Click on Save Group as to save the new group under a different name. The saved group will have a file extension ".grp". This would help in future when you need to upload the group.

\section{Group Builder icons:}

Select one well at a time. Once the well is chosen, a thumb sign indicates the location of the well on the field map.

Select wells through a window. Click on the map and select the region which contain the wells by dragging the cursor, keeping the mouse clicked. Once the region is selected, release the mouse. Two options show up, whether to add the wells to the group or to remove the wells from the group.

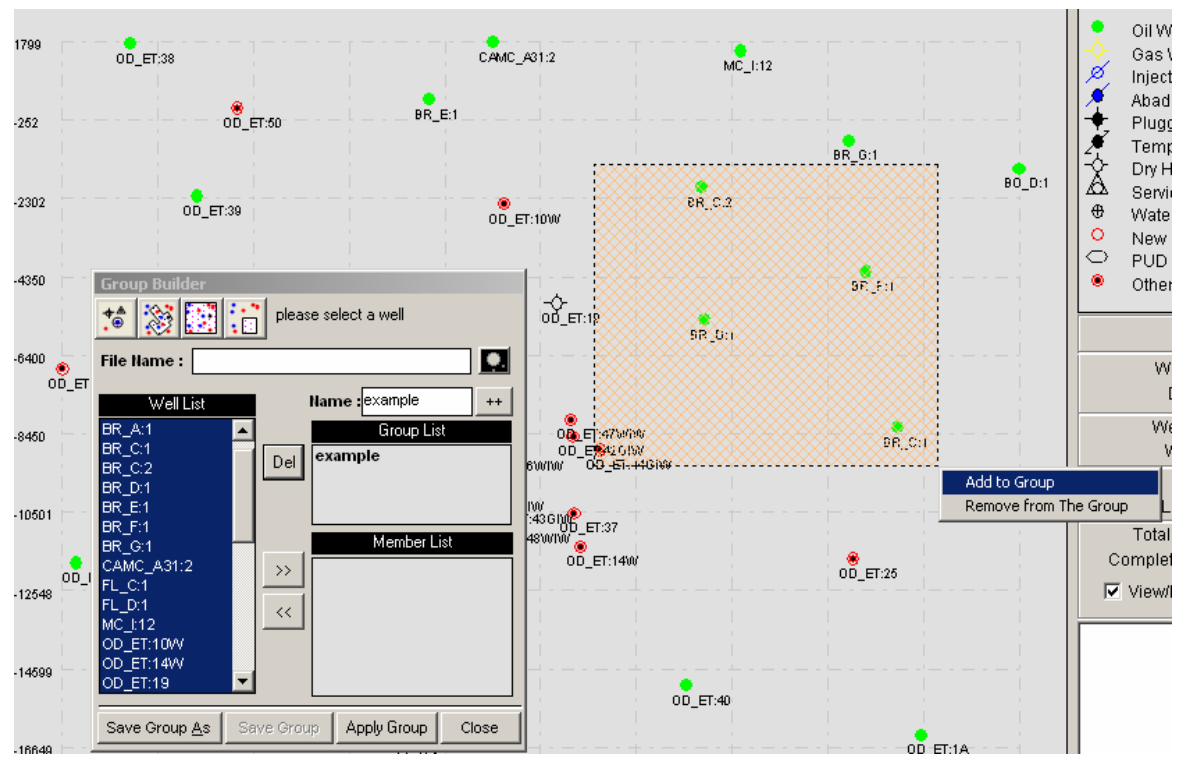

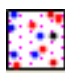

Selects all the wells from the list. Once selected move all the wells to the Member list through ">>" button.

: Deselect All/ Remove the group.

Once having created the group click on Apply Group in order to activate the new group. This would activate the Data Viewer mode as shown in figure below. You can see from the figure that the group that was created is now active. 


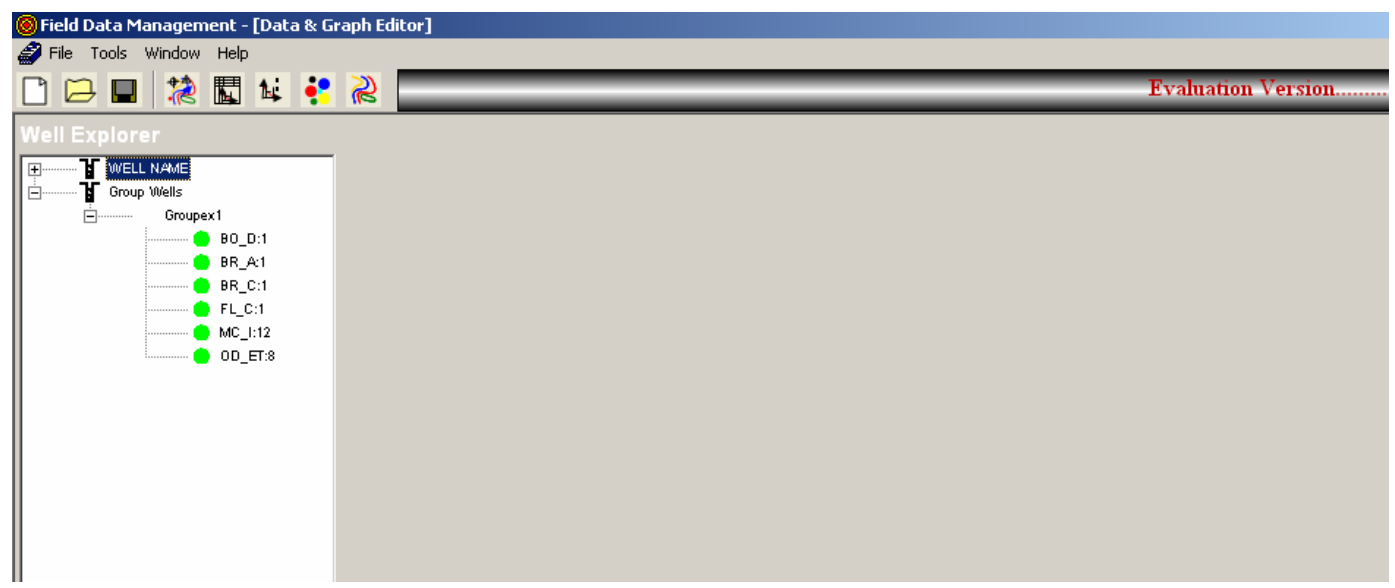

Now you can choose to view the production and pressure plots of each well. This procedure will be described in subsequent pages. 


\section{ICON DESCRIPTION}

This software is very useful in handling data and generating the required outputs. The following describes the purpose of each icon.

$\square$ creates a new project

$\boxminus \quad$ opens a new project

saves the project

on clicking displays the field map

display data in a table format and has options to display production maps, pressure maps and also to update data.

世: perform decline curve analysis on production data

view the bubble map of the given production data.

गี view the isobaric contour (pressure map). 


\section{DATA AND CHART VIEWER}

Let us now explore how to display and handle data in a table format and create production and pressure maps.

On clicking 庶, the data view mode is activated. You will be shown the screen shown below. The left-hand side of the screen describes the type and status of well. The type of well is specified as an integral part of the input data. on the lefthand side window two categories would be shown

- WELL NAME

- Group wells

"Group wells" option is available because we have created the group "Groupex1" earlier. We can also load another group that we have created already. The group can also be created as a part of the input data set. The procedure will be addressed later.

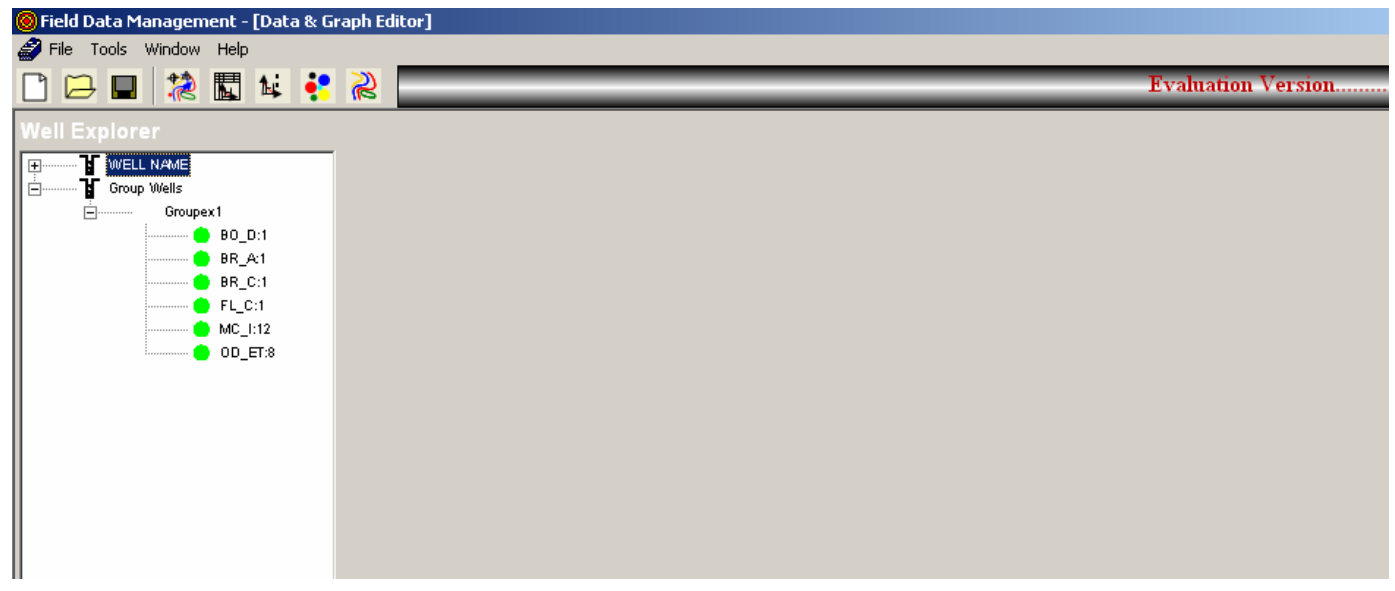

On expanding WELL NAME, the information about the type and status of the wells are shown. Figure below shows the type and names of wells present in the input data. The right-hand window shows the data in a table form. In this example, the production data for well BO_D:1 is shown. 


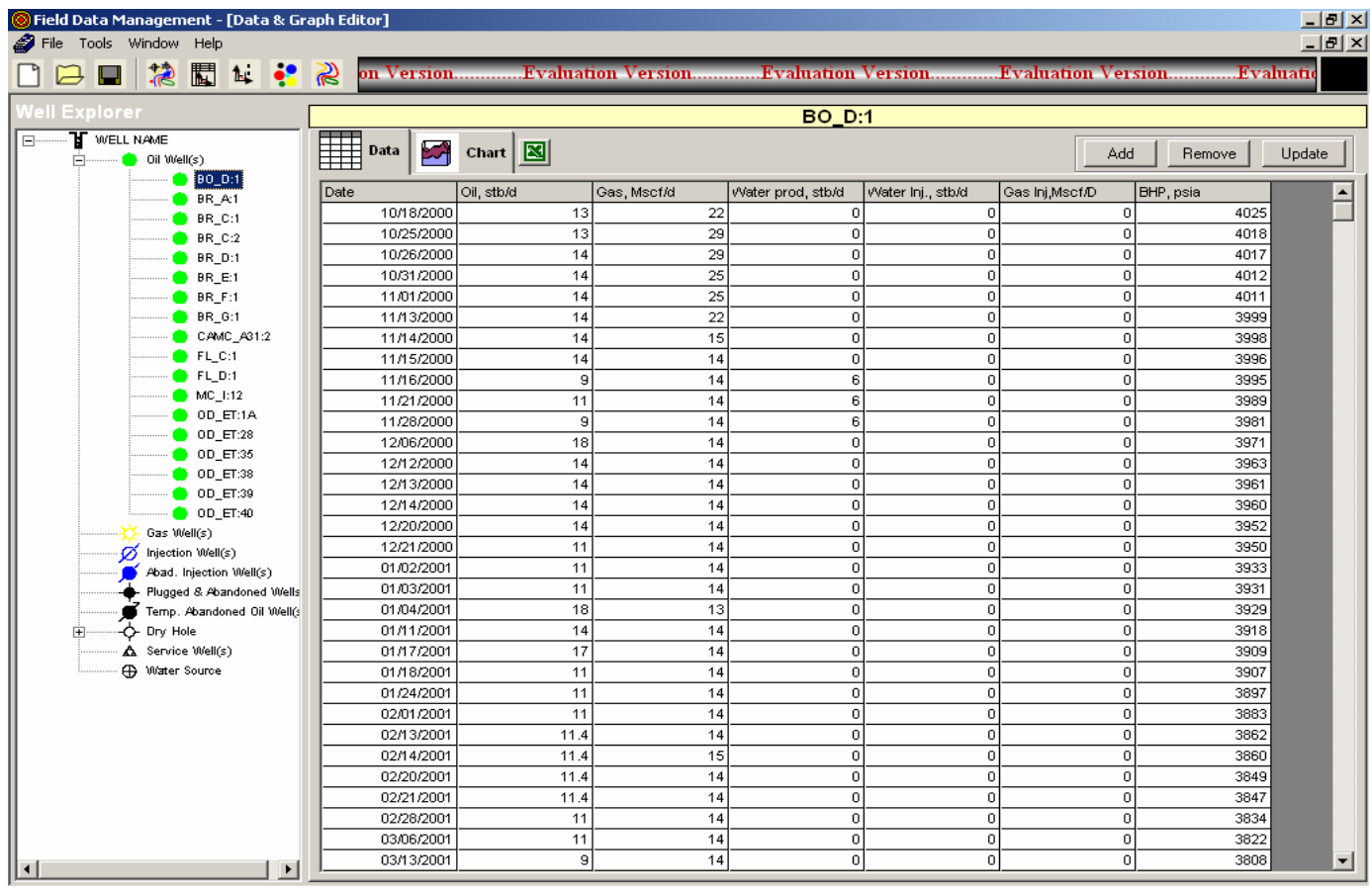

Click on to view production plots of the selected well. The software gives you many options to plot. The two windows located on the right -hand gives the plot options.

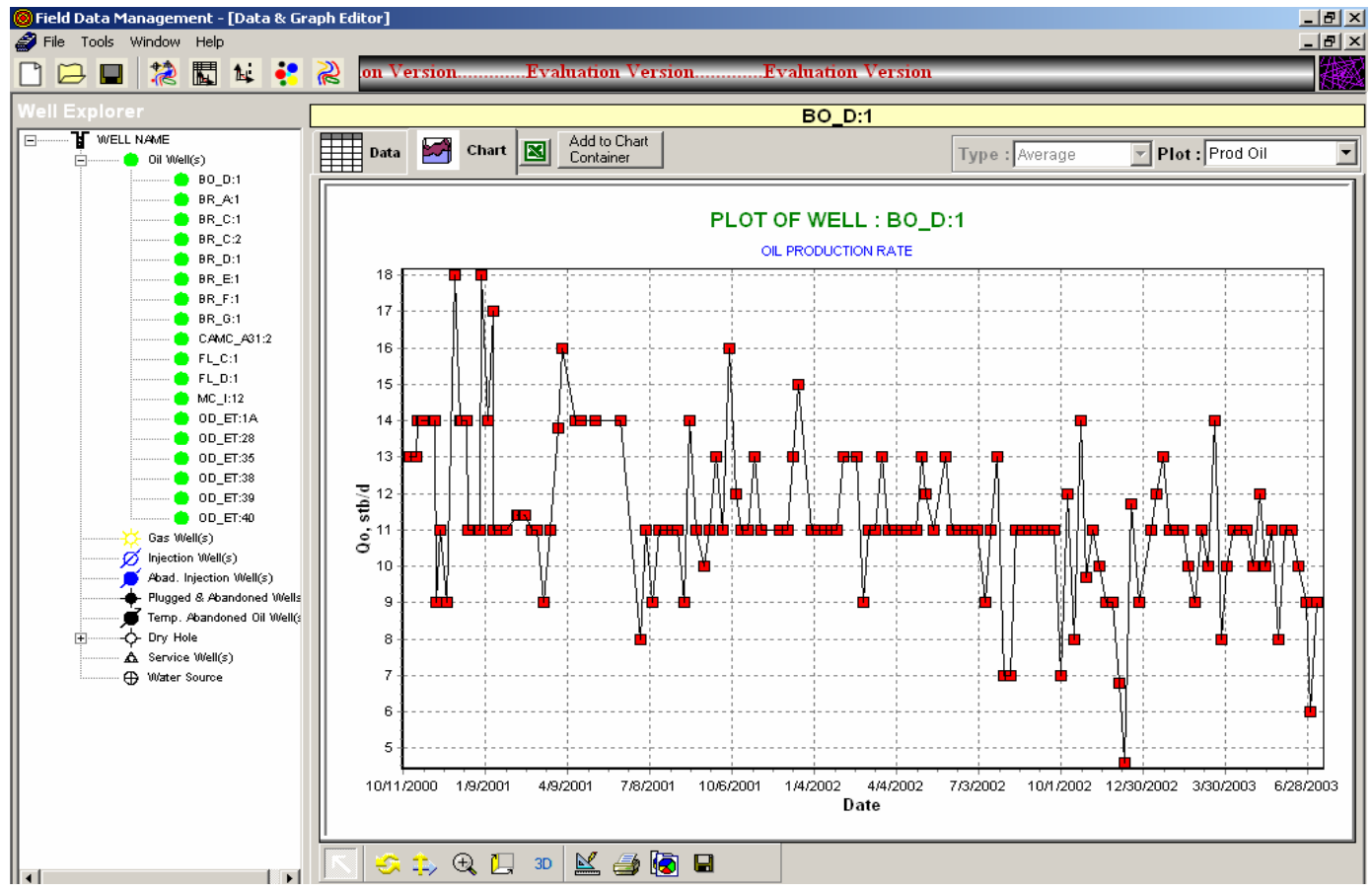


On scrolling down the Plot window the following plot options are available.

- Produced oil

- Produced gas

- Produced water

- Injected water

- Injected gas

- ALL- plot all the data available

- Cumulative oil

- Cumulative water

- Cumulative water injected

- Cumulative gas injected

- Water oil ratio

- Gas oil ratio

- Gas water ratio

- Bottom hole pressure

The Type window is turned inactive here because the data entered is the average daily production rate for wells. The Type window will become active and will have two options, when you click on Groupex1

- Average

- Summation

On clicking 国, the data is transferred to an excel spreadsheet, where data can be updated or changed.

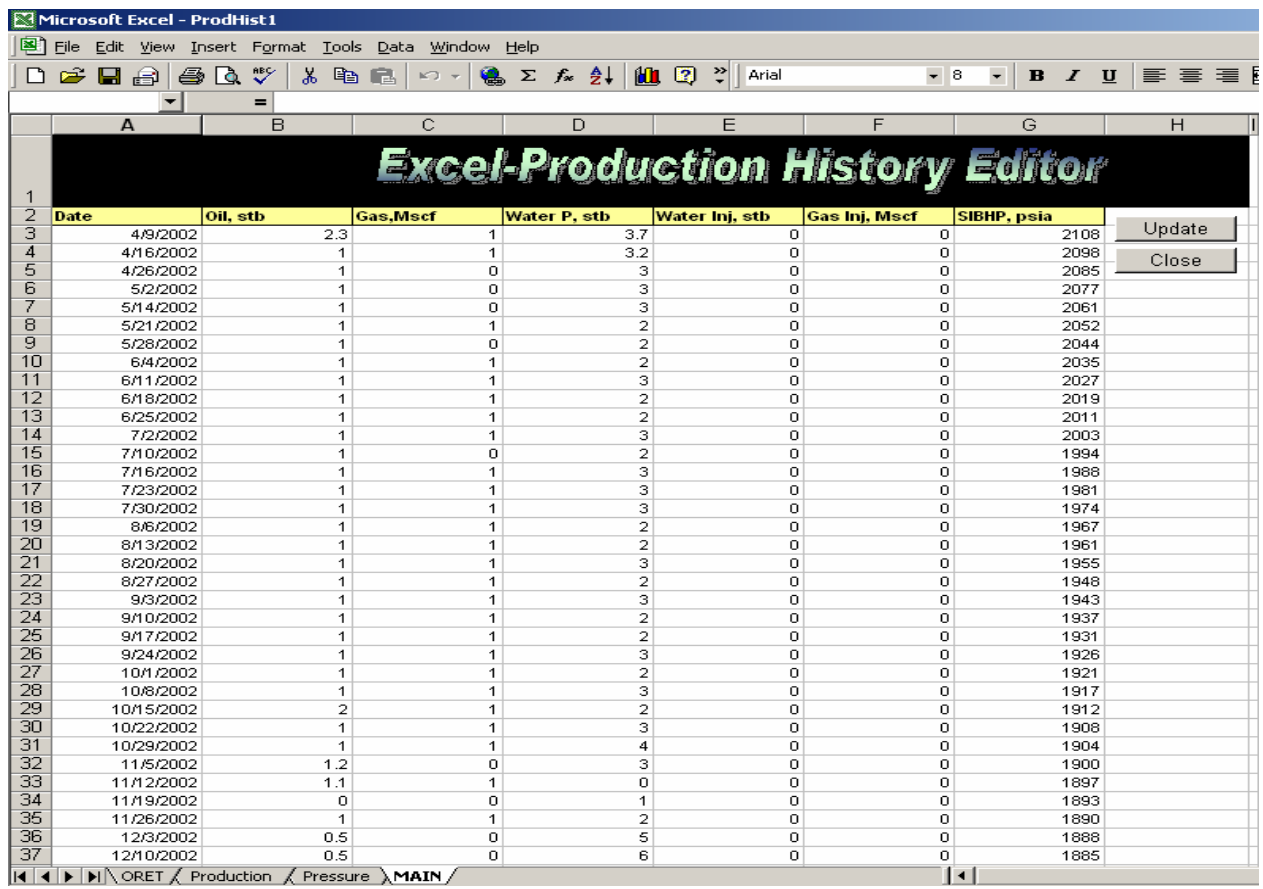


On addition/correction of data hit the update button to update the data. In order to view the updated data, refresh the data by clicking on the well name and then view the plot. Once the update is done, you can close the spreadsheet by hitting the close button.

The software also has options to design your own plots. This is handled by the Add to Chart Container tab. The following exercise will help you familiarize with designing your own plots.

Suppose you want to create a plot of cumulative oil, BHP and produced gas vs. time for well BO_D:1.

1. Choose Cum. Oil in the Plot window. You should now see the plot of cumulative oil vs. time.

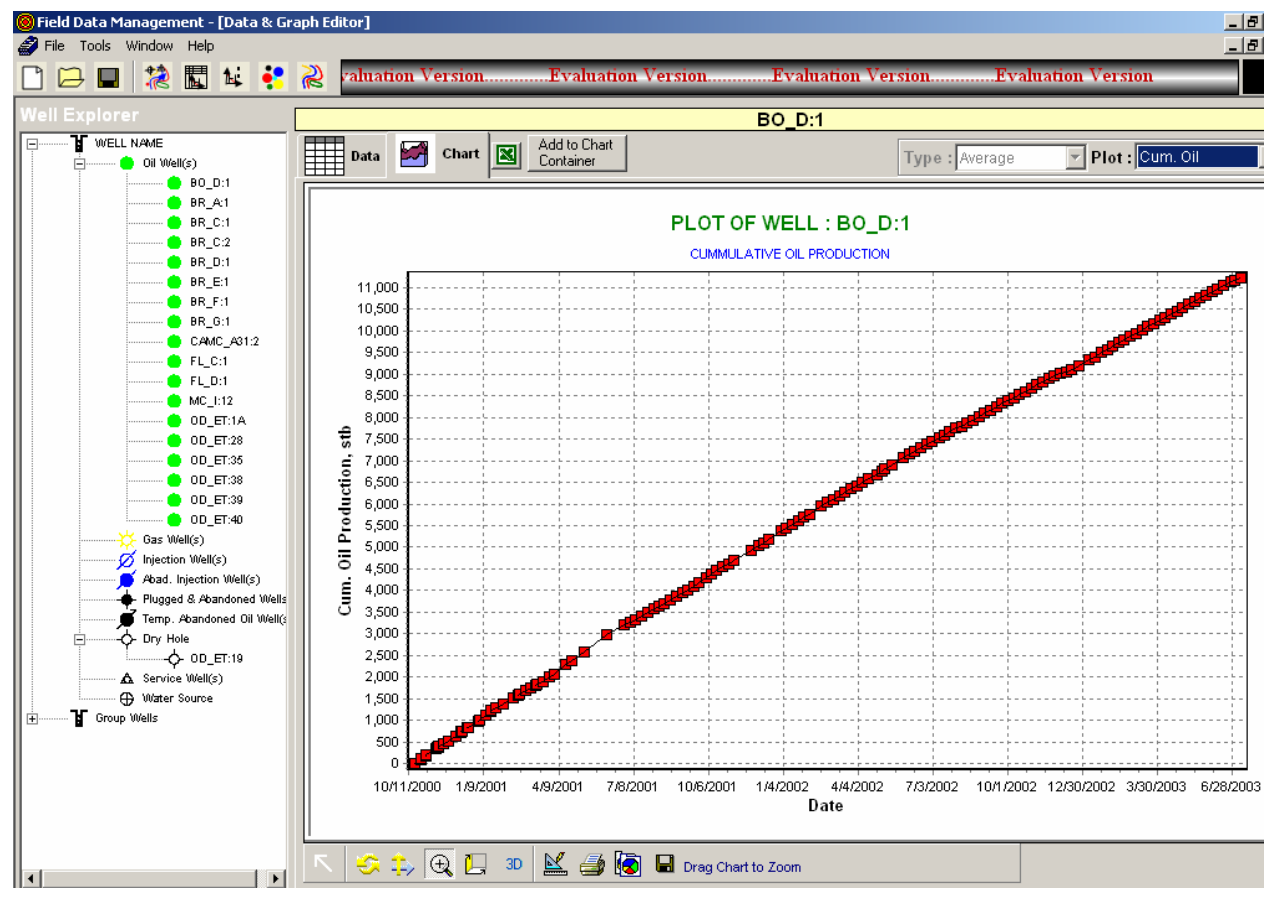

2. Select Tools:Add to Chart Container. You should see the screen shown in following figure. 


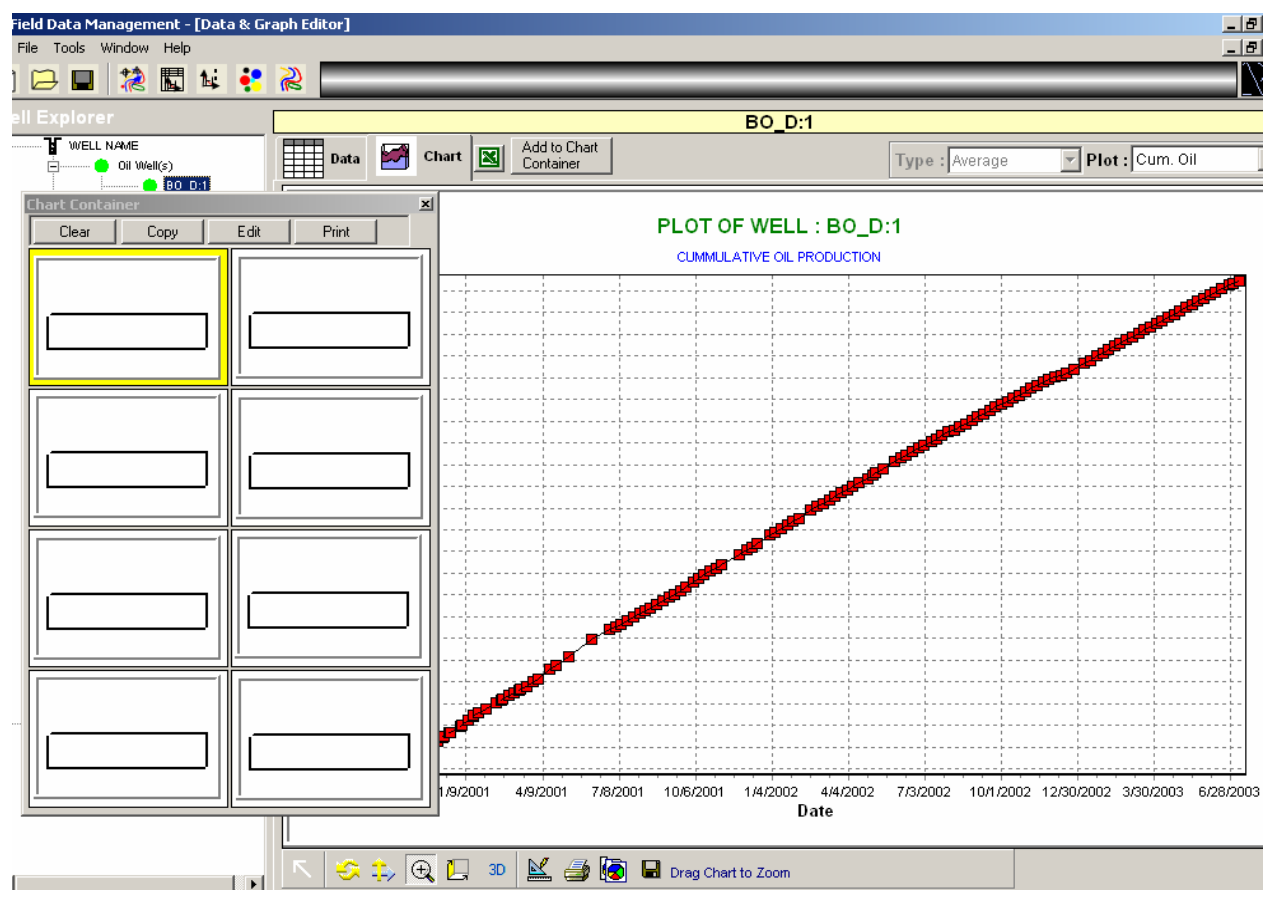

3. Click on Add to Chart Container tab. Now the plot is transferred to the chart container. Similarly choose BHP and Prod. Gas from the Plot window and click on Add to Chart Container tab. Now all the 3 plots are added to the chart. You can view the created chart by double clicking on the chart in the chart container.

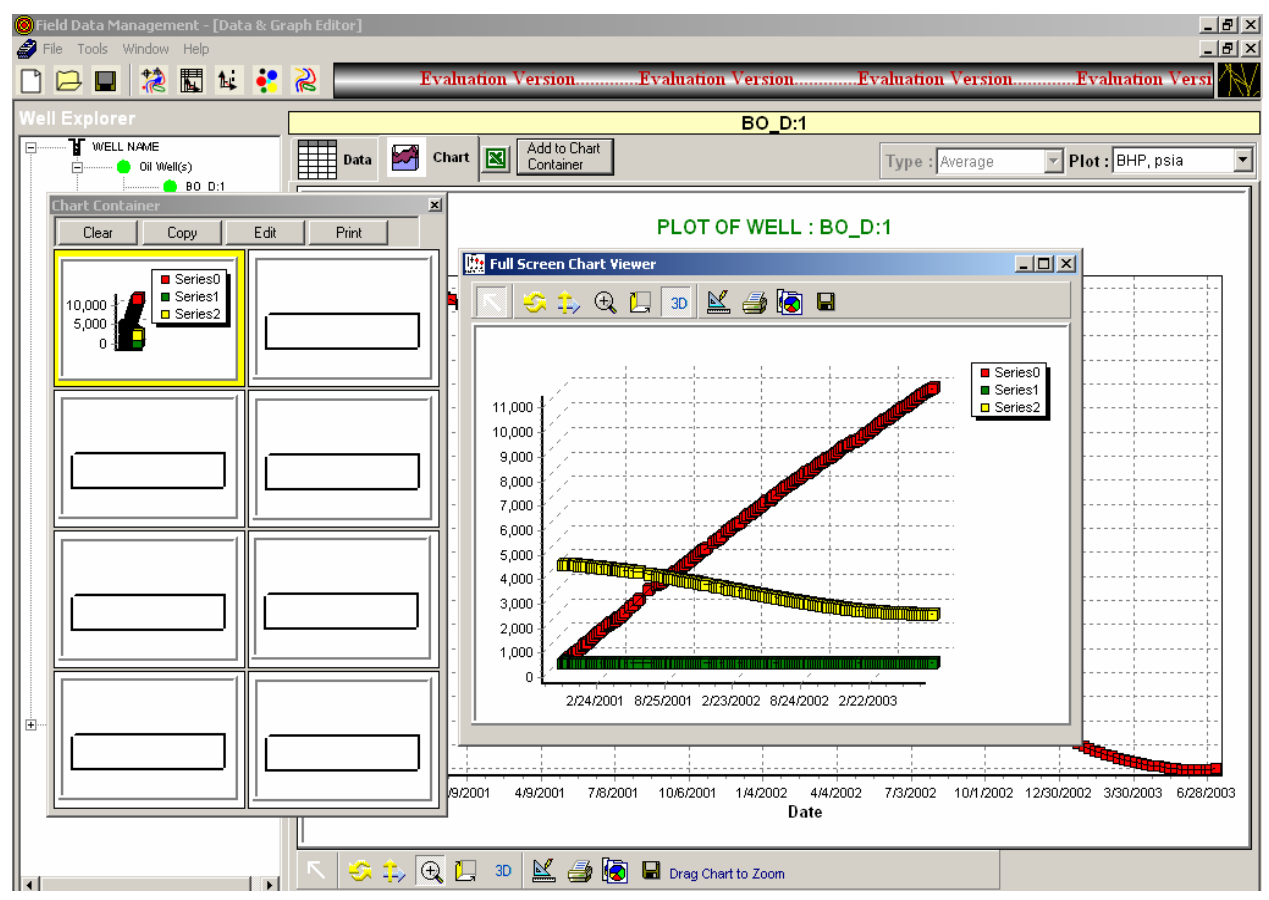

The plots can be edited using the options available in the chart viewer. 


\section{CHART EDITOR}

Chart display features are those, which affect the overall appearance of the Chart. They include those properties and methods that define the color of the Chart background, Titles and their position, margins, borders and bevels, background images, frame and axis visible, pen colors and widths, 3D, walls, etc.

The overall Chart appearance characteristics are a grouped into these categories:

General

Axis

Titles

Legend

Panel

Walls

$\underline{3 \mathrm{D}}$

These are the groupings you will find if you open the Chart Editor and browse the Chart pages. Other properties affect the 'look' of your Chart. These include Series colors and mark characteristics, individual Axis and Grid display properties and labeling. 


\section{Getting started with Chart display properties}

\section{General Chart properties}

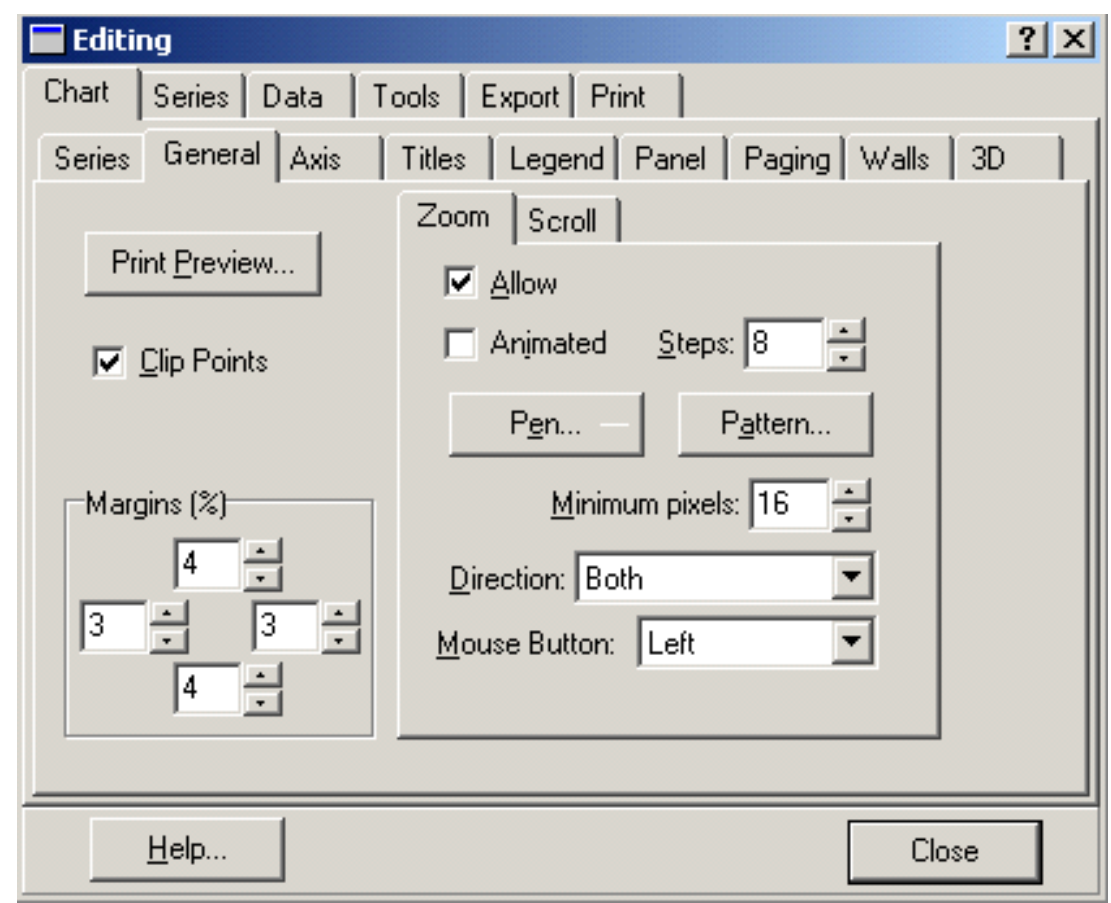

General options include Print Preview and Chart Export . This also has options to zoom the chart to the desired level. 


\section{Axis Properties}

Control of the axes is quite a specialized area at its greatest level of intricacy. Here we will cover the fundamentals of Axis and Frame display.

The second Chart page holds the properties for defining axis and frame characteristics.

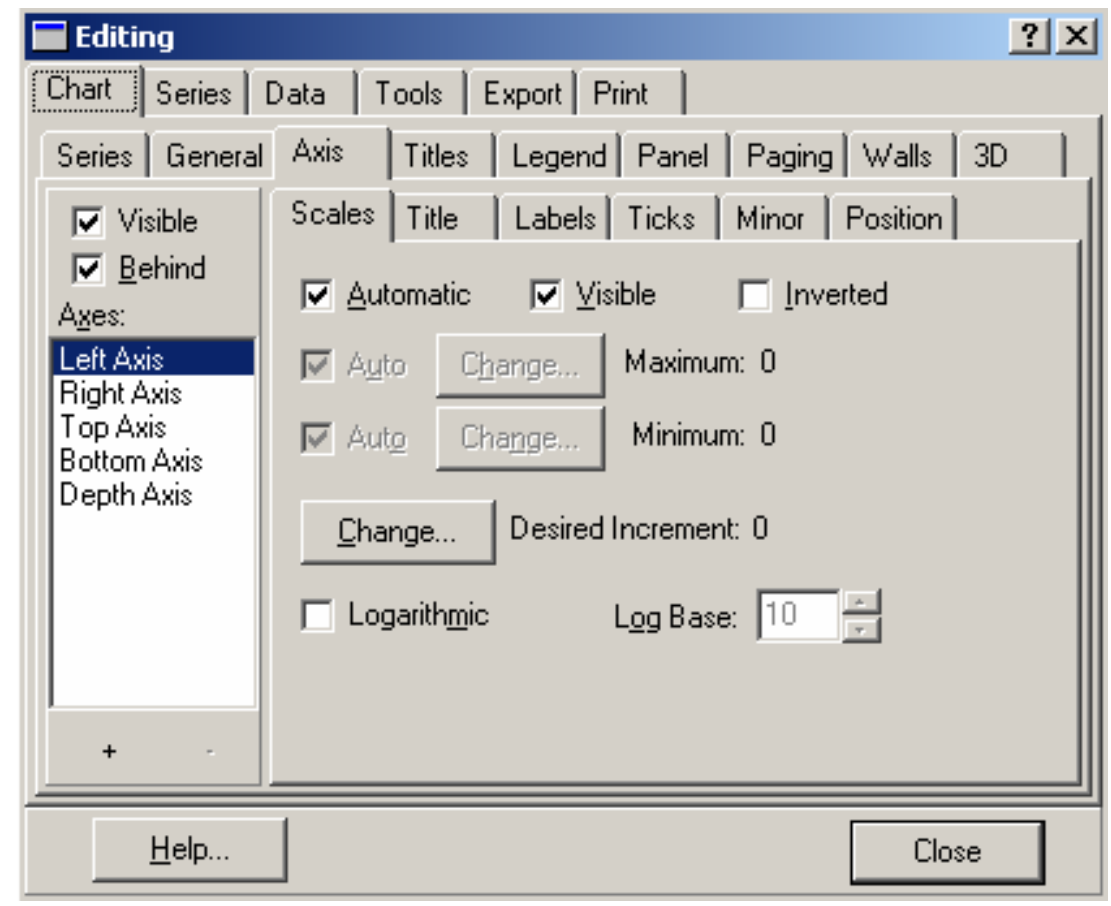

There are 5 axes in the Chart. Left, Top, Right, Bottom and Depth. The Chart Frame displays as a surround to the Chart's plottable area and has no data corresponding features (See BackWall). The Depth initializes by default as not Visible. All other Axes are visible from the moment that a Series is added to the Chart and associated with those Axes (Left and Bottom as default). Custom Axes may be added/removed by using the + and - keys on the dialogue. For a Custom Axis to be visible (as for any other Axis) a Series must be associated with the Axis. 
The key properties to enable display of axes and frame are:

\section{Visible}

This checkbox enables or disables display of all axes. If the Back wall Frame is visible then the Chart surround is still visible when the axes are hidden. The result will be the appearance of a larger Chart as no area is reserved for the axis labels. The Visible property for each Axis (Scales tab) overrides the visible characteristic for each Axis. If you select the Depth Axis in the List then you will note that Depth Axis Scales. Visible is by default 'not visible'.

\section{Axis: Left, Right, Top, Bottom and Depth Visible}

Select the Axis that you wish to display or hide in the Listbox and toggle the Visible checkbox on the Scales tab to control the display for that specific axis.

\section{Titles Properties}

The Titles page of the Chart Editor controls the characteristics of the Chart Titles, Header and Footer.

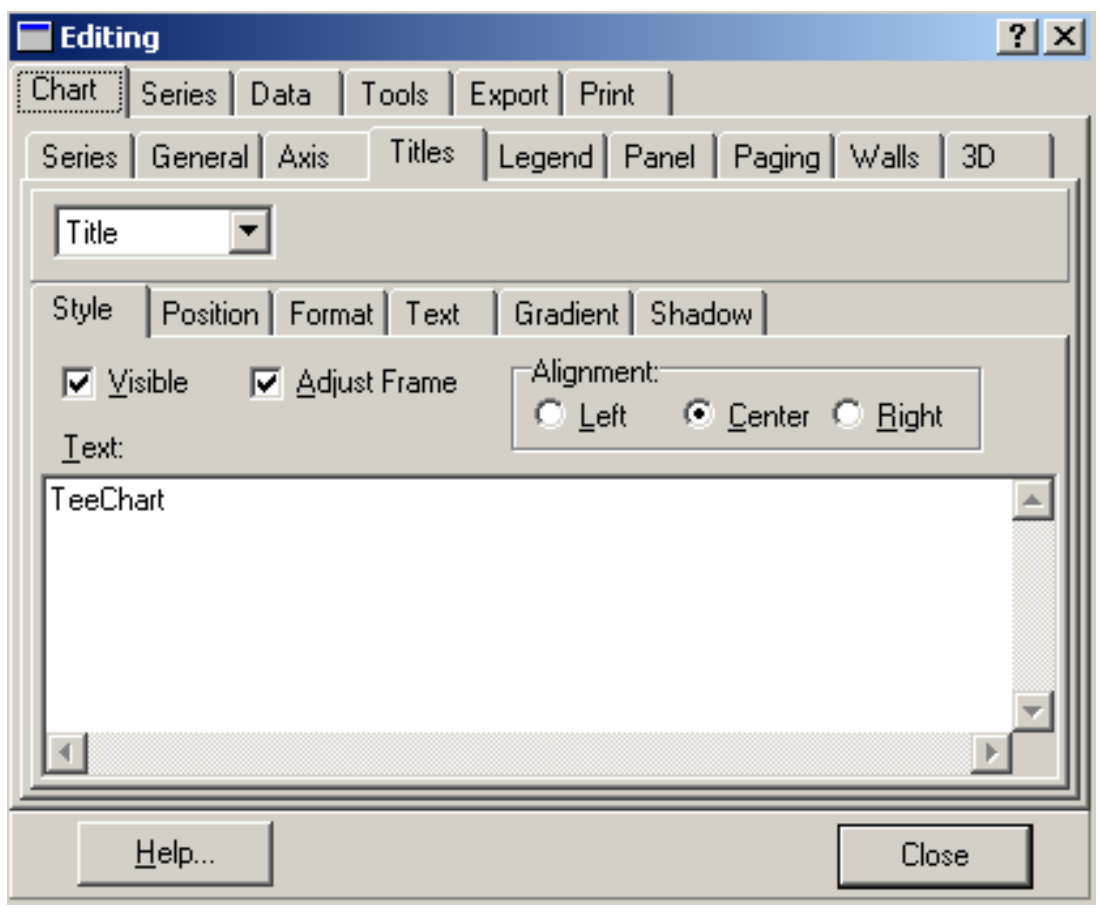




\section{Text}

Use the dropdown Combobox to select either Title (Header), SubTitle, Foot or SubFoot. Enter the required text in the Textbox. You may type multiline titles.

\section{Style Alignment}

Alignment refers to the Header (or Footer) alignment with respect to the Chart area NOT the overall Chart Panel. The Chart area is the plottable area of the Chart plus Axis Labels and Legend.

\section{Position}

Use Position to override the Title or Footer default position and set a custom position (pixels relation to Chart Top, Left).

\section{Format}

Contains the settings for the Title box, e.g. Frame, Background color, etc.

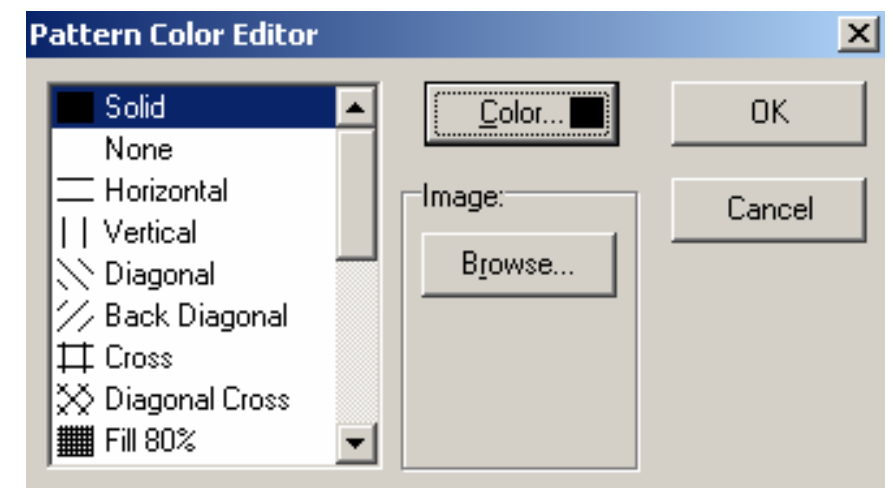

Pattern refers to the background pattern of the Title or Footer box. The default is Clear. The selected Pattern will sit behind the Text.

\section{Text}

Contains the Title Text appearance formatting characteristics.

The Font button will grant access to the Font Dialog window, which allows selection of Windows Fonts and definition of style (italic, bold, etc.) and color. 


\section{Gradient}

Enable/disable a Background Gradient in the Title box and select its direction and colors.

\section{Shadow}

Properties for display of the Header or Footer box Shadow. You may define color and size.

\section{Legend Properties}

Legend contents are a specialist subject that will be dealt with in a later tutorial. In the Legend page of the Chart Editor you may define appearance aspects of the Legend. Important initial steps are to control Legend alignment and visible properties, color, font, frame, shadow, etc..

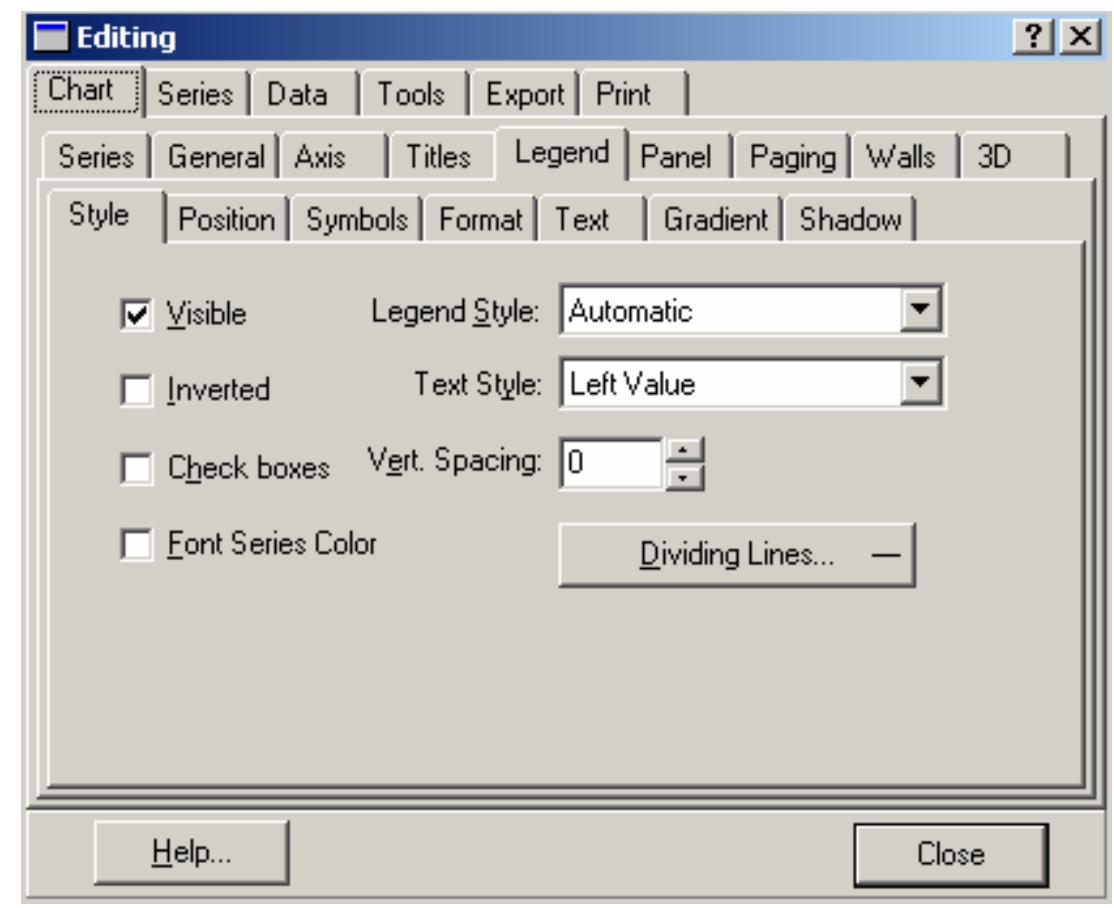

\section{Style}

Toggle On/Off Legend display and set Legend display content characteristics (inclusion of Checkboxes, etc). 


\section{Position}

Sets the default display position or enables custom positioning of the Legend. If the Legend is set to the side (left or right) of the Chart the contents of the Legend, by default, sit as a list from top to bottom. If the Legend sits below or above the Chart then the Legend contents are placed side by side. The default behavior may be overridden by using the Resize Chart option and/or by using Custom positioning.

\section{Symbols}

Size and formatting of the Legend symbols.

\section{Format}

Contains the settings for the Legend box, e.g. Fill Color, Frame color, style and width, Pattern style, color and image, bevel style and size and checkboxes for Round Frame and Transparent.

\section{Text}

Text characteristics for the Legend text contents.

\section{Gradient}

Enable/disable a Background Gradient in the Legend box and select its direction and colors.

\section{Shadow}

Properties for display of the Legend box Shadow. You may define color and size.

\section{Panel Properties}

\section{Border}

You may define Border independently or in conjunction with the Bevel properties. With Border set to Visible True you will obtain a 'sunken' border effect on the outside of the Chart Panel. Mixing Bevel and Border and manipulating Width give almost any combination of 3D effects.

\section{Gradient}

To define a Gradient you must select a StartColor and EndColor (plus, optionally MidColor) and enable as Visible the Gradient. The Gradient will cover the whole Chart panel. Gradient direction defines the direction of color change between Start, Mid and EndColor.

\section{Walls Properties}


This page in the Chart section of the Chart Editor applies properties to the Chart Walls. There are 4 Walls: Left, Right, Bottom and Back that may be represented in $2 \mathrm{D}$ or $3 \mathrm{D}$.

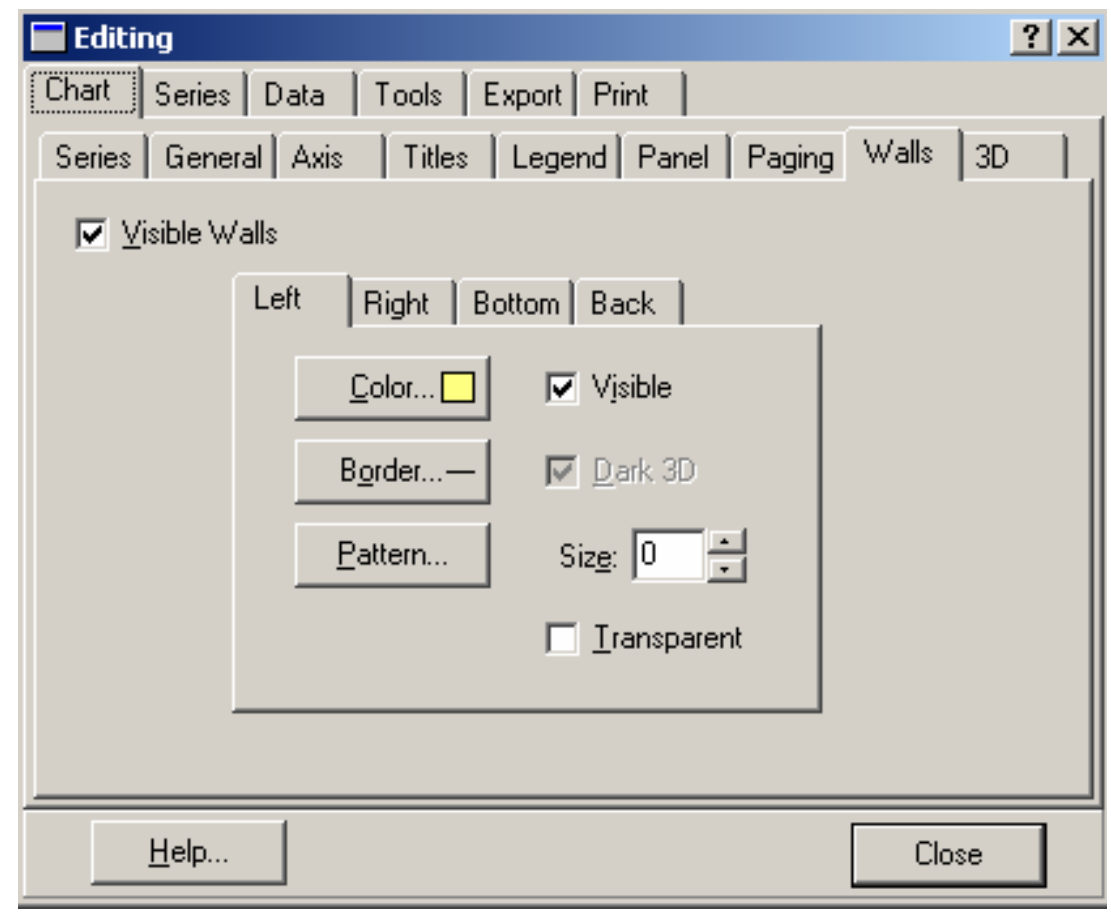

\section{WallBorder}

You can specify the thickness of the walls for the chart here.

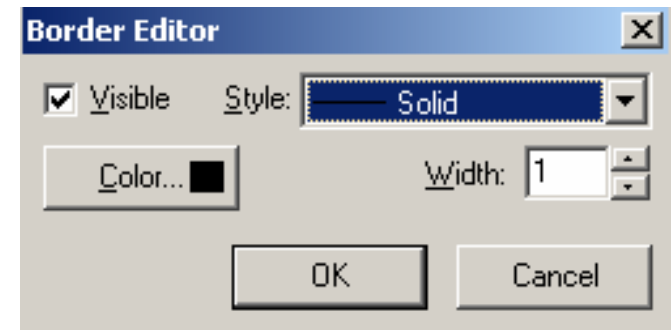




\section{D VIEW}

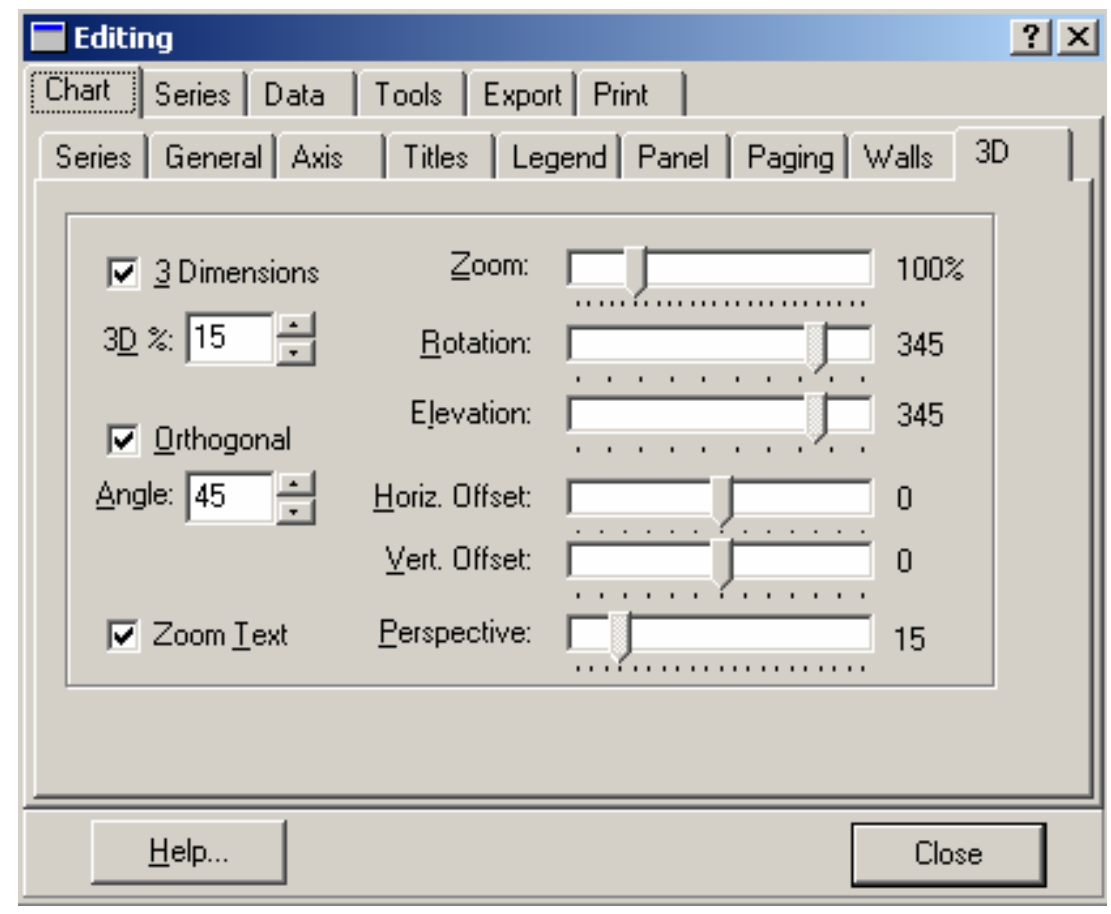

\section{Editor options:}

\section{Dimensions}

Selecting this property (toggling) will change the Chart from a 3D to a 2D Chart in design time and the setting will take effect for runtime.

The Orthogonal Option, when disabled, enables Windows Native 3D mode allowing you to Rotate, Elevate and offset the Chart. The Zoom option allows you to bring forward or move away the whole Chart. Zoom internally in the Chart on Data Series is still available at runtime.

The Perspective property allows you to set a distance perspective as if looking into a room. Perspective offers a visual enhancement for Charts used for presentation purposes. 


\section{INTERPOLATION OPTIONS}

The software also has provisions to interpolate the given data in order to create more data. On of the main features of this software is that it can handle sporadic data. This would extremely be helpful when handling a group of wells, because the production dates of all the wells in a group may not be the same. The interpolation option helps to create a regular data set from a sporadic data set of the selected group.

\section{Select Tools: Interpolation Options}

The interpolation option window will prop up and give you options for the duration of interpolation.

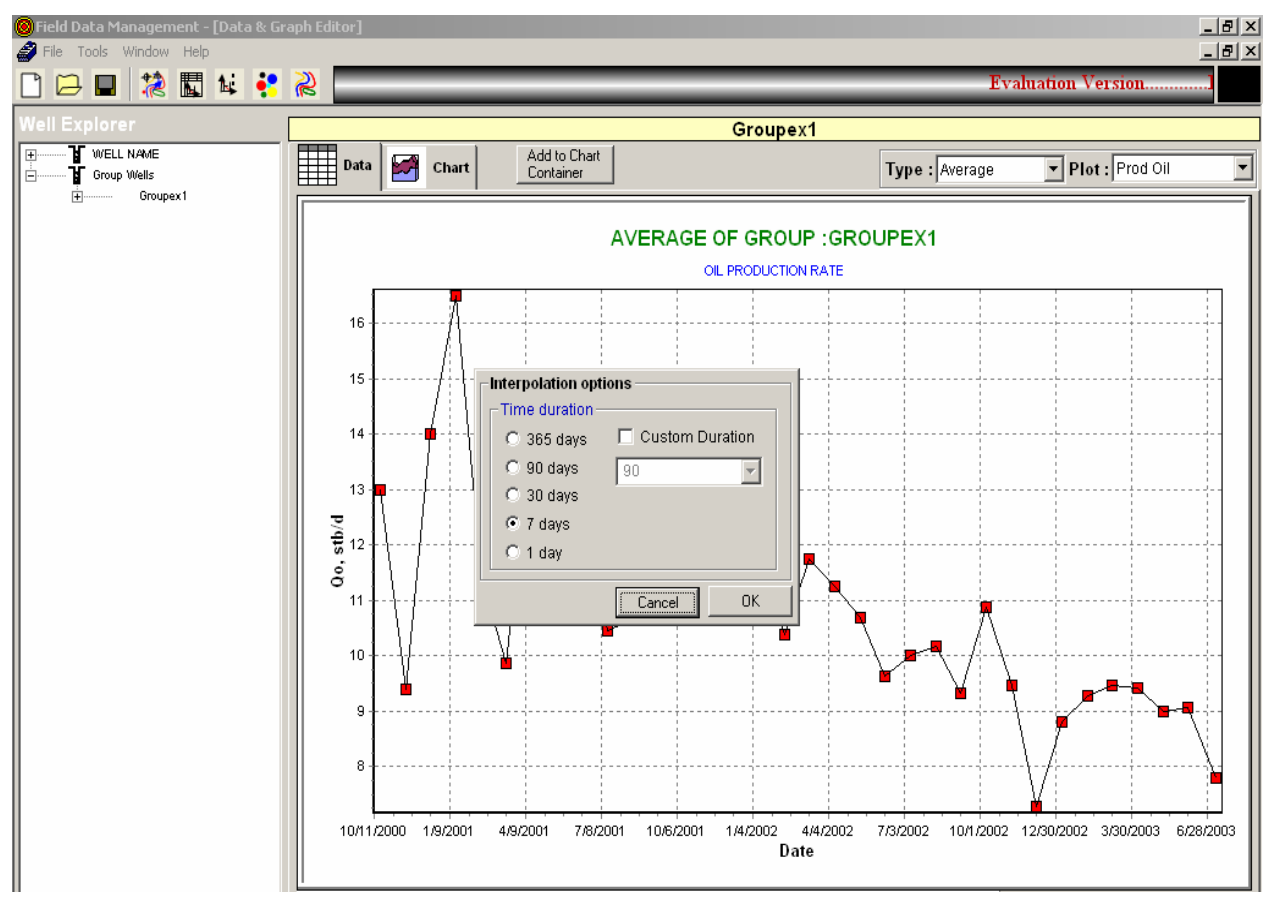

Choose the time duration for interpolation from the window and press OK. For this example select 7 days. The software calculates the interpolated values and plots the result of interpolation. Figure shown below gives the interpolated (every 7 days) plot of oil produced for the group "Groupex1". 


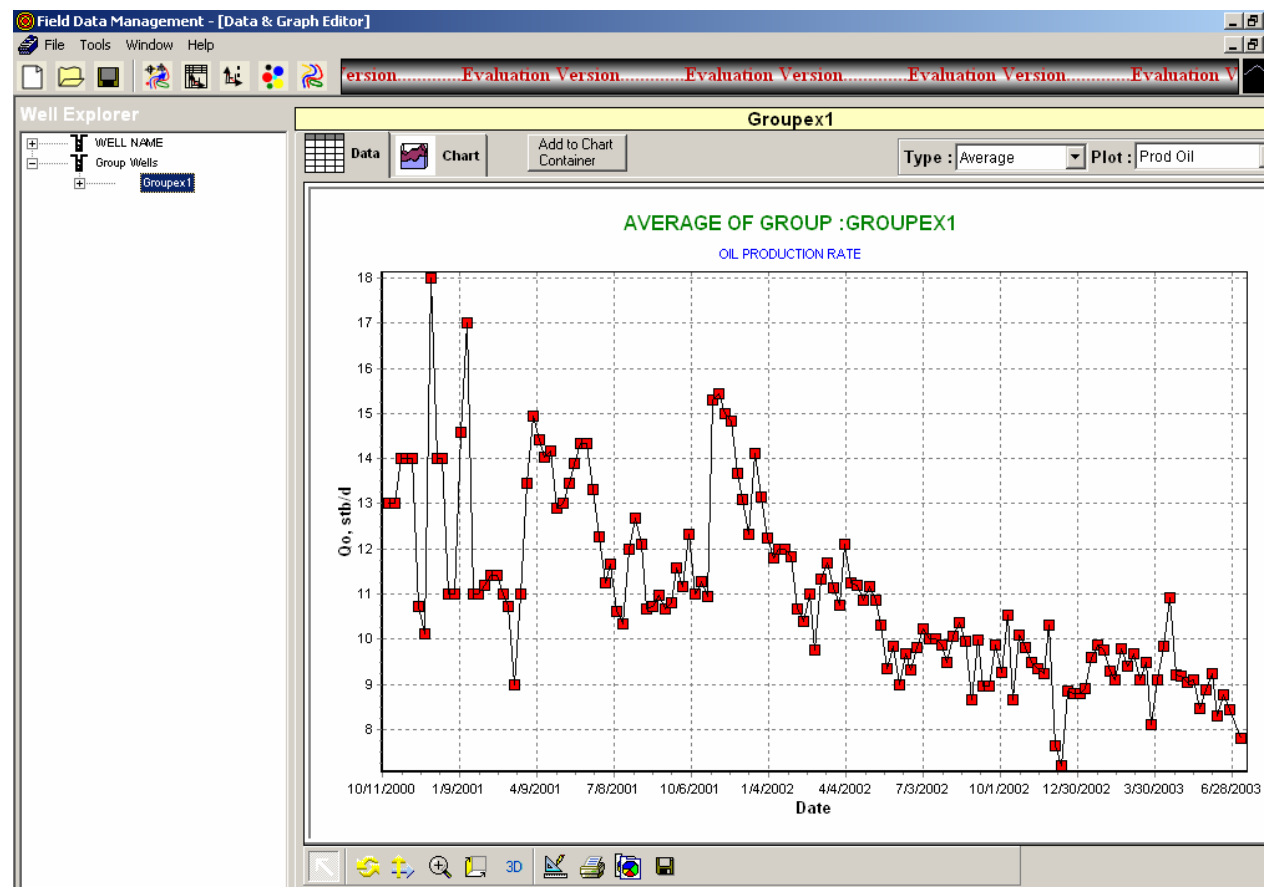

Note: the "Type" window is now active and has the options

- Average

- Summation

This enables to plot the average of the group or the summation of the group. 


\section{DECLINE CURVE ANALYSIS}

The software also performs decline curve analysis. In order to perform decline curve analysis click on this

On click the decline curve analysis mode is activated and the screen would look like the one shown in Figure below. In the figure well BR_C:2 has been highlighted to perform decline curve analysis. The yellow lines at the left and right end of the plot separated by a red-dashed line indicates the range of data to be included in the analysis.

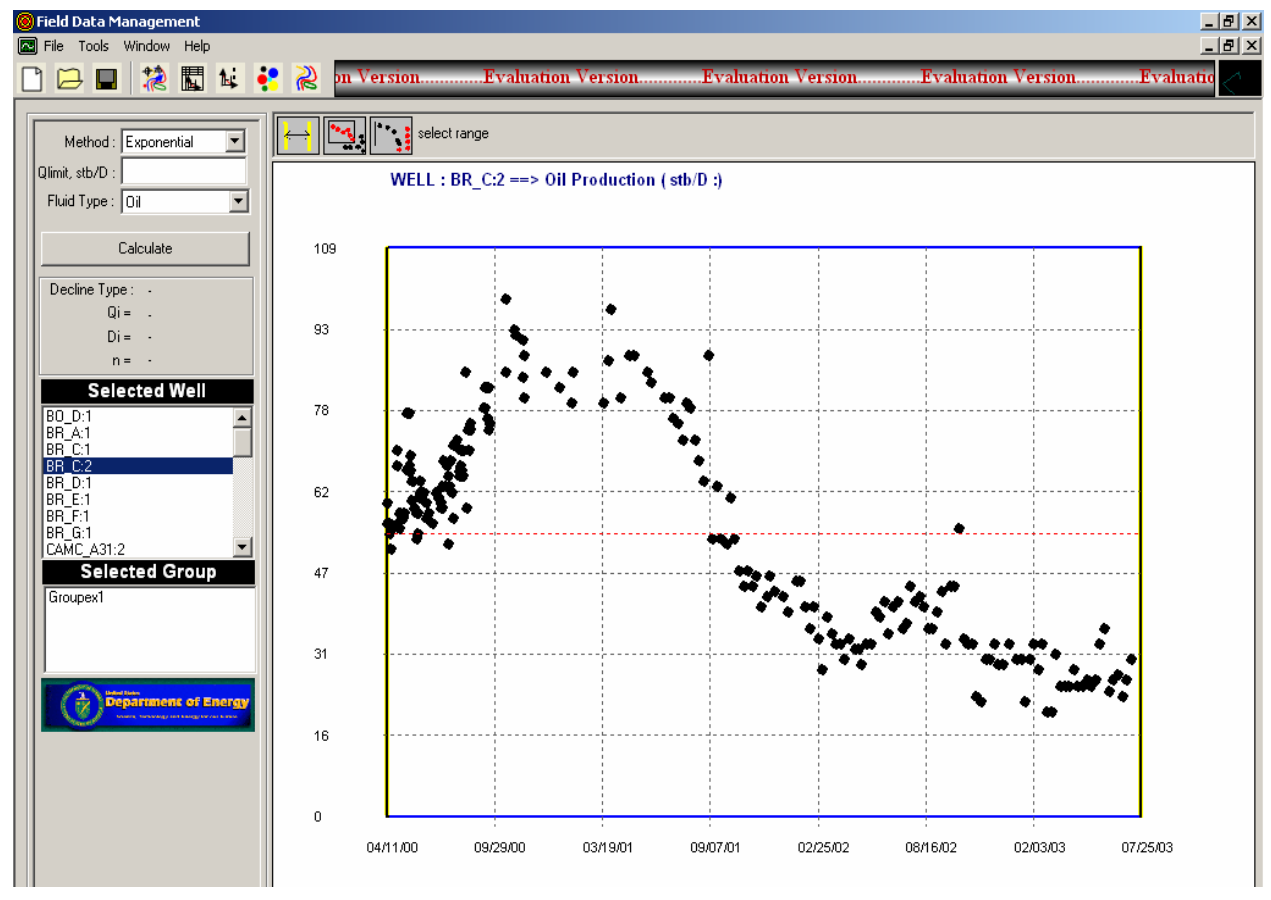

The decline curve analysis has several options. The functions of icons are discussed below.

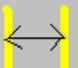

The range of data in the $x$-axis can be adjusted using this icon. Clicking the icon and moving the yellow lines to the required range achieve this.

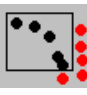

Bad data can be omitted using this option.

The data can be reset through a window range using this option. 
Using the above options a decline curve analysis is performed on well BR_C:2 as shown below. The data points in red indicate neglected data.

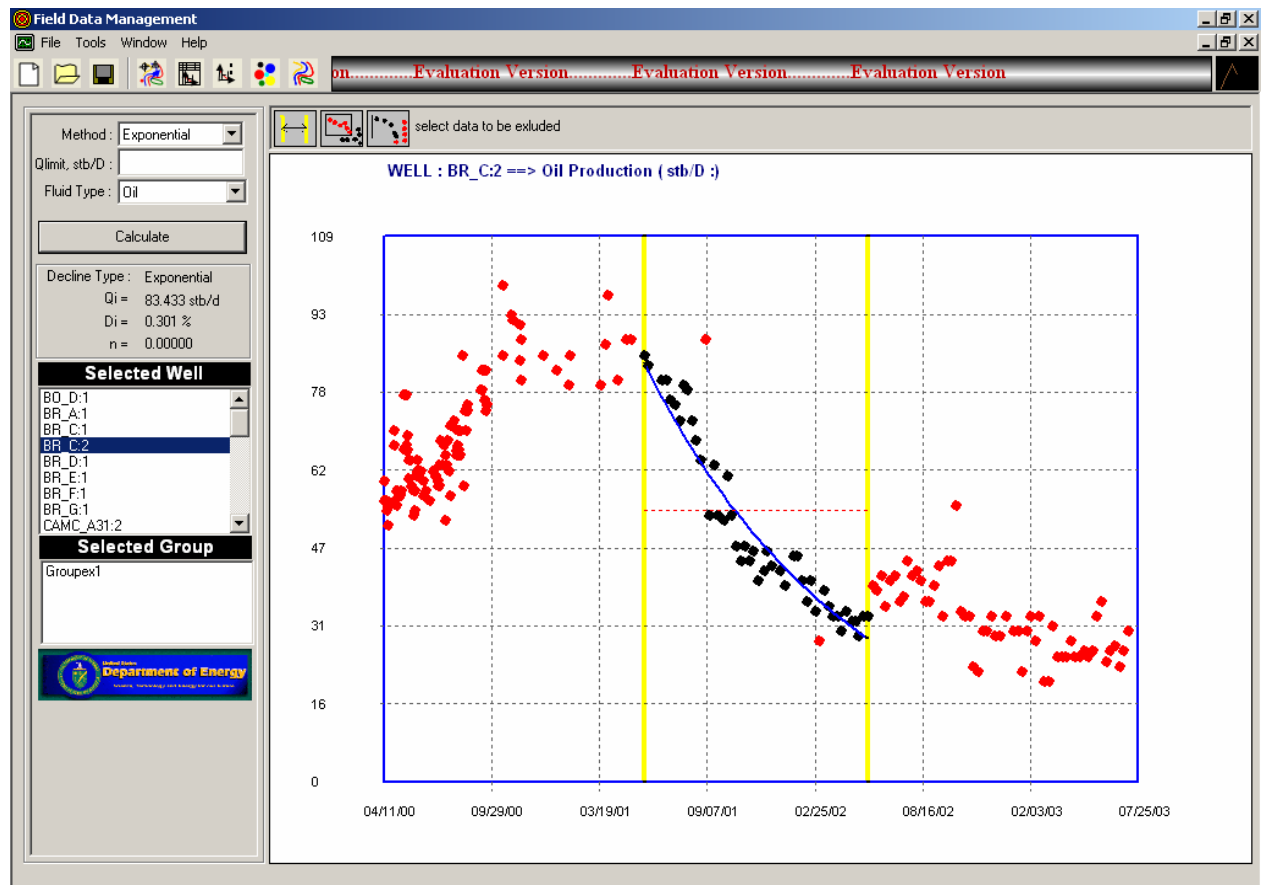

The decline curve analysis in this software has provisions for the type of decline you choose to employ. The methods of decline available are

- $\quad$ Exponential

- Hyperbolic

- Harmonic

A Best Fit Option is also provided to let the software determine the best of the three methods used to analyze decline. You can also specify the Qlimit for decline in the window provided. Press Calculate to perform the decline. On performing the decline the output is shown in the left-hand side window stating,

- $\quad$ Decline type

- Initial rate

- Decline rate

- $\quad$ Decline parameter

There are two windows on the left-hand side, Selected well and Selected Group which enables to analyze decline for one selected well or a group of wells, respectively. 


\section{BUBBLE MAP AND PIE CHARTS}

Procedure for generating bubble map

Click on ${ }^{\bullet}$ to activate the Bubble map mode. You would see the a screen as shown below.

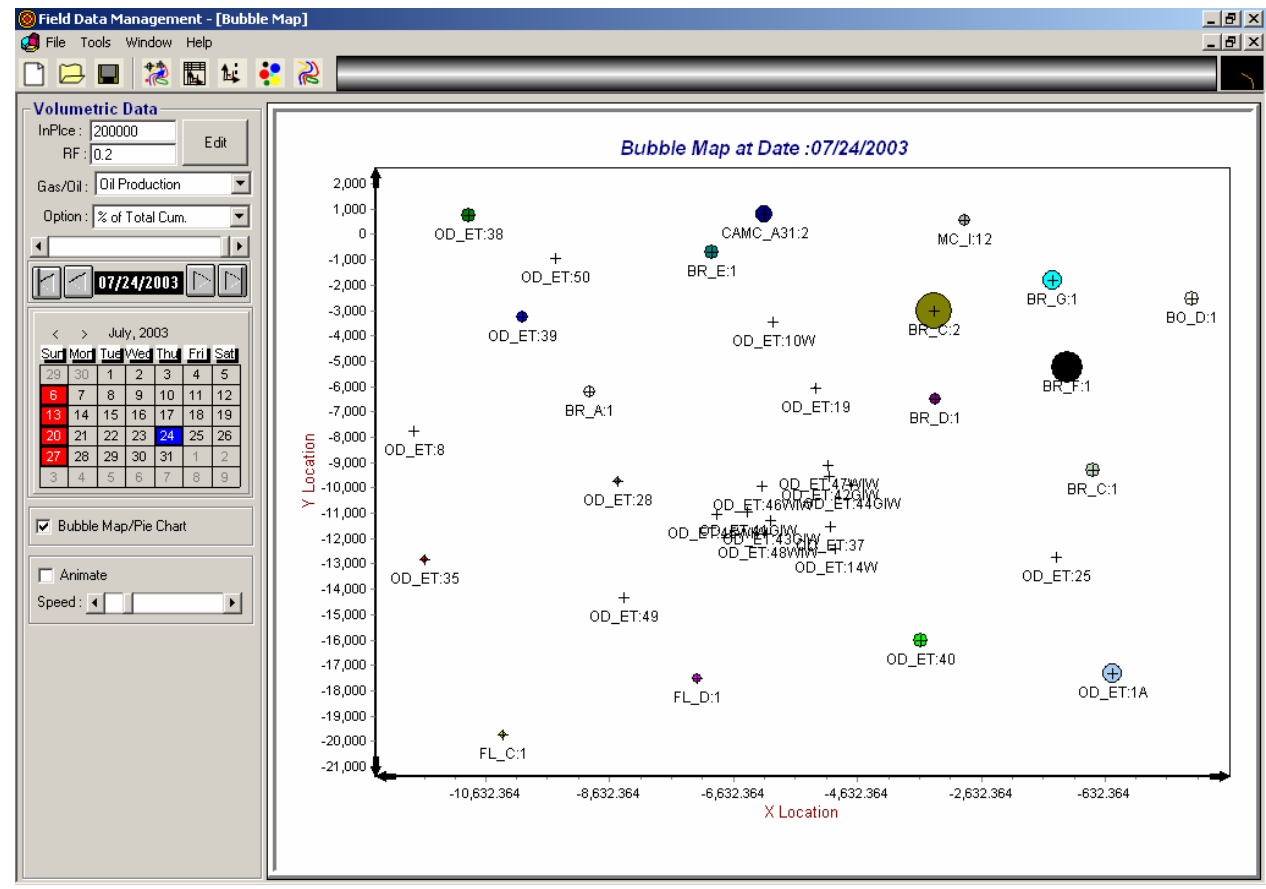

The volumetric data is given on the left-hand side of the window. The features in the volumetric window are,

1. Fluid in place

2. Recovery factor

3. Types

This would include

- Oil production

- Gas production

- Water production

- Water injection

- Gas injection

There are also options to plot the bubble map as

1. $\%$ of Total cumulative production

2. $\%$ of Recoverable reserves

3. $\%$ of Fluid in place 
The bubble map can be plotted at any point in time by scrolling the time bar on the left-hand side of the window or even more specific by choosing the required date on the calendar.

The Bubble Map/Pie chart button can be toggled to view either the Bubble map or the Pie chart. By default the button is checked and the bubble map is visible. In order to view the Pie chart uncheck the button.

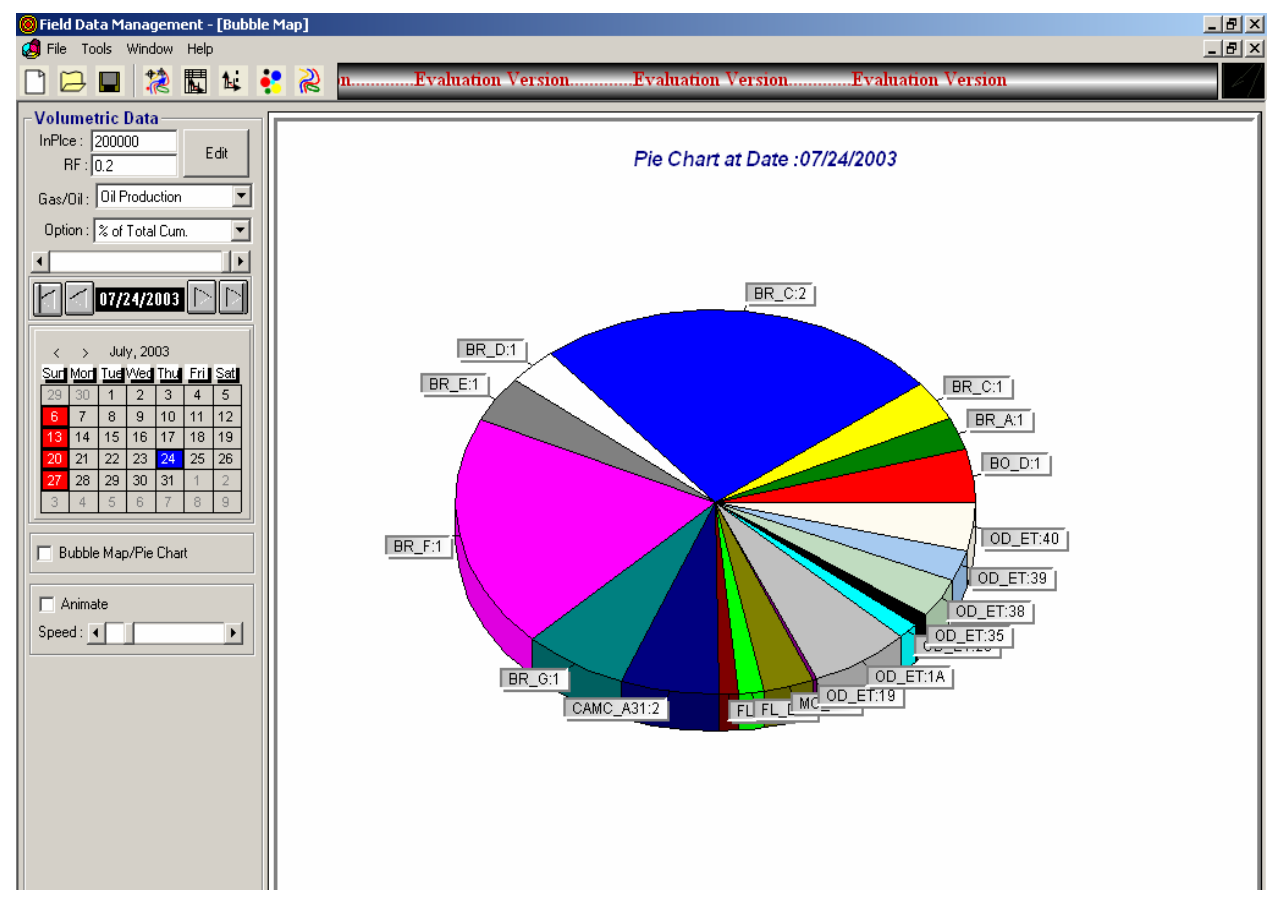

Animation sequence of the formation of bubble map over time could be realized by checking the Animate button. The speed of the animation could be adjusted using the Speed bar.

Note: when this button is checked all other options are disabled. In order to stop the animation, uncheck the Animate button. Also if the speed of animation is very high there could be problems in animation, keep the speed to optimum level. 


\section{ISOBARIC MAPS}

\section{Procedure for generating bubble map}

Click on

Note: As soon as the button is clicked, there would be an error message prompting that there is insufficient data to create a contour map. Press OK to the message. You would then see a field map without any contours. On moving the Time bar located on the left side of the window, the isobaric map becomes visible.

You would see the screen as shown below

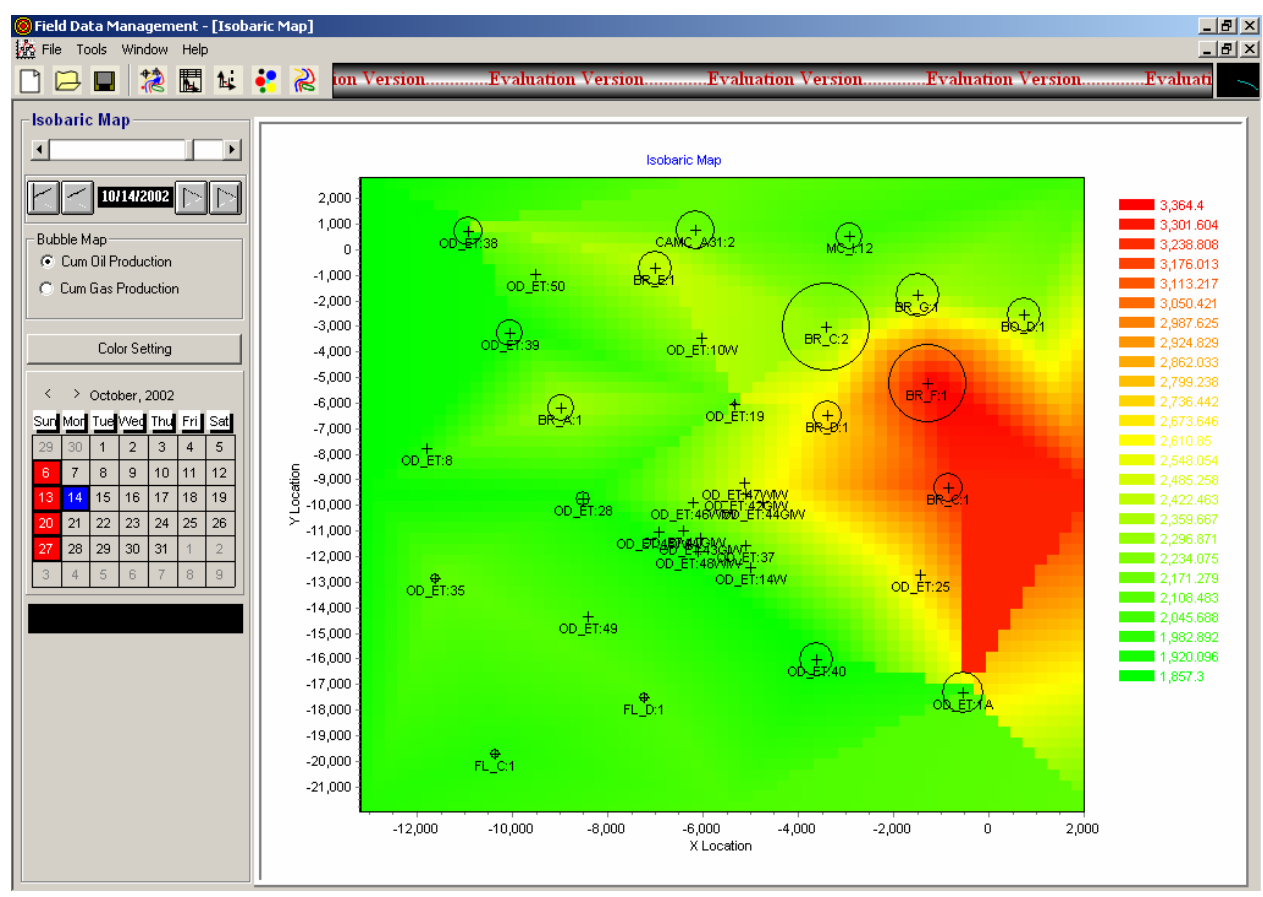

The isobaric map mode also has provisions to include the bubble map along with the isobaric contours. The bubbles ( with no color inside them) represent the production bubble maps. The types of bubble map options presented at the left side of the window are

1. Cumulative oil production

2. Cumulative gas production 
The Color settings button enables to change the basic colors of the map by editing the start, end and middle color.

\section{GAS MATERIAL BALANCE}

The procedure for performing gas material balance is as follows.

\section{Select Windows : Gas Material Balance}

On selecting this option the gas material balance window is activated. You would see the screen shown below. In this example the well BO_D:1 is activated and the Historical Data tab is enabled. This tab plots the gas production history and pressure history of the well.

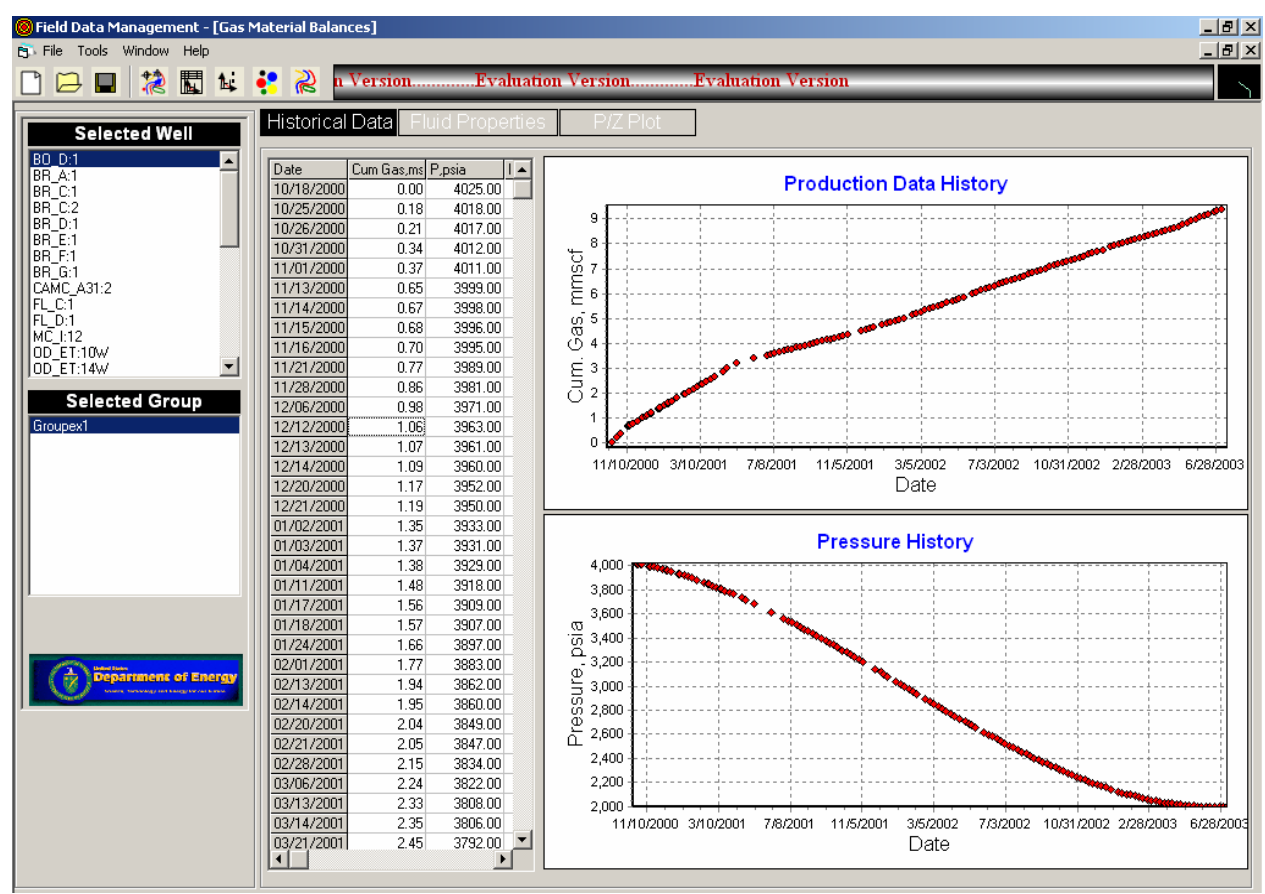


The fluid properties for the analysis could be viewed by pressing the Fluid Properties tab. The general properties of the reservoir can be entered here by enabling the PVT tab.

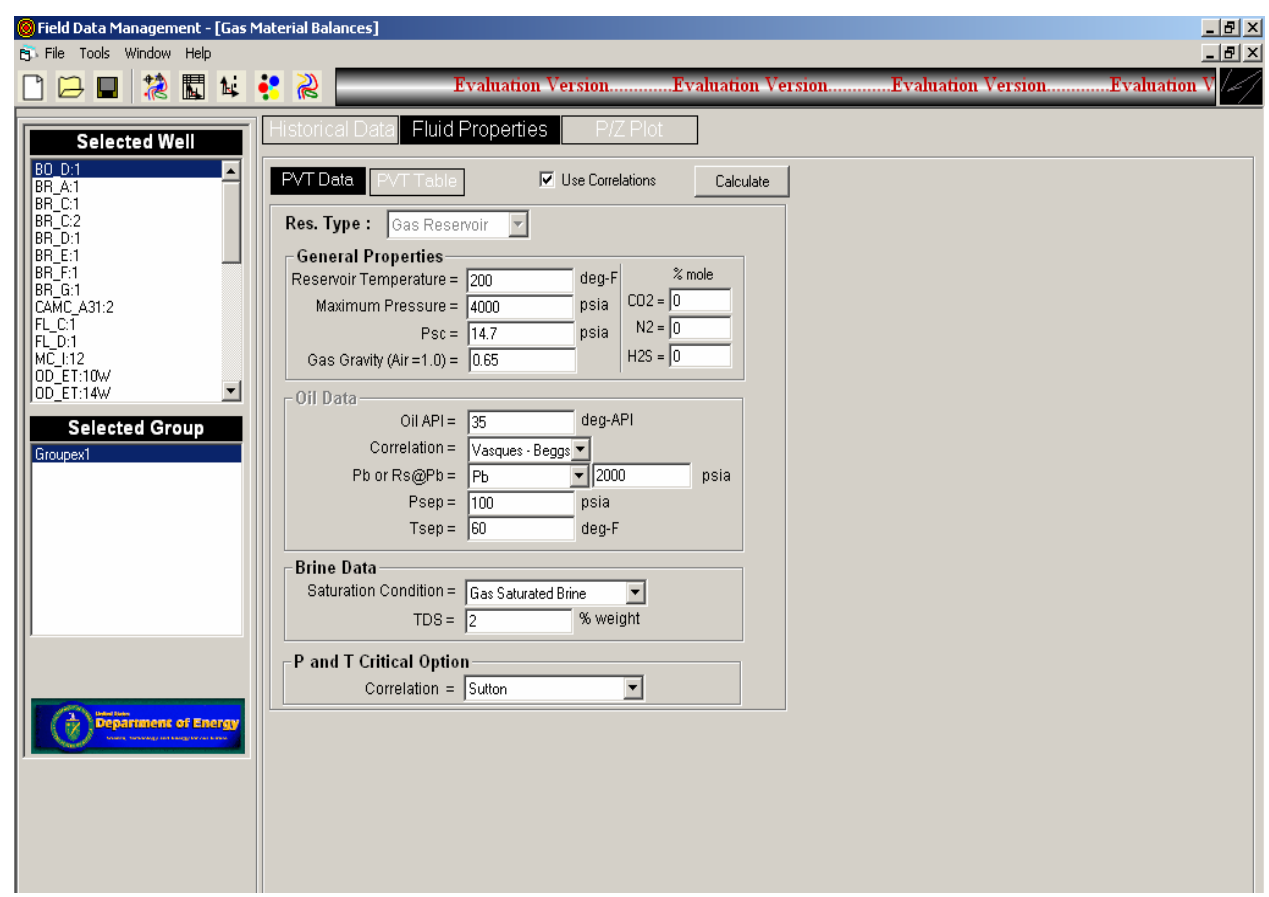

The PVT data table can be viewed by pressing the PVT table tab as shown below. In order to view the PVT properties click on View Chart to explore the chart options. Your can either leave the data unchanged or input your own data.

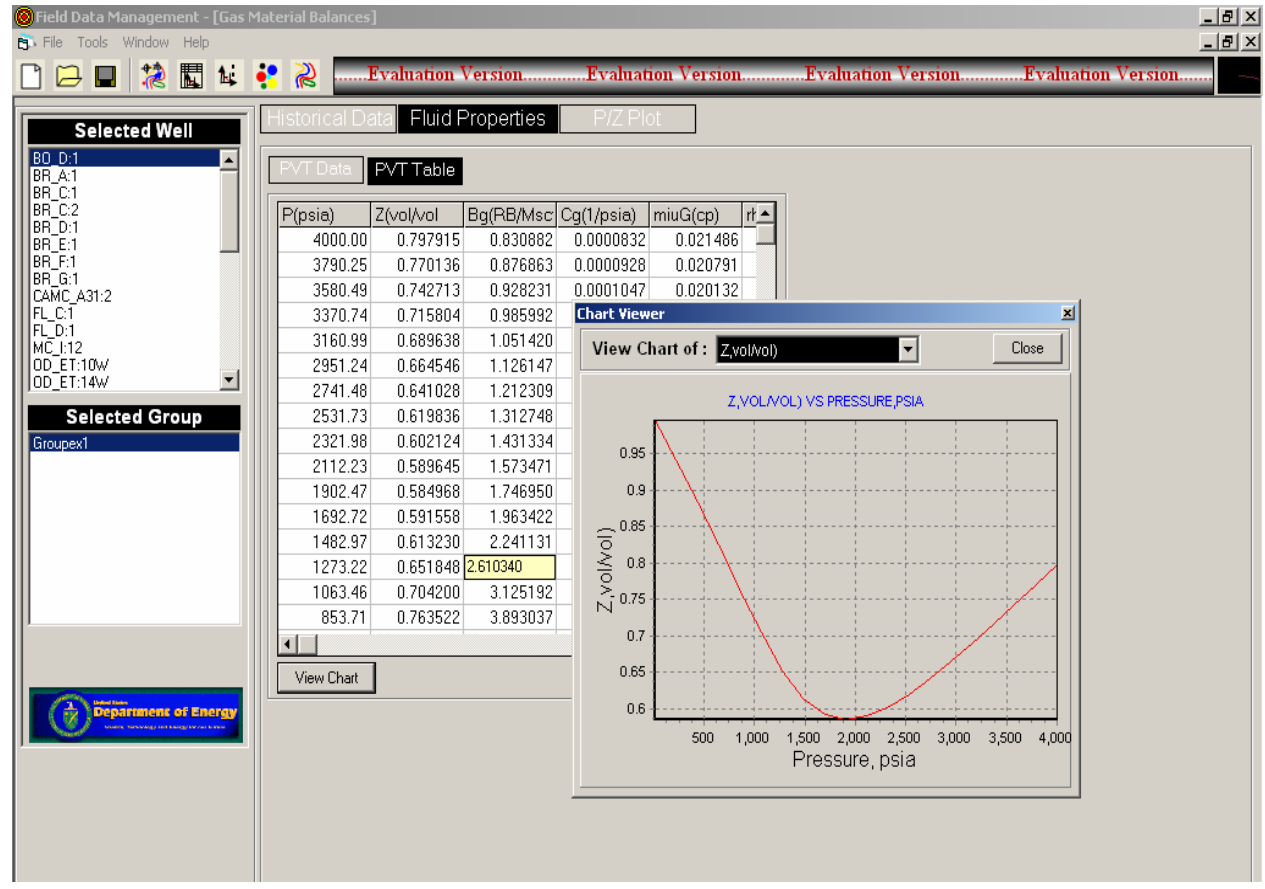


In order to perform the P/Z plot click on P/Z Plot. on clicking the P/Z Plot mode is active . you can enter a value for abandonment pressure ( say 200 psi) and press Enter. On hitting the Enter button you would see the run summary, which indicates the Original Gas in place along with the error estimates.

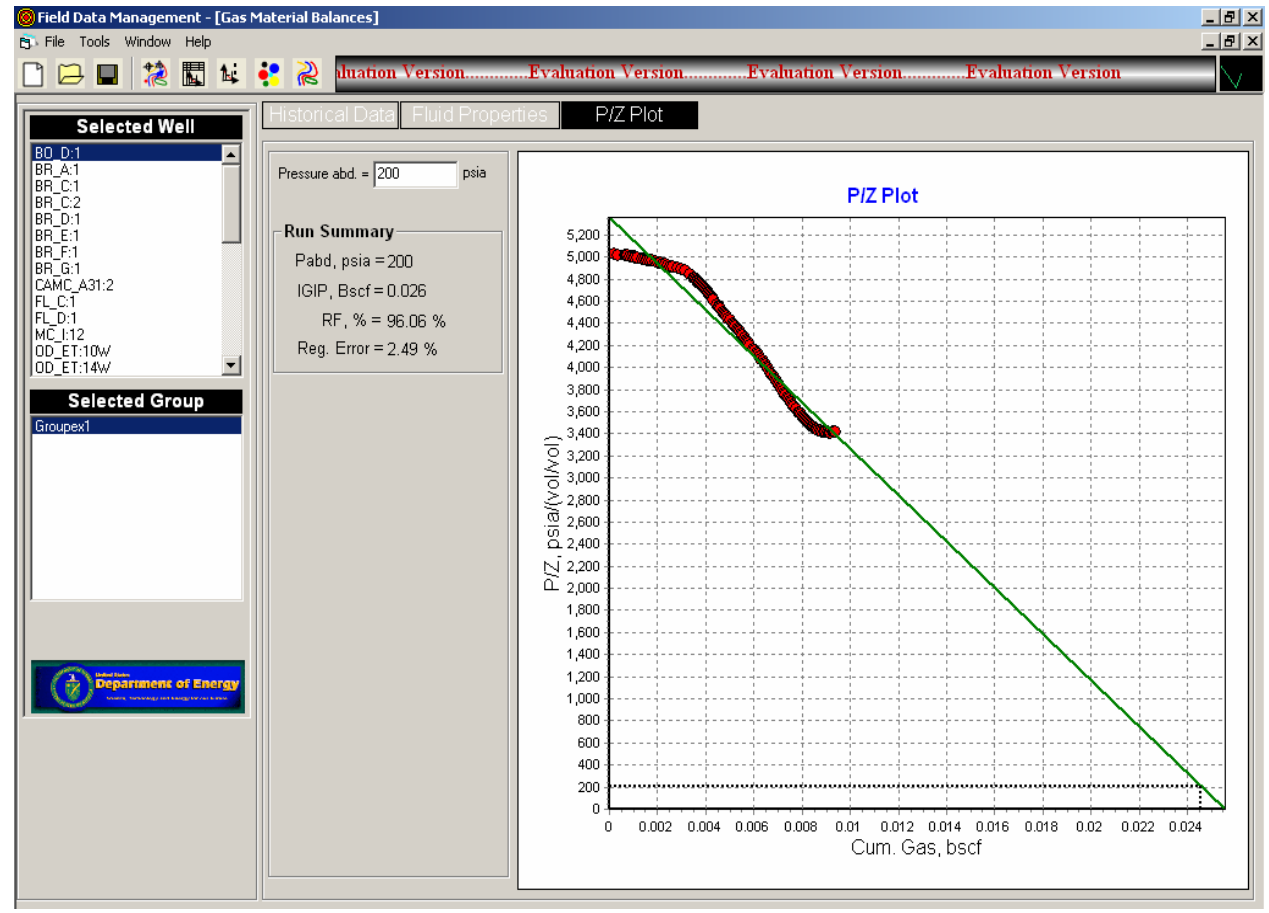




\section{GERMANIA SPRABERRY FIELD DEMONSTRATION STATUS}

The objective of this project is to design and test different waterflood techniques that have never been utilized in the Spraberry Trend Area. The new waterfloods will align injection wells along the fracture trend with production wells. New injection wells will be drilled that will not be artificially fractured to test whether specific zonal isolation is the primary key. Existing producers with massive hydraulic fracture treatments will be converted to injectors to test whether the hydraulic fractures hinder or aid sweep efficiency. An injection pattern, which is adjacent to, and on-trend with a section containing a majority of plugged wells will be dedicated to investigating whether there is still mobile oil in the vicinity of old, abandoned wells and whether this oil can be swept and captured in current producing wells. The results of the Germania Unit Field Demonstration will be provided to identify the preferred management practices and to transfer the information to all Spraberry operators so that other operators can initiate water injection.

The report discussed the on-going field activities in the GSU pilot. We presented a report on maintaining the injection rate based on VRR, polymer treatment and pressure observation results, and gathering the production data for each production wells, predicting the waterflood response and incremental oil recovery due to waterflooding.

\subsection{Voidage Replacement Ratio (VRR)}

The water injection began on Feb 3, 2003 from six injectors. The six injectors consist of three wells converted to water injection (17, 407A and 410A), two wells were returned to water injection (11W and 22W) and a new injection well (214W) as shown in Fig. 3.1. An average of 270 bwpd are injected through each of these six injectors (Fig. 3.2). The amount of water injected was determined based on Voidage Replacement Ratio (VRR). VRR is the ratio between injected reservoir volume and produced reservoir volume, which is expressed in the following equation:

$$
V R R=\frac{B_{W}\left(I_{W}\right)}{B_{o} q_{o}+B_{w} q_{w}+q_{o}\left(G O R-R_{s}\right) B_{g}}
$$

where : $\quad B_{w}=$ Water formation volume factor (STBW/BBL)

$B_{o} \quad=$ Oil formation volume factor (STBO/BBL)

$B_{g} \quad=$ Gas formation volume factor (SCF/BBL)

$I_{w} \quad=$ Water injection rate $(\mathrm{BBL} / \mathrm{DAY})$

$q_{o} \quad=$ Oil production rate $(\mathrm{BBL} / \mathrm{DAY})$

$q_{w} \quad=$ Water production rate (BBL/DAY)

GOR = Gas oil ratio $(\mathrm{SCF} / \mathrm{STB})$

$R_{S} \quad=$ Solution gas $(\mathrm{SCF} / \mathrm{STB})$

In waterflooding practice, VRR is kept to be one, in order to balance the amount of water injected and the amount of fluid produced. If the VRR is greater than 1 , the reservoir is over-injected or vice versa.

The total amount of water that had been injected up to 8/1/2004 was about 900,000 BBLs (Fig. 3.3). If we compare this injection amount to ET O'Daniel, the wells located in the on-trend direction i.e. Brunson F-1 and C-2 have responded to water injection (Fig. 3.4). The Voidage Replacement Ratio 
(VRR) is expected to be one to balance the amount of water being injected and the amount of fluid being produced. Two different injection periods were compared to analyze the effect of VRR. The first injection period was from 1965 to 1989 (Fig. 3.5) and second injection period was from January 2003 to the current time (08/1/2004). The first period exhibited an overall VRR greater than one suggesting that the volume being injected exceeded the total volume being produced. From 1969 to 1975 the average value of VRR was 20, indicating that 20 barrels of water were injected per 1 barrel of fluid produced (oil, water, and gas). This may explain that the high water cut and rapid breakthrough observed in some wells (especially those surrounding the injectors) and is perhaps one of the most responsible factors for the poor performance of the unit during this period. The second period exhibits an overall VRR of one (Fig. 3.6), thus indicating that the energy balance was maintained throughout this pilot. However, after nine new wells had been drilled the VRR dropped below one, indicating less than the total amount of water being injected. Once the injection was kept low for some time, the production rate also decreased. It caused the VRR to once again approach one. At a later period, the VRR dropped back below one as more water was produced. At this period, higher injection rate is needed to sustain the energy balance. As comparison, the VRR in ET O'Daniel was well maintained during the waterflood period (Fig. 3.7).

\subsection{Polymer Treatment}

In order to reduce water production, three wells (406A, 408A and 409A) that had high water production located close to the injectors were treated with polymer. After the polymer squeezing, it is anticipated that the water being injected will find different paths around these wells and increase the overall sweep efficiency of the waterflood project. Tiorco polymer squeezed was performed in Upper Spraberry for these 3 wells consecutively from June 8-10, 2004 as detailed below:

GSU 406A: Gel was pumped in 7 stages with increasing concentrations. Stages 1-5 consisted of MARCIT gel, Stage 6 was a CAPIT gel, and Stage 7 was the Mara-Seal gel. Prior to polymer treatment, this well was an observation well in the GSU waterflood. After waterflood commenced in 2/03, the well went to nearly $100 \%$ water.

GSU 408A: Gel was pumped in 7 stages w/ increasing concentrations. Stages 1-5 consisted of MARCIT gel, Stage 6 was a CAPIT gel, and Stage 7 was the Mara-Seal gel. Prior to polymer treatment, this well was an observation well in the GSU waterflood. After waterflood commenced in $2 / 03$, the well went to nearly $100 \%$ water.

GSU 409A: Gel was pumped in 7 stages w/ increasing concentrations. Stages 1-5 consisted of MARCIT gel, Stage 6 was a CAPIT gel, and Stage 7 was the Mara-Seal gel. Prior to polymer treatment, this well was an observation well in the GSU waterflood. After waterflood commenced in $2 / 03$, the well went to nearly $100 \%$ water.

The fluid level (FL) and pump intake pressure (PIP) were measured every three days in these treatment wells. After the polymer treatment, we expect that FL will increase and PIP will decrease. We also expect that the water being produced will significantly decrease and the sweep efficiency will improve. The treatment results were discussed as followed:

For GSU 406A, we found that FL increases and PIP decreases as expected during early periods and remains constant at later periods indicating the success of polymer treatment (Fig. 3.8). However, no production data is available after polymer treatment to further examine this response. For GSU 408A, we observed there is inconsistency in FL and PIP trends early on indicating ineffective polymer treatment at this period (Fig. 3.9). At later a date, the trends behave as expected. Again, no production 
data is available after polymer treatment to further examine this response. For GSU 409A, we also observed there is inconsistency of FL and PIP trends still to this date indicating ineffective polymer treatment throughout this period (Fig. 3.10). In contrary, single production data taken after the treatment shows a significantly lower water production and higher oil production.

\subsection{Pressure Observation Wells}

In Germania we have a nice opportunity to understand what the heck went on in O'Daniel. We had the BHP for the 38, 39 and 40 but in the end what we really needed was pressure outside the pilot area in the ring of influence stretching SW from the Floyd lease up NE through the Brunson lease. What was really interesting was how some wells responded gang busters while other wells in between the pilot and the good wells showed little or no response. We think this is a result of the heterogeneity in the fracture system however we had no pressure data to back up that hand waving.

If we have two adjacent wells, then we can ascertain the pressure drop as a function of flow rate and distance from the injectors. Then we can do some really neat stuff like pulsing individual injection wells and looking at the response in the two adjacent wells as well as all the other monitoring wells. We can do a very simple rotation of single injection wells, pulse those wells (change the flow rate of one injector or shut the injector in for a few days) and look at pressure response. The two adjacent wells would give us major clues as to what fracture system (out of the 3 sets measured from the O'Daniel horizontal core) the injected water is flowing in. This would eventually allow us to better design injection strategies in the future. The real key to sucessful water injection in Spraberry must be sweep efficiency. That is what we did not learn in O'Daniel since we only had water production data at which to look. We think an aggressive pressure monitoring program in Germania will answer some of those questions.

Shut-in Pressure had been taken at three observation wells (GSU 5, 20 and 317) outside the pilot. Each observation well had been tested for casing leaks. The FL was shot every 2 weeks. During injection period, we expect that FL will decrease and PIP will increase at observation wells. The FL and PIP trends for each observation well are discussed as follows: For GSU 5, we found that FL has a decreasing trend and PIP has an increasing trend as expected (Fig. 3.11). This well has a good response to water injection. For GSU 20, we found that the responses of FL and PIP are delayed. The well starts responding on 8/16/2004 after about 57 days shut-in (Fig. 3.12). For GSU 317, we found that FL has a decreasing trend and PIP has an increasing trend as expected (Fig. 3.13). The well has a good response to water injection. We found that one data set taken in 8/23/2004 has inconsistent trend between FL and PIP.

\subsection{Well Response}

The response of water injection was observed through each production well. We gathered the production data and recorded them in two production database management software, Oil Field Manager $\left(\mathrm{OFM}^{\mathrm{TM}}\right)$ and Field Management Database Software (FMDS). The FMDS software is the software we developed for better managing, predicting and tracking the production for this area. We are able to make the software more general and robust in handling more sporadic data sets. The development of this software is presented in Chapter 2 and the manual of the software is listed in Appendix-A. 
During the year of 2003, several new wells had been drilled in this area as shown in Fig. 3.1. Individual wells were observed, as well as the group of the wells in each track. We observed the water injection response in the track 2, 3 and 4 on a daily basis. Figs. 3.14-3.16 show the production performance in each tract. Most of the sudden increases in oil production are due to the new production wells (Figs. 3.17 - 3.19) not the response from water injection.

Two new wells (213A and 413A) in Tracts 2 and 4 located in off-trend wells and near injection wells are not affected by waterflooding. Even, water production rates decrease significantly with time indicating no response of water injection to these wells. Both wells produced at high initial oil production and later on declined rapidly. Well \#213A produced at initial rate of 229 bopd. Current production rate drops to 25 bopd. Well \#413A produced at an initial rate of 87 bopd. Current production rate drops to 10 bopd. All new wells in Tract 3 show similar water production response as those in Tract 2. Even though oil production decreased with time, the oil production trend was different and oil rate decline was not as dramatic as those in Tract 2. Well \#330A was completed in $11 / 20 / 03$ with initial rate of 35.2 bopd and the production increased to 69.2 bopd on 12/19/03. The current production rate has decreased to 20 bopd. Well \#332A was completed in 10/25/03 with initial rate of 6 bopd and the production increased to 61 bopd on 10/25/03. The current production rate decreases to 10 bopd. Well \#331A was completed on 10/5/03 with initial rate of 47.5 bopd and the production increased to 87 bopd on 10/16/03. The current production rate has decreases to 20 bopd.

We do not see any waterflood responses in the track so far. Therefore, we observe the response in each production well and locate them on the map. Figure 3.20 shows the wells that experienced high water production rates during this water injection period. We observed that the on-trend wells located close to the injectors experience high water production rate as expected. Meanwhile Fig. 3.21 shows the wells that have had good oil response. We found that most of the wells in Track 3 respond well to water injection. It looks like the water pushes the oil more to the $\mathrm{N}$-E direction than to the S-W direction. A detailed production history of these wells can be found in Appendix-B and C.

Using the FMDS software, we combined the sporadic production data from 22 good responded wells. The average and the total of production responses are presented in Figs. 3.22 and 3.23. We performed a decline curve analysis on oil production rate as shown in Fig. 3.24. The base line was taken as exponential decline with $0.092 \%$ decline rate (Fig. 3.25). The incremental oil production is calculated based on cumulative production about the base line. The cumulative incremental oil production on current date is about 30,000 bbls as presented in Fig. 3.26.

\section{5 Summary}

1. The current amount of water injection rate is not enough to support the current production rates based on VRR analysis.

2. Based on pressure response in observation wells, we summarize our findings as follows:

- Despite the inconsistency of the data, the trends of pressure buildup in observation wells are as expected.

- Waterflood response is better in N-E rather than in S-W direction.

- Even though well \#5 is farther in distance than well \#20, well \#5 has a better pressure response. 
3. Based on polymer treatment response as indicated by the response of fluid level and pump intake pressure, we found that:

- GSU 406A shows a successful polymer treatment since early period.

- Even though the GSU 408A shows ineffective polymer trend at early period, it has a good polymer treatment later.

- Until this date, 409A still shows ineffective polymer treatment.

4. The effect of water injection has reached many wells in Tracts 3 but less in Tract 1 . It may be that the water is still in the filling up process. We may need to wait a longer time to see the waterflood response. The current response can be summarized as follows:

- Waterflooding process has affected 22 wells located in the on-trend direction and near the injectors as indicated by an increase in water production.

- Wells located in off-trend location and even near injection wells has still not been affected by water injection.

- The response of new production wells shows that the water production decreases even during water injection indicating that the performance of these wells is not supported by on-going water injection. 


\section{References}

1. Schechter et al.: "Preferred Waterflood Management Practices for the Spraberry Trend Area," SemiAnnual Report (DOE Contract No.: DE-FC26-01BC15274), Oct. 2001 - March 2002.

2. Schechter et al.: "Preferred Waterflood Management Practices for the Spraberry Trend Area," SemiAnnual Report (DOE Contract No.: DE-FC26-01BC15274), April 2002 - Sept. 2002.

3. Schechter et al.: "Preferred Waterflood Management Practices for the Spraberry Trend Area," SemiAnnual Report (DOE Contract No.: DE-FC26-01BC15274), Oct 2002 - March 2003.

4. Schechter et al.: "Preferred Waterflood Management Practices for the Spraberry Trend Area," SemiAnnual Report (DOE Contract No.: DE-FC26-01BC15274), April 2003 - Sept. 2003.

5. Schechter et al.: "Preferred Waterflood Management Practices for the Spraberry Trend Area," SemiAnnual Report (DOE Contract No.: DE-FC26-01BC15274), Oct 2003 - March 2004. 


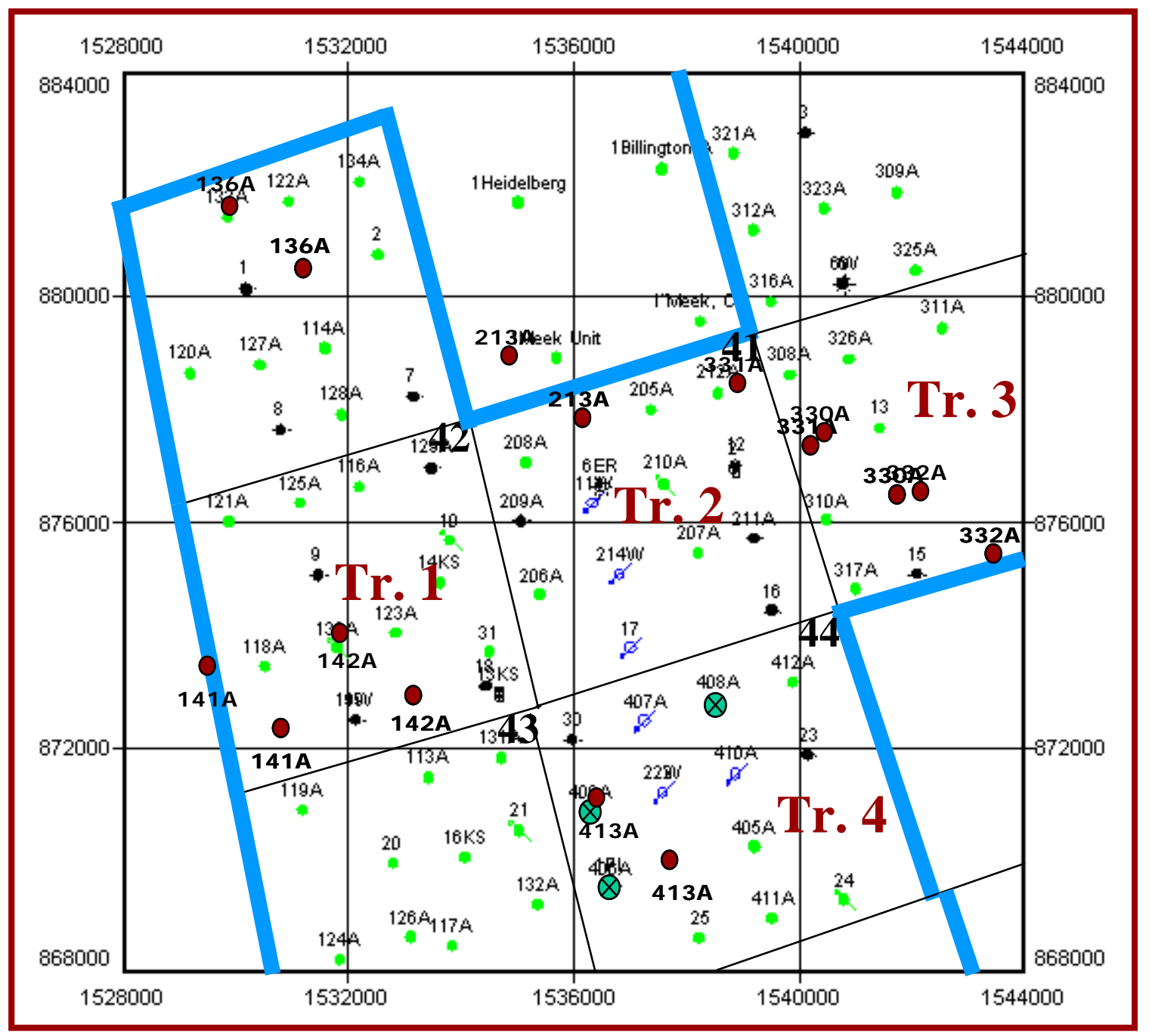

Fig. 3.1 - Location of new wells drilled in 2003 (red dot symbol) 


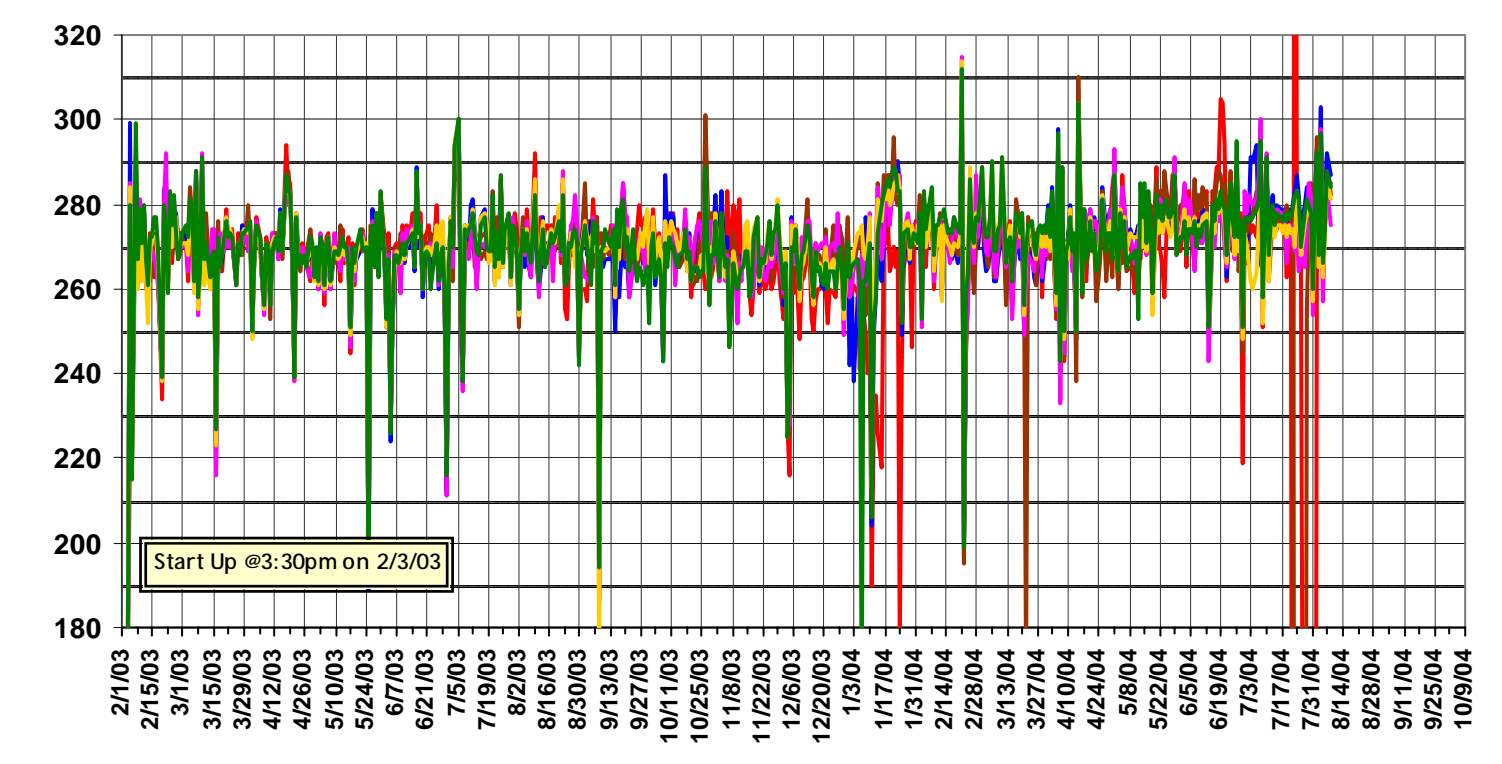

GSU \#11WIW — GSU \#17WIW - GSU \#22WIW - GSU \#214WIW GSU \#407WIW

Fig. 3.2 - GSU daily water injection from six injectors

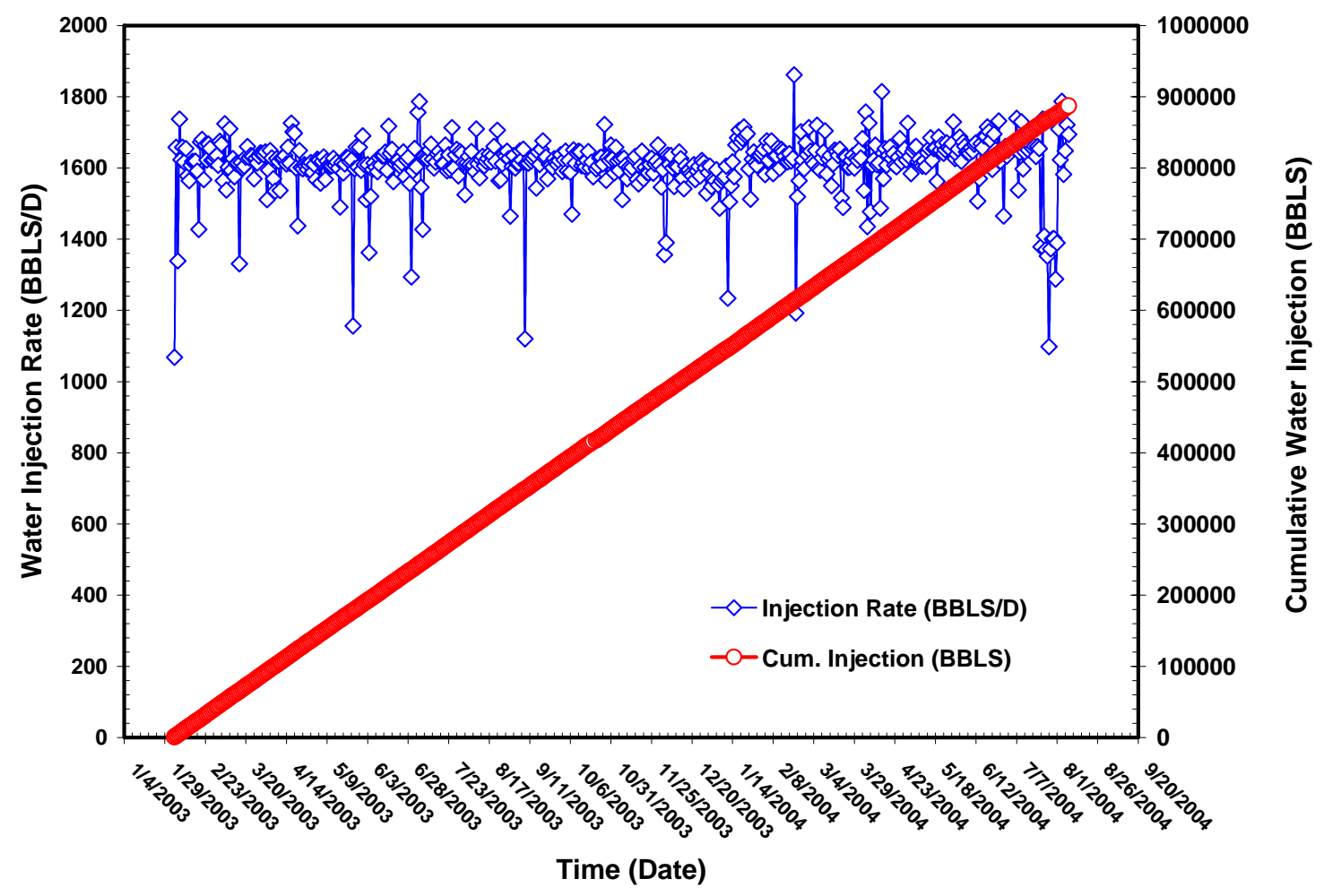

Fig. 3.3 -Total GSU water injection 


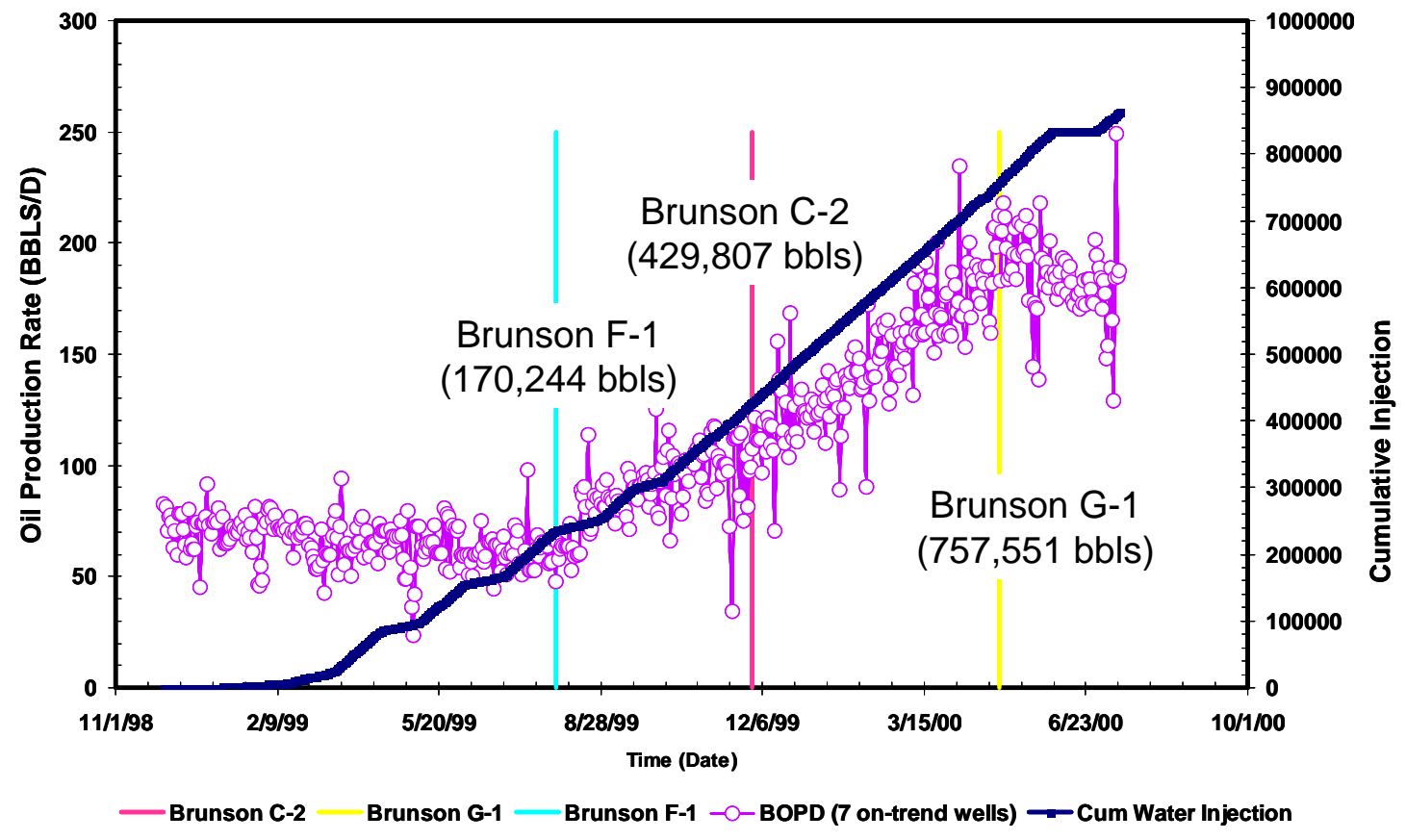

Fig. 3.4 - Volume of water injection until on-trend production wells start responding

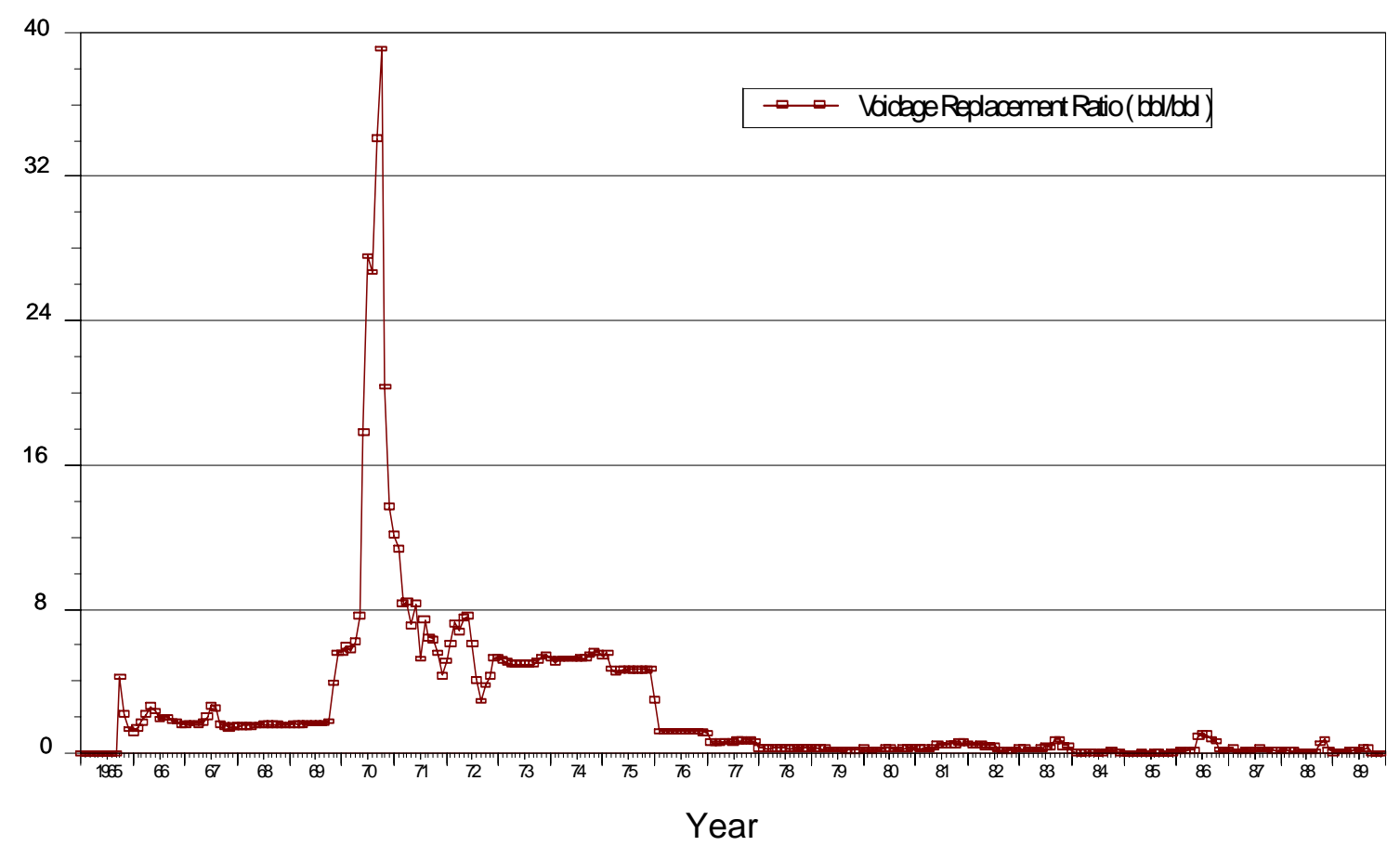

Fig. 3.5 - Voidage Replacement Ratio (1965-1989) 


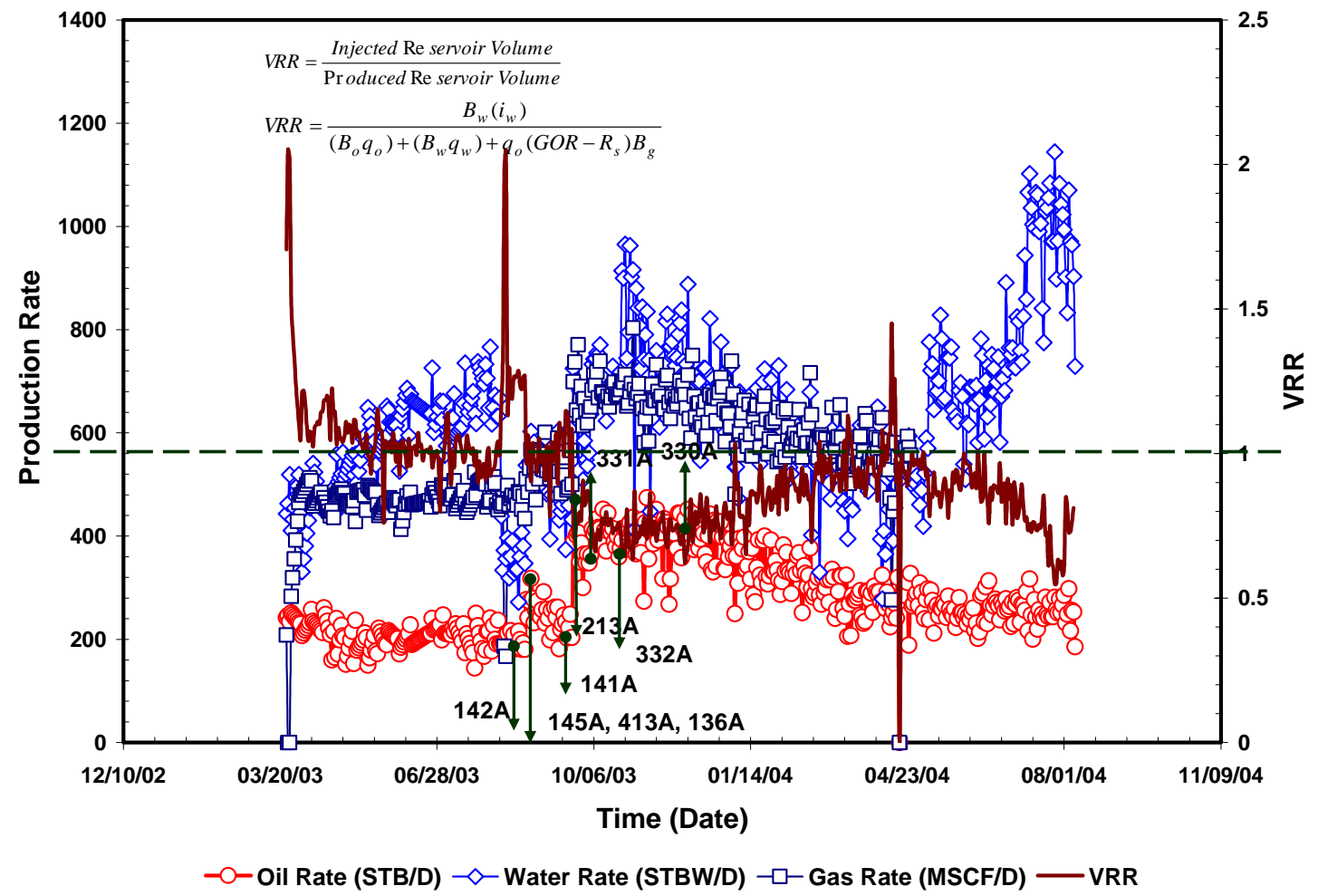

Fig. 3.6 - Voidage Replacement Ratio (VRR) in GSU

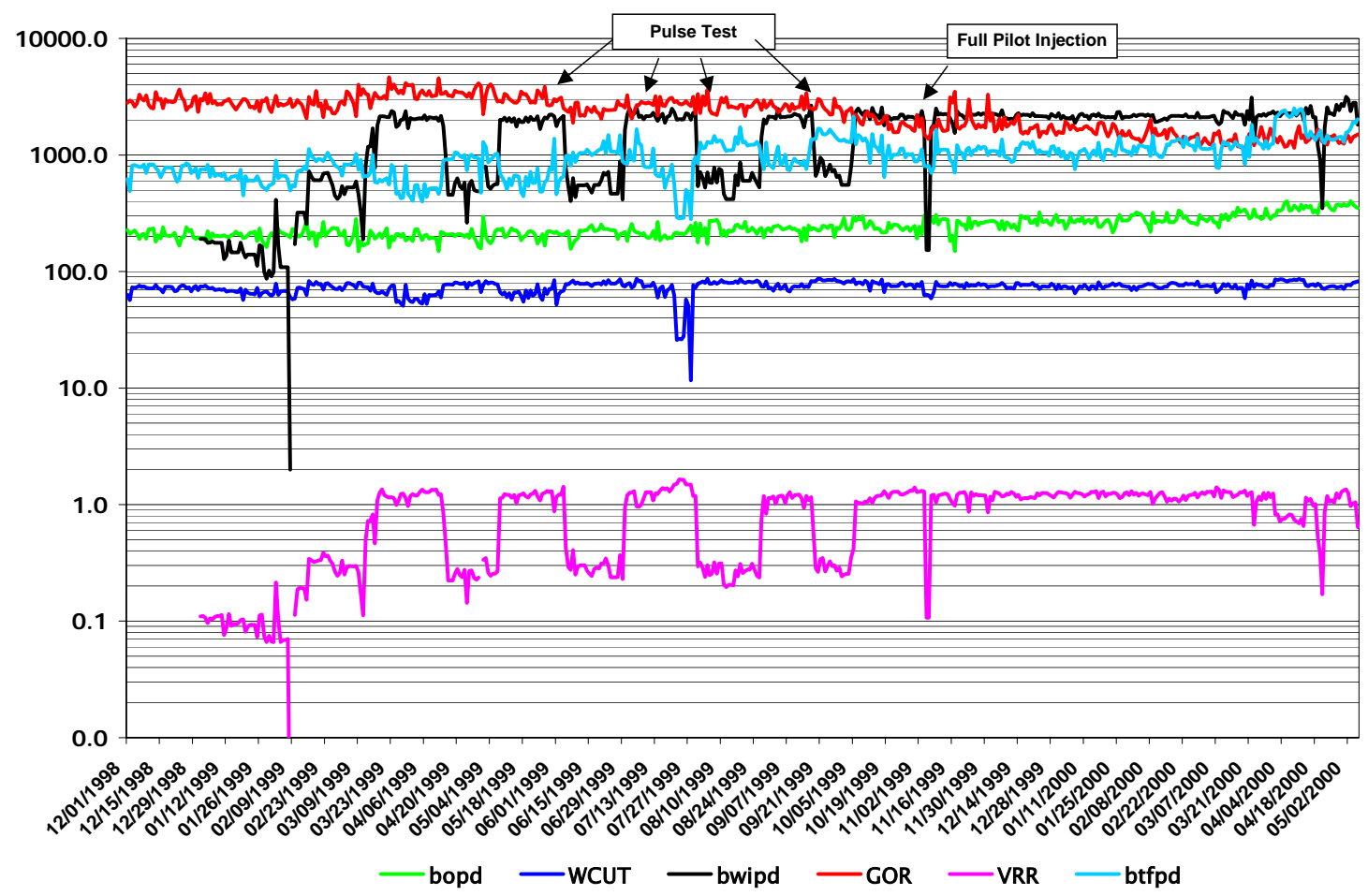

Fig. 3.7 - Voidage Replacement Ratio (VRR). in ET O’Daniel 


\section{GSU 406A}

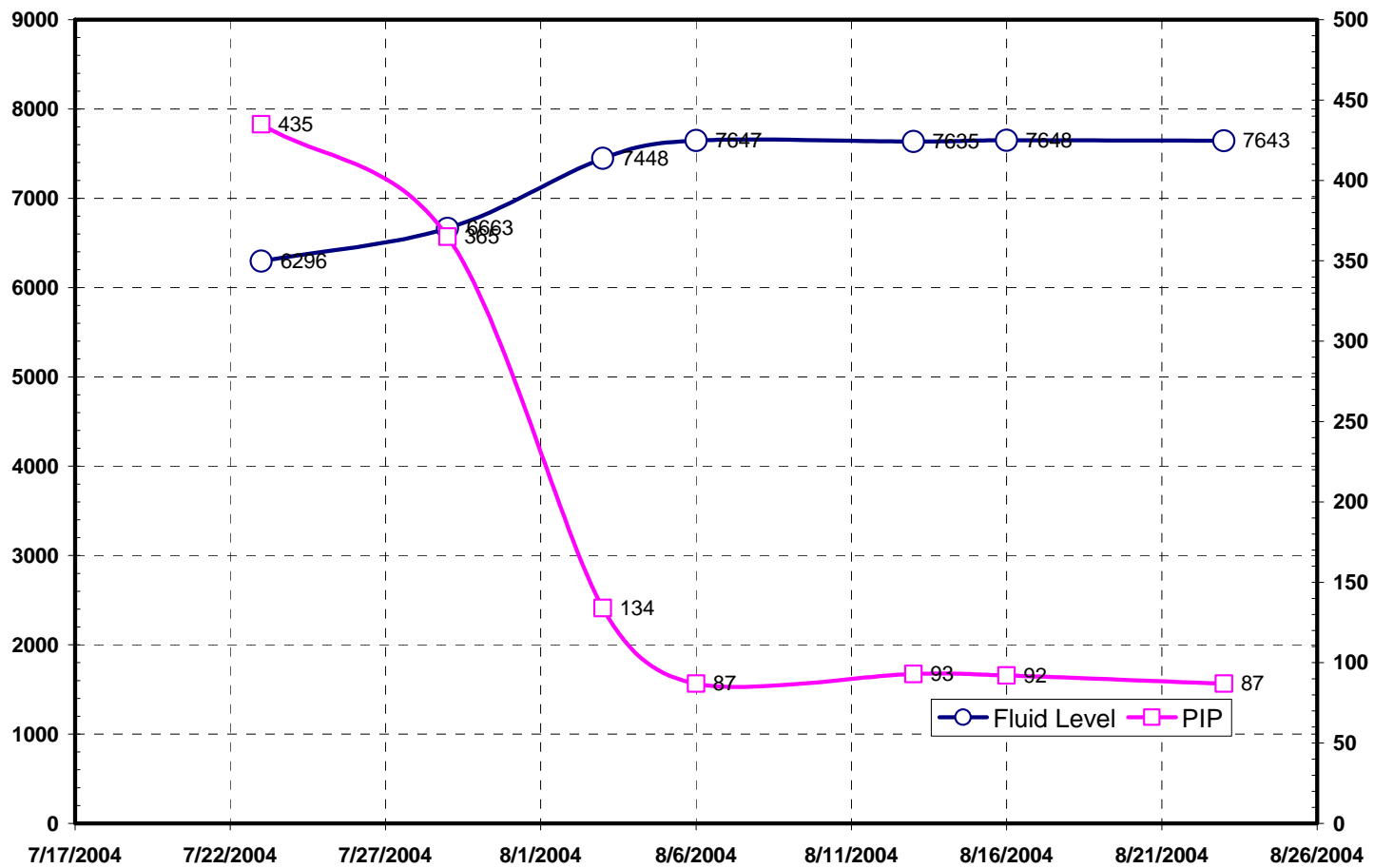

Fig. 3.8 - FL and PIP performances of GSU 406A polymer squeezed well.

\section{GSU 408A}

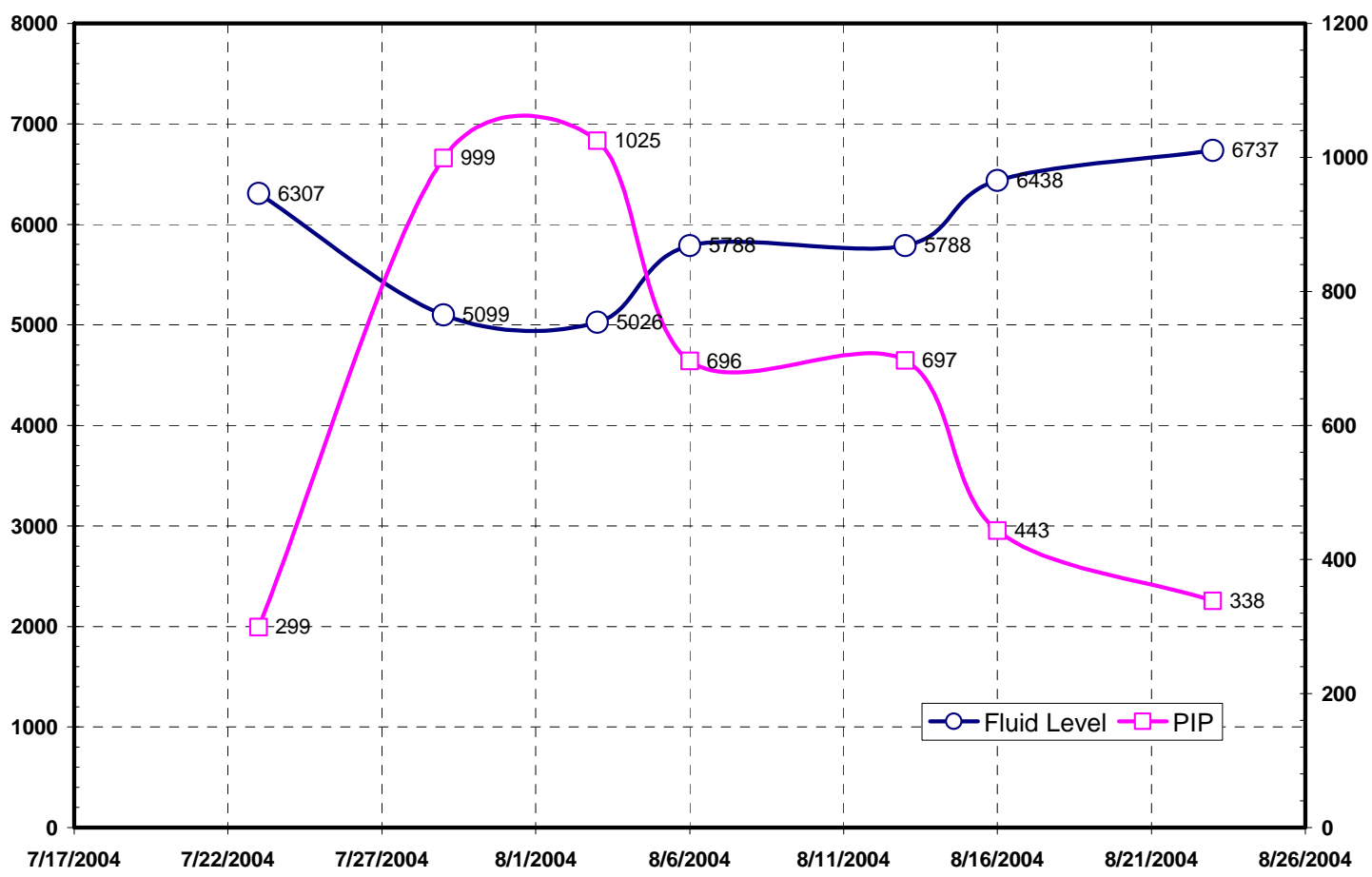

Fig. 3.9 - FL and PIP performances of GSU 408A polymer squeezed well. 


\section{GSU 409A}

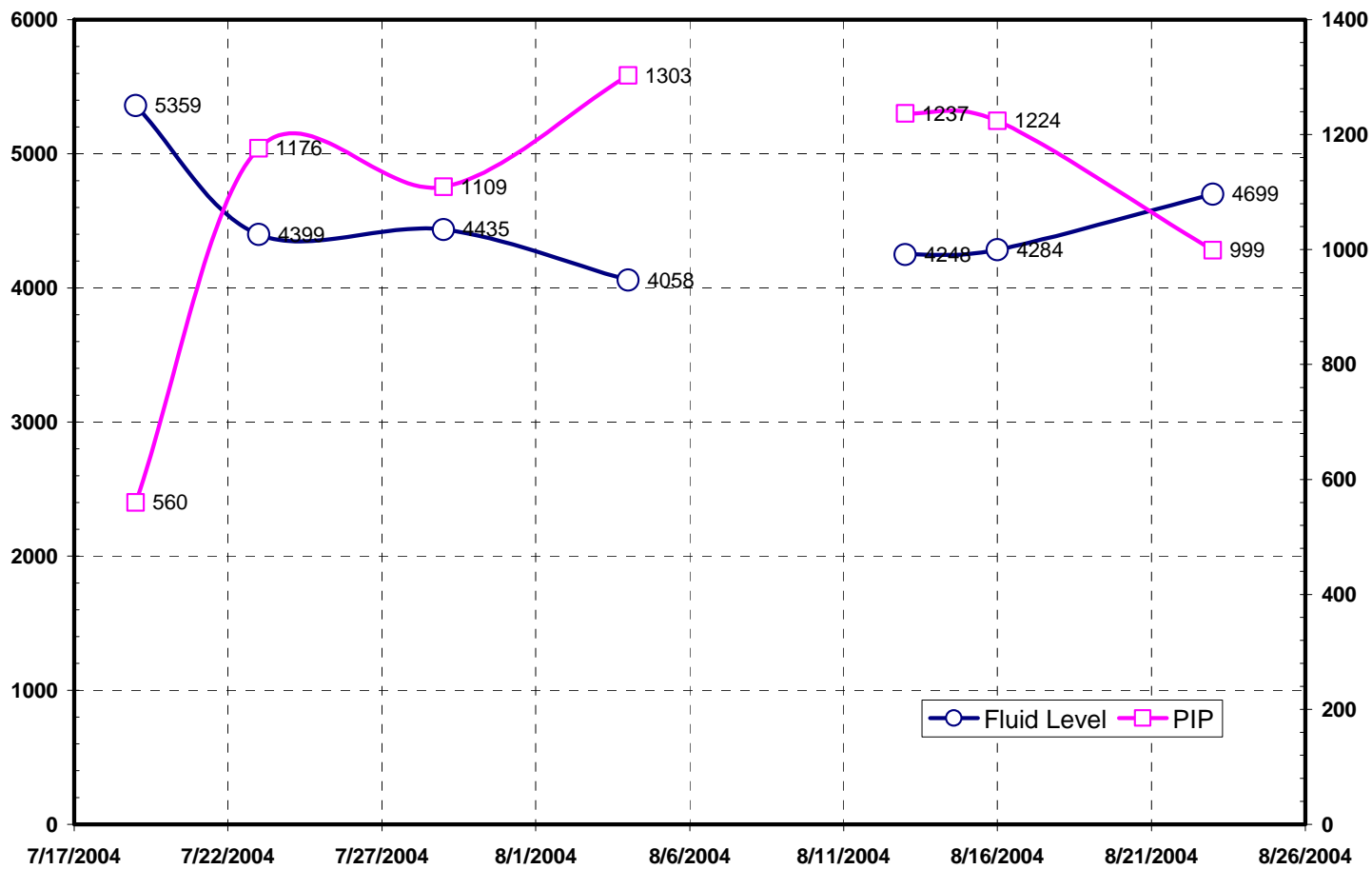

Fig. 3.10 - FL and PIP performances of GSU 409A polymer squeezed well.

\section{GSU 5}

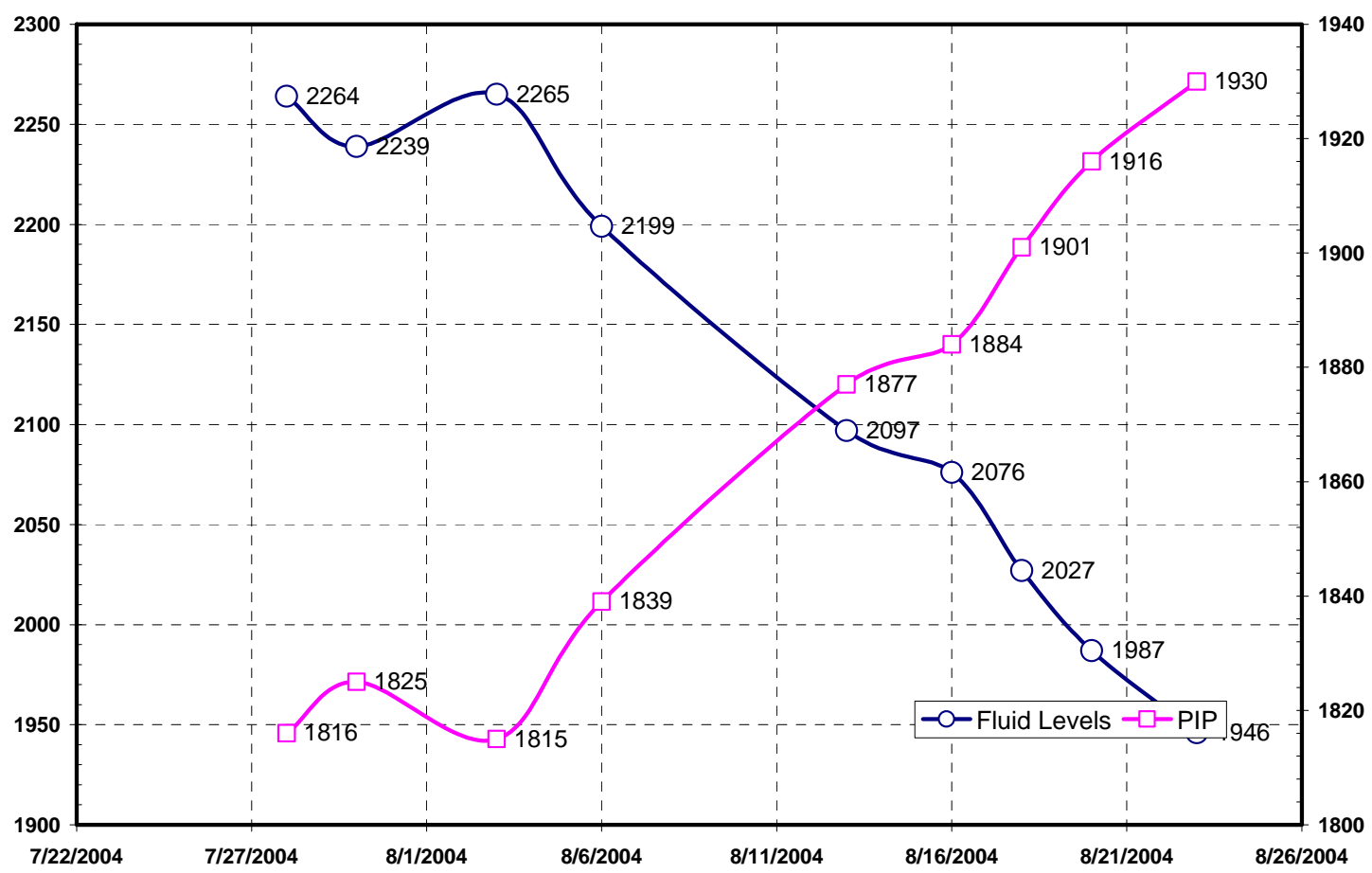

Fig. 3.11 - FL and PIP performances of GSU 5 observation well. 
GSU 20

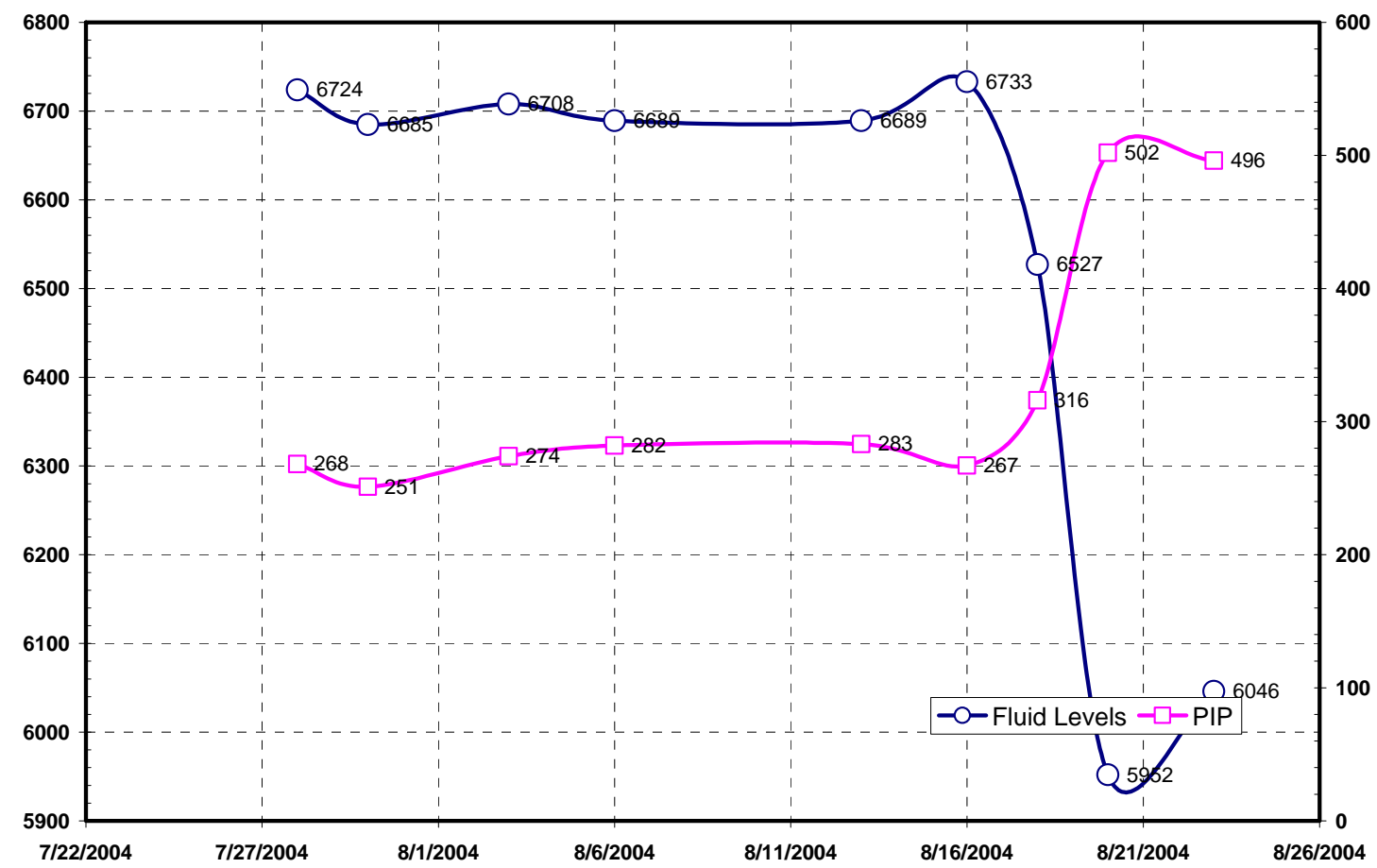

Fig. 3.12 - FL and PIP performances of GSU 20 observation well.

GSU 317

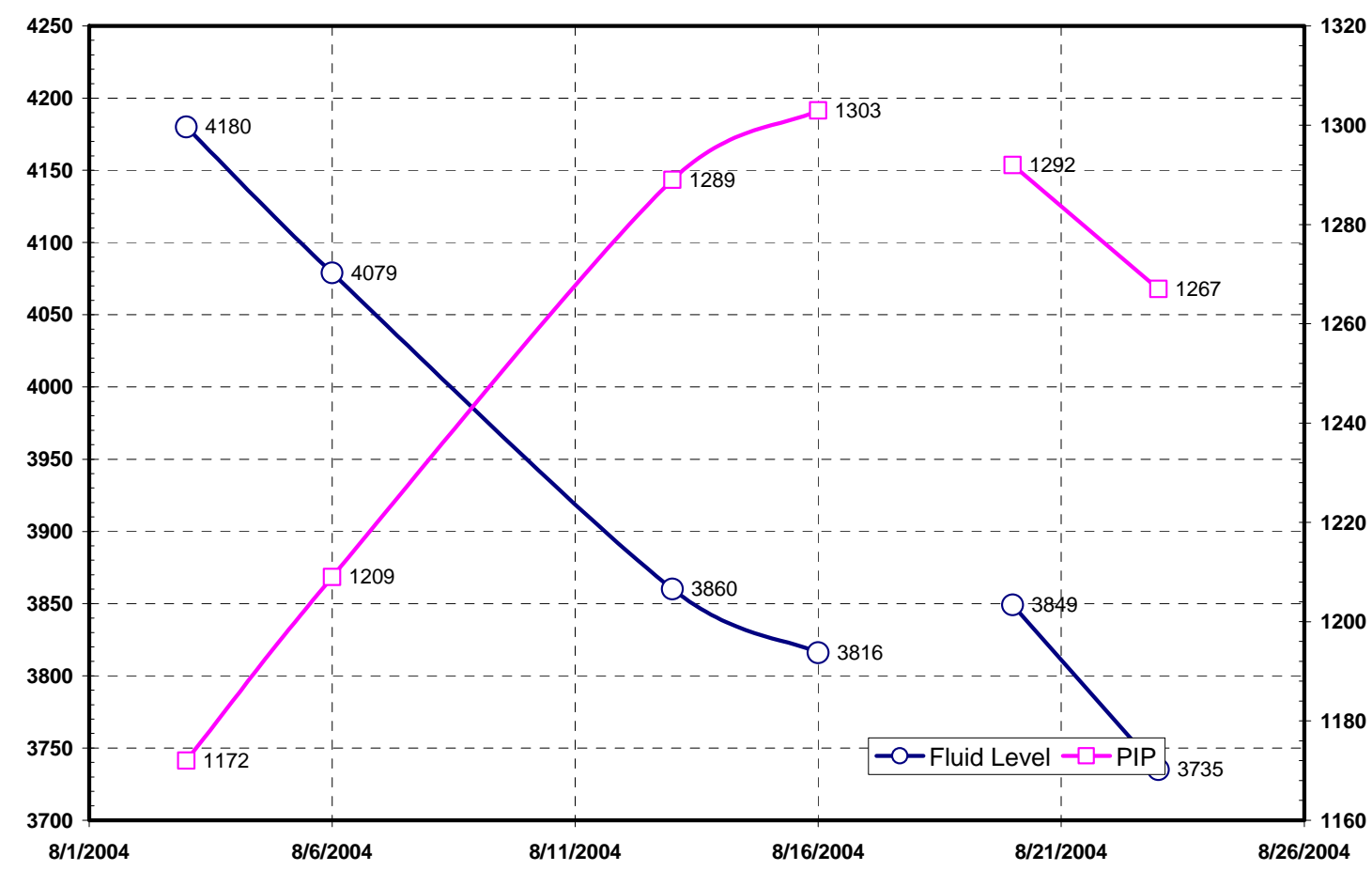

Fig. 3.13 - FL and PIP performances of GSU 20 observation well. 


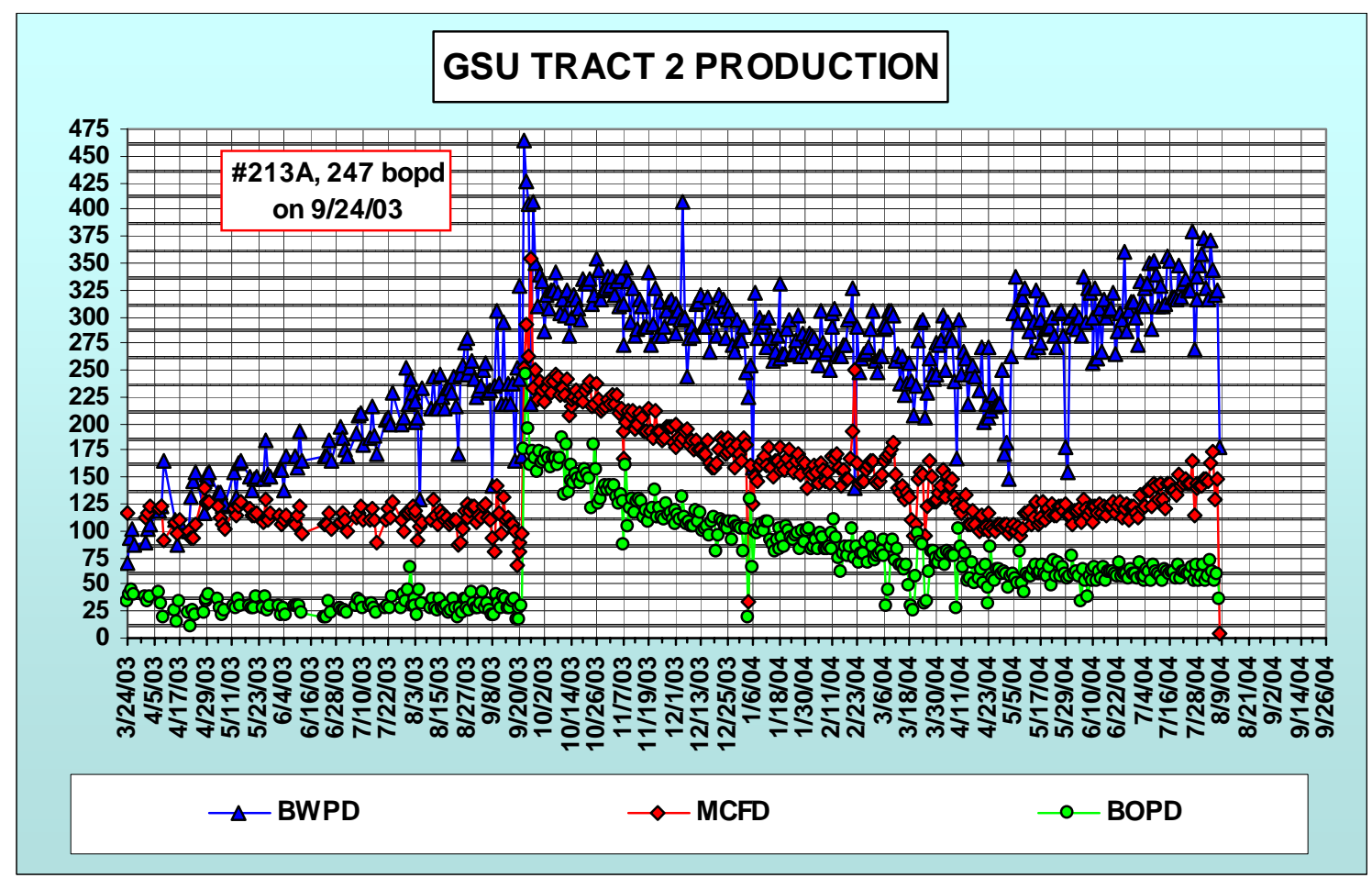

Fig. 3.14 - Production performance in Tract 2.

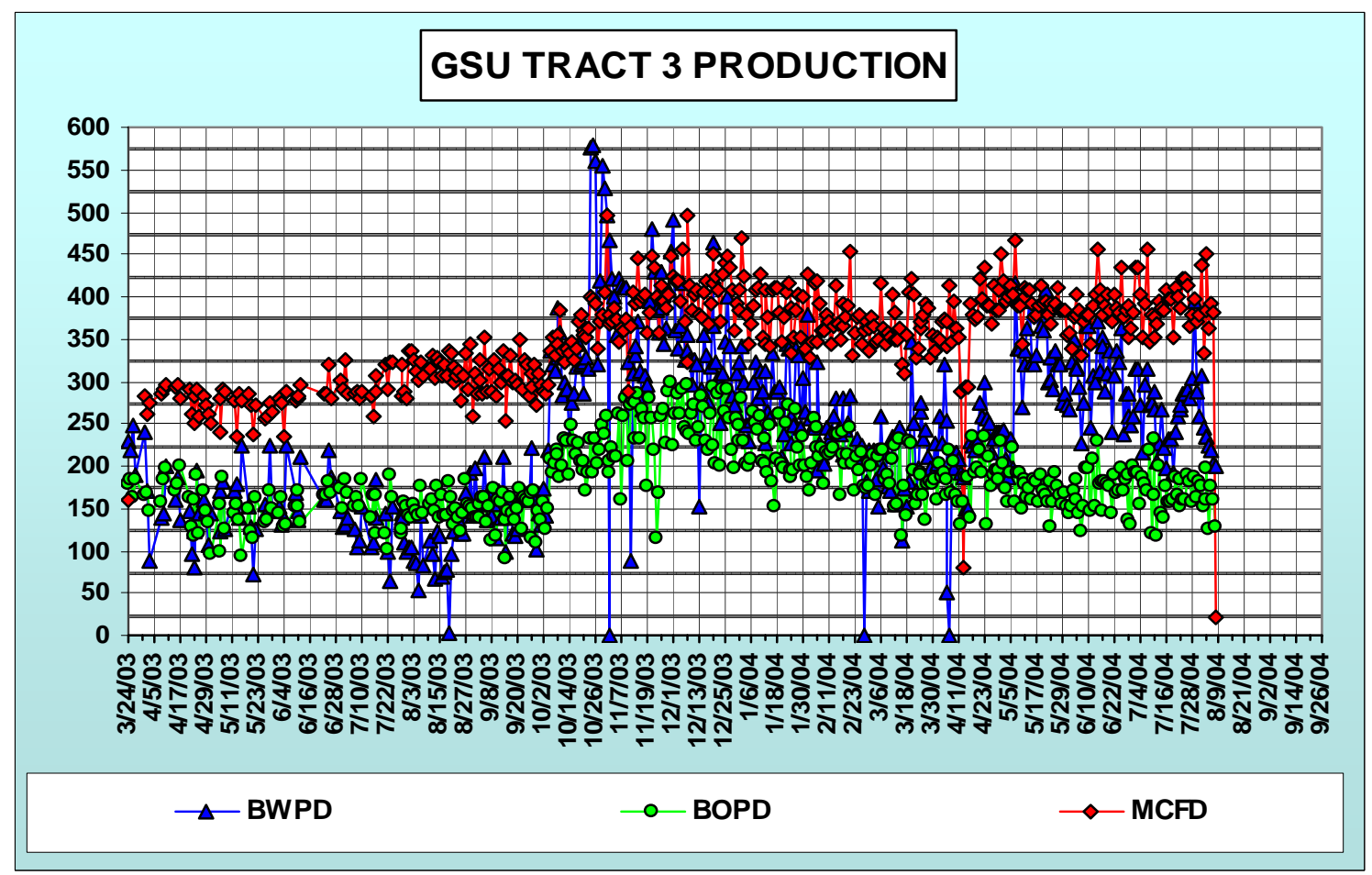

Fig. 3.15 - Production performance in Tract 3. 


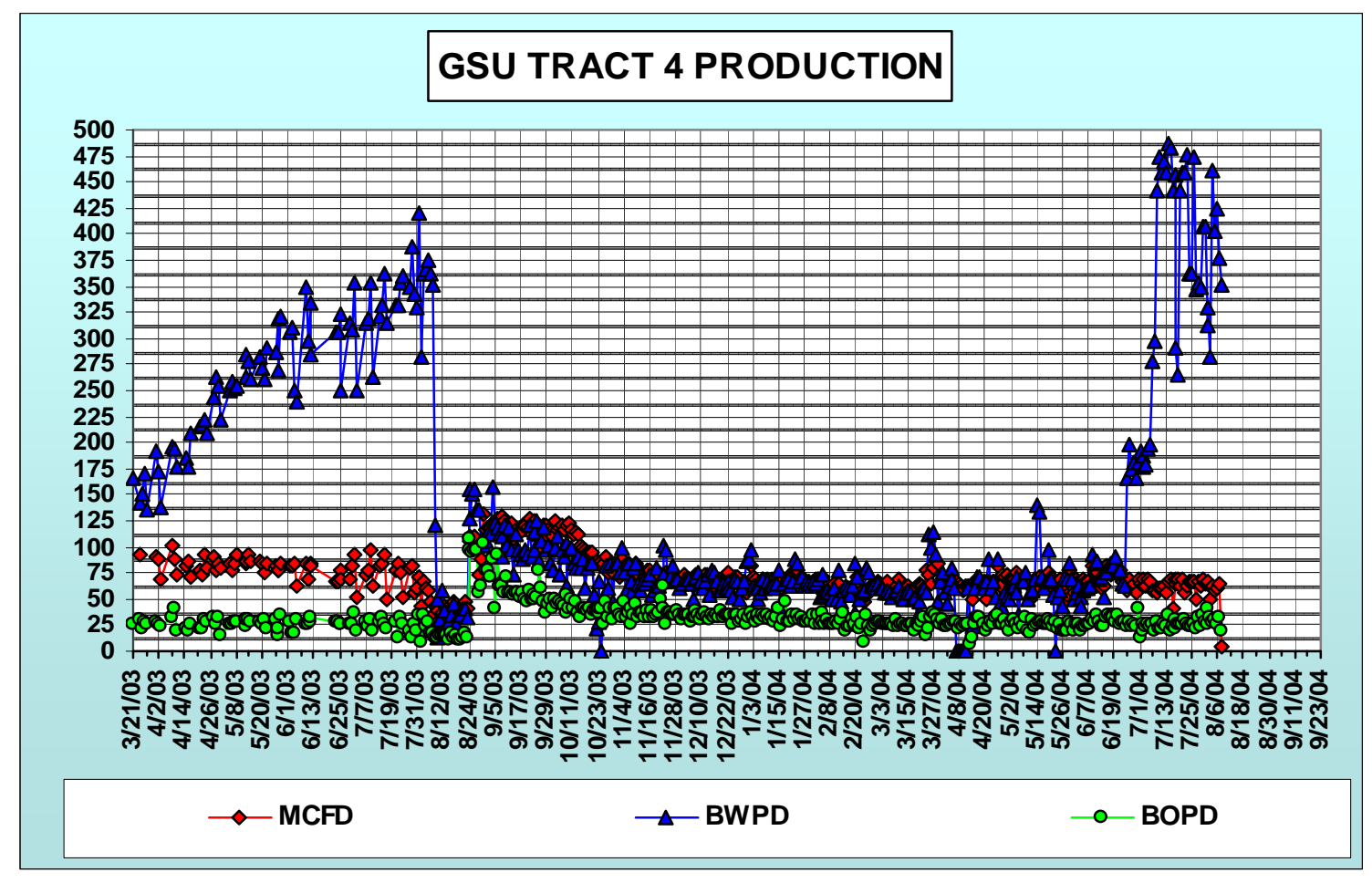

Fig. 3.16 - Production performance in Tract 4
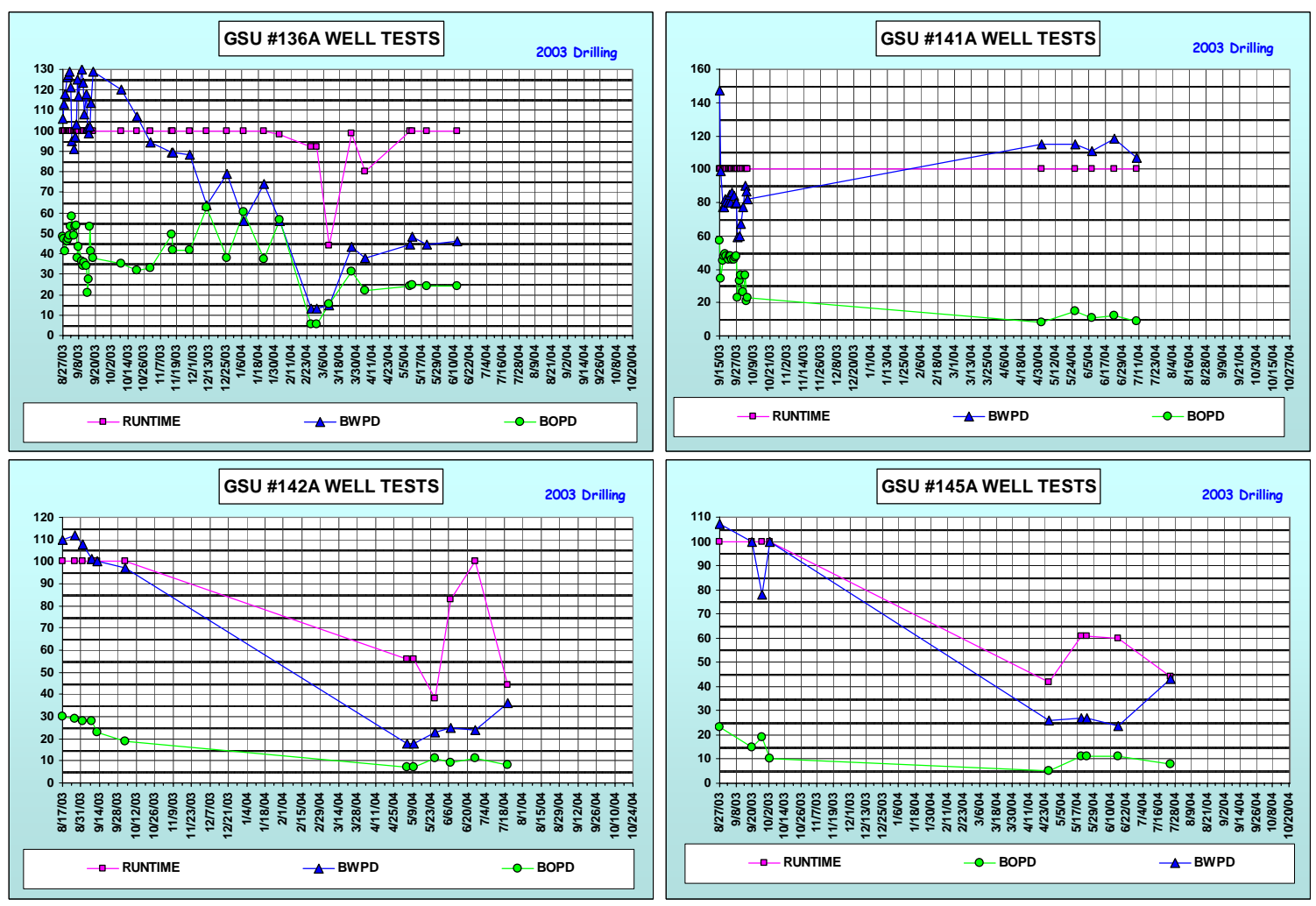

Fig. 3.17 - Performance of new wells in Tract 1 

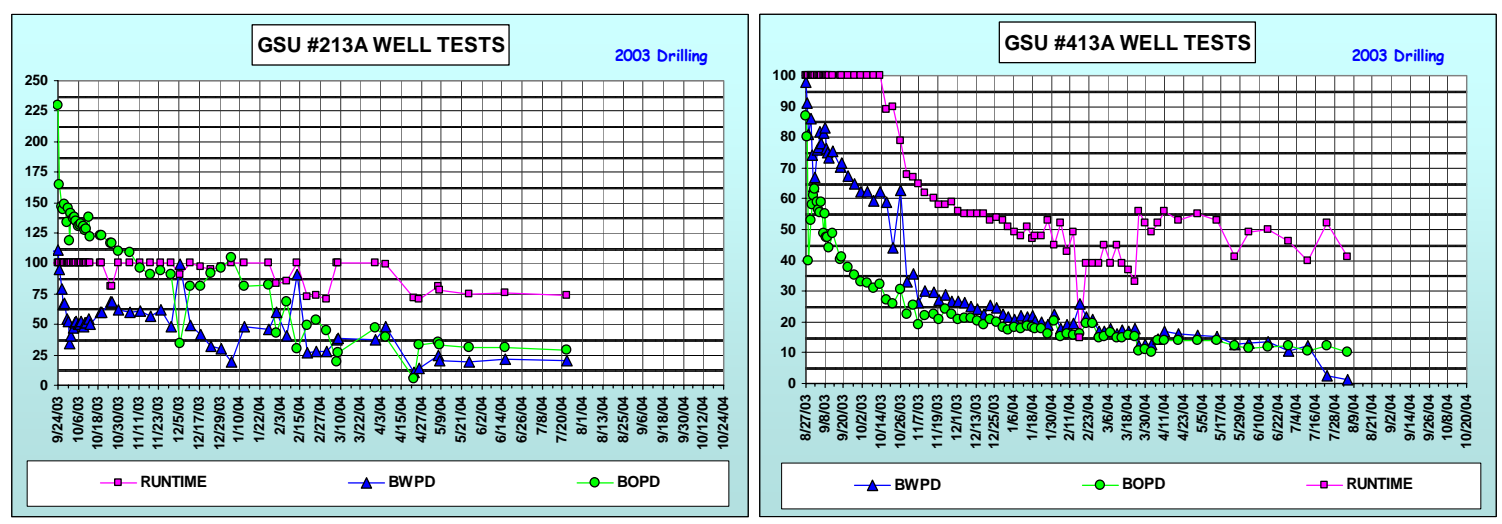

Fig. 3.18 - Performance of new wells in Tracts 2 and 4

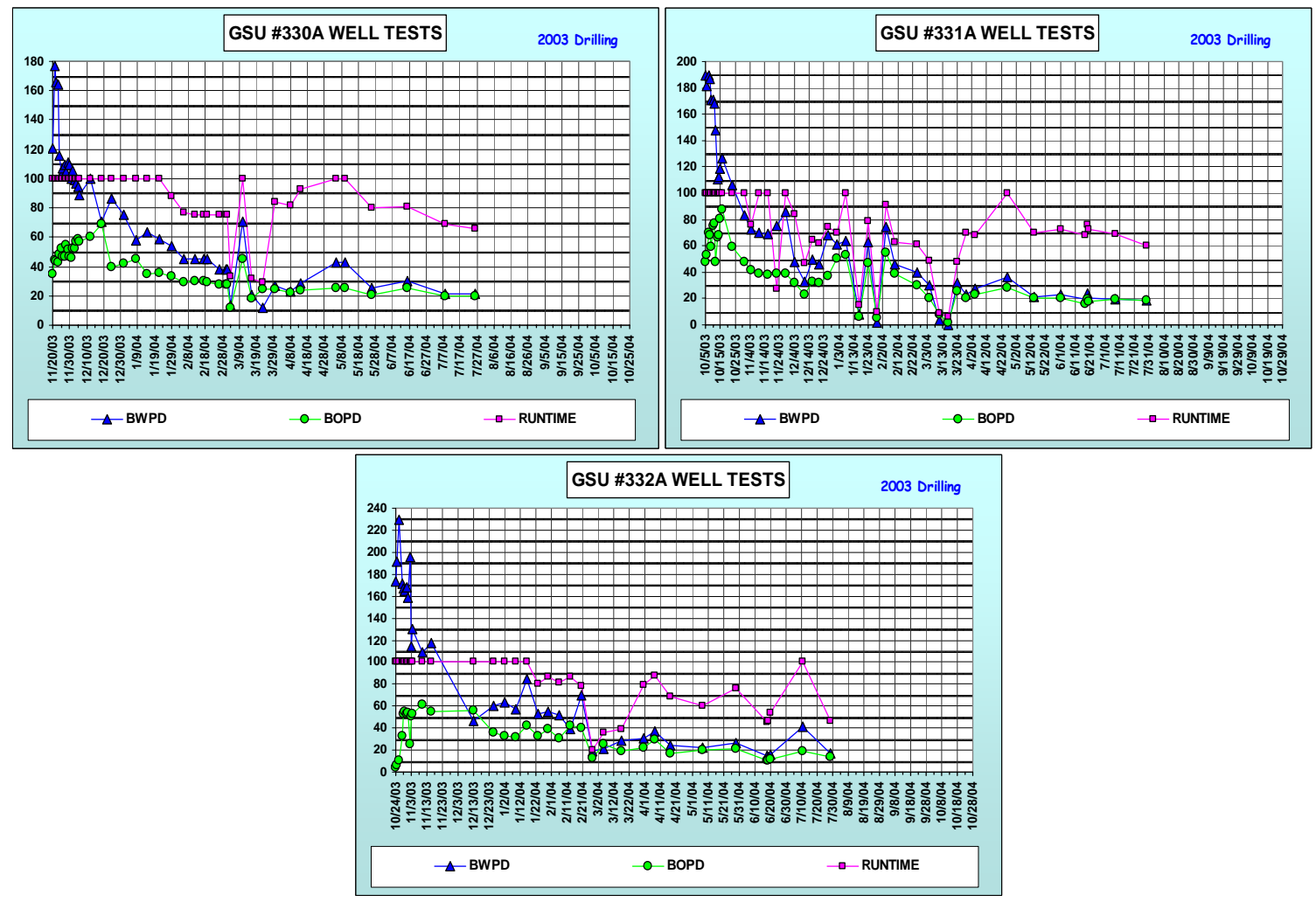

Fig. 3.19 - Performance of new wells in Tract 3 


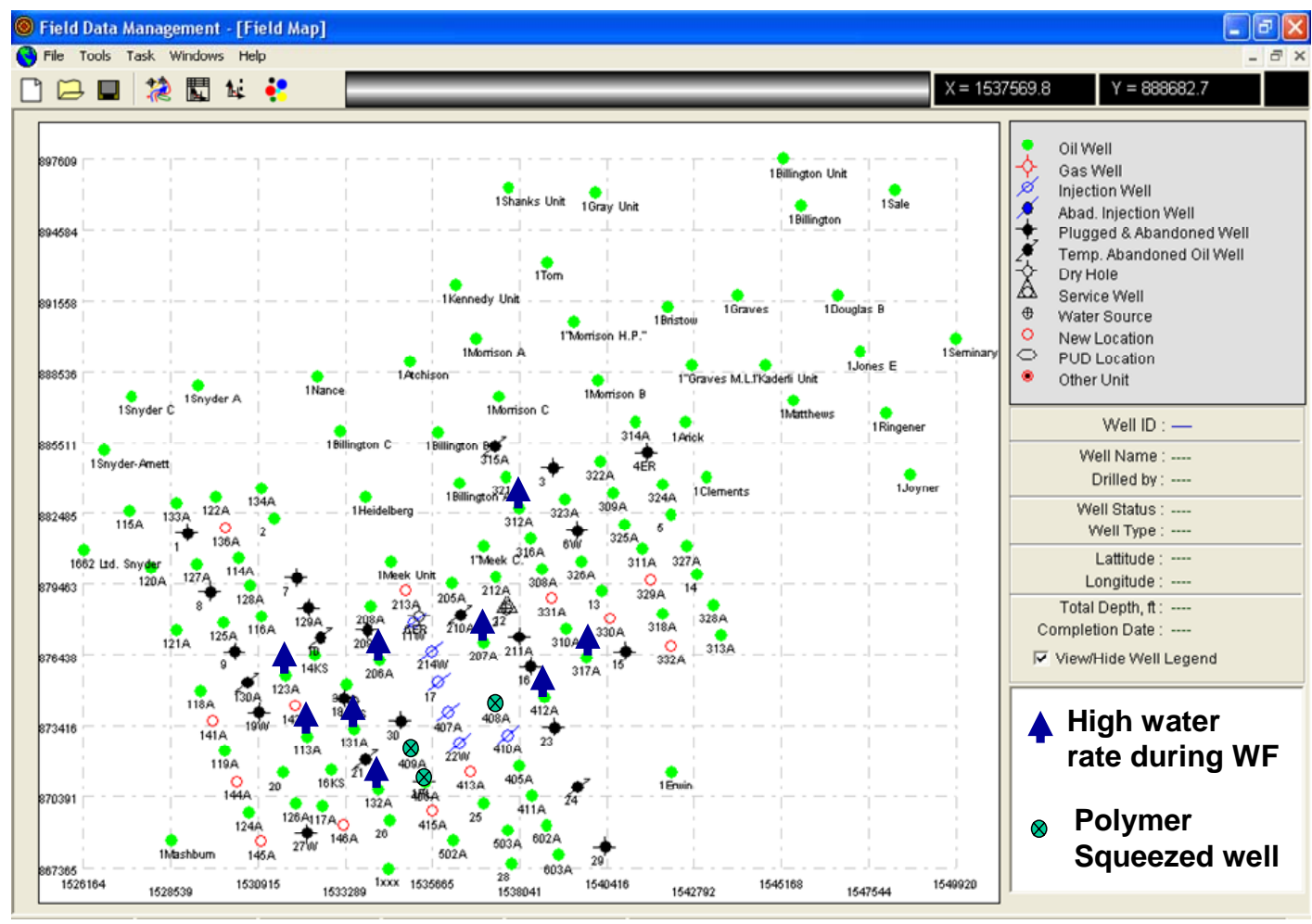

Fig. 3.20 - Wells experience high water production rate

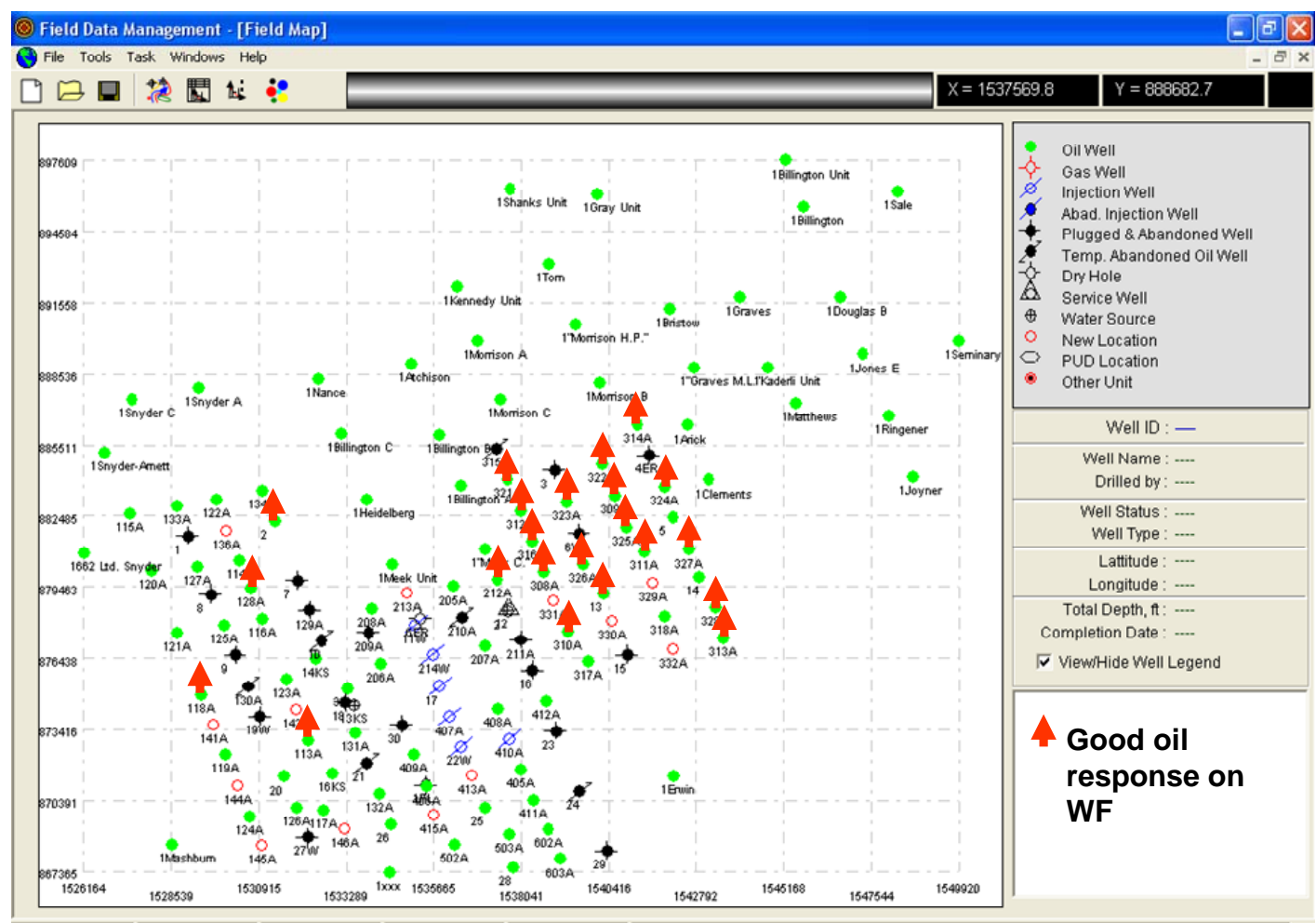

Fig. 3.21 - Wells have good oil responses 


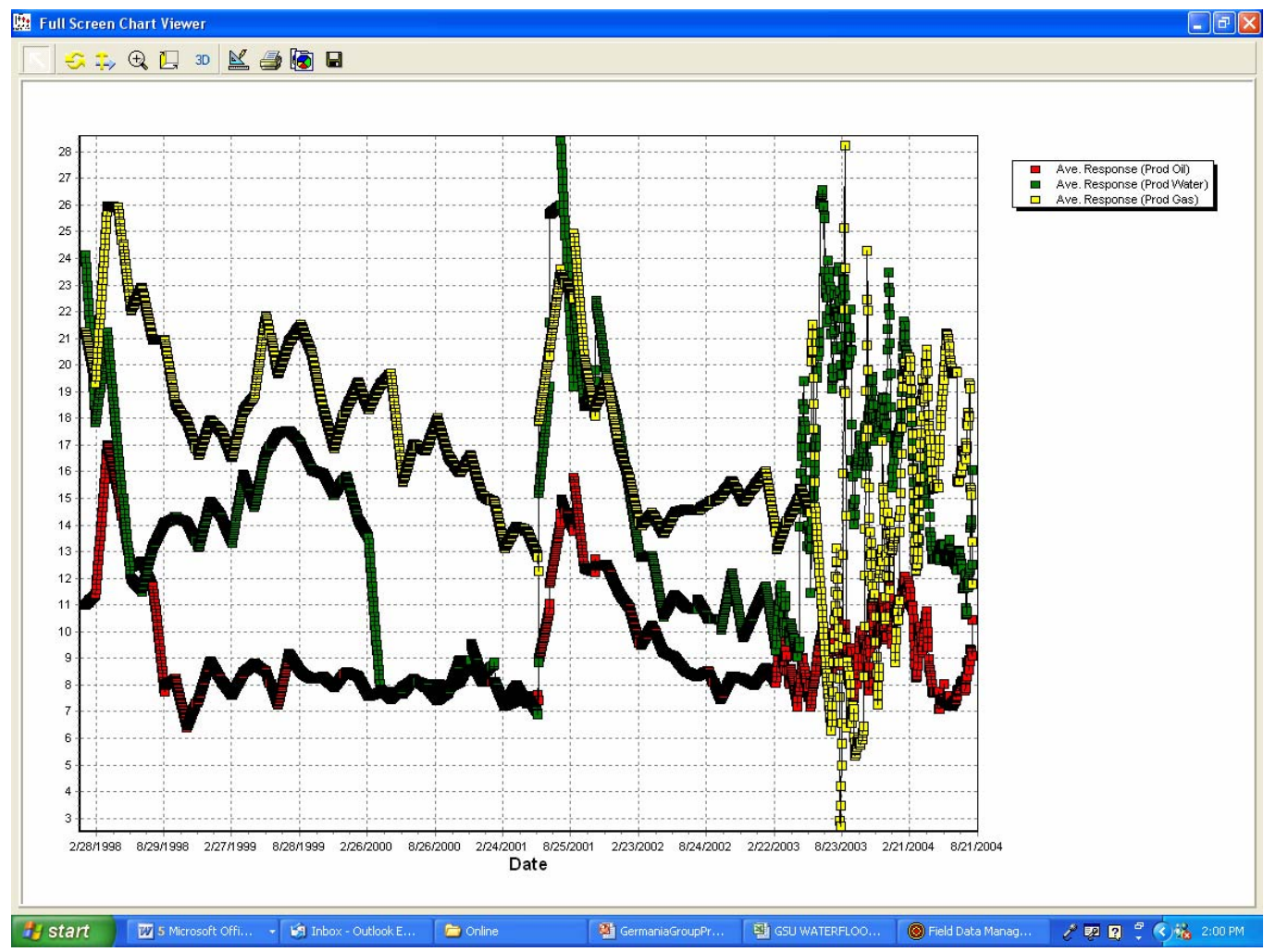

Fig. 3.22 - Average of production response

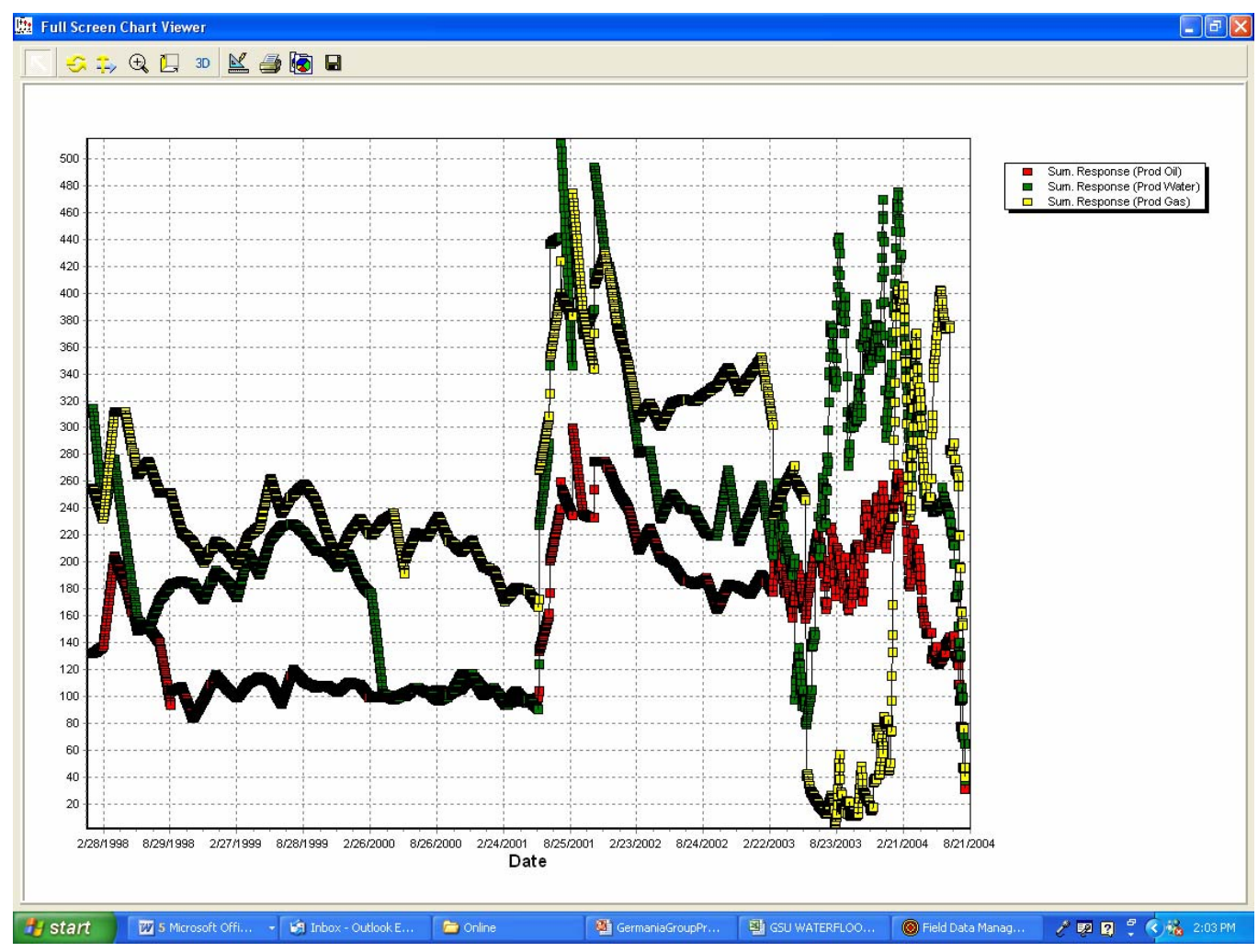

Fig. 3.23 - Total of production response 


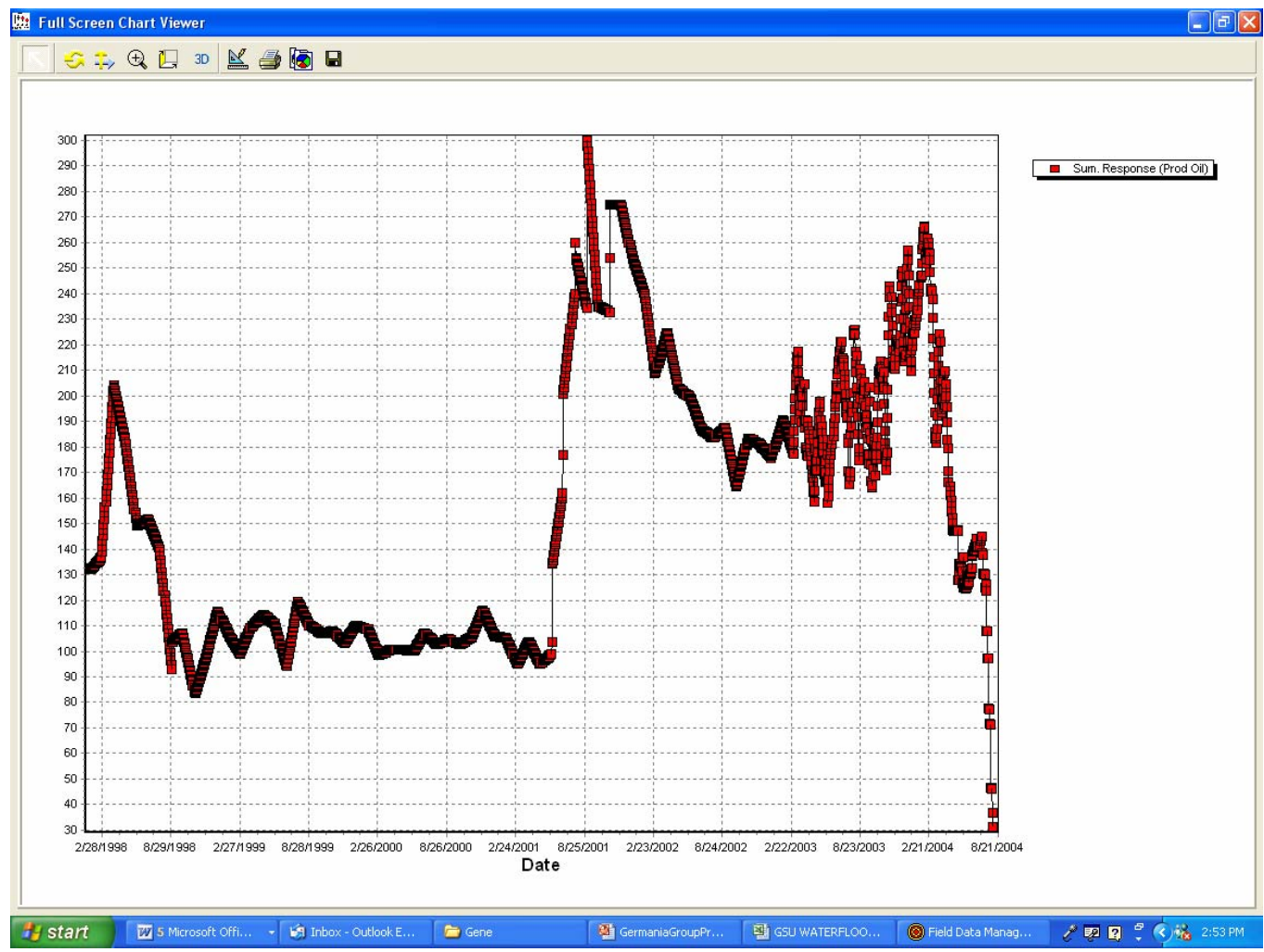

Fig. 3.24 - Total of oil production rate

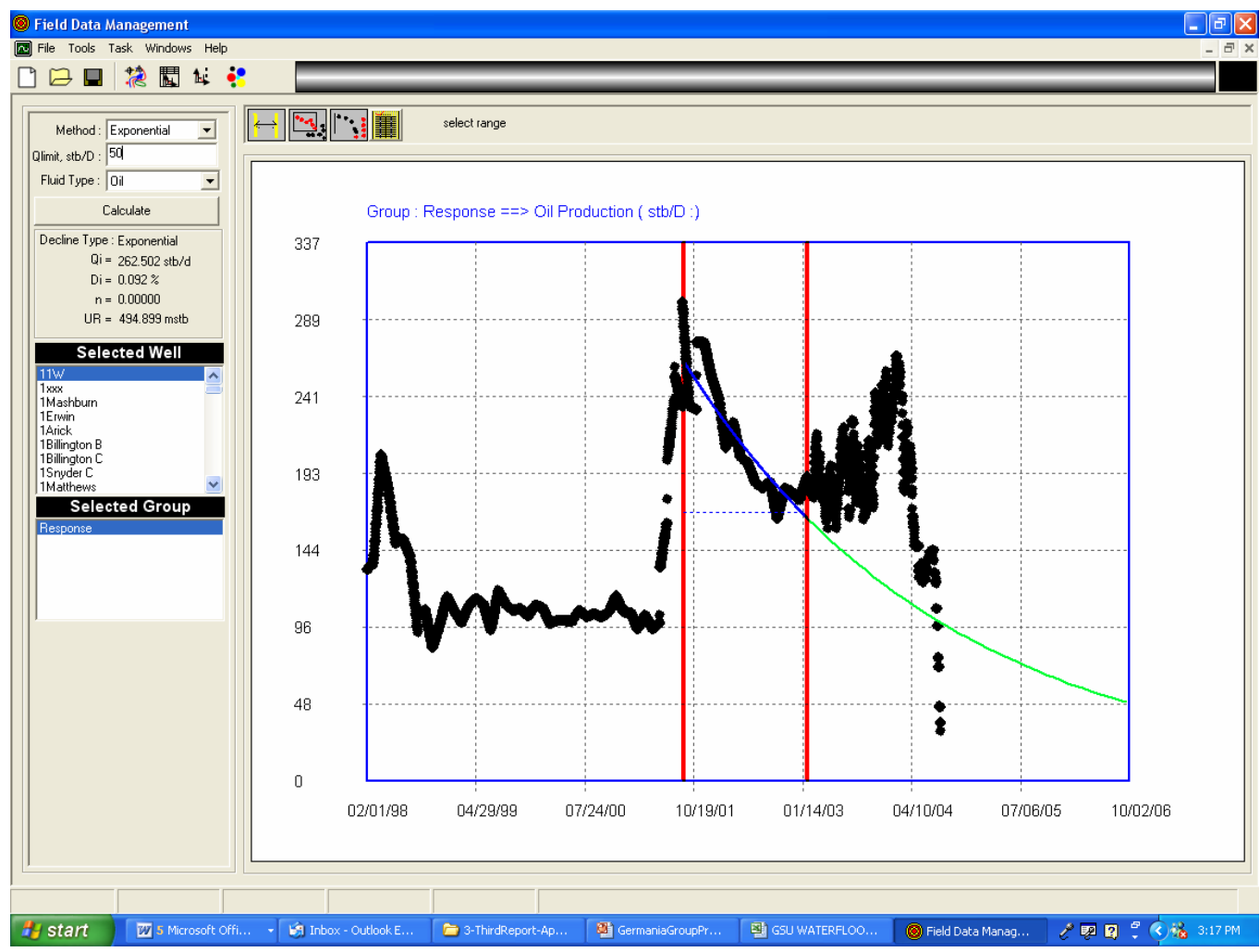

Fig. 3.25 - Decline curve analysis 


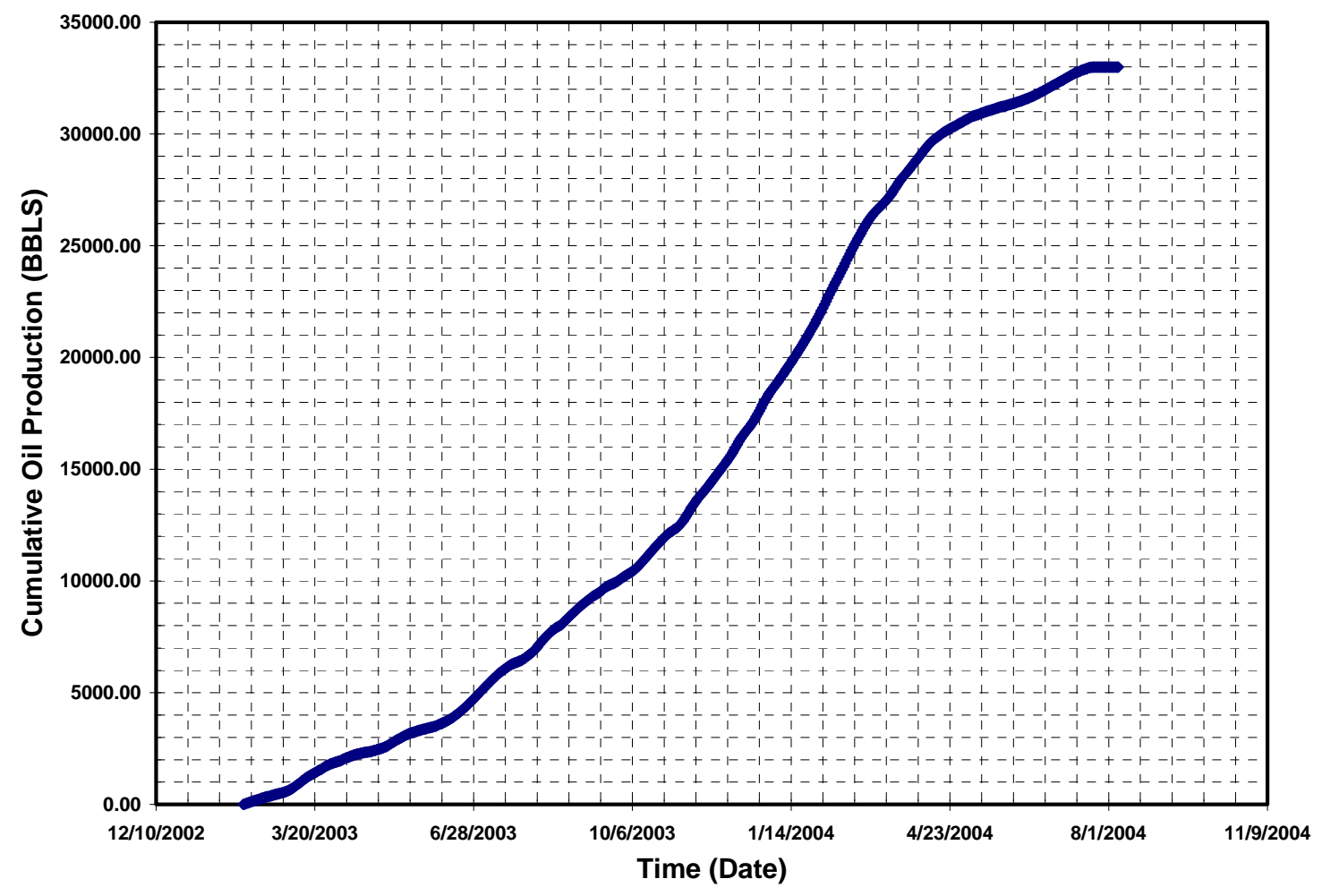

Fig. 3.26 - Cumulative incremental oil production 
Appendix A - Wells produce high water rate during water injection in GSU waterflood pilot
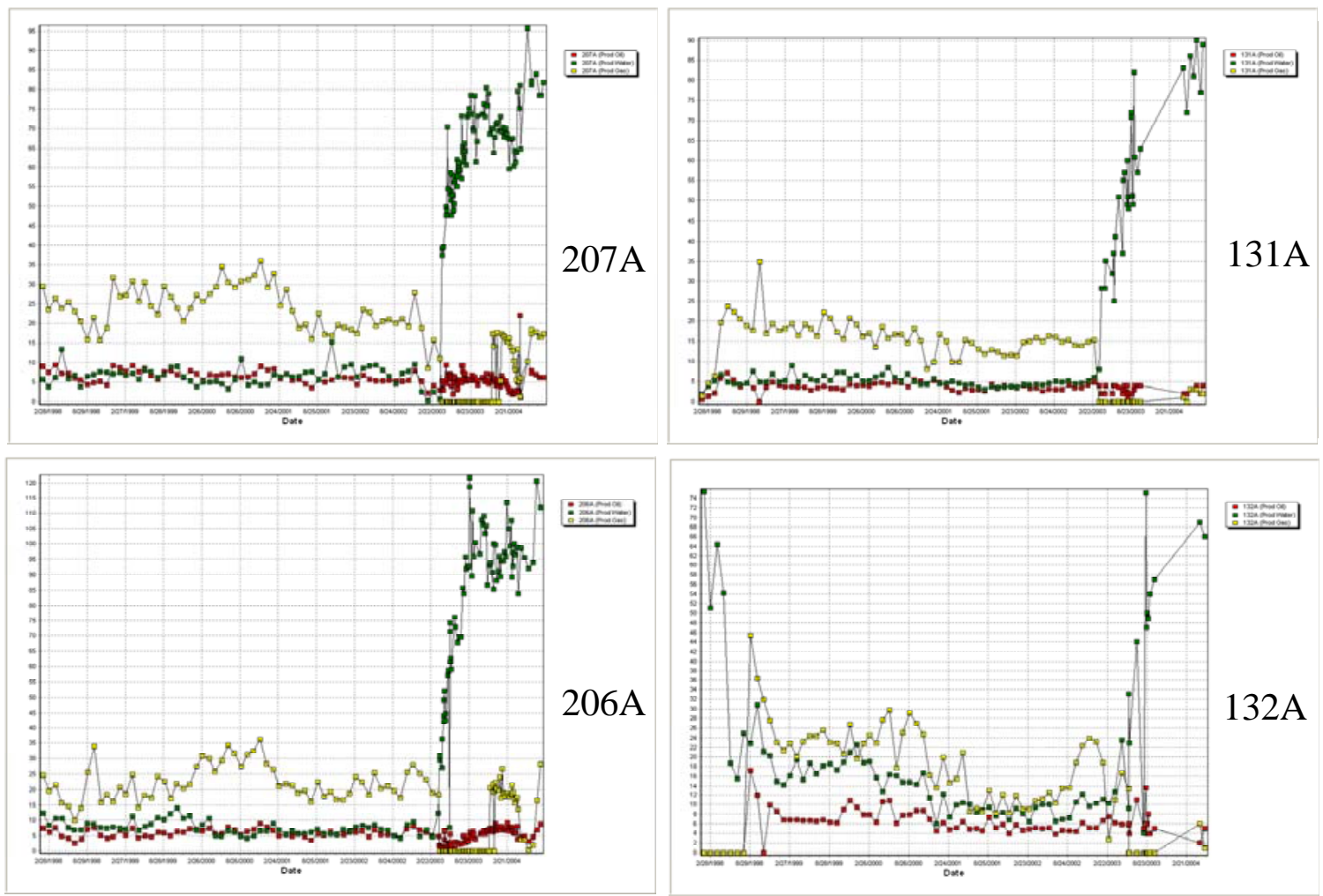

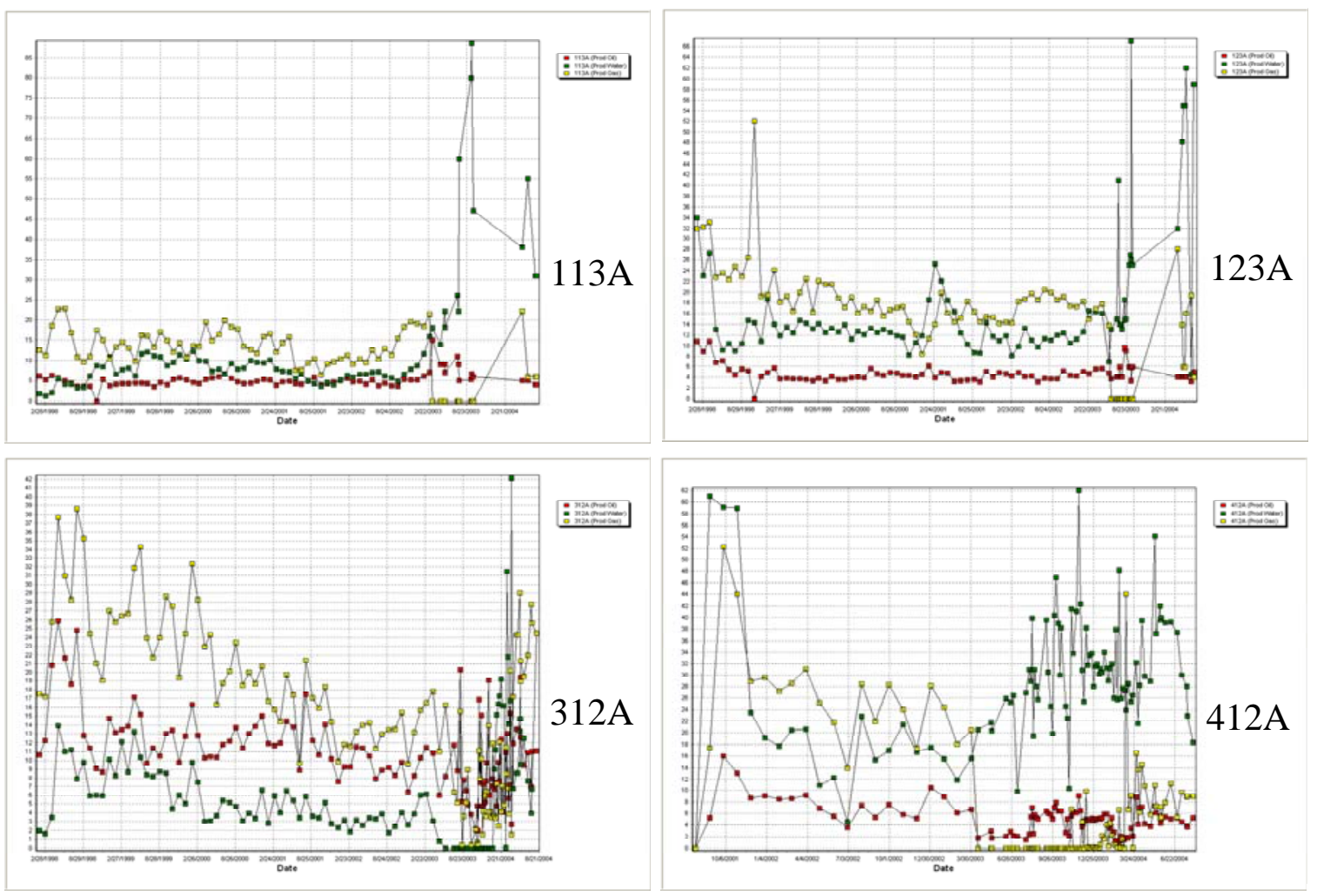
Appendix B - Wells that have oil response during water injection in GSU waterflood pilot
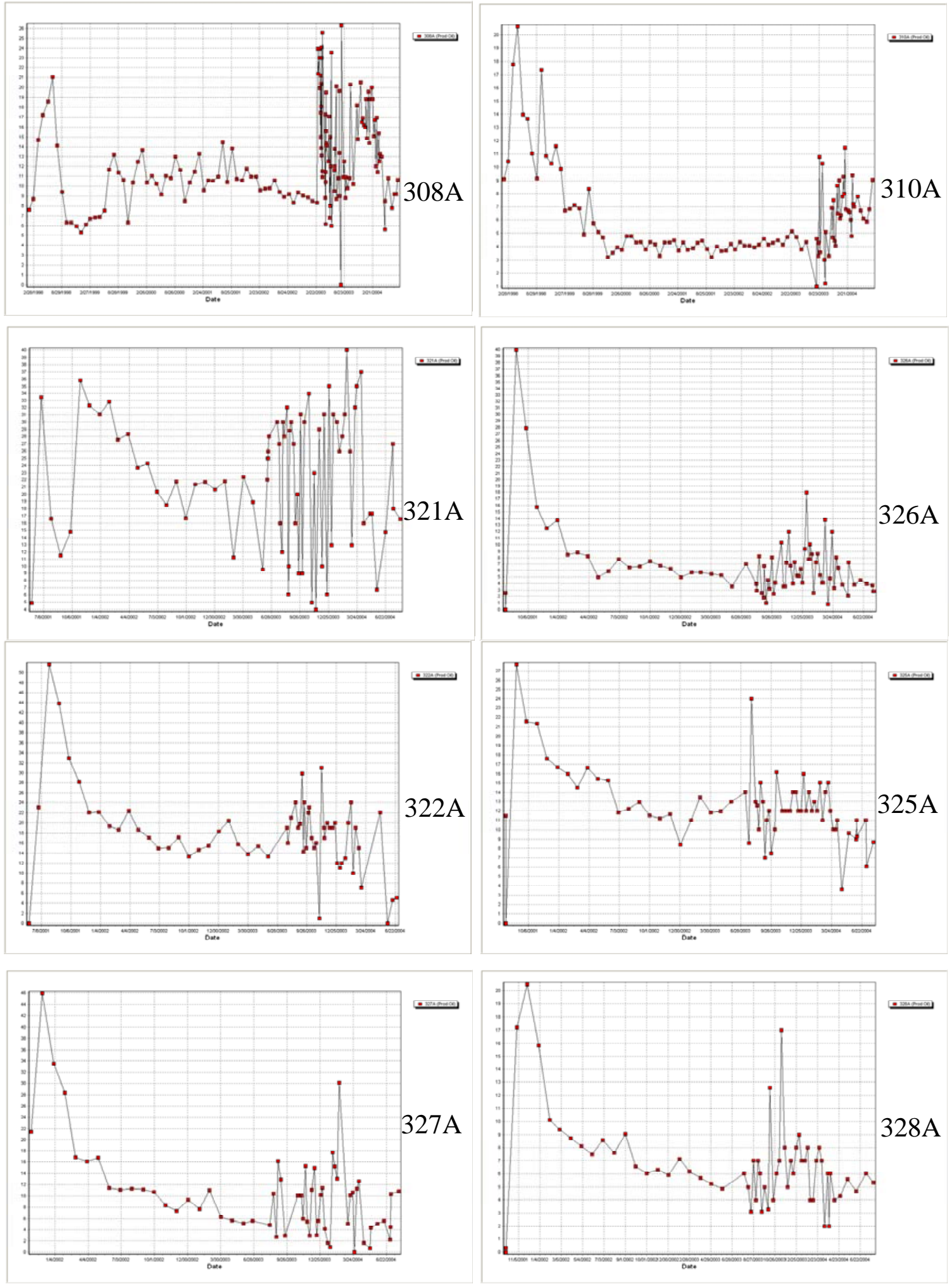

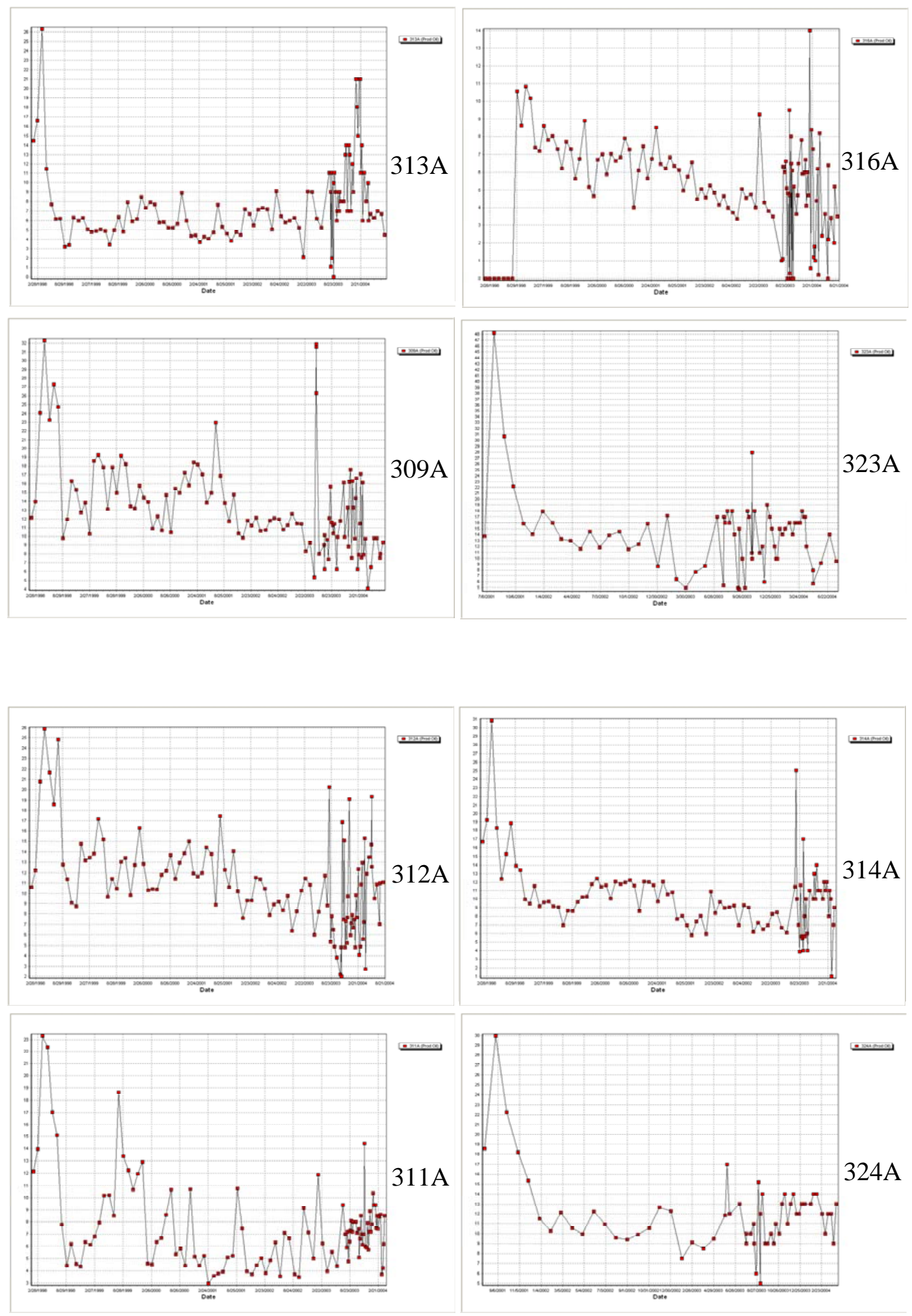

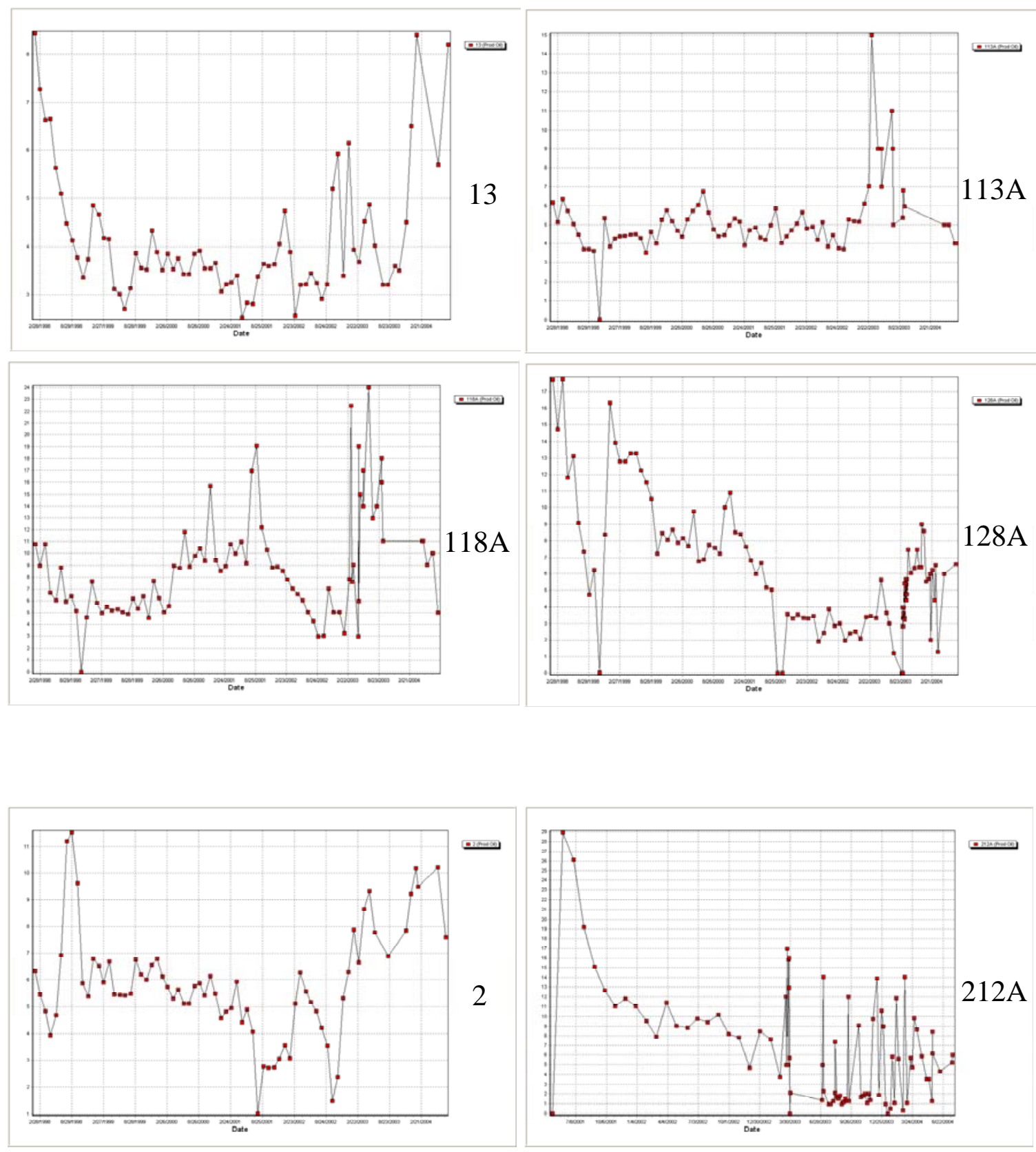
CONTRACT TITLE: Preferred Waterflood Management Practices for the Spraberry Trend Area - PUMP Breakout

\begin{tabular}{|c|c|c|c|c|}
\hline \multicolumn{2}{|c|}{$\begin{array}{l}\text { ID NUMBER: DE-FC26-01BC15274 } \\
\text { B\&R CODE: AC1005000 }\end{array}$} & \multicolumn{3}{|c|}{$\begin{array}{l}\text { CONTRACTOR: Texas Engineering Experiment Station } \\
\text { ADDR: } 322 \text { Wisenbaker Engineering Research Center } \\
\text { College Station, TX } 77843\end{array}$} \\
\hline \multicolumn{2}{|c|}{ DOE PROJECT MANAGER: } & \multicolumn{3}{|c|}{ CONTRACT PROJECT MANAGER: } \\
\hline \multirow{2}{*}{\multicolumn{2}{|c|}{ NAME: Daniel J. Ferguson }} & \multicolumn{3}{|c|}{ NAME: David Schechter } \\
\hline & LOCATION: NPTO & \multicolumn{3}{|c|}{ PHONE: 979/ 845-2275 } \\
\hline \multicolumn{2}{|c|}{ PHONE: 918/ 699-2047 } & \multicolumn{3}{|c|}{ FAX: 979/845-1307 } \\
\hline \multicolumn{2}{|c|}{ E-MAIL: dan.ferguson@npto.doe.gov } & \multicolumn{3}{|c|}{ E-MAIL: schech@spindletop.tamu.edu } \\
\hline $\begin{array}{l}\text { PROJECT SITE } \\
\text { CITY: College Station } \\
\text { CITY: } \\
\text { CITY: }\end{array}$ & $\begin{array}{l}\text { STATE: TX } \\
\text { STATE: } \\
\text { STATE: }\end{array}$ & $\begin{array}{l}\text { PROGRAM: } \\
\text { RESEARCH } \\
\text { PRODUCT I }\end{array}$ & $\begin{array}{l}\text { MANCE PER } \\
\text { Life Extension } \\
\text { UMP } \\
\end{array}$ & \\
\hline \multicolumn{5}{|l|}{ CO-PARTICIPANTS: } \\
\hline \multicolumn{2}{|c|}{ PERFORMER: Pioneer Natural Resources } & CITY: Irving & STATE: TX & CD: \\
\hline \multicolumn{2}{|c|}{ PERFORMER: } & CITY: & STATE: & CD: \\
\hline \multicolumn{2}{|l|}{ PERFORMER: } & CITY: & STATE: & CD: \\
\hline \multicolumn{2}{|l|}{ PERFORMER: } & CITY: & STATE: & CD: \\
\hline
\end{tabular}

\begin{tabular}{|l|r|r|r|}
\hline \multicolumn{1}{|c|}{ FUNDING (1000'S) } & DOE & \multicolumn{2}{c|}{ CONTRACTOR } \\
\hline PRIOR FISCAL YRS & 0 & 0 & 0 \\
FY 2001 CURRENT OBLIGATIONS & 500 & 1567 & 2067 \\
FUTURE FUNDS & 5 & 0 & 5 \\
\hline TOTAL EST'D FUNDS & 505 & 1567 & 2072 \\
\hline
\end{tabular}

OBJECTIVE: The objective of this project is to design and test different waterflood techniques that have never been utilized in the Spraberry Trend Area. The new waterfloods will align injection wells along the fracture trend with production wells. New injection wells will be drilled that will not be artificially fractured to test whether specific zonal isolation is the primary key. Existing producers with massive hydraulic fracture treatments will be converted to injectors to test whether the hydraulic fractures hinder or aid sweep efficiency. An injection pattern, which is adjacent to, and on-trend with a section containing a majority of plugged wells will be dedicated to investigating whether there is still mobile oil in the vicinity of old, abandoned wells and whether this oil can be swept and captured in current producing wells. A comprehensive economic analysis will be provided to identify the preferred management practices and to transfer the information to all Spraberry operators so that other operators can initiate water injection based on the results of the Spraberry Shackelford Unit Field Demonstration. 


\section{PROJECT SUMMARY \\ Background:}

Regions with greatest potential - the naturally fractured Spraberry Trend Area is one of the largest reservoirs in the domestic U.S. and is the largest reservoir in area extent in the world. Production from Spraberry sands is found over a 2,500 sq. mile area and Spraberry reservoirs can be found in an eight county area in west Texas. Over 150 operators produce 65,000 barrels of oil per day (bopd) from the Spraberry Trend Area from more than 9,000 production wells. Recovery is poor, on the order of $7-10 \%$ due to the profoundly complicated nature of the reservoir, yet billions of barrels of hydrocarbons remain. We estimate over $15 \%$ of remaining reserves in domestic Class III reservoirs are in Spraberry Trend Area reservoirs. This tremendous domestic asset is a prime example of an endangered hydrocarbon resource in need of immediate technological advancements before thousands of wells are permanently abandoned.

Integrate solutions to technological, regulatory and data constraints - the technological and data constraints have resulted in a general lack of confidence for water injection in the Spraberry Trend. Regional variations in geology combined with highly permeable, stress-sensitive fractures and very low matrix permeability create intensely difficult technical challenges. The fact that several waterflood projects over the course of 40 years have failed to provide an adequate and definitive answer regarding the technical and economic feasibility of waterflooding is a testament to technological and data constraints. Simply by the magnitude of the number of wells, management practices are of paramount importance when optimizing water injection in the Spraberry Trend Area. Many companies operate wells outside the Spraberry Units and several zones are typically commingled. Regulatory and data acquisition constraints are a serious issue and pose a great challenge for waterflood operations in Spraberry reservoirs. Proper reservoir engineering in a reservoir that is so large and communicates, via the fractures, over great distances poses a complicated technological and data management constraint. Reservoir engineering, by definition, requires precise injection, production and pressure data. Acquisition and control of this data has always been a constraint to providing the optimum method for water injection. The result is large volumes of oil that could have been recovered via water injection that remain untapped. We believe we have reached a fundamental understanding of Spraberry reservoirs. Individual Spraberry wells will never produce large volumes of oil, however, if past constraints, barriers to production and a general lack of confidence in waterflooding can be overcome, even modest improvements in well productivity multiplied over such a vast area would result in rapid increase in production.

Field demonstration - a low risk, high potential demonstration of technological innovations will be completed within two years. A waterflood demonstration is proposed by the Harold Vance Department of Petroleum Engineering in the Spraberry Shackelford Unit with Pioneer Natural Resources as the operator and Exxon/Mobil as supporting owner. This field demonstration will be carefully monitored and may result in a rapid increase in Spraberry production.

\section{ACCOMPLISMENTS:}

Task 1.0 Shackelford and Germania Unit Historical Review

- Reconstruction of Shackelford and Germania Injection/Production Data

- Development of production and database using Oil Field Manager (OFM)

- Development of field management software (FMS)

- Review well bore status in Shackelford Unit

Task 2.0 Review Midkiff Pilot

- Review of Upper and Lower Pilots in the Spraberry Area

Task 3.0 Develop production and database using OFM and Field Data Management software

Task 4.0 Development of optimum injection well patterns based on simulation

- Improving Waterflood and CO2 Pilot Performance in the Naturally Fractured Spraberry Trend Area, West Texas 
Task 5 Refine sub-surface maps for $1 \mathrm{U}$ and $5 \mathrm{U}$ oil saturated intervals

- Germania Unit Characterization using an Analog Field and Old Cased Hole Neutron

Task 6 Field demonstration

- Modify strategy based on response and development of expansion plans

- Germania Unit rate forecasting based on other waterflood pilots in the Spraberry Area

- Evaluation of E.T O’Daniel Pilot

- Evaluation of Waterflooding Performance in Germania Spraberry Unit

- Analysis of Waterflood performance on daily basis

Task 7 History match and verification of simulation results

- Improving Waterflood Performance in the Naturally Fractured Spraberry Trend Area

\section{SCHEDULED MILESTONES:}

\begin{tabular}{|c|c|c|c|c|}
\hline & \multicolumn{4}{|c|}{ Time (months) } \\
\hline & 6 & 12 & 18 & 24 \\
\hline $\begin{array}{l}\text { Task 1. Shackelford and Germania Unit Historical } \\
\text { Review }\end{array}$ & 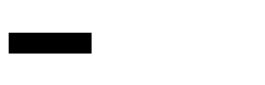 & & & \\
\hline Task 2. Review Midkiff Pilot & 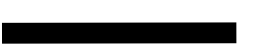 & & & \\
\hline $\begin{array}{l}\text { Task 3. Develop production and database using } \\
\text { OFM and Field Data Management } \\
\text { software* }\end{array}$ & & & & \\
\hline $\begin{array}{l}\text { Task 4. Development of optimum injection well } \\
\text { patterns based on simulation }\end{array}$ & & & & \\
\hline $\begin{array}{l}\text { Task 5. Refine sub-surface maps for } 1 \mathrm{U} \text { and } 5 \mathrm{U} \text { oil } \\
\text { saturated interval }\end{array}$ & & & & \\
\hline Task 6. Field demonstration & & & & \\
\hline $\begin{array}{l}\text { Task 7. History match and verification of } \\
\text { simulation results }\end{array}$ & & & & \\
\hline Task 8. Technology Transfer & & & & \\
\hline
\end{tabular}

* Software developed during this project

Accomplished Milestones

Proposed Milestones

\section{REPORTS:}

1. Putra, E. and Schechter, D.S.: "Review of Upper and Lower Pilots in The Spraberry Area," report included in "Preferred Waterflood Management Practices for the Spraberry Trend Area - PUMP” Semi-Annual Report (DOE Contract No.: DE-FC26-01BC15274), Sept 1, 2001- March 1, 2002.

2. Putra, E., and Schechter, D.S.: “ Germania Unit Rate Forecasting Based on Other Waterflood Pilots," report included in "Preferred Waterflood Management Practices for the Spraberry Trend Area - PUMP" First Annual Report (DOE Contract No.: DE-FC26-01BC15274), April 1- Sept 31, 2002.

3. Lakshman, G., Putra, E., and Schechter, D.S.: “Evaluation of Current E.T O’Daniel CO2 Pilot,” report included in "Preferred Waterflood Management Practices for the Spraberry Trend Area - PUMP” First Annual Report (DOE Contract No.: DE-FC26-01BC15274), April 1- Sept 31, 2002. 
4. Olumide, B.A.: "Germania Unit Characterization using an Analog Field and Old Cased Hole Neutron," report included in "Preferred Waterflood Management Practices for the Spraberry Trend Area - PUMP” Semi-Annual Report (DOE Contract No.: DE-FC26-01BC15274), April 1 - Sept 31, 2003.

5. Hernandez, E.: "Evaluation of Waterflooding Performance in Germania Spraberry Unit," report included in "Preferred Waterflood Management Practices for the Spraberry Trend Area - PUMP" Semi-Annual Report (DOE Contract No.: DE-FC26-01BC15274), Oct. 1, 2003 - March 31, 2004.

\section{TECHNOLOGY TRANSFER ACTIVITIES: Presentations}

On February 17, 2004, we presented "Waterflood and $\mathrm{CO}_{2}$ Performance in the Naturally Fractured Spraberry Trend Area,” presented at SPE Gulf Coast Section, February 17, 2004.

On October 6, 2003, we (Galaviz, J.) presented "Low-Rate Water Injection Enhances Recovery In The Naturally Fractured Spraberry Trend Area” at the international student paper contest at 2003 SPE Annual Technical Conference, CO. Mr. Galaviz won the first price.

On September 18, 2003, we presented the talk "Waterflood and $\mathrm{CO}_{2}$ performance in the Naturally Fractured Spraberry Trend Area," at the Statoil Research Summit 2003, Trondheim, Norway.

On June 2003, we presented the Short Course for Saudi Aramco in Al Khobar, Saudi Arabia - "Reservoir Characterization, Engineering and Enhanced Oil Recovery in Naturally Fractured Reservoirs.”

On April 2003, we presented "Fracture Characterization and Pilot Performance in the E.T. O’Daniel Unit Spraberry Trend Area, West Texas,” at the University of Texas at Austin invited lecture for Society of Petroleum Engineering Chapter and Graduate Seminar.

On March 2003, we presented the Short Course for UNAM/PEMEX in Mexico City, Mexico - "Reservoir Characterization and Engineering in Naturally Fractured Gas and Oil Reservoirs - Part II.”

On June 13, 2002, we presented the "Imbibition and its Relevance to Waterflood Performance in the Naturally Fractured Spraberry Trend Area," at the Rice University and University of Houston invited lecture for Society of Petroleum Engineering Chapter, Duncan Hall, Rice University.

On October 2001, we presented the Short Course for UNAM/PEMEX (National Petroleum Company of Mexico) in Mexico City, Mexico - "Reservoir Characterization and Engineering in Naturally Fractured Gas and Oil Reservoirs - Part I."

On February 2001, we presented the Short Course for for UNAM/PEMEX in Mexico City, Mexico - "Reservoir Characterization and Engineering in Naturally Fractured Gas and Oil Reservoirs - Part I.”

\section{Papers and Publications}

1. Schechter, D.S., Putra, E., Baker, R.O., Knight, W.H., McDonald, W.P., Leonard, P., and Rounding, C.: "CO Pilot Design and Water Injection Performance in the Naturally Fractured Spraberry Trend Area, West Texas," paper SPE 71605 presented at the 2001 Annual Technical Conference and Exhibition, New Orleans, LA, September 30-October 3.

2. Baker, R.O., Bora, R., Schechter, D.S., McDonald, P., Knight, W.H., Leonard, P., and Rounding, C.: "Development of a Fracture Model for Spraberry Field, Texas USA, ” paper SPE 71635 presented at the 2001 Annual Technical Conference and Exhibition, New Orleans, LA, September 30-October 3. 
3. Schechter, D.S., Putra, E., Knight, W.H., Leonard, P., and Baker, R.O.: "Improving Waterflood and CO2 Pilot Performance in the Naturally Fractured Spraberry Trend Area, West Texas,” paper presented at the 2002 Conference on Naturally Fractured Reservoirs, Oklahoma, June 3-4.

4. Chowdhury, T., Dabiri, G., Putra, E., and Schechter, D.S.: "Improving Waterflood Performance in the Naturally Fractured Spraberry Trend Area," paper presented at the 2002 Conference on Naturally Fractured Reservoirs, Oklahoma, June 3-4.

5. Alfred, D., Putra, E., and Schechter, D.S.: "Transcending Conventional Log Interpretation - A More Effective Approach for Spraberry Reservoir,” paper presented at the 2002 Conference on Naturally Fractured Reservoirs, Oklahoma, June 3-4.

6. Galaviz, J., Schechter, D.S. and Putra, E.: "Low-Rate Water Injection Enhances Recovery in the Naturally Fractured Spraberry Trend Area,” paper SPE presented at 2003 International Student Paper Contest, Denver, Colorado, 6-8 October.

\section{Internet Postings on the Project and Software to Download}

A description of our research group can be found at the following Petroleum Engineering Texas A\&M Website: http://pumpjack.tamu.edu/faculty/schechter/baervan/homepage.html. The site lists the publications of our group and allows downloads of several papers, reports, and presentations.

\section{CONTACT INFORMATION:}

NAME: David Schechter

PHONE: 979/ 845-2275

FAX: 979/845-1307

E-MAIL: schech@spindletop.tamu.edu

\section{DIGITAL PICTURES:}

